\title{
Synthesis and Characterizations of Linear and Ladder
}

\section{Conjugated Polymers}

\author{
By \\ Sukanta Kumar Saha

\begin{abstract}
A thesis submitted to
the Faculty of Graduate Studies and Postdoctoral Affairs

in Partial Fulfillment of the Requirements for the Degree of

Doctor of Philosophy

in

Chemistry
\end{abstract} \\ Carleton University \\ Ottawa, ON, Canada
}

August 2017

(C) Copyright, Sukanta Kumar Saha, Ottawa, ON, Canada, 2017 


\section{Abstract}

Semiconducting polymers have gained tremendous attention in the past decades as a potential alternative over Si-based semiconductors. The research on tuning the band gap of semiconducting polymers has mainly focused on the development of new electron donor and acceptor building blocks. Post-polymerization such as annulations and modification of functional groups is considered as an effective approach to the band-gap tuning for semiconducting polymers.

The first part of this thesis describes the synthesis and characterization of ladder and linear-ladder polymers by post-polymerization annulations. 8-Octylnaphtho[2,3c]thiophene-4,9-dione (NTDO) having the two keto groups was incorporated into conjugated linear polymers, which were then treated with Lawesson's Reagent (LR) for post-polymerization annulation. The obtained ladder and linear-ladder polymers had a lower energy band gap and absorption at a longer wavelength in comparison with their precursor polymers. The linear-ladder polymers containing of the electron-donating thiophene units and electron-withdrawing benzothiadiazole units had the absorption onset in the NIR region, e.g., at $1300 \mathrm{~nm}$ and $1000 \mathrm{~nm}$, respectively.

The second part discusses chemical transformations of the NTDO-containing conjugated polymers. The transformation by sequential reduction, dehydration, and esterification on the NTDO homopolymer was successful. The energy band gap decreased, absorption maxima were red-shifted and the absorption spectrum was broadened after chemical transformation. The transformation of the keto group of NTDObased D-A polymers to the corresponding dicyanovinylene group unexpectedly resulted in an increase of the LUMO energy level and therefore an increase in energy band gap. In 
the third part of this thesis, the building block of thieno[3,4-c]pyrrole-4,6-dione (TPD) was incorporated into conjugated linear polymers. Treatment of TPD-containing polymers with LR led to conversion of the keto group to thio-keto instead of annulation by intramolecular coupling. The obtained polymer displayed a hypsochromic shift and a higher energy band gap.

In the final part of this thesis, fluorene-based conjugated polymers containing the AIE-active tetraphenylethene and dicarboxylate pseudocrown as an ion receptor were synthesized and the optical sensing for lead ion was investigated. The study showed that polymer structure, solvent polarity, and nature of probe-analyte complex play collective roles in the dual-mode fluorescence detection for lead ion. 


\section{Acknowledgements}

I would like to offer my regards and profound gratitude to my supervisor Professor Zhi Yuan (Wayne) Wang for selecting the topics and his guidance, encouragement, and suggestions given to me throughout my research work. I also appreciate him for his support during the tough period of my study and research.

I am delighted to get this opportunity for expressing my praise to Dr. Jane Gao for her assistance on various problems during the research period.

I want to thank the past and present members in Dr. Wang's group: Wen Hui Hao, Saif Mia, Di Zhang, Songqiu Zhou and Khama Rani Ghosh for miscellaneous experiences during the research period. Special thanks to Monica Anne Gill for proofreading of my dissertation. I would like to say thanks to Jim Logan for fixing different instruments in our lab and fixing my laptop as well as Tony O’Neil, Keith Bourque for supporting of instrumental technique. A special thanks to Peter Mosher and Mastaneh Azad for giving me better schedule for TA.

I want to express my thanks to my parents who raised me. I want to praise and many thanks to my wife Khama Rani Ghosh for countless support, and motivation during my study and research work. My sweet son, Sreekanta Saha whose smiling and happy face always inspires me and removes all fatigue at the end of the day. Lastly, I want to say thanks to all the members in the department of chemistry, Carleton University for a nice and friendly environment. 


\section{Table of Contents}

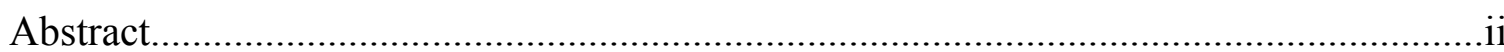

Acknowledgement...............................................................................................

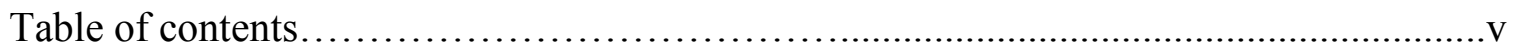

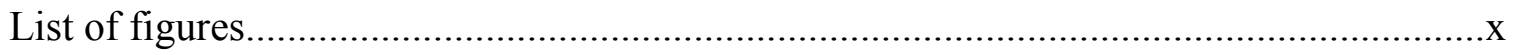

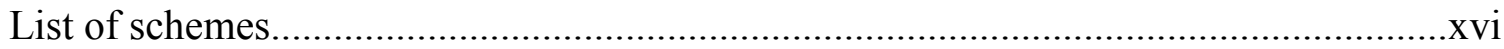

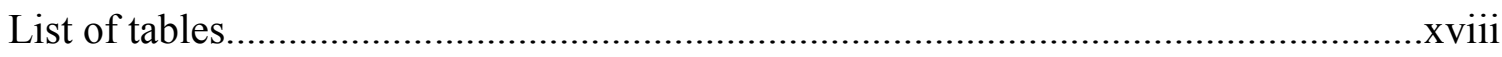

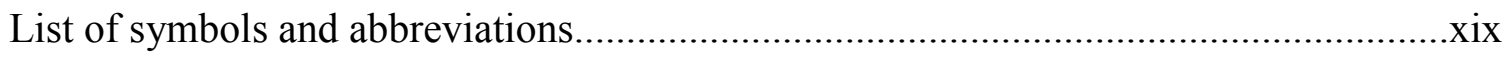

\section{Chapter 1 Introduction}

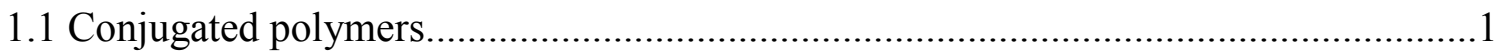

1.2 Band gap tuning in conjugated polymers......................................

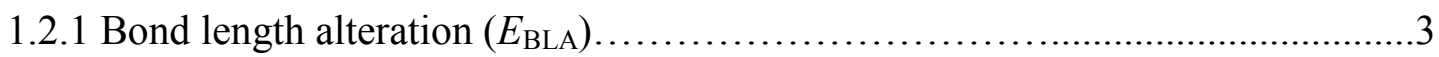

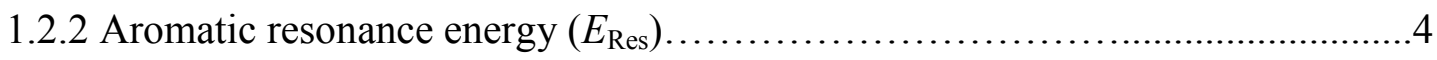

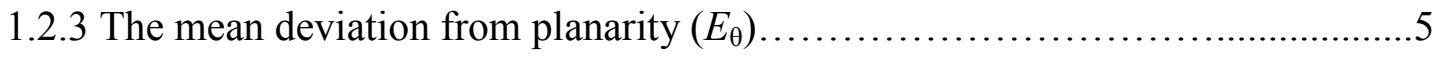

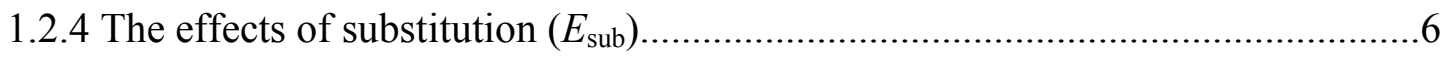

1.2.5 Intermolecular or interchain coupling in the solid state $\left(E_{\text {int }}\right) \ldots \ldots \ldots \ldots \ldots \ldots \ldots \ldots . .7$

1.3 Molecular engineering for low band gap polymers.........................................

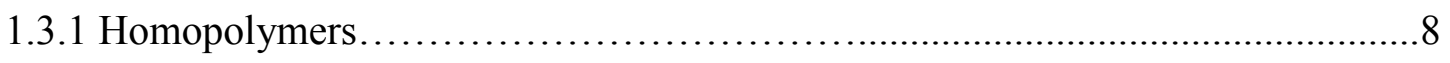

1.3.2 Ladder polymers.......................................................................... 12

1.3.3 Donor-acceptor polymers and energy band gaps.............................. 14

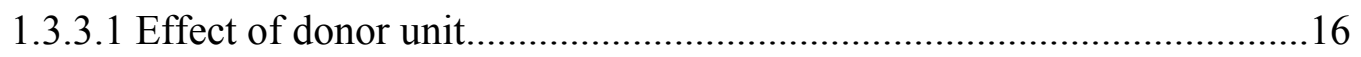

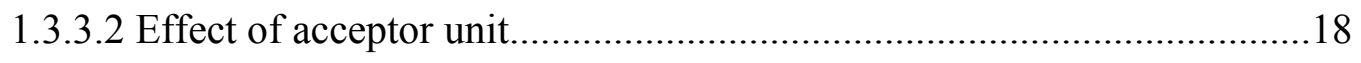

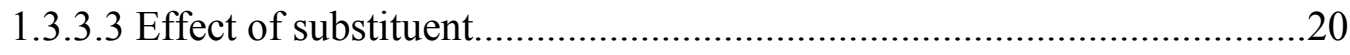

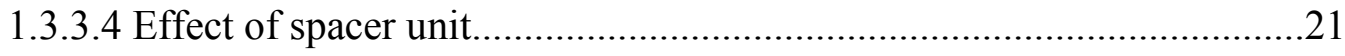


1.3.3.5 Side chain and position effect.

1.3.4 Band gap tuning by addition of tetracyanoethylene and

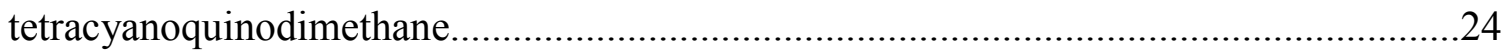

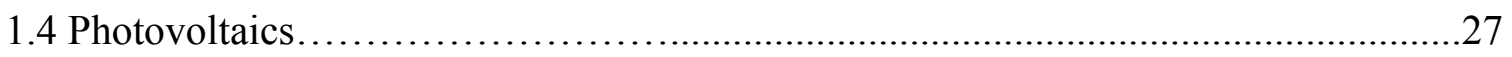

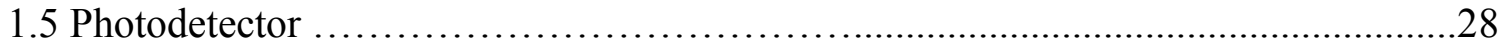

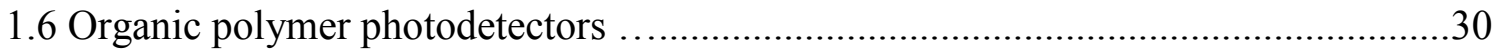

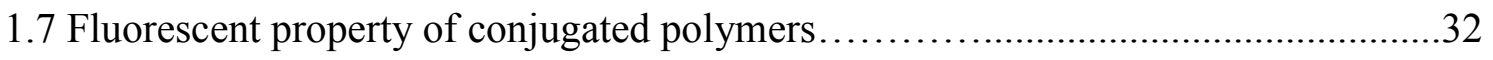

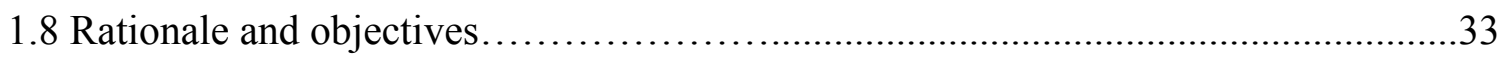

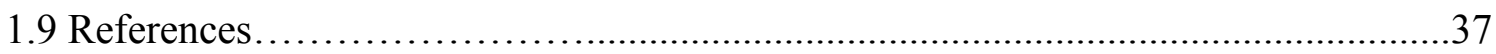

\section{Chapter 2 Design, Synthesis and Characterization of Conjugated}

\section{Ladder Polymers}

2.1 Introduction

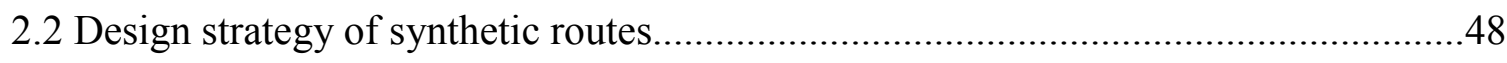

2.3 Synthesis and characterization of 1,3-dibromo-6-octylnaphtho[2,3-c]thiophene-4,9-

dione.

2.4 Synthesis and characterization of ladder polymer.

2.5 Attempted synthesis of ladder polymer by intramolecular McMurry reaction of polymer P1 62

2.6 Attempted synthesis of ladder polymer from a precursor polymer by intramolecular Ullmann reaction. .66

2.7 Linear and linear-ladder conjugated copolymers. 70

2.8 Synthesis and characterization of linear-ladder copolymers containing a thiophene linkage. 
2.9 Synthesis and characterization of linear-ladder copolymers containing a benzothiadiazole linkage.

2.10 Investigation of possible structures of polymers obtained from post-polymerization

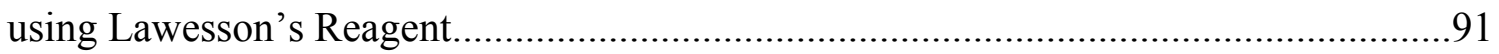

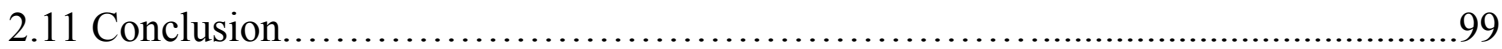

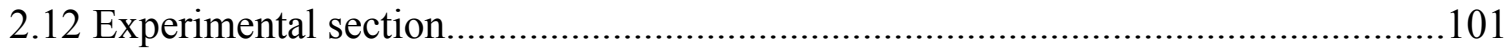

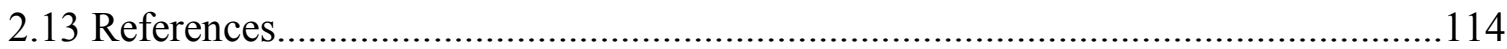

\section{Chapter 3 Syntheses and Transformations of NTDO-Based Polymers}

3.1 Introduction 119

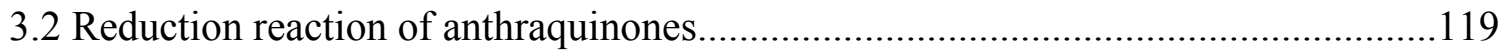

3.3 Synthesis and characterizations of polymers P13-16 ...........................................120

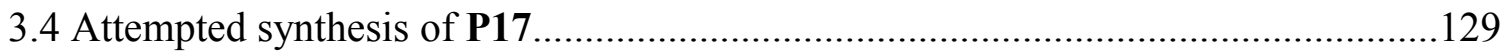

3.5 Synthesis and transformation of NTDO-based copolymers.....................................134

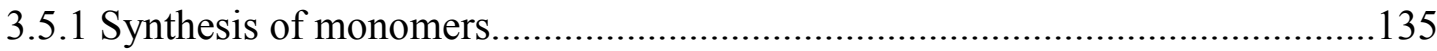

3.5.2 Synthesis and characterization of NTDO-based copolymers...........................136

3.5.3 Synthesis and characterizations of dicyanovinylene-containing polymers by chemical transformation of NTDO-based copolymers..............................................146

3.6 Polymer photodetectors based on dicyanovinylene-containing polymers P22-

P25. .153

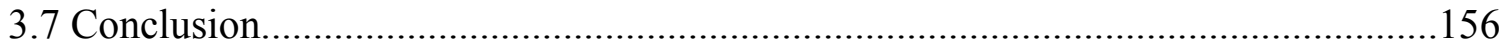

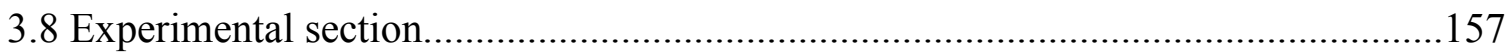

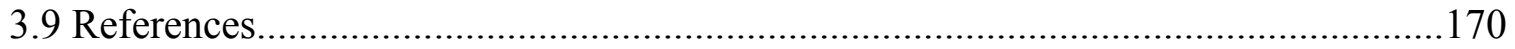




\section{Chapter 4 Synthesis and Characterizations of TPD-Based Compounds and Conjugated Polymers}

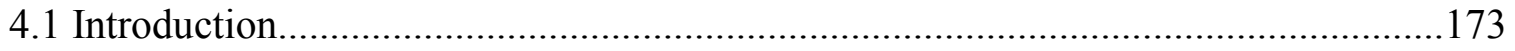

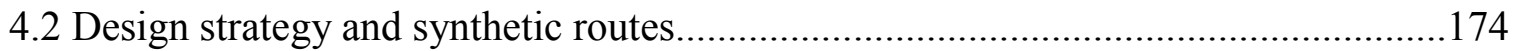

4.3 Synthesis and characterizations of monomer $\mathbf{M 7}$ and homopolymer of TPD-

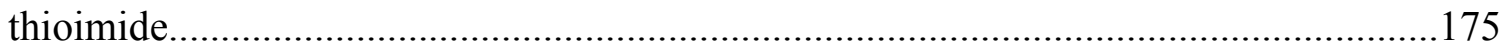

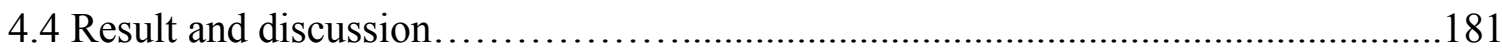

4.5 Synthesis and characterizations of thieno[3,4-c]pyrrole-4,6-dione (TPD)-based

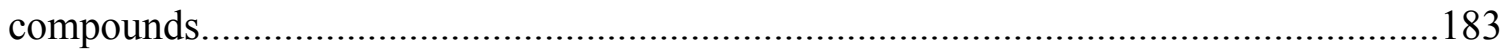

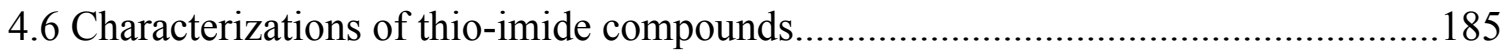

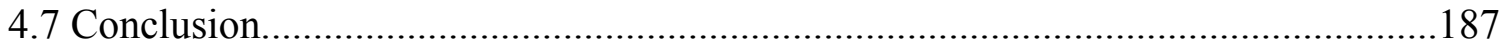

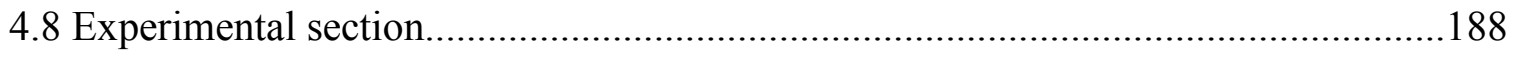

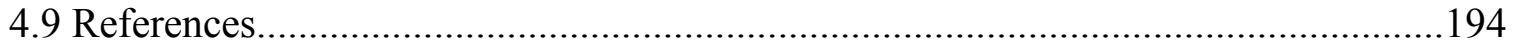

Chapter 5 Highly Sensitive Dual-Mode Fluorescence Detection of Lead Ion in Water Using Aggregation-Induced Emissive Polymers

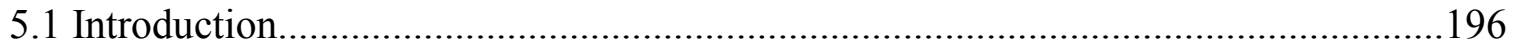

5.1.1 Overview of aggregation induced emission, conjugated polymer and design

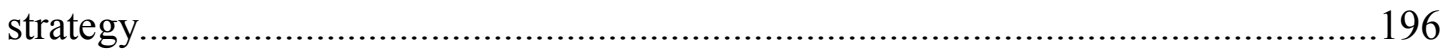

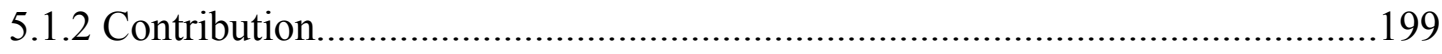

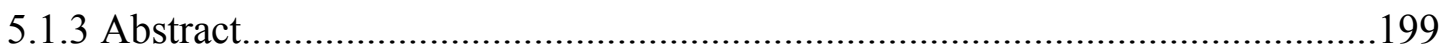

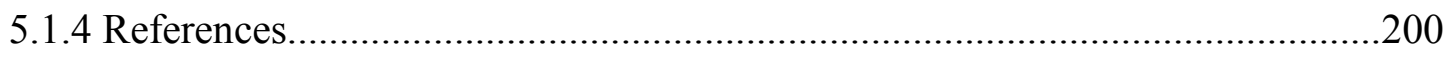

5.2 Article 


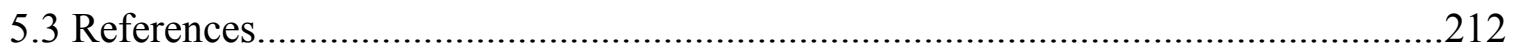

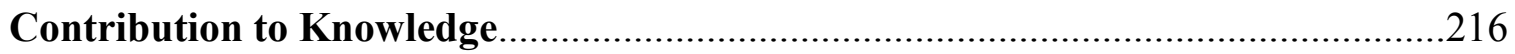

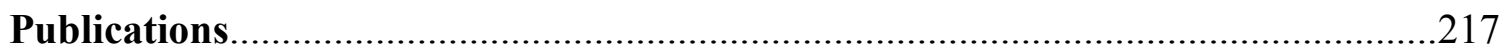

Appendices

Appendix A: Spectra of synthesized compounds and polymers in chapter $2 \ldots \ldots \ldots \ldots \ldots \ldots . . .219$

Appendix B: Spectra of synthesized compounds, polymers and device properties in

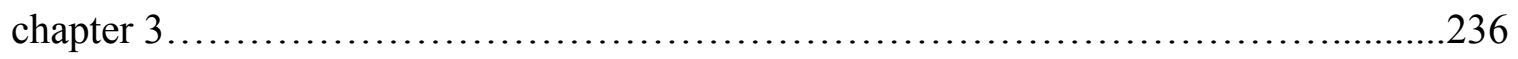

Appendix C: Spectra of synthesized compounds and polymers in chapter $4 \ldots \ldots \ldots \ldots \ldots \ldots . . .255$

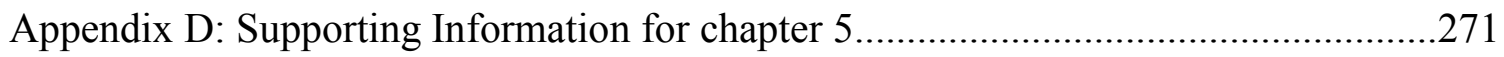




\section{List of Figures}

Figure 1.1 Structural factors affect the energy band gap of semiconducting polymers.......3

Figure 1.2 Aromatic and quinoid form of conjugated polymers..................................4

Figure 1.3 Effect of dihedral angle in P3HT.......................................................

Figure 1.4 Orientation of conjugated polymer in solid state........................................

Figure 1.5 Structures of polythiophenes, polyisothianaphthene and resonance structures..9

Figure 1.6 Structures of thiophene-based p-type homopolymers..................................10

Figure 1.7 Structures of acceptor and donor type homopolymers...............................11

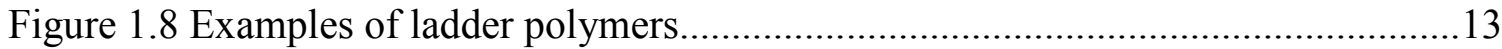

Figure 1.9 Structures of polysquaraines and polycroconaines.................................... 14

Figure 1.10 Molecular orbital of typical donor-acceptor polymer..................................16

Figure 1.11 Structures of various fused heterocyclic donor units...............................18

Figure 1.12 Structures of some strong acceptor units..............................................19

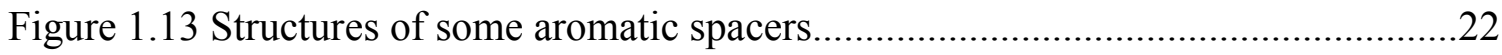

Figure 1.14 Structures of some polymers with side chains at different positions............24

Figure 1.15 Structures of $\mathrm{CN}$-based acceptors and TTF...............................24

Figure 1.16 Polymers before and after addition of TCNE and TCNQ.........................26

Figure 1.17 Structures of donor polymers used in BHJ photodetectors..........................32

Figure 1.18 Structures of TPE-based conjugated polymers........................................33

Figure 2.1 IR spectra of polymers $\mathbf{P 1}$ (a) and $\mathbf{P 2}$ (b) (on $\mathrm{NaCl}$ plate)...........................55

Figure $2.2{ }^{13} \mathrm{C}$ NMR (300 MHz, $\mathrm{CDCl}_{3}$ ) spectra for polymers $\mathbf{P 1}$ (a) and $\mathbf{P 2}$ (b)..........56

Figure 2.3 Absorption spectra of M1 and polymers P1 $\left(7.04 \times 10^{-5} \mathrm{M}\right)$ and P2 $\left(1.08 \times 10^{-}\right.$

${ }^{4} \mathrm{M}$ ) in $\mathrm{CHCl}_{3}$ solution (a) and as thin films on glass substrates (b) ..............................58 
Figure 2.4 Cyclic voltammogram of polymer films of P1 (a) and P2 (b) on ITO in $\mathrm{CH}_{3} \mathrm{CN}$ solution containing $0.1 \mathrm{~mol} / \mathrm{L}$ of $\mathrm{Bu}_{4} \mathrm{NPF}_{6}$ with a scan rate of $100 \mathrm{mV} / \mathrm{s}$

Figure 2.5 (a) IR (on $\mathrm{NaCl}$ plate) and (b) ${ }^{13} \mathrm{C}$ NMR $\left(75 \mathrm{MHz}, \mathrm{CDCl}_{3}\right.$ ) spectra of $\mathbf{P 2}$ obtained by McMurry reaction .64

Figure 2.6 Absorption spectra of polymer $\mathbf{P 2}$ obtained by $\mathrm{McMurry}$ in $\mathrm{CHCl}_{3}$ solution and as a thin film on a glass substrate. .64

Figure 2.7 Cyclic voltammogram of polymer film on ITO in $\mathrm{CH}_{3} \mathrm{CN}$ solution containing $0.1 \mathrm{~mol} / \mathrm{L} \mathrm{Bu}_{4} \mathrm{NPF}_{6}$, with a scan rate of $100 \mathrm{mV} / \mathrm{s}$. .65

Figure 2.8 (a) IR (on $\mathrm{NaCl}$ plate) and (b) ${ }^{13} \mathrm{C} \mathrm{NMR}\left(75 \mathrm{MHz}, \mathrm{CDCl}_{3}\right.$ ) spectra of polymer P3. .67

Figure 2.9 Absorption spectra of polymer $\mathbf{P 3}$ in $\mathrm{CHCl}_{3}$ solution and as thin film on a glass substrate (a) and cyclic voltammogram of the polymer film on ITO in $\mathrm{CH}_{3} \mathrm{CN}$ solution containing in $0.1 \mathrm{~mol} / \mathrm{L} \mathrm{Bu}_{4} \mathrm{NPF}_{6}$ with a scan rate of $100 \mathrm{mV} / \mathrm{s}$ (b) .68

Figure 2.10 Absorption spectrum of polymer $\mathbf{P 2}$ in $\mathrm{CHCl}_{3}$ solution.....................70 Figure 2.11 IR spectra of copolymers (a) P4, (b) P6, (c) P5 and (d) P7 where P4 and P5

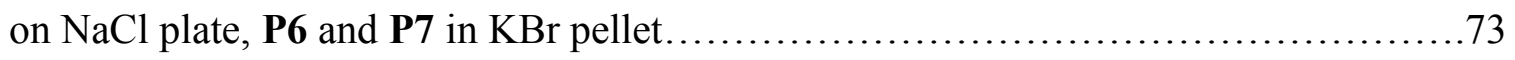
Figure 2.12 Absorption spectra of polymers P4 and P5 in TCB (a) and thin films on glass

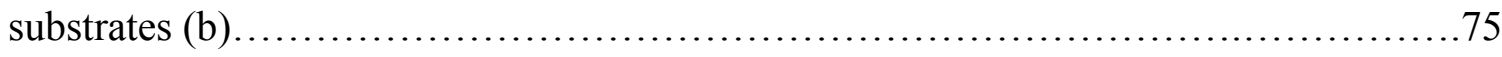

Figure 2.13 Absorption spectra of polymers P6 (TCB) and P7 (TCE) (a) and thin films on

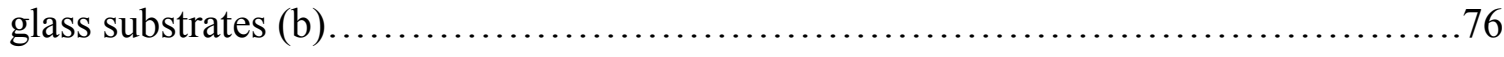

Figure 2.14 Absorption spectra of polymer P7 films in different thicknesses on quartz surface. .77 
Figure 2.15 Cyclic voltammogram of polymers P4 (a), P5 (b), P6 (c) and P7 (d) film on ITO in $\mathrm{CH}_{3} \mathrm{CN}$ solution containing $0.1 \mathrm{~mol} / \mathrm{L} \mathrm{Bu}_{4} \mathrm{NPF}_{6}$ with a scan rate of 100 $\mathrm{mV} / \mathrm{s}$. .79

Figure 2.16 IR spectra of polymers P8 (a), P9 (b), P10 (c), and P11 (d) in $\mathrm{KBr}$ pellet. .83

Figure $2.17{ }^{13} \mathrm{C}$ NMR spectra $\left(75 \mathrm{MHz}, \mathrm{CDCl}_{3}\right)$ for copolymers $\mathbf{P 1}$ (a), P8 (b), and P9 (c). .84

Figure 2.18 Absorption spectra of polymers $\mathbf{P 1}, \mathbf{P 8}$ and $\mathbf{P 9}$ in $\mathrm{CHCl}_{3}$ (a) and on glass substrate (b). .85

Figure 2.19 Absorption spectra of polymers P10 and P11 (a) in solution (b) on a glass substrate .87

Figure 2.20 Cyclic voltammogram of polymers P8 (a), P9 (b) P10, (c) and P11(d) film on ITO in $\mathrm{CH}_{3} \mathrm{CN}$ solution containing $0.1 \mathrm{~mol} / \mathrm{L} \mathrm{Bu}_{4} \mathrm{NPF}_{6}$ with a scan rate of 100 $\mathrm{mV} / \mathrm{s}$. .89

Figure 2.21 IR spectra of polymers P1, P2 and P3 in $\mathrm{KBr}$ pellet..............................93

Figure 2.22 IR spectrum (in KBr pellet) of polymer $\mathbf{P 2}$ after esterification. .94

Figure 2.23 (a) IR (in $\mathrm{KBr}$ pellet) and (b) ${ }^{13} \mathrm{C} \mathrm{NMR} \mathrm{(75} \mathrm{MHz,} \mathrm{CDCl}_{3}$ ) spectra of polymer P12. 95

Figure 2.24 Absorption spectra of polymer P12 in solution and on glass substrate. .96

Figure 2.25 Cyclic voltammogram of polymer $\mathbf{P 1 2}$ film on ITO in $\mathrm{CH}_{3} \mathrm{CN}$ solution

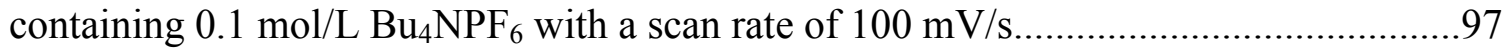

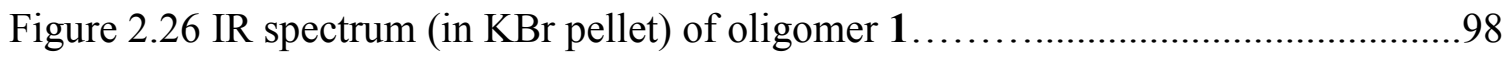

Figure 3.1 IR spectrum of polymer $\mathbf{P 1 3}$ on $\mathrm{NaCl}$ Plate.........................................121 
Figure 3.2 Absorption spectra of polymer $\mathbf{P 1 3}$ in $\mathrm{CHCl}_{3}$ solution and as thin film on a glass substrate (a) and cyclic voltammogram of film on ITO in $\mathrm{CH}_{3} \mathrm{CN}$ solution containing in $0.1 \mathrm{~mol} / \mathrm{L} \mathrm{Bu}_{4} \mathrm{NPF}_{6}$ with a scan rate of $100 \mathrm{mV} / \mathrm{s}(\mathrm{b}) \ldots \ldots \ldots \ldots \ldots \ldots \ldots \ldots \ldots \ldots \ldots \ldots \ldots . . .122$

Figure 3.3 IR spectrum of polymer $\mathbf{P 1 4}$ on $\mathrm{NaCl}$ plate. 123 Figure 3.4 Absorption spectra of polymer $\mathbf{P 1 4}$ in $\mathrm{CHCl}_{3}$ solution and as thin film on glass substrate (a) and cyclic voltammogram of film on ITO in $\mathrm{CH}_{3} \mathrm{CN}$ solution containing in $0.1 \mathrm{~mol} / \mathrm{L} \mathrm{Bu}_{4} \mathrm{NPF}_{6}$ with a scan rate of $100 \mathrm{mV} / \mathrm{s}(\mathrm{b})$. 124

Figure 3.5 IR spectrum of polymer P15 on NaCl Plate. 125

Figure 3.6 Absorption spectra of polymer $\mathbf{P 1 5}$ in $\mathrm{CHCl}_{3}$ solution and as thin film on a glass substrate (a) and cyclic voltammogram of film on ITO in $\mathrm{CH}_{3} \mathrm{CN}$ solution containing in $0.1 \mathrm{~mol} / \mathrm{L} \mathrm{Bu}_{4} \mathrm{NPF}_{6}$ with a scan rate of $100 \mathrm{mV} / \mathrm{s}(\mathrm{b}) \ldots \ldots \ldots \ldots \ldots \ldots \ldots \ldots . . . .126$

Figure 3.7 IR spectrum of polymer P16 on NaCl Plate 127

Figure 3.8 Absorption spectra of polymer $\mathbf{P 1 6}$ in $\mathrm{CHCl}_{3}$ solution and as thin film on a glass substrate (a) and cyclic voltammogram of film on ITO in $\mathrm{CH}_{3} \mathrm{CN}$ solution

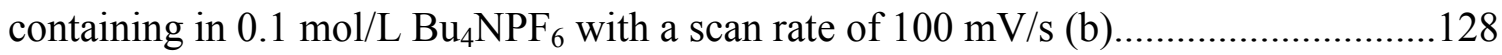

Figure 3.9 IR spectrum of polymer P17 on $\mathrm{NaCl}$ Plate.................................130

Figure 3.10 Absorption spectrum of polymer $\mathbf{P 1 7}$ in $\mathrm{CHCl}_{3}$ solution.........................130

Figure 3.11 IR spectrum of polymer P17 on $\mathrm{NaCl}$ Plate............................................131

Figure 3.12 Absorption spectra of polymer $\mathbf{P 1 7}$ in $\mathrm{CHCl}_{3}$ solution and as thin film on a glass substrate (a) and cyclic voltammogram of film on ITO in $\mathrm{CH}_{3} \mathrm{CN}$ solution containing in $0.1 \mathrm{~mol} / \mathrm{L} \mathrm{Bu}_{4} \mathrm{NPF}_{6}$ with a scan rate of $100 \mathrm{mV} / \mathrm{s}(\mathrm{b})$ 132

Figure 3.13 TGA trace of polymer P16 with a heating rate of $10{ }^{\circ} \mathrm{C} / \mathrm{min}$ under nitrogen atmosphere 133 
Figure 3.14 IR spectrum of polymer $\mathbf{P 1 7}$ in $\mathrm{KBr}$ pellet..... .134

Figure 3.15 IR spectra polymer $\mathbf{P 1 8}$ on $\mathrm{NaCl}$ plate .139

Figure 3.16 (a) ${ }^{1} \mathrm{H}$ NMR (300 MHz, $\mathrm{CDCl}_{3}$ and (b) ${ }^{13} \mathrm{C} \mathrm{NMR}\left(75 \mathrm{MHz}, \mathrm{CDCl}_{3}\right)$ Spectra of polymer P20. 140

Figure 3.17 Absorption of polymers P18 $\left(3.77 \times 10^{-5} \mathrm{M}\right), \mathbf{P 1 9}\left(4.16 \times 10^{-5} \mathrm{M}\right), \mathbf{P 2 0}(3.32$ $\left.\times 10^{-5} \mathrm{M}\right)$ and $\mathbf{P 2 1}\left(4.17 \times 10^{-5} \mathrm{M}\right)$ in $\mathrm{CHC}_{3}$ solution (a) and as thin films on glass substrates (b) 142

Figure 3.18 Cyclic voltammogram of polymers (a) P18, (b) P19, (c) P20 and (d) P21 film on ITO in $\mathrm{CH}_{3} \mathrm{CN}$ solution containing $0.1 \mathrm{~mol} / \mathrm{L} \mathrm{Bu} \mathrm{NPF}_{6}$ with a scan rate of 100 $\mathrm{mV} / \mathrm{s}$ 144

Figure 3.19 IR spectrum of polymer $\mathbf{P 2 2}$ in $\mathrm{KBr}$ pellet...... .148

Figure 3.20 Absorption spectra of polymers P22 $\left(4.30 \times 10^{-5} \mathrm{M}\right), \mathbf{P 2 3}\left(4.15 \times 10^{-5} \mathrm{M}\right)$, P24 $\left(5.09 \times 10^{-5} \mathrm{M}\right)$ and P25 $\left(5.62 \times 10^{-5} \mathrm{M}\right)$ in NMP solution (a) and as thin film on glass substrate (b) .150

Figure 3.21 Cyclic voltammogram of polymers (a) P22, (b) P23 (c) P24 and (d) P25 film on ITO in $\mathrm{CH}_{3} \mathrm{CN}$ solution containing $0.1 \mathrm{~mol} / \mathrm{L}$ of $\mathrm{Bu}_{4} \mathrm{NPF}_{6}$ with a scan rate of 100 $\mathrm{mV} / \mathrm{s}$ .151

Figure 3.22 Device structure of bulk-heterojunction photodetector. 153

Figure 3.23 EQE, responsivity and detectivity of the devices based on polymers P22P25. 155

Figure 4.1 IR, ${ }^{1} \mathrm{H}$ NMR and ${ }^{13} \mathrm{C}$ NMR spectra of polymers P26 (a) and P27 (b). .178 
Figure 4.2 TGA plots of the polymers P26 and P27 with a heating rate of $10{ }^{\circ} \mathrm{C} / \mathrm{min}$ under nitrogen atmosphere. 178

Figure 4.3 Absorption spectra of polymers P26 $\left(3.03 \times 10^{-6} \mathrm{M}\right)$ and P27 $\left(3.23 \times 10^{-6} \mathrm{M}\right)$ in $\mathrm{CHCl}_{3}$ solution (a) and as thin films on glass substrate (b) ..................................179 Figure 4.4 Cyclic voltammograms of films on Pt electrode in $0.1 \mathrm{~mol} / \mathrm{L} \mathrm{Bu}_{4} \mathrm{NP}_{6}, \mathrm{CH}_{3} \mathrm{CN}$ solution with a scan rate of $100 \mathrm{mV} / \mathrm{s}$. Full cycle of $\mathbf{P 2 6}$ (a) and reduction of P27 (b)..180 Figure 4.5 IR, ${ }^{1} \mathrm{H}$ NMR and ${ }^{13} \mathrm{CNMR}$ spectra of compounds $\mathbf{1 0}$ and $\mathbf{1 1} \ldots \ldots \ldots \ldots \ldots . . . . .186$ 


\section{List of Schemes}

Scheme 2.1 Designed synthetic routes to linear and ladder conjugated polymers............48

Scheme 2.2 Synthesis of 1,3-dibromo-6-octylnaphtho[2,3-c]thiophene-4,9-dione..........50

Scheme 2.3 Plausible mechanism for the formation of compound $2 \ldots \ldots \ldots \ldots \ldots \ldots \ldots \ldots \ldots \ldots . . . .50$

Scheme 2.4 Synthesis of homopolymer P1 and ladder polymer P2 ...........................51

Scheme 2.5 General mechanism of Yamamoto coupling reaction................................53

Scheme 2.6 Plausible mechanisms for thionation and cyclization reaction of diketo compound in the presence of Lawesson's Reagent.......................................................54

Scheme 2.7 Attempted synthesis of $\mathbf{P 2}$ by McMurry reaction.....................................63

Scheme 2.8 Attempted synthesis of $\mathbf{P 2}$ from $\mathbf{P 3}$ by intramolecular Ullmann reaction.....66

Scheme 2.9 Synthesis of linear-ladder copolymers P6 and P7 from linear copolymers P4

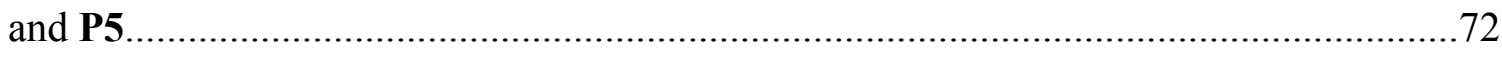

Scheme 2.10 Synthesis of linear-ladder copolymers P10 and P11 from linear copolymers

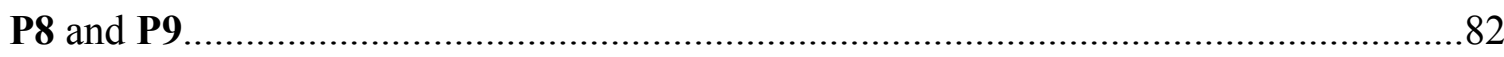

Scheme 2.11 Possible polymer structures for polymers $\mathbf{P 2}$ and P3...........................91

Scheme 2.12 Structures of anthracene containing polydisulfides and thioester...............92

Scheme 2.13 Reduction and esterification in polymer P2 .....................................94

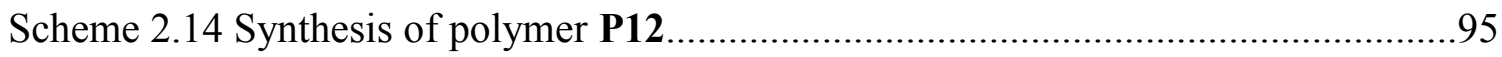

Scheme 2.15 Attempted synthesis of compound $\mathbf{3}$ or oligomer 1 ..............................98

Scheme 2.16 Possible structures for polymer P3/P12 ..............................................99

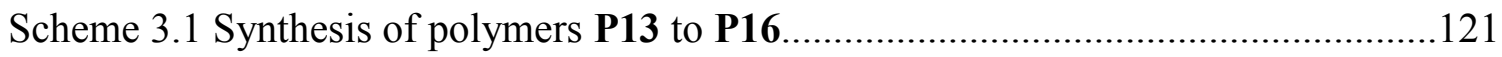

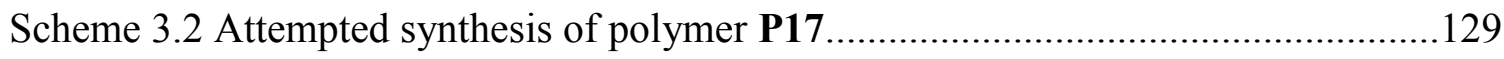

Scheme 3.3 Synthesis of monomers M4, M5 and M6.........................................136 
Scheme 3.4 Synthesis of NTDO-based copolymers P18-P21

Scheme 3.5 General reaction mechanism for palladium catalyst cross-coupling reaction. 138

Scheme 3.6 Synthesis of dicyanovinylene-containing polymers P22-P25 147

Scheme 4.1 Synthetic routes to linear and ladder conjugated polymers derived from TPD. 175

Scheme 4.2 Synthetic route to monomer M7 and homopolymers HTPD (P26) and HTTPD (P27). .176

Scheme 4.3 Synthesis of TPD-based model compounds. 184 


\section{List of Tables}

Table 1.1 Resonance energy and Resonance Energy per Electron..................................5

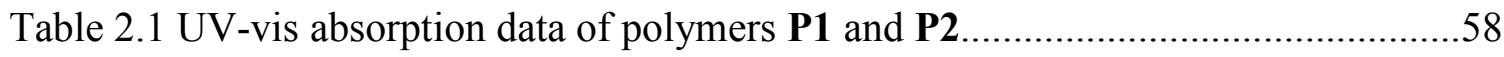

Table 2.2 Optical and electrochemical properties of polymers $\mathbf{P 1}$ and $\mathbf{P 2}$.....................62

Table 2.3 Optical and electrochemical properties of polymers P3.............................69

Table 2.4 Absorption data of polymers P4, P5, P6 and P7 ..................................... 76

Table 2.5. Optical and electrochemical properties of polymers P4-P7........................8 80

Table 2.6. UV-vis absorption of polymers P8, P9, P10 and P11 ..............................88

Table 2.7. Optical and electrochemical properties of polymers P8-P11 ........................90

Table 3.1. UV-vis-NIR absorption data of polymers P18, P19, P20 and P21..............142

Table 3.2. Optical and electrochemical properties of polymers P18-P21.....................145

Table 3.3. UV-vis absorption data for polymers P22, P23, P24 and P25............. 150

Table 3.4. Optical and electrochemical properties of polymers P22-P25....................153

Table 3.5. Summary of device performance based on polymers P22-P25..............156 


\section{List of Symbols and Abbreviations}

ACQ

$\mathrm{AcOH}$

AIE

BDT

BT

BHJ

BLA

CB

$\mathrm{CH}_{3} \mathrm{CN}$

CLPs

CPT

CV

D-A

DCB

DMF

DNT

DP

DPP

DTP

DSC

EDOT

FF
Aggregation-caused quenching

Acetic acid

Aggregation-induced emission

Benzodithiphene

2,1,3-Benzothiadiazole

Bulk heterojunction

Bond length alteration

Chlorobenzene

Acetonitrile

Conjugated ladder polymers

Cyclopentadithiophene

Cyclic voltammetry

Donor-Acceptor

1,2-Dichlorobenzene

$N, N$-Dimethylformamide

2,4-Dinitrotoluene

Dicarboxylate pseudocrown

Diketopyrrolopyrrole

Dithieno[3,2-b:2',3'-d]pyrrole

Differential scanning calorimetric

3,4-Ethylenedioxythiophene

Fill factor 


\begin{tabular}{ll} 
FL & Fluorene \\
FQR & Fluorescence Quenching Response \\
GPC & Gel permeation chromatography \\
HOMO & Highest occupied molecular orbital \\
ICT & Internal charge transfer \\
ITN & Isothianaphthene \\
LR & Lawesson's reagent \\
LUMO & Lowest unoccupied molecular orbital \\
NEP & Noise equivalent power \\
NIR & Near-infrared \\
NTDO & 8-Octylnaphtho[2,3-c]thiophene-4,9-dione \\
OFET & Organic field effect transistor \\
OPD & Organic photodetector \\
OPV & Organic photovoltaic \\
OSC & Organic solar cell \\
P3HT & Poly(3-hexylthiophenes) \\
PCBM & [6,6]-Phenyl-C $61 / 71$-butyric acid methyl ester \\
PCE & Power conversion efficiency \\
PET & Photo-induced electron transfer \\
PITN & Polyisothianaphthene \\
PDI & Polydispersity indices \\
Parts per billion \\
Resonance energy per $\pi$-electron \\
RE & Restrintramolecular rotation \\
\hline
\end{tabular}




\begin{tabular}{ll} 
TCB & $1,2,4-$ Trichlorobenzene \\
TCNE & Tetracyanoethylene \\
TCNQ & $7,7,8,8$-Tetracyanoquinodimethane \\
TLC & Thin layer chromatography \\
TGA & Thermogravimetric analysis \\
THF & Tetrahydrofuran \\
TNT & 2,4,6-Trinitrotoluene \\
TPD & Thieno[3,4-c]pyrrole-4,6-dione \\
TPE & Tetraphenylethene \\
TPT & Bis(2-thienyl)-N-alkyl-pyrrole \\
TT & Thieno[3,4-b]thiophene \\
UV-vis & Ultraviolet-visible \\
$E_{\mathrm{g}}$ & Band gap \\
$E_{\text {ox }}$ & Onset oxidation potential \\
$E_{\text {red }}$ & Onset reduction potential \\
$J_{\text {sc }}$ & Short-circuit current \\
$\lambda_{\text {max }}$ & Maximum absorption wavelength \\
$T_{\mathrm{d}}$ & Decomposition temperature \\
$V_{\text {oc }}$ & Open-circuit voltage \\
\hline
\end{tabular}




\section{Chapter 1 Introduction}

\subsection{Conjugated polymers}

In 1976, Alan Heeger, Alan MacDiarmid and Hideki Shirakawa together discovered the conducting polyacetylene which underwent 11 orders of magnitude increase in conductivity upon charge-transfer oxidative doping. ${ }^{1,2}$ In 2000 , the three scientists were rewarded the Nobel Prize in Chemistry for their discovery and for their work with conjugated polymers. These conjugated polymers are mainly carbon based and are joined by carbon-carbon alternating double and single bonds. In conjugated polymers, each carbon atom is $\mathrm{sp}^{2}$ hybridized and contain one unpaired electron in their $\mathrm{p}_{\mathrm{z}}$ orbital. These $\mathrm{p}_{z}$ orbitals of the carbon atoms along the backbone overlap, which leads to electron delocalization along the backbone. This electron delocalization provides the highway for charge mobility along the backbone of the polymer chain. The $\pi$ bonds are formed by the overlap of the $\mathrm{p}_{\mathrm{z}}$ orbitals that are divided into two bands, the $\pi$ and the $\pi^{*}$ band. The $\pi$ band is filled with electrons and is called the highest occupied molecular orbital (HOMO) while the $\pi^{*}$ band is empty and is called the lowest unoccupied molecular orbital (LUMO). The energy difference between these two bands is the energy gap $\left(E_{\mathrm{g}}\right)$. Since there are no partially filled bands, conjugated polymers behave as typical semiconductors. As the $E_{\mathrm{g}}$ depends on the molecular structure of the repeating units, there is an opportunity to tune the band gap by careful design. ${ }^{3}$ The typical energy band gap for organic semiconducting polymers is in the range of $1.0-3.0 \mathrm{eV}$, whereas insulating polymers have energy band gaps larger than $3.0 \mathrm{eV}{ }^{4}$ This energy band gap depends on the temperature and pressure, and starts to decrease with increasing temperature due to increased atomic vibrations. This implies that the band gap is a major factor in 
determination of the electrical conductivity of organic semiconducting polymers, and is of fundamental importance in the application of organic electronics. ${ }^{4 b}$ Conjugated polymers are used in light-emitting diodes, field-effect transistors, photodetectors, nonvolatile memory, batteries, supercapacitors, solar cells and thermoelectric generators. ${ }^{5}$ Conjugated polymers have very high absorption coefficients. As a result, a very thin layer of polymer film is sufficient to absorb the incident light. This property highlights that they are competitive as photovoltaics and photodetectors to their inorganic counterpart. The small band gap facilitates easy charge injection of electrons into the LUMO and/or holes into the HOMO, and the strong molecular polarity enables effective charge transport of both negative and positive charges. This property makes conjugated polymers promising materials for $\mathrm{p}$-type, $\mathrm{n}$-type and ambipolar field effect transistors. ${ }^{5 \mathrm{c}}$

\subsection{Band gap tuning in conjugated polymers}

The optoelectronic property of a conjugated polymer is highly executed by their energy band gap. The most critical challenge in developing ideal semiconducting polymers is to design and synthesize a conjugated polymer that simultaneously possess good film-forming properties, strong absorption abilities in the spectral range, high charge mobility, and suitable HOMO-LUMO energy levels. An effective tailoring of the polymer's energy band gap and molecular structure allow for control of the intrinsic properties of semiconducting polymers to serve the desired purpose and address the application needs. The band gap of semiconducting $\pi$-conjugated polymers is determined by five factors: 1) bond length alteration $\left.\left(E_{\mathrm{BLA}}\right), 2\right)$ the mean deviation from planarity $\left.\left(E_{\theta}\right), 3\right)$ aromatic resonance energy $\left.\left(E_{\mathrm{res}}\right), 4\right)$ the inductive or mesomeric electronic effects 
of eventual substitution ( $\left.E_{\text {sub }}\right)$ and 5 ) the intermolecular or interchain coupling in the solid state $\left(E_{\text {int }}\right)$. As a result, $E_{\mathrm{g}}$ of a $\pi$-conjugated system is expressed as: $E_{\mathrm{g}}=E_{\mathrm{BLA}}+E_{\text {res }}+$ $E_{\mathrm{sub}}+E_{\theta}+E_{\text {int }}$, as shown in Figure 1.1. ${ }^{6,7}$

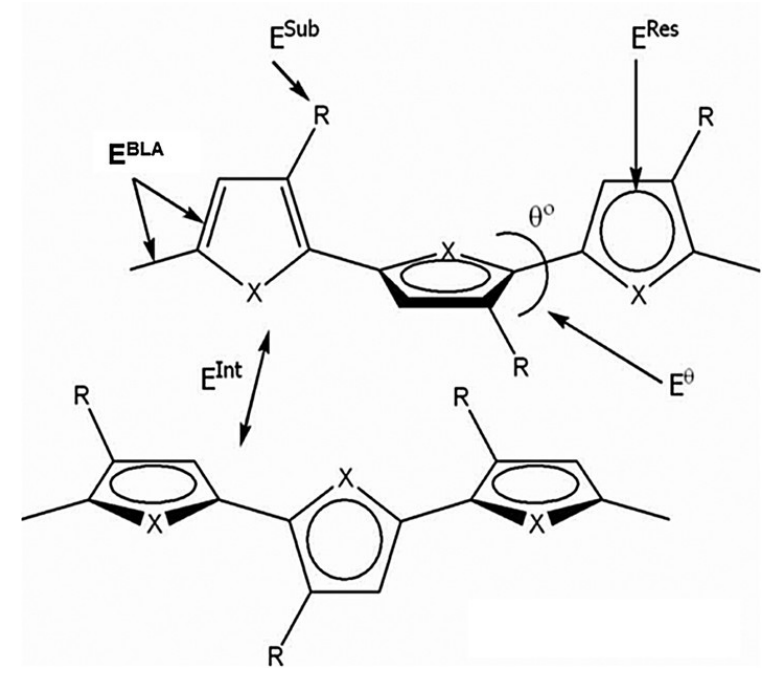

Figure 1.1 Structural factors affect the energy band gap of semiconducting polymers. ${ }^{7}$

\subsubsection{Bond length alteration $\left(E_{\mathrm{BLA}}\right)$}

BLA can be defined as the average of the difference in lengths between adjacent carbon-carbon bonds in a polyene chain. In polyaromatic conjugated systems, there are two resonance structures for the ground state with nondegenerate energy: First, the aromatic structure (single geometric structure), which is formed by the confined $\pi$ electrons and maintain aromaticity; second, the quinoid structure which is originated from the delocalization of flowing $\pi$ electrons along the conjugated chains and simultaneously converts a double bond to a single bond and vice versa, as shown in Figure 1.2. The former is energetically more stable, whereas the latter has higher energy and a narrower band gap, whose contribution is quantified as $E_{\mathrm{BLA}}$. Therefore, the quinoid system has lower ionization potential and larger electron affinity in comparison 
to aromatic form. The more the aromatic ring is sustained in the ground state, the larger the BLA value. On the other hand, the more the quinoid structure is sustained in the ground state, the smaller the BLA value and the more carbon-carbon double bonds between the two adjacent rings. ${ }^{7,8}$

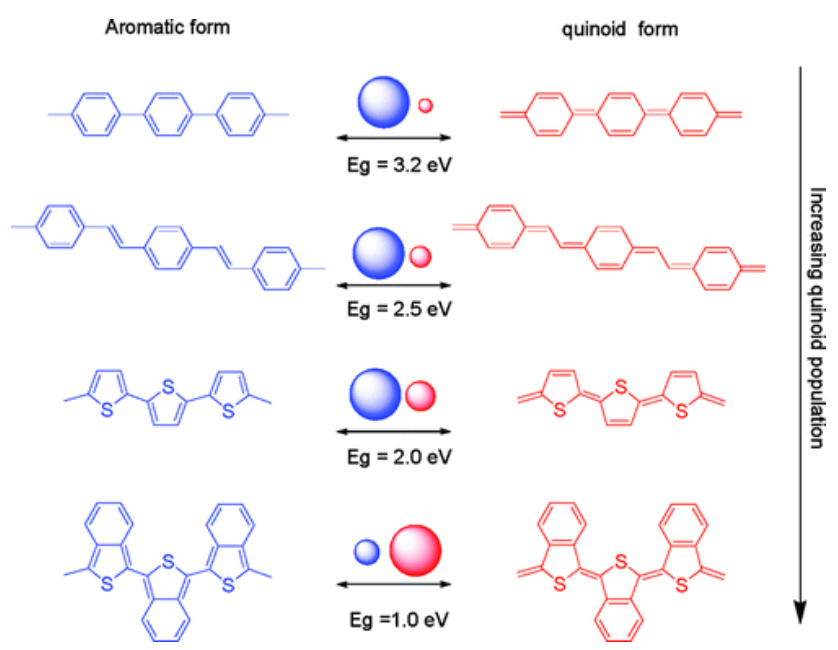

Figure 1.2 Aromatic and quinoid form of conjugated polymers. ${ }^{8 \mathrm{~b}}$

\subsubsection{Aromatic resonance energy $\left(E_{\text {Res }}\right)$}

The aromatic resonance energy is defined as the energy difference between the aromatic structure and a hypothetical reference, consisting of isolated double bonds. In the aromatic ring, $\pi$-electrons are confined in the circle and prevent the electron delocalization along the chain and increase aromaticity. ${ }^{9 a}$ Therefore, energy is required to transform from the aromatic structure to the quinoid structure $\left(E_{\text {res }}\right)$, which is directly dependent on the aromatic stabilization resonance energy. ${ }^{7}$ Hess et al. calculated the resonance energy per $\pi$-electron (REPE) of different types of aromatic compounds. ${ }^{9 b, c}$ They found that the more positive the REPE value, the greater the aromaticity (table 1.1). Therefore, the energy band gaps of the corresponding polymers increases. ${ }^{8 b, 9 b, c}$ 
Table 1.1. Resonance energy and Resonance Energy per Electron.

\begin{tabular}{|l|l|l|l|}
\hline Compound & $\operatorname{Re}(\beta)$ & $\operatorname{REPE}(\beta)$ & $E_{\mathrm{g}}(\mathrm{eV})$ \\
\hline Benzene & 0.390 & 0.065 & 3.2 \\
\hline Pyrrole & 0.233 & 0.039 & 3.1 \\
\hline Thiophene & 0.193 & 0.032 & 2.0 \\
\hline Furan & 0.044 & 0.007 & 2.3 \\
\hline Isothionaphthene & 0.247 & 0.025 & 1.0 \\
\hline
\end{tabular}

\subsubsection{The mean deviation from planarity $\left(E_{\theta}\right)$}

When conjugated polymers exist in nondegenerate states, the repeating units are connected by a single bond which means there is rotational disorder around inter-annular single bonds. As a result, the mean dihedral angle $(\theta)$ between the consecutive units affects their orbital overlap and the electron delocalization along the conjugated backbone. As the dihedral angle between the adjacent ring increases, the ionization potential, and the band gap $\left(E_{\theta}\right)$ increases. ${ }^{10 \mathrm{a}, \mathrm{b}}$ Studies have shown that head to head coupling of poly(3-hexylthiophene) (P3HT) leads to a higher energy band gap with a blue shifted maximum absorption in comparison to head to tail coupling of P3HT due to its nonplanar structure (Figure 1.3). ${ }^{10 \mathrm{c}, \mathrm{d}}$ 


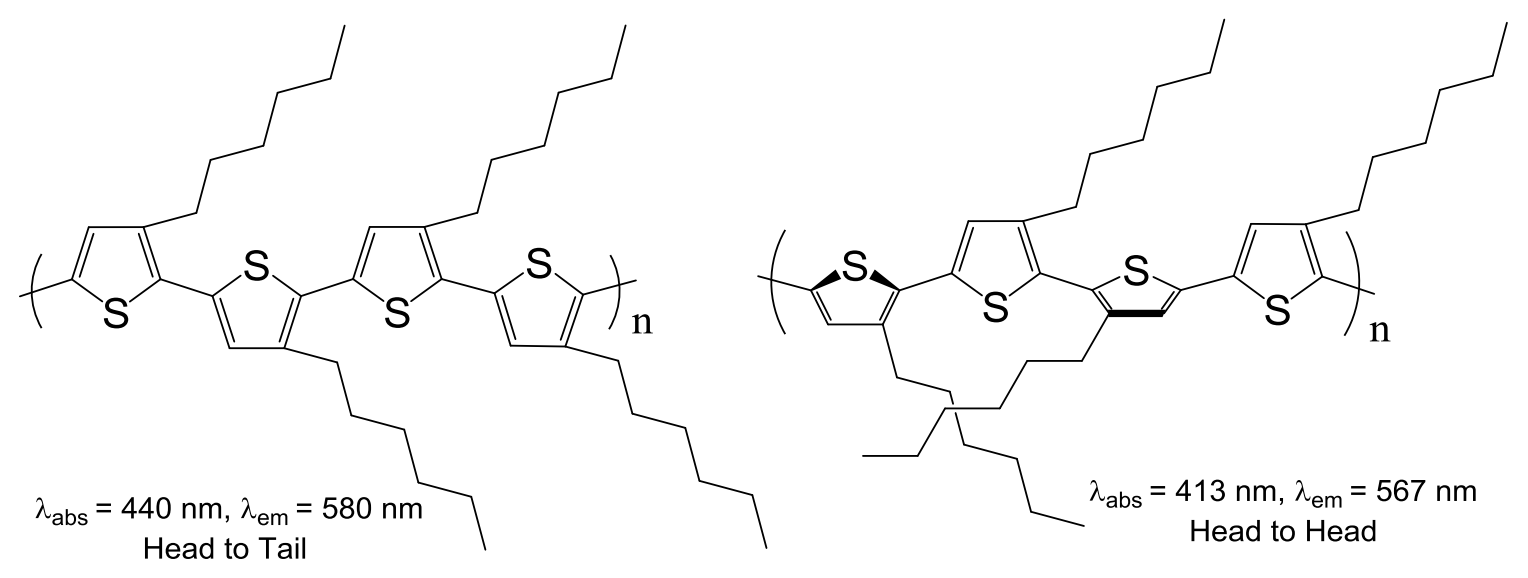

Figure 1.3 Effect of dihedral angle in P3HT.

\subsubsection{The effects of substitution $\left(E_{\text {sub }}\right)$}

Substituents also play an important role in changing the HOMO, LUMO and energy band gap in semiconducting conjugated polymers, via mesomeric or inductive effects. $^{10 b, 11 \mathrm{a}, \mathrm{b}}$ Introducing electron-donating substituents up-shift the HOMO energy level in comparison to the LUMO energy level and reduce the $E_{\mathrm{g}}$. On the other hand, introducing electron-withdrawing substituents down-shift the LUMO energy level in comparison to the HOMO energy level and reduce the $E_{\mathrm{g}}$. These substituent effects are very straight forward and have been extensively studied in conjugated polymers in order to obtain desirable band gaps. The steric effect of the bulkier substituent may dominates the electronic effect and blue shift of the absorption maximum. ${ }^{11 \mathrm{c}}$ Alkyl substituents influence the processability, phase behavior, and structural order charge transport. Branched alkyl chains are more effective in increasing the solubility in comparison to their linear alkyl chain counterpart. However, they can affect the $\pi-\pi$ stacking distance hence charge carrier mobility. ${ }^{11 \mathrm{~d}, \mathrm{e}}$ 


\subsubsection{Intermolecular or interchain coupling in the solid state $\left(E_{\text {int }}\right)$}

An additional factor that influences the energy band gap and charge transfer of semiconducting conjugated polymers is the interchain coupling $\left(E_{\text {int }}\right)$ or interchain interaction. Molecular packing in the solid state greatly affects the $\pi-\pi$ stacking in comparison to the solution state. In solution, polymer chains experiences full rotation with respect to their single bonds whereas in solid state the polymer chains are free of rotation and may possess $\pi-\pi$ stacking (Figure 1.4). As a result, the band gap may decreases in solid state. Moreover, in the solid state, regioregular polymers have higher packing order than random analogs, which also decreases the band gap and increase the charge mobility. ${ }^{5 b, 12}$

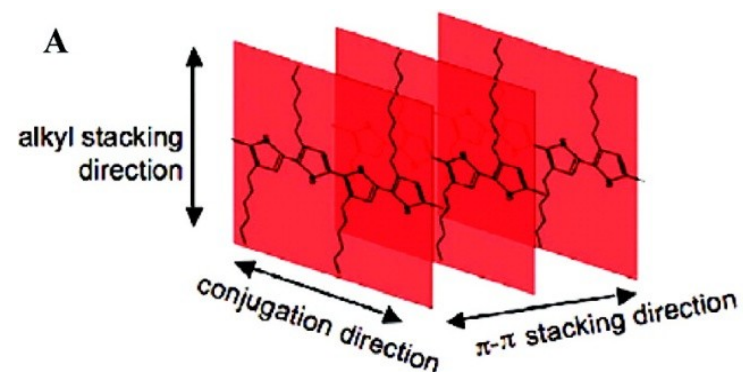

Figure 1.4 Orientation of conjugated polymer in solid state. ${ }^{5 \mathrm{~b}}$

\subsection{Molecular engineering for low band gap polymers}

Generally, conjugated polymers are p-types and n-types. There are several parts in conjugated polymers which influence their optoelectronic properties, among them, the conjugated backbone plays the main role. Different types of conjugated polymers have been developed to synthesize low band gap polymers such as 1) homopolymer 2) donoracceptor polymer 3) ladder polymer 4) acceptor-acceptor polymers 5) others. Different effects such as spacer, donor, and acceptor have also been well studied. 


\subsubsection{Homopolymers}

Homopolymers consist of same aromatic or fused monomer units. The properties of these polymers depend on the intrinsic property of the aromatic or fused monomeric units and intermolecular interaction. Most homopolymers have a band gap greater than $1.9 \mathrm{eV}$. As a result, their light absorption property limits their application. Several groups have studied polythiophene and poly(3-hexylthiophene) (P3HT) (Figure 1.5) homopolymers. Polythiophene has very high band gap and in the presence of an alkyl chain in 3-position of thiophene, the $\pi-\pi^{*}$ transition is lowered and provide a polymer (P3HT) with band gap of $2.0 \mathrm{eV} .^{13,8 \mathrm{~b}}$ Studies have shown that regioregular polymers have lower band gaps with higher absorption maximum around $525 \mathrm{~nm}$ in a blend of PCBM derivatives and absorption coefficient increases. ${ }^{14}$ It was also reported that increasing the regioregularity of P3HT polymer results in a red shift in the absorption. ${ }^{15}$ The highest power conversion efficiency (PCE) of $\mathrm{P} 3 \mathrm{HT}$ polymer blended with $\mathrm{PC}_{61} \mathrm{BM}$ was $4.4 \%$, without optimization. ${ }^{14}$ The PCE can be defined as the ratio of maximum power output $\left(P_{\max }\right)$ to power input $\left(P_{\text {in }}\right)$. The incident light power $(P$ in $)$ is standardized at $1000 \mathrm{~W} \mathrm{~m}^{-2}$ for solar cell testing with a spectral intensity distribution matching that of the sun on the earth's surface at an incident angle of 45 degree, which is called the AM 1.5 spectrum. $^{5 b, 8 d}$ Recently, Hollyday et. al. studied fluorene free OPV with P3HT and they obtained PCE of $6.4 \% .{ }^{16}$ The minimum regioregularity of $\mathrm{P} 3 \mathrm{HT}$ polymer is $95 \%$ for better device performances. ${ }^{14}$ It was also found that increasing the molecular weight of the polymer P3HT the absorption maximum was red shifted due to the important role of chain length in solid state packing. ${ }^{17}$ The molecular weight also has an effect on charge carrier mobility, crystallinity, solubility, thermal stability. It was also reported that P3HT 
polymers as a mixture of different molecular weights from 30,000 to $70,000 \mathrm{~g} / \mathrm{mol}$ and a polydispersity over 2 showed better performance due to the achievement of better morphology. ${ }^{18}$ Polydispersity index is a measure of the distribution of molecular mass in a given polymer sample.

In 1985 Kobayashi et al. synthesized a new homopolymer, polyisothianaphthene (PITN), where the repeating unit was a fused aromatic monomer. ${ }^{19} \mathrm{~A}$ thiophene unit is fused with a benzene ring at the 3- and 4-position (Figure 1.5). The polymer has a band gap $1.1 \mathrm{eV}$, which is much lower (almost half) than the polymer P3HT. This lower band gap can be explained due to many resonance forms including quinoid structure (equal bond length along the pseudopolyene backbone) and selectively maintaining the benzene aromaticity. The doped thin films of PITN have very low optical density in the visible portion of the spectrum (transparent) and conductivity of up to $50 \mathrm{~S} \mathrm{~cm}^{-1}$.

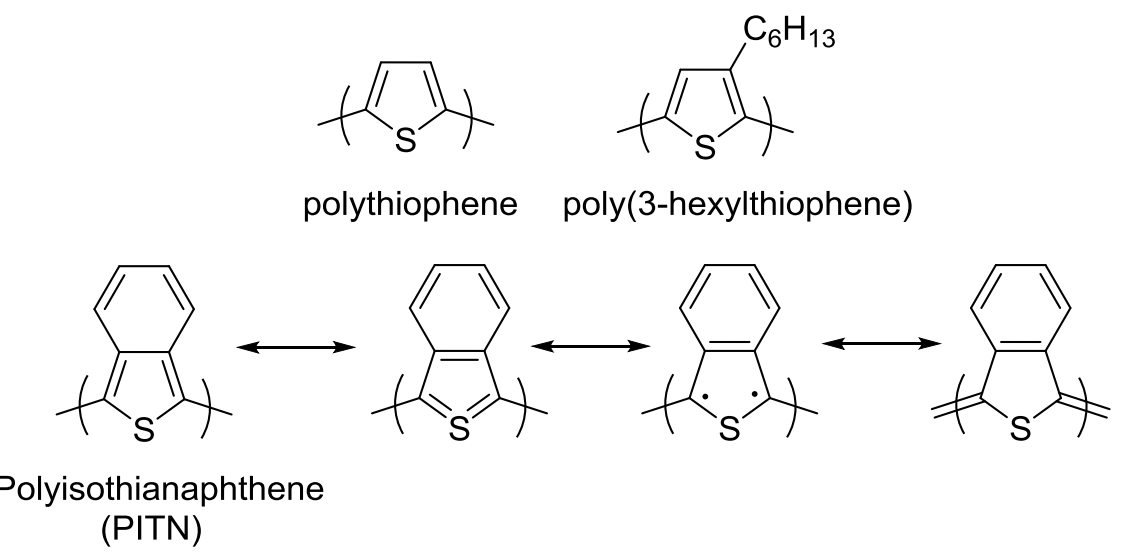

Figure 1.5 Structures of polythiophenes, polyisothianaphthene and resonance structures.

To increase the stability of the PITN polymer, Ikenoue et al. introduced an electron-donating dioxymethylene group at the 5- and 6-positions of the isothianaphthene (ITN) ring. The polymer showed a similar energy band gap, $1.0 \mathrm{eV}$, with a higher redox 
potential. ${ }^{20}$ Based on a similar approach of quinoidization by aromatic exchange, poly(thieno[3,4-b]pyrazine), poly(thieno[3,4-b]-thiophene) with primary thiophene rings fused with another aromatic pyrazine or thiophene ring at the $\beta$ position have also been reported. The polymer with fused pyrazine showed band gap from $0.86-1.02 \mathrm{eV}$ and conductivity of $3.6 \times 10^{-2} \mathrm{~S} \mathrm{~cm}^{-1}$ upon doping. ${ }^{21}$ The polymer with fused thiophenes at the $\beta$ position showed a band gap $1.2 \mathrm{eV}$ with an absorption maximum of $738 \mathrm{~nm}$ and conductivity of $7.2 \times 10^{-2} \mathrm{~S} \mathrm{~cm}^{-1}$ upon doping. ${ }^{22}$ Two different homopolymers of dithieno-thiophene were reported and energy band gaps of 1.12-1.21 eV were obtained with absorption maximal of 650 and $750 \mathrm{~nm}$ (Figure 1.6). ${ }^{23}$

Additionally, planarization between adjacent aromatic units allowed parallel porbitals to increase interaction and extended conjugation to facilitate delocalization. This planarization decreases BLA and reduces the band gap. Rancali et al. tied the adjacent aromatic ring by a covalent bond in a most straightforward way. They rigidified the adjacent bithiophene unit with a $\mathrm{sp}^{3}$ carbon of a ketal group and the polymer showed an energy band gap of $1.2 \mathrm{eV}$. It was also reported that increasing the thiophene units and further rigidification with $\mathrm{sp}^{3}$ carbons further decreases the energy band gap to $1.1 \mathrm{eV}$ (Figure 1.6). ${ }^{24-26}$

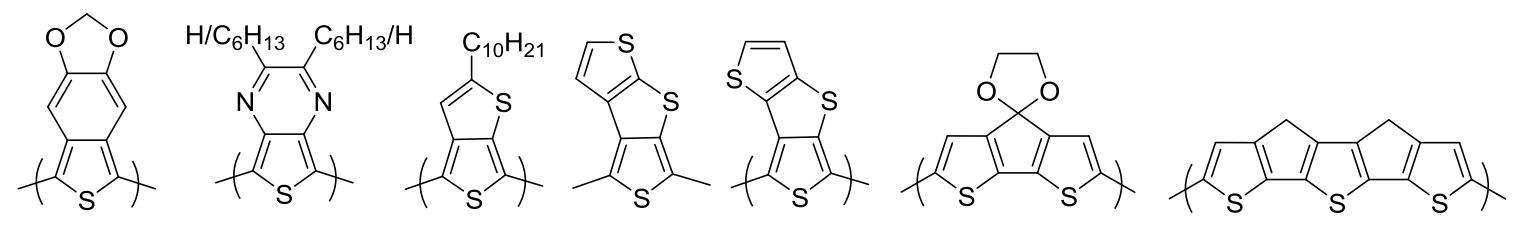

Figure 1.6 Structures of thiophene-based p-type homopolymers.

Diketopyrrolopyrrole (DPP) is a promising electron deficient monomer and its homopolymer has been studied as an organic field effect transistor. DPP contains 
different reactive sites and can be functionalized. Therefore, $\mathrm{N}$ substitution has been used to tune the band gap and properties. The homopolymer of DPP with branched alkyl chain showed a band gap of $1.23 \mathrm{eV}$ and broad absorption from NIR region. Changing the alkyl chain on $\mathrm{N}$ with aromatic group, absorption blue shifted and increased the band gap. Although, the hole and electron mobility was not very high $\left(\sim 0.01 \mathrm{~cm}^{2} / \mathrm{Vs}\right) \cdot{ }^{27,28}$ Replacing the alkyl chain with triethylene glycol the polymer showed an optical band gap $1.2 \mathrm{eV}$ with high electron mobility $\left(3 \mathrm{~cm}^{2} / \mathrm{Vs}\right)$ (Figure 1.7$){ }^{29}$
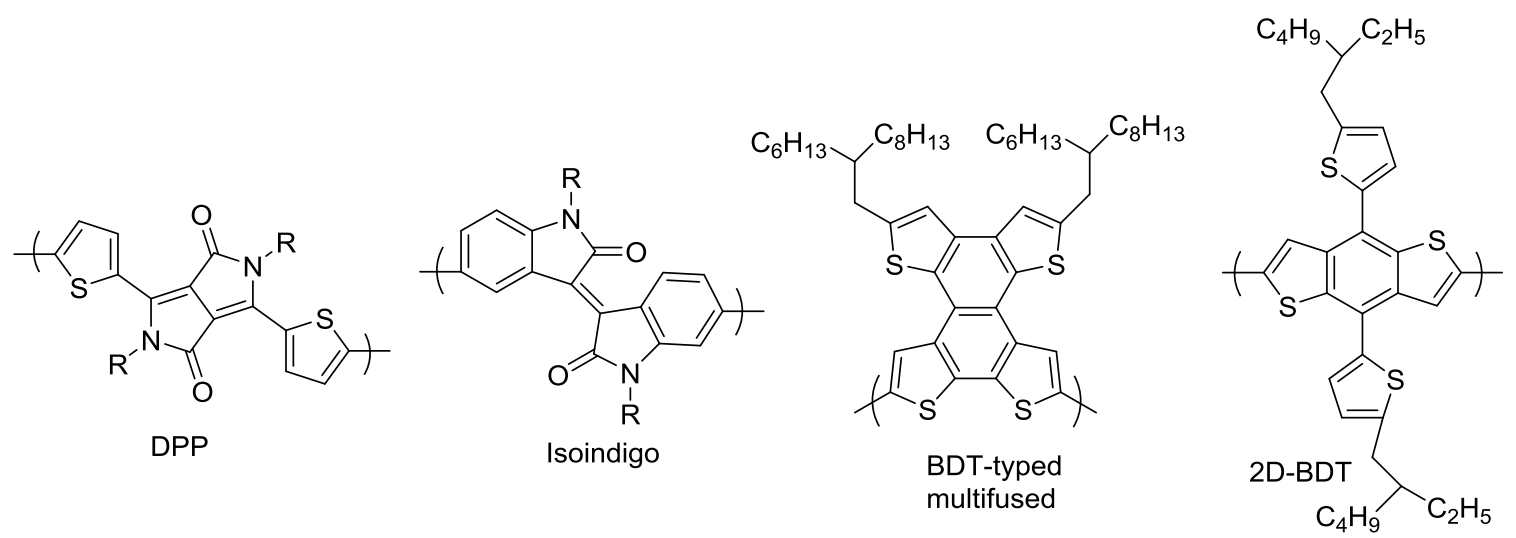

Figure 1.7 Structures of acceptor and donor type homopolymers.

Isoindigo is another type of acceptor monomer and has recently been used as an alternative to PCBM derivatives in organic photovoltaic (OPV). The reported energy band gap of isoindigo homopolymer was $1.77 \mathrm{eV}$ with $\mathrm{HOMO}$ and LUMO energy levels of -3.84 and $-5.54 \mathrm{eV}$. The obtained PCE was $0.5 \%$ with P3HT polymer. ${ }^{30}$ Benzodithiphene (BDT)-typed monomers are extensively studied in bulk heterojunction (BHJ) solar cells. In 2010, Xiao et al. synthesized a BDT-based multifused donor monomer and its homopolymer. They studied its application in BHJ solar cells. The polymer has a wide energy band gap $(2.0 \mathrm{eV})$ and the observed PCE was $1.35 \% .{ }^{31 \mathrm{a}}$ 
Thienyl-substituted benzodithiophene is a 2D-type donor monomer which has recently been used to synthesize homopolymers and its properties in OPV have been studied as an alternative to P3HT. The reported energy band gap was $1.98 \mathrm{eV}$ and the obtained PCE was $6.12 \%$. This high PCE value is due to the higher absorption coefficient of the 2Dtype homopolymer (Figure 1.7). ${ }^{31 \mathrm{~b}}$

\subsubsection{Ladder polymers}

Conjugated ladder polymers (CLPs) are those with all $\pi$-conjugated fused rings in the backbone which distinguish them from conventional conjugated polymers. This unique constitution restricts the torsional rotation in-between the aromatic units along the conjugated backbone. ${ }^{32}$ The earliest ladder polymer was synthesized in the 1970s and after that, many CPLs have been reported with their applications in different fields such as OFET (organic field effect transistor), OLED (organic light emitting diode) and OPV (organic photovoltaics, solar sell). ${ }^{33}$ There are two different approaches to synthesize the CLPs : a) single-step polycondensation of tetra-functional monomers or repetitive DielsAlder reactions ${ }^{33 a, b, 34}$ and b) post-polymerization annulations where a single-stranded conjugated polymer is synthesized and then other functional groups are cyclized to form a second strand of bonds. ${ }^{33, g, \mathrm{i}, \mathrm{j}}$ Although the aromatic heterocyclic ladder polymers have promising intrinsic properties, they have solubility issues. They require strong acidic conditions to process the film which limits their long term stability. ${ }^{33 \mathrm{~b}}$ The other limitation is quantitative conversion in the ring-closing reaction (structural defect). The $\mathrm{n}$ type BBL [poly(benzobisimidazobenzophenanthroline)] polymer has a band gap around $1.8 \mathrm{eV}$ with low LUMO $(\sim-4.4 \mathrm{eV})$ and HOMO $(\sim-5.3 \mathrm{eV})$ energy level. ${ }^{33 \mathrm{~d}}$ Jenekhe et al. 
reported the highest electron mobility from a BBL polymer is $0.1 \mathrm{~cm}^{2} / \mathrm{Vs} .{ }^{33 \mathrm{k}}$ In 2004 , the same group used a BBL polymer for OPV. They prepared bilayer OPV using BBL as the n-type and PPV as the p-type polymer and the obtained PCE was 5.4\%. ${ }^{35}$ In 2011, Xiong et al. synthesized a new n-type ladder conjugated polymer (LCPT) based on perylenediimide and thienothiophene and reported the LUMO energy level is $-3.98 \mathrm{eV} .^{36}$ In 2017, Lee et al. synthesized series of low band gap p-type ladder polymers (cyclopenta-fused polycyclic aromatic hydrocarbon) by post-polymerization annulations. The obtained band gaps are from $1.20 \mathrm{eV}$ to $1.55 \mathrm{eV}$ with higher LUMO $(-3.45 \mathrm{eV}$ to $3.85 \mathrm{eV}$ ) and HOMO energy levels $(-5.18 \mathrm{eV}$ to $-5.50 \mathrm{eV}$ ) (Figure 1.8) compared to parent polymers. ${ }^{32 \mathrm{f}}$

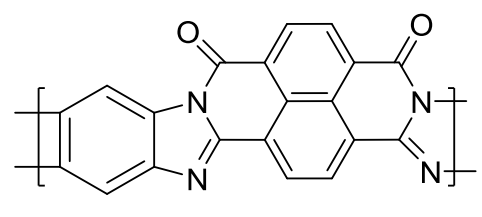

BBL

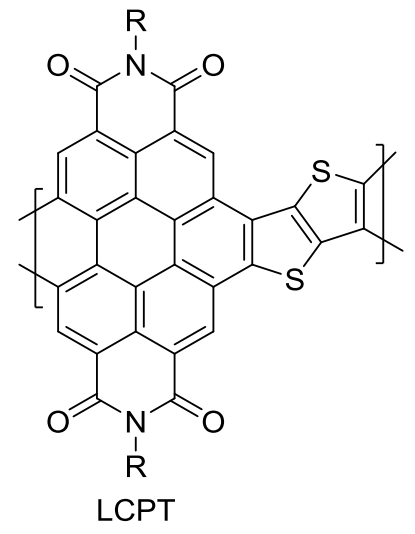

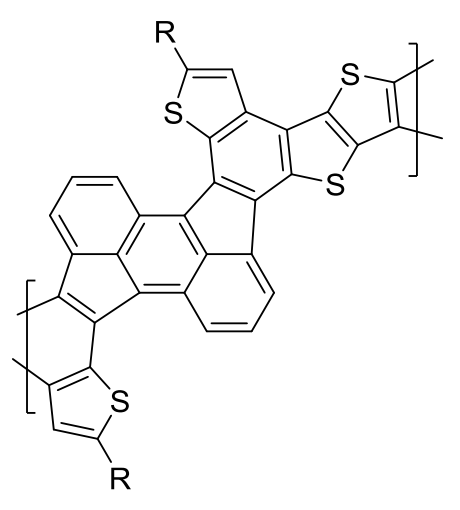

Figure 1.8 Examples of ladder polymers.

The existing dihedral angles between building blocks in conjugated polymers are due to torsional strain and thermodynamic fluctuation, which disturbs the $\pi$-conjugation of the polymer backbone and shortens the effective conjugation lengths along the polymer main chain, thus decreasing the carrier mobility. The dihedral angle also perturbs the intermolecular packing of the polymer in the solid state, resulting in a higher 
energy barrier for the charge carriers and excitons to transport throughout the bulk material. CLPs consist of rigid, planar backbone and continuous $\pi$-conjugation with a zero dihedral angle between each building block, thus a maximum of $\pi$-electron delocalization is expected. As a result, CLPs usually show better electronic properties, better chemical resistance, higher thermal and optical stability than linear conjugated polymers. ${ }^{32}$

\subsubsection{Donor-acceptor polymers and their energy band gaps}

The most studied and acceptable concept to tune the energy band gap of conjugated polymers is D-A-based polymer. This concept was first proposed by Havinga et al. in 1992. Several poly(squaraine-benzobispyrroline/benzobisthiazole), poly(croconaine- benzobispyrroline/benzobisthiazole) and poly(squaraine-trithiophene) were reported using various donor and acceptors. The band gaps were in the range of 1.0 $\mathrm{eV}$ to $0.5 \mathrm{eV}$ (Figure 1.9). ${ }^{37 \mathrm{a}, \mathrm{b}}$
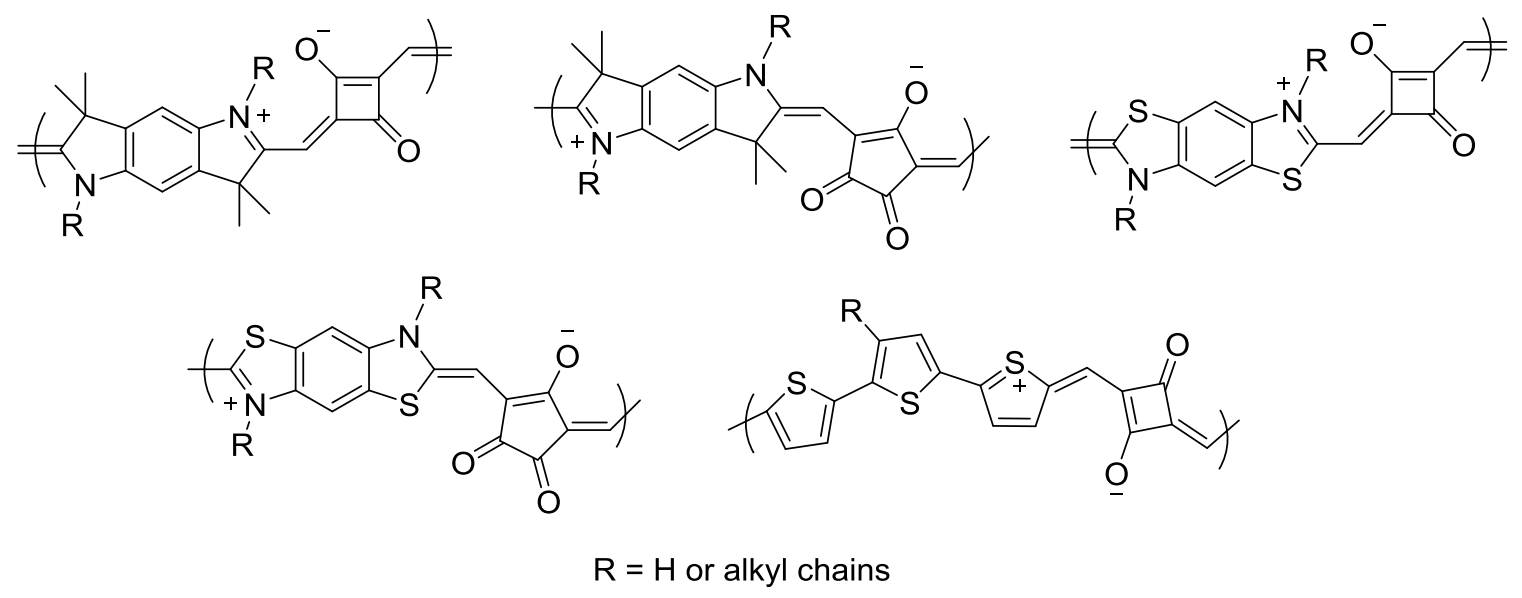

Figure 1.9 Structures of polysquaraines and polycroconaines.

After the introduction of D-A system in 1998, Zhang and Tour synthesized a new D-A polymer with a band gap of $1.0 \mathrm{eV} \cdot{ }^{37 \mathrm{c}}$ In alternating D-A polymers, the interaction 
between the moieties enhances the double bond character between the repeating units, which adopt the low band gap quinoid forms within the polymer backbones. As a result, a conjugated polymer with an alternating sequence of appropriate donor and acceptor units in the main chain leading to a low energy band gap. ${ }^{37 b}$ This low band gap energy can be explained by their localized HOMO-LUMO energy level through the polymer backbone. Generally, electron-rich donor units have higher HOMO and LUMO energy levels in comparison to that of their electron-acceptor counterparts. If the HOMO levels and the LUMO levels of the donor and acceptor moieties are close in energy, the hybridization of energy levels show a low HOMO-LUMO separation. As a result, the obtained molecular orbital energy gap will be small, as depicted in Figure 1.10. This feature opened an important opportunity to tune the band gap and energy level of conjugated semiconducting polymers. Based on this idea, different approaches such as (a) strong donor and strong acceptor (b) weak donor and strong acceptor have been adopted to adjust the polymer band gaps and energy levels. A polymer with strong donor and acceptor unit provide low band gap polymer whereas polymer with weak donor and strong acceptor provide larger band gap with respect to the first concept. ${ }^{5 \mathrm{a}, \mathrm{c}, \mathrm{d}, 38}$ The polymers with weak donor and strong acceptor provide lower HOMO level which ensures higher $V_{\mathrm{oc}}$ and stability of the polymers. ${ }^{38 \mathrm{a}, 39}$ 


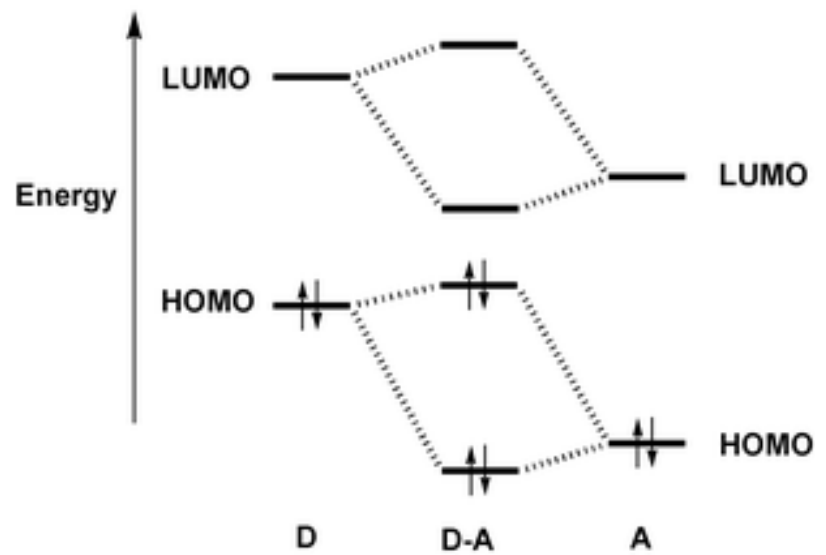

Figure 1.10 Molecular orbital of typical donor-acceptor polymer. ${ }^{37 b}$

Polymers with different energy band gaps absorb at different spectral wavelengths. Moreover, introduction of $\pi$-spacer is another approach to ensure effective conjugation and planarization with appropriate energy band gap and charge transportation. Therefore, selections of suitable donors, acceptors, and spacers have accordingly been subjected to intensive research in the field of organic semiconducting polymers.

\subsubsection{Effect of donor unit}

The electron donating ability of the donor unit has a large impact on the HOMO energy level of the semiconducting conjugated polymers. Research has shown that donor monomers with fused conjugated rings show high-lying HOMO levels and favor narrow band gap for alternating D-A polymers. ${ }^{39}$ Fluorene (FL) is a very common donor unit in alternating D-A conjugated polymers. One central five-membered ring is fused with two benzene rings in FL. Electronically, the FL unit is a weak electron donor due to the relatively electron-deficient benzene units. ${ }^{40}$ And most of the FL-based polymers have 
HOMO level around $-5.5 \mathrm{eV} .^{5 \mathrm{a}}$ However, these polymers are mostly reported with relatively higher energy band gaps. ${ }^{5 a, 38 a}$ When the carbon atom at position 9 in FL is replaced with group 14 atoms such as $\mathrm{Si}$ and $\mathrm{Ge}$, the band gap lowers due to higher hole mobility and the device performance is also increased. ${ }^{38 a, 41}$ To overcome this band-gap challenge, the thiophene analogue of FL, cyclopentadithiophene (CPT) unit where thiophene units fused with a central five-membered ring was developed. The CPT unit is much more electron-rich than the FL; thus, the ICT between the CPT donor unit and the acceptor unit is much stronger, leading to a significantly smaller band gap of related polymers. ${ }^{38 a}$ When the carbon atom at position 4 in CPT is replaced with group 14 atoms such as $\mathrm{Si}$ (DTS) and Ge (DTG), structural order in the solid state and device performance is also increased. ${ }^{38 \mathrm{a}, 42}$ CPT-based polymers have HOMO level around -5.2 $\mathrm{eV}$ and most of the band gaps are around $-1.4 \mathrm{eV}$.

BDT is another strong donor which was reported in the synthesis of low band gap conjugated polymers. The band gaps were tuned effectively using different acceptors. BDT-based polymers showed HOMO levels from -4.6 to $-5.2 \mathrm{eV}$ and the reported band gaps are a wide range of -1.05 to $2.10 \mathrm{eV}$. A fused aromatic structure of more than three units is another approach to obtain highly planar, effective $\pi$-electron delocalization and a better $\pi-\pi$ interaction between the backbone chains in the solid state to broaden light absorption and enhance charge carrier mobility. ${ }^{19}$ Quadrathienonaphthalene (QTN monomer)-based D-A polymers exhibit small band gaps $(1.6 \mathrm{eV})$ as well as low HOMO (-5.4 eV, ideal) levels compare to benzodithiophene (BDT monomer) based polymers (Figure 1.11)..$^{31 \mathrm{a}, 38 \mathrm{a}}$ 


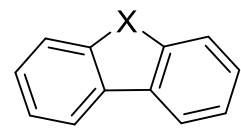

$\mathrm{X}=\mathrm{C}, \mathrm{N}, \mathrm{Si}, \mathrm{Ge}$

$\mathrm{FL}$

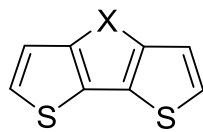

CPT

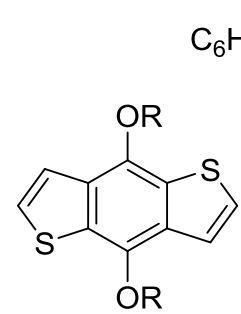

BDT

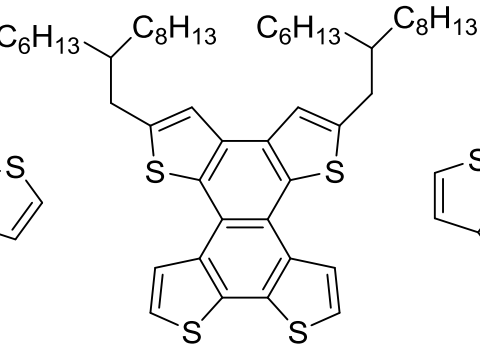

QTN

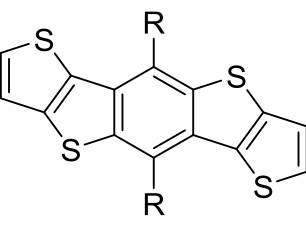

DBD

Figure 1.11 Structures of various fused heterocyclic donor units.

However, when the conjugation is too large especially for fused structures, quinoidal structures in the backbone may be inhibited. The solubility, processability, and miscibility of the polymers with acceptor materials like PCBM derivatives will be negatively affected, which will lead to inferior performance (in OPV). Therefore, a new monomer, DBD, with extended thiophene unit on the BDT was designed. The expanded conjugation of the rigid DBD unit could cause the holes to be more delocalized which lowers the local positive charge density. As a result, the Coulombic interactions between the holes and the electrons would be reduced. This would lead better charge separation at the donor-acceptor interface and enhance the performances. The reported HOMO energy level was $-5.2 \mathrm{eV}$ and energy band gap $1.67 \mathrm{eV} .^{43,5 \mathrm{~d}}$

\subsubsection{Effect of acceptor unit}

Acceptor units have high importance in controlling the energy levels and band gaps. The acceptor monomers are mostly containing aryl or heteroaryl units with one or several electronegative atoms. 2,1,3-Benzothiadiazole (BT) is a common, strong and commercially available acceptor unit in the field of D-A polymers. The study showed that substitution of $\mathrm{S}$ atom to $\mathrm{Se}$ and Te the band gap decreases from 1.59 to 1.46 and $1.06 \mathrm{eV}$ 
in D-A polymer. Replacing the $\mathrm{S}$ atom with heavy atoms down the group 16 , the ionization potential and aromaticity decrease, this influences the band gap. The LUMO energy level stabilizes and the HOMO energy level destabilizes, and as a result, the band gap decreases. On the other hand, replacing the $\mathrm{S}$ atom with $\mathrm{N}$ in the $\mathrm{BT}$ monomer increased the band gap of D-A polymer due to the electron donating nature of the middle N. The lone pair on the middle nitrogen is more basic than $\mathrm{S} .{ }^{45}$ The electron accepting ability of the quinoxaline is lower due to the higher energy level LUMO than the BTcontaining polymer and their energy band gaps are larger. Benzoxadiazole (BX) monomers contain an $\mathrm{O}$ atom instead of $\mathrm{S}, \mathrm{BX}$-based polymers have similar energy levels as BT polymers. However, the device result is not as good as BT-based polymers due to the lower mobilities. ${ }^{46}$ It was also reported that replacing the benzene ring in BT analogues by a pyridine ring the LUMO energy level decrease significantly and the energy band gap becomes smaller. Thiadiazoloquinoxaline (TQ) and bezobisthiadiazole (BBT) are promising acceptor units for use in D-A polymers. The reported absorption onsets of semiconducting polymers containing TQ and BBT monomers are 1950 and $2200 \mathrm{~nm}$ with energy band gap of 0.64 and $0.56 \mathrm{eV}$, respectively. Both polymers showed very high ambipolar mobilities where BBT containing polymer showed better performances (hole and electron mobilities are $1.0 \mathrm{~cm}^{2} \mathrm{~V}^{-1} \mathrm{~s}^{-1}$ and $0.7 \mathrm{~cm}^{2} \mathrm{~V}^{-1} \mathrm{~s}^{-1}$, respectively) (Figure 1.12). ${ }^{47}$

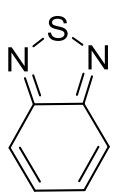

BT

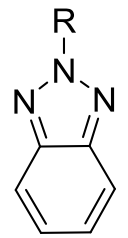

Triazole

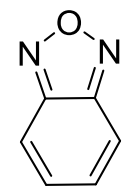

$\mathrm{BX}$

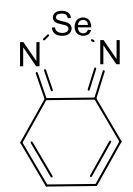

BSe

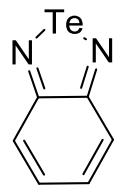

BTe

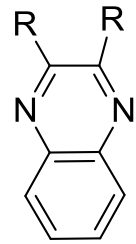

QX

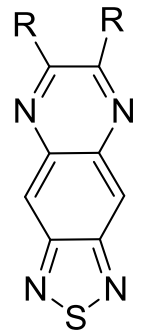

TQ

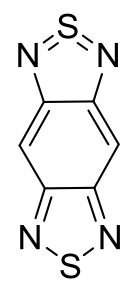

BBT 
Figure 1.12 Structures of some strong acceptor units.

\subsubsection{Effect of substituent}

Varying the substituent on monomers has a great impact on tuning the energy levels, which then influence the band gaps, as well as properties of semiconducting conjugated polymers. The electron donating group (such as alkoxy-) raises the HOMO energy levels and electron withdrawing group (such as cyano-) lowers the LUMO energy level. As a result, the energy gap decreases. ${ }^{48}$ 3,4-Ethylenedioxythiophene (EDOT) is a widely studied electron donating group. The EDOT unit generates noncovalent, intramolecular, sulfur-oxygen interactions which lead to a self-rigidification of the conjugated structure in which it is incorporated. However, its neutral state is unstable under ambient conditions, which limits its applications. ${ }^{10 \mathrm{~b}}$ Increasing the HOMO energy level causes a decrease in the open-circuit voltage $\left(V_{\text {oc }}\right) . V_{\text {oc }}$ is the voltage between the two the electrodes when the current is zero. It is determined by the energy levels difference between HOMO of donor and LUMO of acceptor. Therefore, electron withdrawing substituents are mostly used on acceptor units to decrease the LUMO energy levels and energy band gaps for polymers as well as maintain the higher $V_{\text {oc. }}$ Cyano ($\mathrm{CN})$, fluorine (-F) and nitro $\left(-\mathrm{NO}_{2}\right)$ are the most common substituents used to improve the strength of the acceptor monomers. In 1995, Roncali et al. reported that a cyano group at the vinylene linkage of dithienylethylene could lead to a considerable reduction of the band gap of the corresponding polymers to a value as low as $0.60 \mathrm{eV}$. However,

the polymer suffered poor solubility. ${ }^{49 a, 10 b}$ In 2016 , Heeney et al. synthesized CN substituted BT monomers and their semiconducting polymers. The results showed that 
upon addition of each cyano group the LUMO energy level was lowered by $0.25 \mathrm{eV}$ and the HOMO energy level was lowered by $0.1 \mathrm{eV}$ and the band gap decreased by $0.15 \mathrm{eV}$. The reported band gaps were 1.57 and $1.37 \mathrm{eV}$ with respect to mono- and di-substituted units. ${ }^{49}$ They also reported that the drop in performance for the polymer with two cyano substituents on the BT moiety was caused by very limited yields of electron transfer from the polymer to the fullerene. This was likely caused by poor orbital energy level alignment with the fullerene acceptor $\left(\mathrm{PC}_{71} \mathrm{BM}\right)$. Fluorine is another interesting substituent due to its high electronegativity value (4.0) and the ability to form different intra and intermolecular interactions $(\mathrm{C}-\mathrm{F} \ldots \mathrm{H}, \mathrm{F} \ldots \mathrm{S}$ and $\mathrm{F} \ldots \pi)$. However, it does not influence the energy band gap, it decreased both the HOMO and LUMO energy levels. ${ }^{50}$ Fluoride, ester and sulfonyl, substituted thieno[3,4-b]thiophene (TT) and fluoride substituted BT units in conjugated polymers are well studied. Results suggest that these substituents enhance the device properties. ${ }^{51}$

\subsubsection{Effect of spacer unit}

Generally, the spacers are small $\pi$ conjugated chromophores which link the donors and acceptors in semiconducting polymers. The goal is to affect the desired torsional or dihedral angle between the monomer units of the conjugated backbone chain. Therefore, changes in the optical absorption, molecular energy levels, charge transport, as well as the blend morphology with the acceptor material, all relate to their performance in devices. It was reported that the band gap decreases from 3.20 to $2.60 \mathrm{eV}$ for poly(paraphenylene) to poly(phenylenevinylene) and from 2.0 to $1.70 \mathrm{eV}$ for

polythiophene to poly(2,5-thienylene vinylene) due to the insertion of double bonds. ${ }^{52,10 \mathrm{~b}}$ 
Thiophene is widely used as a spacer in semiconducting polymer due to its planarity and small size, its good thermal photochemical stability and the ease of functionalization for polymerization, as well as enhancement of solubility. ${ }^{53,51 \mathrm{a}}$ In 2012 , Wang et al. reported a comparative study of different $\pi$-spacer such as thiophene, furan and TT (Figure 1.13) and their effects in semiconducting polymer. They concluded that from furan to TT, the molecular chain shape changed from Z-shape to almost a straight line due to the increased $\pi$-orbital overlap between neighboring aromatic spacers. The absorption red shifted from furan to TT. The energy band gaps decreased from furan to TT (1.96 to 1.66 $\mathrm{eV}$ ) due to the increase of the HOMO energy levels. The device property was also increased accordingly. ${ }^{53 a}$

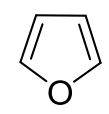

Furan

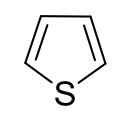

Thiophene

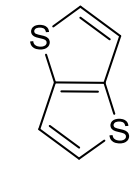

TT

Figure 1.13 Structures of some aromatic spacers.

\subsubsection{Side chain and position effect}

Without the side chains, $\pi$-conjugated semiconducting polymers are difficult to process. Their solubility decreases and their molecular weights become lower. Conjugated polymers with fewer $\pi-\pi$ interchain interactions and non-planar structures are soluble in organic solvents. However, they are mostly used for sensing nitro-aromatics by fluorescence quenching. ${ }^{54}$ On the other hand, semiconducting polymers are for photovoltaic and OFET requires high molecular weight, a planar structure, and better $\pi-\pi$ interchain interaction. In 2010, You et al. reported that the side chain size affects the device performance. Polymer (PNDT-DTBT) with long and branched side chains 
exhibited the highest $V_{\mathrm{oc}}(0.81 \mathrm{~V})$ and lowest charge transport property $\left(J_{\mathrm{sc}}\right)$ for BHJ devices due to the weak intermolecular $\pi-\pi$ interactions among the conjugated polymers while the long linear side chains provided the smallest $V_{\mathrm{oc}}(0.41)$ and $J_{\mathrm{sc}} . J_{\mathrm{sc}}$ is defined as the maximum current density generated when the organic solar cell is in short-circuit Polymers with moderate side chain bulkiness effectively balanced $V_{\mathrm{oc}}$ and $J_{\mathrm{sc}}$, to reach the optimum efficiency. ${ }^{55 a, b}$ On the other hand, Frechet et al. reported that the previously mentioned trend was different for PBDTTPD-based polymers: $V_{\mathrm{oc}}$ remained almost unchanged at $0.85 \pm 0.04 \mathrm{~V}$, regardless of the bulkiness of the side chain. However, a noticeable change of $J_{\mathrm{sc}}$ was observed. Polymer with bulky side chains exhibited smaller $J_{\mathrm{sc}}\left(5.5 \mathrm{~mA} / \mathrm{cm}^{2}\right)$ whereas the polymers with long linear alkyl chain exhibited the highest $J_{\mathrm{sc}}$ (up to $10.6 \mathrm{~mA} / \mathrm{cm}^{2}$ ). The high $J_{\mathrm{sc}}$ was due to a smaller $\pi-\pi$ distance. ${ }^{55 \mathrm{c}, \mathrm{b}}$ The length of the side chain was studied by different groups and different results were observed. ${ }^{56}$ Gadisa et al. proposed that the longer side chains facilitated the formation of the PCBM clusters by establishing efficient electron-percolation pathways which balanced electron/hole mobilities with proper phase separation, leading to a higher fill factor. ${ }^{56 a}$ Egbe et al. did not observe a similar trend and they found lower device property due to phase separation. ${ }^{56 \mathrm{~b}}$ The side chain still play a critical role in solving issues in material synthesis, processing, and device fabrication. You et al. studied the effect of the position of the side chain on PBDT-DTBT. They reported that introduction of alkyl chains at the $\mathrm{R}_{3}$ position induces an increase in dihedral angle 3, keeping the planar structure of DTBT without change of dihedral angle 1 or 2 . The band gap and HOMO level were identical to the polymer without any alkyl chains. The introduction of alkyl chains at the $R_{1}$ and $R_{2}$ position did not change the dihedral angle 3 , but did increase the dihedral angle 1 and 2 . 
As a result, enlarged band gaps and lowered HOMOs (Figure 1.14) were observed. ${ }^{56}$ Therefore, flexible and appropriate side chain introduction in conjugated polymers may provide a better device result.
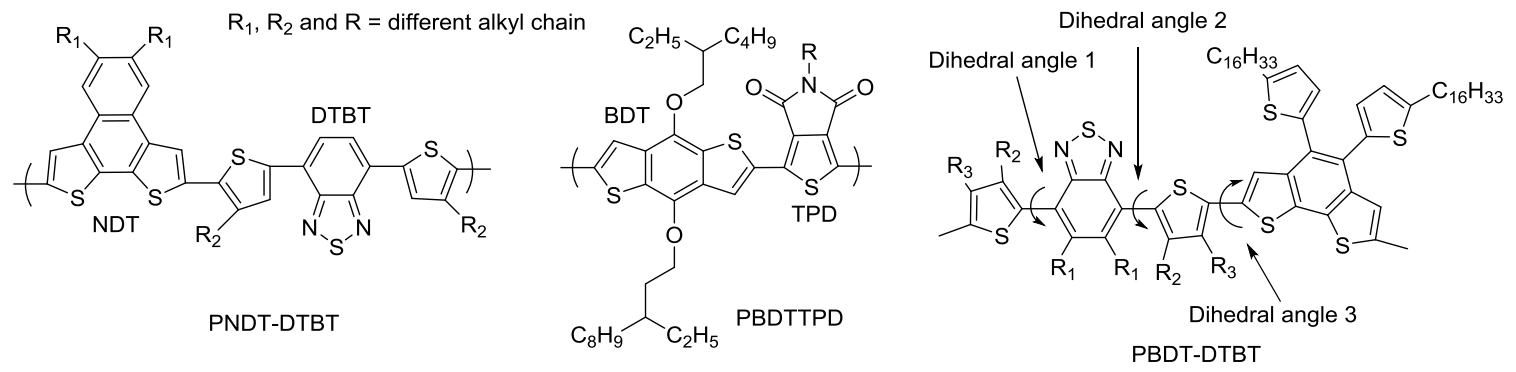

Figure 1.14 Structures of some polymers with side chains at different positions.

\subsubsection{Band gap tuning by addition of tetracyanoethylene and tetracyano- quinodimethane}

Tetracyanoethylene (TCNE) and 7,7,8,8-tetracyanoquinodimethane (TCNQ) are well known as electron acceptors, wherein the latter is stronger than the former (Figure $1.15) .{ }^{57 a}$ The acceptor ability decreases with increasing numbers of benzene rings. ${ }^{57 b}$ Due to the electron accepting nature of these compounds they are used as p-type dopants.

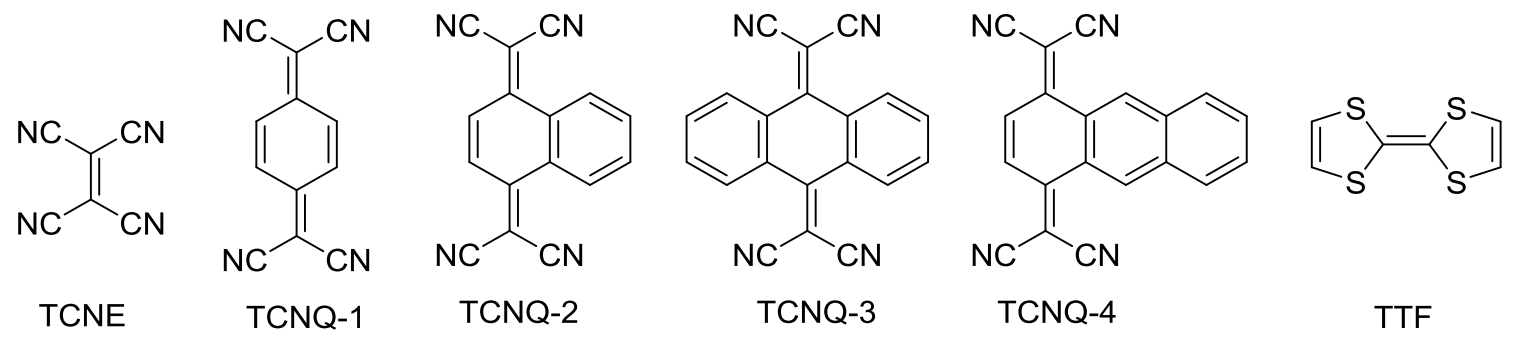

Figure 1.15 Structures of CN-based acceptors and TTF.

TTF-TCNQ-1 charge complex provides highest electric conductivity over any type of organic compound and is as electrically conducting as metals such as copper, $\sigma$ 
$\left(298^{\circ} \mathrm{K}\right)=6 \times 10^{5} \mathrm{~S} \mathrm{~cm}^{-1} .{ }^{57 \mathrm{c}}$ Superconductivity by charge transfer complex was also reported by Jerome et $a .^{57 \mathrm{~d}}$ Addition of TCNE and TCNQ to conjugated polymer systems shows absorption at a longer wavelength. In 2009, Michinobu et al. showed that the ferrocene containing poly(aryleneethynylene) has several maximal absorption peaks from 295 to $435 \mathrm{~nm}$ which moves to $603 \mathrm{~nm}$ with an absorption onset from a longer wavelength with the addition of TCNE in polymer backbone. ${ }^{58 \mathrm{a}}$ The absorption spectrum was broad compare to the parent polymer. In 2010, the same group showed that 3,6 carbazole-based poly(arylenebutadiynylene) has maximal absorption peak at $385 \mathrm{~nm}$ which moves to $515 \mathrm{~nm}$ with an absorption onset from longer wavelength with the addition of TCNE in the polymer backbone (Figure 1.16). ${ }^{58 b}$ In 2008, Michinobu reported that the addition of the TCNE group in fluorene-based polyamine polymer backbones decreased the energy band gap by lowering the LUMO and HOMO energy levels and a new absorption maximal peak appeared at longer wavelength $(478 \mathrm{~nm})$ with an isosbestic point at $418 \mathrm{~nm} .{ }^{58 \mathrm{c}}$ In 2011 , the same group showed that addition of TCNQ in thiophenebased polyamines polymer backbone decrease the energy band gap by lowering the LUMO and HOMO energy levels and a new absorption maximal peak appeared at a longer wavelength $(750 \mathrm{~nm})$ with an isosbestic point at $382 \mathrm{~nm}$. This comparative study showed that addition of TCNQ in thiophene-based polyamine polymer backbones causes a greater decrease in the energy band gap than for TCNE. The LUMO level with TCNQ decreases more than for TCNE (Figure 1.16). The polymer turns to an n-type polymer from a p-type polymer. ${ }^{58 d}$ In 2014 , Yu et al. synthesized a new acceptor monomer containing a dicyanovinylene group. They synthesized a series of polymers with different strengths of donor and acceptor groups with this acceptor unit. The CN-based polymers 
exhibited a more red-shifted absorption maxima with a longer wavelength absorption tail than did the CO-based polymers. The LUMO energy level of the CN-based D-A polymer was lower than for CO-based polymer. The $\mathrm{CN}$-based polymer failed to show any photovoltaic properties due to the orientation of $\mathrm{CN}$ group. The $\mathrm{CN}$ moieties were all in the same direction on the polymer chain, which can generate a large polarization between the ground and excited states. This can cause electron trapping sites and as a result, the polymer did not show any photovoltaic property. ${ }^{58 \mathrm{e}}$
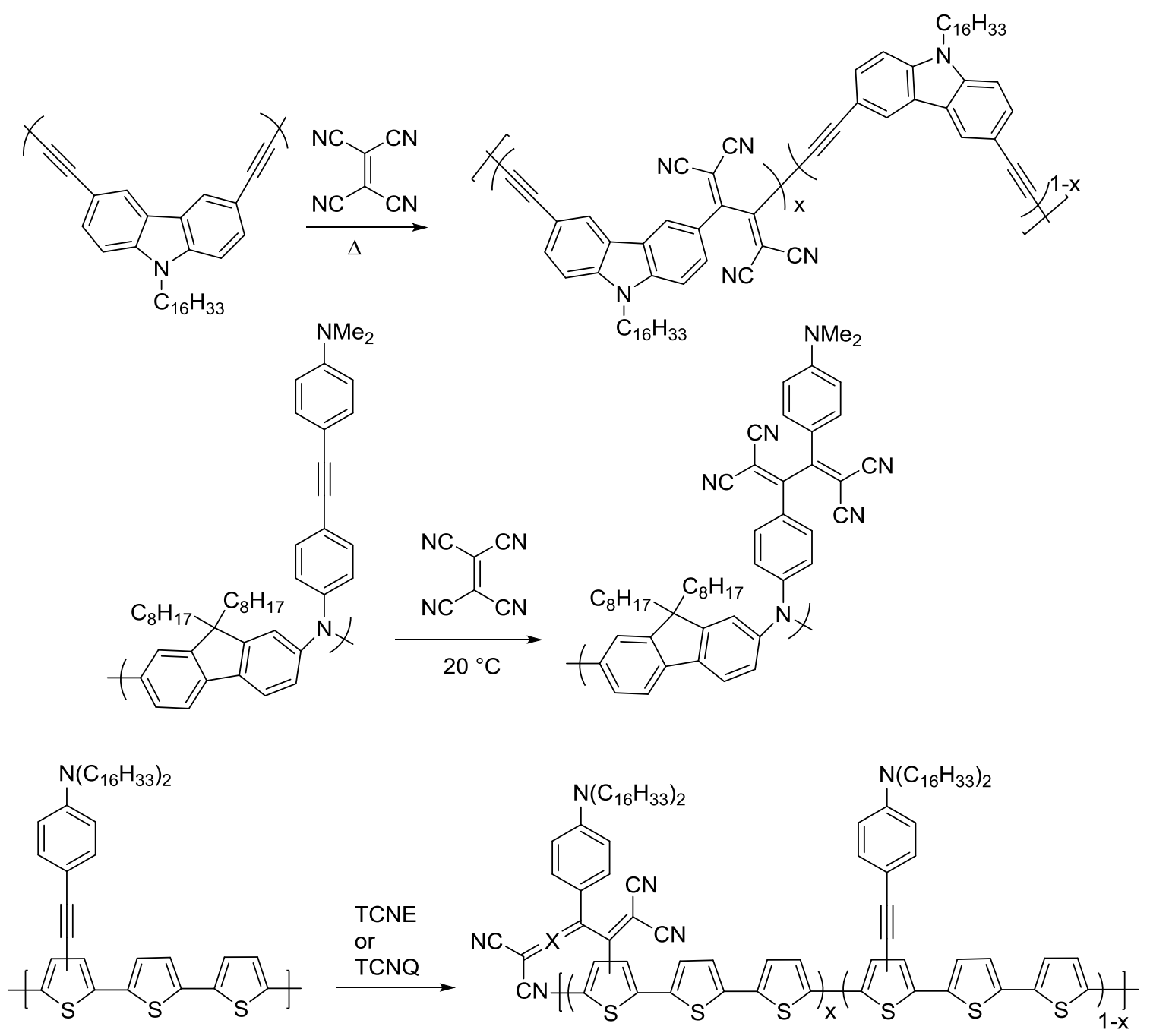

Figure 1.16 Polymers before and after addition of TCNE and TCNQ. 


\subsection{Photovoltaics}

Photovoltaic is the process to convert the sun light into direct current by using semiconducting materials that exhibit the photovoltaic effect. A photovoltaic effect is observed when electrons from the valence band are excited to the conduction band upon exposure to light. In inorganic materials absorption of photons with energies higher than the band gap creates direct free charges (electron and hole) whereas in organic materials it creates excitons which are coulombically bound electron and hole pairs. The binding energy of the exciton is very high, which prevents dissociation of the exciton. Organic materials characteristically have much lower dielectric constant $\left(\varepsilon_{\mathrm{r}} \approx 2-4\right)$ than their inorganic counterparts. ${ }^{38 c, 59}$ Dielectric constant measures the ability of a substance to store electrical energy in an electric field. Metal has high dielectric constant like infinite, if a metal is placed in between two charges no force will go through the metal. Therefore, organic semiconducting polymers provide lower efficiencies in comparison to their inorganic counterpart. In OPV devices, there are two working principle modes. Firstly, the photovoltaic mode, where under the zero bias, the flow of photocurrent of the device is restricted and the voltage builds-up. This voltage build up leads to electrical power release and is the main principle of solar cells. Secondly, under a reverse bias consumes power to detect a signal and the device are generally called photodetector. ${ }^{59 a, d}$ The device architecture for organic solar cells and photodetectors is same. However, the figure of merits is different. Solar cells depend on $V_{\mathrm{oc}}, J_{\mathrm{sc}}$, PCE, solar intensity and large surface area, whereas the photodetectors require small surface area, low noise and low power consumption. ${ }^{59 b, c, e}$ 


\subsection{Photodetector}

A photodetector is a device that converts an optical signal to an electrical signal in the form of current or voltage. Currently, organic photodetectors (OPDs) are in their initial stage of development and require more research to compete with the Si-based photodetectors. The construction of OPD is same as photovoltaic device and the working principle is based on photoelectric effect, absorption of photons - generation of excitons migration of excitons - charge separation and extraction of the separated electrons and holes to the respective electrodes. The typical photodetector performance parameters are spectral response, external quantum efficiency (EQE), responsivity $(\mathrm{R})$, noise equivalent power (NEP), detectivity (D*) and dark current which are described below.

Spectral response: For any given photodetector, OPD's can only respond to a certain wavelength range. When designing a OPD, the first consideration is choice of the proper material that can respond to the photo signal. There are different spectral ranges of OPDs, such as ultraviolet-visible range and near-infrared range. ${ }^{60,61,7}$

External quantum efficiency (EQE): EQE can be defined as the ratio of the number of photogenerated charge carriers, in the form of either photoelectrons or electron-hole pairs, to the number of incident photons. The term gain is used when EQE is larger than $100 \%$. If the gain is 10 then EQE is $1000 \%$. If EQE is less than $100 \%$ then there is no gain. The deviation of obtained spectrum of EQE from real square-shape is due to the recombination. $^{60}$ 
Responsivity (R): $\mathrm{R}$ is defined as the ratio of photocurrent or voltage to the incident-light intensity and it indicates the output signal for a given signal and how effective the device responds to optical signal. $\mathrm{R}=J_{\mathrm{ph}} / L_{\text {light }}$, where $J_{\mathrm{ph}}$ is the photocurrent and $L_{\text {light }}$ is the incident light intensity. It is important to a material to have high $\mathrm{EQE}$ because responsivity is proportional to EQE (quantum yield). ${ }^{60,62,7}$

Noise Equivalent Power (NEP): NEP is defined as the input power required of the input signal for the signal-to-noise ratio (SNR) to be unity at the detector output. It is the minimum impinging power required for a PD to distinguish signal from the noise. A photodetector with smaller NEP can detect weaker light than the photodetector with a larger NEP. NEP $=(\mathrm{A} \Delta \mathrm{f})^{1 / 2} / \mathrm{D}^{*}$, where $\mathrm{A}$ is the effective area of the OPD in $\mathrm{cm}^{2}, \Delta \mathrm{f}$ is the electrical bandwidth in $\mathrm{Hz}$ and $\mathrm{D}^{*}$ is the detectivity measured in units Jones. ${ }^{60,7}$

Detectivity $\left(\mathbf{D}^{*}\right)$ : The detectivity of a OPD indicates the ability to detect a small photo signal. $\mathrm{D}^{*}=(\mathrm{A} \Delta \mathrm{f})^{1 / 2} \mathrm{R} / i_{\mathrm{n}}$ where $\mathrm{R}$ the responsivity in $\mathrm{A} / \mathrm{W}$ and noise current in (in amperes). There are three contributions to noise that limits $\mathrm{D}^{*}$. Among them, the shot noise from the dark current is the major contribution. Therefore, the detectivity can be expressed as $\mathrm{D}^{*}=\mathrm{R} /\left(2 \mathrm{q} J_{\mathrm{d}}\right)^{1 / 2}=\left(J_{\mathrm{ph}} / L_{\text {light }}\right) /\left(2 \mathrm{q} J_{\mathrm{d}}\right)^{1 / 2}$ where, $2 \mathrm{q} J_{\mathrm{d}}$ is the short noise power spectral density, q is the absolute value of electron charge and $J_{\mathrm{d}}$ is the dark current. ${ }^{60,7}$

Dark Current: Dark current is the constant current when there is no radiation. The current measured in the device at reverse bias and without light must be minimized in order to increase the limit of detection and minimize power consumption. ${ }^{61}$ 


\subsection{Organic polymer photodetectors}

Photodetectors from UV to NIR have industrial and scientific applications, including optical communications, remote controls, day/night surveillance and chemical/biological sensing. ${ }^{7}$ Recently, GaN-, Si-, and InGaAs-based inorganic detectors have been reported for the three important sub-bands, $0.25 \mathrm{~mm}$ to $0.4 \mathrm{~mm}$ (UV), $0.45 \mathrm{~mm}$ to $0.8 \mathrm{~mm}$ (visible) and $0.9 \mathrm{~mm}$ to $1.7 \mathrm{~mm}$ (NIR), respectively. The detectivities of silicon photodetectors are in $4 \times 10^{12}$ Jones $(1 \mathrm{Jones}=1 \mathrm{cmHzl} / 2 / \mathrm{W})$ while, detectivities of InGaAs photodetectors are greater than $10^{12}$ Jones when cooled to $4.2 \mathrm{~K}^{60 \mathrm{a}}$ Inorganic photodetectors must be tuned in the optical band gap for broadband (UV, visible to NIR) spectrum sensing, thus highlighting a major limitation. Therefore, organic polymeric materials are an alternative due to their wide range of absorption properties and ease of tuning the band gap.

Huang et al. reported a photodetector for the ultraviolet spectral range where they used a non-conjugated polymer, poly(9-vinylcarbazole). This polymer has a very high band gap of $3.5 \mathrm{eV}$. The detectivity was reported as $3.4 \times 10^{15}$ Jones at $360 \mathrm{~nm}$ and room temperature, which was two to three orders of magnitude higher than that of existing inorganic semiconductor ultraviolet photodetectors. ${ }^{63}$ Fluorene-based polymers are used as photodetectors for the ultraviolet range and the reported EQE is for 7 to $24 \%{ }^{63}$

P3HT is a well studied semiconducting polymer. Chaudhary et. al reported P3HT and fullerene-based OPD with high carrier mobility and EQE about 70\% from a spectral range of $400-700 \mathrm{~nm}^{64 \mathrm{a}} \mathrm{Kim}$ et al. also reported visible spectral range (350 to $650 \mathrm{~nm}$ ) OPD with P3HT in blends with a non-fullerene, solution-processable indigo acceptor with relatively flat EQE of near $80 \%$, R of $0.4 \mathrm{~A} / \mathrm{W}$, and a $D^{*}$ of near $10^{12}$ Jones. $^{64 \mathrm{~b}}$ Yang 
et al. have shown that P3HT and fullerene based OPV devices with junction thicknesses of several micrometers was able to achieve a NIR photo-response by utilizing absorption by charge-transfer states, although the devices were not particularly efficient. ${ }^{64 c}$ In 2015 , photomultiplication (PM)-type polymer photodetectors based on P3HT polymer were reported with very high $\mathrm{EQE} .{ }^{64 d, e}$ A visible spectral range $(350$ to $650 \mathrm{~nm})$ OPD with carbazole-based polymer blended with fullerene was reported for constant EQE near 70\% at $-1 \mathrm{~V}, D^{*}$ of near $10^{13}$ Jones and low dark current, less than $1 \mathrm{nA} / \mathrm{cm}^{2}{ }^{65}$ Especially small molecules have been reported as active materials for red-light sensitive OPDs. Phthalocyanines-, squaraines- and porphyrins-based compounds are very common for red-light $(600-800 \mathrm{~nm})$ sensitive OPD. ${ }^{63}$ Thieonothiadiazole-based polymers showed high detectivity $1.4 \times 10^{12}$ Jones in 700 to $800 \mathrm{~nm}$ with low dark current. However, the EQE was $15 \% .{ }^{66 a}$ Thiadiazolobenzotriazol-based polymer showed spectral response from 400 to $1100 \mathrm{~nm}, D^{*}$ of upto $1.75 \times 10^{13}$ Jones, low dark current of $1.25 \times 10^{-10} \mathrm{~A} / \mathrm{cm}^{2}$ and EQE of $16 \%$ at $800 \mathrm{~nm} .{ }^{66 \mathrm{~b}}$ Development of wide spectral range of polymers for OPDs with high detectivity is still challenging. Yao et al. explored TT-based polymer OPD blended with fullerene derivative with broad spectral range from 400 to $1000 \mathrm{~nm}$. The detector exhibited an average EQE of $10 \%$ for a spectral range of 700-900 nm under $0 \mathrm{~V}$ bias, and an average EQE of $40 \%$ under $-5 \mathrm{~V}$ bias. The response bandwidth was $4 \mathrm{MHz}$ and NEP of $3.85 \times 10^{-12} \mathrm{~W} / \mathrm{H}^{1 / 2}$ at $850 \mathrm{~nm}$ was reported. ${ }^{67 \mathrm{a}}$ Same year Inganäs et al. reported a new polymer based on thiadiazoloquinoxaline pushing the response tail to $1200 \mathrm{~nm} .{ }^{67 b}$ Heeger et al. synthesized a thieonothiadiazole-based low band gap polymer (Figure 1.17) and demonstrated its photodetector properties when blended with a fullerene derivative with a broad spectral response (300 to 1450 nanometers). The 
polymer exhibited detectivity greater than $10^{13}$ Jones at wavelengths from $300 \mathrm{~nm}$ to 1150 $\mathrm{nm}$ and greater than $10^{12}$ Jones from $1150 \mathrm{~nm}$ to $1450 \mathrm{~nm} \cdot{ }^{60 \mathrm{a}}$ This polymer performance was comparable to their inorganic counter parts ( $\mathrm{Si}$ - and GaInAs-based detector). TTbased OPD was reported in 2013 by Simonato et al. with detectivity over $10^{12}$ Jones in the visible spectrum to $800 \mathrm{~nm}$ and with longer stability. ${ }^{67 \mathrm{c}}$

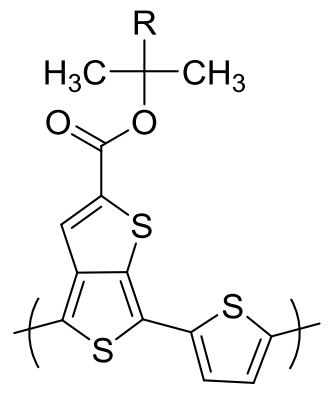

PTT

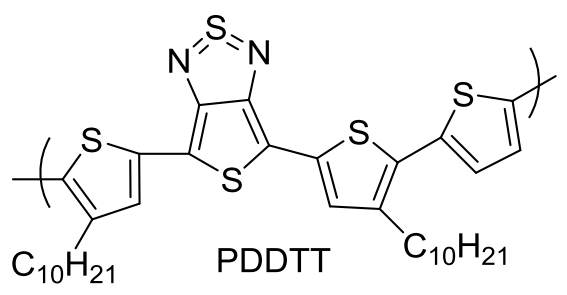

Figure 1.17 Structures of donor polymers used in BHJ photodetectors.

\subsection{Fluorescent property of conjugated polymers}

Conjugated polymers possess exceptional electronic and optical properties due to their delocalized electrons. Conjugated polymers are highly fluorescent in solution and in the solid state. The delocalized electrons are responsible for exciton migration in their excited state through the backbone. This migration increases the frequency of interaction with the bound quencher, resulting in higher sensitivity when compared to their small molecular sensor. Therefore, they have been used as signal-transducing elements for sensory application. Conjugated polymers are well-established in sensing nitroaromatics. $^{68 \mathrm{a}, \mathrm{b}}$ Tetraphenylethene (TPE) is one of the most studied luminophores. TPE and its polymers are weakly emissive in lower volume fraction of poor solvent but it shows higher emission when the poor solvent reaches to higher volume fraction. In the 
presence of poor solvent and in the solid state the intramolecular rotations of its aryl rotors are greatly restricted which then blocks the non-radiative pathway and opens up the radiative channel and makes it abnormally emissive. TPE-based fluorescent conjugated polymers are used for sensing different analytes and nitro-aromatics (Figure 1.18). ${ }^{68 c-e}$
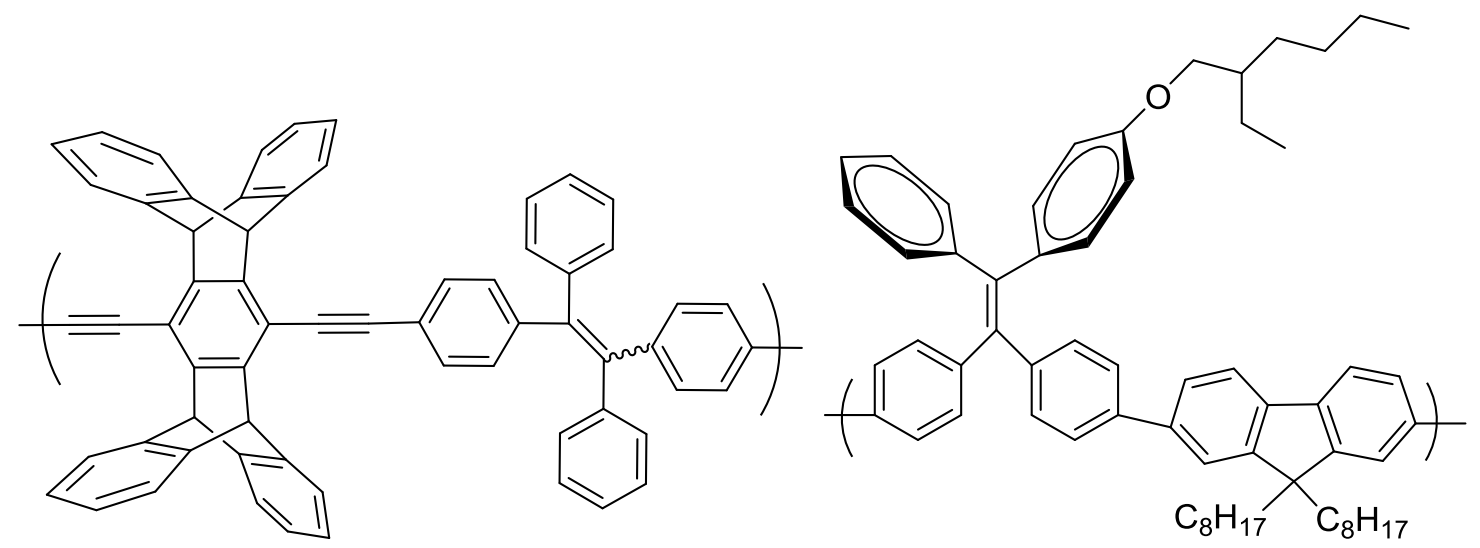

Figure 1.18 Structures of TPE-based conjugated polymers.

\subsection{Rationale and Objectives}

Conjugated polymers possess semiconducting properties caused by the delocalized electrons in an alternating overlapped orbital along the polymer backbone. Different types of conjugated polymers such as the homopolymers of donor and acceptor, donor-acceptor, acceptor-acceptor, and ladder polymer based on their applications (photovoltaic devices, plastic transistors, non-linear optical devices, light-emitting displays and sensing analytes including explosives, isocyanates, and heavy metals) have been reported in the literature. Synthesis of low band gap polymers with an absorption in the NIR region is still challenging to the researchers. Tailoring of band gaps in conjugated polymers is very common to enhance the properties. To get new low band gap polymers, one must design and synthesize new donor or acceptor monomers, which may 
not provide the desired result. However, synthesizing a monomer with a pre-existing large-ladder structure may not provide a high molecular-weight polymer because of solubility issues. Therefore, post-polymerization annulation could be an interesting choice to synthesize a new ladder polymer with a low band gap, a broader absorption spectrum, and fluorescence. Moreover, polymer modification could be another choice to tune the band gap, absorption, and fluorescence properties.

Intramolecular cyclization is an effective way to lock the rotation between monomer units in polymers and produce a new ladder polymer. Previously, Wang et al reported intramolecular cyclization of neighboring keto groups using LR. ${ }^{69 a, b}$ LR in a heating dissociate in disulfide phosphine, which reacts with the carbonyl functional group to form 4-membered rings and decomposes to thioketones. At a high temperature these thioketones formed four-membered $\mathrm{C}-\mathrm{S}$ rings, which decompose to $\mathrm{C}=\mathrm{C}$ bond by evolving $\mathrm{S}_{2}{ }^{69 \mathrm{c}}$ Anthracenes were synthesized from anthraquinones via successive reductions and dehydrations by Criswell and Klanderman. ${ }^{69 d}$ Therefore, sequential reduction, dehydration, and esterification in the keto group of a polymer will produce different polymers with different optoelectronic properties. Further, modified Knoevenagel condensation in the keto group of the polymer will produce different polymers with different optoelectronic properties. Fused, conjugated monomer units can not only tune the electronic properties but also affect the charge mobility and intermolecular interaction.

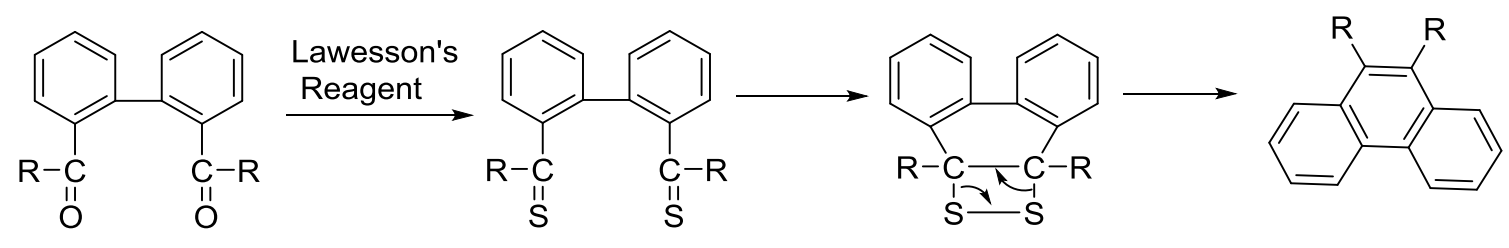


Based on Wang's work, a new monomer with multi-fused rings and 4 functional groups, including 2 keto groups which can form 6-a membered conjugated ring after intramolecular cyclization in its polymer, is required to synthesize a low band gap ladder polymer. The presence of the keto group will offer an opportunity for polymer modification by reduction, dehydration, esterification and Knoevenagel condensation.

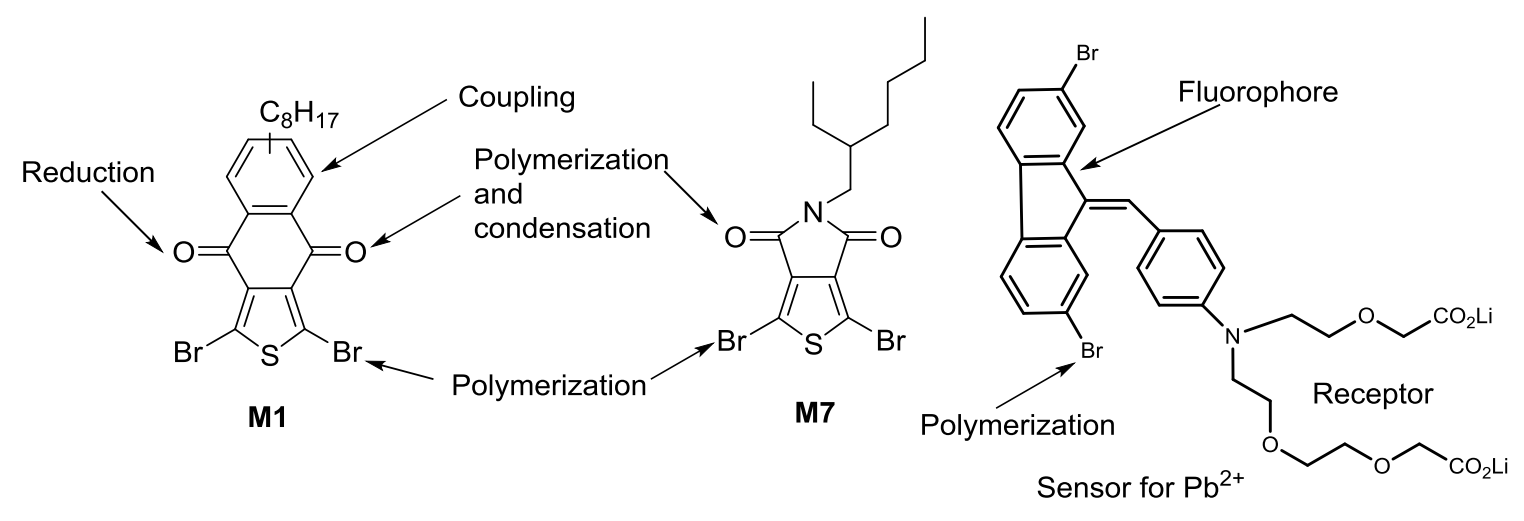

Therefore, this thesis describes to use 1,3-dibromo-8-octylnaptho[2,3c]thiophene-4,9-dione and 1,3-dibromo-5-(2-ethylhexyl)-thieno[3,4-c]pyrrole-4,6-dione as 2 monomers to synthesize polymers. Both monomers contain 2 bromines as a functional group in the thiophene unit and 2 keto groups as another functional group. The polymerization on the thiophene unit will provide a linear polymer, and intramolecular cyclization (using LR or McMurry reaction) will produce a ladder polymer. The ladder polymer can be synthesized by keto-keto coupling, followed by Ullmann reaction on the thiophene unit. The reduction (using $\mathrm{NaBH}_{4}$ ), dehydration (using acid) and esterification (using EtOH) as well as the Knoevenagel condensation (using malononitrile) reaction on the keto group of the polymers will produce new polymers with new properties. Conjugated polymers show a fluorescent property, and this property is used for sensing; therefore, the sensing property of a conjugated polymer can be tuned by introducing any receptor part into the conjugated backbone. 
Based on the above rationale, the objectives of this thesis research are as follows:

1. to synthesize and characterize the conjugated ladder polymers;

2. to adjust the properties of conjugated ladder polymers by synthesizing linear-ladder polymers with different molar ratios of thiophene and benzothiadiazole;

3. to modify the properties of a homopolymer and D-A polymers by reactions on the keto group;

4. to synthesize a new conjugated fluorescent polymer for the detection $\mathrm{Pb}^{2+}$ in water. 


\subsection{References}

1. Shirakawa, H.; Louis, E. J.; MacDiarmid, A. G.; Chiang C. K.; Heeger, A. J. Chem. Commun. 1977, 578.

2. $\quad$ Chiang, C. K.; Fincher, C.R.; Park, Jr., Y.W.; Heeger, A. J.; Shirakawa H.; Louis, E. J. Phys. Rev. Lett. 1977, 39, 1098.

3. Heeger, A. J. Angew. Chem. Int. Ed. 2001, 40, 2591.

4. (a) Xu, T.; Yu, L. Mater. Today, 2014, 17, 11. (b) Costa, J. C. S.; Taveira, R. J. S.; Lima, C. F. R. A. C.; Mendes, A.; Santos, L. M. N. B. F. Optical Mater. 2016, 58, 51. (c) Virkar, A. Introduction to Organic Semiconductors Transistors and Conductors, Investigating the Nucleation, Growth, and Energy Levels of Organic Semiconductors for High Performance Plastic Electronics, Springer Science \& Business Media: New York, USA, 2011; pp 3-4.

5. (a) Grimsdale, A. C.; Chan K. L.; Martin, R. E.; Jokisz, P. G.; Holmes, A. B. Chem. Rev. 2009, 109, 897. (b) Wang, C.; Dong, H.; Hu, W.; Liu, Y.; Zhu, D. Chem. Rev. 2012, 112, 2208. (c) Dou, L.; Liu, Y.; Hong, Z.; Li, G.; Yang, Y. Chem. Rev. 2015, 115, 12633. (d) Lu, L.; Zheng, T.; Wu, Q.; Schneider, A. M.; Zhao, D.; Yu, L. Chem. Rev. 2015, 115, 12666. (e) Yen, H.-J.; Shan, C.; Wang, L.; Xu, P.; Zhou, M.; Wang, H.-L. Polymers, 2017, 9, 25. (f) Muench, S.; Wild, A.; Friebe, C.; Haupler, B.; Janoschka, T.; Schubert, U. S. Chem. Rev. 2016, 116, 9438. (g) Marchioni, F.; Yang, J.; Walker, W.; Wudl, F. J. Phys. Chem. B, 2006, 110, 22202. (h) Cowen, L. M.; Atoyo, J.; Carnie, M. J.; Baran, D.; Schroeder, B. C. ECS J. Solid State Sci. and Tech. 2017, 6, N3080. (i) Kroon, R.; Mengistie, 
D. A.; Kiefer, D.; Hynynen, J.; Ryan, J. D.; Yu, L.; Muller, C. Chem. Soc. Rev. 2016, 45, 6147.

6. Roncali, J. Chem. Rev. 1997, 97, 173.

7. Liu, C.; Wang, K.; Gong, X.; Heeger, A. J. Chem. Soc. Rev. 2016, 45, 4825.

8. (a) Brédas, J. L. J. Chem. Phys. 1985, 82, 3808. (b) Cheng, Y.-J.; Yang, S.-H.; Hsu, C.-S. Chem. Rev. 2009, 109, 5868. (c) Kitamura,C.; Tanaka, S.; Yamashita, Y. Chem. Mater. 1996, 8, 570.

9. (a) Hernandez, V.; Castiglioni, C.; Zopo, M. D.; Zerbi, G. Phys. Rev. B 1994, 50, 9815. (b) Hess, B. A. Jr.; Schaad, L. J. J. Am. Chem. Soc. 1971, 93, 305. (c) Hess, B. A. Jr.; Schaad, L. J. J. Am. Chem. Soc. 1973, 95, 39

10. (a). Brédas, J. L.; Street, G. B.; Themans, B.; André, J. M. J. Chem. Phys. 1985, 83, 1323. (b) Roncali, J. Macromol. Rapid Commun. 2007, 28, 1761. (c) Xu, B.; S. Holdcroft, S. Macromolecules, 1993, 26, 4457. (d) McCullough, R. D.; Lowe, R. D.; Jayraraman, M.; Anderson, D. L. J. Org chem. 1993, 58, 904.

11. (a) Lowe, J. P.; Kafafi, S. A. J. Am. Chem. Soc. 1984, 106, 5837. (b) Huo,L.; Li, Z.; Guo, X.; Wu, Y.; Zhang, M.; Ye, L.; Zhang, S.; Hou, J. Polym. Chem. 2013, 4, 3047. (c) Pal, B.; Yen, W.-C.; Yang, J.-S.; Su, W.-F. Macromolecules, 2007, 40, 8189. (d) Szarko, J. M.; Guo, L.; Liang, Y.; Lee, B.; Rolczynski, B. S.; Strzalka, J.; Zu, T.; Loser, S.; Marks, T. J.; Yu, L.; Chen, L. X. Adv. Mater., 2010, 22, 5468. (e) Biniek, L.; Fall, S.; Chochos, C. L.; Anokhin, D. V.; Ivanov, D. A.; Leclerc, N.; Leveque, P.; Heiser, T. Macromolecules, 2010, 43, 9779.

12. (a) Kim, N.; Lee, B. H.; Choi, D.; Kim, G.; Kim, H.; Kim, J.-R.; Lee, J.;. Kahng, Y. H; Lee, K. Phys. Rev. Lett. 2012, 109, 106405. (b) Su, Y.-W.; Lan, S.-C.; Wei, 
K.-H. Mater. Today. 2012, 15, 554. (c) Woo, C. H.; Thompson, B. C.; Kim, B. J.; Toney, M. F.; Fréchet, J. M. J. J. Am. Chem. Soc. 2008, 130, 16324.

13. Salzner, U.; Lagowski, J. B.; Pickup, P.G.; Poirier, R.A. Synth. Met. 1998, 96, 177

14. Kim, Y.; Cook, S.; Tuladhar, S. M.; Choulis, S. A.; Nelson, J.; Durrant, J. R.; Bradley, D. D. C.; Giles, M.; McCulloch, I.; Ha, C.-S.; Ree, M. Nature Mater. 2006, 5, 197.

15. Mauer, R.; Kastler, M.; Laquai, F. Adv. Funct. Mater. 2010, 20, 2085.

16. Holliday, S.; Ashraf, R. S.; Wadsworth, A.; Baran, D.; Yousaf, S. A; Nielsen, C. B.; Tan, C.-H.; Dimitrov, S. D.; Shang, Z.; Gasparini, N.; Alamoudi, M.; Laquai, F.; Brabec, C. J.; Salleo, A.; Durrant, J. R.; McCulloch, I. Nat. Commun. 2016, 7, 1.

17. Schilinsky, P.; Asawapirom, U.; Scherf, U.; Biele, M.; Brabec, C. J. Chem. Mater. 2005, 17, 2175.

18. (a) Ma, W.; Kim, J. Y.; Lee, K.; Heeger, A. J. Macromol. Rapid Commun. 2007, 28, 1776. (b) Dennler, G.; Scharber, M. C.; Brabec, C. J. Adv. Mater. 2009, 21, 1323.

19. Kobayashi, M.; Colaneri, N.; Boysel, M.; Wudl, F.; Heeger, A. J. J. Chem. Phys. 1985, 82,5717 .

20. Ikenoue,Y.; Wudl, F.; Heeger, A. J. Synth. Met.1991, 40, 1

21. Pomerantz, M.; Chaloner-Gill, B.; Harding, L. O.; Tseng, J. J.; Pomerantz, W. J. Synth. Met. 1993, 55-57, 960.

22. Pomerantz, M.; Xiaormin, G. Synth. Met. 1997, 84, 243. 
23. Arbizzani, C.; Catellani, M.; Cerroni, M. G.; Mastragostoni, M. Synth. Met. 1997, $84,249$.

24. Brisset, H.; Thobie-Gautier, C.; Gorgues, A.; Jubault, M.; Roncali, J. J. Chem. Soc., Chem. Commum. 1994, 1305.

25. Orti, E.; Sanchis, M. J.; Viruela, P. M.; Vituela, R. Synth. Met. 1999, 101, 602.

26. Roncali, J.; Thobie-Gautier, C. Adv. Mater. 1994, 6, 846.

27. Zoombelt, A. P.; Mathijssen, S. G. J.; Turbiez, M. G. R.; Wienka, M. M.; Janssen, R. A. J. J. Mater. Chem. 2010, 20, 2240.

28. Guo, X.; Puniredd, S. R.; He, B.; Marszalek, T.; Baumgarten, M.; Pisula, W.; Müllen, K. Chem. Mater. 2014, 26, 3595.

29. Kanimozhi, C.; Yaacobi-Gross, N.; Chou, K. W.; Amassian, A.; Anthopoulos, T. D. Patil, S. J. Am. Chem. Soc. 2012, 134, 16532.

30. Stalder, R.; Mei, J.; Subbiah, J.; Grand, C.; Estrada, L. A.; So, F.; Reynolds, J. R. Macromolecules, 2011, 44, 6303.

31. (a) Xiao, S.; Stuart, A. C.; Liu, S.; Zhou, H.; You, W. Adv. Funct. Mater. 2010, 20, 635. (b) Kang, T. E.; Kim, T.; Wang, C.; Yoo, S.; Kim, B. J. Chem. Mater. 2015, 27, 2653.

32. Lee, J.; Kalin, A. J.; Yuan, T.; Al-Hashimic, M.; Fang, L.; Chem. Sci. 2017, 8, 2503.

33. (a) Van Deusen, R. L. J. Polym. Sci., Part B: Polym. Lett. 1966, 4, 211. (b) Arnold, F. E.; Van Deusen, R. L J. Appl. Polym.Sci. 1971, 15, 2035. (c) Jenekhe S. A.; Yi. S. Appl. Phys. Lett. 2000, 77, 2635. (d) Alam, M. M.; Jenekhe, S. A. J. Phys. Chem. B, 2002, 106, 11172. (e) Yuan, Z.; Xiao, Y.; Yang, Y.; Xiong, T, 
Macromolecules, 2011, 44, 1788. (f) Bheemireddy, S. R.; Hautzinger, M. P.; Li, T.; Lee, B.; Plunkett, K. N. J. Am. Chem. Soc. 2017, 139, 5801. (g) Dierschke, F.; Grimsdale, A. C.; Mullen, K. Macromol. Chem. Phys. 2004, 205, 1147. (i) Chen, Y.; Huang, W.; Li, C.; Bo, Z. Macromolecules, 2010, 43, 10216. (j) Chmil, K.; Scherf, U. Acta Polymer, 1997, 48, 208. (k) Babel, A.; Jenekhe, S. A. J. Am. Chem. Soc. 2003, 125, 13656.

34. (a) Schluter, A. D.; Loffler, M.; Enkelmann, V. Nature, 1994, 368, 831. (b) Schlicke, B.; Schirmer, H.; Schluter, A. D. Adv. Mater.1995, 7, 544.

35. Alam, M. A.; Jenekhe, S. A. Chem. Mater. 2004, 16, 4647.

36. Yuan, Z.; Xiao, Y.; Yang, Y.; Xiong, T. Macromolecules, 2011, 44, 1788.

37. (a) Havinga, E. E.; ten Hoeve, W.; Wynberg, H. Synth. Met. 1993, 55 299. (b) Ajayaghosh, A. Chem. Soc. Rev. 2003, 32 181. (c) Zhang, Q. T.; Tour, J. M. J. Am. Chem. Soc. 1998, 1205355.

38. (a) Zhou, H.; Yang, L.; You, W. Macromolecules, 2012, 45, 607. (b) Li, Y. Acc. Chem. Res. 2012, 45, 723. (c) Li, G.; Zhu, R.; Yang, Y. Nature Photonics, 2012, 6, 153 (d) Wu, J.-S.; Cheng, S.-W.; Cheng, Y.-J.; Hsu, C.-S. Chem. Soc. Rev. $\mathbf{2 0 1 5}, 44,1113$.

39. Zhou, H.; Yang, L.; Stoneking, S.; You, W. ACS Appl. Mater. Interfaces, 2010, 2,1377 .

40. Zhang, Z.-G.; Wang, J. J. Mater. Chem. 2012, 22, 4178.

41. (a) Wang, E.; Wang, L.; Lan, L.; Luo, C.; Zhuang, W.; Peng, J.; Cao, Y. Appl. Phys. Lett. 2008, 92, 033307. (b) Allard, N.; Aïch, R. d. B.; Gendron, D.; 
Boudreault, P.-L. T.; Tessier, C.; Alem, S.; Tse, S-C.; Tao, Y.; Leclerc, M. Macromolecules, 2010, 43, 2328.

42. (a) Amb, C. M.; Chen, S.; Graham, K. R.; Subbiah, J.; Small, C. E.; So, F.; Reynolds, J. R. J. Am. Chem. Soc. 2011, 133, 10062. (b) Coffin, R. C.; Peet, J.; Rogers, J.; Bazan, G. C. Nature Chem. 2009, 1, 657.

43. Son, H. J.; Lu, L.; Chen, W.; Xu, T.; Zheng, T.; Carsten, B.; Strzalka, J.; Darling, S. B.; Chen, L. X.; Yu, L. Adv. Mater. 2013, 25, 838.

44. Gibson, G. L.; McCormick, T. M.; Seferos, D. S. J. Am. Chem.Soc. 2012, 134, 539.

45. Price, S. C.; Stuart, A. C.; Yang, L.; Zhou, H.; You, W. J. Am. Chem. Soc. 2011, 133, 4625. (b) Zhong, W.; Xiao, J.; Sun, S.; Jiang, X.-F.; Lan, L.; Ying, L.; Yang, W.; Yip, H.-L. Huang, F.; Cao, Y. J. Mater. Chem. C, 2016, 4, 4719.

46. (a) Blouin, N.; Michaud, A.; Gendron, D.; Wakim, S.; Blair, E.; Neagu-Plesu, R.; Belletete, M.; Durocher, G.; Tao, Y.; Leclerc, M. J. Am. Chem. Soc. 2008, 130, 732. (b) Hou, J.; Park, M.-H.; Zhang, S.; Yao, Y.; Chen, L.-M.; Li, J.-H.; Yang, Y. Macromolecules, 2008, 41, 6012.

47. (a) Steckler, T. T.; Henriksson, P.; Mollinger, S.; Lundin, A.; Salleo, A.; Andersson, M. R. J. Am. Chem. Soc. 2014, 136, 1190. (b) Fan J.; Yuen , J. D.; Wang , M.; Seifter, J.; Seo, J.-H.; Mohebbi, A. R.; Zakhidov , D.; Heeger , A.; Wudl, F. Adv. Mater. 2012, 24, 2186.

48. (a) Helbig, M.; Hörhold, H.-H. Makromol. Chem. 1993, 194, 1607 (b) Greenham, N. C.; Moratti, S. C.; Bradley, D. D. C.; Friend, R. H.; Holmes, A. B. Nature, 1993, 365,628 . 
49. (a) Ho, H. A.; Brisset, H.; Frere, P.;. Roncali, J. J. Chem. Soc. Chem.Commun. 1995, 2309. (b) Casey, A.; Dimitrov, S. D.; Shakya-Tuladhar, P.; Fei, Z.; Nguyen, M.; Han, Y.; Anthopoulos, T. D.; Durrant, J. R.; Heeney, M. Chem. Mater. 2016, 28, 5110.

50. (a) Zhou, H.; Yang, L.; Stuart, A. C.; Price, S. C.; Liu, S.; You, W. Angew. Chem. Int. Ed. 2011, 50, 2995. (b) Price, S. C.; Stuart, A. C.; Yang, L.; Zhou, H.; You, W. J. Am. Chem. Soc. 2011, 133, 4625.

51. (a) Yao, H.; Ye, L.; Zhang, H.; Li, S.; Zhang, S.; Hou, J. Chem. Rev. 2016, 116, 7397.(b) Gao, Y.; Liu, M.; Zhang, Y.; Liu, Z.; Yang, Y.; Zhao, L. Polymers, 2017, 9, 39 .

52. Jen, K.-Y.; Maxfield, M. R.; Shacklette, L. W.; Elsenbaumer, R. L. J. Chem. Soc., Chem. Commun. 1987, 309.

53. (a) Wang, X.; Sun, Y.; Chen, S.; Guo, X.; Zhang, M.; Li, X.; Li, Y.; Wang, H. Macromolecules, 2012, 45, 1208. (b) Lan, S.-C.; Yang, P.-A.; Zhu, M.-J.; Yu, C.M.; Jiang, J.-M.; Wei, K.-H. Polym. Chem. 2013, 4, 1132.

54. Ghosh, K. R.; Saha, S. K.; Wang, Z. Y. Polym. Chem. 2014, 5, 5638.

55. (a) Yang, L.; Zhou, H.; You, W.; J. Phys. Chem. C, 2010, 114, 16793. (b) Mei, J.; Bao, Z.; Chem. Mater. 2014, 26, 604. (c) Piliego, C.; Holcombe, T. W.; Douglas, J. D.; Woo, C. H.; Beaujuge, P. M.; Frechet, J. M. J. J. Am. Chem. Soc. 2010, 132, 7595.

56. (a) Gadisa, A.; Oosterbaan, W. D.; Vandewal, K.; Bolsee, J.-C.; Bertho, S.; D’Haen, J.; Lutsen, L.; Vanderzande, D.; Manca, J. V. Adv. Funct. Mater. 2009, 19, 3300. (b) Egbe, D. A. M.; Türk, S.; Rathgeber, S.; Kühnlenz, F.; Jadhav, R.; 
Wild, A.; Birckner, E.; Adam, G.; Pivrikas, A.; Cimrova, V.; Knör, G. N.; Sariciftci, N. S.; Hoppe, H. Macromolecules, 2010, 43, 1261. (c) Zhou, H.; Yang, L.; Xiao, S.; Liu, S.; You, W. Macromolecules, 2010, 43811.

57. (a) Michinobu, T. Chem. Soc. Rev. 2011, 40, 2306. (b) Yamaguchi, S.; Tatemitsu, H.; Sakata, Y.; Misumi, S. Chemistry Letters, 1983, 1229. (c) Ferraris, J.; Cowan, D. O.; Walatka, V.; Perlstein, J. H. J. Am. Chem. Soc. 1973, 95, 948. (d) Jerome, D.; Mazaud, M.; Ribault, M.; Bechgaard, K. J. Phys. Let. 1980, 41, L95.

58. (a) Michinobu, T.; Kumazawa, H.; K. Noguchi, K.; Shigehara, K. Macromolecules, 2009, 42, 5903. (b) Michinobu, T.; Fujita, H.; Materials, 2010, 3, 4773. (c) Michinobu, T. J. Am. Chem. Soc. 2008, 130, 14074. (d) Yuan Y.; Michinobu, T. J. Polym. Sci. Part A: Polym. Chem. 2011, 49, 225.(e) Jung, I. H.; Lo, W.-Y.; Jang, J.; Chen, W.; Zhao, D.; Landry, E. S.; Lu, L.; Talapin, D. V.; Yu, L. Chem. Mater. 2014, 26, 3450.

59. (a) Peumans, P.; Yakimov, A.; Forrest, S. R. J. Appl. Phys. 2003, 93, 3693. (b) Peumans, P.; Bulovic, V.; Forrest, S. R. J. Appl. Phys. 2000, 76, 2650. (c) Peumans, P.; Bulovic, V.; Forrest, S. R. J. Appl. Phys. 2000, 76, 3855. (d) Mazzio, K. A.; Luscombe, C. K. Chem. Soc. Rev. 2015, 44, 78. (e) Mia, S. Development of low band gap donor-acceptor polymers for use in optoelectronics. Ph.D. Dissertation, Carleton University, Ottawa, ON, Canada, April 2016.

60. (a) Gong, X.; Tong, M.; Xia, Y.; Cai, W.; Moon, J. S.; Cao, Y.; Yu, G.; Shieh, C. L.; Nilsson, B; Heeger, A. J. Science, 2009, 325, 1665. (b) Guo, F. Low noise, high detectivity photodetectors based on organic materials. Ph.D. Dissertation. University of Nebraska, Lincoln, Nebraska, May, 2014 
61. Baeg, K.-J.; Binda, M.; Natali, D.; Caironi, M.; Noh, Y.-Y. Adv. Mater. 2013, 25, 4267.

62. Dou, L.; Yang, Y.; You, J.; Hong, Z.; Chang, w.-H.; Li, G.; Yang, Y. Nat. Commun. 2014, 5, 5404.

63. (a) Vuuren, R. D. J.-V.; Armin, A.; Pandey, A. K.; Burn, P. L.; Meredith, P. Adv. Mater. 2016, 28, 4766. (b) Guo, F.; Yang, B.; Yuan, Y.; Xiao, Z.; Dong, Q.; Bi, Y.; Huang, j. Nat. Nanotechnol. 2012, 7, 798

64. (a) Nalwa, K. S.; Cai, Y.; Thoeming, A. L.; Shinar, J.; Shinar, R.; Chaudhary, S.; Adv. Mater. 2010, 22, 4157. (b) Kim, I. K.; Li, X.; Ullah, M.; Shaw, P. E.; Wawrzinek, R.; Namdas, E. B.; Lo, S.-C. Adv. Mater. 2015, 27, 1521. (c) Yang, C.-M.; Tsai, P.-Y.; Horng, S.-F.; Lee, K.-C.; Tzeng, S.-R.; Meng, H.-F.; Shy, J.T.; Shu, C.-F. Appl. Phys. Lett. 2008, 92, 083504. (d) Wang, W.; Zhang, F.; Li, L.; Gao, M.; Hu, B. ACS Appl. Mater. Interfaces, 2015, 7, 22660. (e) Li, L.; Zhang, F.; Wang, J.; An, Q.; Sun, Q.; Wang, W.; Zhang, J.; Teng, F. Sci. Report. 2015, 5, 9181 .

65. Armin, A.; Hambsch, M.; Kim, I. K.; Burn, P. L.; Meredith, P.; Namdas, E. B. Laser Photonics Rev. 2014, 8, 924.

66. (a) Qi, J,; Zhou, X.; Yang, D.; Qiao, W.; Ma, D.; Wang, Z. Y. Adv. Funct. Mater. 2014, 24, 7605. (b) Hu, X.; Dong, Y.; Huan, X.; Gong, X.; Cao, Y.; J. Phys. Chem. C, 2013, 117, 6537.

67. (a) Yao, Y.; Liang, Y.; Shrotriya, V.; Xiao, S.; Yu, L.; Yang, Y. Adv. Mater. 2007, 19, 3979. (b) Perzon, E.; Zhang, F.; Andersson, M.; Mammo, W.; Inganäs, O.; Andersson, M. R. Adv. Mater. 2007, 19, 3308. (c) Saracco, E.; Bouthinon, B.; 
Verilhac, J.-M.; Celle, C.; Chevalier, N.; Mariolle, D.; Dhez, O.; Simonato, J. P. Adv. Mater. 2013, 25, 6534.

68. (a) Thomas, S. W.; Joly, G.D.; Swager, T. M. Chem. Rev. 2007, 107, 1339. (b) Cumming, C. J.; Aker, C.; Fisher, M.; Fox, M.; la Grone, M. J.; Ruest, D.; Rockley, M. G.; Swager, T. M.; Towers, E.; Williams, V. IEEE Trans. Geosci. Rem. Sens. 2001, 39, 1119. (c) Hong, Y.; Lam, J. W. Y Tang, B. Z. Chem. Soc. Rev. 2011, 40, 5361. (d) Ghosh, K. R.; Saha, S. K.; Wang, Z. Y. Polym. Chem. 2014, 5, 5638. (e) Xu, B.; Wu, X.; Li, H.; Tong, H.; Wang, L. Macromolecules 2011, 44, 5089.

69. (a) Zhang, C.; Wang, Z. Y. Macromolecules, 1993, 26, 3330 (b) Zhang, C.; Wang, Z. Y. Macromolecules, 1992, 25, 5851. (c). Ozturk, T.; Ertas, E.; Mert, O. Chem. Rev. 2007, 107, 5210. (d) Criswell, T, R.; Klanderman, B. H. J. Org. Chem. 1974, 39, 771 


\section{Chapter 2 Design, Synthesis and Characterization of Conjugated Ladder Polymers}

\subsection{Introduction}

Naphtho[2,3-c]thiophene-4,9-dione (NTDO) is an electron acceptor that possesses a relatively simple and planar structure. ${ }^{1}$ Recently, different electron donor units have been used together with NTDO monomers to synthesize donor-acceptor (D-A) type conjugated polymers, for applications in bulk heterojunction (BHJ) organic solar cells. ${ }^{2-6}$ Studies show that the NTDO-containing D-A polymers have absorption bands in the wavelengths from 300 to $750 \mathrm{~nm}$ and energy band gaps between 1.93 to $1.67 \mathrm{eV}^{2-6}$ Among those reported to date, the D-A polymer with a strong dithienosilole donor showed the highest absorption maximum at $629 \mathrm{~nm}$. It also had a deeper HOMO energy level $(-5.43 \mathrm{eV})$, higher fill factor $(\mathrm{FF})(64 \%)$ and short-circuit current $\left(J_{\mathrm{sc}}\right)(9.57)^{2}$ values in comparison with those derived from benzo[1,2-b:4,5-b']dithiophene and benzo[1,2b:4,5-b']difuran donors, ${ }^{3}$ thus giving a high PCE $(5.21 \%) .{ }^{2-4}$ However, there was no significant change in $V_{\text {oc }}(0.88-0.89 \mathrm{~V})$. The linear and alkoxy substituents on the NTDObased D-A polymers are expected to affect the optical properties, solubility and hole mobility. The studies showed that D-A polymer with the linear alkyl chain has higher solubility in common organic solvents, higher hole mobility $\left(3.05 \times 10^{-5} \mathrm{~cm}^{2} \mathrm{~V}^{-1} \mathrm{~S}^{-1}\right)$ and longer absorption $(556 \mathrm{~nm})$ compared to the alkoxy chain. Moreover, solar cell devices showed better performance $\left(2.21 \%\right.$ vs $1.52 \%$ PCE with $\mathrm{PC}_{71} \mathrm{BM}$ as an acceptor) for polymers with linear alkyl chains than branched alkyl chains. ${ }^{4,5}$ Clearly, the type and geometry of the side chains on the NTDO-based D-A polymers have a great influence on 
polymer interchain packing, crystallinity, morphology, and fullerene loadings and thus device performance. ${ }^{6}$

\subsection{Design strategy and synthetic routes}

As was discussed in our rationale and objectives, NTDO possesses a fused thiophene unit and two keto groups. Conceivably, the coupling reaction on the thiophene unit and on the keto group can lead to the chain extension or polymerization to form a linear conjugated polymer. The most common route is to polymerize at the thiophene unit. The subsequent intramolecular coupling reaction between the keto groups in the linear polymer would lead to a ladder conjugated polymer that may possess a unique low energy band gap and optical and electrical properties.
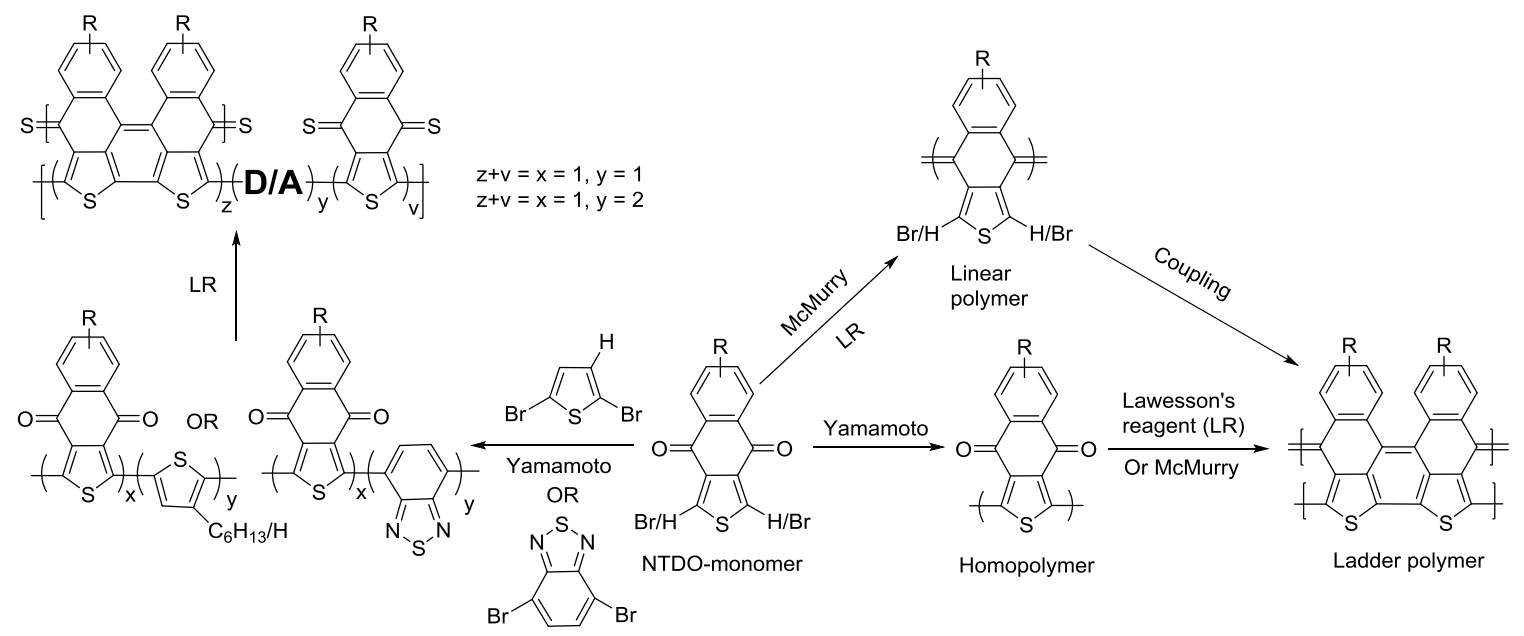

Scheme 2.1 Designed synthetic routes to linear and ladder conjugated polymers.

In this chapter, mainly explored the synthesis of a new series of ladder polymers, first by an intermolecular coupling reaction on the thiophene unit and then by an intramolecular coupling reaction on the keto group. The synthetic routes are shown in 
Scheme 2.1. First, a linear polymer is to be synthesized by Yamamoto coupling reaction on the thiophene unit and subsequent intramolecular cyclization using Lawesson's Reagent (LR) affords a ladder conjugated polymer. ${ }^{7}$ Second, the same ladder polymer can also be prepared first by the keto-keto coupling and the subsequent Ullmann reaction on the thiophene unit. Third, a copolymer having the fused ring and linear units was designed in order to adjust the properties and solubility, which can be synthesized by adjusting the molar ratios of thiophene (donor) and 2,1,3-benzothiadiazole (acceptor) followed by the intramolecular keto-keto coupling reaction.

\subsection{Synthesis and characterization of 1,3-dibromo-6-octylnaphtho[2,3-c]thiophene-}

\section{4,9-dione}

The acceptor monomer, 1,3-dibromo-6-octylnaphtho[2,3-c]thiophene-4,9-dione

(M1), was synthesized according to the reactions in Scheme 2.2. 2,5-Dibromothiophene3,4-dicarboxalic acid (1) was synthesized in 63\% yield by bromination of thiophene-3,4dicarboxylic acid using $N$-bromosuccinimide under the acidic condition. 2,5Dibromothiophene-3,4-dicarboxylic acid chloride (2) was synthesized by a VilsmeierHaack reaction with compound $\mathbf{1}$, oxalyl chloride and a catalytic amount of DMF in $100 \%$ yield. The reaction of DMF and oxalyl chloride is known to form a reactive intermediate imine salt (Vilsmeier-Haack reagent), via an electrophilic substitution reaction. This produces an imino ester salt intermediate that undergoes a nucleophilic substitution reaction to give the desired acid chloride (Scheme 2.3). The Friedel-Crafts reaction of compound $\mathbf{2}$ in dry dichloromethane with n-octylbenzene produced the target monomer $\mathbf{M 1}$ in $83 \%$ yield. $^{4}$ 


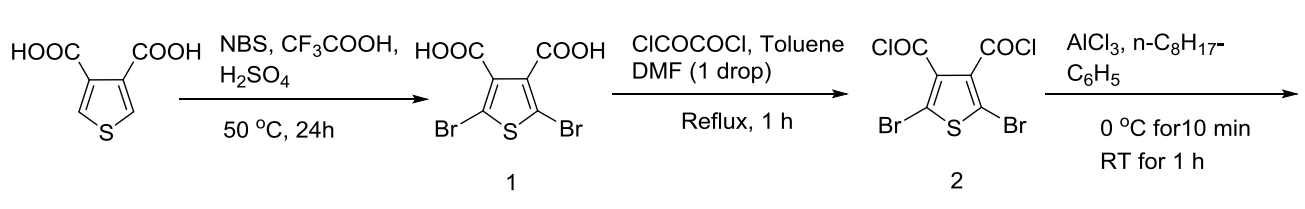

Scheme 2.2 Synthesis of 1,3-dibromo-6-octylnaphtho[2,3-c]thiophene-4,9-dione.

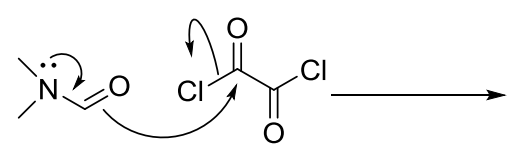<smiles>CCC(=O)C(=O)OCC(CC)N(C)C</smiles>
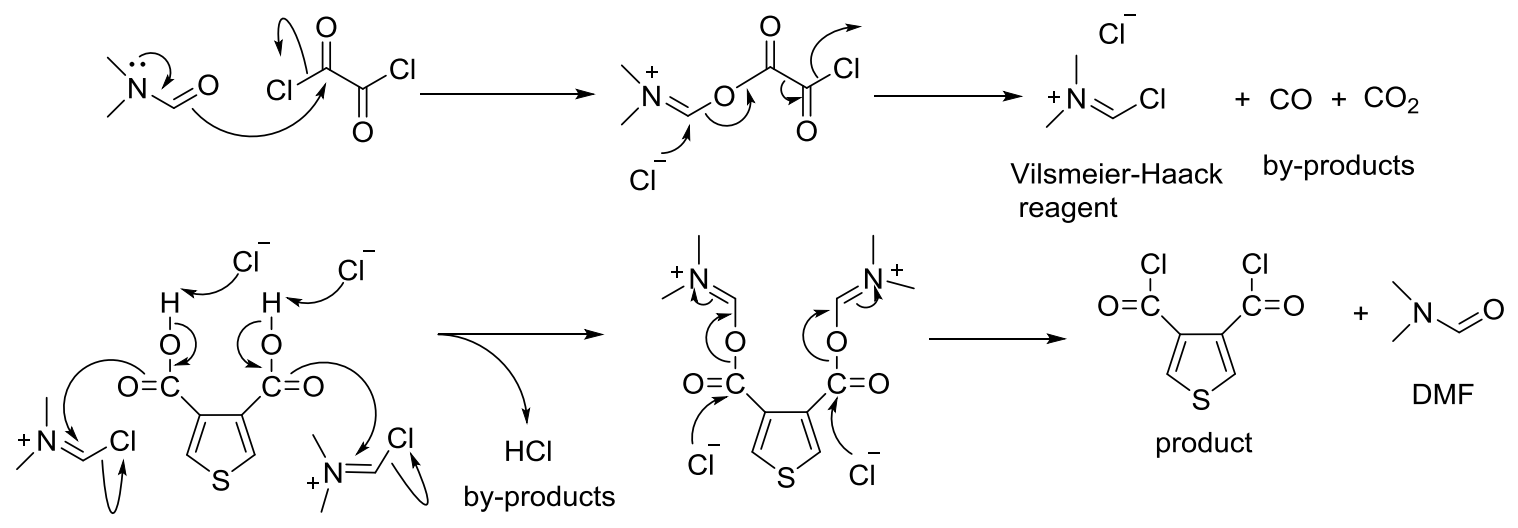

Scheme 2.3 Plausible mechanism for the formation of compound 2.

Monomer M1 was fully characterized by $\mathrm{IR},{ }^{1} \mathrm{H}$ NMR, ${ }^{13} \mathrm{C}$ NMR and mass spectrometry. All spectra are given in "Appendix A". The IR spectrum (Appendix A, Figure S2.2) of M1 displays the characteristic $\mathrm{C}=\mathrm{O}$ band at $1673 \mathrm{~cm}^{-1}$, indicating the successful Friedel-Crafts acylation reaction. The ${ }^{1} \mathrm{H}$ NMR spectrum (Appendix A, Figure S2.3) of M1 shows two doublet peaks at $\delta 8.23$ and $8.11 \mathrm{ppm}$ and one doublet of doublet peak at $\delta 7.61 \mathrm{ppm}$ for the aromatic protons. The multiplet peaks at $\delta 2.84-2.73 \mathrm{ppm}$ are assigned to $\mathrm{Ar}-\mathrm{CH}_{2}$ protons. The other aliphatic peaks are in the region of $\delta 1.69-0.89$ ppm. The ratios between aromatic protons and aliphatic protons are consistent with the structure of the desired compound. The ${ }^{13} \mathrm{C}$ NMR spectrum (Appendix A, Figure S2.4) of M1 shows peaks at $\delta 177.94$ and $177.56 \mathrm{ppm}$ for the carbonyl's carbons, while the substituted aromatic and aromatic carbons are from $\delta 120-150 \mathrm{ppm}$. The mass spectrum 
(Appendix A, Figure S2.5) shows a molecular ion peak at $\mathrm{m} / \mathrm{z} 481.95682$, which corresponds to the desired compound.

\subsection{Synthesis and characterization of ladder polymer}

Linear polymer P1 and ladder polymer P2 were synthesized according to the reactions in Scheme 2.4. Polymer P1 is considered to be a precursor polymer for the target ladder polymer P2. Polymer P1 was obtained in $82 \%$ yield by the Yamamoto reaction in the presence of $\mathrm{Ni}(\mathrm{COD})_{2}$ in toluene at $95^{\circ} \mathrm{C}$. $^{7 \mathrm{a}}$ The polymer was precipitated in methanol and purified by Soxhlet extraction. The obtained polymer was black-amber in color.

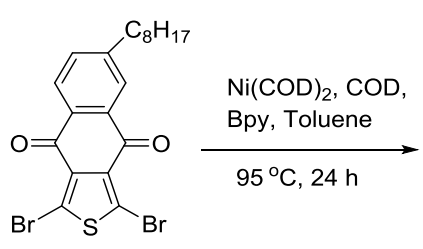

M1

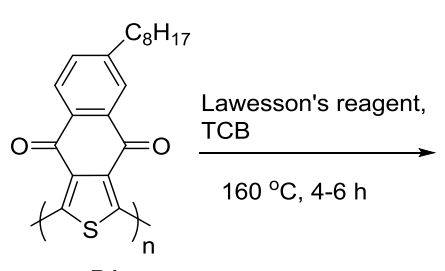

P1

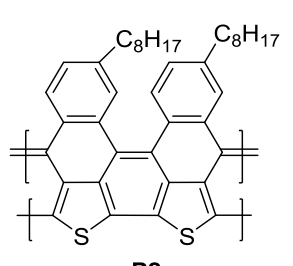

P2

Scheme 2.4 Synthesis of polymer P1 and ladder polymer P2.

The carbon-carbon bond formation has been known for 100 years and copper is the first transition metal used in this reaction. Recently, the transition metal-catalyzed cross-coupling reactions have become a popular and important method in organic synthetic chemistry. The Suzuki, Sonogashira, Heck, Negishi, Kumada, and Stille coupling reactions are used to synthesize pharmaceuticals, natural products, molecular organic materials and conjugated polymers. ${ }^{8}$ The Ullmann reaction is catalyzed by copper; however, the high reaction temperature (around $200{ }^{\circ} \mathrm{C}$ ) limits its scope. ${ }^{9}$ This high-temperature issue is overcome by the Yamamoto coupling reaction, which can be 
carried out at low temperatures of $25-40{ }^{\circ} \mathrm{C}$. Functional groups such as ketone, aldehyde, ester and nitrile, which would not survive with aryl magnesium or aryl lithium reagents, do not interfere with the Yamamoto reaction. ${ }^{10}$

The reaction of aryl bromide using $\mathrm{Ni}(\mathrm{COD})_{2}$ and bipyridine follows $2^{\text {nd }}$-order kinetics with respect to the nickel complex. The rate-determining step of the coupling reaction involves a bimolecular reaction process between the nickel complex rather than the reaction of the nickel complex with aryl bromide. Regarding the halides, the reaction proceeds in an order $\mathrm{I}>\mathrm{Br}>\mathrm{Cl}$. The reaction rate for different para-substituted aryl bromides follows the order of $\mathrm{CH}_{3} \mathrm{CO}<\mathrm{H}<\mathrm{CH}_{3}<\mathrm{OCH}_{3}$. The reaction proceeds through an initial oxidative addition of the substrate to $\mathrm{Ni}(\mathrm{bpy})(\mathrm{COD})$ generated in the reaction to give $\mathrm{NiX}(\mathrm{Ar})(\mathrm{bpy})$, which undergoes a disproportionation reaction and the subsequent formation of a biaryl. The disproportionation process of relatively stable NiX(Ar)(bpy) gives $\mathrm{NiAr}_{2}$ (bpy), which is ready to undergo a facile reductive elimination and is the ratedetermining step of the entire reaction. A polar solvent makes the disproportionation process much easier than in a non-polar solvent and leads to a clean formation of the product. $^{11}$

The mechanism for the Yamamoto reaction involves oxidative addition and reductive elimination but it is uncertain about tansmetallation. A recent study using X-ray absorption fine structure (XAFS) found the presence of [Ni(COD)(bpy)], an intermediate $\left[\mathrm{Ni}(\mathrm{bpy})(\mathrm{Ph}) \mathrm{Br}(\mathrm{DMF})_{2}\right]$ and $\left[\mathrm{Ni}(\mathrm{bpy}) \mathrm{Br}_{2}(\mathrm{DMF})\right]$ during the catalytic reaction, but the absence of dimer intermediate. ${ }^{12}$ As Scheme 2.5 shows, a reactive Ni(COD)bpy complex (I) is formed in situ in the first step. The second step is the oxidative addition of aryl halide to Ni-complex and formation of the intermediate II. The recombination (step 3) 
with the formation of intermediates III and IV is the rate-determining step. The complex IV is not reactive and is formed irreversibly. The reductive elimination (step 4) affords a biaryl product and regenerates the reactive complex I. Only one out of two reactive Nicomplexes is regenerated and can be further used in polymerization.

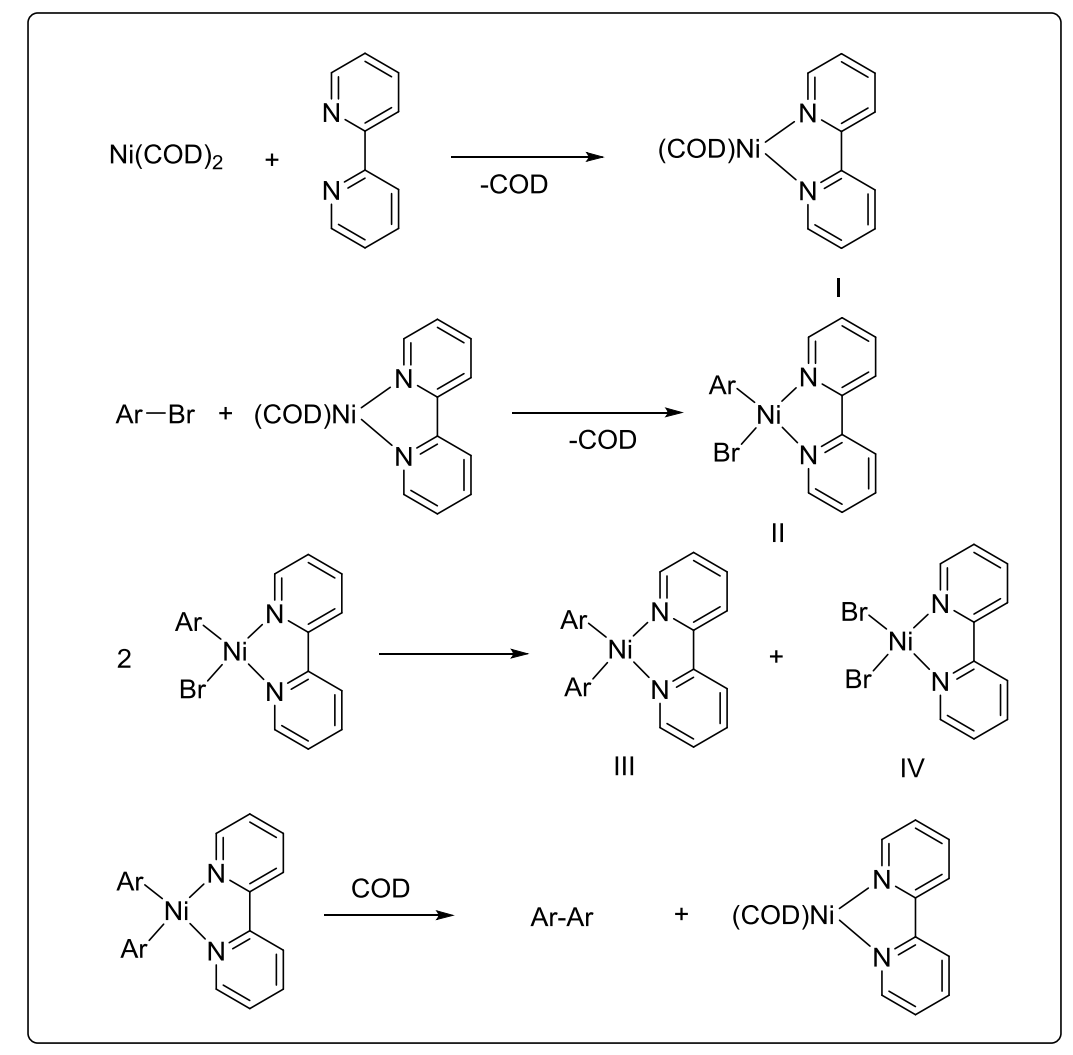

Scheme 2.5 General mechanism of Yamamoto coupling reaction.

The ladder polymer $\mathbf{P 2}$ was obtained by intramolecular cyclization of P1 using LR in $1,2,4$-trichlorobenzene (TCB) at $160{ }^{\circ} \mathrm{C}$ in $94 \%$ yield. ${ }^{7}$ During the reaction, the color of the solution changes to blue-greenish. The polymer was precipitated out in methanol and filtered. The obtained polymer was purified by being heated at reflux in ethanol for $2 \mathrm{~h}$, filtered and washed with ethanol. The resultant polymer was then stirred in different solvents sequentially in acetone, ethyl acetate and hexane to remove any other 
residue from LR. The polymer was then further purified by a Soxhlet extraction in acetone for $24 \mathrm{~h}$. The obtained polymer $\mathbf{P 2}$ was black-greenish in color.
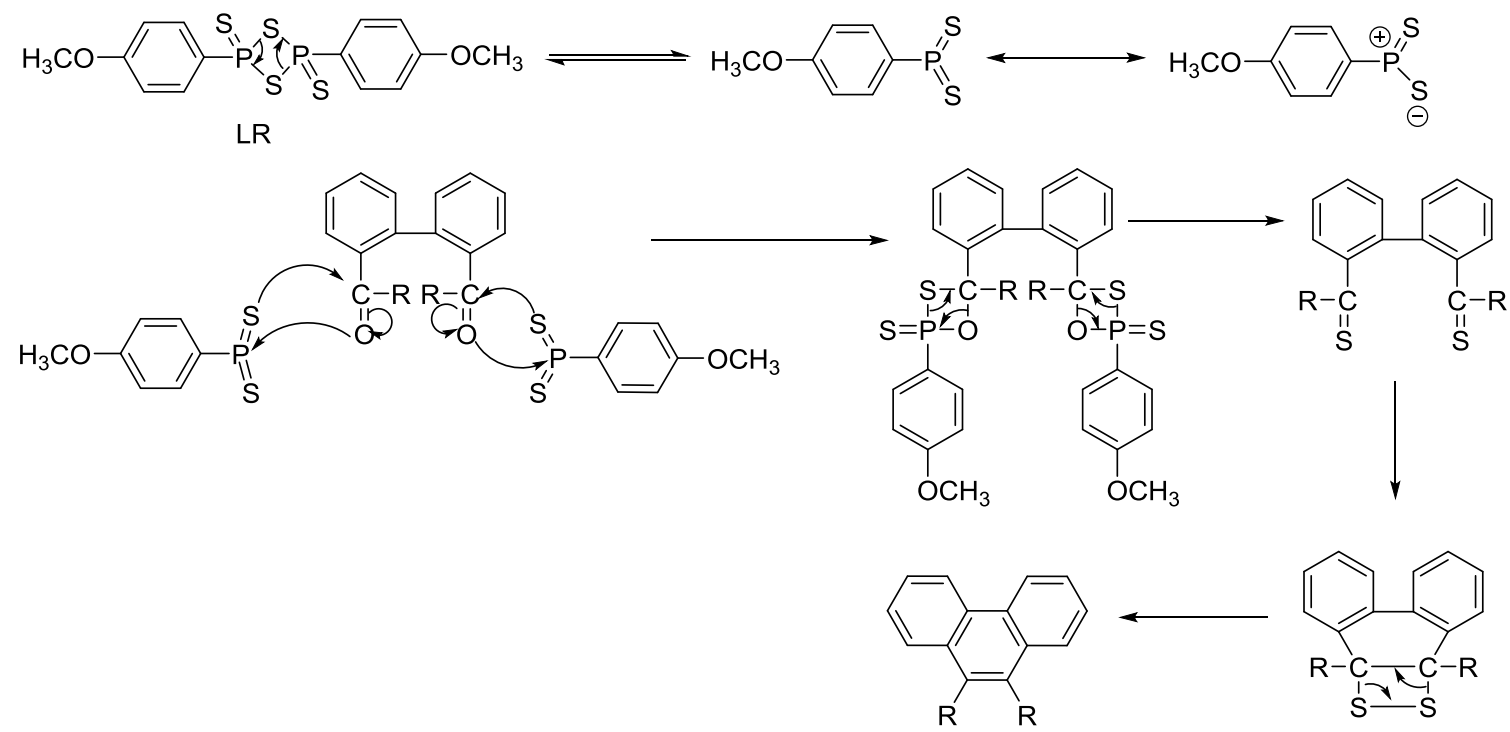

Scheme 2.6 Plausible mechanisms for thionation and cyclization reaction of diketo compound in the presence of Lawesson's Reagent.

Intramolecular cyclization reaction mechanism between keto groups is explained in Scheme 2.6. At high temperature LR involves dissociation equilibrium in the form of a phosphine disulfide intermediate, which then reacts with the carbonyl functional group and forms 4-membered rings. These 4-membered rings then decompose to corresponding thioketones. These thioketones are unstable and at high temperature, they produce sulfur radicals that then form disulfide linkages between the sulfur atoms and subsequently undergo rapid loss of sulfur $\left(\mathrm{S}_{2}\right)$, providing a double bond between the monomer units. ${ }^{13}$

These polymers were characterized by IR, NMR, GPC, TGA, absorption, emission spectroscopy and cyclic voltammetry (CV). The IR spectrum (Figure 2.1a) of polymer P1 shows the sharp, strong peak at $1673 \mathrm{~cm}^{-1}$, which is characteristic for cyclic 
$\mathrm{C}=\mathrm{O}$. The ${ }^{1} \mathrm{H}$ NMR spectrum (Appendix A, Figure S2.6) of $\mathbf{P 1}$ shows broad peaks from $\delta$ 8.15 to $8.10 \mathrm{ppm}$ and $\delta 7.57 \mathrm{ppm}$, corresponding to aromatic protons. The peak at $\delta 2.75$ ppm is assigned to $\mathrm{CH}_{2}$ proton of benzyl and other peaks are at $\delta 1.69,1.46,1.27$ and 0.87 ppm for $\mathrm{CH}_{2}$ and $\mathrm{CH}_{3}$ moieties in the alkyl chain, respectively. The ${ }^{13} \mathrm{C} \mathrm{NMR}$ spectrum (Figure 2.2a) shows two peaks at $\delta 179.60$ and $179.18 \mathrm{ppm}$, indicating the $\mathrm{C}=\mathrm{O}$ moiety of the polymer P1. The broad peaks are from $\delta 150.46$ to $127.66 \mathrm{ppm}$, indicating aromatic carbons for polymer $\mathbf{P 1}$.
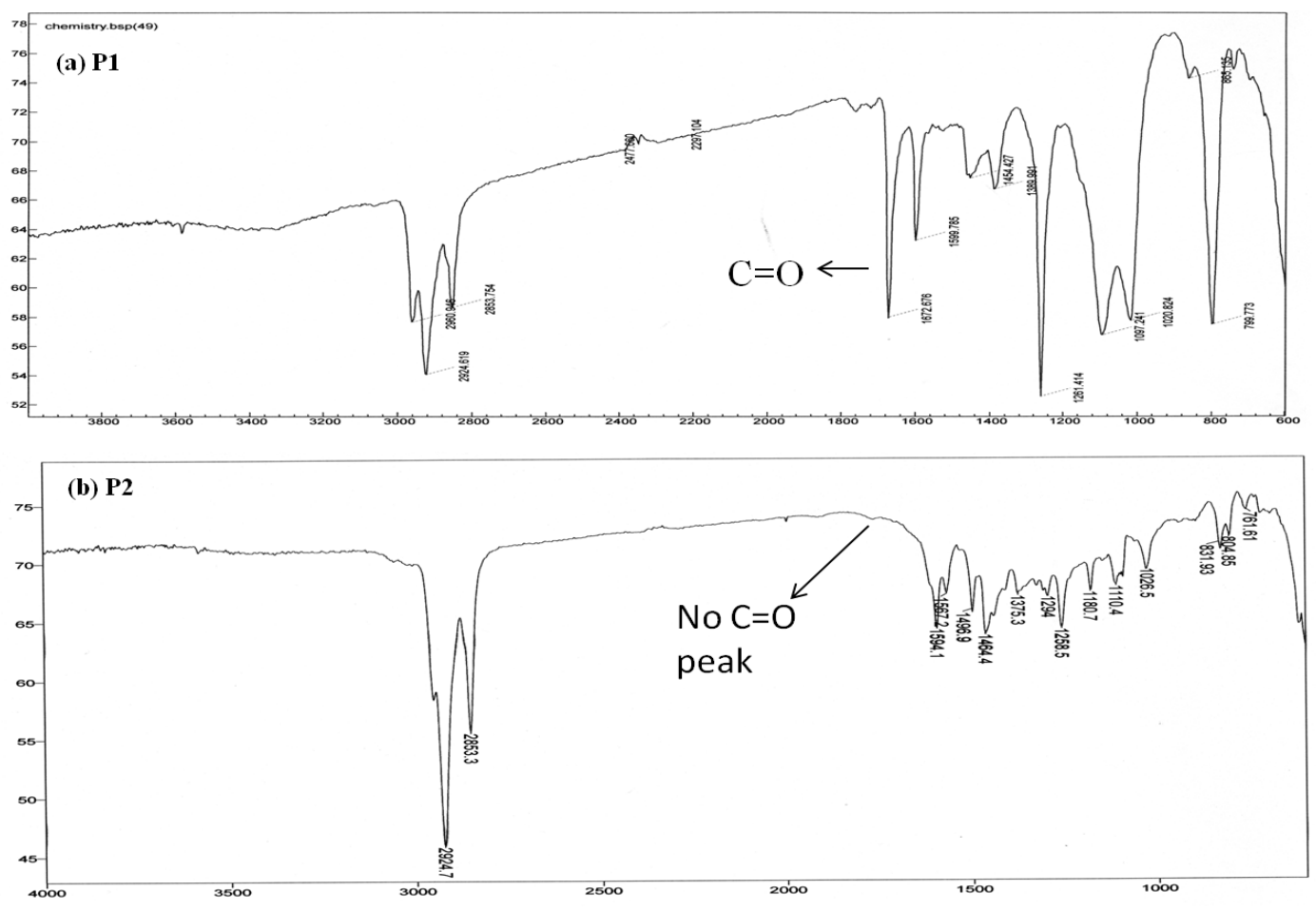

Figure 2.1 IR spectra of polymers $\mathbf{P 1}$ (a) and P2 (b) (on $\mathrm{NaCl}$ plate).

The main purpose of characterizations of polymer P2 is to confirm the success and effectiveness of keto-keto coupling reaction. The IR spectrum of polymer P2 (Figure 2.1b) shows no peak at $1673 \mathrm{~cm}^{-1}$, which indicates the disappearance of $\mathrm{C}=\mathrm{O}$ as a result 
of the keto-keto coupling reaction. The ${ }^{13} \mathrm{C}$ NMR spectrum (Figure 2.2b) of polymer $\mathbf{P 2}$ does not show any peak over $\delta 143 \mathrm{ppm}$, indicating the absence of $\mathrm{C}=\mathrm{O}$ or $\mathrm{C}=\mathrm{S}$.

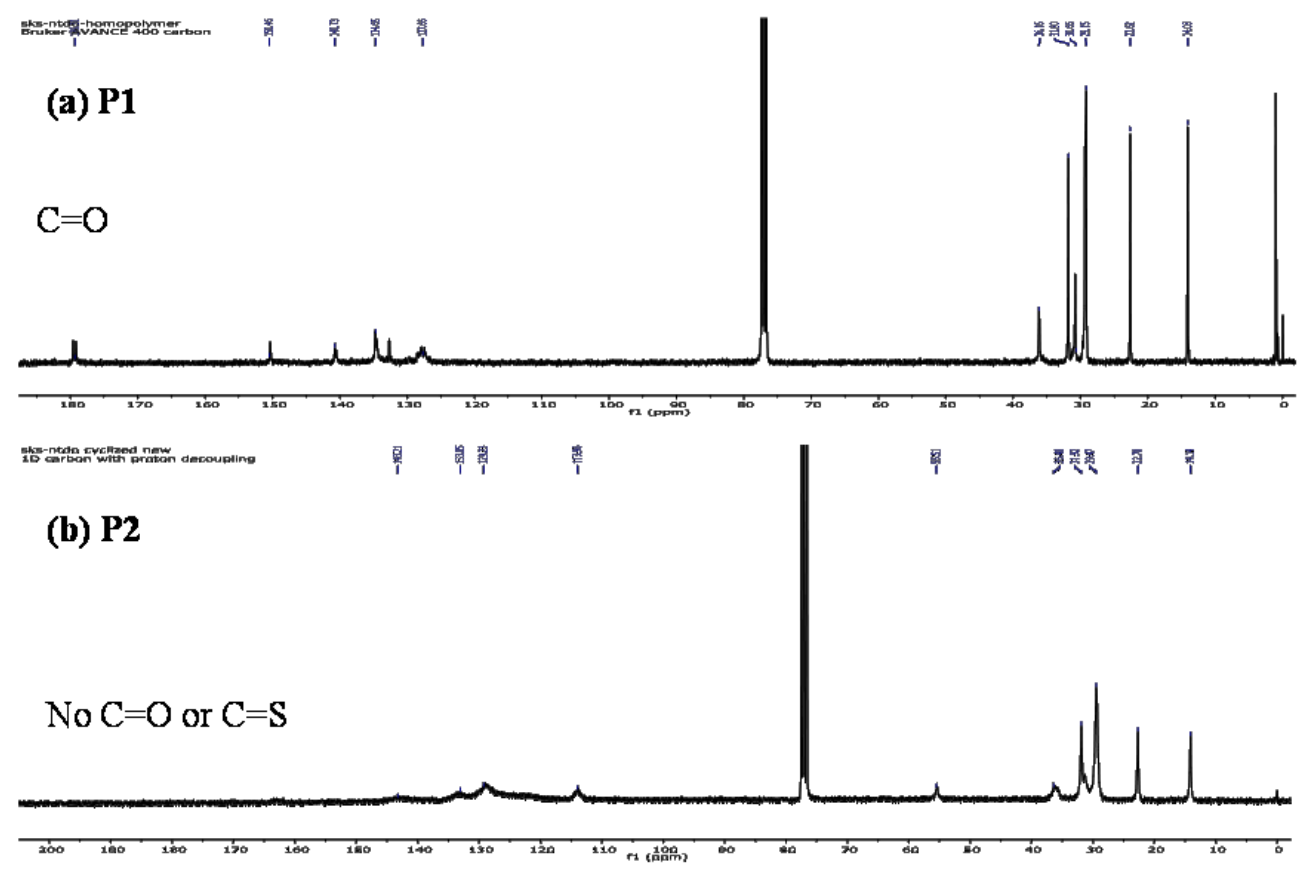

Figure $2.2{ }^{13} \mathrm{C}$ NMR (75 MHz, $\mathrm{CDCl}_{3}$ ) spectra for polymers P1 (a) and P2 (b).

Further, a desulfurization reaction of polymer $\mathbf{P 2}$ in the presence of hydrogen peroxide and thionyl chloride was carried out to understand the quantitative conversion from $\mathbf{P 1}$ to $\mathbf{P 2}$. The IR spectrum of polymer $\mathbf{P 2}$ after desulfurization reaction (Appendix A, Figure S2.8) did not show any appearance of the characteristic $\mathrm{C}=\mathrm{O}$ band, which suggests the absence of possible intermediate $\mathrm{C}=\mathrm{S}$ group in polymer. The keto-keto coupling in polymer $\mathbf{P 2}$ will form a new $\mathrm{C}=\mathrm{C}$ in the polymer backbone. The characteristic $\mathrm{C}=\mathrm{C}$ peak shows in the region of $1600 \mathrm{~cm}^{-1}$ in IR spectrum. However, it is not possible to separate the peak from the IR spectrum of polymer $\mathbf{P 2}$ because of the remaining aromatic $\mathrm{C}=\mathrm{C}$ in the polymer main chain. The expected $\mathrm{C}=\mathrm{C}$ was also not 
distinguishable from the ${ }^{13} \mathrm{C}$ NMR spectrum. Therefore, the effort was made to identify the structure of polymer $\mathbf{P 2}$, which is explained in detail in Section 2.10 below.

Thermal degradation of polymers or in other words, the thermal stability of the polymers was determined by thermogravimetric analysis (TGA). TGA measures the changes in the weight loss of a sample as a function of temperature and time. The resulting thermogram, which is presented in Figure S2.9 (Appendix A), reveals that the onset temperatures for $1 \%$ weight loss for polymers $\mathbf{P 1}$ and $\mathbf{P} 2$ are at 205.6 and $247.1{ }^{\circ} \mathrm{C}$, respectively. It was also observed that the temperature for $5 \%$ weight loss was relatively higher for ladder polymer P2 than linear polymer P1. It indirectly confirms that the intramolecular cyclization or keto-keto coupling was successful to a certain extent. Molecular weight and polydispersity indices (PDI) of polymer P1 were determined by gel permeation chromatography (GPC) relative to polystyrene standard. PDI is used as a measure of the breadth of the molecular weight distribution. PDI is defined as the $M_{w} / M_{n}$ where, $M_{w}$ and $M_{n}$ are the weight average and number average molecular weight, respectively. The larger the PDI, the broader the molecular weight. When PDI is equal to 1 means all the chain lengths are equal, which is called monodisperse polymer. The synthesized polymer P1 was of moderate molecular weight (Appendix A, Figure S2.10), with a PDI of 3.41. A higher Mark-Houwink exponent $(0.8<\alpha<2.0)$ for conjugated ladder polymers than that of linear polymers $(0.5<\alpha<0.8)$ is expected. The conformations and dynamics in solutions are different between conjugated linear and ladder polymers because of the different rigidity of polymer backbone. Traditional solution characterization techniques using polystyrene standards are unable to provide accurate molecular weight for conjugated ladder polymers due to the deviation of hydrodynamic 
volumes of conjugated ladder polymers compared to linear polymers. It is also known that different analytical methods tend to give the different values of molecular weights. ${ }^{14}$ Therefore, molecular weight of polymer $\mathbf{P 2}$ was not determined in this work.
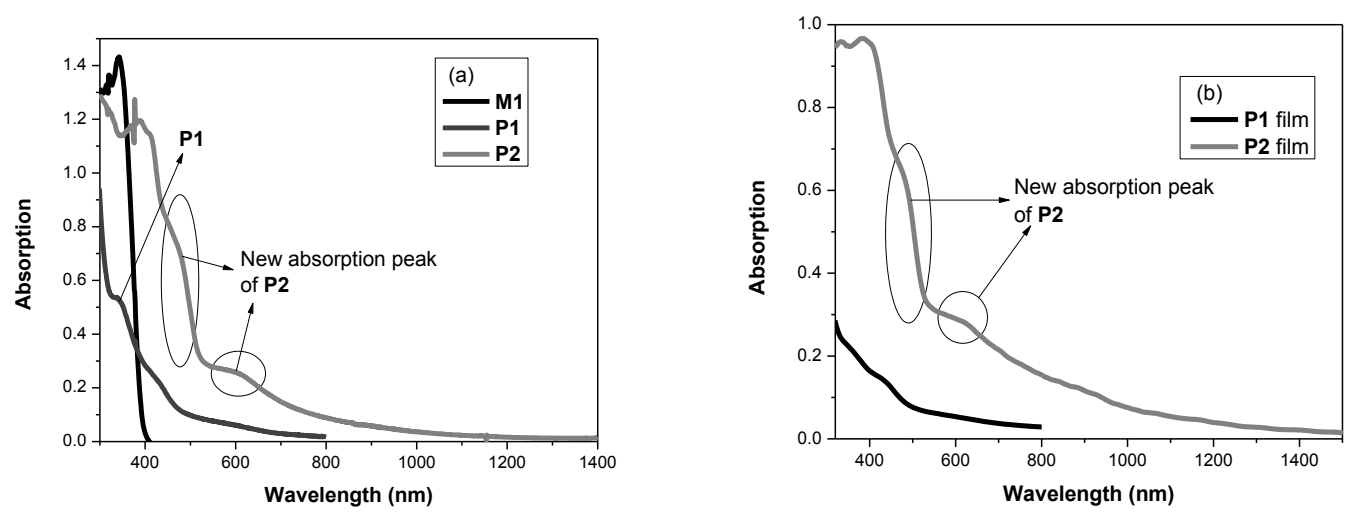

Figure 2.3 Absorption spectra of M1 and polymers P1 $\left(7.04 \times 10^{-5} \mathrm{M}\right)$ and $\mathbf{P 2}\left(1.08 \times 10^{-}\right.$

${ }^{4} \mathrm{M}$ ) in $\mathrm{CHCl}_{3}$ solution (a) and as thin films on glass substrates (b).

Table 2.1. UV-vis absorption data of polymers $\mathbf{P 1}$ and $\mathbf{P 2}$

\begin{tabular}{|c|c|c|c|c|}
\hline \multirow{2}{*}{ Polymer } & \multicolumn{2}{|c|}{$\lambda_{\max }(\mathrm{nm})$} & \multicolumn{2}{c|}{$\lambda_{\text {onset }}(\mathrm{nm})$} \\
\cline { 2 - 5 } & $\begin{array}{c}\text { in } \mathrm{CHCl}_{3} \\
\text { solution }\end{array}$ & film & $\begin{array}{c}\text { in } \mathrm{CHCl}_{3} \\
\text { solution }\end{array}$ & film \\
\hline P1 & 343 & 420 & 660 & 700 \\
\hline P2 & $395,472,600$ & $390,490,600$ & 900 & 1000 \\
\hline
\end{tabular}

Figures 2.3a and 2.3b show the absorption spectra of $\mathbf{P 1}$ and $\mathbf{P 2}$ in $\mathrm{CHCl}_{3}$ solutions and spin-coated thin films on glass substrates, respectively. The polymers are fully soluble in solvents like $\mathrm{CHCl}_{3}$, chlorobenzene, 1,2-dichlorobenzene (DCB) and TCB. Polymer P2 was obtained in 94\% yield and the solubility was in a wide range of solvents, which confirms the higher percentage of intramolecular cyclization. ${ }^{14,15}$ Polymer 
P1 displays a maximal absorption peak at $343 \mathrm{~nm}$ in $\mathrm{CHCl}_{3}$ with an onset from $660 \mathrm{~nm}$. The absorption spectrum of $\mathbf{P 1}$ is broader than that of $\mathbf{M 1}$, due to the extended conjugation in the polymer backbone. The film of $\mathbf{P 1}$ shows broad absorption with a maximum at $420 \mathrm{~nm}$ with an onset from $700 \mathrm{~nm}$. Polymer P2 displays three maximal absorption peaks at 395, 472 and $600 \mathrm{~nm}$ in $\mathrm{CHCl}_{3}$ with an onset from $900 \mathrm{~nm}$. $\mathbf{P 2}$ has broad absorption spectrum compared to P1 because of the increased conjugation and ladder-type backbone. The film of $\mathbf{P} \mathbf{2}$ shows broad absorption with maximal peaks at 390,490 , and $600 \mathrm{~nm}$ and an onset from $1000 \mathrm{~nm} .{ }^{16}$ The maximal absorption peak can be assigned due to $n-\pi^{*}$ and/or $\pi-\pi^{*}$ transition of the aromatic segment while the broader absorption part is may be due to intramolecular charge transfer (ICT) in the polymer chains. ${ }^{17}$ The absorption spectra of the two polymer films were similar to their corresponding spectra in solution with the red shifted, suggesting intermolecular interaction and $\pi-\pi$ stacking of polymer chains. P2 exhibits two broad absorption peaks at 472 and $600 \mathrm{~nm}$ in solution. The peak at $472 \mathrm{~nm}$ may be due to keto-keto coupling. The peak present at $600 \mathrm{~nm}$ is attributed to the conjugated ladder of the polymer. The onset from longer wavelengths (Near-IR) is due to the locking of rotation between the monomer units throughout the polymer backbone and the lower resonance energy after cyclization. The absorption in the solid state becoming broader in comparison to the solution is may be due to the close packing and $\pi-\pi$ stacking of polymer chains. Ideal conjugated ladder polymers are torsional defect-free and possess a maximal $\pi$-electron delocalization. Therefore, a defect-free conjugated ladder polymer should show better device performance than linear polymer. Such a perfect ladder polymer is challenging to synthesize and characterize. The structural defects arise from unreacted sites, side 
reactions and incomplete conversion or ladderization internally in the chain. ${ }^{1} \mathrm{H}$ NMR, ${ }^{13} \mathrm{C}$ NMR and IR spectroscopy is unable to fully determine the structural defect in conjugated ladder polymers. The defect can often impose significant impacts on the optical properties of conjugated ladder polymers. Therefore, the weak absorption of polymer $\mathbf{P 2}$ in the NIR region, which may be due to structural defect derived from the limitation of the reaction process. ${ }^{14}$

The emission spectrum of polymer $\mathbf{P 1}$ in $\mathrm{CHCl}_{3}$ shows two maxima at 468 and $556 \mathrm{~nm}$, whereas the film shows an emission maximum at $428 \mathrm{~nm}$ (Appendix A, Figure S2.11). The emission of $468 \mathrm{~nm}$ may be due to the intramolecular CT. Under such dilute conditions $\left(7.04 \times 10^{-5} \mathrm{M}\right)$, the distance between polymers was too large to permit the transfer of energy and intramolecular CT was favored. Therefore, two emission bands may have originated from intramolecular CT. ${ }^{18 a}$ The large Stokes shift (125 nm and 213 $\mathrm{nm}$ ) in solution for polymer $\mathbf{P 1}$ indicates better intramolecular charge transfer along the polymer backbone or larger non-radiative internal conversion. ${ }^{18 b-d}$ The emission in the solid state is due to less intermolecular $\pi$ - $\pi$ stacking. The absence of emission for polymer $\mathbf{P 2}$ in solution is due to non-radiative transition and in the solid state is the aggregation caused quenching and higher intermolecular $\pi-\pi$ stacking interactions. We found that polymer $\mathbf{P 2}$ is black-amber in color and the absorption is tailing from the longer wavelength. The tailing in the NIR spectral region can be explained by the improper distribution of HOMO and LUMO electron density over the conjugated backbone, therefore, the weak ICT. ${ }^{17 \mathrm{c}}$

The electrochemical characteristics of polymer films on ITO were studied by cyclic voltammetry $(\mathrm{CV})$ with $0.1 \mathrm{~mol} / \mathrm{L} \mathrm{Bu}_{4} \mathrm{NPF}_{6}$ in acetonitrile solution at a scan rate of 
$100 \mathrm{mVS}^{-1}$, where ITO was used as a working electrode, Pt as a counter electrode and $\mathrm{Ag} / \mathrm{Ag}^{+}$as a reference electrode. The HOMO and LUMO energy levels and electrochemical band gaps $\left(\mathrm{E}_{\mathrm{g}}{ }^{\mathrm{CV}}\right)$ of the polymers were calculated from oxidation onset and reduction onset using the equation HOMO or LUMO $=-\mathrm{e}\left(\mathrm{E}_{\mathrm{ox}}\right.$ or $\left.\mathrm{E}_{\text {red }}+4.36\right)(\mathrm{eV}) .{ }^{18 \mathrm{~d}}$ The CV of all the polymers are shown in Figure 2.4. Polymer P1 shows one noticeable oxidation process $(0.46 \mathrm{~V})$ and $\mathbf{P 2}$ shows a reduction process (-1.61 V). Polymer $\mathbf{P} 2$ does not show any oxidation process, which implies that polymer P1 was transformed to the desired polymer structure. However, the non-reversible oxidation process in polymer P1 and reduction processes in polymer $\mathbf{P 2}$ indicate that an irreversible change in chemical structures had took place during the redox process. The optical energy gap for polymers $\mathbf{P} 1$ and $\mathbf{P} 2$ are $1.87 \mathrm{eV}$ and $1.37 \mathrm{eV}$, respectively. The optical energy gap was calculated from absorption onset of polymer in solution. Calculation of LUMO energy level for polymer P1 and HOMO energy level for polymer P2 from optical energy band gaps ( $\mathrm{E}_{\mathrm{g}}{ }^{\mathrm{ppt}}$, from an absorption onset of polymer film) tend to give larger error. However, the LUMO energy level for polymer P1 and HOMO energy level for polymer P2 was calculated from $\mathrm{E}_{\mathrm{g}}{ }^{\text {opt }}$. The HOMO, LUMO, and energy band gap for two polymers are presented in Table 2.2. ${ }^{1}$ Polymer $\mathbf{P 2}$ has an $\mathrm{E}_{\mathrm{g}}{ }^{\mathrm{opt}}$ of $1.24 \mathrm{eV}$ with the $\mathrm{HOMO}$ of $-3.99 \mathrm{eV}$ (optical) and LUMO of $-2.75 \mathrm{eV}(\mathrm{CV})$ energy levels. The formation of a $\mathrm{C}=\mathrm{C}$ bond after intramolecular cyclization will produce a ladder polymer with a high lying HOMO energy level than polymer P1. The result shows that $\mathbf{P 2}$ has a high lying HOMO compared to P1. It was also noticed that the increase of the HOMO energy level was higher than the increase of the LUMO energy level. The LUMO energy level for the n-

${ }^{1} \mathrm{LUMO}_{\mathrm{opt}}=\mathrm{E}_{\mathrm{g}}{ }^{\mathrm{opt}}+\mathrm{HOMO}$ from $\mathrm{CV}$ or $\mathrm{HOMO}_{\mathrm{opt}}=\mathrm{LUMO}$ from CV $-\mathrm{E}_{\mathrm{g}}{ }^{\mathrm{opt}}$ 
type ladder polymer is generally lower than $-4.0 \mathrm{eV}$ and the HOMO energy level is lower than $-5.5 \mathrm{eV}$. The HOMO energy level for the p-type ladder polymer is close to $-5.0 \mathrm{eV}$ and the LUMO energy level may vary depending on the energy band gap. However, the HOMO and LUMO energy levels for polymer $\mathbf{P 2}$ are higher in energy than other ladder polymers in the literature. ${ }^{16,19}$
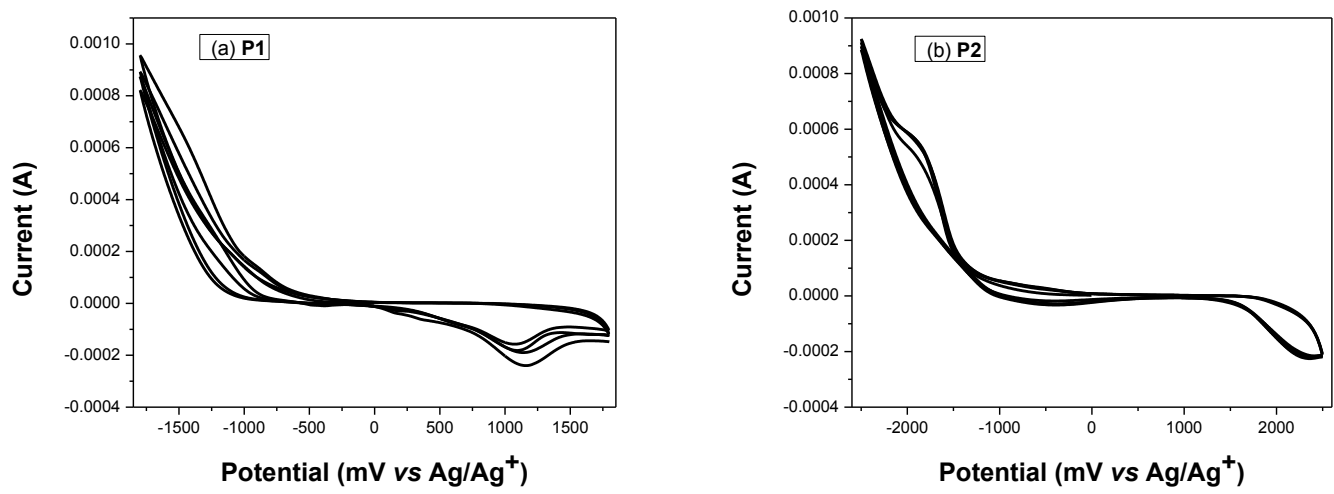

Figure 2.4 Cyclic voltammogram of polymer films of P1 (a) and P2 (b) on ITO in $\mathrm{CH}_{3} \mathrm{CN}$ solution containing $0.1 \mathrm{~mol} / \mathrm{L}$ of $\mathrm{Bu}_{4} \mathrm{NPF}_{6}$ with a scan rate of $100 \mathrm{mV} / \mathrm{s}$.

Table 2.2. Optical and electrochemical properties of polymers $\mathbf{P 1}$ and $\mathbf{P 2}$.

\begin{tabular}{|l|l|l|l|l|}
\hline Polymer & HOMO $(\mathrm{eV})$ & LUMO $(\mathrm{eV})$ & $\mathrm{E}_{\mathrm{g}}{ }^{\mathrm{opt}}(\mathrm{eV})$ & $\mathrm{E}_{\mathrm{g}}{ }^{\mathrm{CV}}(\mathrm{eV})$ \\
\hline P1 & $-4.82(\mathrm{cv})$ & NA (cv), -3.05 (opt) & 1.77 & NA \\
\hline P2 & NA (cv), $-3.99(\mathrm{opt})$ & $-2.75(\mathrm{cv})$ & 1.24 & NA \\
\hline
\end{tabular}

\subsection{Attempted synthesis of ladder polymer by intramolecular McMurry reaction of} polymer P1

Another attempt was made to synthesize polymer $\mathbf{P 2}$ from P1, according to the reaction in Scheme 2.7. The McMurry reaction on polymer P1 was carried out in the 
presence of zinc and titanium tetrachloride for 3 days at the refluxing temperature of THF. ${ }^{20 a}$ The reaction mixture was diluted with $\mathrm{CHCl}_{3}$ and aqueous $\mathrm{NaHCO}_{3}$ and was then filtered through Celite pad. ${ }^{20 \mathrm{~b}}$ The organic layer was collected and the solvent was evaporated. The polymer was obtained as dark amber solid and characterized without any further purification. The obtained polymer was characterized by IR, ${ }^{1} \mathrm{H}$ NMR, CV, and absorption spectroscopy.

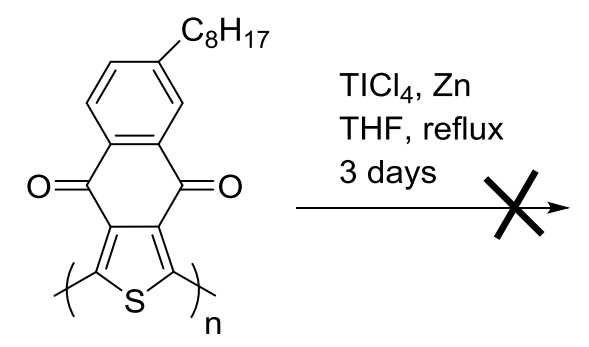

P1

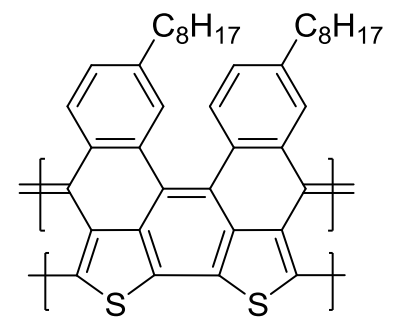

P2

Scheme 2.7 Attempted synthesis of $\mathbf{P 2}$ by McMurry reaction.

The IR spectrum (Figure 2.5a) of the resultant polymer shows a peak at $3422 \mathrm{~cm}^{-}$

${ }^{1}$, which is the characteristic peak for $\mathrm{OH}$. There is a medium peak at $1663 \mathrm{~cm}^{-1}$, which shows the presence of $\mathrm{C}=\mathrm{O}$ from NTDO. The ${ }^{1} \mathrm{H}$ NMR spectrum (Appendix A, Figure S2.12) shows that all the aliphatic protons are broad and they are at the same chemical shift as polymer P1. The aromatic protons are buried at $\delta 7$ to $8 \mathrm{ppm}$. The ${ }^{13} \mathrm{C}$ NMR spectrum (Figure 2.5b) suggests the aliphatic carbons are at the same position as polymer P1 and the aromatic carbons are at $\delta 127$ to $150 \mathrm{ppm}$. However, the presence of $\mathrm{C}=\mathrm{O}$ peak at $180 \mathrm{ppm}$ is difficult to identify. The resultant polymer shows a similar trend of absorption (Figure 2.6) as polymer $\mathbf{P 1}$ with an onset from $700 \mathrm{~nm}$, whereas the film shows broader absorption with an onset from $700 \mathrm{~nm}$. However, the spectra did not show 
any maximal absorption. Any strong absorption in longer wavelength was not observed from absorption spectra.

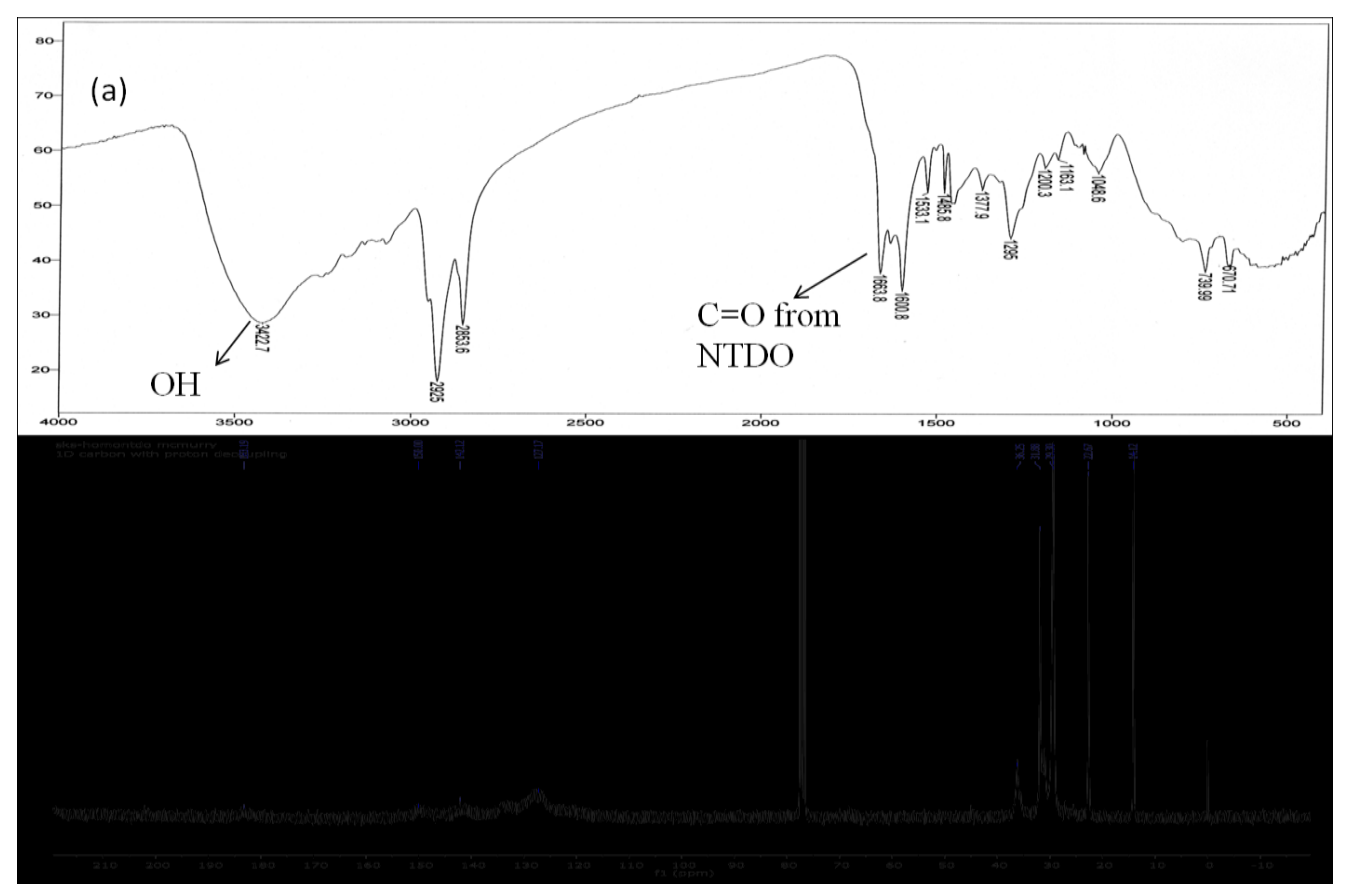

Figure 2.5 (a) IR (on $\mathrm{NaCl}$ plate) and (b) ${ }^{13} \mathrm{C} \mathrm{NMR}\left(75 \mathrm{MHz}, \mathrm{CDCl}_{3}\right.$ ) spectra of $\mathbf{P 2}$ obtained by McMurry reaction.

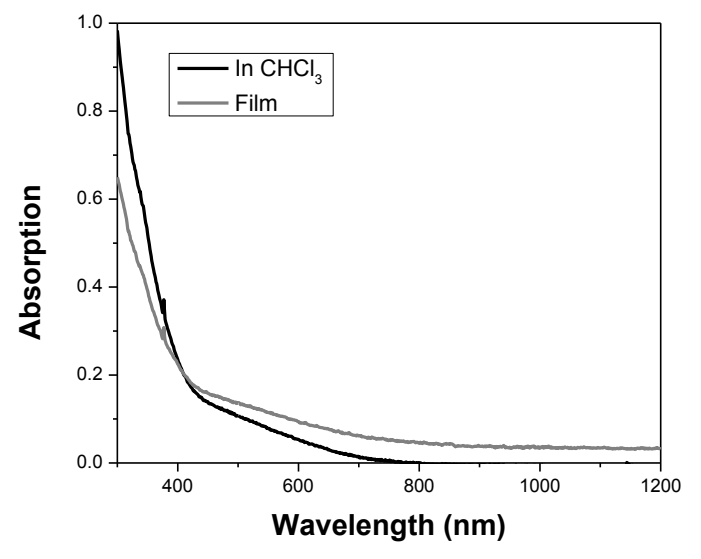

Figure 2.6 Absorption spectra of polymer $\mathbf{P 2}$ obtained by $\mathrm{McMurry}$ in a $\mathrm{CHCl}_{3}$ solution and as a thin film on a glass substrate. 


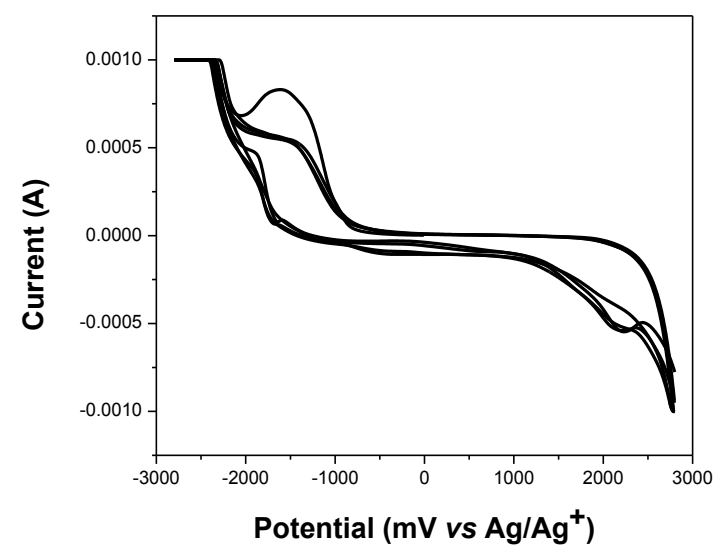

Figure 2.7 Cyclic voltammogram of polymer film on ITO in $\mathrm{CH}_{3} \mathrm{CN}$ solution containing $0.1 \mathrm{~mol} / \mathrm{L} \mathrm{Bu}_{4} \mathrm{NPF}_{6}$, with a scan rate of $100 \mathrm{mV} / \mathrm{s}$.

The resultant polymer shows noticeable oxidation and reduction processes (Figure 2.7). The onset oxidation potential/onset reduction potential is $1.25 /-0.74 \mathrm{~V}$. The HOMO energy level of polymer is $-5.61 \mathrm{eV}$ and the LUMO energy level is $-3.62 \mathrm{eV}$. The electrochemical energy band gap is $1.99 \mathrm{eV}$. The obtained polymer contains both $\mathrm{OH}$ and $\mathrm{C}=\mathrm{O}$ groups in the backbone and may have some new carbon-carbon double bonds that are not distinguishable because of the remaining aromatic $\mathrm{C}=\mathrm{C}$ bond from the polymer. The weak absorption from the longer wavelength may be caused by the loss of conjugation in the polymer backbone. The presence of $\mathrm{OH}$ indicates a breaking of aromaticity in the quinone moiety. No new absorption maximal from the longer wavelength was observed, which reflects inefficient coupling between the keto-keto. The CV shows both oxidation and reduction processes, which indicates the structure of the polymer has changed. The oxidation process indicates the polymer still contains NTDO units, which is an acceptor unit; however, the HOMO energy level is deeper $(-5.61 \mathrm{eV})$ than polymer P1, indicating the obtained polymer may have some ladder units resulting 
in a HOMO-LUMO separation. ${ }^{21}$ Polymer $\mathbf{P 1}$ did not show any reduction process, but the obtained polymer does show a reduction process. From the above spectral analysis, it can be concluded that the resultant polymer is not the target polymer P2. However, the actual structure is not known at this point.

\subsection{Attempted synthesis of ladder polymer from a precursor polymer by intramolecular Ullmann reaction}

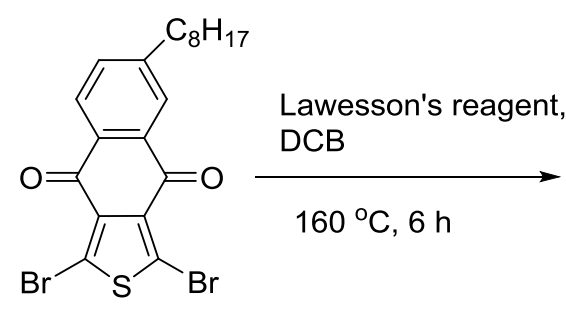

M1

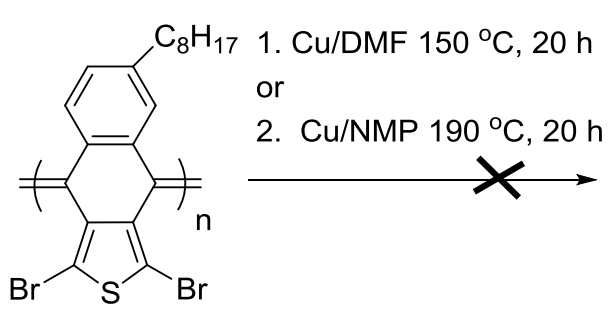

P3

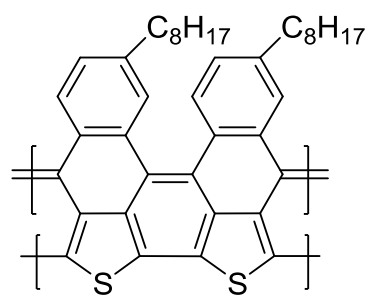

P2

Scheme 2.8 Attempted synthesis of $\mathbf{P 2}$ from $\mathbf{P 3}$ by intramolecular Ullmann reaction.

As was previously discussed, the keto-keto coupling between two keto groups forms a carbon-carbon double bond. Therefore, another approach was taken to synthesize the target ladder polymer $\mathbf{P} 2$ from the precursor polymer P3, by intramolecular Ullmann coupling according to the reactions shown in Scheme 2.8. A linear polymer P3 was obtained using LR in 1,2-dichlorobenzene (DCB) by keto-keto coupling reaction at 160 ${ }^{\circ} \mathrm{C}$ in $86 \%$ yield. ${ }^{7 \mathrm{~b}-\mathrm{e}}$ Polymer $\mathbf{P 3}$ was purified by the same procedure that was explained in Section 2.4. Polymer P3 was black-greenish in color. 


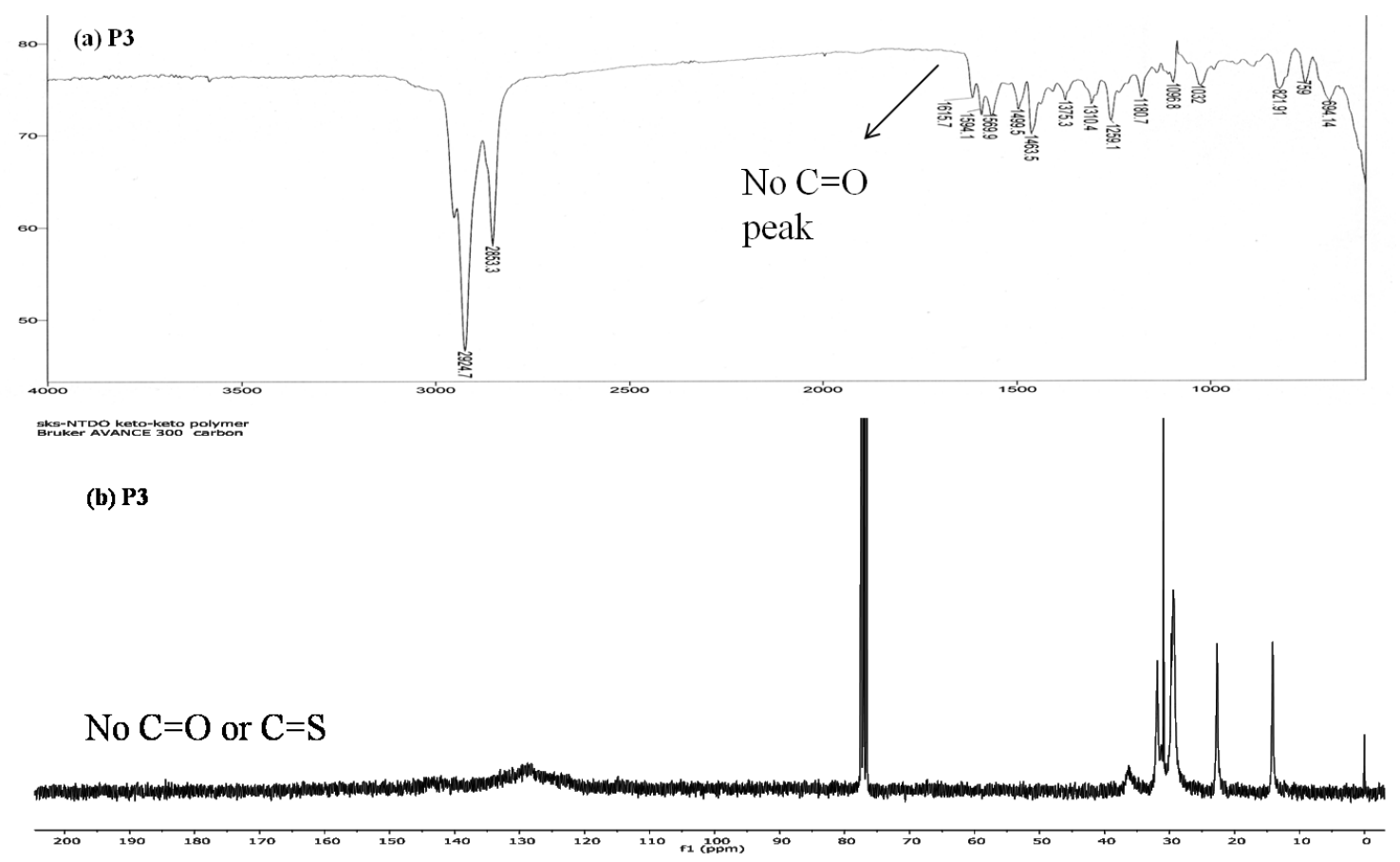

Figure 2.8 (a) IR (on $\mathrm{NaCl}$ plate) and (b) ${ }^{13} \mathrm{C}$ NMR $\left(75 \mathrm{MHz}, \mathrm{CDCl}_{3}\right.$ ) spectra of polymer P3.

The IR spectrum of polymer P3 (Figure 2.8a) shows no peak at $1673 \mathrm{~cm}^{-1}$ for cyclic $\mathrm{C}=\mathrm{O}$, which indicates the complete coupling between the keto groups. The ${ }^{13} \mathrm{C}$ NMR spectrum (Figure 2.8b) of polymer $\mathbf{P 3}$ shows no peak over $\delta 145 \mathrm{ppm}$, which indicates the absence of $\mathrm{C}=\mathrm{O}$ or $\mathrm{C}=\mathrm{S}$ due to the keto-keto coupling in the polymerization.

A new $\mathrm{C}=\mathrm{C}$ bond formed in polymer $\mathbf{P} 3$ because of the keto-keto coupling. The new $\mathrm{C}=\mathrm{C}$ peak in the region of $1600 \mathrm{~cm}^{-1}$ from the IR spectrum (Figure 2.8a) of $\mathbf{P 3}$ was not possible to separate because of the remaining aromatic $\mathrm{C}=\mathrm{C}$ peak in the polymer chain. The expected $\mathrm{C}=\mathrm{C}$ was also not distinguishable from the ${ }^{13} \mathrm{C}$ NMR spectrum.

Thermal degradation of polymer P3 is presented in Figure S2.14 (Appendix A), which reveals that the onset temperatures for 1\% weight loss for polymer P3 is at 223.9 ${ }^{\circ} \mathrm{C}$. This finding indirectly confirms that the keto-keto coupling was successful and that it 
produced a polymer. Molecular weight and polydispersity indices (PDI) of polymer P3 was determined by GPC relative to polystyrene standard. Polymer P3 was of moderate molecular weight (Appendix A, Figure S2.15) with a PDI of 3.91.
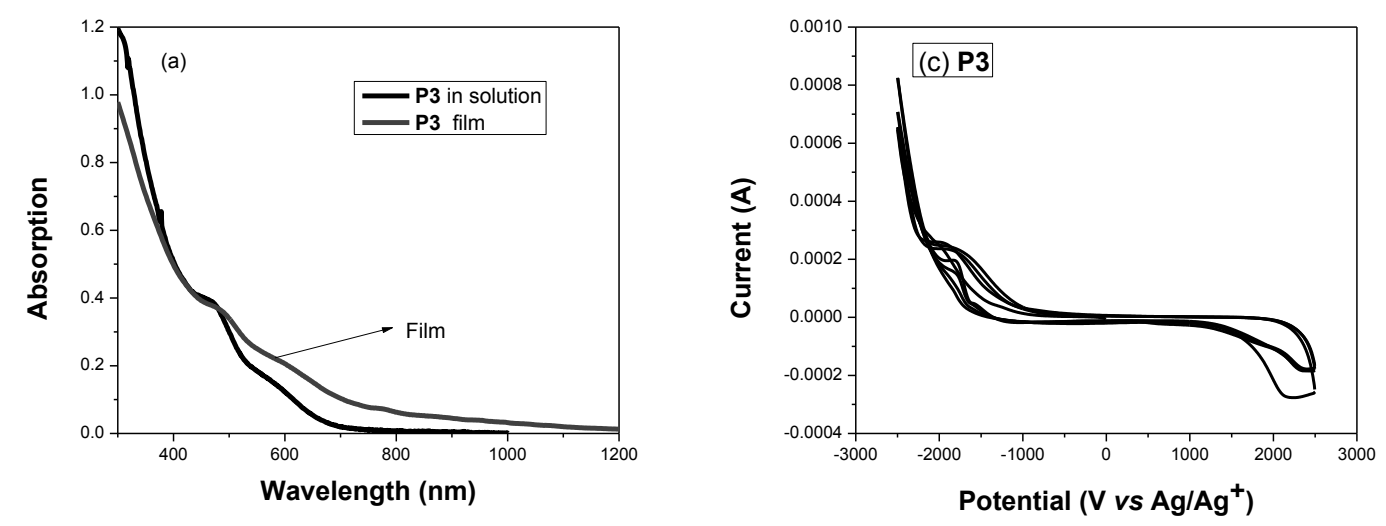

Figure 2.9 Absorption spectra of polymer $\mathbf{P 3}$ in $\mathrm{CHCl}_{3}$ solution and as thin film on a glass substrate (a) and cyclic voltammogram of the polymer film on ITO in $\mathrm{CH}_{3} \mathrm{CN}$ solution containing in $0.1 \mathrm{~mol} / \mathrm{L} \mathrm{Bu}_{4} \mathrm{NPF}_{6}$ with a scan rate of $100 \mathrm{mV} / \mathrm{s}(\mathrm{b})$.

Polymer P3 was highly soluble in a wide range of organic solvents such as THF, $\mathrm{CHCl}_{3}$, chlorobenzene, dichlorobenzene and trichlorobenzene. The absorption spectra of $\mathbf{P 3}$ in $\mathrm{CHCl}_{3}$ solution and a spin-coated thin solid film on a glass substrate are represented in Figure 2.9. Polymer $\mathbf{P 3}$ displays a maximal absorption peak at $469 \mathrm{~nm}$ in $\mathrm{CHCl}_{3}$ with an onset from $690 \mathrm{~nm}$ (Figure 2.9a). The film of P3 displays a maximal absorption peak at $485 \mathrm{~nm}$ with an onset from $900 \mathrm{~nm}$ (Figure 2.9a). The red shift of absorption maximal in the solid state is due to better intramolecular interaction. The film of $\mathbf{P 3}$ shows broad absorption compared to its solution because of the better intermolecular interaction as well as $\pi-\pi$ stacking of polymer chains. The polymer shows no emission in solution and in the solid state may be due to the charge transfer or aggregation caused quenching. 
Hagana et al. synthesized a series of poly(isothianaphthene) (PITN) polymers in presence of $\mathrm{P}_{4} \mathrm{~S}_{10}$ by keto-keto coupling, where monomer units are linked by a double bond. $^{22}$ Those polymers were black or blue in color and the absorption was tailing from the longer wavelength. We found that polymer P3 is black-amber in color and the absorption is tailing from the longer wavelength. The reaction mechanism was described by Vanderzande et al. the keto compound in the presence of $\mathrm{P}_{4} \mathrm{~S}_{10}$ forms thio-keto then free radical polymerization occurs between the sulfur atoms that form the disulfide link through the polymer chain, which subsequently undergoes a rapid loss of sulfur $\left(\mathrm{S}_{2}\right)$ and provides a double bond between the monomer units. ${ }^{23}$ Therefore, the keto-keto coupling by LR took place during the polymer transformation from M1 to P3.

P3 shows a complete oxidation-reduction process in CV (Figure 2.9b). The onset oxidation potential/onset reduction potential of polymer $\mathbf{P} 3$ is $1.01 /-0.98 \mathrm{~V}$. The HOMO energy level of polymer is $-5.37 \mathrm{eV}$ and the LUMO energy level is $-3.38 \mathrm{eV}$. The energy band gap is $1.99 \mathrm{eV}$. The HOMO energy level is very close to the ideal HOMO energy level of D-A polymer, which indicates polymer $\mathbf{P 3}$ is highly electron rich and a donor type in nature. ${ }^{16}$ The large energy band gap may be caused by the higher torsional angle between the monomer units.

Table 2.3. Optical and electrochemical properties of polymers P3.

\begin{tabular}{|l|l|l|l|l|}
\hline Polymer & HOMO $(\mathrm{eV})$ & LUMO $(\mathrm{eV})$ & $\mathrm{E}_{\mathrm{g}}{ }^{\mathrm{pt}}(\mathrm{eV})$ & $\mathrm{E}_{\mathrm{g}}{ }^{\mathrm{CV}}(\mathrm{eV})$ \\
\hline P3 & $-5.37(\mathrm{cv})$ & $-3.38(\mathrm{cv})$ & 1.37 & 1.99 \\
\hline
\end{tabular}

Ullmann reaction was carried out on polymer P3, according to the reaction in Scheme 2.8 , in two different solvents and the resultant polymer was characterized by absorption and IR spectroscopy. The absorption spectrum (Figure 2.10) shows that the 
obtained polymer has the same absorption as polymer P3, which raises a question about the proposed structure for polymer P3. If the structure of polymer $\mathbf{P 3}$ is correct, then an intramolecular free radical reaction will produce the polymer P2. This result gave an opportunity to investigate the polymer structure.

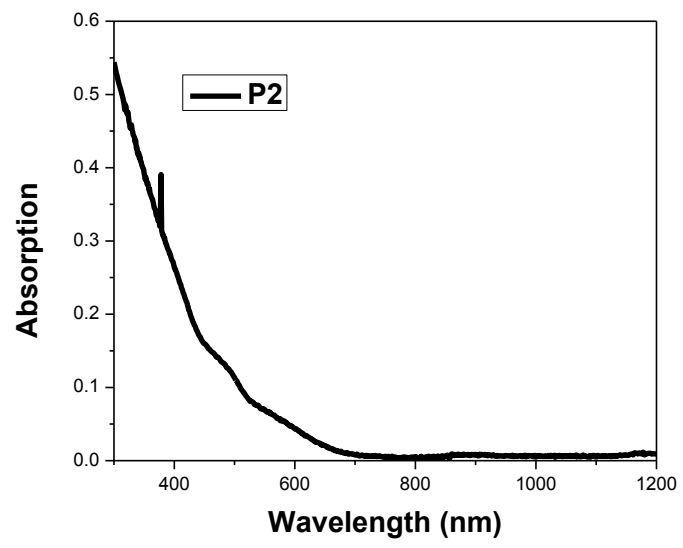

Figure 2.10 Absorption spectrum of polymer $\mathbf{P 2}$ in $\mathrm{CHCl}_{3}$ solution.

\subsection{Linear and linear-ladder conjugated copolymers}

The copolymers having both linear and ladder conjugated units are expected to have properties that are different from a linear polymer and a ladder polymer and thus is a subject of this thesis research. Two different series of conjugated D-A polymers with thiophene as a donor and benzothiadiazole (BT) as an acceptor were synthesized and characterized. Thiophene and BT were chosen due to their availability and the properties of the corresponding conjugated polymers. ${ }^{24}$ Thiophene has less aromatic characteristics, meaning it has less aromatic stabilization energy resulting in higher electron delocalization and the narrowing of the bandgap. ${ }^{25-29}$ Moreover, it has a good stability in the doped and undoped state, along with structural versatility. BT has been used for constructing organic electronics due to its electron deficient nature. ${ }^{30-33}$ The imine 
functionality with relatively low energy $\pi^{*}$-orbitals gives BT a relatively high electron affinity. BT has a quasi-quinoidal structure (i.e., with localized, relatively short $\pi$-bonds in the benzo ring) rather than a $10 \pi$ electron heteroaromatic system; this can increase in electronic coupling between substituents in the 4- and 7-positions relative to that found across 1,4-substituted aromatic moieties in both small molecules and polymers. ${ }^{31}$ Currently, the D-A polymer with a BT unit is reported to have the highest open-circuit voltage $\left(V_{\text {oc }}\right)$ of $1.02 \mathrm{~V},,^{33}$ internal quantum efficiency (IQE) of $100 \%{ }^{34}$ and the hole mobility of $5.5 \mathrm{~cm}^{2} \mathrm{~V}^{-1} \mathrm{~s}^{-1} \cdot{ }^{35}$ Han et al. synthesized a BT-based acceptor polymer, which shows a strong optical absorption in the Near-IR (NIR) region (up to $1100 \mathrm{~nm}$ ) and weak absorption in the visible range $(400 \sim 600 \mathrm{~nm}){ }^{36}$

\subsection{Synthesis and characterization of linear-ladder copolymers containing a thiophene linkage}

Two random copolymers, $\mathbf{P 4}$ and $\mathbf{P 5}$ were synthesized as a precursor polymer for target linear-ladder copolymers $\mathbf{P 6}$ and P7. The polymers were synthesized according to the reactions in Scheme 2.9. The linear copolymers P4 and P5 were synthesized by the Yamamoto reaction with the two different molar ratios of M1 and thiophene in the presence of stoichiometric amount of $\mathrm{Ni}(\mathrm{COD})_{2}$ in toluene at $95{ }^{\circ} \mathrm{C}$ in $87.5 \%$ and $97.2 \%$ yield, respectively. The obtained polymers were dark brown solids. The polymers were purified by Soxhlet extraction. Intramolecular cyclization of polymers P4 and P5 using LR in DCB at $160{ }^{\circ} \mathrm{C}$ resulted in the linear-ladder copolymers $\mathbf{P 6}$ and P7. During the reaction, the color of the solution changed from reddish to greenish, which confirmed the 
cyclization of polymer. The polymers were purified by following the procedure described in Section 2.4 for polymer P2. The obtained polymers $\mathbf{P 6}$ and $\mathbf{P 7}$ were dark in color.

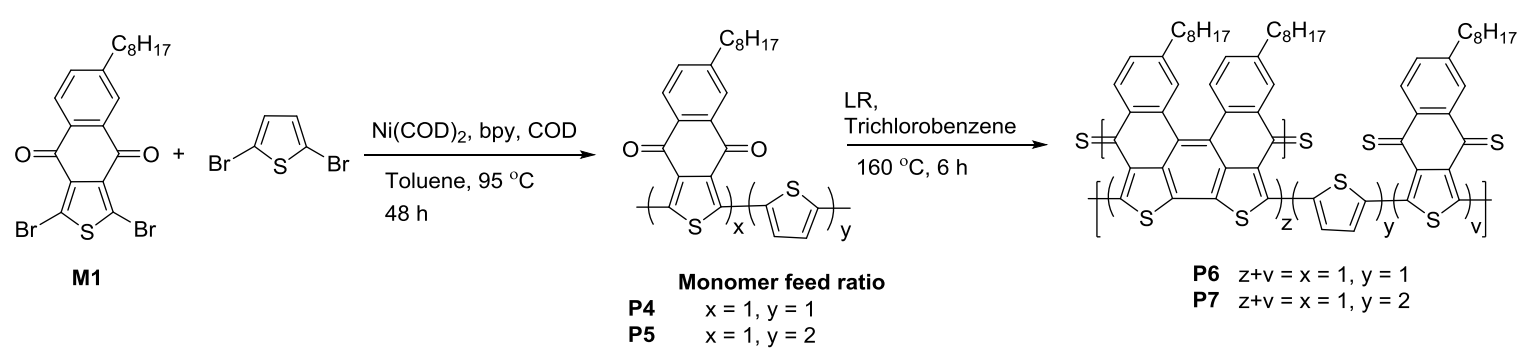

Scheme 2.9 Synthesis of linear-ladder copolymers $\mathbf{P 6}$ and $\mathbf{P 7}$ from linear copolymers P4 and P5.

The polymers were characterized by IR, NMR, TGA, absorption, emission spectroscopy and CV. The IR spectra (Figures 2.11a and c) of copolymers P4 and P5 show a sharp and intense peak at $1671 \mathrm{~cm}^{-1}$, which is the characteristic peak for cyclic $\mathrm{C}=\mathrm{O}$, indicating the polymers have NTDO moiety. The peak intensity for the $\mathrm{CO}$ moiety in copolymer $\mathbf{P 5}$ decreases when compared to copolymer $\mathbf{P 4}$, indicating the feed in ratio of thiophene unit was consistent with the IR spectrum. It was also impossible to differentiate between copolymers $\mathbf{P 4}$ and $\mathbf{P 5}$ by the ${ }^{1} \mathrm{H}$ NMR spectra (Appendix A, Figure S2.16). Both polymers show the same broad peaks at $\delta 8.16,8.06$ and $7.57 \mathrm{ppm}$, which are assigned as aromatic peaks. The peak at $\delta 2.75 \mathrm{ppm}$ is assigned to the $\mathrm{CH}_{2}$ proton of benzyl and all other peaks are at $\delta 1.66,1.53,1.27$ and $0.87 \mathrm{ppm}$, indicating $\mathrm{CH}_{2}$ and $\mathrm{CH}_{3}$ moieties in the alkyl chain, respectively. The ${ }^{1} \mathrm{H}$ NMR spectra of copolymers P4 and P5 are similar to that of polymer P1. However, it is not possible to isolate the thiophene protons from the ${ }^{1} \mathrm{H}$ NMR spectra of copolymers P4 and P5. We believe that the thiophene proton is embedded at position $\delta 7.57 \mathrm{ppm}$ with the other aromatic protons of the polymers. It was also observed that when the molar ratio of 
thiophene units in the polymers increased, the solubility decreased in $\mathrm{CHCl}_{3}$, likely because of the planarity of the thiophene segments and the decrease of alkyl long chains.

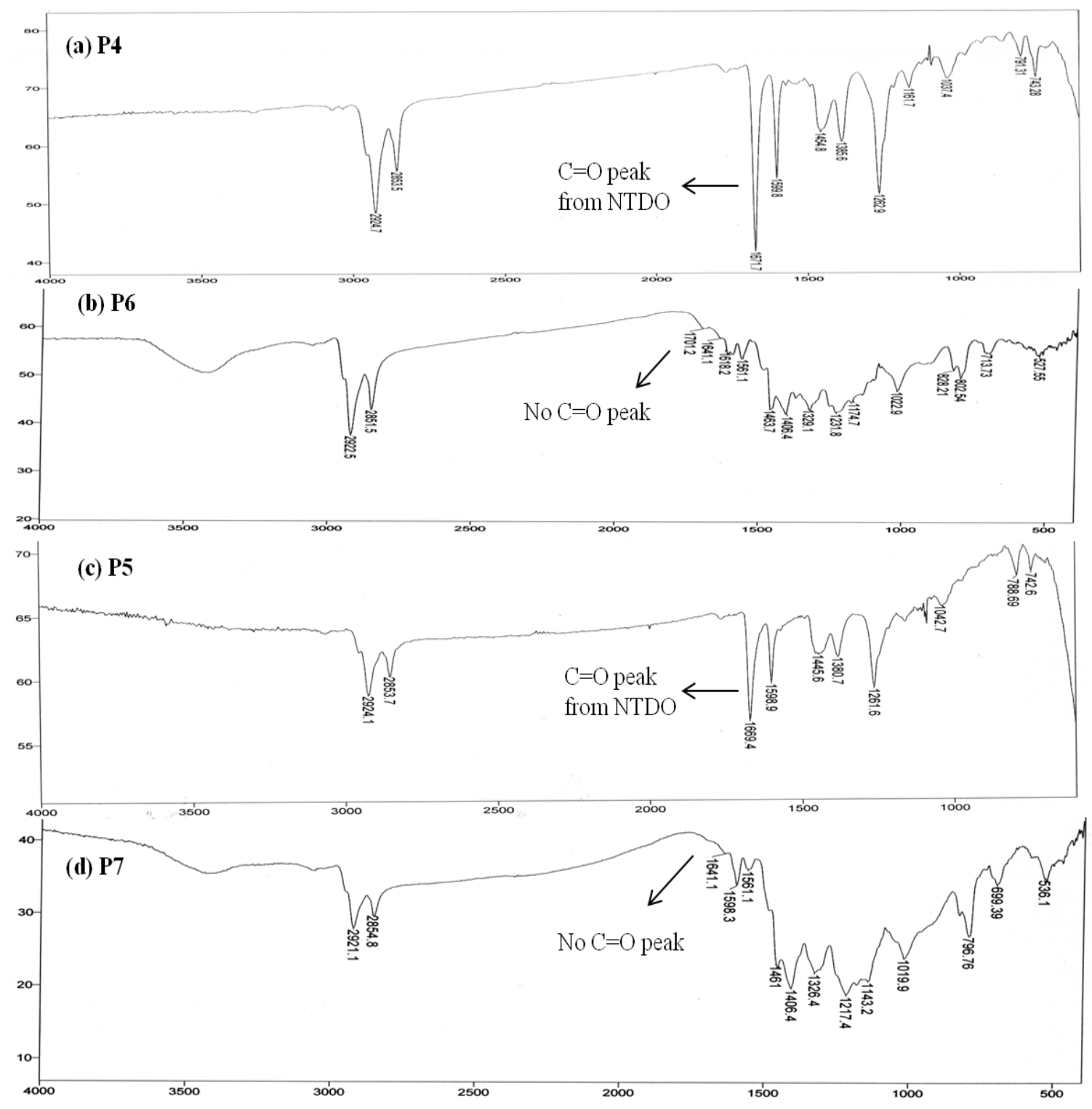

Figure 2.11 IR spectra of copolymers (a) P4, (b) P6, (c) P5 and (d) P7, where P4 and P5 are on $\mathrm{NaCl}$ plate and $\mathbf{P 6}$ and $\mathbf{P 7}$ are in $\mathrm{KBr}$ pellet.

The IR spectra of polymers P6 and P7 (Figure 2.11b and d) show no peak at 1671 $\mathrm{cm}^{-1}$, which indicates the absence of $\mathrm{C}=\mathrm{O}$ and either the intramolecular cyclization or 
formation of $\mathrm{C}=\mathrm{S}$. However, the peak for $\mathrm{C}=\mathrm{S}$ moiety was not observed in the IR spectra of polymers $\mathbf{P 6}$ and $\mathbf{P 7}$. The peak for $\mathrm{C}=\mathrm{S}$ moiety varies from 1160 to $1030 \mathrm{~cm}^{-1}$. The precursor copolymers P4 and P5 have several weak peaks in this region, as do polymers P6 and P7.

The thermal stability of the polymers was examined by thermogravimetric analysis, as presented in Figure S2.17 (Appendix A) and reveals that the decomposition ( $\mathrm{T}_{\mathrm{d}}$ ) onset for polymers P4, P5, P6 and P7 are 306.4, 292.4, 207.6, and $181.3{ }^{\circ} \mathrm{C}$, respectively with $1 \%$ weight loss. It was also observed that the temperature for $5 \%$ weight loss was following the same trend, $\mathbf{P 4}>\mathbf{P 5}>\mathbf{P 6}>\mathbf{P} 7$, indicating the thermal stability of the polymers decreased after the cyclization reaction. This might be caused by the higher number of the $\mathrm{C}=\mathrm{S}$ segments and fewer ladder segments inside the polymer backbone. As the thiophene units increase from polymer $\mathbf{P 6}$ to $\mathbf{P 7}$, the decomposition temperature decreases because of a higher probability of the $\mathrm{C}=\mathrm{S}$ segments. Interestingly, the decomposition temperature for copolymer P4 was higher than P1, whereas $\mathbf{P 5}$ has a lower decomposition temperature in compared to P4. This may be due to the inter and intramolecular interactions caused by the change of the monomer sequence in the polymer backbone. $^{37}$

Copolymers $\mathbf{P 4}$ and $\mathbf{P 5}$ are fully soluble in $\mathrm{CHCl}_{3}$, chlorobenzene, $\mathrm{DCB}$ and TCB, whereas the polymer $\mathbf{P 6}$ is fully soluble in TCB and $\mathbf{P 7}$ is fully soluble in 1,1,2,2tetrachloroethelene (TCE). The absorption spectra of P4, P5 and P6 in TCB and P7 in TCE, as well as the spin-coated thin films on glass substrates were taken. The absorptions (P4 and P5) were taken in the same solvent to show the difference in the absorptions spectra. Polymer P4 displayed a maximal absorption peak at $433 \mathrm{~nm}$ in TCB with an 
onset from $660 \mathrm{~nm}$ (Figure 2.12a). Copolymer P5 displays a maximal absorption peak at $472 \mathrm{~nm}$ in TCB with an onset from $660 \mathrm{~nm}$ (Figure 2.12a). P4 shows a bathocromic shift compared to P1 because of the donor thiophene unit into the polymer backbone. Copolymer $\mathbf{P 5}$ shows a bathocromic shift and a broader absorption compared to $\mathbf{P 4}$ and P1, which indicates it has more planar geometry and better internal charge transfer caused by the donor thiophene segments. With increasing the thiophene segment in random copolymers, $\pi$-conjugation and ICT also increased. The films of P4 and P5 show broader absorptions, compared to their spectra in solution with a red shift (Figure 2.12b). The films of P4 and P5 show absorption peaks at 445 and $492 \mathrm{~nm}$, respectively, with the onset of $730 \mathrm{~nm}$. The maximal absorption bands can be assigned due to $\mathrm{n}-\pi^{*}$ and/or $\pi-\pi^{*}$ transition of the aromatic segment or is due to intramolecular charge transfer. The absorption spectra of polymers in the solid film were red shifted and had a larger area from $730 \mathrm{~nm}$, suggesting intermolecular interactions, more planar structure, close packing, and $\pi-\pi$ stacking. The normalized emission spectra of copolymers P4 and P5 in TCB shows an emission maximum at 538 and $556 \mathrm{~nm}$ (Appendix A, Figure S2.18), whereas the films do not have any emission.
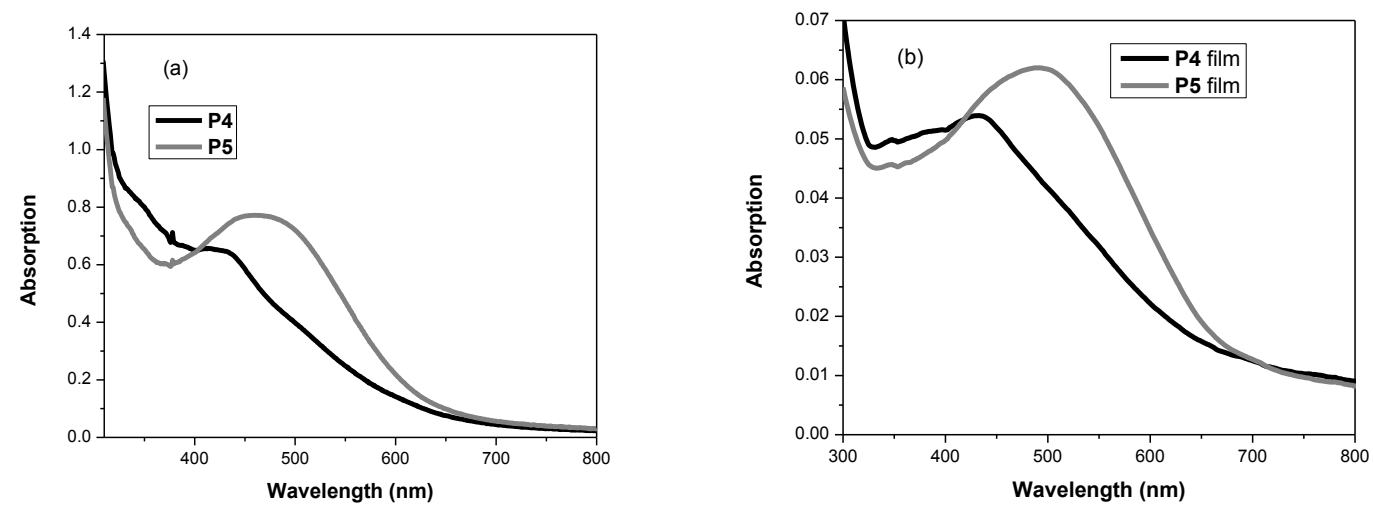
Figure 2.12 Absorption spectra of polymers P4 and P5 in TCB (a) and thin films on glass substrates (b).
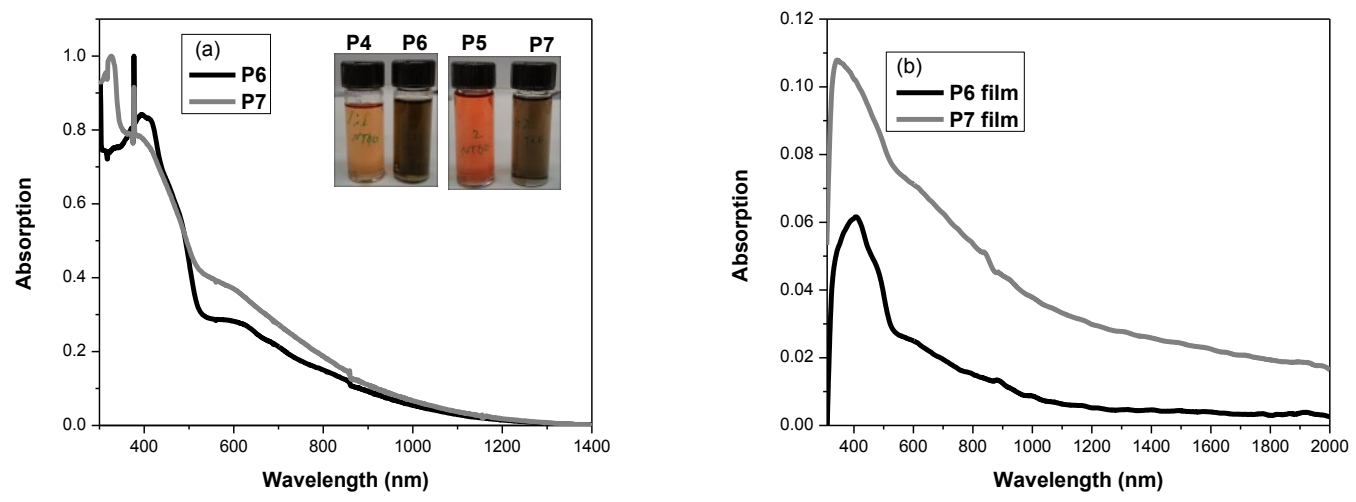

Figure 2.13 Absorption spectra of polymers P6 (TCB) and P7 (TCE) (a) and thin films on glass substrates (b).

Table 2.4. Absorption data of polymers P4, P5, P6 and P7.

\begin{tabular}{|c|c|c|c|c|}
\hline \multirow{2}{*}{ Polymer } & \multicolumn{2}{|c|}{$\lambda_{\max }(\mathrm{nm})$} & \multicolumn{2}{c|}{$\lambda_{\text {onset }}(\mathrm{nm})$} \\
\cline { 2 - 5 } & in solution & film & in solution & film \\
\hline P4 & $433(\mathrm{TCB})$ & 445 & $660(\mathrm{TCB})$ & 730 \\
\hline P5 & $472(\mathrm{TCB})$ & 492 & $660(\mathrm{TCB})$ & 730 \\
\hline P6 & $395,600(\mathrm{TCB})$ & 418 & $1000(\mathrm{TCB})$ & 1100 \\
\hline P7 & $\begin{array}{c}324,392,600 \\
\text { (TCE) }\end{array}$ & 350 & $1000(\mathrm{TCE})$ & 1300 \\
\hline
\end{tabular}

Polymer P6 displays two maximal absorption peaks at 395 and $600 \mathrm{~nm}$ in TCB with an onset from $1000 \mathrm{~nm}$ (Figure 2.13a). Polymer P7 displays similar maximal absorption peak at 324, 392 and $600 \mathrm{~nm}$ in TCE with an onset from $1000 \mathrm{~nm}$. However, 
the absorption spectrum for polymer $\mathbf{P} 7$ is broader in the longer wavelength than polymer P6 (Figure 2.13a). The film of P6 and P7 show broader absorption, similar to their corresponding solution spectra. The film of $\mathbf{P 6}$ shows an absorption peak at $418 \mathrm{~nm}$ and a wide absorption spectrum with an onset from $1100 \mathrm{~nm}$, whereas the film of $\mathbf{P} 7$ shows an absorption peak at $350 \mathrm{~nm}$ and a wide absorption spectrum with an onset from $1300 \mathrm{~nm}$ (Figure 2.13b). Polymer P7 has a higher number of thiophene segments that are planar, as a result, the planarity of $\mathbf{P 7}$ is higher than $\mathbf{P 6}$, so $\mathbf{P 7}$ shows absorption in the longer wavelength. It can also be said that increasing the ladder segments' electron delocalization somehow hindered and increased torsional angle, resulting in lower absorption onset.

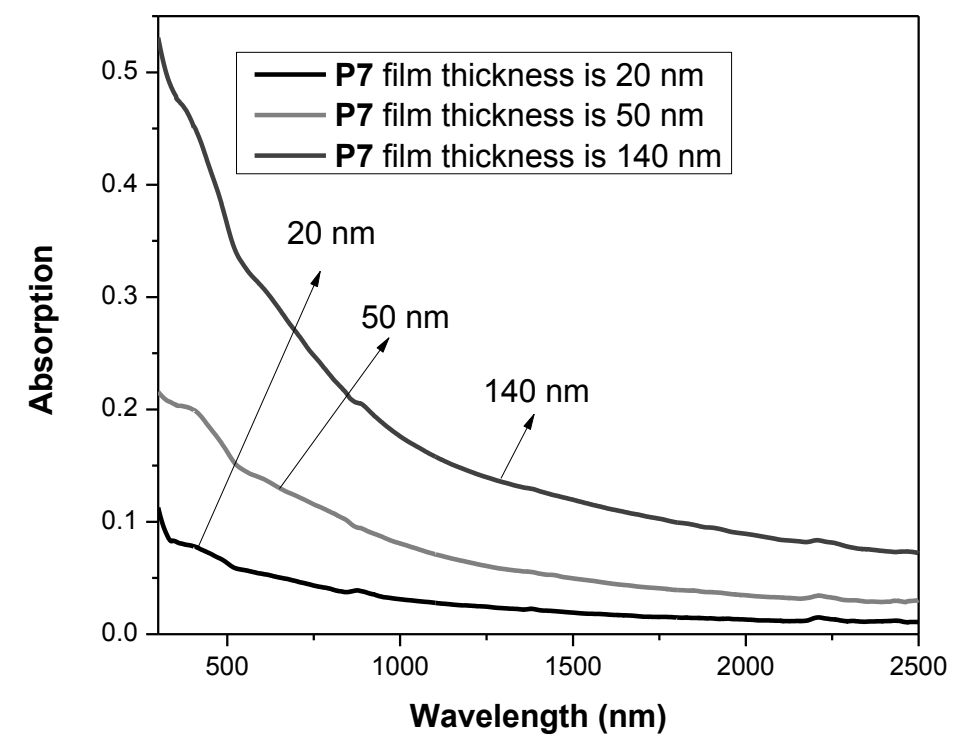

Figure 2.14 Absorption spectra of polymer $\mathbf{P 7}$ films in different thicknesses on quartz surface. 
To understand the broad absorption of polymer $\mathbf{P 7}$ in the solid state, different thicknesses of film were prepared on a quartz surface (Figure 2.14). Quartz does not have any absorption in NIR region. The absorption spectra show that the film's thickness increases, the absorption intensity in the NIR also increases, which reflects that the polymer film has absorption in NIR region. The NIR absorption indicates the ICT band is located in the NIR region, thus enabling the polymers to absorb the NIR insolation. However, the weak intensity in the NIR region could be due to the localized electron density over both the HOMO and LUMO energy levels. ${ }^{15 \mathrm{c}}$ The broad absorption spectra of polymers in the solid state suggests intermolecular interaction, close packing, and $\pi-\pi$ stacking among the polymer chains. The emission spectrum of polymer P6 in TCB shows a shoulder at $500 \mathrm{~nm}$ and a maximum at $556 \mathrm{~nm}$ whereas the polymer $\mathbf{P 7}$ in TCE shows a shoulder at $454 \mathrm{~nm}$ and a maximum at $527 \mathrm{~nm}$ (Figure S2.19). The solid films of polymers P6 and P7 do not show any emission because of aggregation caused quenching.

The HOMO and LUMO energy levels and electrochemical band gaps $\left(\mathrm{E}_{g}\right)$ of the polymers P4 to $\mathbf{P 7}$ were calculated from oxidation onset and reduction onset potentials. Their HOMO and LUMO energy levels are summarized in Table 2.5 and their CV are shown in Figure 2.15. Polymers, P4 and P5 show two noticeable reduction processes each. The onsets are $-0.26,-1.60 \mathrm{~V}$, and $-0.45,-1.53 \mathrm{~V}$, respectively in $\mathrm{CV}$. As the donor ratio increases from polymer $\mathbf{P 4}$ to $\mathbf{P 5}$, the first reduction potential increases but the second reduction potential decreases. Polymers P6 and P7 show both oxidation and reduction processes. The onset oxidation potentials/onset reduction potentials of polymers P6 and P7 are $0.85 /-1.26 \mathrm{~V}$ and $0.80 /-0.57,-1.67 \mathrm{~V}$ (Table 2.5 ), respectively. The HOMO energy levels of polymers are $-5.21 \mathrm{eV}$ and $-5.16 \mathrm{eV}$, which are raised (less 
negative) from $\mathbf{P 6}$ to $\mathbf{P 7}$. The LUMO energy levels are $-3.10 \mathrm{eV}$ and, -3.79 , lowered from $\mathbf{P 6}$ to P7. The optical energy gap for both the polymers $\mathbf{P 4}$ and $\mathbf{P 5}$ is $1.87 \mathrm{eV}$.
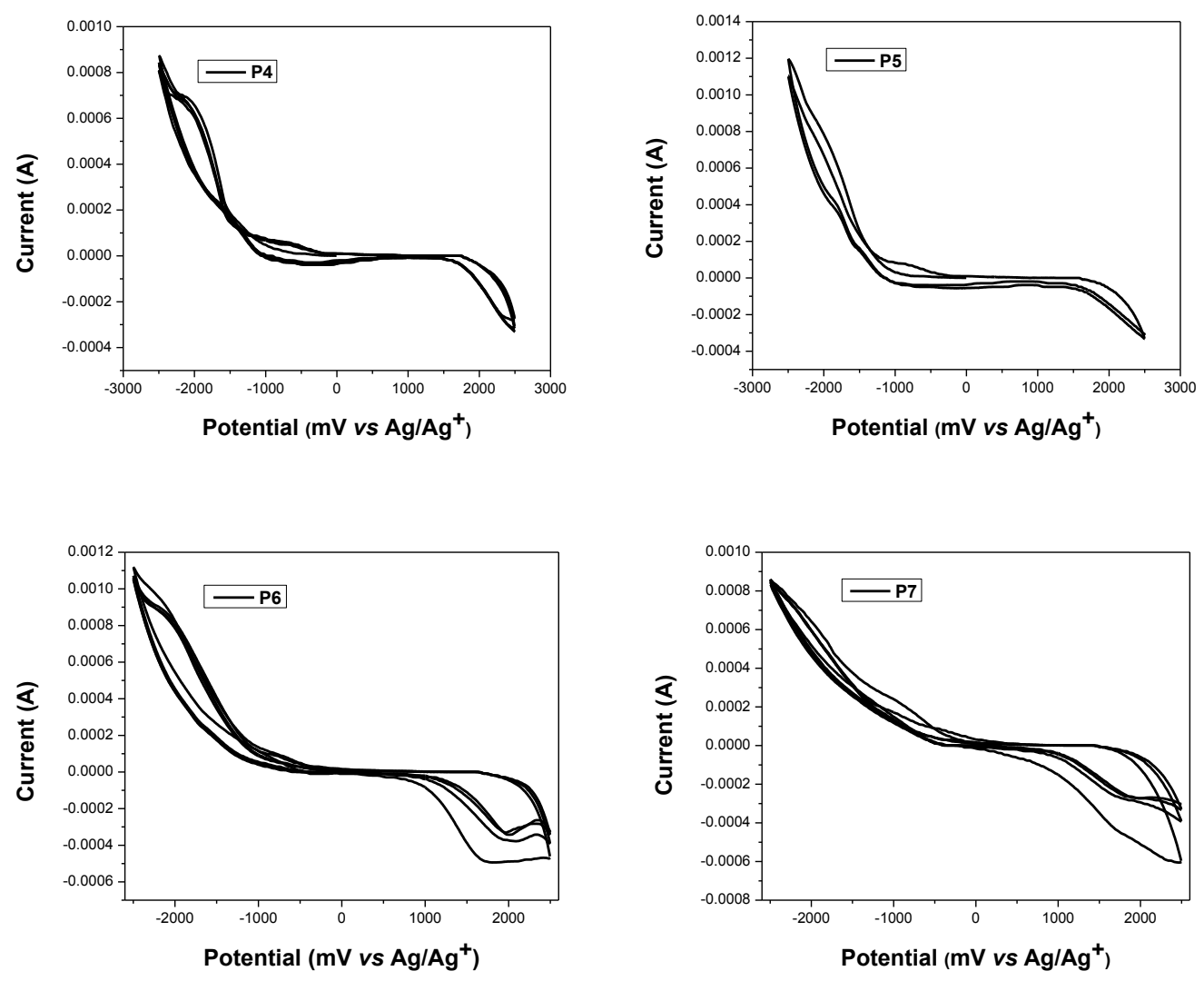

Figure 2.15 Cyclic voltammogram of polymers P4 (a), P5 (b), P6 (c) and P7 (d) film on ITO in $\mathrm{CH}_{3} \mathrm{CN}$ solution containing $0.1 \mathrm{~mol} / \mathrm{L} \mathrm{Bu}_{4} \mathrm{NPF}_{6}$ with a scan rate of $100 \mathrm{mV} / \mathrm{s}$.

Incorporation of thiophene units in linear polymer backbone the HOMO and LUMO energy levels of thiophene and NTDO hybridized, as a result, they show a lower HOMO-LUMO separation and thus the energy band gaps of $\mathbf{P 4}$ and $\mathbf{P 5}$ are lower than polymer P1. Generally, as donor amounts increase in a conjugated polymer chain, the polymer becomes more p-type in nature and shows a higher HOMO energy level. There is a higher HOMO energy level in $\mathbf{P 5}$ than $\mathbf{P 4}$, which might be attributed to the existence 
of the consecutive thiophene segments in the random polymer or better $\pi$-electron delocalization.

Table 2.5. Optical and electrochemical properties of polymers P4-P7.

\begin{tabular}{|l|l|l|l|l|}
\hline Polymer & HOMO $(\mathrm{eV})$ & LUMO $(\mathrm{eV})$ & $\mathrm{E}_{\mathrm{g}}{ }^{\mathrm{ppt}}(\mathrm{eV})$ & $\mathrm{E}_{\mathrm{g}}^{\mathrm{CV}}(\mathrm{eV})$ \\
\hline $\mathbf{P 4}$ & $\begin{array}{l}\text { NA (cv), }-5.78,-4.43 \\
\text { (opt) }\end{array}$ & $\begin{array}{l}-4.11,-2.76(\mathrm{cv}) \\
\text { Onset, }-0.25,-1.60\end{array}$ & 1.67 & NA \\
\hline P5 & $\begin{array}{l}\text { NA (cv), -5.58, -4.50 } \\
\text { (opt) }\end{array}$ & $-3.91,-2.83(\mathrm{cv})$ & 1.67 & NA \\
\hline P6 & $\begin{array}{l}-5.21(\mathrm{cv}) \\
\text { Onset, }-0.45,-1.53\end{array}$ & $-3.10(\mathrm{cv})$ & 1.13 & 2.11 \\
\hline P7 & $\begin{array}{l}-5.16(\mathrm{cv}) \\
\text { Onset } 0.80\end{array}$ & $-3.79,-2.69(\mathrm{cv})$ & 0.95 & 1.37 \\
\hline
\end{tabular}

The LUMO energy level increases inversely as the donor unit increases. Here, we observed that when the donor thiophene unit increased from polymer P4 to P5, both the energy levels increased and there was no change in the energy band gap. ${ }^{38}$ The coupling reaction of keto groups of polymers created more donor units because of the new $\mathrm{C}=\mathrm{C}$ bond formation. Therefore, the HOMO energy level increased in $\mathbf{P 6}$ and P7. The LUMO energy level of $\mathbf{P 6}$ increased more than $\mathbf{P 7}$ due to the higher number of ladder units. The discrepancy in the higher electrochemical band gap compared to the optical band gap in film-based CV was previously reported for polymers and small compounds due to substantial differences in the exciton-binding energy of cyclized systems. ${ }^{16}$ The optical 
band gaps decreased from ladder polymer $\mathbf{P 2}$ to linear-ladder polymers $\mathbf{P 6}$ and $\mathbf{P 7}$ due to better hybridization and lower separation of HOMO and LUMO energy levels. This also indicates that with increasing less aromatic segments with smaller structures in the ladder type polymer backbone, the band gap decrease may be due to better $\pi$-conjugation, less torsional angle and planarity.

\subsection{Synthesis and characterization of linear-ladder copolymers containing a benzothiadiazole linkage}

Two random copolymers P8 and P9 with $\mathbf{M 1}$ and benzothiadiazole in different molar ratios were synthesized as precursor polymers for the target linear-ladder polymers P10 and P11. All polymers were synthesized according to the reactions in Scheme 2.10. The linear copolymers P9 and P10 were synthesized by the Yamamoto reaction as described in Section 2.6 for polymer $\mathbf{P 4}$ and $\mathbf{P 5}$ in $70.0 \%$ and $73.3 \%$ yield, respectively. The obtained copolymers were brown solids. Intramolecular cyclization using LR afforded linear-ladder polymers P10 and P11 with a benzothiadiazole linkage in the main polymer chain. The intramolecular cyclization was carried out according to the procedure described in Section 2.8 for polymers P5 and P6. The obtained polymers were black solids. The resultant polymers $\mathbf{P 8}$ and $\mathbf{P 9}$ in the presence of BT unit are more electron deficient because of the electron deficient nature of BT monomers. The energy band gap will decrease as the LUMO energy level lowers compared to polymer P1. The cyclization reaction in polymers $\mathbf{P 8}$ and $\mathbf{P 9}$ will create more of a D-A-type nature instead of an $\mathrm{n}$ type. As a result, the energy band gap will decrease by increasing the HOMO energy 
level. Therefore, these two linear-ladder polymers (P10 and P11) may exhibit lower energy band gaps than other polymers (P8 and $\mathbf{P 9})$.

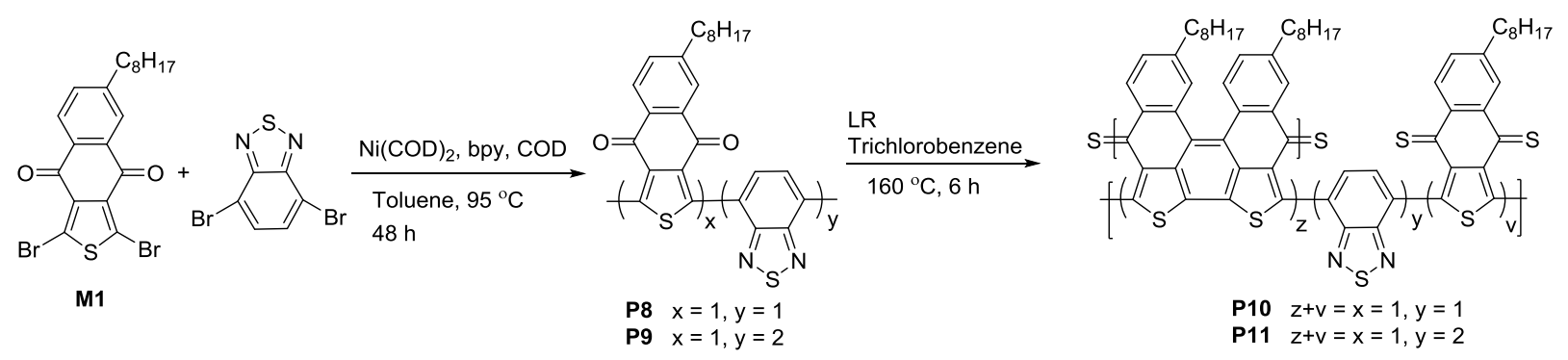

Scheme 2.10 Synthesis of linear-ladder copolymers P10 and P11 from linear copolymers

$\mathbf{P 8}$ and $\mathbf{P 9}$.

The polymers were characterized by IR, NMR, TGA, absorption, emission spectroscopy and CV. The IR spectra (Figure 2.16a and b) of copolymers P8 and P9 show sharp and strong peaks at $1672 \mathrm{~cm}^{-1}$, which is the characteristic peak for cyclic $\mathrm{C}=\mathrm{O}$, indicating the copolymers have the NTDO unit. The peak intensity for the $\mathrm{C}=\mathrm{O}$ moiety in copolymers $\mathbf{P 8}$ and $\mathbf{P 9}$ did not change with feed in ratio. It is also not possible to differentiate between polymers $\mathbf{P 8}$ and $\mathbf{P 9}$ by ${ }^{1} \mathrm{H}$ NMR (Appendix A, Figure S2.20) and ${ }^{13} \mathrm{C}$ NMR spectra (Figure 2.17). The ${ }^{1} \mathrm{H}$ NMR spectra show the aromatic and aliphatic peaks at the same positions for both polymers. The copolymers $\mathbf{P 8}$ and $\mathbf{P 9}$ show the same broad peaks at $\delta 8.16,8.06$ and $7.57 \mathrm{ppm}$, which are assigned to aromatic protons. The peak at $\delta 2.75 \mathrm{ppm}$ is assigned as $\mathrm{CH}_{2}$ proton of benzyl and all other peaks are at $\delta 1.66,1.53,1.27$ and $0.87 \mathrm{ppm}$, indicating $\mathrm{CH}_{2}$ and $\mathrm{CH}_{3}$ moieties in the alkyl chain, respectively. The ${ }^{13} \mathrm{C}$ NMR spectra (Figure $2.17 \mathrm{~b}$ and c) show two peaks at $\delta$ 179.69 and 179.18 ppm, indicating the $\mathrm{C}=\mathrm{O}$ moiety of copolymers $\mathbf{P 8}$ and $\mathbf{P 9}$. All peaks 
are broad from $\delta 150.46$ to $127.66 \mathrm{ppm}$, indicating aromatic carbon for copolymers $\mathbf{P 8}$ and P9.
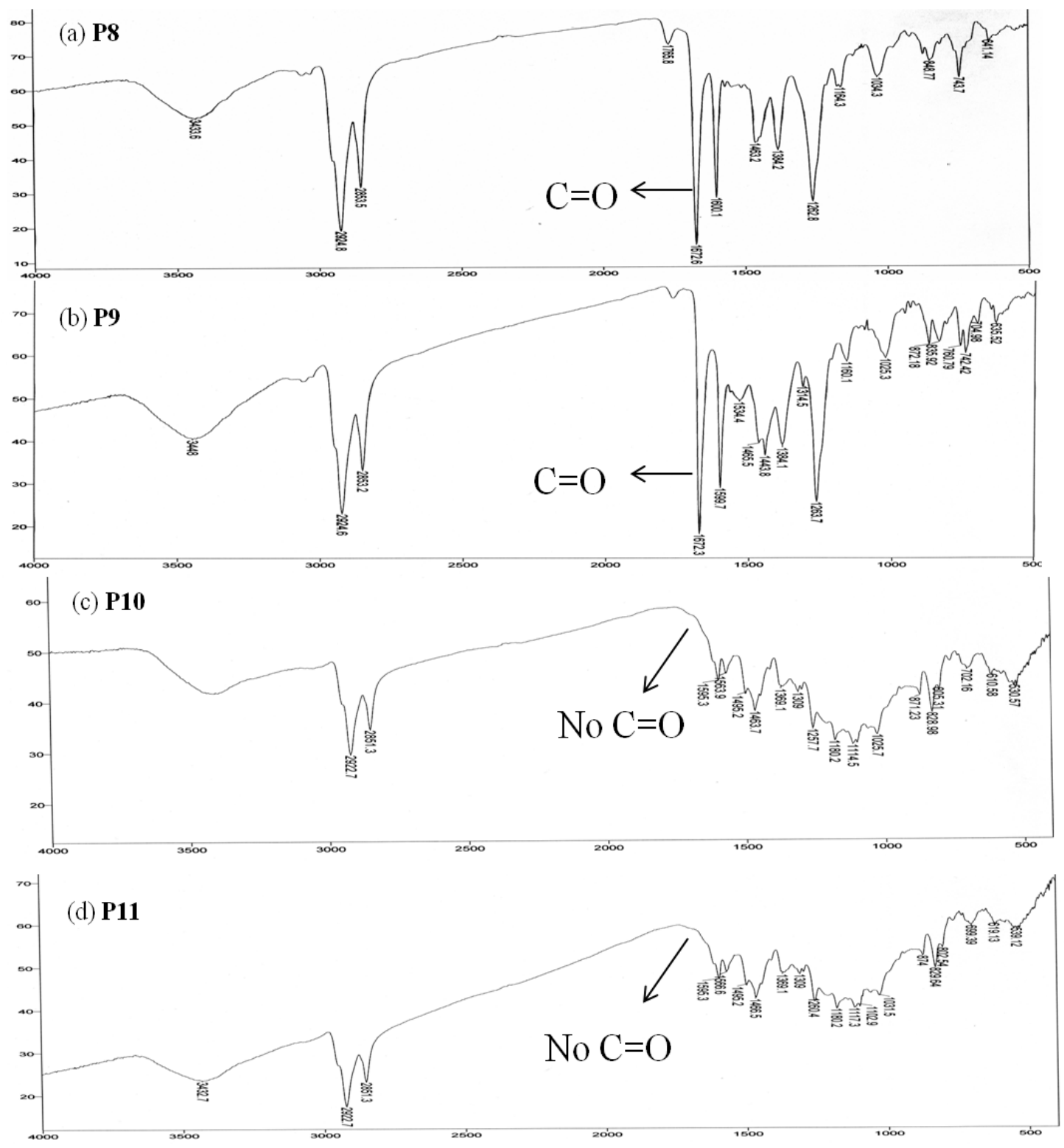

Figure 2.16 IR spectra of polymers P8 (a), P9 (b), P10 (c), and P11 (d) in KBr pellet.

The IR spectra of polymers P10 and P11 (Figures 2.16c and d) show no peak at $1671 \mathrm{~cm}^{-1}$, indicating the absence of $\mathrm{C}=\mathrm{O}$, which suggests either the intramolecular cyclization or the formation of $\mathrm{C}=\mathrm{S}$. However, the peak for $\mathrm{C}=\mathrm{S}$ moiety (from 1160 to 
$1030 \mathrm{~cm}^{-1}$ ) was not identified in the IR spectra of polymers P10 and P11. The precursor polymers P8 and P9 have several weak peaks in this region, as do polymers P10 and P11. sck

(a) $\mathbf{P 1}$

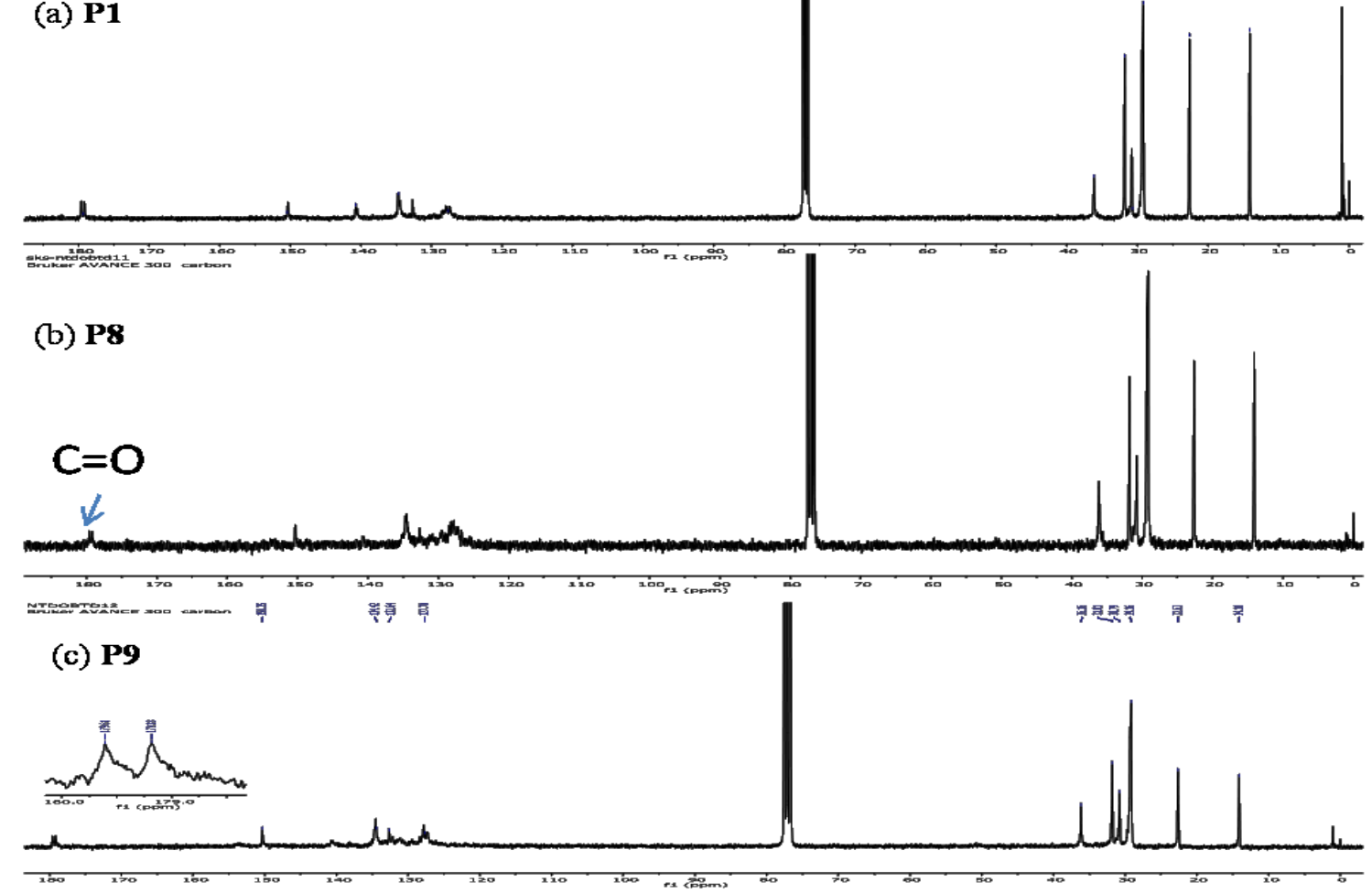

Figure $2.17{ }^{13} \mathrm{C}$ NMR spectra ( $75 \mathrm{MHz}, \mathrm{CDCl}_{3}$ ) of polymers $\mathbf{P 1}$ (a), $\mathbf{P 8}$ (b), and $\mathbf{P 9}$ (c).

The thermal stability of the polymers was investigated by thermogravimetric analysis, as presented in Figure S2.21 (Appendix A), which shows that the decomposition ( $\mathrm{T}_{\mathrm{d}}$ ) onset for polymers P8, P9, P10, and P11 are 257.95, 274.14, 193.97 and $169.80{ }^{\circ} \mathrm{C}$, respectively, with $1 \%$ weight loss. It was also observed that the temperature for $5 \%$ weight loss followed the same trend, $\mathbf{P 9}>\mathbf{P 8}>\mathbf{P 1 0}>\mathbf{P 1 1}$. The $T_{d}$ onset temperature increases as the feed in ratio of benzothiadiazole increases for linear copolymers. However, the $T_{d}$ decreases after cyclization. The linear-ladder polymer P11 has lower thermal stability than $\mathbf{P 1 0}$, which might be due to the higher number of $\mathrm{C}=\mathrm{S}$ units and 
fewer ladder segments inside the main backbone. Moreover, the higher thermal stability of copolymers $\mathbf{P 8}$ and $\mathbf{P 9}$ compared to $\mathbf{P 1}$ may be due to the rigid structure of the benzothiadiazole units and fewer aliphatic units.
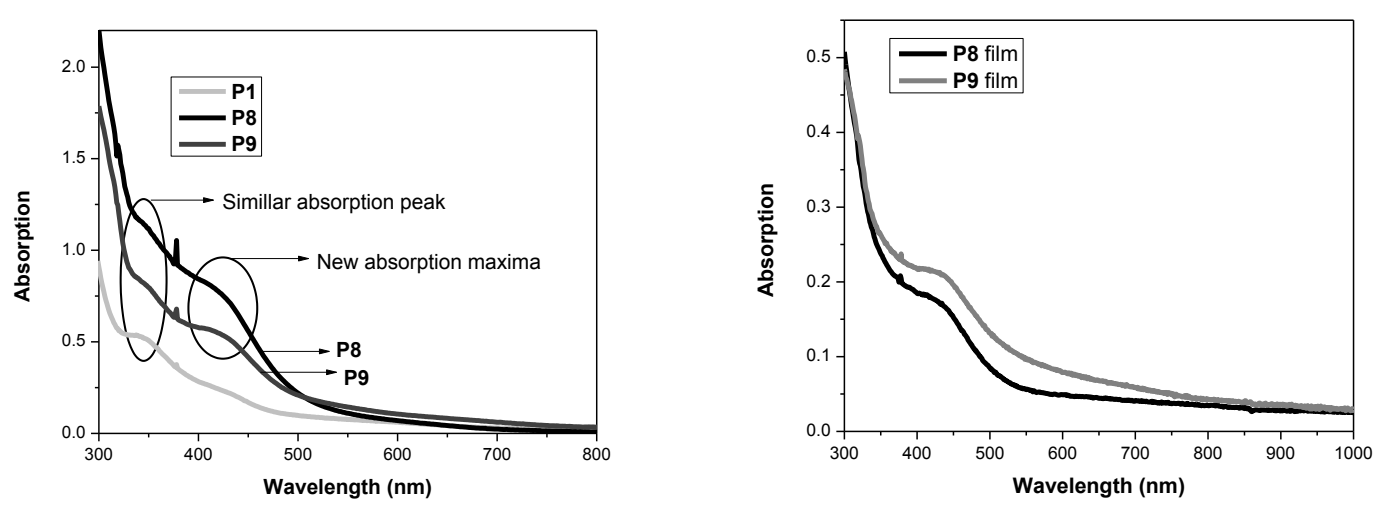

Figure 2.18 Absorption spectra of polymers $\mathbf{P 1}, \mathbf{P 8}$ and $\mathbf{P 9}$ in $\mathrm{CHCl}_{3}$ (a) and on glass substrates (b).

Copolymers $\mathbf{P 8}$ and $\mathbf{P 9}$ are fully soluble in solvents such as $\mathrm{CHCl}_{3}$, chlorobenzene, dichlorobenzene and trichlorobenzene whereas polymer P10 is fully soluble in chlorobenzene and P11 is fully soluble in $\mathrm{N}$-methylpyrrolidinone (NMP). Absorption spectra of $\mathbf{P 8}$ and $\mathbf{P 9}$ in $\mathrm{CHCl}_{3}, \mathbf{P 1 0}$ in chlorobenzene and $\mathbf{P 1 1}$ in NMP, as well as the spin-coated thin solid films on glass substrates were taken. The polymers P1, P8 and P9 display similar absorption spectra with a maximal peak at $343 \mathrm{~nm}$. Moreover, copolymers $\mathbf{P 8}$ and $\mathbf{P 9}$ show a new maximal absorption peak in $\mathrm{CHCl}_{3}$ at 415 and 408 $\mathrm{nm}$, respectively, with onset from $660 \mathrm{~nm}$ (Figure 2.18a), which implies that polymers $\mathbf{P 8}$ and $\mathbf{P 9}$ are new polymers with different ratios of NTDO and BT units. P8 shows more of a bathochromic shift compared to $\mathbf{P 1}$ and $\mathbf{P 9}$. This red shift can be explained by the introduction of BT units, which is a higher electron deficient and rigid monomer than the 
NTDO unit. The bathochromic shift indicates that there is $\pi$-conjugation and ICT between NTDO and BT segments in polymers. ${ }^{39}$ Even though P9 has more BT units than P8, the absorption is blue-shifted. The hypsochromic shift of polymer P9 may be due to the decrease of $\pi$-conjugation and ICT as the BT monomer increases. However, the absorption intensity is weak over $500 \mathrm{~nm}$ which may be due to the ICT being weaker and the electron density not being well distributed over HOMO and LUMO orbitals. The electron density is mostly residing in one of the HOMO of a monomer and one of the LUMO of another monomer. ${ }^{17 \mathrm{c}, 39}$ The films of P8 and P9 show similar absorption spectra to their corresponding solution spectra with a red shift (Figure 2.18b). The films of P8 and $\mathbf{P 9}$ show absorption peaks at 430 and $415 \mathrm{~nm}$, respectively, with an onset of $700 \mathrm{~nm}$. Moreover, the polymer P9 shows broader absorption in film than P8, which may be due to the better $\pi$ - $\pi$ stacking in the solid film. The emission spectra of polymers $\mathbf{P 8}$ and $\mathbf{P 9}$ in $\mathrm{CHCl}_{3}$ show a maximum at 542 and $536 \mathrm{~nm}$ (Figure $\mathrm{S} 2.22$ ), whereas the solid film does not have any emission. This can be explained by the better $\pi-\pi$ stacking and aggregation caused quenching in the solid state.

The absorption spectra of polymers P10 and P11 are shown in Figure 2.19. The absorption spectra for polymers are broader in the solid state than in the solution. Neither of the polymers shows any maximal absorption peak; however, the onsets are from 800 $\mathrm{nm}$ in solution. The polymer films of P10 and P11 show onsets from $1000 \mathrm{~nm}$. The absorption spectra for polymers in the solid film are broader in the longer wavelengths than in polymers P8 and P9 (Figure 2.19). The broad absorption spectra of polymers P10 and P11 suggest they have better intermolecular interaction, close packing, as well as $\pi-\pi$ stacking than do polymers P8 and P9. The broader absorption and red-shifted onset of 
polymers P10 and P11 is due to the formation of ladder segments by keto-keto coupling. Ladder segments increase the D-A type construction thus $\pi$-conjugation and ICT between the ladder and BT segment which influence intrinsic and photophysical properties. The absorption intensity of polymer P11 is stronger in shorter wavelength and weaker in longer wavelengths because of the higher number of BT segments and lower number of ladder units. The emission spectra of polymer P10 in CB shows a maximum at $556 \mathrm{~nm}$ whereas the polymer P11 shows a maximum at $520 \mathrm{~nm}$ (Figure S2.23). This larger Stokes-shift also indicates the polymer P10 has better ICT than polymer P11. The solid film of polymer P10 and P11 do not show any emission due to aggregation caused quenching or charge transfer quenching.
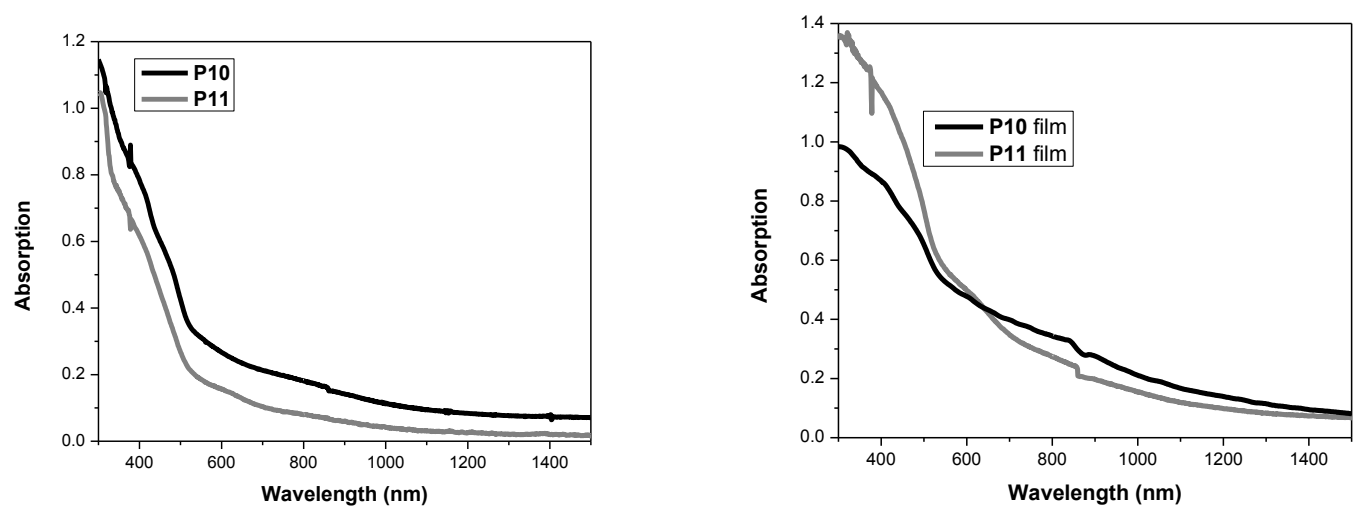

Figure 2.19 Absorption spectra of polymers P10 and P11 (a) in solution and (b) on glass substrates. 
Table 2.6. UV-vis absorption of polymers P8, P9, P10 and P11.

\begin{tabular}{|c|c|c|c|c|}
\hline \multirow{2}{*}{ Polymer } & \multicolumn{2}{|c|}{$\lambda_{\max }(\mathrm{nm})$} & \multicolumn{2}{c|}{$\lambda_{\text {onset }}(\mathrm{nm})$} \\
\cline { 2 - 5 } & in solution & film & in solution & film \\
\hline P8 & $343,415\left(\mathrm{CHCl}_{3}\right)$ & 430 & $660\left(\mathrm{CHCl}_{3}\right)$ & 700 \\
\hline P9 & $343,408\left(\mathrm{CHCl}_{3}\right)$ & 415 & $660\left(\mathrm{CHCl}_{3}\right)$ & 700 \\
\hline P10 & NA & NA & $800(\mathrm{CB})$ & 1000 \\
\hline P11 & NA & NA & $800(\mathrm{NMP})$ & 1000 \\
\hline
\end{tabular}

The HOMO and LUMO energy levels of the polymers are summarized in Table 2.7 and their CV are shown in Figure 2.20. Polymers P8 and P9 show three noticeable reduction processes; the onsets are $-1.26,-2.16,-2.54 \mathrm{~V}$, and $-0.74,-1.85,-2.40 \mathrm{~V}$, respectively, in CV. The polymer $\mathbf{P 8}$ shows one oxidation process, whereas $\mathbf{P 9}$ shows two oxidation processes; the onsets are 1.10 and $0.46,1.30 \mathrm{~V}$, respectively. Increasing the BT ratio from polymer $\mathbf{P 8}$ to $\mathbf{P 9}$ causes the reduction potential to decrease gradually and leads to a lower LUMO. Increasing the BT unit the LUMO level decreased, indicating the BT unit's strong electron accepting ability. The deeper HOMO energy level of P8 compared to $\mathbf{P 9}$ also indicates better $\pi$-conjugation and ICT between the monomer segments when their ratio is 1:1. The HOMO energy level indicates that, when acceptor segments are at a 1:1 ratio, a deeper HOMO energy level like in a D-A polymer is achievable. Polymers P10 and P11 show both oxidation and reduction processes. The onset oxidation potential/onset reduction potential of polymers P10 and P11 are 1.00/$0.39,-1.06 \mathrm{~V}$ and $0.85 /-0.75 \mathrm{~V}$, (Table 6), respectively. The HOMO energy levels of the polymers are $-5.36 \mathrm{eV}$ and $-5.16 \mathrm{eV}$; they rise from P10 to P11. The LUMO energy levels are $-3.97 \mathrm{eV}$ and, $-3.61 \mathrm{eV}$, raised from P10 to P11. The HOMO energy level 
increased and the LUMO energy level decreased of polymer P10, which is due to the presence of donor type ladder segments. This indicates better hybridization of HOMO and LUMO orbitals. Therefore, the band gap is decreased. This low energy band gap can also be explained by effective conjugation and planarity caused by the presence of ladder and BT segments. However, the HOMO energy level of P11 decreased compared to P9 which is unusual. The LUMO energy level of P11 did not change, thus indicating the saturation of the LUMO energy level
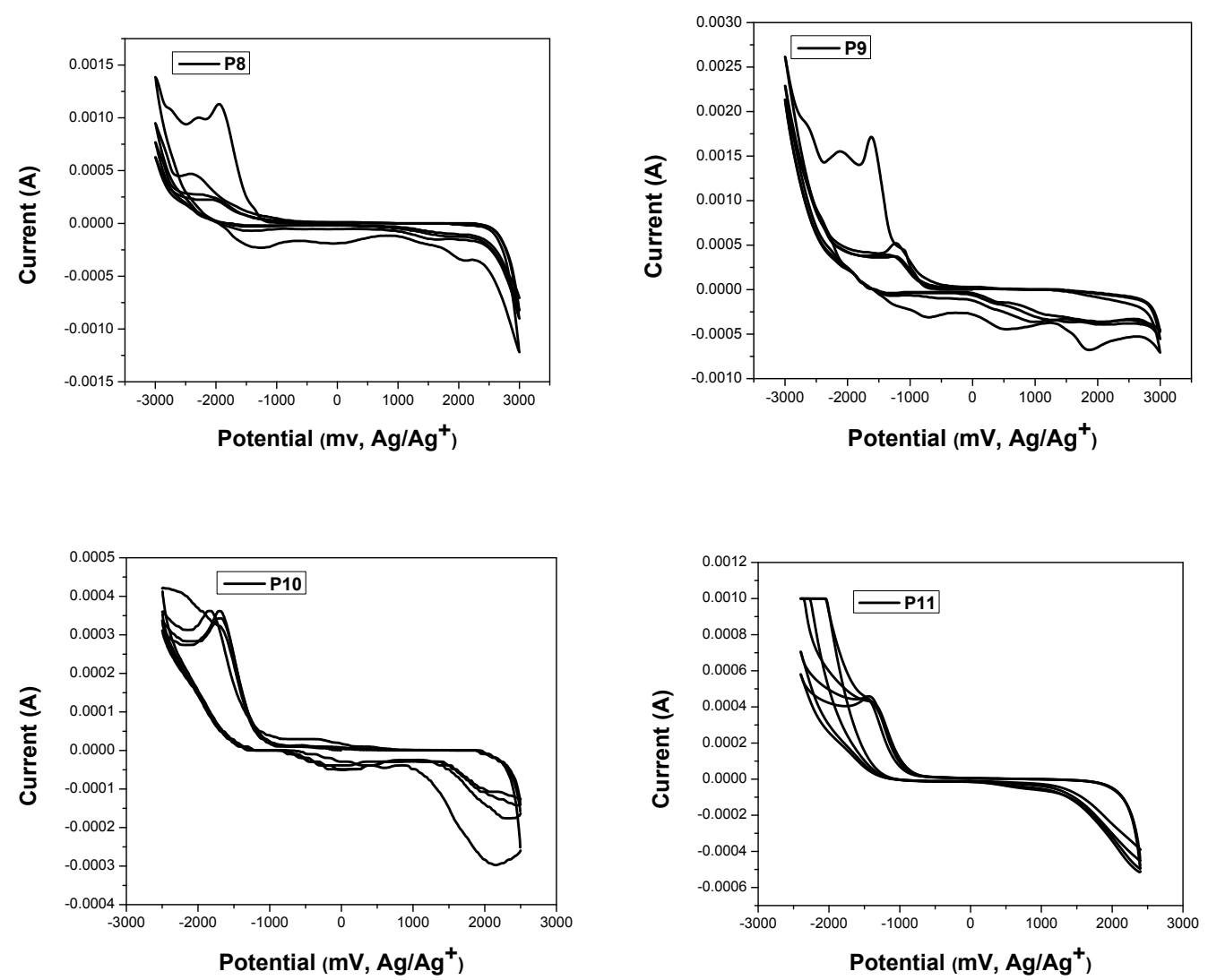

Figure 2.20 Cyclic voltammogram of polymers P8 (a), P9 (b) P10, (c) and P11(d) film on ITO in $\mathrm{CH}_{3} \mathrm{CN}$ solution containing $0.1 \mathrm{~mol} / \mathrm{L} \mathrm{Bu}_{4} \mathrm{NPF}_{6}$ with a scan rate of $100 \mathrm{mV} / \mathrm{s}$. 
Table 2.7. Optical and electrochemical properties of polymers P8-P11.

\begin{tabular}{|c|c|c|c|c|}
\hline Polymer & HOMO (eV) & LUMO (eV) & $\mathrm{E}_{\mathrm{g}}^{\mathrm{opt}}(\mathrm{eV})$ & $\mathrm{E}_{\mathrm{g}}^{\mathrm{CV}}(\mathrm{eV})$ \\
\hline P8 & $\begin{array}{l}-5.46,(\mathrm{cv}) \\
\text { Onset } 1.10,\end{array}$ & $\begin{array}{l}-3.10,-2.26,-1.82 \\
(\mathrm{cv}) \\
\text { Onset, }-1.26,-2.10,- \\
2.54\end{array}$ & 1.77 & 2.36 \\
\hline P9 & $\begin{array}{l}-4.82,-5.66(\mathrm{cv}) \\
\text { Onset } 0.46,1.30\end{array}$ & $\begin{array}{l}-3.62,-2.51, \quad 1.96 \\
(\mathrm{cv}) \\
\text { Onset, }-0.74,-1.85,- \\
2.40\end{array}$ & 1.77 & 1.20 \\
\hline P10 & $\begin{array}{l}-5.36(\mathrm{cv}) \\
\text { Onset }-1.00\end{array}$ & $\begin{array}{l}-3.97,-3.27(\mathrm{cv}) \\
\text { Onset }-0.39,-1.09\end{array}$ & 1.24 & 1.39 \\
\hline P11 & $\begin{array}{l}-5.16(\mathrm{cv}) \\
\text { Onset } 0.80\end{array}$ & $\begin{array}{l}-3.61(\mathrm{cv}) \\
\text { Onset }-0.75\end{array}$ & 1.24 & 1.55 \\
\hline
\end{tabular}

NTDO-based linear copolymers with thiophene donor monomers show more red shifting compared to the acceptor monomer units, BT, which can be attribute to the intramolecular charge transfer between NTDO and thiophene segments. Linear polymers with thiophene donor segments show lower optical band gaps. Moreover, the thiophenecontaining linear copolymers show deeper HOMO and LUMO energy levels. They also show higher thermal stability due to the thiophene donor units. 


\subsection{Investigation of possible structures of polymers obtained from post- polymerization using Lawesson's Reagent}

As we know, if the rotation between the monomer units in the polymer chain is frozen by a conjugation, then the absorption maximum will occur at a longer wavelength and the absorption intensity will be strong. ${ }^{16,40}$ On the other hand, our synthesized ladder polymer shows very weak absorption intensity at a longer wavelength. This prompted us to further investigate the polymer structure. We performed the elemental analysis of polymers P2 and P3. Generally, elemental analysis did not provide the correct composition of the polymers because it is suitable only for small compounds. However, we carried out the process to get an idea about the compositions of the elements. The elemental analysis indicated that polymer P2 contained 19.39 to $19.69 \%$ of sulfur element whereas polymer P3 contained 24.99\%. According to our proposed structure, the elemental analysis of polymers P2 and P3 should not exceed 10.95 and 7.14\%, respectively. The elemental analysis indicated that polymer P2 may have extra sulfur and P3 may have more than one sulfur atom in the polymer backbone. The possible structures of polymers $\mathbf{P 2}$ and $\mathbf{P 3}$ are presented in Scheme 2.11 as $\mathbf{P 2}$ ' and $\mathbf{P 3}$ '.

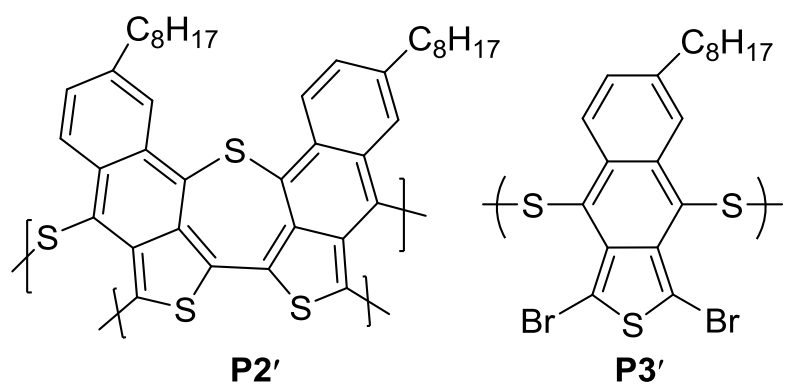

Scheme 2.11 Possible polymer structures for polymers P2' and P3'. 
In 1985, Lakshmikantham et al. synthesized an anthracene disulfide polymer from an anthraquinone monomer using LR. ${ }^{41}$ In 2008, Bartels et al. synthesized a similar poly(2,3-dimethylanthracene)disulfide from 2,3-dimethylanthraquinone using LR. The reduction reaction on the disulfide polymer using $\mathrm{NaBH}_{4}$ followed by in situ esterification in presence of acetic anhydride provided the small molecule 2,3-dimethyl9,10-dithioacethylanthracene (Scheme 2.12). ${ }^{42}$
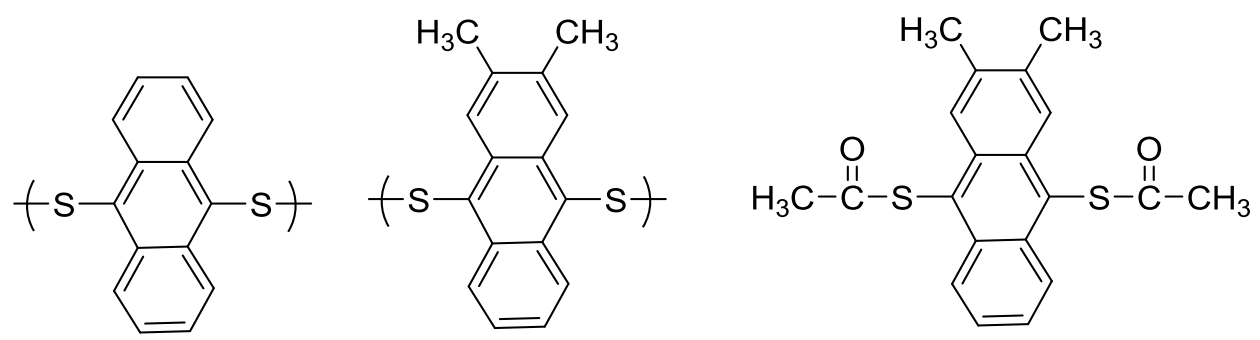

Scheme 2.12 Structures of anthracene containing polydisulfides and thioester.

To investigate the disulfide bond in our polymers $\mathbf{P 2}$ and P3, IR spectra for the polymers were taken in $\mathrm{KBr}$. The disulfide bond shows a characteristic peak at 500 to $550 \mathrm{~cm}^{-1}$. Figure 2.21 shows the IR spectra of polymers P1, P2 and P3. Polymers P2 and P3 show a weak peak at 522 and $527 \mathrm{~cm}^{-1}$, whereas P1 does not show any peak in this range, indicating that both polymers may contain a disulfide bond in the polymer backbone. For further confirmation of the structure of the polymer, a reduction reaction was carried out on polymer P2. Instead of performing the reduction reaction on polymer P3, a new polymer P12 was synthesized. The reduction reaction was then carried out in polymer P12. 

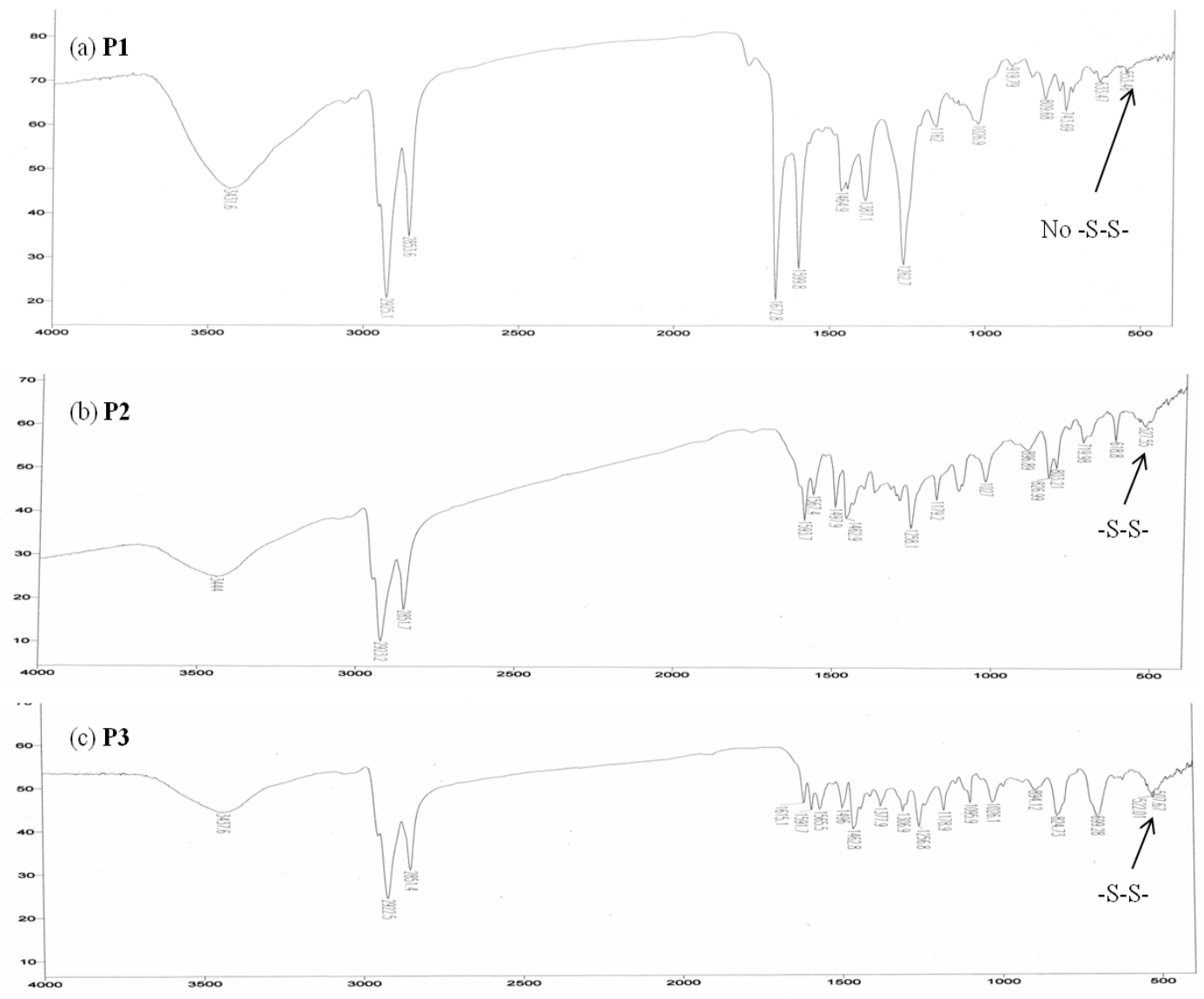

Figure 2.21 IR spectra of polymers P1, P2 and P3 in KBr pellet.

A reduction reaction followed by esterification in polymer P2 was carried out according to the reaction in Scheme 2.13 to get a polymer with ester group to confirm the structure of polymer P2. ${ }^{42}$ However, the reaction was not successful to get a polymer with ester group in it. The polymer was characterized by IR spectroscopy. Figure 2.22 shows the IR spectrum after the reaction. The obtained polymer does not have any $\mathrm{C}=\mathrm{O}$ peak at $1730 \mathrm{~cm}^{-1}$, which indicates the esterification did not take place. This confirms that the polymer P2 may not have any disulfide bond in the backbone. Moreover, the elemental analysis shows that sulfur is 19.39 to $19.69 \%$ in composition, which is close to 
the calculated value (19.75\%). This suggests that the structure proposed in Scheme 2.11 for $\mathbf{P 2}$ may be the correct structure.

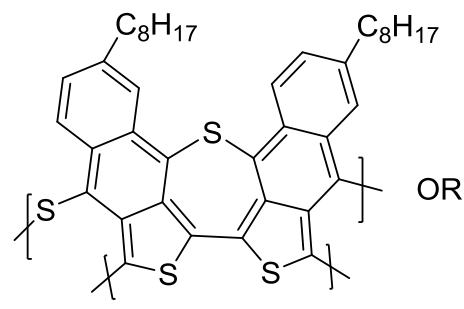

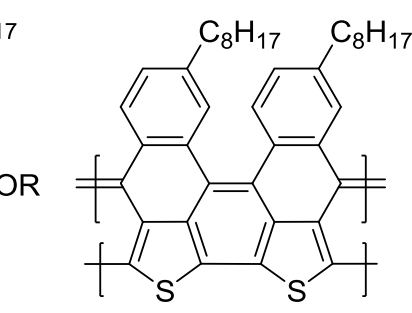

1. $\mathrm{NaBH}_{4}$ aq. $\mathrm{NaOH} 20 \%$, RT

$72 \mathrm{~h}$ 2. $\mathrm{Ac}_{2} \mathrm{O}, \mathrm{RT}$

P2' or P2

Scheme 2.13 Reduction and esterification in polymer P2.

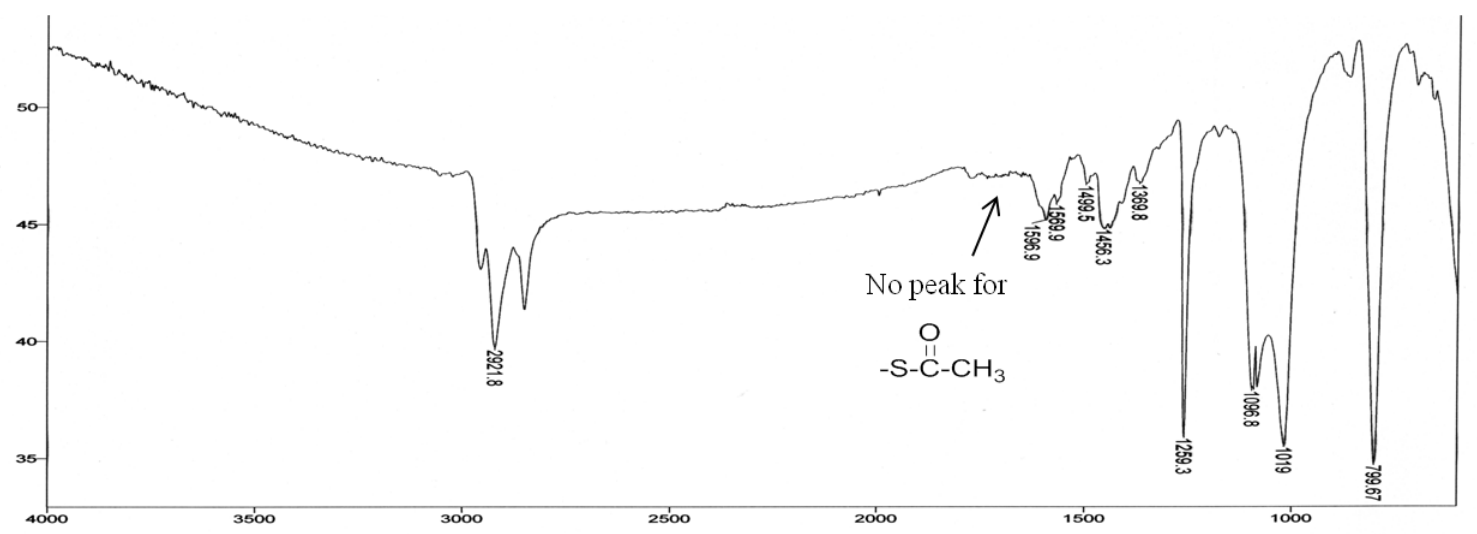

Figure 2.22 IR spectrum (in KBr pellet) of polymer $\mathbf{P} 2$ after esterification.

A linear polymer P12 was synthesized according to the reactions in Scheme 2.14 by the debromination of monomer $\mathbf{M 1}$ in the presence of $\mathrm{Zn} / \mathrm{EtOH}+\mathrm{AcOH}$ followed by keto-keto coupling reaction at $160{ }^{\circ} \mathrm{C}$ using LR in DCB in $86 \%$ yield. ${ }^{7,8}$ The obtained polymer was purified as described in Section 2.4. 


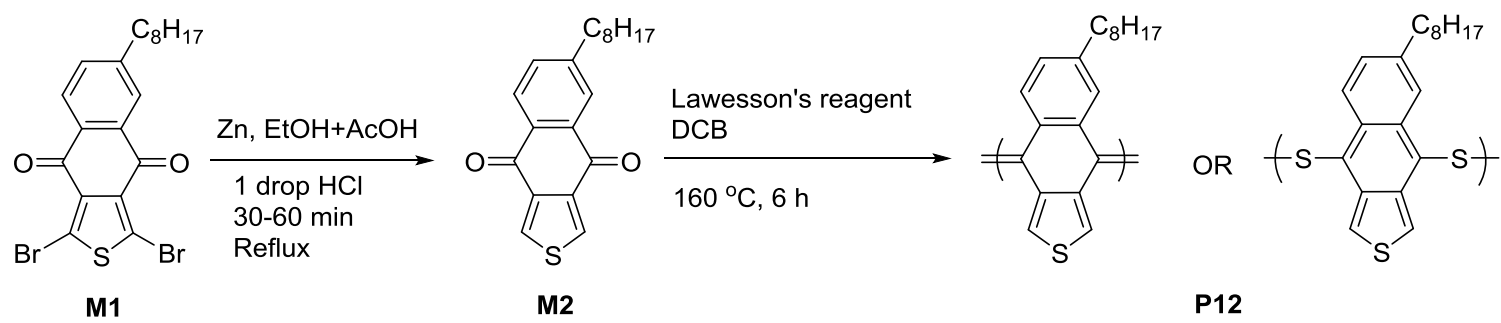

Scheme 2.14 Synthesis of polymer P12.

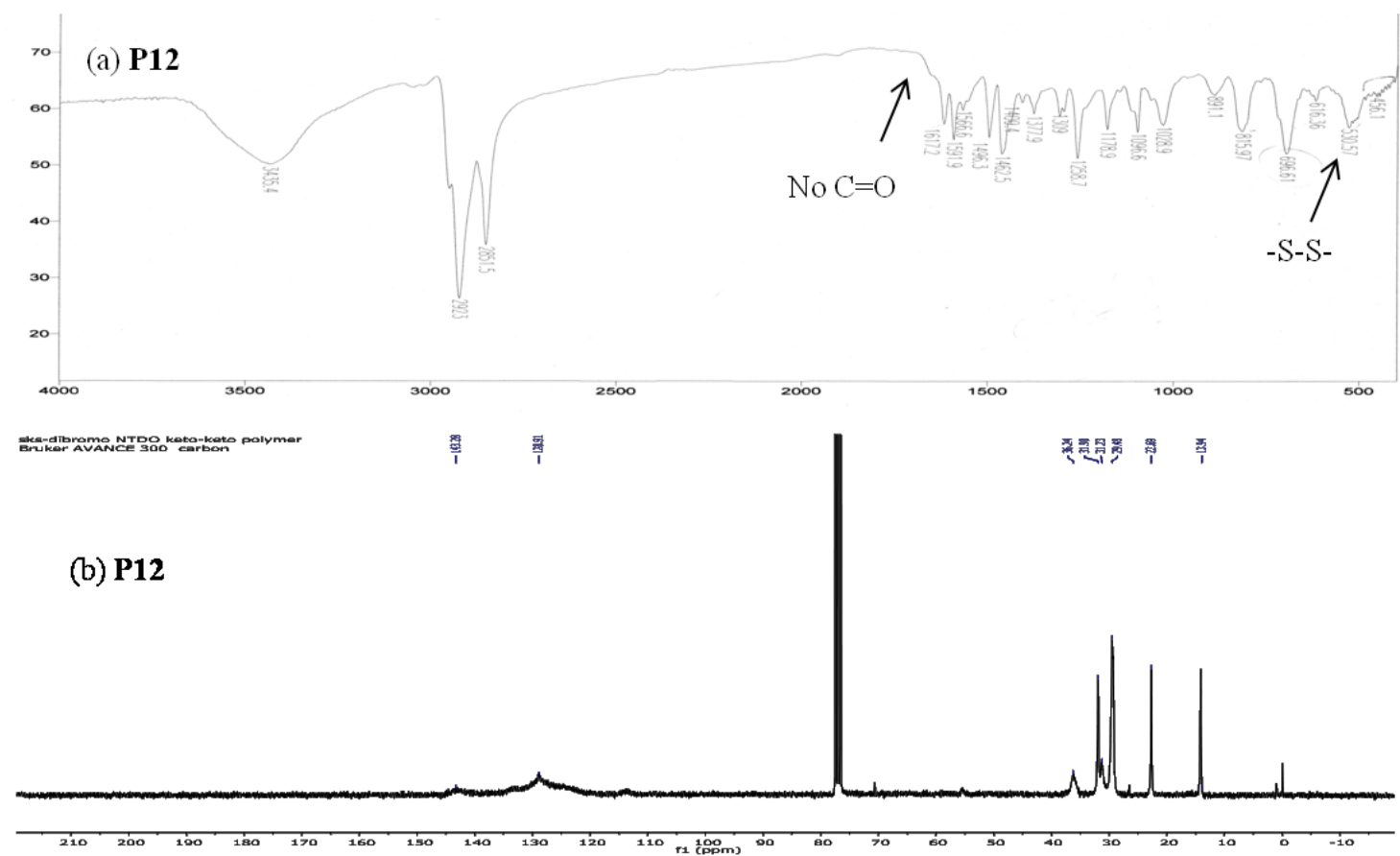

Figure 2.23 (a) IR (in $\mathrm{KBr}$ pellet) and (b) ${ }^{13} \mathrm{C}$ NMR $\left(75 \mathrm{MHz}, \mathrm{CDCl}_{3}\right)$ spectra of polymer P12.

Polymer P12 was characterized by IR, NMR, GPC, absorption, emission spectroscopy and CV. The IR spectrum of polymer P12 (Figure 2.23a) shows no peak at $1673 \mathrm{~cm}^{-1}$ for cyclic $\mathrm{C}=\mathrm{O}$, suggesting coupling between keto groups. The weak peak at $530 \mathrm{~cm}^{-1}$ suggests the disulfide bond in the polymer backbone. The ${ }^{1} \mathrm{H}$ NMR spectrum (Appendix A, Figure S2.28) shows broad peaks in the aromatic region which are 
embedded over $\delta 7.5 \mathrm{ppm}$. All of the aliphatic peaks are broad and range from $\delta 2.60$ ppm to $0.90 \mathrm{ppm}$. The ${ }^{13} \mathrm{C}$ NMR spectrum (Figure 2.23b) of polymer P12 does not show any peak over $145 \mathrm{ppm}$, which indicates the disappearance of $\mathrm{C}=\mathrm{O}$ or $\mathrm{C}=\mathrm{S}$ and the completion of keto-keto coupling polymerization. The elemental analysis shows that the polymer P12 has 24.07 to $24.99 \%$ of sulfur element. Thermogravimetric analysis, as presented in Figure S2.29 (Appendix A) reveals that the decomposition onset for polymer P12 is $262.9^{\circ} \mathrm{C}$, with $1 \%$ weight loss. It was also observed that the $\mathrm{T}_{\mathrm{d}}$ for $5 \%$ weight loss is $332.6{ }^{\circ} \mathrm{C}$, which is higher than polymer P3. Polymer P12 shows a moderate molecular weight (Appendix A, Figure S2.30) with a PDI of 2.30. The polymerization reaction time was shorter so as to control the solubility of the polymer. Absorption spectra of P12 in $\mathrm{CHCl}_{3}$ solution and as a spin-coated thin solid film on a glass substrate are shown in Figure 2.24. The polymer is fully soluble in a wide range of solvents like $\mathrm{CHCl}_{3}$, chlorobenzene, dichlorobenzene and trichlorobenzene. Polymer P12 shows similar absorption spectra to polymer P3. Polymer P12 displays a maximal absorption peak at $473 \mathrm{~nm}$ in $\mathrm{CHCl}_{3}$ with an onset from $690 \mathrm{~nm}$. The film of $\mathbf{P 3}$ shows broad absorption with a maximal at $486 \mathrm{~nm}$ with an onset from $700 \mathrm{~nm}$. The polymer shows no emission in solution or solid state.

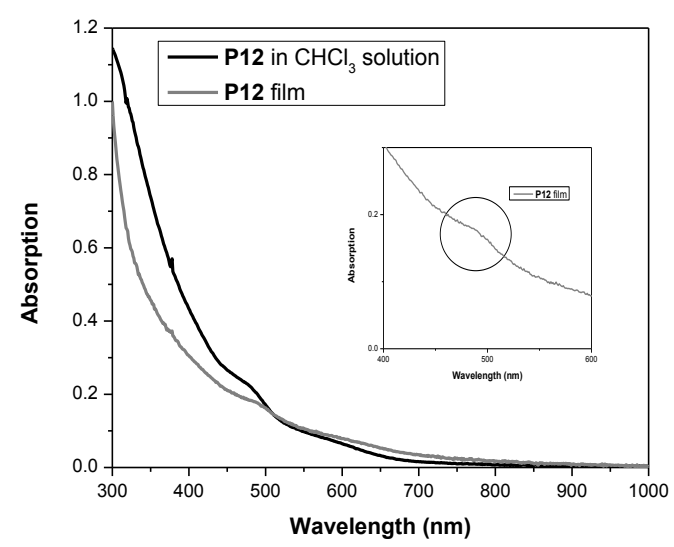


Figure 2.24 Absorption spectra of polymer P12 in solution and on glass substrate.

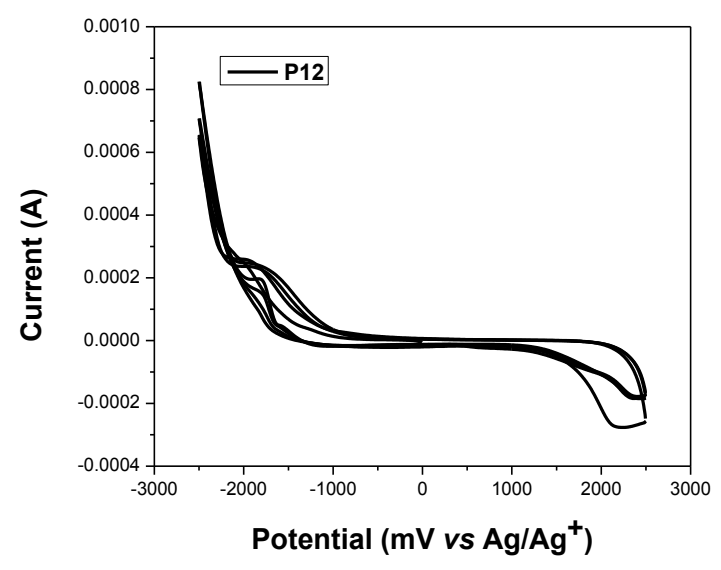

Figure 2.25 Cyclic voltammogram of polymer P12 film on ITO in $\mathrm{CH}_{3} \mathrm{CN}$ solution containing $0.1 \mathrm{~mol} / \mathrm{L} \mathrm{Bu}_{4} \mathrm{NPF}_{6}$ with a scan rate of $100 \mathrm{mV} / \mathrm{s}$.

Polymer P12 shows a noticeable oxidation and reduction process (Figure 2.25). The onset oxidation potential/onset reduction potentials are 1.30/-0.99 V. The HOMO energy level of the polymer is $-5.66 \mathrm{eV}$ and the LUMO energy level is $-3.37 \mathrm{eV}$. The energy band gap is $2.29 \mathrm{eV}$, which is higher than polymer P3. The HOMO energy level of P12 is deeper than P3. This indicates the bromine has electron-donating nature in P3 polymer; therefore, the HOMO energy level is high lying. In absence of bromine, polymer P12 shows a deeper HOMO level. The LUMO energy level is not affected much, therefore the energy band gap increased for polymer P12.

A reduction reaction followed by esterification on polymer P12 was carried out according to reaction in Scheme 2.15 to obtain compound 3, which confirms the structure of polymers P3/P12. ${ }^{42}$ However, the reaction was unsuccessful to get compound 3. After the reaction, an oligomer was found that does not move on TLC plate, and IR shows the thioester peak. The oligomer was characterized by IR and absorption spectra. 

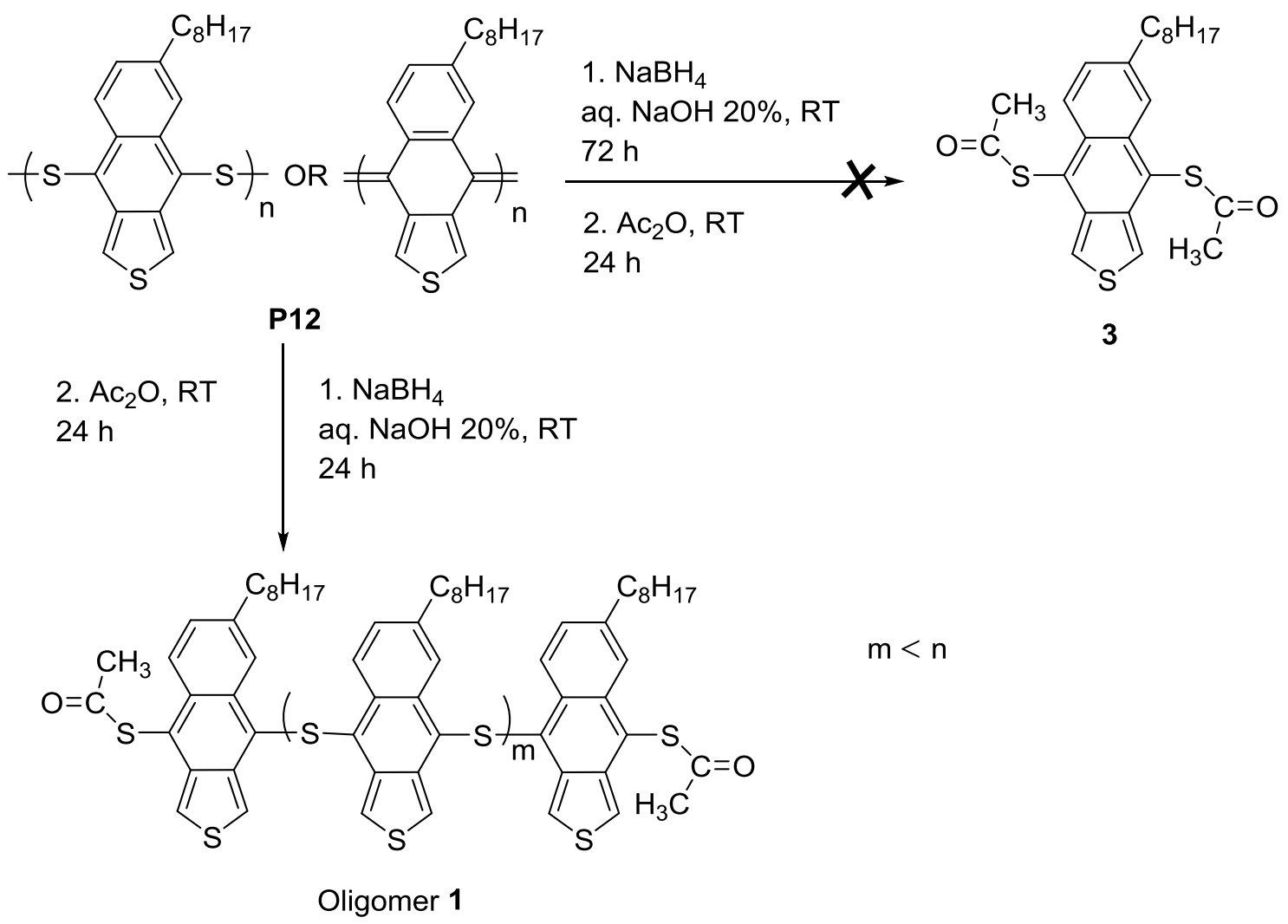

Scheme 2.15 Attempted synthesis of compound $\mathbf{3}$ or oligomer $\mathbf{1}$.

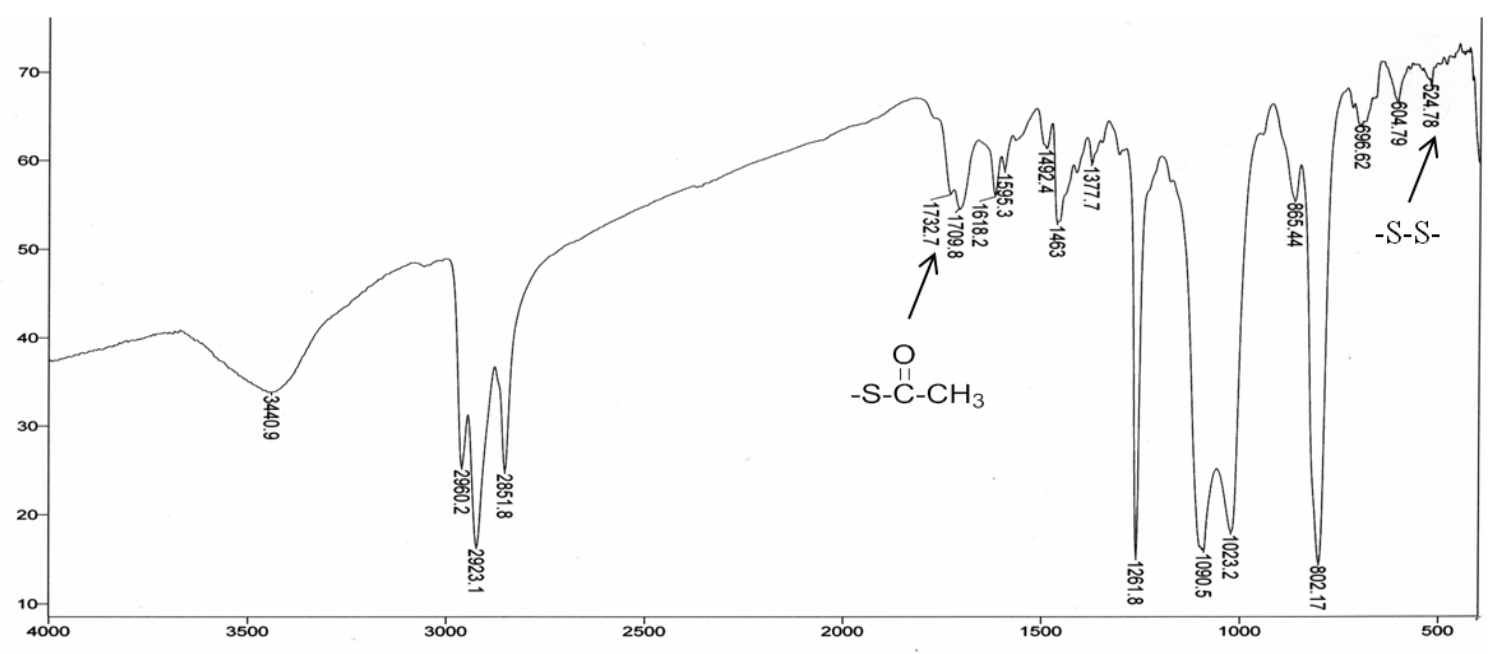

Figure 2.26 IR spectrum (in $\mathrm{KBr}$ pellet) of oligomer $\mathbf{1}$. 
Figure 2.26 shows the IR spectrum for oligomer 1. Polymer P12 does not have any $\mathrm{C}=\mathrm{O}$ peak at $1730 \mathrm{~cm}^{-1}$. The IR spectrum after the reaction, however, shows weak peaks at 1732 and $1709 \mathrm{~cm}^{-1}$, indicating the esterification was successful. The ${ }^{1} \mathrm{H}$ NMR spectrum (Appendix, Figure S2.31a) shows broad peaks, which suggests it is not a small compound. However, the ${ }^{13} \mathrm{C}$ NMR spectrum (Appendix A, Figure S2.32b) does not show any peak at 170 to $180 \mathrm{ppm}$. The aromatic carbon peaks are observed at 127 to 142 ppm. This result suggests that polymers P3 and P12 may contain both the disulfide and sulfur linkage in the polymer backbone. During the reduction reaction, the disulfide bonds break and undergo esterification, which was observed by IR spectroscopy. The oligomer was obtained due to the sulfur linkage between the monomer units, which provides broad NMR peaks. These data provides strong evidence that polymers P3 and P12 contain disulfide and sulfur bonds in the polymer backbone. The possible structure is provided in Scheme 2.16

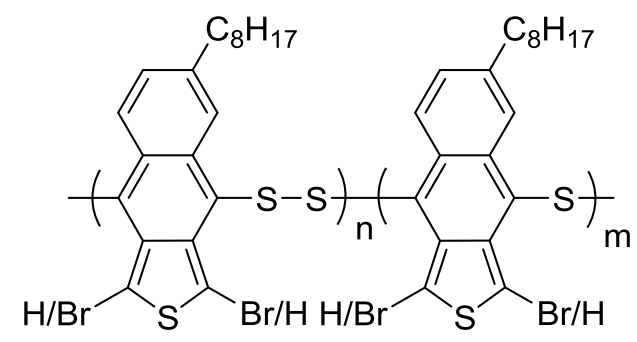

Where $m>n$

Scheme 2.16 Possible structures for polymer P3/P12.

\subsection{Conclusion}

The synthesis of ladder polymers by Yamamoto coupling followed by cyclization in the presence of Lawesson's Reagent was successful. However, the desired polymer 
structure (P2) was still not obtained. The formation of a carbon-carbon double bond between the cyclic keto was not successful. It may form a C-S-C bond (P2'), which may influence the aromaticity, resulting in weakened absorption intensity. The obtained ladder polymer P2' shows the resultant absorption onset is in longer wavelengths (1000 nm), especially, in NIR region. However, the absorption intensity in The NIR region is very weak, though it is stronger in the visible region. The alternative method to synthesize the ladder polymer $\mathbf{P 2}$ by the Yamamoto reaction followed by the McMurry reaction was not successful. The structure of the obtained polymer was unknown. The resultant polymer contains $\mathrm{OH}$ and a cyclic $\mathrm{C}=\mathrm{O}$ group in the polymer backbone, which may influence the aromaticity, weakening the absorption intensity. The last method was to synthesize polymer P2 by coupling between keto groups followed by Ullmann reaction was not satisfactory. The structure of the precursor polymer P3 may not be right; therefore, the method followed to obtain polymer P2 was failed. The obtained polymer showed similar absorption of P3. The effect of donor and acceptor in the ladder polymer was also studied. Increasing the donor unit from 1-molar to 2-molar ratio in linear-ladder conjugated polymers resulted in absorption onset in longer wavelengths (1100 and 1300 nm, respectively) compared to polymer P2'. The absorption intensity followed a similar pattern to P2': it was weak in the NIR region and stronger in the visible region. Increasing the acceptor unit of a linear-ladder conjugated polymer did not influence the absorption as much as that which was influenced by donor unit. The resultant onset is weak in the NIR region, similar to polymer P2' and stronger in visible region. 


\subsection{Experimental section}

\section{Materials}

Trifluoroacetic acid $\left(\mathrm{CF}_{3} \mathrm{COOH}\right), \quad N$-bromosuccinimide, oxalyl chloride, aluminium chloride, n-octylbenzene, titanium tetrachloride, 2,5-dibromothiophene, Lawesson's Reagent, 1,5-cyclooctadiene, 2,2'-bipyridine and $\mathrm{Ni}(\mathrm{COD})_{2}$ were purchased from Aldrich Chemicals and used as received. Thiophene-3,4-dicarboxalic acid was purchased from Matrix Scientific and used as received.

\section{Purification of solvents}

$N, N$-Dimethylformamide (DMF), dichloromethane and toluene were dried and distilled over $\mathrm{CaH}_{2}$ under an atmosphere of dry argon. Tetrahydofuran (THF) was distilled over benzophenone and $\mathrm{Na}$ under an atmosphere of dry argon.

\section{Chromatography}

Thin layer chromatography (TLC) was used to monitor the progress of the reaction and to check the purity of the synthesized products. The material used for TLC was silica gel, $60 \mathrm{G} / \mathrm{UV}_{254}$ (Macherey-Nagel). Column chromatography was used for the separation of pure product from a reaction mixture. Crude products were often purified by column chromatography. The chromatography column was prepared using slurry silica gel (Silicycle Chemical Division, 70-230 mesh) as the stationary phase. 


\section{General methods}

${ }^{1} \mathrm{H}$ and ${ }^{13} \mathrm{C}$ NMR spectra were recorded on a Bruker Avance Digital $300 \mathrm{MHz}$ (300 and $75 \mathrm{MHz}$ for ${ }^{1} \mathrm{H}$ and ${ }^{13} \mathrm{C} \mathrm{NMR}$, respectively). Resonances were quoted on the $\delta$ scale relative to tetramethylsilane (TMS, $\delta=0$ ) as an internal standard. For ${ }^{1} \mathrm{H}$ NMR spectra, the following abbreviations have been used: $\mathrm{s}=$ singlet, $\mathrm{d}=$ doublet, $\mathrm{t}=$ triplet, $\mathrm{q}$ $=$ quartet, $\mathrm{m}=$ multiplet. Infrared measurements were performed on a Varian $1000 \mathrm{FT}-\mathrm{IR}$ Scirinitar series spectrophotometer. The measuring mode was \% $\mathrm{T}$ (percentage transmittance). The bands were expressed in $\mathrm{cm}^{-1}$ (per centimeter). Mass spectra were measured with a Micromass Quattro LC ESI (EI). Thermogravimetric analysis was carried out under nitrogen atmosphere on a Hi-Res TGA 2950 thermogravimetric analyzer with a heating rate of $10{ }^{\circ} \mathrm{C} \mathrm{min}^{-1}$. Standard differential scanning calorimetric analysis was carried out in nitrogen on a TA DSC Q100 with a heating rate $10{ }^{\circ} \mathrm{C} \mathrm{min}^{-1}$. The UV-vis-NIR spectra were recorded on a Perkin-Elmer Lambda 900 UV-vis-NIR spectrometer at room temperature. The fluorescence spectra of all samples were measured in a quartz cuvette with a path length $1 \mathrm{~cm}$ in Shimadzu RF-1501 spectrofluorometer.

\section{2,5-Dibromothiophene-3,4-dicarboxalic acid (1)}

To a solution of thiophene-3,4-dicarboxalic acid $(2.00 \mathrm{~g}, 11.6 \mathrm{mmol})$ in $\mathrm{H}_{2} \mathrm{SO}_{4} / \mathrm{CF}_{3} \mathrm{COOH}(14.0 \mathrm{~mL} / 36.0 \mathrm{~mL})$ was added $N$-bromosuccinimide $(6.20 \mathrm{~g}, 34.8$ mmol) in one portion. After stirring for $24 \mathrm{~h}$ at $70{ }^{\circ} \mathrm{C}$ in dark, the mixture was poured into ice. Diethyl ether was added to the mixture and shaken vigorously. The organic portion was separated and extracted three times by diethyl ether. The combined organic portions 
were dried over magnesium sulfate. The solvent was removed under reduced pressure. The crude product was further purified by recrystalization in water as gray crystalline solid (1) (2.40 g, 63.0\% yield). ${ }^{1} \mathrm{H}$ NMR (300 MHz, DMSO-d 6 ): No peak.

\section{2,5-Dibromothiophene-3,4-dicarboxalic acid chloride (2)}

In a flame dried, two-neck round-bottom flask, oxalyl chloride $(2.0 \mathrm{~mL}, 22.8$ mmol) was slowly added to 2,5-dibromothiophene-3,4-dicarboxylic acid (1.00 g, 2.67 mmol) and DMF (1 drop) in dry toluene $(10 \mathrm{~mL})$. The mixture was heated to reflux for 1 $\mathrm{h}$, and then cooled to room temperature. The volatiles were removed under reduced pressure and the product was used for the next step without any further purification, 1.11 $\mathrm{g}(100 \%$ yield $)$.

\section{1,3-Dibromo-8-octylnaptho[2,3-c]thiophene-4,9-dione (M1)}

A solution of 2,5-dibromothiophene-3,4-dicarboxylic acid chloride (2) (1.25 g, $3.42 \mathrm{mmol})$ in dichloromethane $(6.0 \mathrm{~mL})$ was slowly added to a suspension of aluminium chloride $(1.82 \mathrm{~g}, 13.7 \mathrm{mmol})$ in dichloromethane $(10 \mathrm{~mL})$ maintained at $0{ }^{\circ} \mathrm{C}$. The mixture was stirred at $0{ }^{\circ} \mathrm{C}$ for $10 \mathrm{~min}$ and $\mathrm{n}$-octylbenzene $(0.650 \mathrm{~g}, 3.42 \mathrm{mmol})$ was added slowly. The mixture was stirred for $60 \mathrm{~min}$ at room temperature and then poured into ice. Dichloromethane was added to the mixture and shaken vigorously. The organic portion was separated and extracted three times by dichloromethane. The combined organic portions were dried over magnesium sulfate. The solvent was removed under reduced pressure. The crude product was purified by column chromatography on silica gel using ethyl acetate and hexane $(1: 10 \mathrm{v} / \mathrm{v})$ as eluting solvent. The compound was 
further purified by recrystallization in hexane and obtained as a yellow solid M1 $(0.1 .38$ g, 83.0\% yield). ${ }^{1} \mathrm{H}$ NMR (300 MHz, $\left.\mathrm{CDCl}_{3}\right): \delta 8.23(\mathrm{~d}, J=8.0 \mathrm{~Hz}, 1 \mathrm{H}), 8.11(\mathrm{~d}, J=1.6$ $\mathrm{Hz}, 1 \mathrm{H}), 7.61(\mathrm{dd}, J=8.0,1.8 \mathrm{~Hz}, 1 \mathrm{H}), 2.84-2.73(\mathrm{~m}, 2 \mathrm{H}), 1.69$ (dd, $J=14.9,7.3 \mathrm{~Hz}$, $2 \mathrm{H}), 1.31(\mathrm{~d}, J=14.4 \mathrm{~Hz}, 10 \mathrm{H}), 0.89(\mathrm{t}, J=6.7 \mathrm{~Hz}, 3 \mathrm{H}) .{ }^{13} \mathrm{C} \mathrm{NMR}\left(75 \mathrm{MHz}, \mathrm{CDCl}_{3}\right): \delta$ $177.94,177.56,150.63,134.67,134.41,132.39,127.82,127.21,120.87,120.65,36.15$, 31.83, 30.83, 29.38, 29.19, 22.65, 14.11. HRMS calculated for $\left[\mathrm{M}^{+}\right]$: 481.95507, found 481. 95682; IR ( $\mathrm{NaCl}$ plate, $\left.\mathrm{cm}^{-1}\right): 2924,2853,1596,1467,1443,1361,1254,1178$, $1139,1038,981,743,725,629$.

\section{8-Octylnaphtho[2,3-c]thiophene-4,9-dione (M2)}

Monomer M1 (1.00 g, $2.06 \mathrm{mmol})$ was added to a solution of ethanol (13 mL), acetic acid $(3.0 \mathrm{~mL})$, and 1 drop of $\mathrm{HCl}$ and heated until dissolution of the solid. Then zinc powder $(0.276 \mathrm{~g}, 4.22 \mathrm{mmol})$ was added to the mixture and was refluxed for 30 to 60 min. The progress of the reaction was monitored by TLC (Hexane:ethyl acetate, 10:1 $\mathrm{v} / \mathrm{v}$ ). After cooling to room temperature, the reaction mixture was filtered and the filtrate was extracted with dichloromethane. The organic extract was dried over $\mathrm{MgSO}_{4}$ and the solvent was removed by rotary evaporator. The compound was purified by silica gel column chromatography using hexane and ethyl acetate $(5: 1 \mathrm{v} / \mathrm{v})$ as eluting solvent. The

monomer M2 was obtained as a brownish solid (0.400 g, 59.1\% yield). ${ }^{1} \mathrm{H}$ NMR (300 $\left.\mathrm{MHz}, \mathrm{CDCl}_{3}\right): \delta 8.37(\mathrm{~m}, 2 \mathrm{H}), 8.25(\mathrm{~s}, 1 \mathrm{H}), 8.13(\mathrm{~s}, 1 \mathrm{H}), 7.61(\mathrm{~s}, 1 \mathrm{H}), 2.79(\mathrm{~s}, 2 \mathrm{H}), 1.71$ $(\mathrm{s}, 2 \mathrm{H}), 1.32(\mathrm{~s}, 10 \mathrm{H}), 0.90(\mathrm{~s}, 3 \mathrm{H}) .{ }^{13} \mathrm{C} \mathrm{NMR}\left(75 \mathrm{MHz}, \mathrm{CDCl}_{3}\right): \delta$ 179.63, 179.22, $150.36,137.44,134.79,134.32,132.60,132.42,127.94,127.42,36.17,31.83,30.96$, 
29.38, 22.64, HRMS calculated for $\left[\mathrm{M}^{+}\right]$: 326.13405 found 326.13269. IR ( $\mathrm{NaCl}$ plate, $\left.\mathrm{cm}^{-1}\right): 2922,2852,1666,1597,1515,1455,1268,862,732$.

\section{Polymer P1}

Under an argon atmosphere, a solution of $\mathrm{Ni}(\mathrm{COD})_{2}(2.00 \mathrm{~g}, 7.27 \mathrm{mmol}), \mathrm{COD}$ $(1.0 \mathrm{~mL}, 8.16 \mathrm{mmol})$ and bipyridine $(1.13 \mathrm{~g}, 7.27 \mathrm{mmol})$ in toluene $(8.00 \mathrm{~mL})$ was heated to $80{ }^{\circ} \mathrm{C}$. Then, a solution of monomer $\mathbf{M 1}(1.50 \mathrm{~g}, 3.09 \mathrm{mmol})$ in $4 \mathrm{~mL}$ of toluene was added to the reaction solution and the reaction mixture was heated at $100{ }^{\circ} \mathrm{C}$ in dark for $24 \mathrm{~h}$. The solution was then poured into methanol with vigorous stirring. After stirring 2 h, a black-amber precipitate as polymer P1 was collected by filtration. The polymer P1 was purified by dissolution in $\mathrm{CHCl}_{3}$ and re-precipitated using methanol. The material was further purified by a Soxhlet extraction in acetone for $24 \mathrm{~h}$. The resultant polymer was dissolved in $\mathrm{CHCl}_{3}$ and precipitated with methanol and collected by filtration. After drying under vacuum at $60{ }^{\circ} \mathrm{C}$ for $5 \mathrm{~h}$, the polymers were obtained $(0.970 \mathrm{~g}, 95.5 \%$ yield) as black-amber powder. ${ }^{1} \mathrm{H}$ NMR (300 MHz, $\left.\mathrm{CDCl}_{3}, \mathrm{ppm}\right): \delta$ 8.15-7.56 (m, br, all Ar-H) 2.75 (br, $\left.\mathrm{Ar}-\mathrm{CH}_{2}\right), 1.66\left(\mathrm{CH}_{2}\right.$ moiety from aliphatic part), $1.37\left(\mathrm{br}, \mathrm{CH}_{2}\right), 0.87\left(\mathrm{CH}_{3}\right) .{ }^{13} \mathrm{C}$ NMR (101 MHz, $\left.\mathrm{CDCl}_{3}\right): \delta 179.44,178.98,150.16,140.73,134.65,132.75,127.57$,

36.02, 31.80, 30.79, 29.15, 22.79, 14.08. IR ( $\mathrm{NaCl}$ plate, $\left.\mathrm{cm}^{-1}\right): 2960,2924,2853,1672$ $(\mathrm{C}=\mathrm{O}), 1599,1454,1389,1261,1097,1020,799$.

Polymer $\mathbf{P} 2$ by LR

In a flame dried three-neck round-bottom flask under argon atmosphere, polymer P1 (0.190 g, $0586 \mathrm{mmol})$ was added to 1,2-dichlorobenzene $(5.0 \mathrm{~mL})$ and stirred until 
dissolution. The solution was degassed and purged with argon for $30 \mathrm{~min}$, the solution was heated to $130{ }^{\circ} \mathrm{C}$ and Lawesson's reagent $(0.237 \mathrm{~g}, 0.586 \mathrm{mmol})$ was added. The mixture was heated to $160{ }^{\circ} \mathrm{C}$ for $6 \mathrm{~h}$ under argon. During the reaction, the color changed from amber to blue-greenish. The solution was then poured into methanol with vigorous stirring. After stirring $2 \mathrm{~h}$, a dark greenish precipitate as polymer $\mathbf{P 2}$ was collected by filtration. The polymer was further purified by refluxing in ethanol for $2 \mathrm{~h}$, filtered and washed with ethanol. The resultant polymer was then stirred in different solvents sequentially acetone, ethyl acetate and hexane to remove any other residue from LR. Then the polymer was further purified by a Soxhlet extraction in acetone for $24 \mathrm{~h}$. The polymer was dissolved in $\mathrm{CHCl}_{3}$ and re-precipitated with methanol. After drying under vacuum at $60{ }^{\circ} \mathrm{C}$ for $5 \mathrm{~h}$, the polymers were obtained $(0.170 \mathrm{~g}, 95.8 \%$ yield) as dark black-greenish powder. ${ }^{1} \mathrm{H}$ NMR (300 MHz, $\left.\mathrm{CDCl}_{3}, \mathrm{ppm}\right): \delta$ 8-7 (br, all Ar-H) 2.61 (br, Ar- $\left.\mathrm{CH}_{2}\right), 1.28\left(\mathrm{CH}_{2}\right.$ moiety from aliphatic part), $0.90\left(\mathrm{br}, \mathrm{CH}_{3}\right) .{ }^{13} \mathrm{C} \mathrm{NMR}(75 \mathrm{MHz}$, $\left.\mathrm{CDCl}_{3}\right): \delta 143.00,133.62,128.96,113.69,55.32,35.95,31.92,29.47,22.70,14.14 . \mathrm{IR}$ $\left(\mathrm{NaCl}\right.$ plate, $\left.\mathrm{cm}^{-1}\right): 3026,2924,2853,1594,1567,1499,1464,1296,1258,1180,1113$, 1032, 823, 804. IR (KBr pallet, $\left.\mathrm{cm}^{-1}\right): 3024,2921,2851,1593,1567,1497,1462,1258$, 1179, 1111, 1027, 826, 803, 719, 618, 527. Anal. calculated for $\mathrm{C}_{40} \mathrm{H}_{40} \mathrm{~S}_{4}: \mathrm{S}, 19.75$. Found: S, 19.39 to 19.69 .

\section{Polymer $\mathbf{P} 2$ by McMurry Reaction}

In a flame-dried three-neck flask under argon atmosphere, an ice-cooled $\left(-5^{\circ} \mathrm{C}\right)$ suspension of zinc powder $(0.805 \mathrm{~g}, 12.4 \mathrm{mmol})$ in THF $(10 \mathrm{~mL})$ was prepared and titanium tetrachloride $(1.35 \mathrm{~mL}, 12.3 \mathrm{mmol})$ was added to the suspension. The resulting 
mixture was refluxed for $2 \mathrm{~h}$. The mixture was again cooled to -5 to $0{ }^{\circ} \mathrm{C}$, charged with pyridine $(0.25 \mathrm{~mL}, 3.10 \mathrm{mmol})$ and stirred for $10 \mathrm{~min}$. The mixture was allowed to warm to room temperature and a solution of polymer P1 $(0.200 \mathrm{~g}, 0.617 \mathrm{mmol})$ in THF (5.0 $\mathrm{mL}$ ) was slowly added to the mixture and the mixture was then refluxed for $48 \mathrm{~h}$. After cooling to room temperature, the mixture was diluted with saturated aqueous sodium hydrogen carbonate solution and chloroform and the solution was stirred for $5 \mathrm{~h}$. The mixture was filtered through a Celite pad, and the filtrate was separated into organic and aqueous layers. The aqueous layer was extracted twice with chloroform and the combined organic layer was dried over $\mathrm{MgSO}_{4}$. The solvent was removed under reduced pressure and the product $(0.190 \mathrm{~g})$ was obtained as dark amber solid which was characterized without any further purification. ${ }^{1} \mathrm{H} \mathrm{NMR}\left(300 \mathrm{MHz}, \mathrm{CDCl}_{3}, \mathrm{ppm}\right): \delta 8-7$ (br, all Ar-H) 2.81 (br, $\left.\mathrm{Ar}-\mathrm{CH}_{2}\right), 1.30\left(\mathrm{CH}_{2}\right.$ moiety from aliphatic part), $0.90\left(\mathrm{br}, \mathrm{CH}_{3}\right) .{ }^{13} \mathrm{C} \mathrm{NMR}(75$ $\left.\mathrm{MHz}, \mathrm{CDCl}_{3}\right): \delta 183.19,149.73,141.55,127.50,35.96,31.88,30.96,29.39,22.67$, 14.12. IR (KBr pallet, $\mathrm{cm}^{-1}$ ): 3422 (br, OH), 2925, 2853, 1663, 1600, 1533, 1485, 1492, $1463,1377,1295,1048,739,670$.

Polymer P3

Polymer P3 was synthesized according to the procedure described in polymer P2. Monomer M1 (0.200 g, $0.415 \mathrm{mmol}), \quad$ LR $(0.170 \mathrm{~g}, 0.416 \mathrm{mmol})$ and 1,2dichlorobenzene $(5.0 \mathrm{~mL})$. Obtained polymer is dark black-amber solid $(0.160 \mathrm{~g}, 86.0 \%$ yield). ${ }^{1} \mathrm{H}$ NMR (300 MHz, $\left.\mathrm{CDCl}_{3}, \mathrm{ppm}\right): \delta$ 9-7 (br, all Ar-H) 2.83 (br, Ar- $\mathrm{CH}_{2}$ ), 1.28 $\left(\mathrm{CH}_{2}\right.$ moiety from aliphatic part), $0.90\left(\mathrm{br}, \mathrm{CH}_{3}\right) .{ }^{13} \mathrm{C} \mathrm{NMR}\left(75 \mathrm{MHz}, \mathrm{CDCl}_{3}\right): \delta 143.16$, 128.15, 36.09, 31.88, 31.00, 29.51, 22.84, 13.95. IR ( $\mathrm{NaCl}$ plate, $\left.\mathrm{cm}^{-1}\right): 3026,2924$, 
2853, 1615, 1594, 1569, 1495, 1463, 1375, 1310, 1259, 1180, 1086, 1032, 821, 759, 694.

IR (KBr pallet, $\left.\mathrm{cm}^{-1}\right): 3024,2921,2852,1615,1591,1565,1496,1462,1377,1306$, 1256, 1178, 1095, 1026, 894, 824, 699, 522. Anal. Found: S, 24.99.

\section{General procedure for desulfurization}

A solution of polymer $(0.500 \mathrm{mmol})$ in appropriate amount of $\mathrm{CHCl}_{3}, \mathrm{H}_{2} \mathrm{O}_{2}(30 \%$, $2.00 \mathrm{mmol}), \mathrm{SOCl}_{2}(1.00 \mathrm{mmol})$ and $1.0 \mathrm{~mL}$ of ethanol was stirred at room temperature for 30 minutes. The polymer was poured into cooled methanol and stirred for $2 \mathrm{~h}$. The precipitated was filtered and dried under argon at $135^{\circ} \mathrm{C}$ for $2 \mathrm{~h}$.

\section{Polymer P4}

Polymer P4 was synthesized according to the procedure described in polymer P1. Monomer M1 (0.200 g, $0.415 \mathrm{mmol})$, 2,5-dibromothiophene (0.100 g, $0.415 \mathrm{mmol})$, $\mathrm{Ni}(\mathrm{COD})_{2}(0.500 \mathrm{~g}, 1.81 \mathrm{mmol}) \mathrm{COD}(0.30 \mathrm{~mL})$ bypyridine $(0.284 \mathrm{~g}, 1.81 \mathrm{mmol})$ and toluene $(8.0 \mathrm{~mL})$. The obtained polymer is a dark brown solid $(0.148 \mathrm{~g}, 87.5 \%$ yield $) .{ }^{1} \mathrm{H}$ NMR (300 MHz, $\left.\mathrm{CDCl}_{3}, \mathrm{ppm}\right): \delta$ 8.15-7.56 (m, br, all Ar-H) 2.74 (br, $\left.\mathrm{Ar}-\mathrm{CH}_{2}\right), 1.66$ $1.521 .37\left(\mathrm{CH}_{2}\right.$ from aliphatic part), $0.87\left(\mathrm{CH}_{3}\right)$. IR $\left(\mathrm{NaCl}\right.$ plate, $\left.\mathrm{cm}^{-1}\right): 3026,2924,2853$, $1671,1599,1454,1385,1262,1161,1037,821,791,743$.

\section{Polymer P5}

Polymer P5 was synthesized according to the procedure described in polymer P1. Monomer M1 (0.250 g, $0.518 \mathrm{mmol})$, 2,5-dibromothiophene $(0.250 \mathrm{~g}, 1.03 \mathrm{mmol})$, $\mathrm{Ni}(\mathrm{COD})_{2}(0.452 \mathrm{~g}, 1.64 \mathrm{mmol}) \mathrm{COD}(0.30 \mathrm{~mL})$ bypyridine $(0.218 \mathrm{~g}, 1.64 \mathrm{mmol})$ and 
toluene $(9.0 \mathrm{~mL})$. The obtained polymer is a dark brown solid $(0.245 \mathrm{~g}, 97.2 \%$ yield $) .{ }^{1} \mathrm{H}$ NMR (300 MHz, $\left.\mathrm{CDCl}_{3}, \mathrm{ppm}\right): \delta$ 8.15-7.56 (m, br, all Ar-H) 2.75 (br, $\mathrm{Ar}-\mathrm{CH}_{2}$ ), 1.66 $1.541 .29\left(\mathrm{CH}_{2}\right.$ moiety from aliphatic part), $0.88\left(\mathrm{CH}_{3}\right)$. IR $\left(\mathrm{NaCl}\right.$ plate, $\left.\mathrm{cm}^{-1}\right): 3026$, 2924, 2853, 1669, 1598, 1456, 1380, 1261, 1042, 788, 742.

\section{Polymer P6}

Polymer P6 was synthesized according to the procedure described in polymer P2. Polymer P4 (0.100 g, $0.245 \mathrm{mmol})$, LR (0.100 g, $0.245 \mathrm{mmol})$ and 1,2-dichlorobenzene $(5.0 \mathrm{~mL})$. The obtained polymer is a black solid $(0.0800 \mathrm{~g}) . \mathrm{IR}\left(\mathrm{KBr}\right.$ pallet, $\left.\mathrm{cm}^{-1}\right): 3024$, 2922, 2851, 1618, 1561, 1463, 1406, 1329, 1231, 1022, 802, 713, 527.

Polymer P7

Polymer P7 was synthesized according to the procedure described in polymer $\mathbf{P 2}$. Polymer P4 (0.150 g, $0.307 \mathrm{mmol})$, LR (0.100 g, $0.245 \mathrm{mmol})$ and 1,2-dichlorobenzene $(5.0 \mathrm{~mL})$. The obtained polymer is a black solid $(0.130 \mathrm{~g}) . \mathrm{IR}\left(\mathrm{KBr}\right.$ pellet, $\left.\mathrm{cm}^{-1}\right): 3022$, 2921, 2854, 1598, 1561, 1461, 1406, 1326, 1217, 1143, 1019, 796, 699, 536.

\section{Polymer P8}

Polymer P8 was synthesized according to the procedure described in polymer P1. Monomer M1 (0.300 g, $0.622 \mathrm{mmol}), 4,7$-dibromobenzo[c][1,2,5]thiadiazole $(0.182 \mathrm{~g}$, $0.622 \mathrm{mmol}), \mathrm{Ni}(\mathrm{COD})_{2}(0.510 \mathrm{~g}, 1.85 \mathrm{mmol}) \mathrm{COD}(0.300 \mathrm{~mL})$ bypyridine $(0.289 \mathrm{~g}$, $1.85 \mathrm{mmol})$ and toluene $8.0 \mathrm{~mL}$. The obtained polymer is brown solid $(0.200 \mathrm{~g}, 70.0 \%$ yield). ${ }^{1} \mathrm{H}$ NMR $\left(300 \mathrm{MHz}, \mathrm{CDCl}_{3}, \mathrm{ppm}\right): \delta 8.15-7.56(\mathrm{~m}, \mathrm{br}$, all Ar-H) 2.74 (br, Ar- 
$\left.\mathrm{CH}_{2}\right), 1.661 .521 .37\left(\mathrm{CH}_{2}\right.$ moiety from aliphatic part), $0.87\left(\mathrm{CH}_{3}\right) .{ }^{13} \mathrm{C} \mathrm{NMR}(75 \mathrm{MHz}$, $\left.\mathrm{CDCl}_{3}\right): \delta 179.60,179.19,150.36,134.49,128.05,36.15,31.81,30.67,29.16,22.64$, 13.94. IR (KBr pellet, $\left.\mathrm{cm}^{-1}\right): 3026,2924,2853,1672,1600,1432,1384,1264,1034,848$, 743,641 .

Polymer P9

Polymer $\mathbf{P 9}$ was synthesized according to the procedure described in polymer P1. Monomer M1 (0.200 g, $0.415 \mathrm{mmol})$, 4,7-dibromobenzo[c][1,2,5]thiadiazole $(0.242 \mathrm{~g}$, $0.830 \mathrm{mmol}), \mathrm{Ni}(\mathrm{COD})_{2}(0.510 \mathrm{~g}, 1.85 \mathrm{mmol}) \mathrm{COD}(0.30 \mathrm{~mL})$ bypyridine $(0.289 \mathrm{~g}, 1.85$

$\mathrm{mmol})$ and toluene $8 \mathrm{~mL}$. The obtained polymer is brown solid $\left(0.180 \mathrm{~g}, 73.3 \%\right.$ yield). ${ }^{1} \mathrm{H}$ NMR (300 MHz, $\left.\mathrm{CDCl}_{3}, \mathrm{ppm}\right): \delta$ 8.59-7.56 (m, br, all Ar-H) 2.74 (br, $\left.\mathrm{Ar}_{-}-\mathrm{CH}_{2}\right), 1.65$ $1.28\left(\mathrm{CH}_{2}\right.$ moiety from aliphatic part), $0.87\left(\mathrm{CH}_{3}\right) .{ }^{13} \mathrm{C} \mathrm{NMR}\left(75 \mathrm{MHz}, \mathrm{CDCl}_{3}\right): \delta 179.60$, $179.19,150.36,134.49,132.64,127.65 .05,36.15,31.81,30.67,29.16,22.64,14.31$. IR $\left(\mathrm{KBr}\right.$ pellet, $\left.\mathrm{cm}^{-1}\right): 3026,2924,2853,1672,1600,1432,1384,1262,1164,1034,848$, 743,641 .

Polymer P10

Polymer P10 was synthesized according to the procedure described in polymer P2. Polymer P8 (0.100 g, $0.218 \mathrm{mmol})$, LR (0.090 g, $0.168 \mathrm{mmol})$ and 1,2dichlorobenzene $(5.0 \mathrm{~mL})$. The obtained polymer is black solid $(0.090 \mathrm{~g})$. IR ( $\mathrm{KBr}$ pellet, $\left.\mathrm{cm}^{-1}\right): 3022,2923,2852,1596,1566,1464,1254,1217,1180,1022,828,7192,533$. 


\section{Polymer P11}

Polymer P11 was synthesized according to the procedure described in polymer P2. Polymer P9 (0.100 g, $0.168 \mathrm{mmol})$, LR (0.070 g, $0.168 \mathrm{mmol})$ and 1,2dichlorobenzene $(5.0 \mathrm{~mL})$. The obtained polymer is a black solid $(0.090 \mathrm{~g})$. IR $(\mathrm{KBr}$ pellet, $\left.\mathrm{cm}^{-1}\right): 3022,2922,2851,1595,1563,1495,1463,1369,1309,1257,1180,1114$, $1025,828,702,530$.

\section{Polymer P12}

Polymer P12 was synthesized according to the procedure described in polymer P2. Monomer M2 (0.326 g, $1.00 \mathrm{mmol})$, LR (0.4040 g, $1.00 \mathrm{mmol})$ and 1,2dichlorobenzene $(6.0 \mathrm{~mL})$. Obtained polymer is a dark black-amber solid $(0.250 \mathrm{~g}, 84.5 \%$ yield). ${ }^{1} \mathrm{H}$ NMR (300 MHz, $\left.\mathrm{CDCl}_{3}, \mathrm{ppm}\right): \delta 8-7$ (br, all Ar-H) 2.81 (br, $\mathrm{Ar}-\mathrm{CH}_{2}$ ), 1.30 $\left(\mathrm{CH}_{2}\right.$ moiety from aliphatic part), $0.90\left(\mathrm{br}, \mathrm{CH}_{3}\right) .{ }^{13} \mathrm{C} \mathrm{NMR}\left(75 \mathrm{MHz}, \mathrm{CDCl}_{3}\right): \delta 142.71$, 128.91, 36.24, 31.90, 31.03, 29.48, 22.69, 14.14.IR. IR ( $\mathrm{KBr}$ pellet, $\left.\mathrm{cm}^{-1}\right): 3024,2923$, $2851,1617,1591,1566,1496,1462,1377,1309,1258,1178,1096,1028,891,815,696$, 530. Anal. Found: S, 24.09

\section{Reduction and esterification of polymer P2}

It was followed the same procedure as described for oligomer $\mathbf{1} /$ compound $\mathbf{3}$. The starting P2 was $0.100 \mathrm{~g}$ and the resultant product was found $0.0950 \mathrm{~g}$. IR ( $\mathrm{NaCl}$ plate, $\left.\mathrm{cm}^{-1}\right): 2962,2921,2853,1596,1569,1499,1456,1369,1269,1096,1019,799$. 


\section{Oligomer $\mathbf{1}$ /compound $\mathbf{3}$}

To a solution of polymer P12 $(0.0730 \mathrm{~g})$ in THF $(8.0 \mathrm{~mL})$ under argon atmosphere $2 \mathrm{~mL}$ of $20 \%$ aqueous $\mathrm{NaOH}$ was added. An aqueous alkaline solution of sodium borohydride ( $0.0930 \mathrm{~g}, 2.46 \mathrm{mmol}$ in $2.0 \mathrm{~mL}$ of $10 \% \mathrm{NaOH}$ aqueous solution) was added dropwise to the polymer solution. After half an hour same amount of sodium borohydride was added to the reaction mixture. The reaction was stirred at room temperature for $72 \mathrm{~h}$. The dark black solution was then poured into ice water and stirred for $1 \mathrm{~h}$. The resulting mixture was extracted by dichloromethane and dried over $\mathrm{MgSO}_{4}$. The solvent was removed under reduced pressure. Then a solution of resultant product was prepared in THF and $\mathrm{CHCl}_{3}(1: 1$ ratio, $6.0 \mathrm{~mL})$. The solution was then added dropwise to a solution of acetic anhydride $(1.0 \mathrm{~mL})$ in THF $(2.0 \mathrm{ml})$ at $0{ }^{\circ} \mathrm{C}$. The reaction was stirred at room temperature for $24 \mathrm{~h}$. The dark black solution was then poured into ice water and stirred for $1 \mathrm{~h}$. The resulting mixture was extracted by dichloromethane and dried over $\mathrm{MgSO}_{4}$. The solvent was removed under reduced pressure and obtained the product $(0.0600 \mathrm{~g})$ as dark black solid which was characterize without any further purification. ${ }^{1} \mathrm{H}$ NMR (300 $\left.\mathrm{MHz}, \mathrm{CDCl}_{3}, \mathrm{ppm}\right): \delta 8-7$ (br, all Ar-H) 2.81 (br, $\left.\mathrm{Ar}-\mathrm{CH}_{2}\right), 1.30\left(\mathrm{CH}_{2}\right.$ moiety from aliphatic part), $0.90\left(\mathrm{br}, \mathrm{CH}_{3}\right) .{ }^{13} \mathrm{C} \mathrm{NMR}\left(75 \mathrm{MHz}, \mathrm{CDCl}_{3}\right): \delta 141.60,127.50,35.97$, 31.91, 29.46, 22.71, 13.95. IR (KBr pellet, $\mathrm{cm}^{-1}$ ): 2960, 2923, 2851, 1737, 1709, 1618, $1595,1492,1463,1377,1261,1090,1023,865,802,696,524$.

\section{Film formation}

Films coated on glass substrates were prepared by the spin-coating process. Polymer films were spin-coated from different solvents onto a glass plate (microscope slide $20 \times$ 
$20 \times 1 \mathrm{~mm}$ ) and spun at $1000 \mathrm{rpm}$ by using a Chemat KW-4B spin-Coater. Then the films were dried for $2-4 \mathrm{~h}$ at $80{ }^{\circ} \mathrm{C}-120{ }^{\circ} \mathrm{C}$ depends on solvent under argon atmosphere. To prepare $40-100 \mathrm{~nm}$ thick films, $8-10 \mathrm{mg}$ of polymers in 1.0-1.2 $\mathrm{mL}$ of solvent were used. 


\subsection{References}

1. Zhang, Z.-G.; Wang, J. J. Mater. Chem. 2012, 22, 4178.

2. Cui, C.; Fan, X.; Zhang, M.; Zhang, J.; Min, J.; Li. Y. Chem. Commun. 2011, 47, 11345.

3. Chen, X.; Liu, B.; Zou, Y.; Xiao, L.; Guo, X.; He, Y.; Li, Y. J. Mater. Chem. 2012, 22, 17724.

4. Chen, X.; Liu, B.; Zou, Y.; Tang, W.; Li, Y.; Xiao, D. RSC Adv. 2012, 2, 7439.

5. Cui, C.; Fan, H.; Guo, X.; Zhang, M.; He, Y.; Zhan, X.; Li, Y. Polym. Chem. 2012, 3, 99 .

6. Yuan, J.; Liu, Y.; Dong, H.; Shi, X.; Liu, Z.; Li, Y.; Ma, W. Polym. Chem. 2015, 6,7550 .

7. (a) Saha, S. K.; Ghosh, K. R.; Hao, W.; Wang, Z. Y.; Chiniforooshan, Y.; Ma, D.; Bock, W. J. J. Mater. Chem. A. 2014, 2, 5024. (b) Kim, G.; Kang, S.-J.; Dutta, G. K.; Han, Y.-K.; Shin, T. J.; Noh, Y.-Y.; Yang, C. J. Am. Chem. Soc. 2014, 136, 9477. (c) Han, J.; Qi, J.; Zheng, X.; Wang, Y.; Hu, L.; Guo, C.; Wang, Y.; Li, Y.; Ma, D.; Qiao, W.; Wang, Z. Y. J. Mater. Chem. C, 2017, 5, 159. (d) Zhang, C.; Wang, Z. Y. Macromolecules, 1993, 26, 3330. (e) Zhang, C.; Wang, Z. Y. Macromolecules, 1992, 25, 5851.

8. (a) Miyaura, N.; Yamada, K.; Suzuki, A. Tetrahedron Lett. 1979, 36, 3437. (b) Kotha, S.; Lahiri, K.; Dhurke, K. Tetrahedron, 2002, 58, 9633. (b) Sonogashira, K.; Tohda, Y.; Hagihara, N. Tetrahedron Lett. 1975, 50, 4467. (c) Sonogashira, K. J. Organomet. Chem. 2002, 653, 46. (d) Heck, R. F.; Nolley, J. P. Jr. J. Org. Chem. 1972, 37, 2320. (e) Johannes, G. D. V. Rev. Can. J. Chem. 2001, 79, 1086. (f) King, O. A.; Okukado, N. O.; Negishi, E-I. J. C. S. Chem. Comm. 1977, 683. 
(g) Tamao, K.; Sumitani, K.; Kumada, M. J. Am. Chem. Soc. 1972, 94, 4374. (h) Stille, J. K. Angew. Chem. Int. Ed. Engl. 1986, 25, 508 (i) Espinet, P.; Antonio, M. E. Angew. Chem. Int. Ed. 2004, 43, 4704. (J) Saha, S. K. Fluorescence turn-on sensory compounds and polymers for the detection of lead ion in water, M. Sc. Thesis, Carleton University, Ottawa, ON, Canada, 2012.

9. (a) Fanta, P. E. Chem. Rev. 1946, 38, 139. (b) Fanta, P. E. Chem. Rev. 1964, 64, 613.

10. Semmelhack, M. F.; Helquist, M. P.; Jones, L. D. J. Am. Chem. Soc. 1971, 93, 5908.

11. Yamamoto, T.; Shoichiro Wakabayashi, S.; Osakada, K. J. Organomet. Chem. 1992, 428, 223.

12. Asakura, H.; Shishido, T.; Tanaka, T. J. Phys. Chem. A. 2012, 116, 4029.

13. Ozturk, T.; Ertas, E.; Mert, O. Chem. Rev. 2007, 107, 5210.

14. Lee, J.; Kalin, A. J.; Yuan,T.; Al-Hashimic, M.; Fang, L. Chem. Sci. 2017, 8, 2503

15. Scherf, U. J. Mater. Chem. 1999, 9, 1853

16. Bheemireddy, S. R.; Hautzinger, M. P.; Li, T.; Lee, B.; Plunkett, K. N. J. Am. Chem. Soc. 2017, 139, 5801.

17. (a) Oh, H. S.; Kim, T.-D.; Koh, Y.-H.; Lee, K.-S.; Cho, S.; Cartwrightb, A.; Paras N. Prasad, P. N. Chem. Commun. 2011, 47, 8931. (b) Qian, G.; Li, X.; Wang, Z. Y. J. Mater. Chem. 2009, 19, 522. (c) Ge, C.-W.; Mei, C.-Y.; Ling, J.; Zhao, F.G.; Li, H.-J.; Liang, L.; Wang, J.-T.; Yu, J.-C.; Shao, W.; Xie, Y.-S.; Li, W.-S. J. Polym. SCi. Part A: Polym. Chem. 2014, 52, 2356. 
18. (a) Kim, J.; Kwon, Y. S.; Shin, W. S.; Moon, S.-J.; Park, T. Macromolecules, 2011, 44, 1909. (b) Zhou, Y.; Xiao, Y.; Chi, S. Qian, X. Org. Lett. 2008, 10, 633. (c) He, B.; Nie, H.; Chen, L.; Lou, X.; Hu, R.; Qin, A.; Zhao, Z.; Tang, B. Z. Org. Lett. 2015, 17, 6174. (d) Qian,G.; Abu, H.; Wang, Z. Y. J. Mater. Chem. 2011, 21,7678 .

19. (a) Jenekhe S. A.; Yi. S. Appl. Phys. Lett. 2000, 77, 2635. (b) Alam, M. M.; Jenekhe, S. A. J. Phys. Chem. B 2002, 106, 11172. (c) Yuan, Z.; Xiao, Y.; Yang, Y.; Xiong, T, Macromolecules, 2011, 44, 1788. (d) Usta, H.; Risko, C.; Wang, Z.; Huang, H.; Deliomeroglu, M. K.; Zhukhovitskiy, A.; Facchetti, A.; Marks, T. J. J. Am. Chem. Soc. 2009, 131, 5586. (e) Dierschke, F.; Grimsdale, A. C.; Mullen, K. Macromol. Chem. Phys. 2004, 205, 1147.

20. (a) Ghosh, K. R.; Saha, S. K.; Gao, J. P.; Wang, Z. Y Wang, Chem. Commun. 2014, 50, 176. (b) Hicks, F.; Brookhart, M. U. S. Patent 0027957 A1, 2002

21. (a) Zhou, H.; Yang, L.; You, W. Macromolecules, 2012, 45, 607. (b) Liu, D.; Yang, B.; Jang, B.; Xu, B.; Zhang, S.; He, C.; Woo, H. Y.; Hou, J. Energy Environ. Sci. 2017, 10, 546.

22. Hagana, A. J.; Moratti, S. C.; Sage, I. C. Synth. Met. 2001, 119, 147.

23. Paulussen, H.; Haitjema, H.; van Asselt, R.; Mylle, P.; Adriaensens, P.; Gelan, J.; Vanderzande, D. Polymer, 2000, 41, 3121.

24. (a) Guo, X.; Baumgarten, M.; Müllen, K. Progress in Polymer Science, 2013, 38, 1832. (b) Wu, J.-S.; Cheng, S.-W.; Cheng, Y.-J.; Hsu, C.-S. Chem. Soc. Rev. 2015, 44, 1113. (c) Cheng, Y.-J.; Yang, S.-H.; Hsu, C.-S. Chem. Rev. 2009, 109, 
5868. (d) Liu, C.; Wang, K.; Xiong Gong, X.; Heeger, A. J. Chem. Soc. Rev. 2016, 45,4825 .

25. (a) Roncali, J. Chem. Rev., 1992, 92, 711. (b) Roncali, J. Macromol. Rapid Commun. 2007, 28, 1761. (c) Dennler, G.; Scharber, M. C.; Brabec, C. J. Adv. Mater. 2009, 21, 1323.

26. Chan, H. Z.; Choonng, S. Prog. Polym. Sci. 1998, 23, 1167.

27. Cheng, Y.-J.; Yang, S. H.; Hsu, C.-S. Chem. Rev. 2009, 109, 5868.

28. Liu, C.; Wang, K.; Gong, X.; Heeger, H. J. Chem. Soc. Rev. 2016, 45, 4825.

29. Wu, J.-S.; Cheng, S.-W.; Cheng, Y.-J.; Hsu, C.-S. Chem. Soc. Rev. 2015, 44, 1113.

30. Chen, J.; Cao, Y. Acc. Chem. Res. 2009, 42, 1709.

31. Parker, T. C.; Patel, D. G.; Moudgil, K.; Barlow, S.; Risko, C.; Bredas, J.-L.; Reynoldsac, J. R.; Marder, S. R. Mater. Horiz. 2015, 2, 22.

32. Wang, Y.; Michinobu, T. J. Mater. Chem. C, 2016, 4, 6200.

33. Li, W.; Qin, R.; Zhou, Y.; Andersson, M.; Li, F.; Zhang, C.; Li, B.; Liu, Z.; Bo, Z.; Zhang, F. Polymer, 2010, 51, 3031.

34. Park, S. H.; Roy, A.; Beaupre, S.; Cho, S.; Coates, N.; Moon, J. S.; Moses, D.; Leclerc, M.; Lee, K.; Heeger. A. J. Nature Photonics, 2009, 3, 297.

35. Wang, S.; Kappl, M.; Liebewirth, I.; Muller, M.; Kirchhoff, K.; Pisula, W.; Mullen, K. Adv. Mater. 2012, 24, 417.

36. Han, H.; Nam, S.; Seo, J.; Lee, C.; Kim, H.; Bradley, D. D. C.; Ha, C.-S.; Kim, Y. Sci. Rep. 2015, 5, 16457. 
37. Kang, T. E.; Choi, J.; Cho, H.-H.; Yoon, S. C.; Kim, B. J. Macromolecules, 2016, 49, 2096.

38. (a) Fua, Y.; Wanga, X.; Chena, M.; Shen, W. Molecular Simulation, 2014, 40, 439. (b) Kang, T. E.; Choi, J.; Cho, H.-H.; Yoon, S. C.; Kim, B. J. Macromolecules, 2016, 49, 2096.

39. (a) Ge, C.-W.; Mei, C.-Y.; Ling, J.; Wang, J.-T.; Zhao, F.-G.; Liang, L.; Li, H.-J.; Xie, Y.-S.; Li, W.-S. J. Polym. SCi. Part A: Polym. Chem. 2014, 52, 1200. (b) Kim, G.; Han, A.-R.; Lee, H. R.; Lee, J.; Oh, J. H.; Yang, C. Chem. Commun., 2014, 50, 2180.

40. (a) Kass, K.-J.; Forster, M.; Scherf, U. Angew. Chem. Int. Ed. 2016, 55, 7816.

41. Lakshmikantham, M. V.; Levinson, M.; Menachery, M.; Cava, M. P. J. Org. Chem. 1986, 51, 412.

42. Pawin, G.; Wong, K. L.; Kwon, K.-Y.; Frisbee, R. J.; Rahman, T. S.; Bartels, L. J. Am. Chem. Soc. 2008, 130, 15244. 


\section{Chapter 3 Syntheses and Transformations of NTDO-based Polymers}

\subsection{Introduction}

Since NTDO has an anthraquinone unit, reduction reaction in the keto group and the subsequent transformation of NTDO-based polymers would lead to a new series of polymers. Moreover, the condensation reaction or modified Knoevenagel condensation on the keto group of NTDO-based polymers would yield new polymers, possibly with interesting properties. In this part of the research we carried out the chemical transformations of NTDO-based polymers and characterized the resulting new polymers by reduction and condensation reactions.

\subsection{Reduction reaction of anthraquinones}

In 1974 Criswell and Klanderman developed a method for the conversion of certain 9,10-anthraquinones to anthracenes via successive reductions and dehydrations. ${ }^{1}$ The reduction was carried out in the presence of alcoholic sodium borohydride. Several halo- and methyl-substituted anthracenes had also been synthesized and reported by that procedure. They found that a three-step procedure involving two successive reductions and one dehydration in the presence of alcoholic (methanol or 2-propanol) sodium borohydride converts anthraquinones to anthracenes in $65-95 \%$ yield. The reaction was described as a straightforward synthesis which generated these intermediates 9,10dihydroxy-9,10-dihydroanthracenes, anthrones, and 9-hydroxy-9,10-dihydroanthracenes intermediates. This alcoholic sodium borohydride reduction methodology was reported as a successful method for the synthesis of anthracene from anthraquinone over Lithium aluminium hydride. ${ }^{2,3}$ In 1997, Hodge followed the same procedure and synthesized 2,6- 
dibromoanthracene from 2,6-dibromoanthraquinone in $65 \%$ yield. ${ }^{4}$ In 1971, Rabideau synthesized 2,3,6,7-tetramethoxyantracene from 2,3,6,7-tetramethoxyantraquinone in the presence of $\mathrm{Zn}$ and $10 \% \mathrm{NaOH}$ in very low yield. ${ }^{5}$ In 2006, Balaban et. al. synthesized 2,3,6,7-tetraalkoxyanthracene from 2,3,6,7-tetraalkoxyanthraquonone in the presence of $\mathrm{Zn}$ and $10 \% \mathrm{NaOH}$ in $56 \%$ yield. They also performed stepwise reduction on 2,3,6,7tetraalkoxyanthraquonone in the presence of $\mathrm{Zn} / \mathrm{NaOH}$ followed by sodium borohydride which provided 2,3,6,7-tetraalkoxyanthracene in $75 \%$ yield. $^{6}$

\subsection{Synthesis and characterizations of polymers P13-16}

Based on the reduction reaction in 9,10-anthraquinones discussed in section 3.2, similar reactions were intended to conduct on the homopolymer P1, which possesses a similar diketo group. Newly obtained polymers will provide different optical, electrochemical and solubility properties. Polymers P13 to P16 were synthesized according to the reactions in Scheme 3.1. Polymer P1 was reduced under alcoholic sodium borohydride and the obtained polymer $\mathbf{P 1 3}$ in 95\% yield was characterized by IR spectroscopy, absorption spectroscopy, and CV. The IR spectrum (Figure 3.1) of polymer P13 shows broad peak at $3399 \mathrm{~cm}^{-1}$, which is the characteristic peak for $\mathrm{OH}$. The weak peak at $1669 \mathrm{~cm}^{-1}$ which is the characteristic peak for $\mathrm{C}=\mathrm{O}$, indicates most of the keto group in homopolymer $\mathbf{P 1}$ has been reduced and converted to $\mathrm{OH}$. 


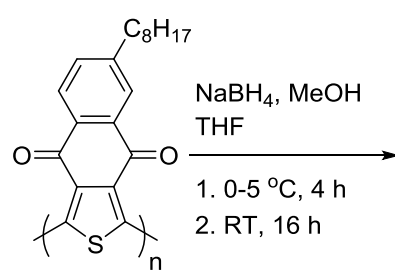

P1

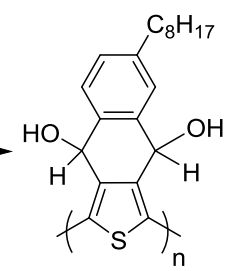

P13

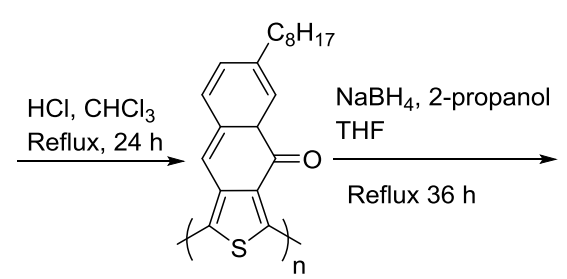

P14

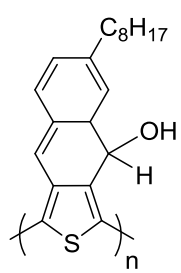

P15

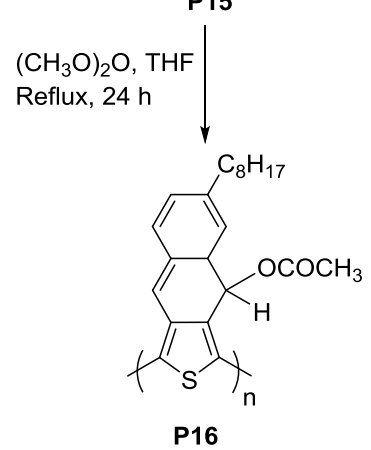

Scheme 3.1 Synthesis of polymers P13 to P16.

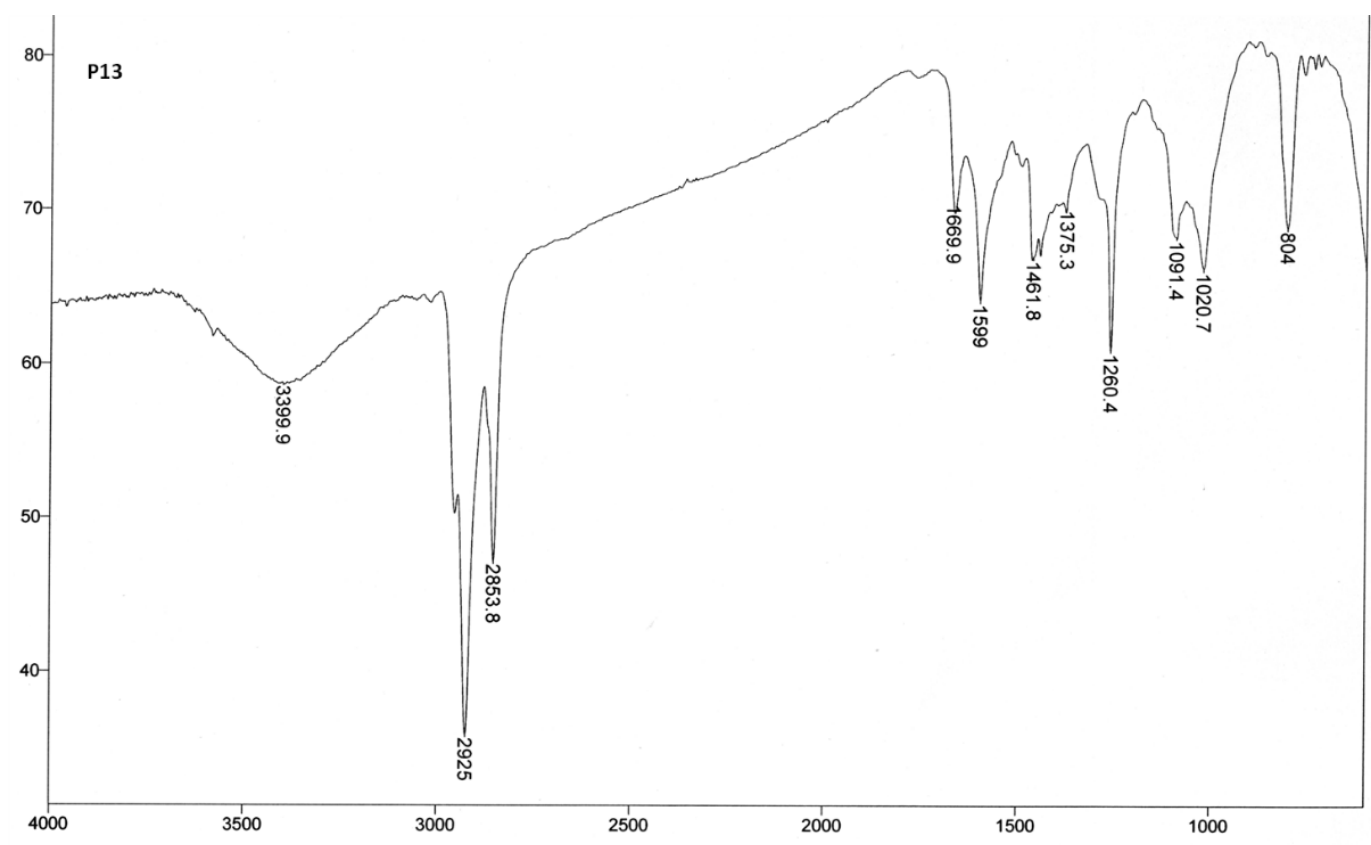

Figure 3.1 IR spectrum of polymer $\mathbf{P 1 3}$ on $\mathrm{NaCl}$ plate. 

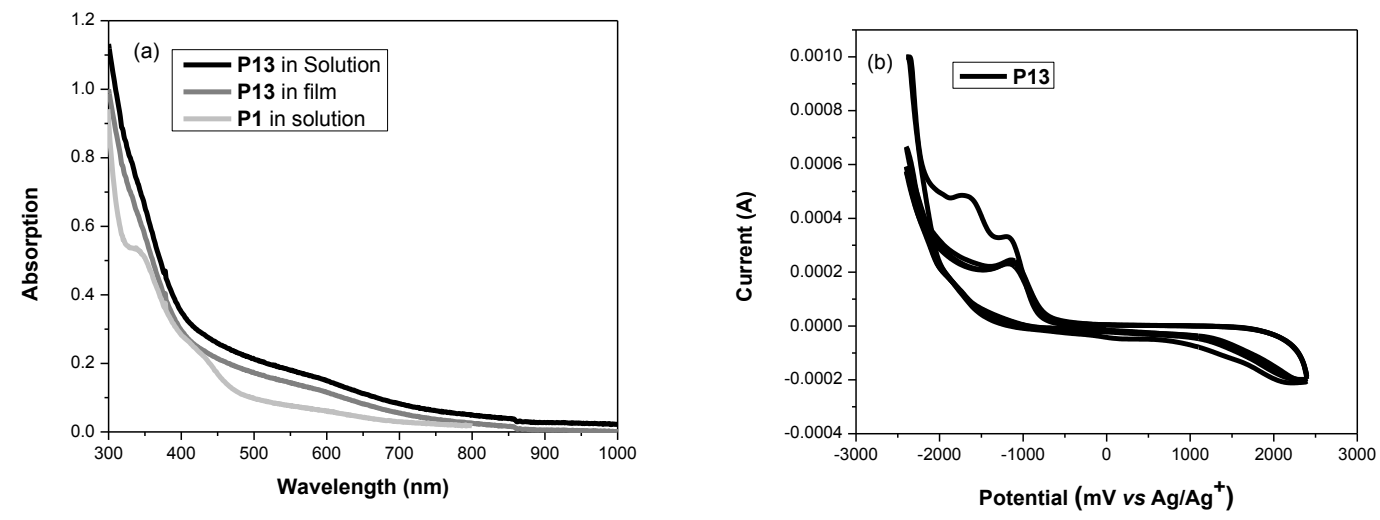

Figure 3.2 Absorption spectra of polymer $\mathbf{P 1 3}$ in $\mathrm{CHCl}_{3}$ solution and as thin film on a glass substrate (a) and cyclic voltammogram of film on ITO in $\mathrm{CH}_{3} \mathrm{CN}$ solution containing in $0.1 \mathrm{~mol} / \mathrm{L} \mathrm{Bu}_{4} \mathrm{NPF}_{6}$ with a scan rate of $100 \mathrm{mV} / \mathrm{s}(\mathrm{b})$.

Polymer P13 is highly soluble in solvents such as $\mathrm{CHCl}_{3}$, THF, chlorobenzene, dichlorobenzene and trichlorobenzene. Absorption spectra of $\mathbf{P 1 3}$ in $\mathrm{CHCl}_{3}$ solutions and a spin-coated thin solid film on a glass substrate were acquired. Polymer P13 does not display any maximal absorption peak in solution or in solid film. It shows absorption onset from $700 \mathrm{~nm}$ (Figure 3.2a) in solution and solid film. Polymer P1 has a maximal absorption peak whereas polymer P13 does not show any maximal absorption peak. This is a strong indication that the polymer structure has been changed after the reduction reaction. The absence of maximal absorption peak of P13 may be due to the loss of conjugation in polymer structure.

The HOMO and LUMO energy levels and the electrochemical band gap $\left(\mathrm{E}_{g}\right)$ of polymer P13 were calculated from the oxidation onset and reduction onset potentials. Figure $3.2 \mathrm{~b}$ shows the CV of polymer P13. Polymer P13 displays two observable reduction processes in the first cycle, while in the late cycles it has only one. Moreover, 
the reduction process is not reversible, indicating the structural change during the process. The reduction onset is at $-0.66 \mathrm{~V}$ in $\mathrm{CV}$, the LUMO energy level of P13 is -3.70 . The homopolymer P1 shows oxidation potential in CV, whereas P13 shows reduction potential, which suggests the reduction reaction on the polymer was successful.

Polymer P14 was synthesized according to the reaction in Scheme 3.1. Polymer P14 was dehydrated under an acidic condition and polymer P14 was obtained in 95\% yield and was characterized by IR, absorption spectroscopy and CV. The IR spectrum (Figure 3.3) of polymer P14 shows no broad peak at $3399 \mathrm{~cm}^{-1}$, which suggests the dehydration was successful. The peak intensity at $1664 \mathrm{~cm}^{-1}$ did not increase significantly after the dehydration reaction compared to polymer P13.

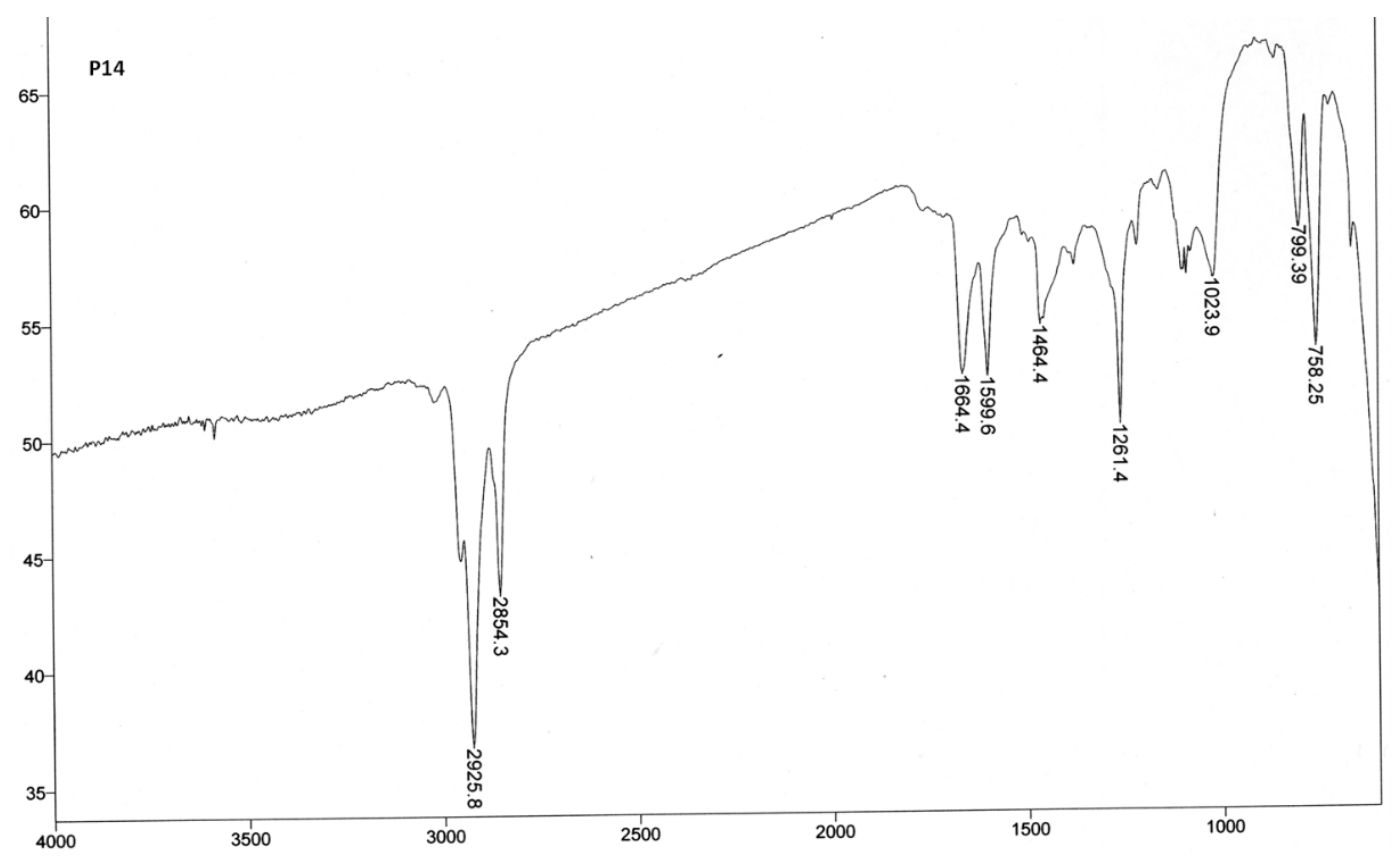

Figure 3.3 IR spectrum of polymer P14 on $\mathrm{NaCl}$ plate. 

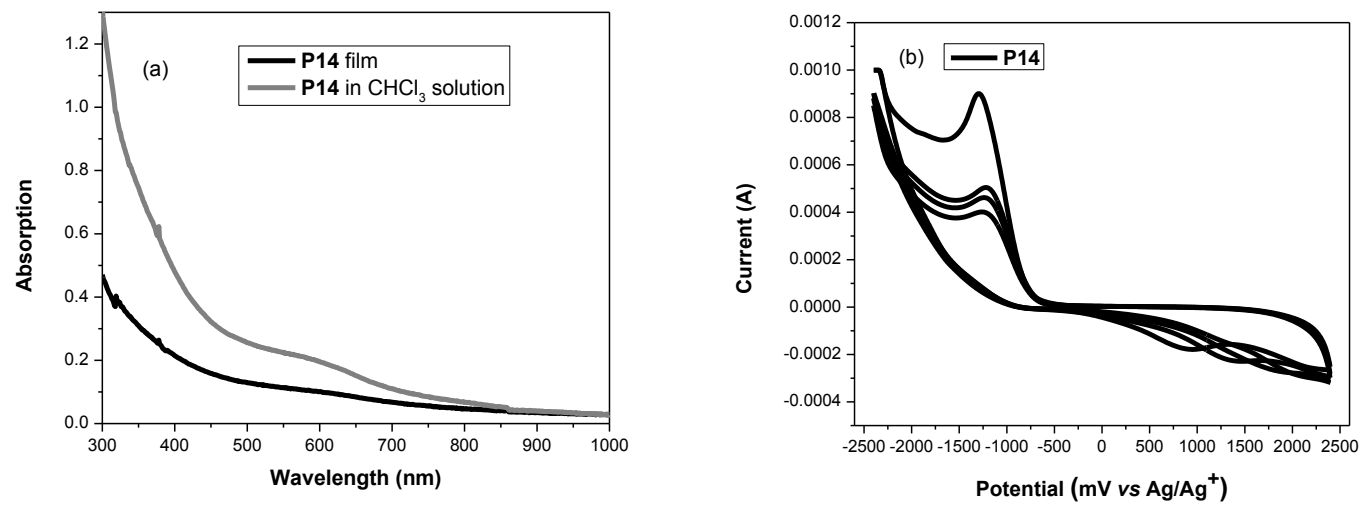

Figure 3.4 Absorption spectra of polymer $\mathbf{P 1 4}$ in $\mathrm{CHCl}_{3}$ solution and as thin film on glass substrate (a) and cyclic voltammogram of film on ITO in $\mathrm{CH}_{3} \mathrm{CN}$ solution containing in $0.1 \mathrm{~mol} / \mathrm{L} \mathrm{Bu}_{4} \mathrm{NPF}_{6}$ with a scan rate of $100 \mathrm{mV} / \mathrm{s}(\mathrm{b})$.

Polymer P14 is fully soluble in a wide range of solvents, including $\mathrm{CHCl}_{3}, \mathrm{THF}$, chlorobenzene, dichlorobenzene and trichlorobenzene. Absorption spectra of P14 in $\mathrm{CHCl}_{3}$ solutions and a spin-coated thin solid film on a glass substrate were taken. Polymer P14 displays a maximal absorption peak at $585 \mathrm{~nm}$ with an onset from $740 \mathrm{~nm}$ (Figure 3.4a). Polymer P13 shows no maximal absorption peak in solution whereas polymer P14 shows the absorption maximal. Moreover, the absorption maximal of P14 is in longer wavelength than P1, which suggests the polymer structure has changed after the dehydration reaction. The polymer film does not show any maximal absorption but does show an onset from $750 \mathrm{~nm}$.

The HOMO and LUMO energy levels and electrochemical $\mathrm{E}_{g}$ of polymer P14 were calculated from the oxidation onset and reduction onset potentials. Figure $3.4 \mathrm{~b}$ shows the CV of polymer P14. Polymer P14 shows both noticeable oxidation and reduction processes. The onset oxidation potential/onset reduction potential of polymer 
P14 is $0.24 /-0.70 \mathrm{~V}$. Therefore, the calculated HOMO level is $-4.60 \mathrm{eV}$ and the LUMO is $-3.66 \mathrm{eV}$. The obtained energy band gap is $0.94 \mathrm{eV}$. The change in the current for polymer P14 during $2^{\text {nd }}$ cycle is may be due to the polymer dissolving into the solution during electrochemical reaction. The obtained oxidation and reduction potentials indicate that polymer P14 is a new polymer and the dehydration reaction on polymer P14 was successful.

Polymer P14 was reduced under alcoholic sodium borohydride according to the reaction in Scheme 3.1 and the polymer was obtained P15 in 95\% yield and was characterized by IR, absorption spectroscopy and CV. The IR spectrum (Figure 3.5) of polymer P15 shows a broad peak at $3391 \mathrm{~cm}^{-1}$, which is the characteristic peak for $\mathrm{OH}$. The presence of a weak peak at $1664 \mathrm{~cm}^{-1}$, indicates polymer P15 still has some keto group and most of the keto groups in polymer P14 were reduced to $\mathrm{OH}$.

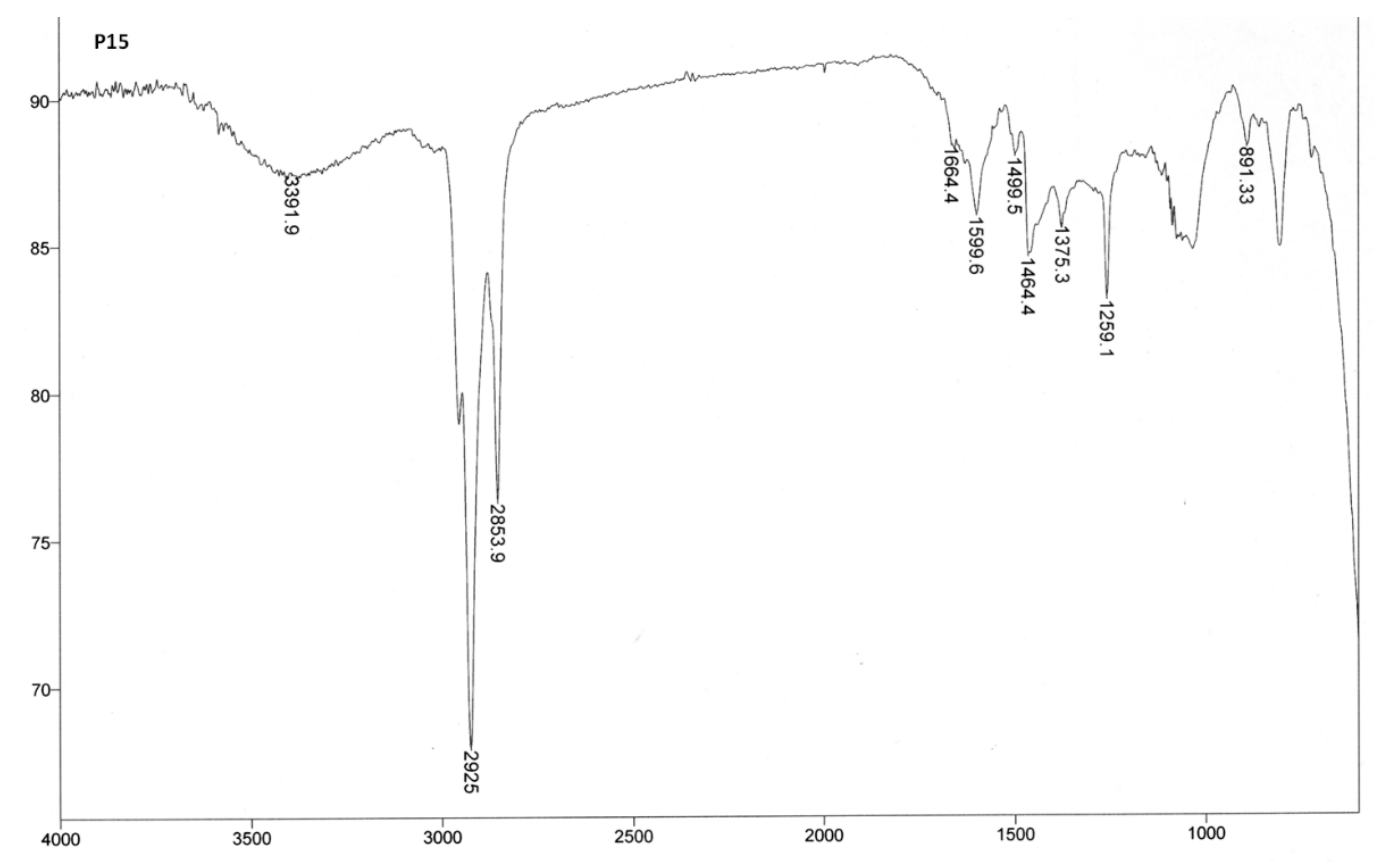

Figure 3.5 IR spectrum of polymer $\mathbf{P 1 5}$ on $\mathrm{NaCl}$ plate. 

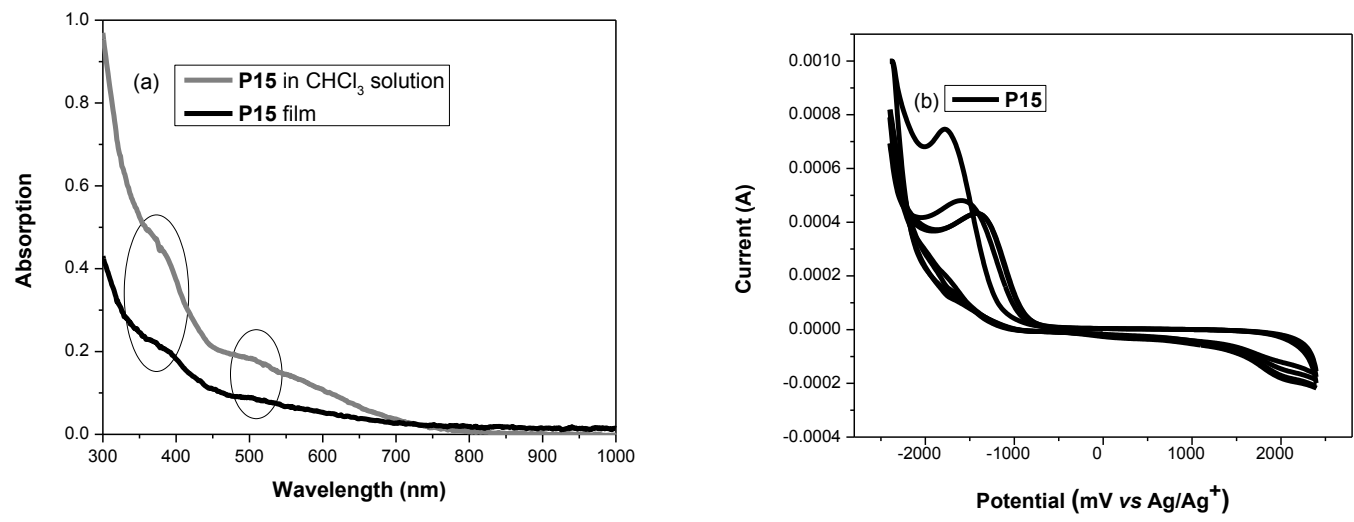

Figure 3.6 Absorption spectra of polymer $\mathbf{P 1 5}$ in $\mathrm{CHCl}_{3}$ solution and as thin film on a glass substrate (a) and cyclic voltammogram of film on ITO in $\mathrm{CH}_{3} \mathrm{CN}$ solution containing in $0.1 \mathrm{~mol} / \mathrm{L} \mathrm{Bu}_{4} \mathrm{NPF}_{6}$ with a scan rate of $100 \mathrm{mV} / \mathrm{s}(\mathrm{b})$.

Polymer P15 is readily soluble in solvents such as $\mathrm{CHCl}_{3}$, THF, chlorobenzene, 1,2-dichlorobenzene and 1,2,4-trichlorobenzene. Absorption spectra of $\mathbf{P 1 5}$ in $\mathrm{CHCl}_{3}$ solutions and a spin-coated thin solid film on a glass substrate were acquired. Polymer P15 displays two maximal absorption peaks at $500 \mathrm{~nm}$ and $375 \mathrm{~nm}$ with an onset from $710 \mathrm{~nm}$ (Figure 3.6a). The absorption of polymer P15, which is $85 \mathrm{~nm}$ blue-shifted compared to polymer P14, may be due to the break of conjugation in the monomer unit and the loss of the electron-withdrawing nature from $\mathrm{C}=\mathrm{O}$ to $\mathrm{OH}$. Moreover, the absorption maximal of $\mathbf{P 1 5}$ is at a longer wavelength than P1. This result indicates the polymer structure has been changed after the reduction reaction. The polymer film shows same absorption maximal peak as in solution with an onset from $710 \mathrm{~nm}$.

The HOMO and LUMO energy levels and electrochemical $\mathrm{E}_{g}$ of polymer P15 were calculated from the oxidation onset and reduction onset potentials. Figure $3.6 \mathrm{~b}$ shows the CV of polymer P15. Polymer P15 shows both noticeable oxidation and 
reduction processes. The onset oxidation potential/onset reduction potential of polymer P15 is $1.40 /-0.92 \mathrm{~V}$. Therefore, the calculated HOMO is $-5.76 \mathrm{eV}$ and the LUMO is -3.44 $\mathrm{eV}$. The obtained energy band gap is $2.32 \mathrm{eV}$. The obtained energy band gap is higher than P14. The oxidation potential, reduction potential and electrochemical energy bandgap indicate that the new polymer P15 was successfully obtained by the reduction of polymer P14.

The esterification of P15 afforded polymer P16 (Scheme 3.1), which was then characterized by IR, absorption spectroscopy and CV. The IR spectrum (Figure 3.7) of polymer P16 does not show broad peak at $3391 \mathrm{~cm}^{-1}$, which indicates that the $\mathrm{OH}$ group of polymer P15 successfully took part in the esterification. The weak peaks at 1773 and $1737 \mathrm{~cm}^{-1}$ are characteristic for the ester's $\mathrm{C}=\mathrm{O}$. This confirms the structure of polymer P16. However, a very weak peak at $1664 \mathrm{~cm}^{-1}$ for the $\mathrm{C}=\mathrm{O}$ group is also observed in the IR spectrum of polymer P16, which suggests polymer P16 still contains keto group in the structure from previous polymers.

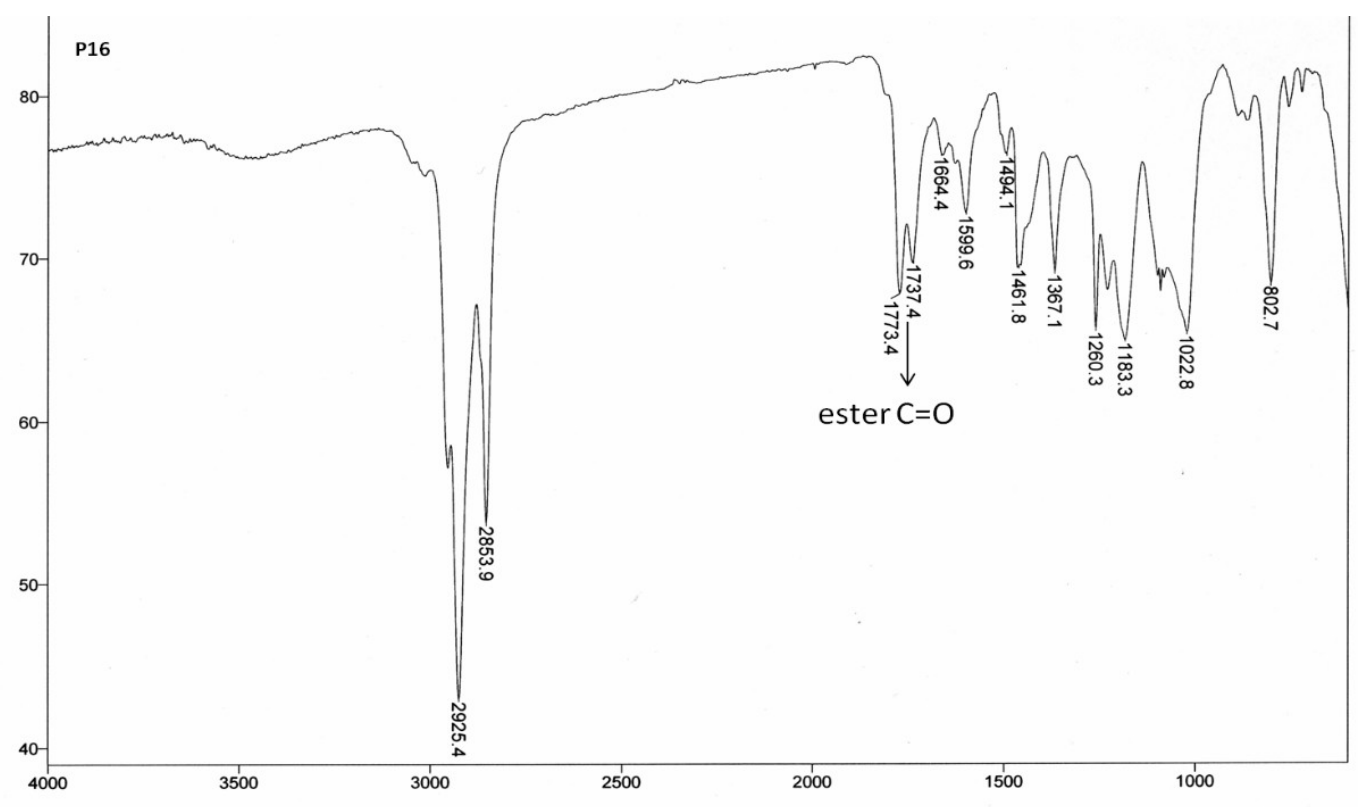

Figure 3.7 IR spectrum of polymer $\mathbf{P 1 6}$ on $\mathrm{NaCl}$ plate. 

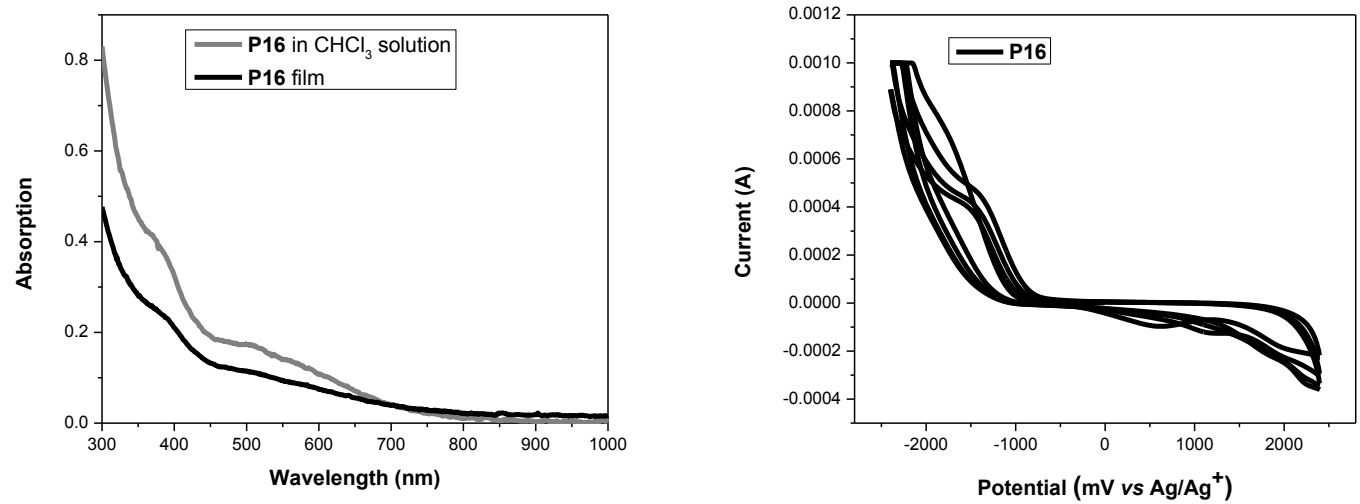

Figure 3.8 Absorption spectra of polymer $\mathbf{P 1 6}$ in $\mathrm{CHCl}_{3}$ solution and as thin film on a glass substrate (a) and cyclic voltammogram of film on ITO in $\mathrm{CH}_{3} \mathrm{CN}$ solution containing in $0.1 \mathrm{~mol} / \mathrm{L} \mathrm{Bu}_{4} \mathrm{NPF}_{6}$ with a scan rate of $100 \mathrm{mV} / \mathrm{s}(\mathrm{b})$.

Polymer P16 is fully soluble in solvents such as $\mathrm{CHCl}_{3}$, THF, chlorobenzene, dichlorobenzene and trichlorobenzene. Absorption spectrum of $\mathbf{P 1 6}$ in $\mathrm{CHCl}_{3}$ solutions and a spin-coated thin solid film on a glass substrate were taken. Polymer P16 displays two maximal absorption peaks at $505 \mathrm{~nm}$ and $370 \mathrm{~nm}$ with an onset from $700 \mathrm{~nm}$ (Figure 3.8a). The absorption maximal of polymer P16 is $5 \mathrm{~nm}$ red-shifted, compared to the longer wavelength of polymer P15 and $5 \mathrm{~nm}$ blue-shifted, compared to the shorter wavelength of polymer P15. This result indicates the polymer structure has been changed after the esterfication reaction. The polymer film shows the same absorption maximal peaks with an onset from $710 \mathrm{~nm}$.

The HOMO and LUMO energy levels and electrochemical $\mathrm{E}_{g}$ of polymer P16 were calculated from the oxidation onset and reduction onset potentials. Figure $3.8 \mathrm{~b}$ shows the CV of polymer P16. Both noticeable oxidation and reduction processes were observed for polymer P16. The onset oxidation potential/onset reduction potentials of 
polymer P16 is $0.10 /-0.84 \mathrm{~V}$. Therefore, the calculated HOMO is $-4.46 \mathrm{eV}$ and the LUMO is $-3.52 \mathrm{eV}$. The obtained energy band gap is $0.94 \mathrm{eV}$. The obtained energy band gap is lower than P15. The LUMO energy level decreased and the HOMO energy level increased compared to polymer P15. The obtained oxidation potential, reduction potential and electrochemical $E_{g}$ indicates polymer P16 is a new polymer and the esterification reaction of polymer $\mathbf{P 1 5}$ was successful.

\subsection{Attempted synthesis of P17}

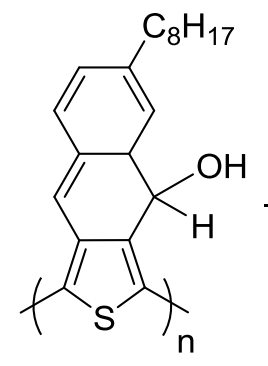

P15
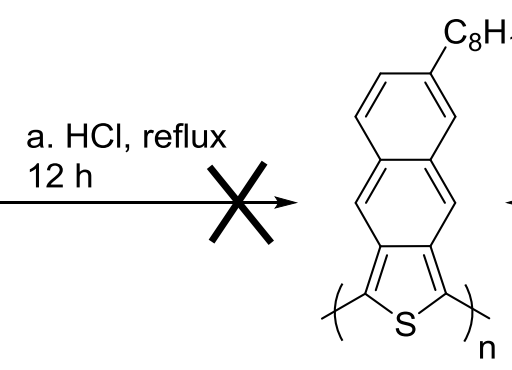

P17

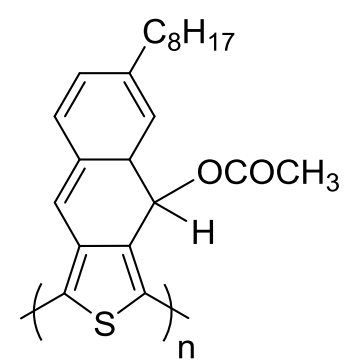

P16

Scheme 3.2 Attempted synthesis of polymer P17.

The synthesis of polymer P17 was attempted according to the reactions in Scheme 3.2. Polymer P17 was attempted to synthesize by three different pathways. The first methodology was the dehydration of polymer P15 in an acidic condition which will provide the target polymer P17. The dehydration reaction of polymer P15 was carried out in the presence of conc. HCl. The obtained polymer was characterized by IR, and absorption spectroscopy. 


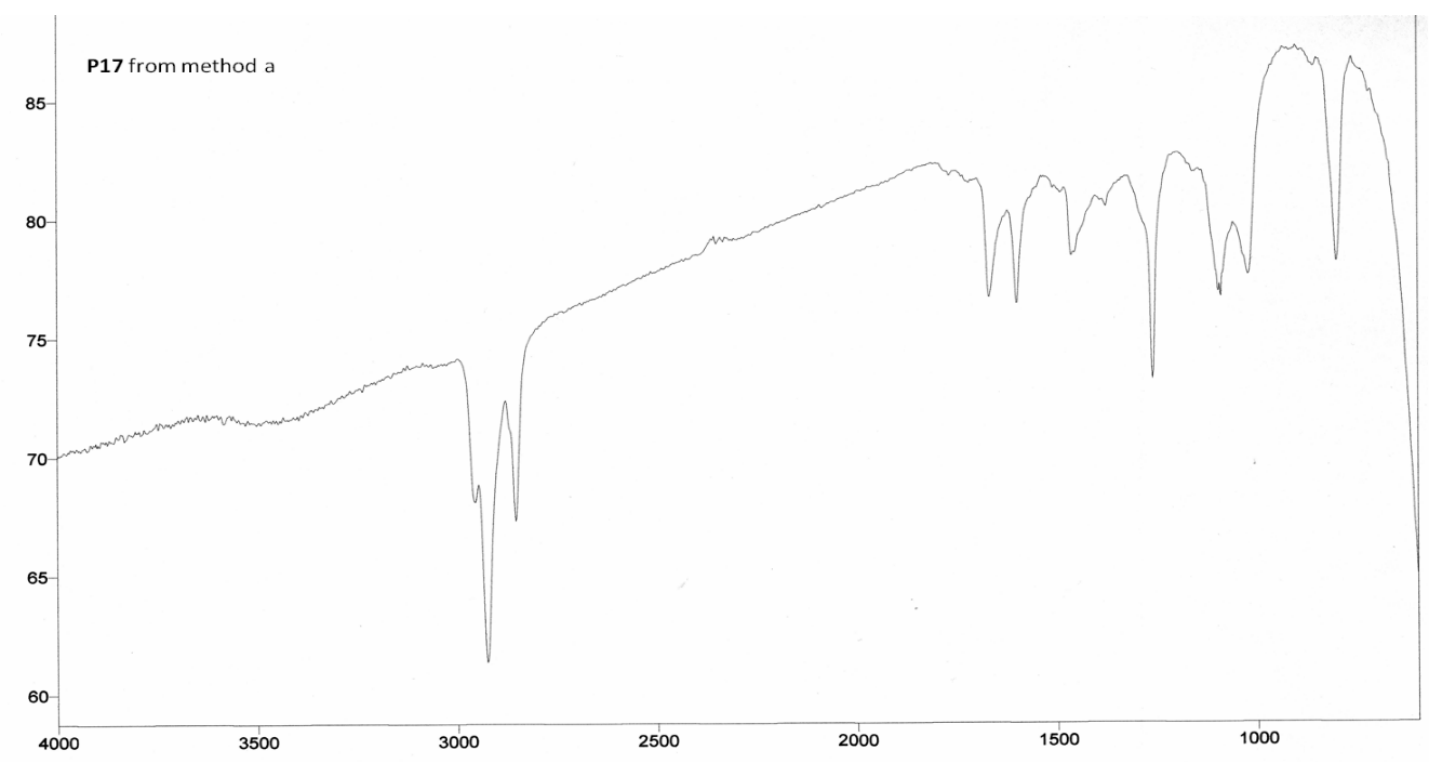

Figure 3.9 IR spectrum of polymer $\mathbf{P 1 7}$ on $\mathrm{NaCl}$ plate.

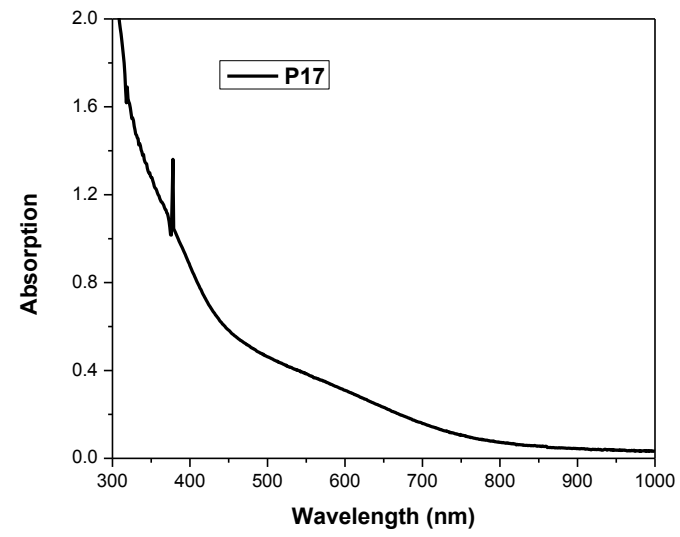

Figure 3.10 Absorption spectrum of polymer $\mathbf{P 1 7}$ in $\mathrm{CHCl}_{3}$ solution.

The IR spectrum (Figure 3.9) of polymer P17 does not show broad peak at 3391 $\mathrm{cm}^{-1}$, which indicates dehydration was successful. However, the weak peak at $1664 \mathrm{~cm}^{-1}$ indicates some of the keto group is still present in the polymer backbone. Absorption spectrum of $\mathbf{P 1 7}$ in $\mathrm{CHCl}_{3}$ solution was taken. Polymer P17 displays broad absorption 
with an onset from $750 \mathrm{~nm}$ (Figure 3.10). The maximal absorption of polymer P17 is not distinguishable.

Based on this result, another approach (path b) was taken to obtain P17 from P16. As we know ester is a very good leaving group therefore, we attempted to synthesis P17 by dehydration reaction in polymer P16. The reaction was carried out in the presence of trifluoroacetic acid. The obtained polymer was characterized by IR and absorption spectroscopy.

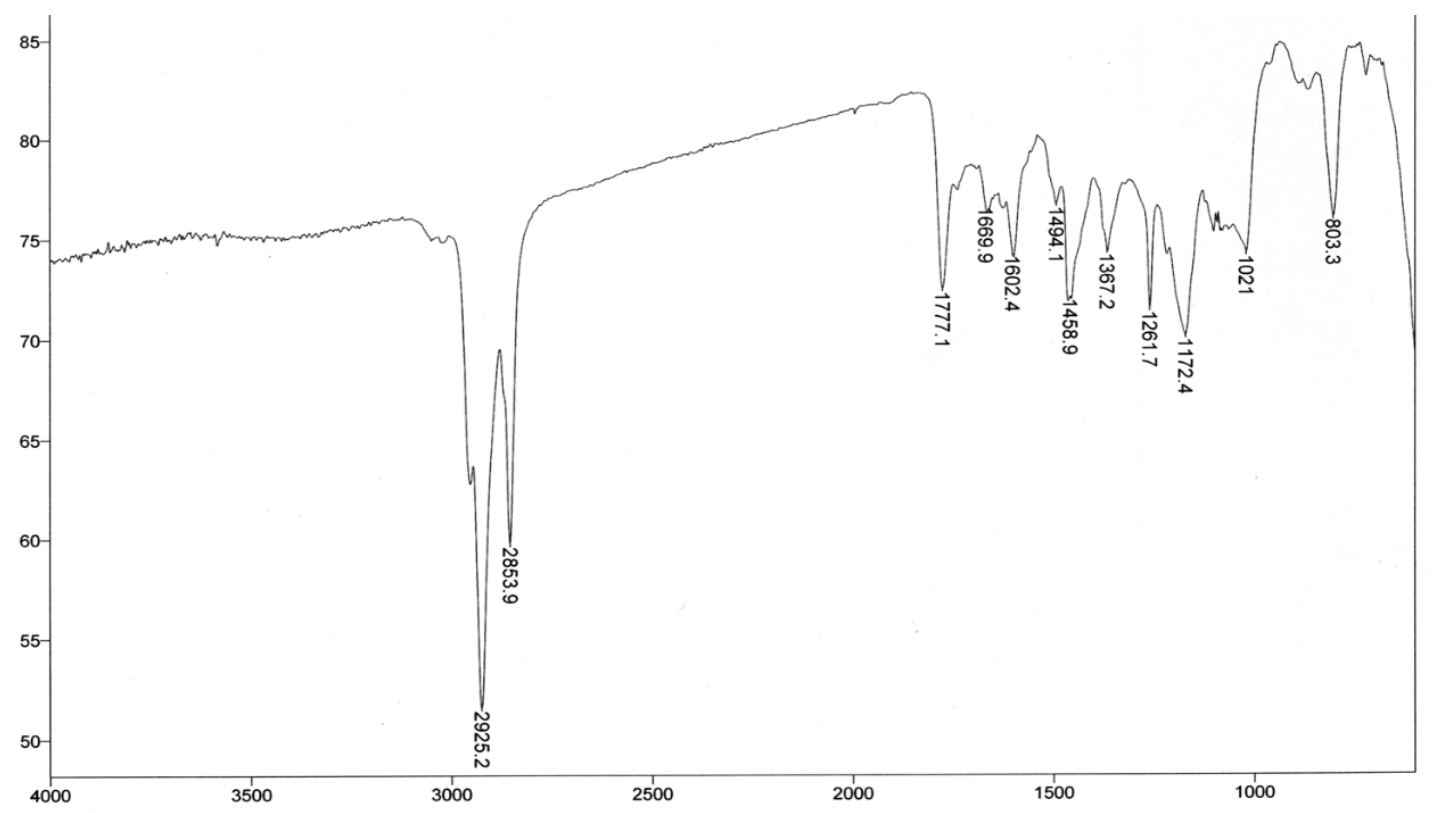

Figure 3.11 IR spectrum of polymer P17 on $\mathrm{NaCl}$ Plate.

Figure 3.11 shows the IR spectroscopy of target polymer P17. The peak at 1777 $\mathrm{cm}^{-1}$, which is the characteristic peak for ester $\mathrm{C}=\mathrm{O}$ and this indicates the dehydration reaction was unsuccessful. Moreover, the absorption spectra in Figure 3.12a show that polymer P17 has two maximal peaks, at $565 \mathrm{~nm}$ and $375 \mathrm{~nm}$ in $\mathrm{CHCl}_{3}$ with an onset from $740 \mathrm{~nm}$. The absorption maximal for the longer wavelength is $60 \mathrm{~nm}$ red-shifted 
compared to polymer P16. The polymer film shows the same absorption maximal peak for the short wavelength and the absorption peak for the longer wavelength is much broader. Figure $3.12 \mathrm{~b}$ shows both observable oxidation and reduction processes of polymer P17. The onset oxidation potential/onset reduction potential of polymer P17 are at $1.40 /-0.92 \mathrm{~V}$. Therefore, the calculated HOMO is $-5.76 \mathrm{eV}$ and the LUMO is $-3.44 \mathrm{eV}$. The obtained energy band gap is $2.32 \mathrm{eV}$. The obtained energy band gap is higher than that of P16. From these analyses, it concluded that the polymer contained esteric groups in its monomer unit and was not our target polymer.
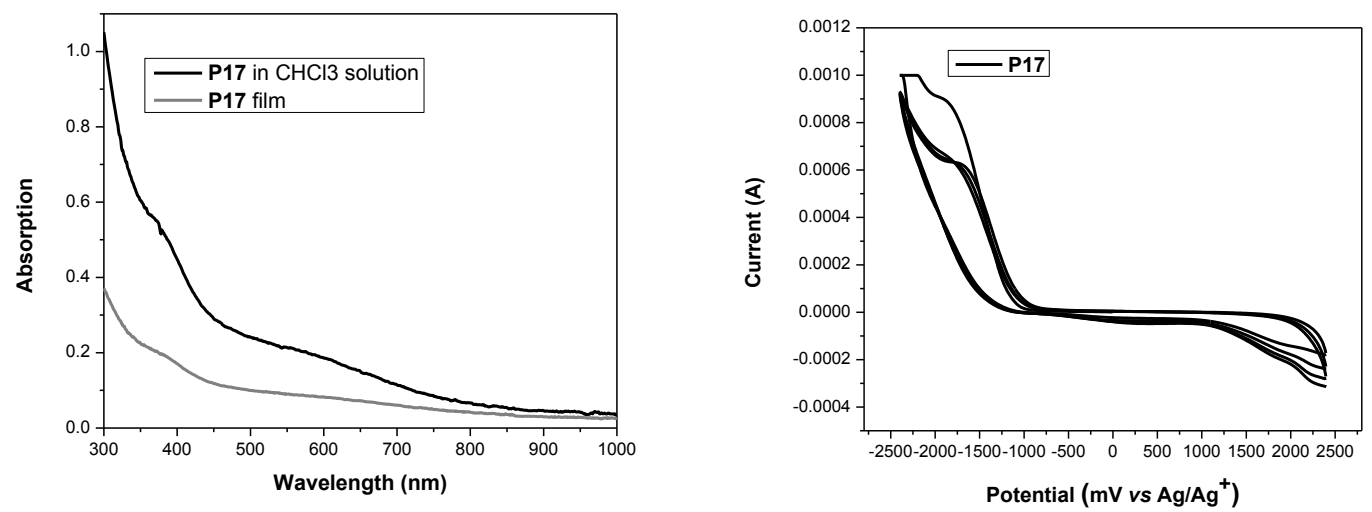

Figure 3.12 Absorption spectra of polymer $\mathbf{P 1 7}$ in $\mathrm{CHCl}_{3}$ solution and as thin film on a glass substrate (a) and cyclic voltammogram of film on ITO in $\mathrm{CH}_{3} \mathrm{CN}$ solution containing $0.1 \mathrm{~mol} / \mathrm{L} \mathrm{Bu}_{4} \mathrm{NPF}_{6}$ with a scan rate of $100 \mathrm{mV} / \mathrm{s}(\mathrm{b})$.

Another approach was then followed (path c) to synthesize polymer P17 by heating the polymer P16 at high temperature. In 1997, Hodge reported that a precursor polymer having an ester group can be converted to its anthracene homopolymer by removal of diester group via heating at $260{ }^{\circ} \mathrm{C} .{ }^{4}$ The thermogram of polymer P16 (Figure 3.13) reveals that the onset temperature is $173{ }^{\circ} \mathrm{C}$ with $1 \%$ weight loss. The $5 \%$ weight 
loss is at $270{ }^{\circ} \mathrm{C}$. Polymer $\mathbf{P 1 6}$ contains a long alkyl chain. Heating at this temperature might result in the loss of both the long alkyl chain and the ester group.

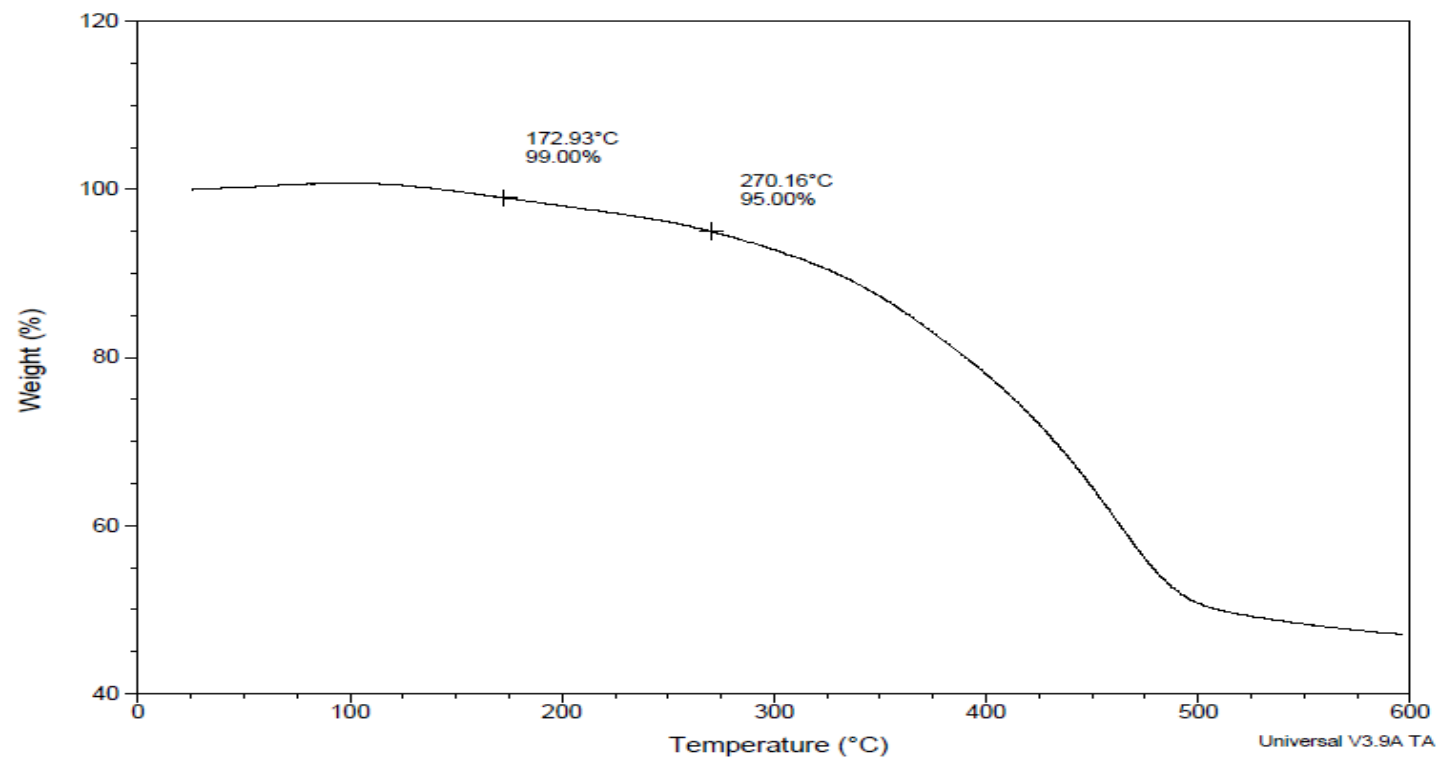

Figure 3.13 TGA trace of polymer $\mathbf{P 1 6}$ with a heating rate of $10^{\circ} \mathrm{C} / \mathrm{min}$ under nitrogen atmosphere.

The polymer film on a glass substrate under an argon atmosphere was heated at $260{ }^{\circ} \mathrm{C}$ for $6 \mathrm{~h}$ and the IR spectrum was taken as a $\mathrm{KBr}$ pellet due to solubility issues. The obtained polymer film was not soluble in any organic solvents. It was partially soluble in NMP upon heating. The IR spectrum in Figure 3.14 shows the peak at $1775 \mathrm{~cm}^{-1}$, which indicates the ester group remains there in the polymer. However, the solubility decreased upon heating. These results indicate that polymer P16 might have lost the long alkyl chain and thus the obtained polymer is not our target polymer P17. 


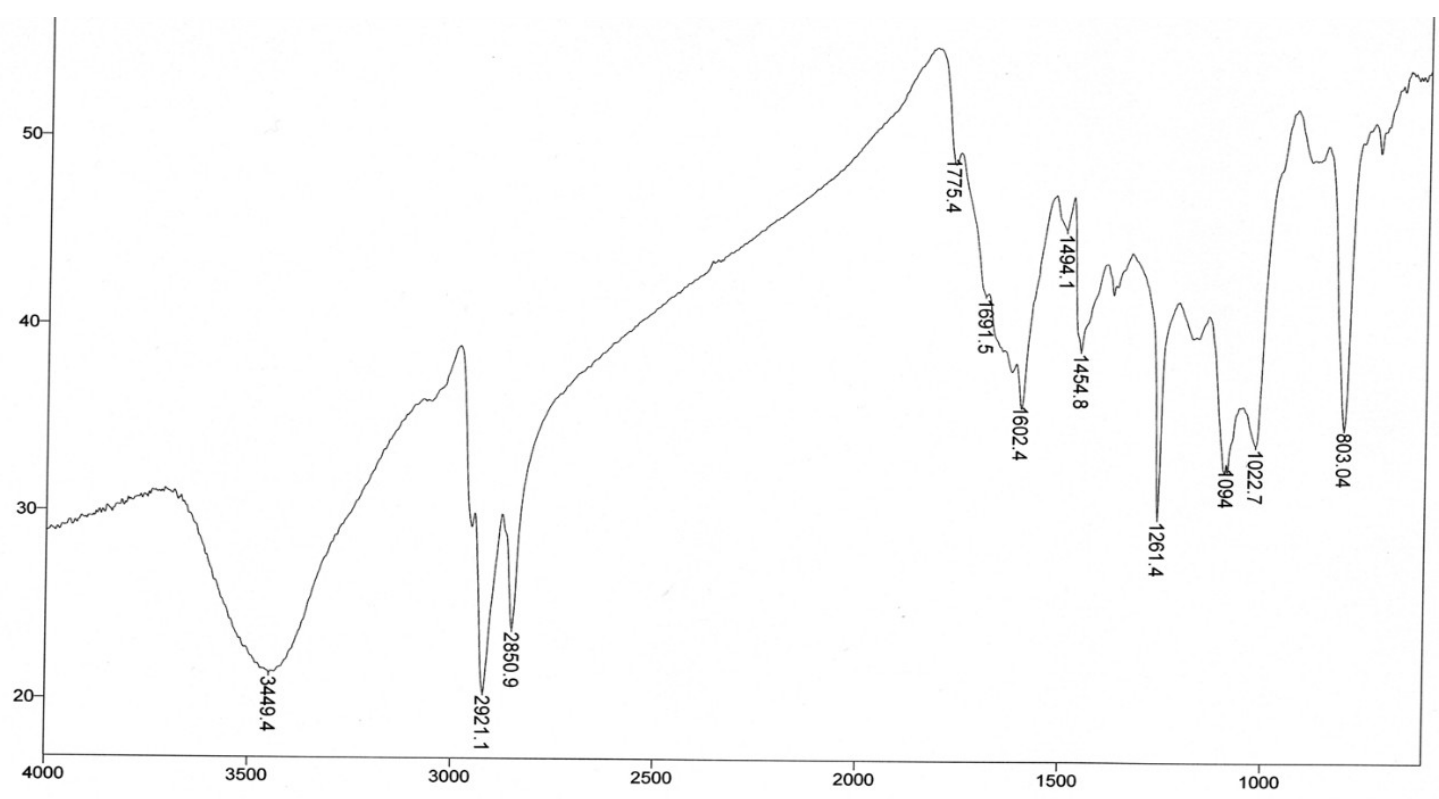

Figure 3.14 IR spectrum of polymer P17 in KBr pellet.

\subsection{Synthesis and transformation of NTDO-based copolymers}

The strength of donor units in D-A conjugated polymers influences the HOMO and LUMO levels and energy band gaps. Stronger donors maintain a high lying HOMO energy level in D-A polymers, resulting in narrower energy band gaps. When the donor strength is stronger, so is the ICT between the donor and acceptor, leading to a lower energy band gap and absorption shifts at longer wavelength as well as the device properties are enhanced. ${ }^{7}$ Fluorene is a weak electron donor due to its electron deficient benzene ring. BDT is a stronger donor unit than fluorene due to the flanking thiophene units. These thiophene units provide much less steric hindrance with adjacent acceptor units, leading to a more planar backbone thus decreasing the energy band gap. DTP is the strongest donor among them when the benzo unit is replaced by the pyrrole unit and thiophene units are fused with the pyrrole. The electron donating ability increases due to the extra lone pair of electrons on pyrrole $\mathrm{N}$, which participates in conjugation. 
Moreover, the planar structure, low oxidation potentials and good conjugation, make them excellent building-blocks for conjugated polymeric systems. ${ }^{7-10}$ The structural property of TPT as a donor monomer over other monomers has not been emphasised in literature, however, broad absorption spectrum from $700 \mathrm{~nm}$ was reported. $N$-alkyl TPTbased polymer film was reported with red shifting over $N$-aryl TPT-based polymer due to better $\pi-\pi$ interaction. ${ }^{11-14}$

\subsubsection{Synthesis of monomers}

Monomers M4 to M6 were synthesized according to the reactions in Scheme 3.3 and the published literature. ${ }^{10,14,15}$ Thiophene, monomer M3, compounds 6 (benzo[1,2b:4,5-b']dithiophene-4,8-dione) and $\mathbf{8}$ (3,3'-dibromo-2,2'-bithiophene) are commercially available. The reduction of compound $\mathbf{4}$ was conducted in the presence of zinc dust and sodium hydroxide and then $O$-alkylation was carried out in the presence of bromooctane which produced compound $\mathbf{5}$ in $80 \%$ yield. Compound 7 was converted to its organo-tin monomer (M4) in the presence of n-butyllithium (n-BuLi) and trimethyltin chloride in $85 \%$ yield. The Friedel-Crafts reaction of succinyl dichloride in dry dichloromethane with $\mathrm{AlCl}_{3}$ and thiophene produced the diketo compound 6 in 95\% yield which was then underwent an acid-catalyzed condensation with dodecylamine and produced compound 7 . Compound 7 was converted to its organo-tin monomer (M5) in the presence of $\mathrm{n}-\mathrm{BuLi}$ and trimethyltin chloride in $89 \%$ yield. Compound 9 was synthesized via a BuchwaldHartwig amination with a branched primary amine (2-ethylhexylamine) and 3,3'dibromo-2,2'-bithiophene in the presence of a Pd-catalyst in $69 \%$ yield. Compound 9 was converted to its organo-tin monomer (M6) in the presence of n-BuLi and trimethyltin chloride in $89 \%$ yield. 


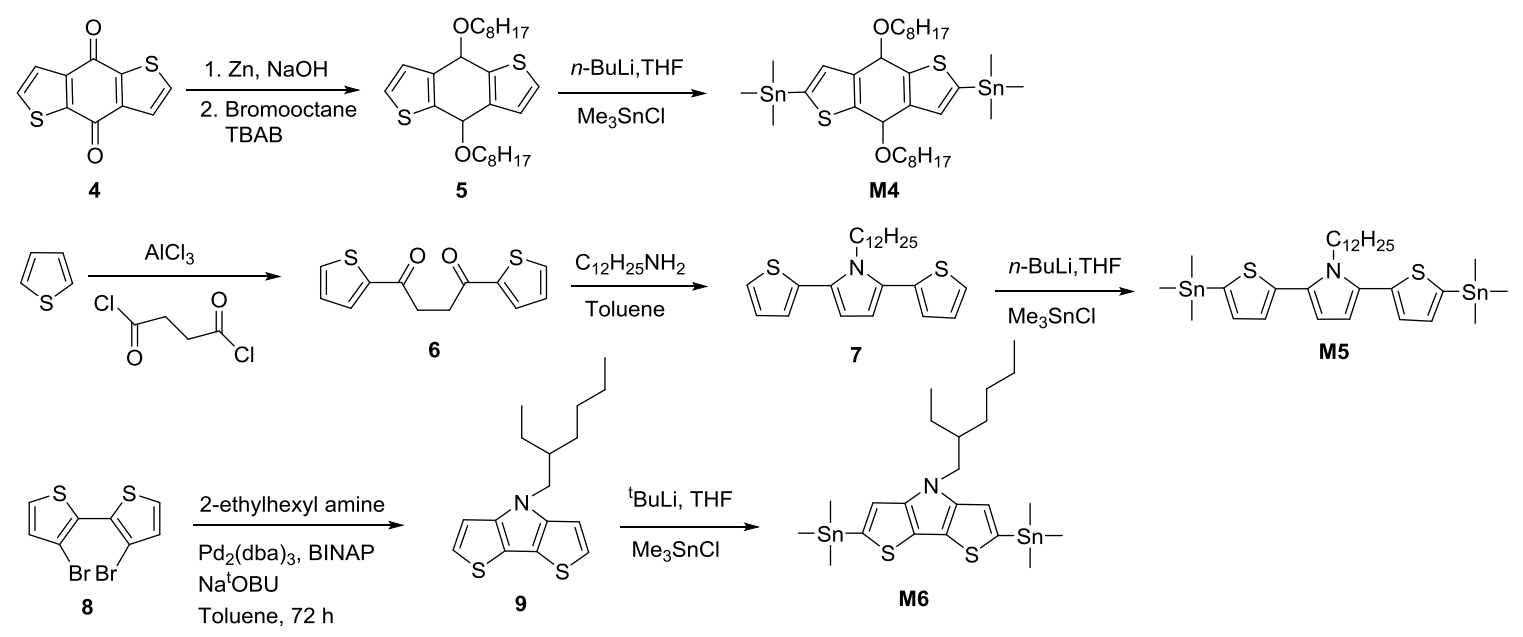

Scheme 3.3 Synthesis of monomers M4 M5 and M6.

\subsubsection{Synthesis and characterization of NTDO-based copolymers}

A series of NTDO-based D-A copolymers; NTDO-FL was synthesized via the Suzuki cross-coupling reaction and NTDO-TPT, NTDO-BDT and NTDO-DTP were synthesized by the Stille cross-coupling reaction under an argon atmosphere using a palladium catalyst according to the reactions in Scheme 3.4. Different strengths of donor monomers were selected to control the absorption. The relative strength of the donors is $\mathrm{FL}<\mathrm{BDT}<\mathrm{TPT}<\mathrm{DTP}$. The polymer P18 was obtained in $91 \%$ yield by polymerization reaction between monomer $\mathbf{M 1}$ and 9,9-dioctylfluorene-2,7-diboronic acid bis(1,3propanediol)ester in the presence of palladium catalyst in toluene and aqueous potassium carbonate. The polymers P19 to P21 were obtained in 94.0, 93.4 and $82.5 \%$ yields, respectively, by polymerization reactions between M1 to M4, M5 and M6 with 1:1 mole ratio in the presence of palladium catalyst in toluene. All the polymers were precipitated in methanol and purified by a Soxhlet extraction in acetone for $24 \mathrm{~h}$. The obtained polymers were yellow, dark purple and dark blue solids, respectively. 

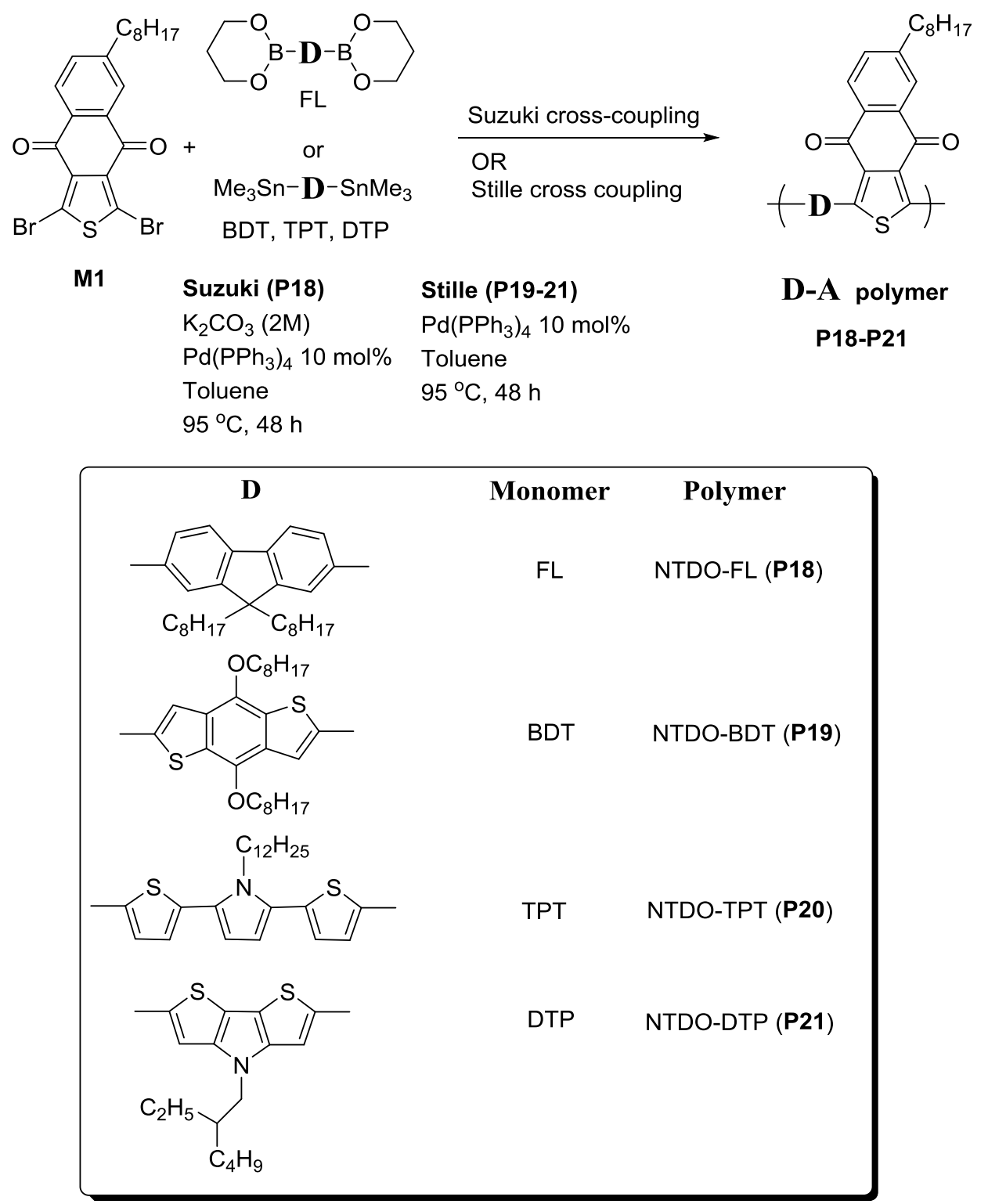

Scheme 3.4 Synthesis of NTDO-based polymers P18-P21.

Palladium-catalyzed cross-coupling reactions, which include Suzuki crosscoupling and Stille cross-coupling, are popular and efficient synthetic methods for carbon-carbon bond formation. ${ }^{16}$ These reactions can tolerate a wide range of functionality. The catalytic cycles are divided into four main sequences: oxidative addition, transmetallation, cis/trans isomerization and reductive elimination (Scheme 
3.5). In the first step of the catalytic cycle, zerovalent Pd forms Ar-Pd-X, a stable trans- $\sigma$ palladium (II) complex, by oxidative addition of the arylhalide compound, which is considered to be the rate-determining step in the catalytic cycle. In this step, the oxidation state of the transition metal changes from palladium (0) to palladium (II) which is why this is called the oxidative addition step. Electron-withdrawing groups facilitate oxidative addition than those of donating groups. Oxidative addition initially gives a cis complex that rapidly isomerizes to its trans isomer. The palladium (II) intermediate then undergoes a transmetallation step in which the nucleophile (Ar') is transferred from the organometallic reagent to the palladium (II) complex. The tansmetallation step produces a trans-adduct which then isomerizes to its cis-adduct intermediate. This step is very crucial in forwarding the reductive elimination process. The steric hinderance from the isomerization forces facilitates the reductive elimination and yields the expected product (Ar- Ar') and the zerovalent palladium catalyst is regenerated for another cycle.

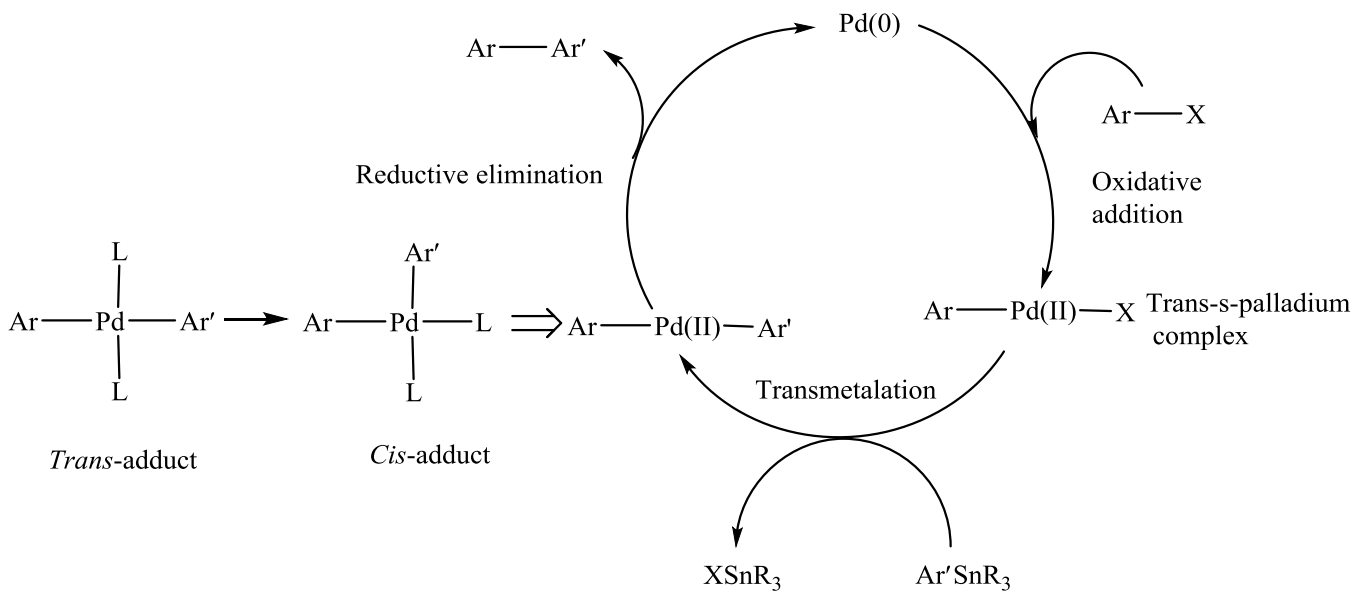

Scheme 3.5 General reaction mechanism for palladium catalyst cross-coupling reaction.

All the synthesized polymers P18 to P21 were characterized by IR, NMR, absorption, emission spectroscopy, GPC, and CV. The IR spectrum of polymer P18 is 
represented in Figure 3.15 and the rest of the spectra of polymers P19 to P21 are shown in Appendix B (Figure S3.12). In Figure 3.15, the sharp and strong peak at $1671 \mathrm{~cm}^{-1}$ is the characteristic peak for a cyclic $\mathrm{C}=\mathrm{O}$, suggesting that polymer $\mathrm{P} 18$ has NTDO moiety. Interestingly, for polymer P18 to $\mathbf{P 2 1}$, as the donor strength in the polymer increases, the peak for the $\mathrm{C}=\mathrm{O}$ stretch moves from higher frequency to lower frequency (1671 to 1652 $\left.\mathrm{cm}^{-1}\right)$.

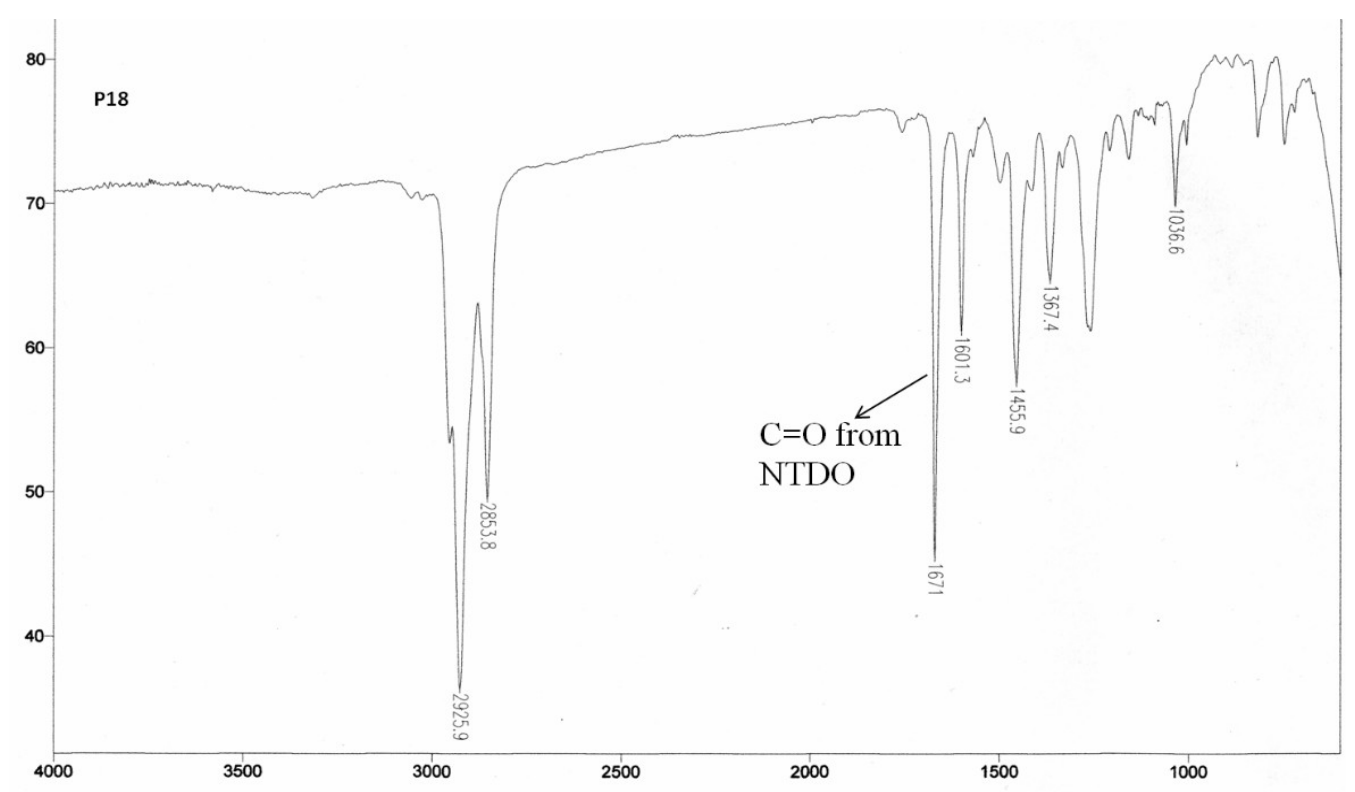

Figure 3.15 IR spectra polymer $\mathbf{P 1 8}$ on $\mathrm{NaCl}$ plate.

The ${ }^{1} \mathrm{H}$ NMR spectrum of polymer $\mathbf{P 2 0}$ is shown in Figure 3.13a; the remaining spectra for other polymers are shown in Appendix B (Figures S3.13, S3.15 and S3.17.). Polymer P20 shows a broad peak at $\delta 2.78 \mathrm{ppm}$, which is assigned to benzyl $\mathrm{CH}_{2}$ of the NTDO monomer and the broad peak at $\delta 4.26 \mathrm{ppm}$, which belongs to the $\mathrm{N}-\mathrm{CH}_{2}$ of the pyrrole unit of the TPT monomer, indicates the formation of polymer. Polymers P19 and P21 show peaks at $\delta 4.35$ (which belongs to the $\mathrm{OCH}_{2}$ moiety of the BDT monomer) and $4.10 \mathrm{ppm}$ (which belongs to the $\mathrm{N}-\mathrm{CH}_{2}$ moiety of the DTP monomer) along with peaks at 
$\delta 2.78 \mathrm{ppm}$, which indicate they are the right polymers. Polymer P18 does not show any peak over $\delta 2.78$ to $6.5 \mathrm{ppm}$, which confirms it has the right structure. All other peaks at $\delta$ 6.5 to $8.5 \mathrm{ppm}$ are assigned as aromatic peaks of the polymers and the other peaks are at $\delta$ 2.00 to $0.80 \mathrm{ppm}$, indicating $\mathrm{CH}_{2}$ and $\mathrm{CH}_{3}$ moieties from alkyl chains, respectively.

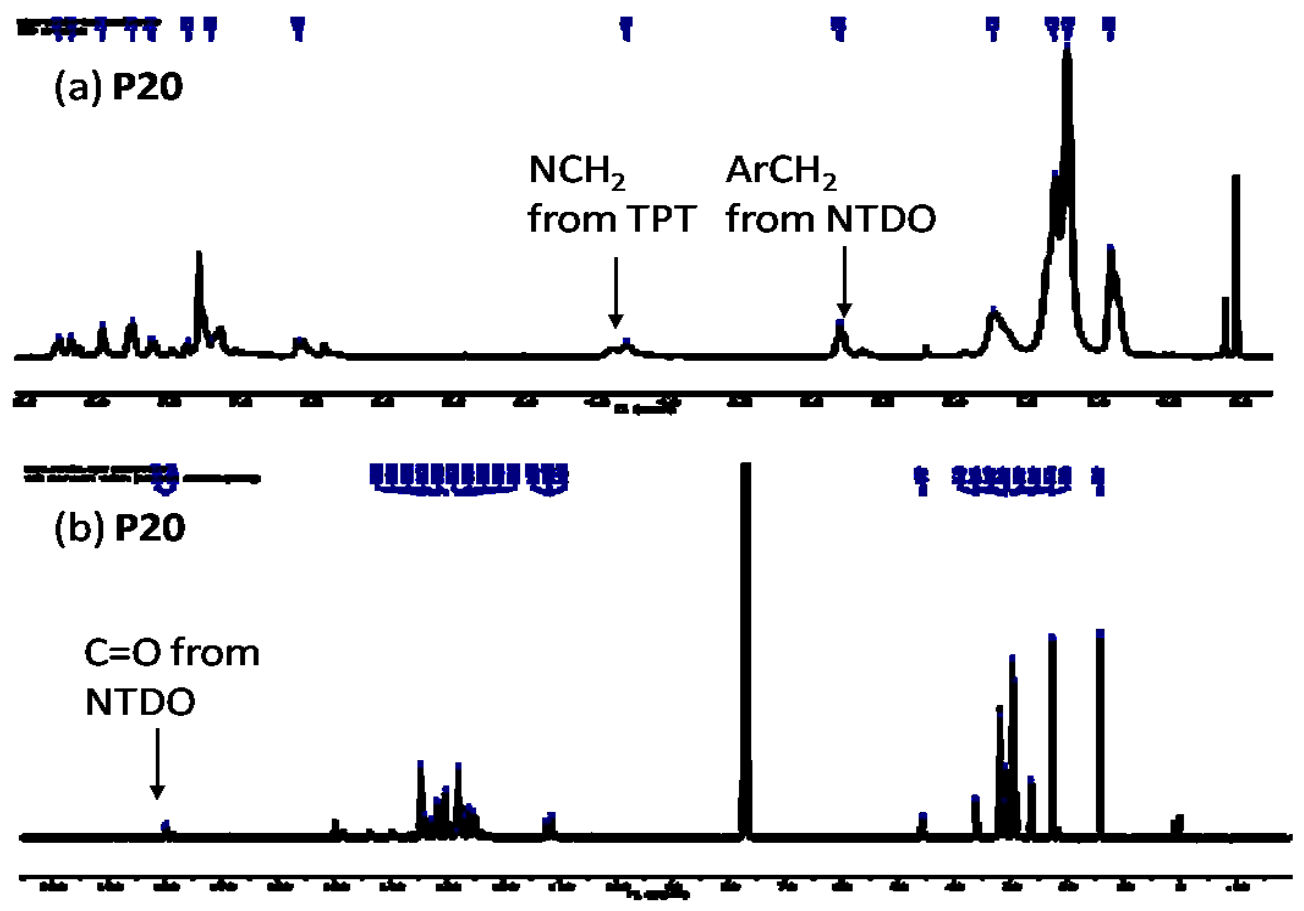

Figure 3.16 (a) ${ }^{1} \mathrm{H} \mathrm{NMR}\left(300 \mathrm{MHz}, \mathrm{CDCl}_{3}\right.$ ) and (b) ${ }^{13} \mathrm{C} \mathrm{NMR}\left(75 \mathrm{MHz}, \mathrm{CDCl}_{3}\right)$ spectra of polymer P20.

The ${ }^{13} \mathrm{C}$ NMR spectrum (Figure 3.13b) shows two peaks at $\delta 179.60$ and 179.18 ppm, indicating the $\mathrm{C}=\mathrm{O}$ moiety of the polymer, which is coming from NTDO monomer. The synthesized polymers (P18 to P21) show a moderate molecular weight (Appendix B, Figure S3.19-S3.22) with a lower polydispersity of 1.96, 1.94, 1.68 and 1.74, respectively. Polymers with dicyanovinylene groups are known to be poorly soluble, which can affect the film-forming property. With this in mind, the parent polymers P18 to $\mathbf{P 2 1}$ were synthesized. It is also known that GPC provides a relative molecular weight. 
The polymers P18 to P21 form uniform and smooth films on glass substrates by spin coating or casting, indicating a reasonably high molecular weight. Figure S3.23 shows the TGA, where the decomposition onset for polymers P18, P19, P20 and P21 is 397.3, 228.3, 242.1 and $310.0^{\circ} \mathrm{C}$, respectively, with $1 \%$ weight loss. It was also observed that the temperature for $5 \%$ weight loss are $414.6,296.9,278.6$ and $370.9{ }^{\circ} \mathrm{C}$, respectively, indicating that the polymers have high thermal stability.

Polymers P18-P21 are fully soluble in solvents such as $\mathrm{CHCl}_{3}$, chlorobenzene, dichlorobenzene and trichlorobenzene. Absorption spectra of P18-P21 in $\mathrm{CHCl}_{3}$ solutions (Figure 3.17a) and spin-coated thin solid films (Figure 3.17b) on glass substrates were taken. The maximal absorption peaks for polymers P18-P21 in $\mathrm{CHCl}_{3}$ were at 395, 522, 515 and $590 \mathrm{~nm}$, respectively. The onsets are at 514, 695, 778, and 867 nm, respectively. Polymer P18 shows the shortest absorption maximal and polymer P21 shows the longest maximal absorption peak and absorption onset among the synthesized polymers. This can be attributed to the strength of the donor monomer. ${ }^{7}$ Polymer P19 shows a longer absorption maximal and a shorter onset when compared to polymer P20. The longer absorption onset for polymer P20 when compared to polymer P19 may be due to the better intramolecular charge transfer or the quinoid structure. The obtained absorption of polymer P19 is quite similar to the reported polymer (PBDTNTDO-C3) in the literature, where the BDT unit contained a branched alkyl chain. ${ }^{17}$ Polymer P19 shows $6 \mathrm{~nm}$ red shift in solid state compared to the reported polymer, which may be due to the better intermolecular interaction in the presence of linear alkyl chain. The films of P18-P21 show absorption peaks are at 395, 563, 595 and $612 \mathrm{~nm}$, respectively. 

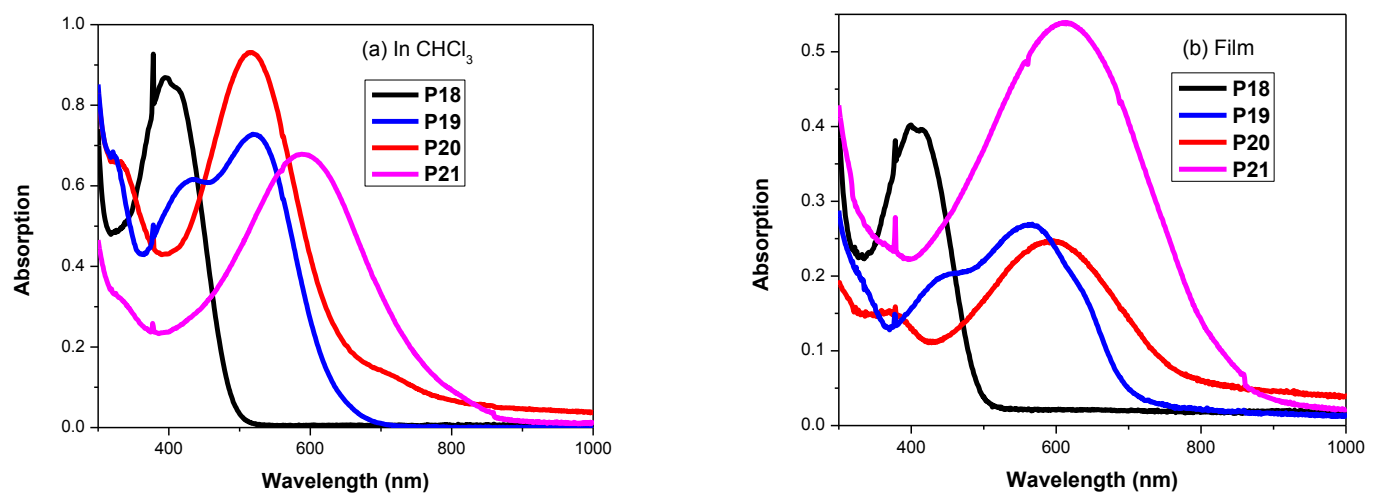

Figure 3.17 Absorption of polymers P18 $\left(3.77 \times 10^{-5} \mathrm{M}\right), \mathbf{P 1 9}\left(4.16 \times 10^{-5} \mathrm{M}\right), \mathbf{P 2 0}(3.32$ $\left.\times 10^{-5} \mathrm{M}\right)$ and $\mathbf{P 2 1}\left(4.17 \times 10^{-5} \mathrm{M}\right)$ in $\mathrm{CHCl}_{3}$ solution (a) and as thin films on glass substrates (b).

Table 3.1. UV-vis-NIR absorption data of polymers P18, P19, P20 and P21.

\begin{tabular}{|c|c|c|c|c|}
\hline \multirow{2}{*}{ Polymer } & \multicolumn{2}{|c|}{$\lambda_{\max }(\mathrm{nm})$} & \multicolumn{2}{c|}{$\lambda_{\text {onset }}(\mathrm{nm})$} \\
\cline { 2 - 5 } & $\begin{array}{c}\text { in } \mathrm{CHCl}_{3} \\
\text { solution }\end{array}$ & film & $\begin{array}{c}\text { in } \mathrm{CHCl}_{3} \\
\text { solution }\end{array}$ & film \\
\hline P18 & 395 & 395 & 514 & 514 \\
\hline P19 & 522 & 563 & 695 & 721 \\
\hline P20 & 515 & 595 & 778 & 800 \\
\hline P21 & 590 & 612 & 867 & 890 \\
\hline
\end{tabular}

The onsets are 514, 721, 800, and $890 \mathrm{~nm}$, respectively. The film of polymer P18 showed the shortest absorption maximal and polymer P21 showed the longest maximal absorption peak. A similar trend was observed for the absorption onset among the synthesized polymers. $^{7}$ The absorption of polymer $\mathbf{P 1 8}$ in solution and film did not change, in the solid state, it is possible that the intermolecular interaction was not 
effective. Polymer P20 shows a longer absorption maximal and onset compared to polymer P19, which can be explained by the stronger intermolecular interaction, a planar structure, closer packing and the $\pi$ - $\pi$ stacking in the solid film. The film of polymer P21 displays the largest maximal absorption peak and onset among the synthesized polymers. Among the polymers, $\mathbf{P 2 0}$ shows the highest red shift in the solid state compared to its solution which is $80 \mathrm{~nm}$. Overall, the red shift of the absorption maximal in solid state can be explained by stronger intermolecular interaction, more planar structure, closer packing and increased $\pi-\pi$ stacking. When the donor strength is stronger, so is the ICT between the donor and acceptor and absorption shifts to a longer wavelength which was observed in the synthesized polymers P18-P21. ${ }^{18}$ The stronger absorption intensity at longer wavelengths is due to the better electron distribution over HOMO and LUMO and therefore the stronger ICT. ${ }^{19}$ Polymers P18-P21 are all emissive in solution. The polymer $\mathbf{P 1 8}$ is emissive in the solid state and all other polymers are non-emissive in the solid states. The emission spectra are represented in Appendix B (Figure S3.24). The emission maxima are at 542, 587665 and $686 \mathrm{~nm}$, respectively. Polymer P18 shows an emission maximal at $552 \mathrm{~nm}$ in the solid state. The absorption of polymer P18 did not red shift in solid state which indicates it does not have enough $\pi-\pi$ stacking as a result charge transfer quenching was not so effective. Therefore, the polymer shows emission in the solid state.

The HOMO and LUMO energy levels of polymers are summarized in Table 3.2 and their CV are shown in Figure 3.18. The polymers exhibit noticeable oxidation and reduction processes. The onset oxidation potential/onset reduction potential of polymers P18, P19, P20 and P21 are $0.50 /-0.90,0.42 /-0.87,0.40 /-0.74$, and $0.28 /-0.85 \mathrm{~V}$, respectively (Table 3.2). The HOMO energy levels of polymers are $-4.86,-4.78,-4.76 \mathrm{eV}$ 
and -4.64 eV raised from P18-P21. The LUMO energy levels are -3.34, -3.49, -3.62, and $-3.51 \mathrm{eV}$, respectively. The obtained HOMO energy levels are higher lying than the NTDO-based polymers reported in the literature. However, the LUMO energy levels are similar to the reported LUMO energy levels. ${ }^{17 \mathrm{~b}}$
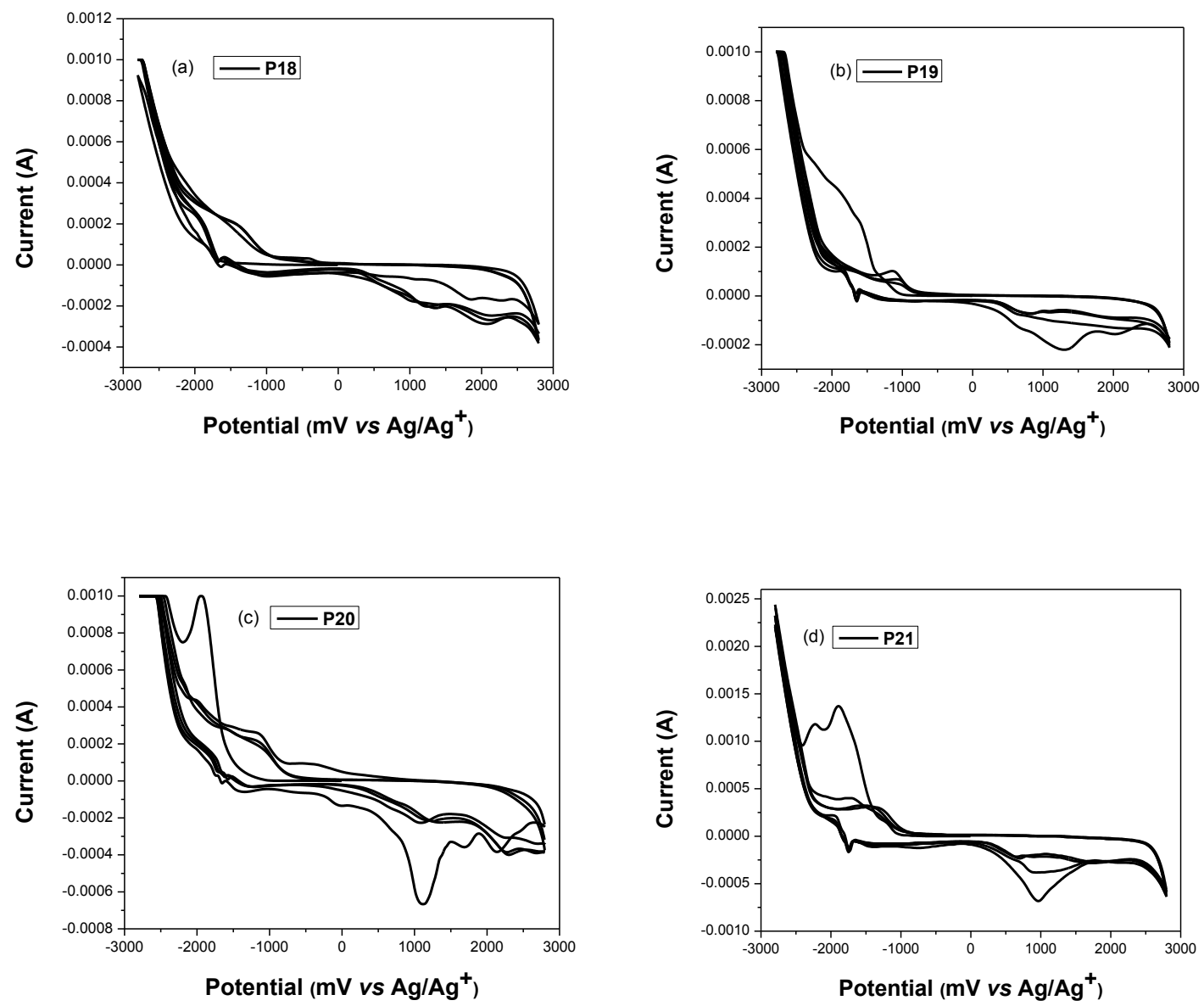

Figure 3.18 Cyclic voltammogram of polymers P18 (a), P19 (b), P20 (c) and P21 (d) film on ITO in $\mathrm{CH}_{3} \mathrm{CN}$ solution containing $0.1 \mathrm{~mol} / \mathrm{L} \mathrm{Bu}_{4} \mathrm{NPF}_{6}$ with a scan rate of 100 $\mathrm{mV} / \mathrm{s}$.

As the donor strength increases, the HOMO levels shift up from polymers P18P21. The HOMO energy levels of polymers P19 and P20 are almost same. The optical 
energy band gap of polymer P19 and the reported polymer PBDTNTDO-C3 are very close. ${ }^{17 a}$ However, the electrochemical energy band gap is smaller for P19 than the reported polymer due to the higher HOMO energy level. The LUMO energy levels gradually decrease from polymers P18-P20. The LUMO energy level of polymer P21 increased $0.09 \mathrm{eV}$ when compared to P20. The electrochemical energy band gap decreases from polymers P18-P21, which is consistent with the optical energy band gap. Stronger donors maintain high lying HOMO energy levels in D-A polymers, resulting in narrower energy band gaps. We observed a similar phenomenon in polymers P18-P21; as the donor strength increased the HOMO energy levels also increased. When the HOMO levels and the LUMO levels of the donor and acceptor moieties are close in energy, hybridized energy levels are in low HOMO-LUMO separation. Therefore, we observed a decrease in LUMO energy levels from polymers P18-P21.

Table 3.2. Optical and electrochemical properties of polymers P18-P21.

\begin{tabular}{|l|l|l|l|l|}
\hline Polymer & HOMO $(\mathrm{eV})$ & LUMO $(\mathrm{eV})$ & $\mathrm{E}_{\mathrm{g}}{ }^{\text {ppt }}(\mathrm{eV})$ & $\mathrm{E}_{\mathrm{g}}^{\mathrm{CV}}(\mathrm{eV})$ \\
\hline $\mathbf{P 1 8}$ & $\begin{array}{l}-4.86(\mathrm{cv}) \\
\text { Onset, } 0.50\end{array}$ & $\begin{array}{l}-3.46(\mathrm{cv}) \\
\text { Onset, }-0.90\end{array}$ & 2.41 & 1.40 \\
\hline $\mathbf{P 1 9}$ & $\begin{array}{l}-4.78(\mathrm{cv}) \\
\text { Onset } 0.42\end{array}$ & $\begin{array}{l}-3.49(\mathrm{cv}) \\
\text { Onset }-0.87\end{array}$ & 1.72 & 1.29 \\
\hline $\mathbf{P 2 0}$ & $\begin{array}{l}-4.76(\mathrm{cv}) \\
\text { Onset, } 0.40\end{array}$ & $\begin{array}{l}-3.62(\mathrm{CV}) \\
\text { Onset, }-0.74\end{array}$ & 1.55 & 1.14 \\
\hline P21 & $-4.64(\mathrm{cv})$ & $-3.51(\mathrm{cv})$ & 1.39 & 1.13 \\
\hline
\end{tabular}




\subsubsection{Synthesis and characterizations of dicyanovinylene-containing polymers by chemical transformation of NTDO-based copolymers}

The CN group is a stronger electron-withdrawing group than the keto group. Addition of the $\mathrm{CN}$ group or substituting the keto with dicyanovinylene moiety lowers the LUMO energy levels and thus the band gap decreases in a conjugated polymer system. $^{20}$ Roncali et al. reported that a cyano group at the vinylene linkage of dithienylethylene decreases the band gap of the corresponding polymers to a value as low as $0.60 \mathrm{eV}$. However, the polymer was suffering with the limited solubility. ${ }^{21} \mathrm{CN}$ substituted semiconducting polymers have also been reported in the literature with lower LUMO (lowered by $0.25 \mathrm{eV}$ ) and HOMO (lowered by $0.10 \mathrm{eV}$ ) energy levels and band gaps (decreased by $0.15 \mathrm{eV}$ ). The reported band gaps were 1.57 and $1.37 \mathrm{eV}$ with respect to mono- and di-substitute units. ${ }^{21}$

Tetracyanoethylene (TCNE) and 7,7,8,8-tetracyanoquinodimethane (TCNQ) are well known as electron acceptors and the latter is stronger than the former (Scheme 3.2). ${ }^{22}$ The addition of TCNE and TCNQ to conjugated polymer systems results in absorption at longer wavelength. Michinobu et al. reported that addition of TCNE to the polymer backbone the absorption spectrum becoming broad and red shifted at both maximal and onset. ${ }^{23,24}$ Addition of a nitrile group in the polymer decreases the solubility. They also reported that the addition of a TCNE group to polymer backbone decreases the energy band gap by lowing the LUMO and HOMO energy levels. ${ }^{25}$ The comparative study showed that the addition of TCNQ in polymer backbones causes a greater decrease in the energy band gap than for TCNE. The LUMO level with TCNQ decreases more than for TCNE and the polymer turns to an n-type polymer from a p-type polymer. ${ }^{26}$ 


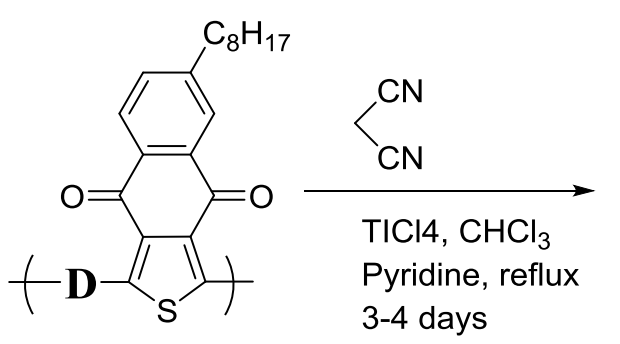

D-A polymer

P18-P21

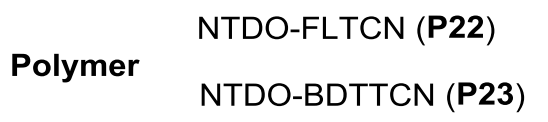

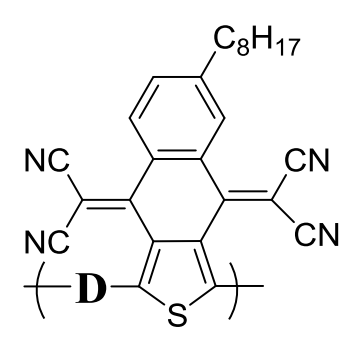

D-A polymer

P22-P25

NTDO-DTPTCN (P25)

Scheme 3.6 Synthesis of dicyanovinylene-containing polymers P22-P25.

A series of D-A polymers containing dicyanovinylene moieties were synthesized from NTDO-based D-A polymers P18-P21 via modified Knoevenagel condensation reaction with malononitrile in the presence of $\mathrm{TiCl}_{4}$ and pyridine according to the reactions in Scheme 3.6. ${ }^{27}$ The obtained polymers were precipitated in water and collected by filtration. The polymers were then stirred in diethyl ether $(30 \mathrm{~mL})$ for half an hour to remove the excess amount of malononitrile and the polymers were collected by filtration. The resultant polymers were then further purified by a Soxhlet extraction in $\mathrm{CHCl}_{3}$ for $24 \mathrm{~h}$ to remove any unreacted starting polymers. The synthesized polymers P22 to P25 were characterized by IR, absorption, emission spectroscopy, TGA and CV. The IR spectrum of polymer P22 is shown in Figure 3.19 and the remaining IR spectra for polymers P23 to $\mathbf{P 2 5}$ are shown in Appendix B (Figures S3.25-S3.27). The IR spectrum of polymer P22 shows no peak at $1672 \mathrm{~cm}^{-1}$, which indicates the disappearance of $\mathrm{C}=\mathrm{O}$ and suggests the condensation reaction was successful. In Figure 3.13, the peak at $2215 \mathrm{~cm}^{-1}$ is the characteristic peak for $\mathrm{CN}$, indicating the condensation reaction on $\mathrm{CO}$ 
of the NTDO moiety was successful. A new, strong peak at $1626 \mathrm{~cm}^{-1}$ appeared, which suggests the formation of $\mathbf{C}=\mathbf{C}(\mathrm{CN})_{2}$ moieties by condensation. The increase of absorption frequency in $\mathrm{C}=\mathrm{C}$ is due to the strong electron-withdrawing group, $\mathrm{CN}$ (inductive effect). Figure S3.28 (Appendix B) shows that the decomposition onsets for polymers P22, P23, P24 and P25 are 334.6, 313.5, 280.9 and $198.7^{\circ} \mathrm{C}$, respectively, with $5 \%$ weight loss, indicating the polymers have high thermal stability.

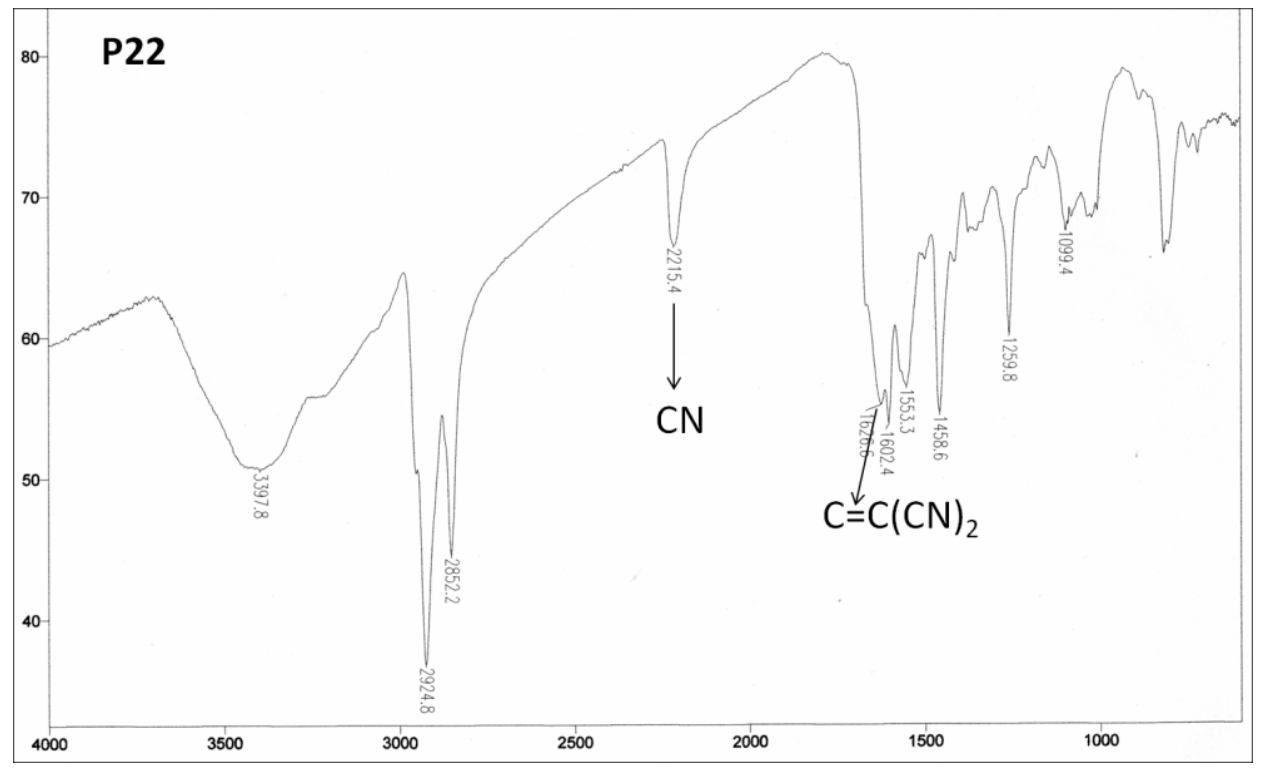

Figure 3.19 IR spectrum of polymer P22 in KBr pellet.

Polymers P22-P25 are soluble only in NMP. Absorption spectra of P22-P25 in NMP solutions (Figure 3.20a) and solid film (Figure 3.20b) on glass substrates were taken. In solution, polymers P22 and P23 show two different absorption maximal peaks each, they are at 337, 425 and 419, 513 nm, respectively. Polymers, P24 and P25 show absorption maximal peaks in solution at 522 and $585 \mathrm{~nm}$, respectively. The onsets are 600, 700, 760 and 900 nm, respectively. Polymer P22 displays the shortest absorption maximal, while polymer P25 shows the longest maximal absorption peak and absorption 
onset among the synthesized polymers. In solution, the absorption onsets for polymers P22-P25 are longer than their parent polymers P18-P22. This indicates that the presence of dicyanovinylene moiety in the acceptor unit strengthens its electron-accepting ability and therefore there is, better $\pi$-conjugation and ICT between the donor and acceptor units. In solution the absorption maximals are red-shifted for polymers $\mathbf{P 2 2}$ and P24 in comparison to their parent polymers P18 and P20. On the other hand, they are blueshifted for polymers $\mathbf{P 2 3}$ and $\mathbf{P 2 5}$ in comparison to their parent polymers. The presence of dicyanovinylene moieties may increase the dihedral angle in solution between the donor and acceptor unit of polymers $\mathbf{P 2 3}$ and $\mathbf{P 2 5}$, resulting in blue-shifting of $\lambda_{\max }$. The absorption spectra for polymers P22-P25 are especially broad in the shorter wavelength range compared to their parent polymers and the films of P22-P25 show broader absorption peaks compared to their solutions. The broader absorption is due to better intra and intermolecular interaction between the polymer backbones. The polymer films of P22 and P23 show two absorption peaks each at 337, 431.and 365, $552 \mathrm{~nm}$, respectively Polymer P24 has a broader absorption peak in the solid state and the absorption maximal is difficult to identify. The polymer film of $\mathbf{P 2 5}$ shows a maximal absorption peak at 585 nm. The absorption onset for polymer P22 did not change in the solid state whereas the onset is shorter $(870 \mathrm{~nm})$ for polymer $\mathbf{P 2 5}$ in the solid state. The absorption onset for polymers P23 and P24 are in longer wavelength in solid state compared to their solution. This absorption behaviour of polymers P23 and P25 requires further research. The presence of dicyanovinylene moiety may influence the dihedral angle thus inconsistency of absorption was observed. 

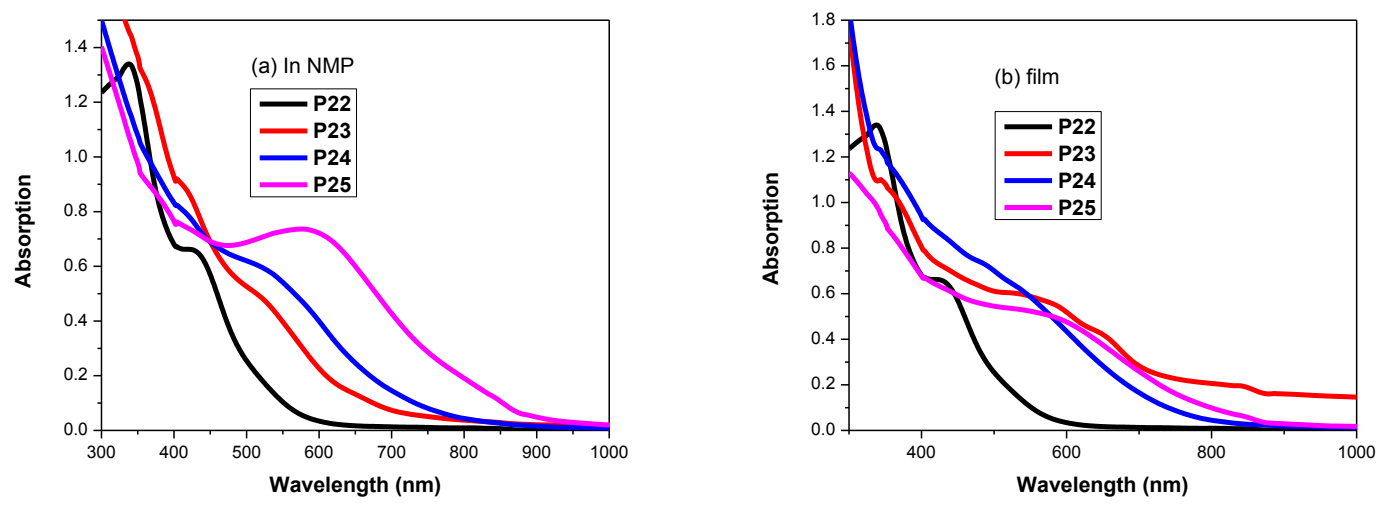

Figure 3.20 Absorption spectra of polymers P22 $\left(4.30 \times 10^{-5} \mathrm{M}\right), \mathbf{P 2 3}\left(4.15 \times 10^{-5} \mathrm{M}\right)$, P24 $\left(5.09 \times 10^{-5} \mathrm{M}\right)$ and P25 $\left(5.62 \times 10^{-5} \mathrm{M}\right)$ in NMP solution (a) and as thin films on glass substrates (b).

Table 3.3. UV-vis absorption data for polymers P22, P23, P24 and P25.

\begin{tabular}{|c|c|c|c|c|}
\hline \multirow{2}{*}{ Polymer } & \multicolumn{2}{|c|}{$\lambda_{\max }(\mathrm{nm})$} & \multicolumn{2}{c|}{$\lambda_{\text {onset }}(\mathrm{nm})$} \\
\cline { 2 - 5 } & in NMP solution & film & in NMP solution & film \\
\hline P22 & 337,425 & 337,431 & 600 & 600 \\
\hline P23 & 419,513 & 365,552 & 700 & 730 \\
\hline P24 & 522 & NA & 760 & 800 \\
\hline P25 & 585 & 585 & 900 & 870 \\
\hline
\end{tabular}

Polymers P22-P25 are emissive in solution and non-emissive in solid states. The emission spectra are represented in Appendix B (Figure S3.29). Polymer P22 shows the emission maxima at $483 \mathrm{~nm}$ and a shoulder at $533 \mathrm{~nm}$. Polymers P23-P25 do not have emissions when the excitation wave length are the same as their absorption maximals. They show emission maxima at 541, 542 and $540 \mathrm{~nm}$ in excitation wavelength at $500 \mathrm{~nm}$. 

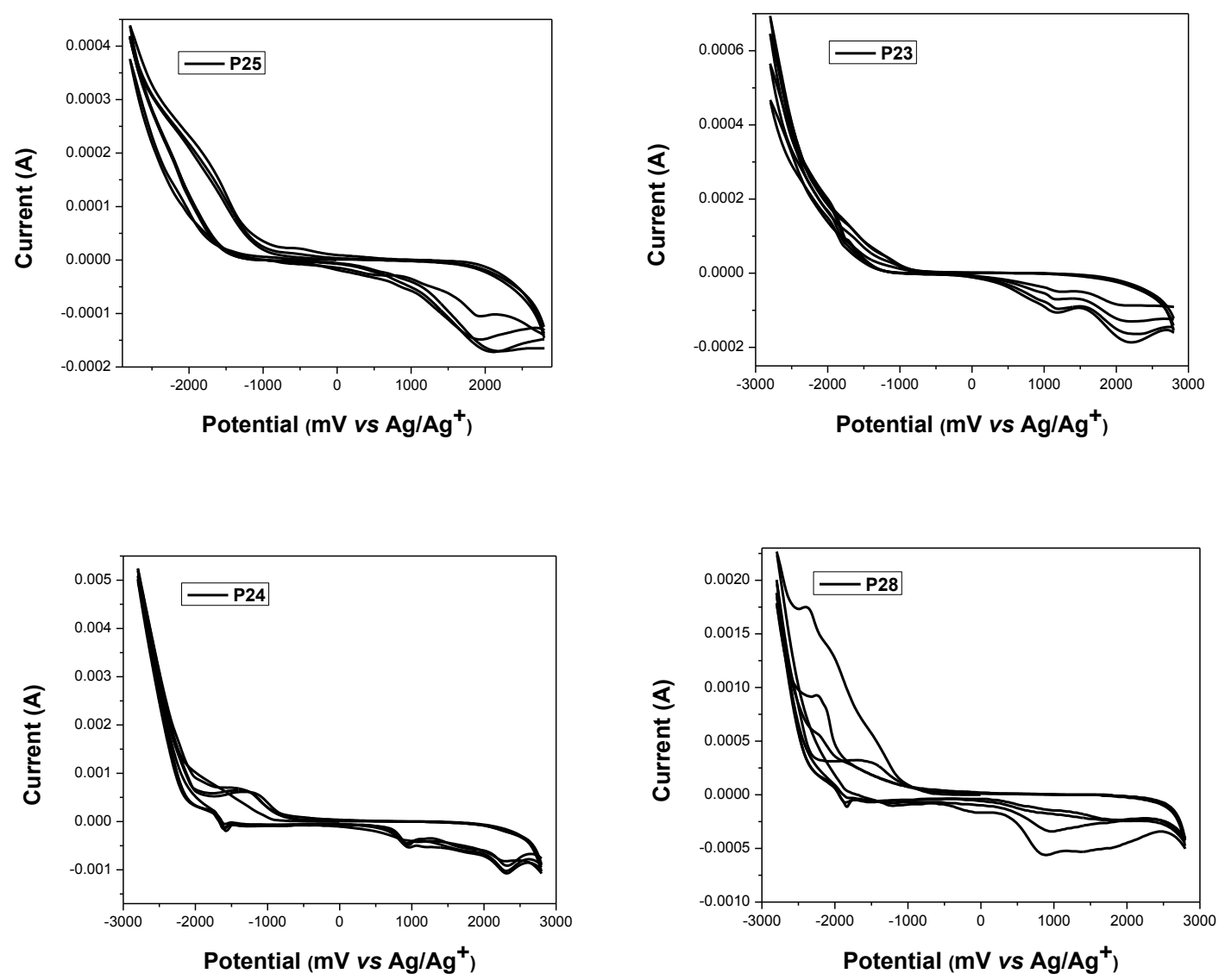

Figure 3.21 Cyclic voltammogram of polymers P22 (a), P23 (b), P24 (c) and P25 (d) film on ITO in $\mathrm{CH}_{3} \mathrm{CN}$ solution containing $0.1 \mathrm{~mol} / \mathrm{L}$ of $\mathrm{Bu}_{4} \mathrm{NPF}_{6}$ with a scan rate of 100

$$
\mathrm{mV} / \mathrm{s} \text {. }
$$

The HOMO and LUMO energy levels of polymers are summarized in Table 3.4 and their CV are shown in Figure 3.21. Polymers P22, P24 and P25 show noticeable oxidation and reduction processes, whereas polymer $\mathbf{P 2 3}$ shows only oxidation process in $\mathrm{CV}$. The onset oxidation potential/onset reduction potential of polymers P22, P24 and P25 are $0.70 /-1.10,0.64 /-0.88$ and $0.34 /-1.08$, respectively. The onset oxidation potential of polymer P23 is 0.48 . The HOMO energy levels of the polymers are $-5.06,-4.84,-5.00$ and -4.70 eV. For polymers P22-P25 the HOMO energy level is lower than their parent 
polymers. The LUMO energy levels for these polymers are $-3.26,-3.14,-3.48$ and, -3.28 $\mathrm{eV}$, respectively. The LUMO energy levels increase for polymers P22-P25 compared to their parent polymers. This increasing trend in their LUMO energy levels affects their electrochemical energy band gaps. Generally, as acceptor strengths in D-A polymer increase, the LUMO energy level decrease more than the HOMO energy level. We observed that the HOMO energy level decreased compared to their parent polymers. The orientation of the two dicyanovinylene and their effects on torsional angles may influence the LUMO energy levels. While the electrochemical energy band gaps increased for polymers P22-P25 compared to their parent polymers the optical energy band gaps ( $\left.\mathrm{E}_{\mathrm{g}}{ }^{\mathrm{opt}}\right)$ for polymers P22-P24 are lower than those of their parent polymers. The optical energy gap for polymer P23 is $1.77 \mathrm{eV}$. Figure S3.30 shows the colors of the polymers in solution before and after the reactions. The color of the polymers became darker after the reactions which indicate the reactions were successful. 
Table 3.4. Optical and electrochemical properties of polymers P22-P25.

\begin{tabular}{|c|c|c|c|c|}
\hline Polymer & HOMO (eV) & LUMO (eV) & $\mathrm{E}_{\mathrm{g}}{ }^{\text {opt }}(\mathrm{eV})$ & $\mathrm{E}_{\mathrm{g}}^{\mathrm{CV}}(\mathrm{eV})$ \\
\hline P22 & $\begin{array}{l}-5.06(\mathrm{cv}) \\
\text { Onset, } 0.70\end{array}$ & $\begin{array}{l}-3.26(\mathrm{cv}) \\
\text { Onset, }-1.10\end{array}$ & 2.07 & 1.80 \\
\hline $\mathbf{P} 23$ & $\begin{array}{l}-4.84(\mathrm{cv}) \\
\text { Onset } 0.48\end{array}$ & $\begin{array}{l}\text { NA (cv), }-3.14 \\
\text { (optical) }\end{array}$ & 1.70 & NA \\
\hline P24 & $\begin{array}{l}-5.00(\mathrm{cv}) \\
\text { Onset, } 0.64\end{array}$ & $\begin{array}{l}-3.48(\mathrm{cv}) \\
\text { Onset, }-0.88\end{array}$ & 1.55 & 1.52 \\
\hline P25 & $\begin{array}{l}-4.70(\mathrm{cv}) \\
\text { Onset } 0.34\end{array}$ & $\begin{array}{l}-3.28(\mathrm{cv}) \\
\text { Onset }-1.08\end{array}$ & 1.43 & 1.42 \\
\hline
\end{tabular}

\subsection{Polymer photodetectors based on dicyanovinylene-containing polymers P22-P25}

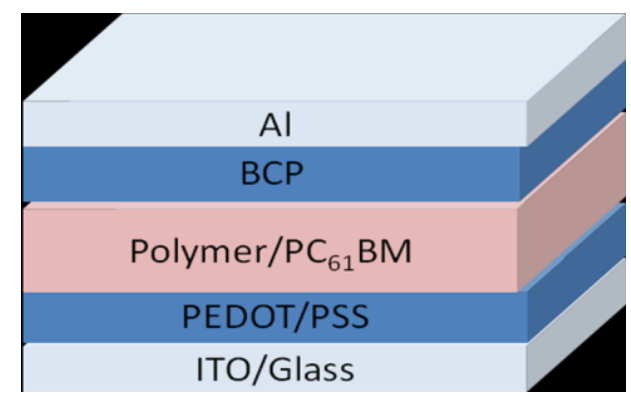

Figure 3.22 Device structure of bulk-heterojunction photodetector.

The photodetector properties of polymers P22-P25 were studied. The device configuration of ITO/PEDOT:PSS (30 nm)/active layer (80 nm)/BCP (3nm)/Al (120 nm) was used (Figure 3.22). The active layers were prepared from the polymers and $\mathrm{PC}_{61} \mathrm{BM}$ in a weight ratio of 1:2. The current density-voltage $(\mathrm{J}-\mathrm{V})$ characteristics of the polymers 
are summarized in Table 3.5. Asymmetry behavior was observed under reverse and forward bias with the polymers show relatively low dark current. Among all, the device based on polymer $\mathbf{P 2 5}$ shows the lowest dark current of $9.48 \times 10^{-9} \mathrm{~A} \mathrm{~cm}^{-2}$. EQE is an important figure of merit that is defined as the ratio of the number of photo-generated electrons flowing in the external circuit and the incident photons. The EQE curves are represented in the Appendix B (Figures S3.31-S3.34) and a summary has been represented in Figure 3.23 and Table 3.5. All four polymers show EQE from $0.47 \%$ to $4.3 \%$ over the wavelength from 350 to $380 \mathrm{~nm}$ and very weak over the 400 to $900 \mathrm{~nm}$. Among all, the device based on polymer P25 shows a steady EQE from 359 to $655 \mathrm{~nm}$. The photo-generated electron and hole are separated and transported by internal and external electric fields, which indicates the efficiency of the charge separation and their transportation in the device application. The lower EQE indicates either a lower film formation property or a higher phase separation in the active layer that traps the electrons. Generally, the responsitivity is expressed as a function of the wavelength of the incident radiation which is represented in the following equation:

$$
R=n \frac{q}{h f} \approx \frac{\lambda_{\mu m}}{1.23985\left(\mu m X \frac{W}{A}\right)}
$$

where $n$ is the quantum efficiency, $\mathrm{q}$ is the electron charge, $\mathrm{f}$ is the frequency of the optical signal, and h is Planck's constant. The polymers P22-P25 display maximum responsitivity of $4.63(371 \mathrm{~nm}), 1.54(552 \mathrm{~nm}), 12.30(370 \mathrm{~nm})$, and $11(612 \mathrm{~nm}) \mathrm{mA} \mathrm{W} \mathrm{W}^{-1}$, respectively. The polymers P22-P24 show responsitivity from $300 \mathrm{~nm}$ to $800 \mathrm{~nm}$ whereas polymer P25 shows responsitivity up to $900 \mathrm{~nm}$. The polymers P22 and P24 show higher responsitivity from 360 to $375 \mathrm{~nm}$, and lower responsitivity over the spectral 
region, which correlates to absorption in this region. Polymer P23 shows steady responsitivity over 371 to $610 \mathrm{~nm}$. Polymer P25 shows three different maximum responsitivities at 391, 465 and $612 \mathrm{~nm}$. All these polymers show that as the applied bias increases the responsivity also increases, which is due to field-induced extinction dissociation. The detectivity, calculated in Jones $(\mathrm{cm} \mathrm{Hz} / W)$, measures the relative noise levels present in the device. The higher the detectivity, the better the device can detect signal from noise. All the polymers show detectivity from $10^{9}$ to $10^{10}$ Jones at $-0.1 \mathrm{~V}$ bias from the visible to the near infrared region. However, when applying a higher voltage, we observed a lower detectivity due to poor film stability and high current leakage (Appendix B).
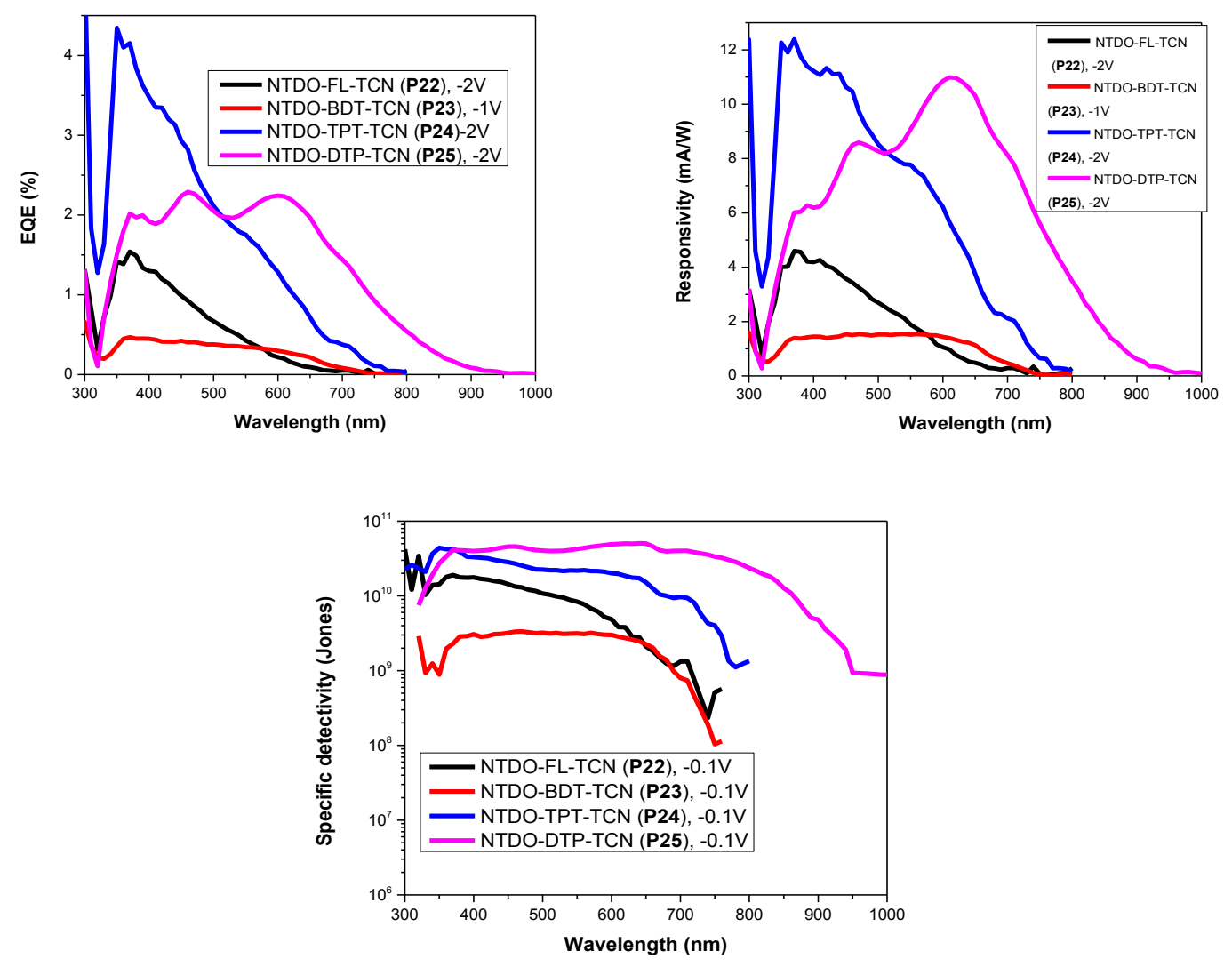

Figure 3.23 EQE, responsivity and detectivity of the devices based on polymers P22-P25. 
Table 3.5. Summary of device performance based on polymers P22-P25.

\begin{tabular}{|l|l|l|l|}
\hline Polymer & $\begin{array}{l}\text { Dark Current } \\
\left(\mathrm{A} / \mathrm{cm}^{2}\right)\end{array}$ & $\begin{array}{l}\text { Responsivity } \\
(\mathrm{mA} / \mathrm{W})\end{array}$ & $\begin{array}{l}\text { Detectivity } \\
(\text { Jones })\end{array}$ \\
\hline P22 & $1.79 \times 10^{-8}$ & $4.63(371 \mathrm{~nm})$ & $1.9 \times 10^{10}$ \\
\hline $\mathbf{P 2 3}$ & $1.11 \times 10^{-7}$ & $1.54(552 \mathrm{~nm})$ & $3.19 \times 10^{9}$ \\
\hline $\mathbf{P 2 4}$ & $1.55 \times 10^{-8}$ & $12.30(370 \mathrm{~nm})$ & $4.26 \times 10^{10}$ \\
\hline P25 & $9.48 \times 10^{-9}$ & $11(612 \mathrm{~nm})$ & $4.80 \times 10^{10}$ \\
\hline
\end{tabular}

\subsection{Conclusion}

A series of newly functionalized polymers $\mathbf{P 1 3}$ to $\mathbf{P 1 7}$ were synthesized from the homopolymer P1. All the polymers are well characterized by IR, absorption spectroscopy and cyclic voltammogram. The complete conversion of polymer P1 to P17 was not possible due to the limitation of the reduction process. All functionalized polymers contained some of the keto groups, which were confirmed by the IR spectroscopy. The changes of electrochemical energy levels and energy band gap in each polymer also suggest the structural changes after the reaction. The obtained polymers showed redshifted absorption maximal and broad absorption in $\mathrm{UV}$-Vis region compared to polymer P1. A series of NTDO-based donor-acceptor polymers was synthesized by cross-coupling reactions and characterized. The synthesized polymers showed a wide range of absorption with an onset from the NIR region. The absorption maximal red-shifted with increases the donor strength, where P18 has lowest absorption maximal and P21 has longest absorption maximal. The energy band gap decreased from polymer P18 to P21. 
Increasing the donor strength in NTDO-based D-A polymers decreased the energy band gap. The condensation reaction on the keto group of NTDO-based donor-acceptor polymers was successfully conducted using malononitrile. The polymers (P22-P25) showed broader absorption compared to the parent polymers (P18-P21). The electrochemical band gap increased due to the up shift of LUMO energy level. The photodetector properties of polymers P22-P25 were studied. Lower EQE, responsitivity and detectivity properties were observed, which is due to the higher leakage current and low film forming properties of polymers P22-P25.

\subsection{Experimental section}

\section{Materials}

Acetic anhydride, sodium borohydride, 2-propanol tifluoroacetic acid, titanium tetrachloride, tetrakis(triphenylphosphine)palladium(0), malononitrile, pyridine, potassium carbonate, Tris(dibenzylideneacetone)dipalladiuum(0): 2,2'-bis(diphenylphosphino)-1,1'-binaphthyl (BINAP):Sodium tert-butoxide $\quad(0.05: 0.15: 2), \quad$ trimethyltin chloride, n-butyllithium solution (2.5 $\mathrm{M}$ in hexane), tert-butyllithium solution (1.7 $\mathrm{M}$ in pentane), 9,9-dioctylfluorene-2,7-diboronic acid bis(1,3propanediol)ester, 2ethylhexylamine, succinyl dichloride, dodecylamine, aluminum chloride, and benzo[1,2b:4,5-b']dithiophene-4,8-dione were purchased from Aldrich Chemicals and used as received. 3,3'-Dibromo-2,2'-bithiophene was purchased from BioFine International and used as received. 


\section{Polymer P13}

Polymer P1 (0.400 g, 1.23mmol) was dissolved in tetrahydrofuran (10 mL) and then $4 \mathrm{~mL}$ of methanol was added to the solution. The solution was stirred while cooling to $0-5{ }^{\circ} \mathrm{C}$ with an ice bath. Solid sodium borohydride $(0.465 \mathrm{~g}, 12.3 \mathrm{mmol})$ was added in small portions over $45 \mathrm{~min}$. The reaction mixture was stirred for $4 \mathrm{~h}$ in ice bath. Then the reaction mixture was warmed to room temperature and stirred for $16 \mathrm{~h}$. The reaction mixture was poured into an ice-water mixture and stirred. Chloroform was added to the mixture and shaken vigorously. The organic portion was separated and extracted three times by chloroform. The combined organic portions were dried over magnesium sulfate. The solvent was removed under reduced pressure. The obtained polymer was dried at 80 ${ }^{\circ} \mathrm{C}$ under an argon atmosphere and was characterised as it is. The polymer was obtained as dark solid (0.386 $\mathrm{g}, 95.0 \%$ yield). IR ( $\mathrm{NaCl}$ plate, $\left.\mathrm{cm}^{-1}\right): 3393,2925,2853,1669$, $1599,1461,1375,1260,1091,1020,804$.

\section{Polymer P14}

Polymer P13 (0.350 g, $1.06 \mathrm{mmol})$ was dissolved in $10 \mathrm{~mL}$ of chloroform then 5 $\mathrm{mL}$ of $6 \mathrm{M} \mathrm{HCl}$ was added to the solution. The reaction mixture was heated to reflux for 6 h. The reaction mixture was then poured into water and stirred. Chloroform was added to the mixture and shaken vigorously. The organic portion was separated and extracted three times by chloroform. The combined organic portions were dried over magnesium sulfate. The solvent was removed under reduced pressure. The obtained polymer was dried at 80 ${ }^{\circ} \mathrm{C}$ under an argon atmosphere and was characterised as it is. The polymer was obtained 
as dark solid (0.312 $\mathrm{g}, 95.1 \%$ yield $)$. IR ( $\mathrm{NaCl}$ plate, $\left.\mathrm{cm}^{-1}\right): 2925,2854,1664,1599$, $1464,1261,1023,799,758$.

\section{Polymer P15}

Polymer P14 (0.280 g, $0.909 \mathrm{mmol})$ was dissolved in tetrahydrofuran (10 mL) and then $4 \mathrm{~mL}$ of 2-propanol was added to the solution. Solid sodium borohydride $(0.400$ $\mathrm{g}, 10.5 \mathrm{mmol}$ ) was added in small portions over $45 \mathrm{~min}$. The reaction mixture was refluxed for $36 \mathrm{~h}$. The reaction mixture was poured into an ice-water mixture and stirred. Then $10 \mathrm{~mL}$ of conc. $\mathrm{HCl}$ was added to the mixture and stirred for $1 \mathrm{~h}$. Chloroform was added to the resulting mixture and shaken vigorously. The organic portion was separated and extracted three times by chloroform. The combined organic portions were dried over magnesium sulfate. The solvent was removed under reduced pressure. The obtained polymer was dried at $80{ }^{\circ} \mathrm{C}$ under an argon atmosphere and was characterised as it is. The polymer was obtained as dark solid $(0.270 \mathrm{~g}, 95.7 \%$ yield $) . \mathrm{IR}\left(\mathrm{NaCl}\right.$ plate, $\left.\mathrm{cm}^{-1}\right): 3391$, 2925, 2853, 1664, 1599, 1499, 1464, 1375, 1259, 1091, 1020, 891.

\section{Polymer P16}

Polymer P15 (0.140 g, $0.451 \mathrm{mmol})$ was dissolved in tetrahydrofuran $(5.0 \mathrm{~mL})$ and then $3.0 \mathrm{~mL}$ of acetic anhydride was added to the solution and stirred it at room temperature for $16 \mathrm{~h}$. The reaction mixture was poured into water and stirred for 30 minutes. Chloroform was added to the resulting mixture and shaken vigorously. The organic portion was separated and extracted three times by chloroform. The combined organic portions were dried over magnesium sulfate. The solvent was removed under 
reduced pressure. The obtained polymer was dried at $80{ }^{\circ} \mathrm{C}$ under an argon atmosphere and was characterised as it is. The polymer was obtained as dark solid $(0.150 \mathrm{~g}, 94.3 \%)$. IR ( $\mathrm{NaCl}$ plate, $\left.\mathrm{cm}^{-1}\right): 2925,2853,1774,1737,1664,1599,1494,1461,1367,1260$, $1183,1022,802$.

\section{Polymer P17 from P15}

Polymer P15 (0.100 g, $0.322 \mathrm{mmol})$ was dissolved in tetrahydrofuran (5 mL) and then $3 \mathrm{~mL}$ of conc. $\mathrm{HCl}$ was added to the solution and heated at $60{ }^{\circ} \mathrm{C}$ for $12 \mathrm{~h}$. The reaction mixture was then poured into water and stirred for 30 minutes. Chloroform was added to the resulting mixture and shaken vigorously. The organic portion was separated and extracted three times by chloroform. The combined organic portions were dried over magnesium sulfate. The solvent was removed under reduced pressure. The obtained polymer was dried at $80{ }^{\circ} \mathrm{C}$ under an argon atmosphere and was characterised as it is. The

polymer was obtained as dark solid (0.095 g). IR ( $\mathrm{NaCl}$ plate, $\left.\mathrm{cm}^{-1}\right)$ : 2925, 2853, 1664, $1599,1499,1464,1375,1259,1091,1020,804$.

Polymer P17 from P16 (path b)

Polymer P16 (0.070 g, $0.199 \mathrm{mmol})$ was dissolved in tetrahydrofuran $(5 \mathrm{~mL})$ and then $3 \mathrm{~mL}$ of trifluoroacetic acid was added to the solution and the reaction mixture was heated at reflux for $6 \mathrm{~h}$. The reaction mixture was then poured into water and stirred for 30 minutes. Chloroform was added to the resulting mixture and shaken vigorously. The organic portion was separated and extracted three times by chloroform. The combined organic portions were dried over magnesium sulfate. The solvent was removed under 
reduced pressure. The obtained polymer was dried at $80{ }^{\circ} \mathrm{C}$ under an argon atmosphere and was characterised as it is. The polymer was obtained as dark solid $(0.065 \mathrm{~g})$. IR $\left(\mathrm{NaCl}\right.$ plate, $\left.\mathrm{cm}^{-1}\right): 2925,2853,1777,1669,1602,1494,1458,1367,1261,1172,1021$, 803.

\section{4,8-Bis(octyloxy)benzo[1,2-b:4,5- $\left.b^{\prime}\right]$ dithiophene (5)}

Benzo[1,2-b:4,5-b']dithiophene-4,8-dione 8 (2.00 g, $9.10 \mathrm{mmol})$, zinc dust (1.56 g, $24.0 \mathrm{mmol})$ and $\mathrm{NaOH}$ aqueous solution $(30 \mathrm{~mL}, 20 \mathrm{wt} \%)$ were added into a dry 100 $\mathrm{mL}$ three-neck flask. The mixture was heated to reflux for $1 \mathrm{~h}$. Then, octyl bromide (4.40 g, $23.0 \mathrm{mmol}$ ) and a catalytic amount of tetrabutylammonium bromide (TBAB) were added into the flask. After heating at reflux for $12 \mathrm{~h}$, the mixture was poured into $150 \mathrm{~mL}$ of cold water, and extracted three times by dichloromethane. The combined organic layers were washed with water and dried over anhydrous $\mathrm{Na}_{2} \mathrm{SO}_{4}$. The compound was purified by silica gel column chromatography using hexane and acetone (7:3 v/v) as eluting solvent. Compound 5 was obtained as white solid (3.61 g, 88.4\% yield). ${ }^{1} \mathrm{H}$ NMR (300 MHz, $\left.\mathrm{CDCl}_{3}\right): \delta(\mathrm{ppm}) 7.51(\mathrm{~d}, J=5.5 \mathrm{~Hz}, 2 \mathrm{H}), 7.39(\mathrm{~d}, J=5.5 \mathrm{~Hz}, 2 \mathrm{H}), 4.30(\mathrm{t}, J$ $=6.6 \mathrm{~Hz}, 4 \mathrm{H}), 1.90(\mathrm{~m}, 4 \mathrm{H}), 1.58(\mathrm{~m}, 4 \mathrm{H}), 1.50-1.33(\mathrm{~m}, 16 \mathrm{H}), 0.90(\mathrm{~m}, 6 \mathrm{H}) .{ }^{13} \mathrm{C} \mathrm{NMR}$ (75 MHz, $\left.\mathrm{CDCl}_{3}\right): \delta 144.46,131.53,130.33,125.98,120.32,73.97,31.70,30.55,29.43$, $26.08,22.67,14.12$. 


\section{(4,8-Bis(octyloxy)benzo[1,2-b:4,5-b']dithiophene-2,6-diyl)bis(trimethylstannane)}

(M4)

In a flame dried three-neck flask under argon atmosphere, compound 5 (1.00 g, $2.23 \mathrm{mmol})$ was dissolved in $15 \mathrm{~mL}$ of anhydrous tetrahydrofuran. The solution was cooled to $-78{ }^{\circ} \mathrm{C}$ and $1.9 \mathrm{~mL}$ of n-butyllithium solution (4.75 mmol, $2.5 \mathrm{M}$ in hexane) was added dropwise. The reaction mixture was stirred at $-78{ }^{\circ} \mathrm{C}$ for $1 \mathrm{~h}$ and was then stirred at room temperature for an additional $1 \mathrm{~h}$. The reaction mixture was again cooled to $-78{ }^{\circ} \mathrm{C}$ and trimethyltin chloride solution $(4.5 \mathrm{~mL}, 4.5 \mathrm{mmol}, 1 \mathrm{M}$ in THF) was added dropwise. The mixture was allowed to warm to room temperature and was stirred overnight. The reaction was poured into ice-water and extracted by dichloromethane three times. The organic extraction was washed by water and then dried by anhydrous magnesium sulfate. After removal of the solvent under vacuum, the residue was recrystallized by methanol yielded colorless needle crystal $(1.42 \mathrm{~g}, 81.0 \%)$ as monomer M4. ${ }^{1} \mathrm{H}$ NMR (300 MHz, $\left.\mathrm{CDCl}_{3}\right): \delta 7.54(\mathrm{~s}, 2 \mathrm{H}), 4.33(\mathrm{t}, J=6.5 \mathrm{~Hz}, 4 \mathrm{H}), 1.90(\mathrm{~m}, 4 \mathrm{H})$, $1.57(\mathrm{~m}, 4 \mathrm{H}), 1.33(\mathrm{~m}, 16 \mathrm{H}), 0.91(\mathrm{~m}, 6 \mathrm{H}), 0.47(\mathrm{~s}, 18 \mathrm{H}) .{ }^{13} \mathrm{C} \mathrm{NMR}\left(75 \mathrm{MHz}, \mathrm{CDCl}_{3}\right) \delta$ $143.10,140.31,133.89,132.91,128.01,73.75,31.68,30.44,29.63,26.34,22.79,14.09$, $5.79,-8.34,-10.72$

\section{1-Dodecyl-2,5-di(thiophen-2-yl)-1H-pyrrole (7)}

In a flame-dried $150 \mathrm{~mL}$ round bottom flask, compound 6 (6 g, $24 \mathrm{mmol})$ was dissolved in $70 \mathrm{~mL}$ dry toluene and $3 \mathrm{~mL}$ of propionic acid. Dodecylamine $(5 \mathrm{~g}, 27$ mmol) was then added and the solution was refluxed for 3 days using a Dean-Stark trap to remove water. The solvent was then removed by vacuum and then extracted using 
$\mathrm{CH}_{2} \mathrm{Cl}_{2}$. It was washed three times with $25 \mathrm{~mL}$ of $\mathrm{NaHCO}_{3}$, water and brine. The organic layer was dried over $\mathrm{MgSO}_{4} \cdot \mathrm{CH}_{2} \mathrm{Cl}_{2}$ was removed under vacuum and the resulting oil was purified by silica gel flash chromatography using hexane. The resulting oil was crystallized out using ethanol to give a light yellow needle $(7.00 \mathrm{~g}, 77.0 \%) .{ }^{1} \mathrm{H}$ NMR (400 $\left.\mathrm{MHz}, \mathrm{CDCl}_{3}\right): \delta 7.34(\mathrm{dd}, J=4.8,1.6 \mathrm{~Hz}, 2 \mathrm{H}), 7.09(\mathrm{~m}, 4 \mathrm{H}), 6.36(\mathrm{~s}, 2 \mathrm{H}), 4.14(\mathrm{~m}, 2 \mathrm{H})$, $1.58(\mathrm{~m}, 2 \mathrm{H}), 1.38-1.06(\mathrm{~m}, 18 \mathrm{H}), 0.91(\mathrm{t}, J=6.9 \mathrm{~Hz}, 3 \mathrm{H}) .{ }^{13} \mathrm{C} \mathrm{NMR}(100 \mathrm{MHz}$ $\left.\mathrm{CDCl}_{3}\right): \delta 135.04,128.28,127.23,125.89,125.18,110.72,45.15,31.93,31.15,29.67-$ $29.29,28.91,26.38,22.70,14.14$.

\section{1-Dodecyl-2,5-bis(5-(trimethylstannyl)thiophen-2-yl)-1H-pyrrole (M5)}

In a flame dried three-neck flask under argon atmosphere, compound 7 (0.399 g, $1.00 \mathrm{mmol}$ ) was dissolved in $15 \mathrm{~mL}$ of anhydrous tetrahydrofuran. The solution was cooled to $-78{ }^{\circ} \mathrm{C}$ and $0.88 \mathrm{~mL}$ of n-butyllithium solution $(2.2 \mathrm{mmol}, 2.5 \mathrm{M}$ in hexane) was added dropwise. The reaction mixture was stirred at $-78^{\circ} \mathrm{C}$ for $1 \mathrm{~h}$ and then stirred at room temperature additional $1 \mathrm{~h}$. The reaction mixture was again cooled to $-78{ }^{\circ} \mathrm{C}$ and trimethyltin chloride solution $(2.1 \mathrm{~mL}, 2.10 \mathrm{mmol}, 1 \mathrm{M}$ in THF) was added dropwise. The mixture was allowed to warm to room temperature and stirred overnight. The reaction was poured into ice-water and extracted by dichloromethane three times. The organic extract was dried over $\mathrm{MgSO}_{4}$ and the solvent was removed by rotary evaporator.

The monomer M5 was obtained as brownish solid (0.648 g, 89.3\% yield). ${ }^{1} \mathrm{H}$ NMR (300 $\left.\mathrm{MHz}, \mathrm{CDCl}_{3}\right): \delta 7.19(\mathrm{~m}, 2 \mathrm{H}), 6.34(\mathrm{~m}, 2 \mathrm{H}), 4.16(\mathrm{~m}, 2 \mathrm{H}), 1.62(\mathrm{~m} \mathrm{4H}), 1.26(\mathrm{~m}, 18 \mathrm{H})$, $0.90(\mathrm{~m}, 3 \mathrm{H}), 0.41 .{ }^{13} \mathrm{C}$ NMR $\left(75 \mathrm{MHz}, \mathrm{CDCl}_{3}\right): \delta 140.89,137.48,135.32,128.57$, 
$126.67,110.39,45.16,31.93,31.17,29.65,29.54,29.42,29.36,28.90,26.39,22.70$, $14.14,-5.83,-8.19,-10.55$.

\section{4-(2-ethylhexyl)-4H-dithieno[3,2-b:2',3'-d]pyrrole (9)}

In a flame dried three-neck flask under argon atmosphere, dibromobithiophene (2.00 g, $6.17 \mathrm{mmol}) \mathrm{Pd}_{2}(\mathrm{dba})_{3}: \mathrm{BINAP}: \mathrm{NatOBu}(0.05: 0.15: 2)(1.00 \mathrm{~g}), \mathrm{Pd}_{2}(\mathrm{dba}) 3(0.070$

g), BINAP $(0.500 \mathrm{~g}) \mathrm{Na}^{\mathrm{t}} \mathrm{OBu}(0.900 \mathrm{~g})$ and alkyl-amine $(0.875 \mathrm{~g}, 6.79 \mathrm{mmol})$ were dissolved in toluene and the solution was degassed and purged with argon for $30 \mathrm{~min}$. The mixture was then refluxed for $24 \mathrm{~h}$. After cooling the solution to room temperature it was poured into water and extracted three times by dichloromethane. The organic extract was dried over $\mathrm{MgSO}_{4}$ and the solvent was removed by rotary evaporator. The compound was purified by silica gel column chromatography using hexane as eluting solvent. The compound 9 was obtained as brown oil $\left(1.26 \mathrm{~g}, 69.8 \%\right.$ yield). ${ }^{1} \mathrm{H}$ NMR $(300 \mathrm{MHz}$, $\left.\mathrm{CDCl}_{3}\right): \delta 7.13(\mathrm{~d}, J=5.3 \mathrm{~Hz}, 2 \mathrm{H}), 7.01(\mathrm{~d}, J=5.3 \mathrm{~Hz}, 2 \mathrm{H}), 4.08(\mathrm{~m}, 2 \mathrm{H}) 1.97(\mathrm{~m}, 1 \mathrm{H})$, $1.34(\mathrm{~m} \mathrm{8H}), 0.92(\mathrm{~m}, 6 \mathrm{H}) .{ }^{13} \mathrm{C}$ NMR $\left(75 \mathrm{MHz}, \mathrm{CDCl}_{3}\right): \delta 145.20,122.79,114.53$, $111.09,51.34,40.47,30.65,28.77,24.03,22.99,14.04,10.69$.

\section{4-(2-ethylhexyl)-2,6-bis(trimethylstannyl)-4H-dithieno[3,2-b:2',3'-d]pyrrole (M6)}

In a flame dried three-neck flask under argon atmosphere, compound $9(0.634 \mathrm{~g}$, $2.17 \mathrm{mmol}$ ) was dissolved in $15 \mathrm{~mL}$ of anhydrous tetrahydrofuran. The solution was cooled to $0{ }^{\circ} \mathrm{C}$ and $3.29 \mathrm{~mL}$ of tert-butyllithium solution (4.45 mmol, $1.7 \mathrm{M}$ in pentane) was added dropwise. The reaction mixture was stirred at $0{ }^{\circ} \mathrm{C}$ for $1 \mathrm{~h}$ and then stirred at room temperature additional $1 \mathrm{~h}$. The reaction mixture was again cooled to $0{ }^{\circ} \mathrm{C}$ and 
trimethyltin chloride solution ( $4.55 \mathrm{~mL}, 4.55 \mathrm{mmol}, 1 \mathrm{M}$ in THF) was added dropwise. The mixture was allowed to warm to room temperature and stirred overnight. The reaction was poured into ice-water and extracted by dichloromethane three times. The organic extract was dried over $\mathrm{MgSO}_{4}$ and the solvent was removed by rotary evaporator.

The monomer M6 was obtained as dark brownish oil (1.20 g, 89.5\% yield). ${ }^{1} \mathrm{H}$ NMR (300 MHz, $\left.\mathrm{CDCl}_{3}\right): \delta 6.98(\mathrm{~s}, 2 \mathrm{H}), 4.07(\mathrm{~m}, 2 \mathrm{H}), 2.00(\mathrm{~m}, 1 \mathrm{H}) 1.34(\mathrm{~m}, 8 \mathrm{H}), 0.94(\mathrm{~m}, 6 \mathrm{H})$, $0.42(\mathrm{~s}, 18 \mathrm{H}) .{ }^{13} \mathrm{C}$ NMR $\left(75 \mathrm{MHz}, \mathrm{CDCl}_{3}\right): \delta 148.33,135.52,122.55,120.12,118.03$, $111.34,51.19,40.36,30.48,28.45,23.98,23.13,14.09,10.72,0.04,-5.66,-8.11,-10.57$.

\section{Polymer P18}

To a mixture of monomer M1 (0.300 g, $0.618 \mathrm{mmol})$ and 9,9-dioctylfluorene-2,7diboronic acid bis $(1,3$-propanediol $)$ ester $(0.345 \mathrm{~g}, 0.618 \mathrm{mmol})$ in toluene $(8.0 \mathrm{~mL})$ was added $3 \mathrm{~mL}$ of $2 \mathrm{M}$ aqueous solution of $\mathrm{K}_{2} \mathrm{CO}_{3}$. After degassed and purged with argon for $30 \mathrm{~min}, \mathrm{Pd}\left(\mathrm{PPh}_{3}\right)_{4}(0.0700 \mathrm{~g}, 0.0700 \mathrm{mmol})$ was added. The mixture was heated at $90{ }^{\circ} \mathrm{C}$ for $48 \mathrm{~h}$ under argon. The solution was then poured into methanol with vigorous stirring. After stirring $2 \mathrm{~h}$ a yellow precipitated as polymer P18 was collected by filtration. The polymer was purified by being dissolved in $\mathrm{CHCl}_{3}$ and re-precipitated into methanol and then was further purified by a Soxhlet extraction in acetone for $24 \mathrm{~h}$. The resultant polymer was dissolved in $\mathrm{CHCl}_{3}$ and precipitated with methanol. After drying under vacuum at $60{ }^{\circ} \mathrm{C}$ overnight, the polymers were obtained ( $0.600 \mathrm{~g}, 91.0 \%$ yield) as yellow powder. ${ }^{1} \mathrm{H} \mathrm{NMR}\left(300 \mathrm{MHz}, \mathrm{CDCl}_{3}\right.$, ppm): $\delta 8.18,8.08,7.89,7.79,7.56,2.78,2.10$, $1.64,1.29,1.21,0.90,0.82 .{ }^{13} \mathrm{C}$ NMR $\left(75 \mathrm{MHz}, \mathrm{CDCl}_{3}\right): \delta \quad 180.10,179.72,152.38$, $152.25,151.28,149.56,141.82,135.27,133.94,133.27,131.30,128.87,127.56,126.98$, 
$124.89,119.78,55.58,40.13,36.18,31.89,30.91,30.19,29.33,24.00,22.66,14.21$. IR $\left(\mathrm{NaCl}\right.$ plate, $\left.\mathrm{cm}^{-1}\right): 2925,2853,1671,1601,1455,1367,1036$

\section{General procedure for Stille coupling reaction}

In a flame-dried 50-mL round-bottom flask under an argon atmosphere, organotin monomer and NTDO monomer M1 in 1:1 mole ratio were dissolved in $8.0-10 \mathrm{~mL}$ of toluene. After degassed and purged with argon for $30 \mathrm{~min}, 10 \mathrm{~mol} \%$ of $\mathrm{Pd}\left(\mathrm{PPh}_{3}\right)_{4}$ was added. The mixture was heated at $90{ }^{\circ} \mathrm{C}$ for $48 \mathrm{~h}$ under argon. The solution was then poured into methanol with vigorous stirring and the precipitate was collected by filtration. The polymer was purified via Soxhlet extraction in acetone for $24 \mathrm{~h}$. The resultant polymers were dissolved in $\mathrm{CHCl}_{3}$ and reprecipitated in methanol and collected by filtration. The polymers were dried under vacuum at $80^{\circ} \mathrm{C}$ overnight

\section{Polymer P19}

Polymer P19 was synthesized according to the procedure described for Stille cross coupling reaction. Monomer M1 $(0.400 \mathrm{~g}, 0.829 \mathrm{mmol})$, M4 $(0.638 \mathrm{~g}, 0.829$ $\mathrm{mmol}), \mathrm{Pd}\left(\mathrm{PPh}_{3}\right)_{4}(0.0950 \mathrm{~g}, 0.0829 \mathrm{mmol})$ and toluene $(10 \mathrm{~mL})$. The obtained polymer was dark purple solid (0.600 g, 94.0\% yield). ${ }^{1} \mathrm{H}$ NMR (300 MHz, $\left.\mathrm{CDCl}_{3}\right): \delta 8.45,8.26$, $8.15,7.65,7.41,7.21,4.46,2.78,2.64,1.96,1.33,0.90 .{ }^{13} \mathrm{C}^{\mathrm{NMR}}\left(75 \mathrm{MHz}, \mathrm{CDCl}_{3}\right): \delta$ $179.43,178.06,149.56,144.05,134.76,130.44,128.25,127.70,120.53,74.36,36.11$, $31.88,30.81,29.46,26.12,22.70,14.13$. IR ( $\mathrm{NaCl}$ plate, $\left.\mathrm{cm}^{-1}\right): 2924,2854,1663,1602$, $1467,1362,1263,1177,1065$ 


\section{Polymer P20}

Polymer P20 was synthesized according to the procedure described for Stille cross coupling reaction. Monomer M1 (0.394 g, $0.812 \mathrm{mmol}), \mathbf{M 5}$ (0.648 g, 0.893 $\mathrm{mmol}), \mathrm{Pd}\left(\mathrm{PPh}_{3}\right)_{4}(0.0950 \mathrm{~g}, 0.0829 \mathrm{mmol})$ and toluene $(10 \mathrm{~mL})$. The obtained polymer was dark purple solid $\left(0.550 \mathrm{~g}, 93.4 \%\right.$ yield). ${ }^{1} \mathrm{H} \mathrm{NMR}\left(300 \mathrm{MHz}, \mathrm{CDCl}_{3}\right): \delta 8.26,8.17$, $7.95,7.74,7.61,7.35,7.19,6.58,4.28,2.79,1.72,1.29,1.21,0.90 .{ }^{13} \mathrm{C}$ NMR $(75 \mathrm{MHz}$, $\left.\mathrm{CDCl}_{3}\right): \delta 180.07,179.67,134.63,134.11,132.70,131.76,130.18,128.22,127.90$ $126.96,126.12,125.48,112.36,111.80,111.43,45.57,36.23,31.86,31.22,30.97,29.67$, 29.37, 26.40, 22.68, 14.12. IR ( $\mathrm{NaCl}$ plate, $\left.\mathrm{cm}^{-1}\right): 2923,2852,1652,1600,1457,1423$, 1370, 1259, 1096, 1037, 796, 692.

Polymer P21

Polymer P21 was synthesized according to the procedure described for Stille cross coupling reaction. Monomer M1 $(0.587 \mathrm{~g}, 1.21 \mathrm{mmol}), \mathbf{M 5}(0.750 \mathrm{~g}, 1.21 \mathrm{mmol})$, $\mathrm{Pd}\left(\mathrm{PPh}_{3}\right)_{4}(1.39 \mathrm{~g}, 0.112 \mathrm{mmol})$ and toluene $(10 \mathrm{~mL})$. The obtained polymer was dark blue solid (0.730 g, 82.5\% yield). ${ }^{1} \mathrm{H}$ NMR (300 MHz, $\left.\mathrm{CDCl}_{3}\right): \delta 7.92,7.70,7.09,4.07$, 2.61, 1.27, 0.90. ${ }^{13} \mathrm{C}$ NMR (75 MHz, $\left.\mathrm{CDCl}_{3}\right): \delta 178.46,149.42,147.06,144.50,133.34$, $129.78,128.36,114.54,110.77,51.19,40.42,36.04,31.88,31.35,30.95,30.60,29.36$, 28.62, 24.07, 22.99, 22.67, 14.11, 10.74. IR ( $\mathrm{NaCl}$ plate, $\left.\mathrm{cm}^{-1}\right): 2925,2855,1652,1600$, $1458,1422,1377,1262$. 


\section{General procedure for modified Knoevenagel condensation reaction}

In a flame-dried 100-mL, three neck, round-bottom flask under an argon atmosphere, malononitrile (10 mol equivalent), $\mathrm{TiCl}_{4}$ (10 mol equivalent) and pyridine $(10 \mathrm{~mol}$ equivalent) were added to the polymer solution chloroform $(20 \mathrm{~mL})$ and the mixture was heated at reflux for $96 \mathrm{~h}$. Every 24 hours an additional $10 \mathrm{~mol}$ equivalent of malononitrile, $\mathrm{TiCl}_{4}$ and pyridine were added to the reaction mixture. The reaction mixture was poured into ice-water and stirred for half an hour. The resultant polymer was then collected by filtration. The polymer was then stirred in diethyl ether $(30 \mathrm{~mL})$ for 30 min to remove the excess amount of malononitrile and the polymer was collected by filtration. The polymer was further purified by a Soxhlet extraction in $\mathrm{CHCl}_{3}$ for $24 \mathrm{~h}$ to remove any unreacted starting polymer. The resultant solid polymer was then collected from Soxhlet and dried under vacuum at $80{ }^{\circ} \mathrm{C}$ overnight.

\section{Polymer P22}

Polymer P22 was synthesized according to the procedure described in modified Knoevenagel condensation reaction. Polymer P18 (0.300 g, 0.412 mmol), malononitrile (0.278 g, $4.20 \mathrm{mmol}), \mathrm{TiCl}_{4}(0.84 \mathrm{~mL}, 4.20 \mathrm{mmol})$ and pyridine $(0.35 \mathrm{~mL}, 4.20 \mathrm{mmol})$ and $\mathrm{CHCl}_{3}(25 \mathrm{~mL})$. The obtained polymer was dark powder $(0.320 \mathrm{~g}, 94.1 \%$ yield $)$. IR $\left(\mathrm{KBr}\right.$ pellet, $\left.\mathrm{cm}^{-1}\right): 2215,1626$

\section{Polymer P23}

Polymer P23 was synthesized according to the procedure described in modified Knoevenagel condensation reaction. Polymer P19 (0.200 g, $0.260 \mathrm{mmol}$ ), malononitrile 
$(0.171 \mathrm{~g}, 2.60 \mathrm{mmol}), \mathrm{TiCl}_{4}(0.52 \mathrm{~mL}, 2.60 \mathrm{mmol})$ and pyridine $(0.21 \mathrm{~mL}, 2.60 \mathrm{mmol})$ and $\mathrm{CHCl}_{3}(20 \mathrm{~mL})$. The obtained polymer was dark powder $(0.220 \mathrm{~g}, 90.9 \%$ yield $)$. IR $\left(\mathrm{KBr}\right.$ pellet, $\left.\mathrm{cm}^{-1}\right): 2215,1626$

Polymer P24

Polymer P24 was synthesized according to the procedure described in modified Knoevenagel condensation reaction. Polymer P20 (0.200 g, $0.277 \mathrm{mmol})$, malononitrile (0.182 g, $2.77 \mathrm{mmol}), \mathrm{TiCl}_{4}(0.55 \mathrm{~mL}, 2.77 \mathrm{mmol})$ and pyridine $(0.22 \mathrm{~mL}, 2.77 \mathrm{mmol})$ and $\mathrm{CHCl}_{3}(20 \mathrm{~mL})$. The obtained polymer was dark powder $(0.210 \mathrm{~g}, 92.9 \%$ yield $)$. IR (KBr pellet, $\left.\mathrm{cm}^{-1}\right): 2209,1626$.

\section{Polymer P25}

Polymer P25 was synthesized according to the procedure described in modified Knoevenagel condensation reaction. Polymer P21 (0.400 g, $0.653 \mathrm{mmol})$, malononitrile (0.430 g, $6.53 \mathrm{mmol}), \mathrm{TiCl}_{4}(1.22 \mathrm{~mL}, 6.53 \mathrm{mmol})$ and pyridine $(0.51 \mathrm{~mL}, 6.53 \mathrm{mmol})$ and $\mathrm{CHCl}_{3}(30 \mathrm{~mL})$. The obtained polymer was dark powder $(0.400 \mathrm{~g}, 86.5 \%$ yield $)$. IR $\left(\mathrm{KBr}\right.$ pellet, $\left.\mathrm{cm}^{-1}\right): 2211,1631$ 


\subsection{References}

1. Criswell, T, R.; Klanderman, B. H. J. Org. Chem. 1974, 39, 771.

2. Nystrom, R. F.; Brown, W. G. J, Amer. Chem. Soc. 1948, 70, 3738.

3. $\quad$ Boyland, E.; Manson, D.; J. Chem. Soc. 1951, 1837.

4. Hodge, P.; Power, G. A.; Rabjohns, M. A. Chem. Commun. 1997, 73.

5. Rabideau, P. W. J. Org. Chem. 1971, 39, 2723.

6. Balaban, T. S.; Eichhöfera, A.; Krischeb, M. J.; Lehna, J.-M. Helvetica Chimica Acta, 2006. 89, 333.

7. Zhou, H.; Yang, L.; You, W. Macromolecules, 2012, 45, 607.

8. Zhang, Z.-G.; Wang, J. J. Mater. Chem. 2012, 22, 4178.

9. $\quad$ Ogawa, K.; Rasmussen, S. C. J. Org. Chem. 2003, 68, 2921.

10. Zhou, E.; Nakamura, M.; Nishizawa, T.; Zhang, Y.; Wei, Q.; Tajima, K.; Yang, C.; Hashimoto, K. Macromolecules, 2008, 41, 8302.

11. Tamilavan, V.; Song, M.; Jin, S.-H.; Hyun, M. H. Macromol. Res, 2013, 21, 406.

12. Tamilavan, V.; Song, M.; Jin, S.-H.; Hyun, M. H. Polymer, 2011, 52, 2384.

13. Tamilavan, V.; Shakthivel, P.; Li, Y.; Song, M.; Kim, C.-H.; Jin, S.-H.; Hyun, M. H. J. Polym. Sci. Part A Polym. Chem. 2010, 48, 3169.

14. Agneeswari, R.; Roh, K. H.; Tamilavan, V.; Lee, D. Y.; Cho, S.; Jin, Y.; Park, S. H.; $\quad$ Hyun, M. H. Polym. Bull. 2015, 72, 1899.

15. Hou, J.; Park, M.-H.; Zhang, S.; Yao, Y.; Chen, L.-M.; Li, J.-H.; Yang, Y. Macromolecules, 2008, 41, 6012.

16. (a) Miyuara, N.; Suzuki, A. Chem. Rev. 1995, 95, 2457. (b) Espinet, P.; Echavarren, A. M. Angew. Chem. Int. Ed. 2004, 43, 4704. (c) Cordovilla, C.; 
Bartolome, C.; Martínez-Ilarduya, J. M.; Espinet, P. ACS Catal. 2015, 5, 3040.

(d) Seechurn, C. C. C. J.; Kitching, M. O.; Colacot, T. J.; Snieckus, V. Angew. Chem. Int. Ed. 2012, 51, 5062.

17. (a) Chen, X.; Liu, B.; Zou, Y.; Xiao, L.; Guo, X.; Heb, Y.; Li, Y. J. Mater. Chem. 2012, 22, 17724. (b) Ie, Y.; Aso, Y. Polymer. Journal. 2017, 49, 13.

18. Kim, N.; Lee, B. H.; Choi, D.; Kim, G.; Kim, H.; Kim, J.-R.; Lee, J.;. Kahng, Y. H; Lee, K. Phys. Rev. Lett. 2012, 109, 106405. (b) Su, Y.-W.; Lan, S.-C.; Wei, K.-H. Mater. Today. 2012, 15, 554.

19. (a) Yuan, M.; Yang, P.; Durban, M. M.; Luscombe, C. K. Macromolecules, 2012, 45, 5934. (b) Ge, C.-W.; Mei, C.-Y.; Ling, J.; Wang, J.-T.; Zhao, F.-G.; Liang, L.; Li, H.-J.; Xie, Y.-S.; Li, W.-S. J. Polym. SCi. Part A: Polym. Chem.

20. Jung, I. H.; Lo, W.-Y.; Jang, J.; Chen, W.; Zhao, D.; Landry, E. S.; Lu, L.; Talapin, D. V.; Yu, L. Chem. Mater. 2014, 26, 3450.

21. (a) Roncali, J. Macromol. Rapid Commun. 2007, 28, 1761. (b) Ho, H. A.; Brisset, H.; Frere, P.; Roncali, J. J. Chem. Soc. Chem.Commun. 1995, 2309. (c) Casey, A.; Dimitrov, S. D.; Shakya-Tuladhar, P.; Fei, Z.; Nguyen, M.; Han, Y.; Anthopoulos, T. D.; Durrant, J. R.; Heeney, M. Chem. Mater. 2016, 28, 5110.

22. Michinobu, T. Chem. Soc. Rev. 2011, 40, 2306.

23. Michinobu, T.; Kumazawa, H.; K. Noguchi, K.; Shigehara, K. Macromolecules, 2009, 42, 5903.

24. Michinobu, T.; Fujita, H.; Materials, 2010, 3, 4773.

25. Michinobu, T. J. Am. Chem. Soc. 2008, 130, 14074.

26. Yuan Y.; Michinobu, T. J. Polym. Sci. Part A: Polym. Chem. 2011, 49, 225. 
27. Bures, F.; Schweizer, W. B.; Boudon, C.; Gisselbrecht, J.-P.; Gross, M.; Diederich, F. Eur. J. Org. Chem. 2008, 994. 


\section{Chapter 4 Synthesis and Characterizations of TPD-based Compounds and Conjugated Polymers}

\subsection{Introduction}

Thieno[3,4-c]pyrrole-4,6-dione (TPD) came to an attention as a unique building block unit due to its rigidity, good solubility and favorable 3D arrangement in the solid state. ${ }^{1}$ TPD can form a quinoidal thiophene-maleimide structure in the excited state, which lowers the energy band gap in TPD-based polymers. ${ }^{2,3}$ TPD is a moderate electron acceptor. ${ }^{4}$ The electron-accepting ability is judged by the LUMO energy level of the unit: the lower the LUMO energy level, the stronger the electron-accepting ability. ${ }^{4}$ Recently, different electron donor units have been used with TPD to synthesize donor-acceptor (DA) type polymers and their applications in bulk-heterojunction organic solar cells have been studied. ${ }^{1,5}$ TPD-based polymers were synthesized by Yamamoto cross-coupling, ${ }^{6}$ Ullmann coupling ${ }^{7,8}$ and direct heteroarylation. ${ }^{3}$ TPD-based D-A polymers have been studied in bulk heterojunction solar cells with the highest power conversion efficiency (PCE) of over $8 \%$ (inverted cell) with longer stability. ${ }^{5 \mathrm{e}, 9}$ The highest reported hole mobility in the TPD-based polymer is $1.4 \mathrm{~cm}^{2} \mathrm{~V}^{-1} \mathrm{~s}^{-1}$ due to the edge-on orientation and $1.16 \mathrm{~cm}^{2} \mathrm{~V}^{-1} \mathrm{~s}^{-1}$ due to the face-on orientation. ${ }^{10}$

Substituent effects of the branched and linear alkyl chains on the N atom of TPDbased copolymers have also been reported, in particular on the $\pi$-stacking distances between the polymer backbones and optical properties. ${ }^{3,8,5 \mathrm{~h}}$ The $\pi$-stacking distance is reduced from branched alkyl chain to linear alkyl chain, as a result, face-on orientation is enhanced, which helps to charge transport and increase PCE. ${ }^{5 \mathrm{~h}}$ TPD homopolymers with branched alkyl chains have absorption at a longer wavelength but the optical band gap 
does not change much. ${ }^{3}$ In a D-A copolymer system, the absorption is red-shifted for linear alkyl chain. However, the optical band gap is quite similar. ${ }^{5 h}$ Results show that homopolymer with branched alkyl chain has higher solubility in common organic solvents compared to the linear alkyl chain. The photodetector properties of TPD-based donor-acceptor polymers were studied by Zhang et al. They reported the dark current at $0.2 \mathrm{~V}$ is $5.9 \times 10^{-9}$, the EQE is over $50 \%$ in the spectral range of 500 to $640 \mathrm{~nm}$ and the detectivity is over $10^{13}$ Jones in the spectral range of 350 to $640 \mathrm{~nm}^{.11}$

\subsection{Design strategy and synthetic routes}

Monomer M7 (1,3-dibromo-5-(2-ethylhexyl)-thieno[3,4-c]pyrrole-4,6-dione) possesses a fused thiophene unit and two keto groups in the pyrrole moiety and was chosen for this research work. Polymerization on the thiophene unit and on the keto group can lead to a ladder conjugated polymer. TPD has been incorporated into polymers mainly by the polymerization on the thiophene unit. To the best of our knowledge, there are no reports on the synthesis of ladder polymers by intramolecular cyclization using Lawesson's Reagent (LR) on TPD-based polymers. It would be an advantage to develop an efficient method to synthesize a low band gap ladder polymer derived from M7.

We intended to explore a new series of linear and ladder polymers first by intermolecular coupling reaction on the thiophene unit of a TPD monomer and subsequently by intramolecular coupling reaction on the keto group in the linear polymer. The synthetic routes are proposed as shown in Scheme 4.1. Firstly, the linear polymer is to be synthesized by the Yamamoto reaction on the thiophene unit followed by the 
intramolecular cyclization using LR. Secondly, linear and ladder copolymers were designed to adjust the solubility and optoelectronic properties.
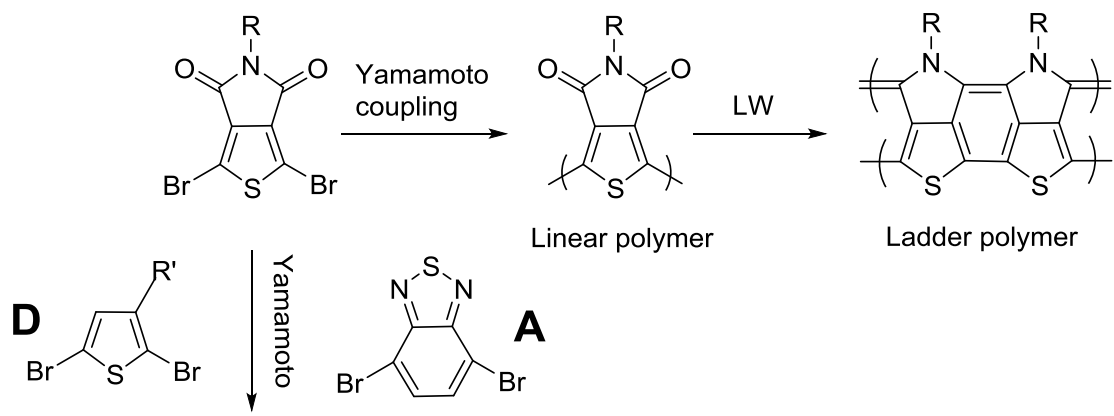

Linear polymer

Ladder polymer
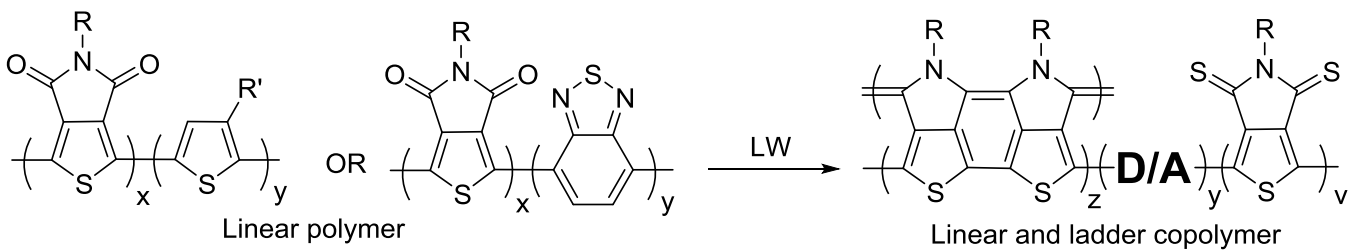

Scheme 4.1 Synthetic routes to linear and ladder conjugated polymers derived from TPD.

\subsection{Synthesis and characterizations of monomer M7 and homopolymer of TPD- thioimide}

The acceptor monomer $\mathbf{M 7}$ was synthesized according to the reactions in Scheme 4.2 in $63 \%$ yield. The neat condensation reaction between thiophene-3,4-dicarboxylic acid and 2-ethylhexylamine at $190-210{ }^{\circ} \mathrm{C}$ produced compound 10. Monomer M7 was synthesized in $63 \%$ yield by bromination with $N$-bromosuccinimide (NBS) under an acidic condition. Monomer M7 was characterized by IR, NMR spectroscopy and melting point. The IR spectrum (Figure S4.5) of M7 shows several peaks at 1768 and $1696 \mathrm{~cm}^{-1}$ which are the characteristic asymmetric and symmetric peaks for cyclic 5-membered imide. The ${ }^{1} \mathrm{H}$ NMR spectrum (Figure S4.6) of $\mathbf{M 7}$ does not show any aromatic peaks over $7 \mathrm{ppm}$, indicating the thiophene protons at position 2 and 5 are substituted by bromine. The doublet peak at $\delta 3.50 \mathrm{ppm}$ and the multiplet peak at $\delta 1.85 \mathrm{ppm}$, which are 
assigned to $\mathrm{NCH}_{2}-\mathrm{CH}$ and $\mathrm{NCH}_{2}-\mathbf{C H}$ protons, respectively. The ${ }^{13} \mathrm{C}$ NMR spectrum (Figure 4.7) shows a peak at $\delta 160.76 \mathrm{ppm}$, indicating the imide $\mathrm{C}=\mathrm{O}$ moiety of the monomer M7. The obtained melting point is in the range of $115-117^{\circ} \mathrm{C}$, as reported in the literature. ${ }^{5 \mathrm{~h}}$

Polymer P27 was synthesized by the polymerization of monomer M7 in $80 \%$ yield (Scheme 4.2) by the Yamamoto reaction using $\mathrm{Ni}(\mathrm{COD})_{2}$ as a catalyst in toluene, followed by the thionation of keto with LR at $160{ }^{\circ} \mathrm{C}$. The obtained polymer P27 was purified by the procedure described in Chapter 2 Section 2.4. The obtained polymer was a dark powder.

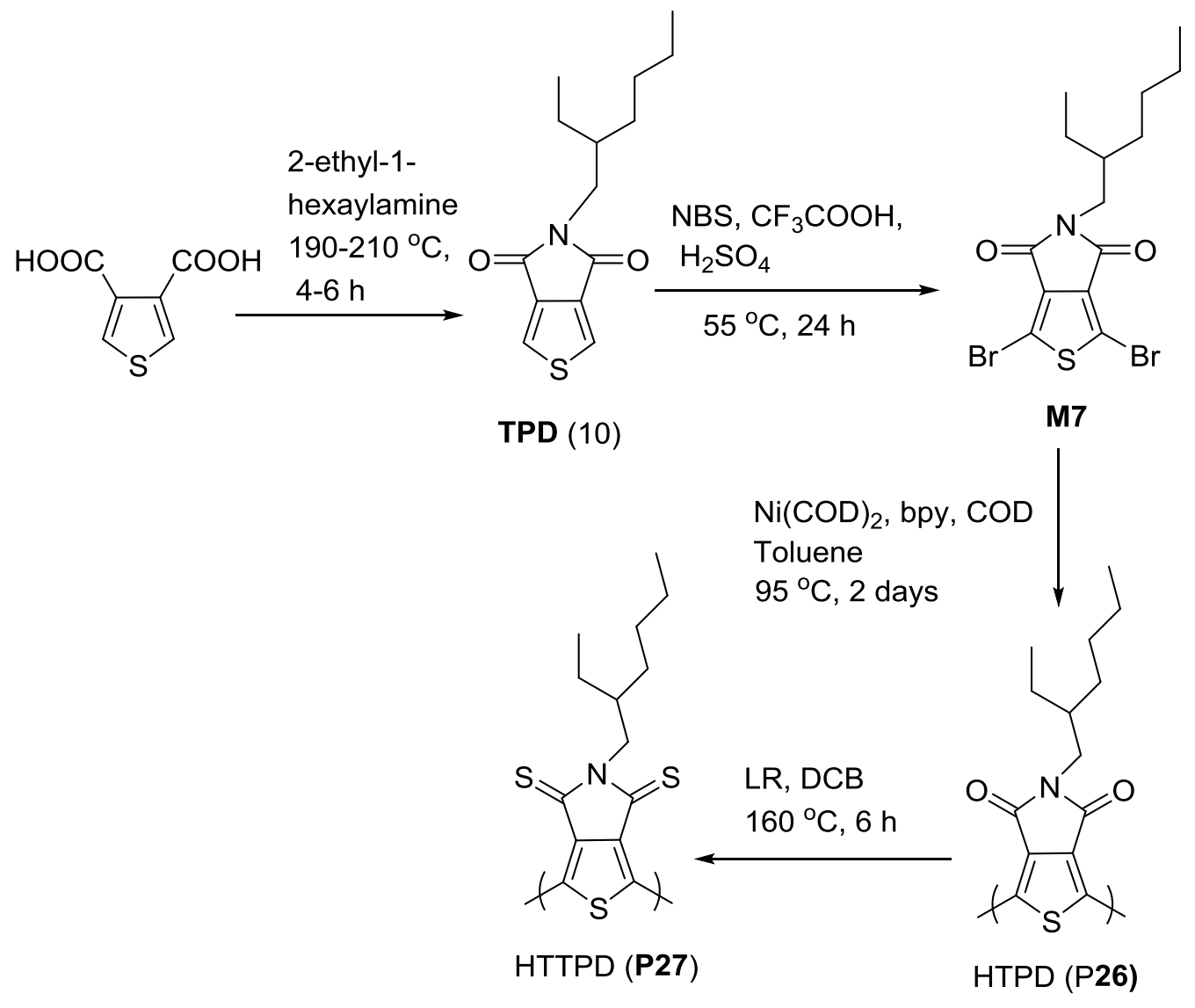

Scheme 4.2 Synthetic route to monomer M7 and homopolymers HTPD (P26) and HTTPD (P27). 
Polymers P26 and P27 were characterized by IR, NMR, TGA, absorption, emission spectroscopy and CV. The IR spectrum (Figure 4.1a) of polymer P26 shows the sharp weak peak at $1780 \mathrm{~cm}^{-1}$ and the sharp strong peak at $1706 \mathrm{~cm}^{-1}$, which are the characteristic asymmetric and symmetric peaks of imide. The ${ }^{1} \mathrm{H}$ NMR spectrum (Figure 4.1a) of polymer P26 shows broad peaks in the aliphatic region, indicating the polymer's formation. The ${ }^{13} \mathrm{C}$ NMR spectrum (Figure 4.1a) shows peaks at $\delta 162.35$ and 162.66 ppm, indicating the imide $\mathrm{C}=\mathrm{O}$ moiety of polymer P26. The IR spectrum (Figure 4.1b) of polymer P27 shows no strong peaks at 1780 to $1706 \mathrm{~cm}^{-1}$ which confirms the absence of imide CO. The strong peaks at 1345 and $1260 \mathrm{~cm}^{-1}$ are the characteristic peaks for imide $\mathrm{C}=\mathrm{S}$ moiety, indicating the imide $\mathrm{C}=\mathrm{O}$ converted to $\mathrm{C}=\mathrm{S} .{ }^{1} \mathrm{H}$ NMR (Figure 4.1b) shows the paramagnetic shift of $\mathrm{NCH}_{2}-\mathrm{CH}$ and $\mathrm{NCH}_{2}-\mathbf{C H}$ moieties at $\delta 3.53$ and $1.85 \mathrm{ppm}$ to $\delta$ 4.30 and $2.18 \mathrm{ppm}$. This paramagnetic shift is emphasizing the presence of higher polarisable atom than oxygen which deshields the $\mathrm{NCH}_{2}-\mathrm{CH}$ and $\mathrm{NCH}_{2}-\mathbf{C H}$ moieties. The ${ }^{13} \mathrm{C}$ NMR spectrum (Figure 4.1b) shows peaks at $\delta 189.59,188.46$, and $187.62 \mathrm{ppm}$, indicating the presence of higher polarizable atom and a stronger deshielding effect than oxygen. ${ }^{12,13}$ This provides the confirmation that polymer $\mathbf{P 2 7}$ contains the $\mathrm{C}=\mathrm{S}$ group. The thermal stability of the polymers was compared by thermogravimetric analysis as presented in Figure 4.2, which reveals that the onset temperatures of $1 \%$ weight loss for polymers P26 and P27 are 264.1 and $157.5^{\circ} \mathrm{C}$, respectively. It was also noticed that the temperature for $5 \%$ weight loss followed the same trend (P26 $>$ P27), indicating the thermal stability of polymer $\mathbf{P 2 7}$ decreased, which may be due to the $\mathrm{C}=\mathrm{S}$ group. Polymer P26 has a higher $\mathrm{T}_{\mathrm{d}}$ value due to the rigid imide structure. Polymer P26 showed very high molecular weight (Figure S4.11) with a PDI of 2.07. 

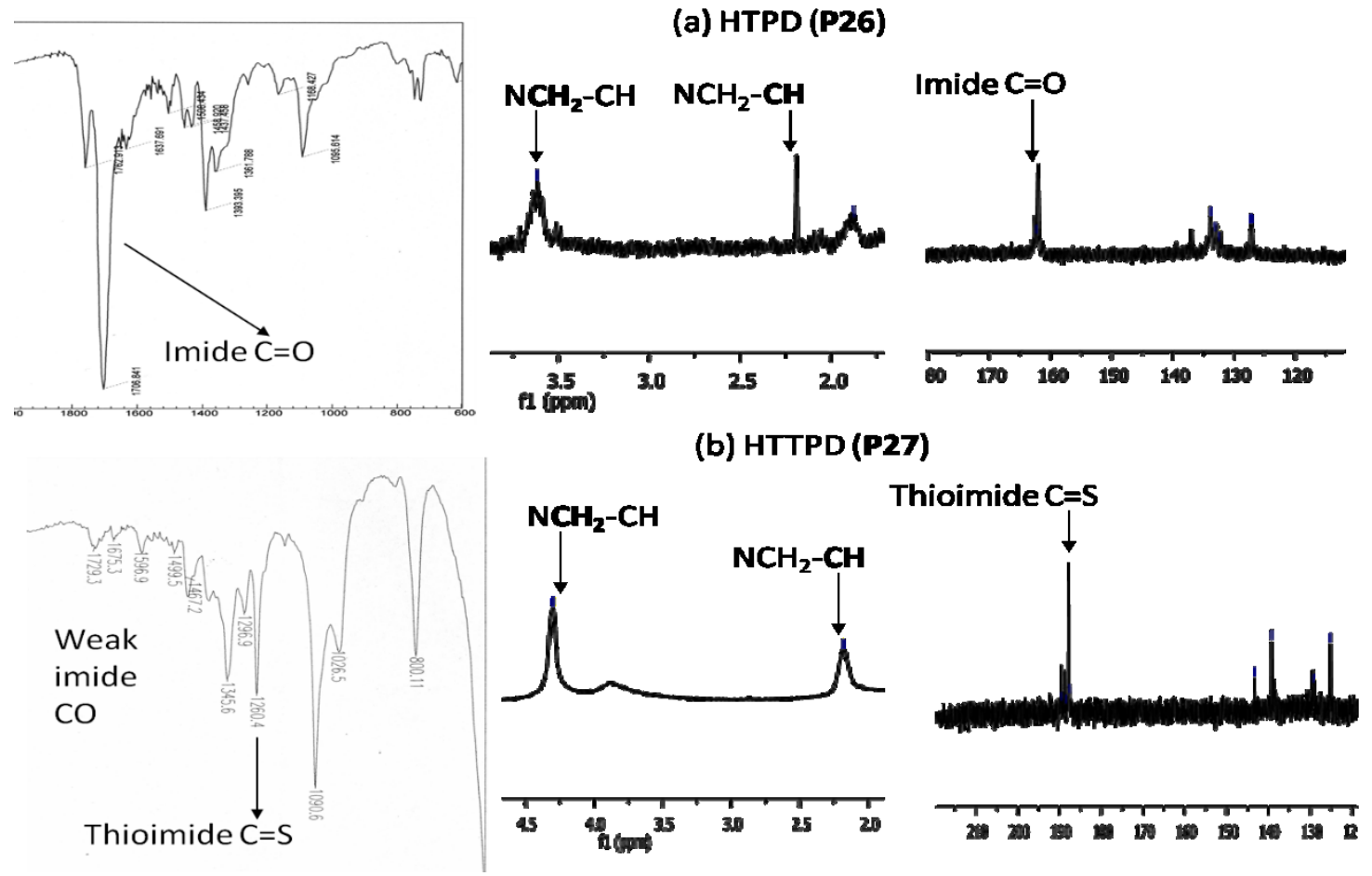

Figure 4.1 IR, ${ }^{1} \mathrm{H}$ NMR and ${ }^{13} \mathrm{C}$ NMR spectra of polymers P26 (a) and P27 (b).

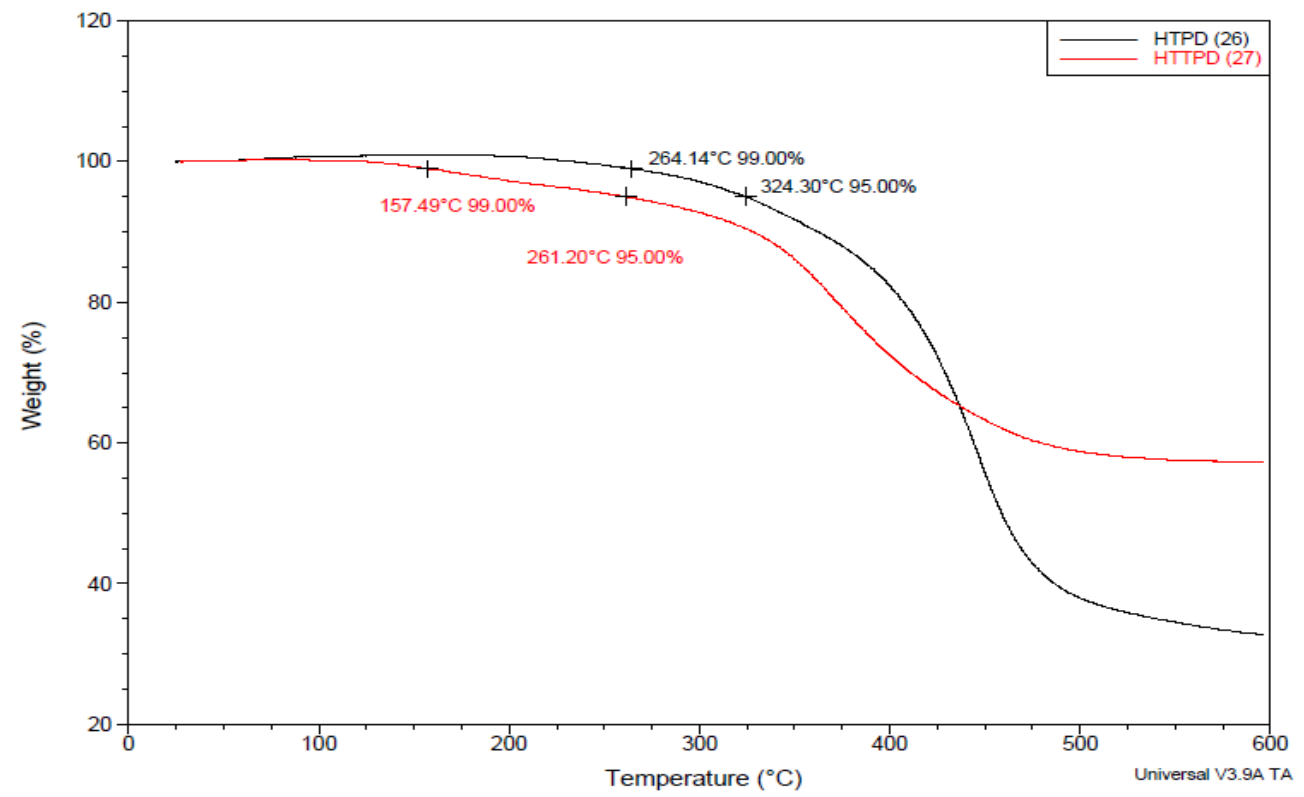

Figure 4.2 TGA plots of the polymers $\mathbf{P 2 6}$ and $\mathbf{P 2 7}$ with a heating rate of $10^{\circ} \mathrm{C} / \mathrm{min}$ under nitrogen atmosphere. 

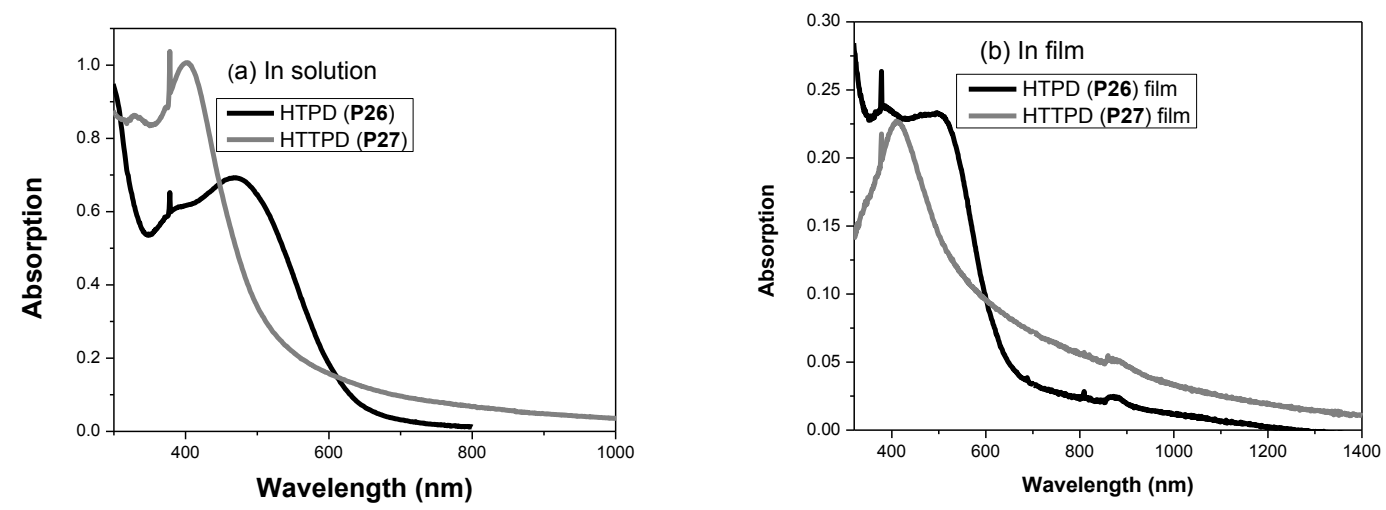

Figure 4.3 Absorption spectra of polymers P26 $\left(3.03 \times 10^{-6} \mathrm{M}\right)$ and P27 $\left(3.23 \times 10^{-6} \mathrm{M}\right)$ in $\mathrm{CHCl}_{3}$ solution (a) and as thin films on glass substrate (b).

Polymers P26 and P27 were fully soluble in organic solvents such as $\mathrm{CHCl}_{3}$, chlorobenzene, dichlorobenzene and trichlorobenzene. Absorption spectra of P26 and $\mathbf{P 2 7}$ in $\mathrm{CHCl}_{3}$ solutions and spin-coated thin solid films on glass substrates were taken. Polymer P26 displays a maximal absorption peak at $470 \mathrm{~nm}$ in $\mathrm{CHCl}_{3}$ with an onset from $660 \mathrm{~nm}$ (Figure 4.3a), whereas polymer P27 shows a maximal absorption peak at $402 \mathrm{~nm}$ with an onset from $650 \mathrm{~nm}$. The film of P26 shows a (Figure 4.3b) maximal absorption peak at $501 \mathrm{~nm}$ with an onset from $700 \mathrm{~nm}$, whereas the polymer P27 shows a maximal absorption peak at $415 \mathrm{~nm}$ with an onset from $680 \mathrm{~nm}$. This blue shift of absorption can be explained by the lower electron-withdrawing nature of thioimide moiety and larger sulfur atom. This less electron-withdrawing nature increases the difference between HOMO and LUMO energy levels hence the energy band gap. The emission spectra of polymer P26 (Figure S4.12) in $\mathrm{CHCl}_{3}$ shows a shoulder at $580 \mathrm{~nm}$ and a maximum at $543 \mathrm{~nm}$ whereas polymer $\mathbf{P 2 7}$ in $\mathrm{CHCl}_{3}$ shows no emission. The solid films of polymers 
P26 and P27 do not show any emission due to the aggregation caused and/or chargetransfer induced quenching.
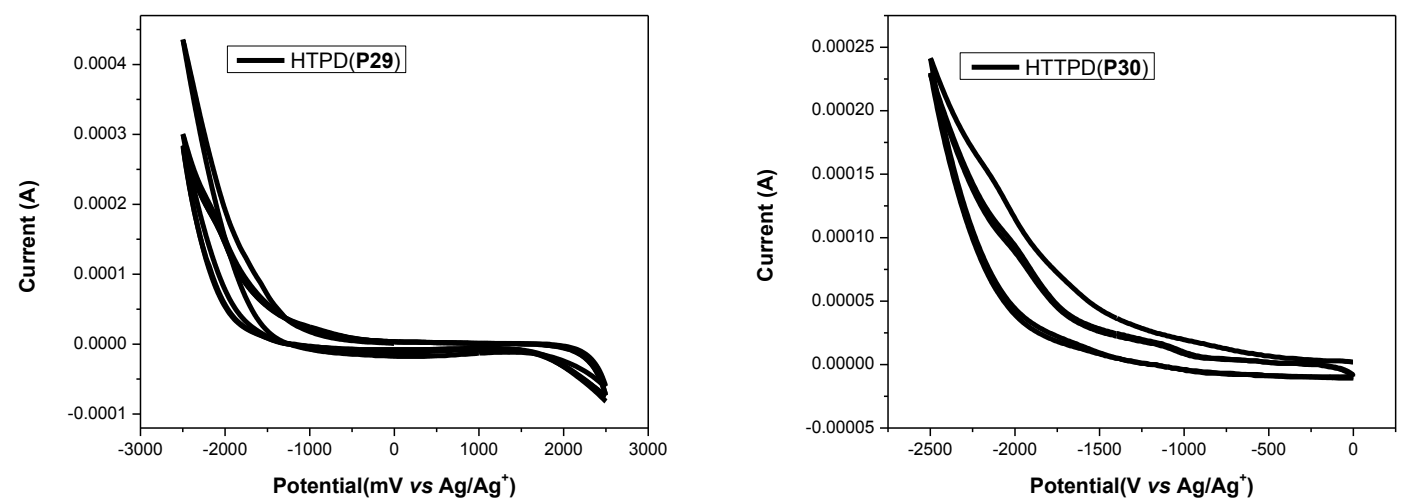

Figure 4.4 Cyclic voltammograms of polymer films on Pt electrode in $0.1 \mathrm{~mol} / \mathrm{L} \mathrm{Bu}_{4} \mathrm{NP}_{6}$, $\mathrm{CH}_{3} \mathrm{CN}$ solution with a scan rate of $100 \mathrm{mV} / \mathrm{s}$. Full cycle of $\mathbf{P 2 6}$ (a) and reduction of $\mathbf{P 2 7}$ (b).

The CV of polymers P26 and P27 are shown in Figure 4.4. Polymer P27 shows two reduction processes, whereas $\mathbf{P 2 6}$ has no observable oxidation or reduction process. The reduction onsets of polymer P27 are -0.95 and -1.79 in $\mathrm{CV}$. The LOMO energy levels of the polymer P27 are $-3.41 \mathrm{eV}$ and $-2.57 \mathrm{eV}$. The optical energy band gaps of P26 and P27 are $1.77 \mathrm{eV}$ and $1.82 \mathrm{eV}$, respectively. The optical energy gap of P26 and $\mathbf{P 2 7}$ are $1.87 \mathrm{eV}$ and $1.90 \mathrm{eV}$, respectively. From the optical energy band gaps, it can be concluded that the increase of band gap of $\mathbf{P 2 7}$ is due to the presence of $\mathrm{C}=\mathrm{S}$ moiety instead of $\mathrm{C}=\mathrm{O}$ 


\subsection{Result and discussion}

Our goal was to synthesize a ladder polymer from TPD monomer M7 by Yamamoto reaction followed by keto-keto coupling using LR. A homopolymer P26 was successfully synthesized from monomer M7, which was successfully characterized by spectroscopic analysis. Polymer P27 was obtained by treating P26 with LR. The IR spectrum of polymer P27 does not show any new strong $C=C$ peak in the region of 1600 $\mathrm{cm}^{-1}$, which indicates there was no keto-keto coupling reaction using LR. The absence of imide $\mathrm{C}=\mathrm{O}$ confirms the reaction between the polar $\mathrm{C}=\mathrm{O}$ and $\mathrm{LR}$. In 2001, Orzeszko et al. reported their investigation of the thionation reaction of cyclic imides at refluxing conditions in toluene using LR. ${ }^{13}$ They selected different imides based on phenyl and naphthyl units. They observed that the polarity of $\mathrm{CO}$ and steric hindrance in the imide structure influenced the thionation reaction and provided mono- and bis-thioimide. Naphthyl-based imides provided mostly bisthioimide product. They did not find any monothioimide products for naphthyl imides. On the other hand, phenyl-based imides provided a mixture of products in low yields. The bulky substituent on nitrogen produced mostly mono-substituted thioimide.

The resultant polymer after the reaction between polymer P26 and LR at high temperature $\left(160{ }^{\circ} \mathrm{C}\right)$ did not show any imide $\mathrm{C}=\mathrm{O}$ peak in the IR spectrum, which indicates the thiophene imide in polymer backbone was polar enough and that the branched alkyl chain did not hinder the reaction pathway. The higher percentage of yield indicates that a reaction at a higher temperature and longer time (6h compared to $1 \mathrm{~h}$ ) may facilitate the thionation reaction. Ozturk et al. reported that thionation is successful at refluxing temperatures of solvents such as benzene, pyridine, THF, dimethoxyethane, 
$\mathrm{CH}_{2} \mathrm{Cl}_{2}$, and $\mathrm{CS}_{2}$ in an inert atmosphere. ${ }^{14}$ On the other hand, the reaction of keto with LR at high temperature yielded $\mathrm{C}=\mathrm{C}$ bond via thionation. ${ }^{15}$ However, the absence of $\mathrm{C}=\mathrm{C}$ double bond in the IR spectrum for polymer P27 indicates no coupling between keto groups of imide at this higher temperature and longer reaction time. The strong peaks at 1345 and $1260 \mathrm{~cm}^{-1}$ strongly recommend the thioimide. Polonski et al. reported that paramagnetic shift of $\mathrm{C}=\mathrm{S}$ over $190 \mathrm{ppm}$ in ${ }^{13} \mathrm{C}$ NMR spectrum. ${ }^{12}$ Polymer $\mathbf{P 2 7}$ shows peaks at 187 to $189 \mathrm{ppm}$ and no peak from 160 to $180 \mathrm{ppm}$, which also recommends the thioimide formation. Cyclization on polymer P26 will provide a ladder polymer, therefore, the solubility should change and the polymer may require highly polar aprotic solvents. However, the change of solubility was not observed. Polymer P27 was highly soluble in the same solvent as P26. Ladder polymers usually display lower band gaps and bathochromic shifts compared to their parent polymers, whereas polymer P27 shows higher optical band gap (1.82 eV compared to $1.77 \mathrm{eV})$ and hypsochromic shift (402 nm compared to $470 \mathrm{~nm}$ in solution). ${ }^{16,17}$ The thermal stability was also reported to be higher in ladder polymers compared to parent polymers but P27 shows lower thermal stability. ${ }^{15 a}$ Therefore, it can be said that the reaction on polymer P26 using LR at a high temperature produced a thioimide homopolymer rather than the ladder polymer. Moreover, the stability of the thioimide compared to thioketone compounds gave us an opportunity to synthesize a model compound similar to polymer P27 to investigate the polymer structure. $^{14}$ 


\subsection{Synthesis and characterizations of thieno[3,4-c]pyrrole-4,6-dione (TPD)-based compounds}

As we were not successful with intramolecular keto-keto coupling using LR in imide homopolymer P26, we intended to make a model compound that possesses two TPD units. An intramolecular reaction of this model compound would provide the information that either the chemical structure of the imide forms thioimide or a cyclized product by keto-keto coupling. Compound $\mathbf{1 3}$ as a model for TPD homopolymer was synthesized and investigated the feasibility of the intramolecular keto-keto coupling of the TPD unit in the polymer. TPD-based compounds and their thio-analogue were synthesized according to the reactions in Scheme 4.3. Compound $\mathbf{1 0}$ was treated with LR at high temperature in an argon atmosphere and dry condition, which produced compound 11 in $89 \%$ yield. Selective debromination on monomer $\mathbf{M 7}$ using zinc and ethanol provided compound $\mathbf{1 2}$ in $77 \%$ yield. The Yamamoto cross-coupling reaction of compound 12 produced dimer 13 in 66\% yield. Compound 13 was treated with LR at a high temperature in an argon atmosphere and dry condition which produced compound 14 in $69 \%$ yield. Neither further heating of compound 14 nor the reaction of compound 14 with LR provided any cyclized or polymer product.Compound 13 was treated with LR and we obtained compound $\mathbf{1 4}$ instead of cyclized product or polymer. To confirm this reaction, we again treated compound $\mathbf{1 4}$ under two different conditions, (a) heating at 200 ${ }^{\circ} \mathrm{C}$ in TCB and (b) using LR. Neither of the method provided any cyclized product or polymers, which confirmed the TPD compounds do not participate in the keto-keto coupling. Moreover, another reaction was completed with TPD (compound 10) and LR, similar to the model reaction in Section 2.11. In Chapter 2 we synthesized monomer M2 
and carried out a reaction with LR to understand the keto-keto coupling. A similar reaction was conducted with TPD and LR. The result showed that it ended up by forming thio-TPD 11 rather than a polymer. Orzeszko et al. reported that less hindered imides provide higher percentage of yield with dithio products and hindered imide produced mixtures of products. Compound $\mathbf{1 1}$ was obtained with higher percentage of yield than compound 14 when compound 10 and 13 were treated with LR, respectively. However, the yield for the obtained polymer P27 from P26 was higher than the small compounds. Therefore, the yield could vary. Leclerc et al. reported that TPD dimers in the solid state possess interaction between the carbonyl of the pyrroledione moiety and the sulfur of the thiophene unit. Therefore, two neighboring TPD units adopt an anticoplanar conformation. ${ }^{3,18}$ To the best of our knowledge, the ladderization by intramolecular cyclization of keto does suffer any steric strain has not been mentioned in literature. ${ }^{15,19}$

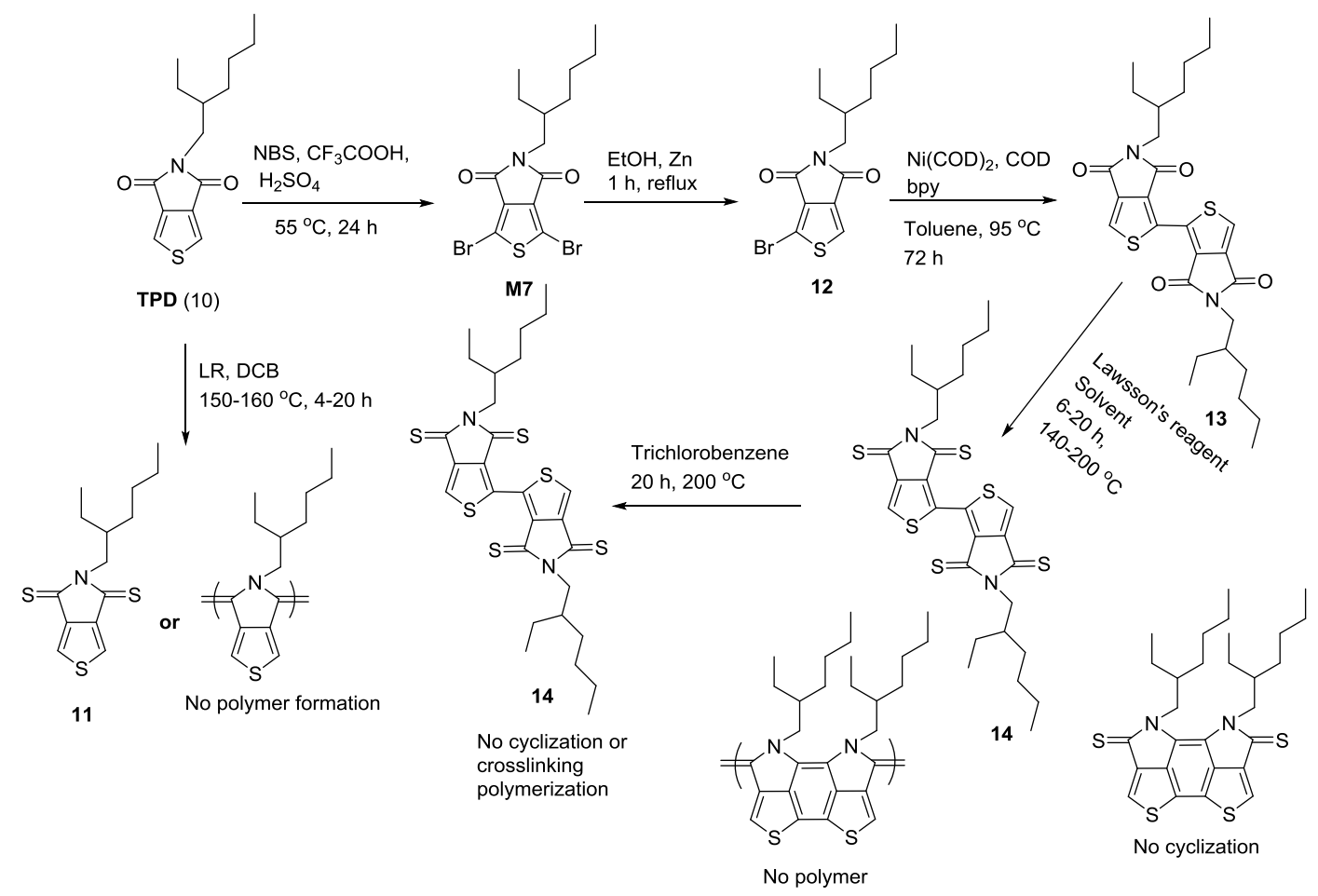

Scheme 4.3 Synthesis of TPD-based model compounds. 


\subsection{Characterizations of thio-imide compounds}

Compounds 11, 13, and 14 were synthesized according to the reaction in Scheme 4.3. These compounds were characterized by IR, NMR, and mass spectroscopy. The IR spectrum (Figure 4.5a) of compound 10 shows several peaks at 1768, 1721, and $1690 \mathrm{~cm}^{-}$

${ }^{1}$, which are the characteristic asymmetric and symmetric peaks for cyclic 5-membered imide. The ${ }^{1} \mathrm{H}$ NMR spectrum (Figure 4.5a) of compound $\mathbf{1 0}$ shows a singlet peak at $\delta$ 7.82 a doublet peak at $\delta 3.57$ and a multiplet peak at $\delta 1.82 \mathrm{ppm}$ which are assigned to aromatic, $\mathrm{NCH}_{2}-\mathrm{CH}$ and $\mathrm{NCH}_{2}-\mathbf{C H}$ protons. The ${ }^{13} \mathrm{C}$ NMR spectrum (Figure 4.5a) shows a peak at $\delta 162.95 \mathrm{ppm}$, indicating the imide $\mathrm{C}=\mathrm{O}$ moiety of compound $\mathbf{1 0}$. The IR spectrum (Figure 4.5b) of compound 11 shows no peaks at 1768 to $1690 \mathrm{~cm}^{-1}$, which indicates the disappearance of imide $\mathrm{CO}$ and the formation of thioimide. The strong peak at $1345 \mathrm{~cm}^{-1}$ is the characteristic peak for the $\mathrm{C}=\mathrm{S}$ moiety, which suggests the imide $\mathrm{C}=\mathrm{O}$ converted to $\mathrm{C}=\mathrm{S}$. The ${ }^{1} \mathrm{H}$ NMR spectrum (Figure $4.5 \mathrm{~b}$ ) of compound $\mathbf{1 1}$ displays the aromatic peak at $\delta 7.83 \mathrm{ppm}$, which is at the same position as compound $\mathbf{1 0}$. However, the doublet peaks and multiplet peaks for $\mathrm{NCH}_{2}-\mathrm{CH}$ and $\mathrm{NCH}_{2}-\mathbf{C H}$ moieties show a paramagnetic shift at $\delta 4.28$ and $2.19 \mathrm{ppm}$ due to the polarizing effect/deshielding effect of the sulfur atom. The ${ }^{13} \mathrm{C}$ NMR spectrum (Figure 4.5b) shows a peak at $\delta 190.84 \mathrm{ppm}$, indicating the thioimide, $\mathrm{C}=\mathrm{S}$ moiety of compound 11. The mass spectrum (Appendix $\mathrm{C}$, Figure S4.19) shows a molecular ion peak at $\mathrm{m} / \mathrm{z} 297.07193$, which corresponds to the desired compound $\mathbf{1 1 .}$

The IR spectrum (Appendix C, Figure S4.24) of compound 13 shows two peaks at 1759 and $1694 \mathrm{~cm}^{-1}$, which can be assigned to the asymmetric and symmetric peaks for the cyclic 5-membered imide. The ${ }^{1} \mathrm{H}$ NMR spectrum (Appendix C, Figure S4.25) of 
compound 13 displays a singlet peak at $\delta 7.81$ a doublet peak at $\delta 3.53$ and a multiplet peak at $\delta 1.81 \mathrm{ppm}$, which are assigned as aromatic, $\mathrm{NCH}_{2}-\mathrm{CH}$ and $\mathrm{NCH}_{2}-\mathbf{C H}$ protons. The aromatic proton and $\mathrm{NCH}_{2}-\mathbf{C H}$ proton ratio is $1: 1$ which indicates a dimer and thus the structure of compound $\mathbf{1 3}$ is correct. The ${ }^{13} \mathrm{C}$ NMR spectrum (Appendix C, Figure S4.26) shows two peaks at $\delta 162.92$ and $162.17 \mathrm{ppm}$, indicating the imide, $\mathrm{C}=\mathrm{O}$ moiety of the compound 13. The two different $\mathrm{C}=\mathrm{O}$ peaks indicate the dimer structure of compound 13. The mass spectrum (Appendix C, Figure S4.27) shows a molecular ion peak at m/z 528.20786, which corresponds to the desired compound $\mathbf{1 3}$.

(a) Compound 10
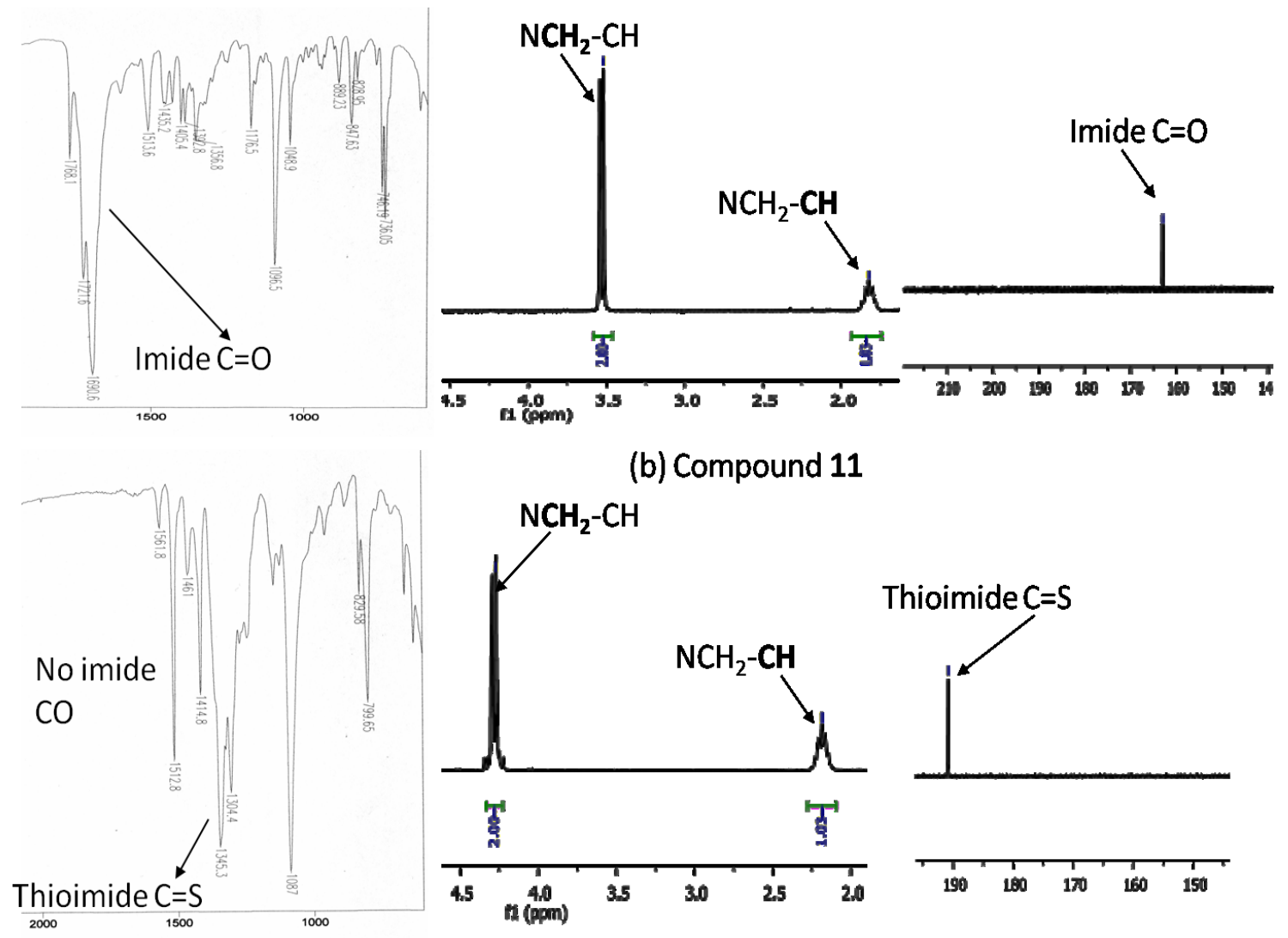

Thioimide $\mathrm{C}=\mathrm{S}$

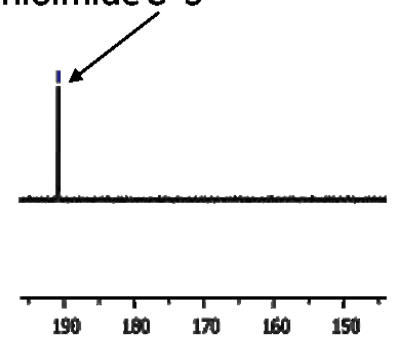

Figure 4.5 IR, ${ }^{1} \mathrm{H}$ NMR and ${ }^{13} \mathrm{CNMR}$ spectra of compounds $\mathbf{1 0}$ and $\mathbf{1 1 .}$ 
For compound 14 the absence of the peak at 1759 to $1694 \mathrm{~cm}^{-1}$ and appearances of the peak at $1345 \mathrm{~cm}^{-1}$ in the IR spectrum (Appendix C, Figure S4.28) confirms the formation of thioimide. The strong peak at $1345 \mathrm{~cm}^{-1}$ is the characteristic peak for the $\mathrm{C}=\mathrm{S}$ moiety, which indicates the imide $\mathrm{C}=\mathrm{O}$ converted to $\mathrm{C}=\mathrm{S}$. The ${ }^{1} \mathrm{H}$ NMR spectrum (Appendix C, Figure S4.29) of compound $\mathbf{1 4}$ shows the peak at $\delta 7.90 \mathrm{ppm}$ which is the aromatic proton of thiophene. The doublet peaks and the multiplet peaks for $\mathrm{NCH}_{2}-\mathrm{CH}$ and $\mathrm{NCH}_{2}-\mathbf{C H}$ moieties display a paramagnetic shift at $\delta 4.29$ and $2.19 \mathrm{ppm}$ due to the polarizing effect/deshielding effect of the sulfur atom. The ${ }^{13} \mathrm{C}$ NMR spectrum (Appendix C, Figure S4.30) shows two peaks at $\delta 189.61,188.47 \mathrm{ppm}$, indicating the thioimide, $\mathrm{C}=\mathrm{S}$ moiety of the compound $\mathbf{1 4}$. Two $\mathrm{C}=\mathrm{S}$ peaks also confirm the compound $\mathbf{1 4}$ is a dimer. The mass spectrum (Figure S4.31) shows a molecular ion peak at m/z 592.11766, which corresponds to the desired compound 14.

\subsection{Conclusion}

The formation of a carbon-carbon double bond between the keto groups of imide compound is not possible. The imide compound forms thioimide in the presence of Lawesson's Reagent at high temperature over $160{ }^{\circ} \mathrm{C}$ in dry condition and under argon. Model compounds 11 and $\mathbf{1 4}$ represent the thionation of imides in the presence of LR. The conversion of TPD-homopolymer to its thio-analogue provides high energy band gap polymer. We planned to synthesize and study the solubility and opto-electronic properties of ladder polymer from the homopolymer of TPD and linear ladder-polymers with rigid fused rings and linear units. However, our model compounds demonstrated that it is not 
possible to synthesize ladder polymer from the homopolymer of TPD, therefore, we did not study the other polymers.

\subsection{Experimental section}

\section{Thieno[3,4-c]pyrrole-4,6-dione (TPD, 10)}

In a flame dried two-neck round-bottom flask under an argon atmosphere, thiophene-3,4-dicarboxalic acid $(1.00 \mathrm{~g}, 5.80 \mathrm{mmol})$ and 2-ethylhexaylamine $(0.800 \mathrm{~g}$, $6.20 \mathrm{mmol}$ ) were added. The neat reaction mixture was gradually warmed to $190-210{ }^{\circ} \mathrm{C}$ in an oil bath for 4-6 $\mathrm{h}$. The reaction mixture was cooled and purified by column chromatography on silica gel using hexane as an eluting solvent. Compound $\mathbf{1 0}$ was obtained as white solid $(0.830 \mathrm{~g}, 54.0 \%$ yield $)$ after removing the solvent. ${ }^{1} \mathrm{H}$ NMR (300 $\left.\mathrm{MHz}, \mathrm{CDCl}_{3}\right): \delta 7.82(\mathrm{~s}, 2 \mathrm{H}), 3.53(\mathrm{~d}, J=7.3 \mathrm{~Hz}, 2 \mathrm{H}), 1.83(\mathrm{dd}, J=12.6,6.4 \mathrm{~Hz}, 1 \mathrm{H})$,

$1.44-1.19(\mathrm{~m}, 8 \mathrm{H}), 0.98-0.83(\mathrm{~m}, 6 \mathrm{H}) .{ }^{13} \mathrm{C} \mathrm{NMR}\left(75 \mathrm{MHz}, \mathrm{CDCl}_{3}\right): \delta 162.95,136.84$ $125.42,42.35,38.31,30.39,28.32,23.95,23.00,14.24,10.40$. HRMS calculated for $\left[\mathrm{M}^{+}\right]$ 265.11365, found 265.11141. IR ( $\mathrm{NaCl}$ plate, $\left.\mathrm{cm}^{-1}\right): 3081,2954,2923,2857,1766,1693$, $1605,1514,1096$.

\section{1,3-Dibromo-5-(2-ethylhexyl)-thieno[3,4-c]pyrrole-4,6-dione (M7)}

To a solution of TPD $(0.800 \mathrm{~g}, 3.02 \mathrm{mmol})$ in $\mathrm{H}_{2} \mathrm{SO}_{4} / \mathrm{CF}_{3} \mathrm{COOH}(3.5 \mathrm{~mL} / 12 \mathrm{~mL})$ was added $N$-bromosuccinimide $(2.15 \mathrm{~g}, 12.1 \mathrm{mmol})$ in one portion. After stirring for 24 h at $55^{\circ} \mathrm{C}$ in dark, the mixture was poured into ice. Dichloromethane was added to the mixture and shaken vigorously. The organic portion was separated and extracted three times by dichloromethane. The combined organic portions were dried over magnesium 
sulfate. The solvent was removed under reduced pressure. The crude product was further purified column chromatography on silica gel using dichloromethane and hexane (1:1 v/v) as eluting solvent. Compound M7 was obtained as pale brownish fleecy solid (0.830 g, $63.0 \%$ yield) after removing the solvent. ${ }^{1} \mathrm{H}$ NMR $\left(300 \mathrm{MHz}, \mathrm{CDCl}_{3}\right): \delta 3.50(\mathrm{~d}, J=$ $7.2 \mathrm{~Hz}, 2 \mathrm{H}), 1.85-1.71(\mathrm{~m}, 1 \mathrm{H}), 1.43-1.17(\mathrm{~m}, 8 \mathrm{H}), 0.91(\mathrm{td}, J=7.0,3.9 \mathrm{~Hz}, 6 \mathrm{H}) .{ }^{13} \mathrm{C}$ NMR $\left(75 \mathrm{MHz}, \mathrm{CDCl}_{3}\right): \delta 160.76,134.72,112.93,42.62,38.17,30.50,28.52,23.82$, 22.94, 14.19, 10.30. IR (KBr pellet, $\left.\mathrm{cm}^{-1}\right):$ 2964, 2930, 2858, 1768, 1696, 1577, 1536. Melting point $115^{\circ} \mathrm{C} .{ }^{5 \mathrm{~h}}$

\section{Thieno[3,4-c]pyrrole-4,6-dithione (11)}

A solution of compound $\mathbf{1 0}(0.100 \mathrm{~g}, 0.380 \mathrm{mmol})$ in 1,2-dichlorobenzene was degassed and purged with argon for $30 \mathrm{~min}$ then Lawesson's reagent $(0.380 \mathrm{~g}, 0.950$ mmol) was added. The mixture was heated at $150-160{ }^{\circ} \mathrm{C}$ for $6 \mathrm{~h}$ under argon. The solution was cooled and extracted three times with dichloromethane as well as dried over $\mathrm{Na}_{2} \mathrm{SO}_{4}$. The crude product was purified by column chromatography using hexane and acetone $(9: 1 \mathrm{v} / \mathrm{v})$ as the eluting solvent. A reddish solid (11) was obtained after evaporating the solvent $\left(0.100 \mathrm{~g}, 89.2 \%\right.$ yield). ${ }^{1} \mathrm{H}$ NMR $\left(300 \mathrm{MHz}, \mathrm{CDCl}_{3}\right): \delta 7.82$ (s, 2H), 4.29 (dd, $J=7.5,1.6 \mathrm{~Hz}, 2 \mathrm{H}), 2.29-2.09(\mathrm{~m}, 1 \mathrm{H}), 1.46-1.18(\mathrm{~m}, 10 \mathrm{H}), 0.99-$ 0.79 (m, 6H) ${ }^{13} \mathrm{C}$ NMR (75 MHz, $\left.\mathrm{CDCl}_{3}\right): \delta 190.85,142.34,124.50,48.22,37.95,30.63$, 28.93, 24.02, 22.80, 14.08, 10.84. IR ( NaCl plate, $\left.\mathrm{cm}^{-1}\right): 2957,2926,2859,1561,1512$, 1461, 1414, 1345, 1304, 1087. HRMS calculated for $\left[\mathrm{M}^{+}\right]$297.06796, found 297.07193. 


\section{1-Bromo-5-(2-ethylhexyl)-thieno[3,4-c]pyrrole-4,6-dione (12)}

In a dry three-neck round bottom flask, monomer M7 (1.21 g, $2.89 \mathrm{mmol})$ was added to a solution of ethanol $(18 \mathrm{~mL})$, acetic acid $(5.0 \mathrm{~mL})$, and 2 drops of $\mathrm{HCl}$ and then heated until the dissolution of the solid. Zinc powder $(0.208 \mathrm{~g}, 3.18 \mathrm{mmol})$ was then added, and the mixture was refluxed for $1 \mathrm{~h}$. The mixture was monitored by TLC (hexane: acetone, 1:0.05 v/v). After cooling to room temperature, the mixture was filtered through a Celite pad and the filtrate extracted twice with dichloromethane and the combined organic layer was dried over $\mathrm{MgSO}_{4}$. The solvent was removed under reduced pressure. The resulting crude product was purified by silica gel column chromatography using hexane as eluting solvent. Compound 12 was obtained as white powder $(0.77 \mathrm{~g}$, $77.0 \%$ yield) after removing the solvent. ${ }^{1} \mathrm{H} \mathrm{NMR}\left(300 \mathrm{MHz}, \mathrm{CDCl}_{3}\right): \delta 7.74(\mathrm{~s}, 1 \mathrm{H})$, $3.53(\mathrm{dd}, J=7.2,3.3 \mathrm{~Hz}, 2 \mathrm{H}), 1.81(\mathrm{~s}, 1 \mathrm{H}), 1.29$ (d, $J=10.8 \mathrm{~Hz}, 10 \mathrm{H}), 0.92$ (dd, $J=9.9$, $4.8 \mathrm{~Hz}, 6 \mathrm{H}) .{ }^{13} \mathrm{C} \mathrm{NMR}\left(75 \mathrm{MHz}, \mathrm{CDCl}_{3}\right): \delta 163.22,136.55,125.39,42.39,37.96,30.39$, 28.60, 23.94, 23.02, 13.94, 10.43. HRMS calculated for $\left[\mathrm{M}^{+}\right]$343.02416, found 343.02706 .

\section{Compound 13}

Under an argon atmosphere, a solution of $\mathrm{Ni}(\mathrm{COD})_{2}(0.890 \mathrm{~g}, 3.23 \mathrm{mmol})$, COD $(0.35 \mathrm{~g}, 3.23 \mathrm{mmol})$ and bypiridine $(0.500 \mathrm{~g}, 3.23 \mathrm{mmol})$ in toluene $(8.0 \mathrm{~mL})$ was heated to $80{ }^{\circ} \mathrm{C}$. Then, a solution of $\mathbf{1 2}(0.700 \mathrm{~g}, 2.04 \mathrm{mmol})$ in $3.0 \mathrm{~mL}$ of toluene was added to the reaction and the mixture was heated at $90{ }^{\circ} \mathrm{C}$ in dark for $72 \mathrm{~h}$. The solution was cooled and extracted three times with dichloromethane as well as dried over $\mathrm{Na}_{2} \mathrm{SO}_{4}$. The crude product was purified by column chromatography using hexane and acetone 
(1.7:0.4) as the eluting solvent. A light yellow solid (13) was obtained after evaporating all the solvents and was further purified by recrystallization in hexane $(0.300 \mathrm{~g}, 55.6 \%$ yield). ${ }^{1} \mathrm{H}$ NMR (300 MHz, $\left.\mathrm{CDCl}_{3}\right): \delta 7.82(\mathrm{~s}, 2 \mathrm{H}), 3.53(\mathrm{~d}, J=4 \mathrm{~Hz}, 3 \mathrm{H}), 1.81(\mathrm{~d}, J=$ $5.9 \mathrm{~Hz}, 2 \mathrm{H}), 1.44-1.20(\mathrm{~m}, 20 \mathrm{H}), 1.00-0.80(\mathrm{~m}, 12 \mathrm{H}) .{ }^{13} \mathrm{C} \mathrm{NMR}\left(75 \mathrm{MHz}, \mathrm{CDCl}_{3}\right) \delta$ $162.92,162.17,136.84,133.80,132.51,126.46,42.78,38.21,30.61,28.57,23.99,23.01$, 14.10, 10.33. HRMS calculated for $\left[\mathrm{M}^{+}\right] 528.21164$, found 528.20786. IR $\left(\mathrm{KBr}, \mathrm{cm}^{-1}\right)$ : 2959, 2930, 2867, 1759, 1694, 1553, 1505, 1460, 1393, 1339, 1099.

\section{Compound 14}

A solution of compound $\mathbf{1 3}(0.110 \mathrm{~g}, 0.208 \mathrm{mmol})$ in 1,2,4-trichlorobenzene (4 $\mathrm{mL})$ was degassed and purged with argon for 30 min then Lawesson's reagent $(0.380 \mathrm{~g}$, $0.950 \mathrm{mmol}$ ) was added. The mixture was heated at $150-160{ }^{\circ} \mathrm{C}$ for $6 \mathrm{~h}$ under argon. The solution was cooled and extracted three times with dichloromethane as well as dried over $\mathrm{Na}_{2} \mathrm{SO}_{4}$. The crude product was purified by column chromatography using hexane and acetone $(5: 1 \mathrm{v} / \mathrm{v})$ as the eluting solvent. A dark solid (14) which is yellow in solution was obtained after evaporating all the solvents $\left(0.850 \mathrm{~g}, 69.1 \%\right.$ yield). ${ }^{1} \mathrm{H}-\mathrm{NMR}(300 \mathrm{MHz}$, $\left.\mathrm{CDCl}_{3}\right): \delta 7.90(\mathrm{~d}, J=5.9 \mathrm{~Hz}, 2 \mathrm{H}), 4.29(\mathrm{~d}, J=7.5 \mathrm{~Hz}, 4 \mathrm{H}), 2.27-2.09(\mathrm{~m}, 2 \mathrm{H}), 1.49-$ $1.16(\mathrm{~m}, 20 \mathrm{H}), 1.00-0.82(\mathrm{~m}, 12 \mathrm{H}) .{ }^{13} \mathrm{C}-\mathrm{NMR}\left(75 \mathrm{MHz}, \mathrm{CDCl}_{3}\right):{ }^{13} \mathrm{C}$ 189.61, 188.47, $143.32,137.99,130.13,124.55,48.00,37.93,30.66,28.63,24.04,23.05,14.23,11.00$. EI-MS calculated for $\left[\mathrm{M}^{+}\right]$592.12027, found 592.11766. IR ( $\mathrm{NaCl}$ plate, $\left.\mathrm{cm}^{-1}\right)$ : 2956, 2925, 2863, 1529, 1510, 1458, 1405, 1355, 1296, 1088. 


\section{Polymer P26}

Under an argon atmosphere, a solution of $\mathrm{Ni}(\mathrm{COD})_{2}(0.560 \mathrm{~g}, 2.03 \mathrm{mmol})$, COD $(1.00 \mathrm{~mL}, 8.16 \mathrm{mmol})$ and bypiridine $(0.317 \mathrm{~g}, 2.03 \mathrm{mmol})$ in toluene $(8.0 \mathrm{~mL})$ was heated to $80{ }^{\circ} \mathrm{C}$. Then, a solution of monomer $\mathbf{M} 7(0.350 \mathrm{~g}, 0.864 \mathrm{mmol})$ in $4.0 \mathrm{~mL}$ of toluene was added to the reaction solution and the reaction mixture was heated at $100{ }^{\circ} \mathrm{C}$ in dark for $72 \mathrm{~h}$. The solution was then poured into methanol with vigorous stirring. After stirring 2 h, a dark purple precipitate as polymer P26 was collected by filtration. Polymer P26 was purified by dissolution in $\mathrm{CHCl}_{3}$ and reprecipitated using methanol. The material was further purified by a Soxhlet extraction in acetone for $24 \mathrm{~h}$. The resultant polymer was dissolved in $\mathrm{CHCl}_{3}$ and precipitated out in methanol. After drying under vacuum at $60{ }^{\circ} \mathrm{C}$ for $5 \mathrm{~h}$, the polymers were obtained $(0.180 \mathrm{~g}, 79.3 \%$ yield $)$ as dark

purple powder. P26. ${ }^{1} \mathrm{H}$ NMR $\left(300 \mathrm{MHz}, \mathrm{CDCl}_{3}\right): \delta 3.61,1.87,1.34,0.94 .{ }^{13} \mathrm{C} \mathrm{NMR}(75$ $\left.\mathrm{MHz}, \mathrm{CDCl}_{3}\right): \delta 162.66,162.35,133.90,133.06,132.44,127.19,42.68,38.11,30.51$, 28.51, 23.83, 23.01, 14.09, 10.38. IR (KBr pellet, $\left.\mathrm{cm}^{-1}\right): 1762,1706$.

\section{Polymer P27}

In a flame dried three-neck flask under argon atmosphere, polymer P26 (0.100 g, $0380 \mathrm{mmol})$ was added to 1,2-dichlorobenzene $(5.0 \mathrm{~mL})$ and stirred until dissolution. The solution was degassed and purged with argon for $30 \mathrm{~min}$, the solution was heated to $130{ }^{\circ} \mathrm{C}$ and Lawesson's reagent $(0.307 \mathrm{~g}, 0.760 \mathrm{mmol})$ was added. The mixture was heated to $160{ }^{\circ} \mathrm{C}$ for $6 \mathrm{~h}$ under argon. During the reaction, the color changed from amber to blue-greenish. The solution was then poured into methanol with vigorous stirring. After stirring $2 \mathrm{~h}$, a dark precipitate as polymer P27 was collected by filtration. The 
polymer was further purified by refluxing in ethanol for $2 \mathrm{~h}$, filtered and washed with ethanol. The resultant polymer was then stirred in different solvents sequentially acetone, ethyl acetate and hexane to remove any other residue from LR. Then the polymer was further purified by a Soxhlet extraction in acetone for $24 \mathrm{~h}$. The resultant polymer was dissolved in $\mathrm{CHCl}_{3}$ and precipitated out in methanol. After drying under vacuum at $60{ }^{\circ} \mathrm{C}$ for $5 \mathrm{~h}$, the polymers were obtained $\left(0.100 \mathrm{~g}, 89.2 \%\right.$ yield) as dark powder. ${ }^{1} \mathrm{H}$ NMR (300 $\left.\mathrm{MHz}, \mathrm{CDCl}_{3}, \mathrm{ppm}\right): \delta 4.30,2.18,1.35,0.92 .{ }^{13} \mathrm{C} \mathrm{NMR}\left(75 \mathrm{MHz}, \mathrm{CDCl}_{3}\right): \delta 189.59$, $188.46,187.62,143.30,139.19,129.22,125.10,47.97,37.95,30.66,28.64,24.04,23.08$, 14.19, 11.00. IR ( $\mathrm{NaCl}$ plate, $\left.\mathrm{cm}^{-1}\right): 1090$ 


\subsection{References}

1. Pron, A.; Berrouard, P.; Leclerc, M. Macromol. Chem. Phys. 2013, 214, 7.

2. Zhang, Y.; Hau, S. K.; Yip, H.-L.; Sun, Y.; Acton, O.; Jen, A. K.Y. Chem. Mater. 2010, 22, 2696.

3. Berrouard, P.; Dufresne, S.; Pron, A.; Veilleux, J.; Leclerc, M. J. Org. Chem. 2012, 77, 8167.

4. Zhang, Z.-G.; Wang, J. J. Mater. Chem. 2012, 22, 4178.

5. (a) He, P.; Qiao, X.-L.; Qian, Q.; Li, H.-X. Chinese Chem. Lett. 2016, 27, 1277.

(b) Zou, Y.; Najari, A.; Berrouard, P.; Beaupré, S.; Réda Aïch, B.; Tao,Y.; Leclerc, M. J. Am. Chem. Soc. 2010, 132, 5330. (c) Chu, T.-Y.; Lu, J.; Beaupré, S.; Zhang, Y.; Pouliot, J.-R.; Wakim,S.; Zhou, J.; Leclerc, M.; Li, Z.; Ding, J.; Tao, Y. J. Am. Chem. Soc. 2011, 133, 4250. (d) Amb, C. M.; Chen, S.; Graham, K. R.; Subbiah, J.; Small, C. E.; So, F.; Reynolds, J. R. J. Am. Chem. Soc. 2011, 133, 10062. (e) Small, C. E.; Chen, S.; Subbiah, J.; Amb, C. M.; Tsang, S.W.;Lai, T.-H.; Reynolds, J. R.; So, F. Nature Photon. 2012, 6, 115. (f) Lin, Z.; Bjorgaard, J.; Gul Yavuz, A.; Iyer, A.; Kose, M. E. RSC Adv. 2012, 2, 642. (g) Najari, A.; Berrouard, P.; Ottone, C.; Boivin, M.; Zou, Y.; Gendron, D.; Caron, W.-O.; Legros, P.; Allen, C. N.; Sadki, S.; Leclerc, M. Macromolecules, 2012, 45, 1833. (h) Piliego, C.; Holcombe, T. W.; Douglas, J. D.; Woo, C. H.; Beaujuge, P. M.; Fréchet, J. M. J. J. Am. Chem. Soc. 2010, 132, 7595. (i) Zhang, G.; Fu, Y.; Zhang, Q.; Xie, Z. Chem. Commun. 2010, 46, 4997. (j) Li, Z.; Tsang, S.-W.; Du, X.; Scoles, L.; Robertson, G.; Zhang, Y.; Toll, F.; Tao, Y.; Lu, J.; Ding, J. Adv. Funct. Mater. 2011, 21, 3331. 
6. Guo, X.; Ortiz, R. P.; Zheng, Y.; Kim, M.-G.; Zhang, S.; Hu, Y.; Lu, G.; Facchetti, A.; Marks, T. J. J. Am. Chem. Soc. 2011, 133, 13685.

7. Pomerantz, M.; Amarasekara, A. S. Synth. Met. 2003, 135-136, 257.

8. Nielsen, C. B.; Bjørnholm, T. Org. Lett. 2004, 6, 3381.

9. Cabanetos, C.; El Labban, A.; Bartelt, J. A.; Douglas, J. D.; Mateker, W. R.; Frechet, J. M. J.; McGehee, M. D.; Beaujug, P. M. J. Am. Chem. Soc. 2013, 135, 4656.

10. Qiao, X.; Wu, Q.; Wu, H.; Wang, D.; Li, H. Polym. Chem. 2016, 7, 807.

11. Zhang, L.; Yang, T.; Shen, L.; Fang, Y.; Dang, L.; Zhou, N.; Guo, X.; Hong, Z.; Yang, Y.; Wu, H.; Huang, J.; Liang, Y. Adv. Mater. 2015, 27, 6496.

12. Milewska, M. J.; Bytner, T.; Polonski, T. Synthesis 1996, 1485.

13. Orzeszko, A.; Maurin, J. K.; Melon-Ksyta, D. Z. Naturforsch., B: Chem. Sci. 2001, 56, 1035.

14. Ozturk, T.; Ertas, E.; Mert, O. Chem. Rev. 2007, 107, 5210.

15. (a) Zhang, C.; Wang, Z. Y. Macromolecules, 1993, 26, 3330. (b) Chmil, K.; Scherf, U. Acta Polymer, 1997 48, 208.

16. Bheemireddy, S. R.; Hautzinger, M. P.; Li, T.; Lee, B.; Plunkett, K. N. J. Am. Chem. Soc. 2017, 139, 5801.

17. Chen, Y.; Huang, W.; Li, C.; Bo, Z. Macromolecules, 2010, 43, 10216.

18. Berrouard, P.; Grenier, F.; Pouliot, J.-R.; Gagnon, E.; Tessier,C.; Leclerc, M. Org. Lett. 2011, 13, 38.

19. Lee, J.; Kalin, A. J.; Yuan,T.; Al-Hashimic, M.; Fang, L. Chem. Sci. 2017, 8, 2503 


\section{Chapter 5 Highly Sensitive Dual-Mode Fluorescence Detection of Lead Ion in Water Using Aggregation-Induced Emissive Polymers}

\subsection{Introduction}

\subsubsection{Overview of aggregation induced emission, conjugated polymer and design strategy}

Light emits from luminophore. In the aggregate state, they may show reduced, unchanged, or enhanced emission, in comparison to their dilute solutions. In the high concentration solution the luminescence is quenched this phenomenon is widely known as aggregated-caused quenching (ACQ). ACQ is a very common phenomenon for aromatic hydrocarbon and its derivatives. In the aggregate state, the molecules are located in the immediate vicinity and the aromatic rings of the adjoining luminophores (like perylene containing planar aromatic ring), especially those with disc or rod-like shapes, experience intense intermolecular $\pi-\pi$ stacking interactions. The excited states of such aggregates often back to the ground state via non-radiative channels, resulting in the emission quenching of the luminophores. This property limits the application of such luminophores. ${ }^{1,2}$

In 2001, Tang et al. discovered a phenomenon on luminophore, in which aggregation worked opposite way in compare to ACQ phenomenon. ${ }^{3,4}$ They found that a series of silole derivatives were non-emissive in good solvent (THF) and became highly luminescent in aggregated state when poor solvent reaches to $\sim 80$ volume $\%$ or cast into solid films. Since the light emission was induced by aggregate formation, they termed the process "aggregation induced emission" (AIE). These aggregates are named luminogens. 
Hexaphenylsilole (HPS) is a propeller shaped non-planar molecule. In a good solvent, six phenyl rings (rotors) in an HPS molecule can easily undergo dynamic intramolecular rotations against its silacyclopentadiene or silole stator, which nonradiatively annihilates its excited state and renders its molecule non-luminescent. In the aggregates, the HPS possess a propeller shape which inhibits $\pi$ - $\pi$ stacking process and the intramolecular rotations of its aryl rotors are greatly restricted. This restriction of intramolecular rotations (RIR) block the non-radiative pathway and opens up the radiative channel which makes the HPS molecules emissive in the aggregate state. ${ }^{5}$ Tetraphenylethene (TPE) is one of the AIEgen has been studied extensively and intensively. In TPE compound the central olefin is the stator which is surrounded by four peripheral phenyl rings (rotors). The TPE compound in good solvent emits almost no light due to the dynamic rotations of the aromatic rotors against the olefinic stator around the single-bond. In the excited state, the central olefinic double bond can be opened and generate two diphenylmethylene units. The friction caused by their random rotational or twisting motions against each other with the solvent media transforms the photonic energy to thermal energy, leading to radiationless relaxation of the excitons. Upon aggregation the highly twisted molecular conformation inhibits $\pi-\pi$ stacking process and the intramolecular rotations of its aryl rotors are greatly restricted. This RIR process blocks the non-radiative pathway and opens up the radiative channel of the TPE which makes it dramatic emissive. ${ }^{6}$ TPE-based polymers are weakly emissive in good solvent due to the steric effect of polymer chain. In the aggregated state intramolecular rotations become more difficult which enhance the emission dramatically. TPE-based polymers are synthesized and applied for sensing. ${ }^{7,8}$ Tang et al. explained extensively about restriction 
of intramolecular vibration, restriction of intramolecular motion and other methodologies for emission. ${ }^{6,2}$ In most of the sensing the highly emissive state has been used for turn-off detection of analyties.TPE and other AIE-active compounds are weakly emissive in lower volume fraction of poor solvent but it shows higher emission when poor solvent reaches to higher volume fraction. Therefore, we believe, there are two windows which can be used for both the turn-on and turn-off detection of analytes.

Almost three decades conjugated polymers have been using as signal-transducing elements for sensory applications due to their signal amplification properties and the versatility in their molecular design. ${ }^{9-12}$ This method based on upon changes in fluorescence intensity, energy transfer, electron transfer, wavelength (excitation and emission), and lifetime. ${ }^{13}$ The ability of the conjugated polymer's to produce signal gain in response to an interaction with target analytes is one of the main features that have accelerated the use of fluorescent conjugated polymers as sensors. This polymer is containing the $\pi$ electron along the polymer chain and these electrons are available for delocalization all over the backbone. Excitons in CPs are very mobile and able to migrate throughout the whole polymer backbone. Therefore, migration of excitons increases the frequency of interaction with the bound quencher; as a result, the sensitivity becomes higher.

Therefore, a polymer having a conjugated backbone and AIE compound would be an effective system to demonstrate higher sensitive sensor. In this part of the thesis, we explored how both the windows can be used for the detection of $\mathrm{Pb}^{2+}$ ion in water. Moreover, to demonstrate highly selective and higher sensitivity with dual mode fluorescence detection of $\mathrm{Pb}^{2+}$ ion in water we require a selective probe. Previously, we 
synthesized a fluorescence sensor and studied the fluorescence turn-on detection of $\mathrm{Pb}^{2+}$ in aqueous medium. We introduced that sensor into the backbone of the AIE active conjugated polymer to complete the sensor.

\subsubsection{Contribution}

The following is a full version of the article "Highly Sensitive Dual-Mode Fluorescence Detection of Lead Ion in Water Using Aggregation-Induced Emissive Polymers" published on July 31, 2014 in Macromolecular Rapid Communication and reproduced with permission under license from WILEY-VCH Verlag GmbH \& Co. KGaA, Weinheim Copyright Clarence Centre (see Appendix D). This includes detailed supporting information with optimization data and tables. The synthesis of polymer and most of the titration was carried out by me and some of the titration works were carried

out by another student, Khama Rani Ghosh. Some of the difficulties were discussed and solved by Dr. Jian Ping Gao.

\subsubsection{Abstract}

A series of fluorene-based conjugated polymers containing the AIE-active tetraphenylethene and dicarboxylate pseudocrown as a receptor exhibits a unique dualmode sensing ability for selective detection of lead ion in water. Fluorescence turn-off and turn-on detections are realized in $80-90 \%$ and $20 \%$ water in tetrahydrofuran (THF), respectively, for lead ion with a concentration as low as $10^{-8} \mathrm{M}$. 


\subsubsection{References}

1. Photophysics of Aromatic Molecules; Birks, J. B., Ed.; Wiley: London, 1970.

2. Mei, J.; Leung, N. L. C.; Kwok, R. T. K.; Lam, J. W. Y.; Tang, B. Z. Chem. Rev. 2015, 115, 11718.

3. Luo, J.; Xie, Z.; Lam, J. W. Y.; Cheng, L.; Chen, H.; Qiu, C.; Kwok, H. S.; Zhan, X.; Liu, Y.; Zhu, D.; Tang, B. Z. Chem. Commun. 2001, 1740.

4. Tang, B. Z.; Zhan, X.; Yu, G.; Lee, P. P. S.; Liu, Y.; Zhu, D. J. Mater. Chem. 2001, 11, 2974.

5. Hong, Y.; Lam, J. W. Y.; Tang, B. Z. Chem. Commun. 2009, 4332.

6. Hong, Y.; Lam, J. W. Y Tang, B. Z. Chem. Soc. Rev. 2011, 40, 5361.

7. Qin, A.; Jim, C. K. W.; Tang, Y.; Lam, J. W. Y.; Liu, J.; Mahtab, F.; Gao, P.; Tang, B. Z. J. Phys. Chem. B, 2008, 112, 9281.

8. (a) Wu, W.; Ye, S.; Huang, L.; Xiao, L.; Fu, Y.; Huang, Q.; Yu, G.; Liu, Y.; Qin, J.; Li, Q.; Li, Z.; J. Mater. Chem. 2012, 22, 6374. (b) Hu, R.; Maldonado, J. L.; Rodriguez, M.; Deng, C.; Jim, C. K. W.; Lam, J. W. Y.; Yuen, M. M. F.; RamosOrtiz, G.; Tang, B. Z. J. Mater. Chem. 2012, 22, 232. (c) Wang, J.; Mei, J.; Yuan, W.; Lu, P.; Qin, A.; Sun, J.; Ma Y.; Tang, B. Z. J. Mater. Chem. 2011, 21, 4056. (d)Wu, W.; Ye, S.; Yu, G.; Liu, Y.; Qin J.; Li, Z. Macromol. Rapid Commun. 2012, 33, 164. (e) Xu, B.; Wu, X.; Li, H.; Tong H.; Wang, L. Macromolecules, 2011, 44, 5089. (f) Ghosh, K. R.; Saha, S. K.; Gao, J. P.; Wang, Z. Y. Chem. Commun, 2014, 50, 716. (g) Ghosh, K. R.; Saha, S. K.; Wang, Z. Y. Polym. Chem. 2014, 5, 5638.

9. Thomas, S.W.; Joly, G. D.; Swager, T. M. Chem. Rev. 2007, 107, 1339. 
10. Zhou, Q.; Swager, T. M. J. Am. Chem. Soc. 1995, 117, 7017.

11. Cumming, C. J.; Aker, C.; Fisher, M.; Fox, M.; la Grone, M. J.; Reust, D.; Rockley, M. G.; Swager, T. M.; Towers, E.; Williams, V. IEEE Trans. Geosci. Rem. Sens. 2001, 39, 1119.

12. Toal S. J.; Trogler, W. C. J. Mater. Chem. 2006, 16, 2871.

13. McQuade, D. T.; Pullen, A. E.; Swager, T. M. Chem. Rev. 2000, 100, 2537. 


\subsection{Article}

A simple and rapid technique for detection of a low level of lead $\left(\mathrm{Pb}^{2+}\right)$ contamination in water is critical, because even with a minimal exposure of $\mathrm{Pb}^{2+}$ could lead to a number of adverse health effect, including physical or mental development delay in children, kidney and brain damage in adults. ${ }^{[1,2]}$ World Health Organization and Environmental Protection Agency (EPA) have strictly defined the concentration limit of $\mathrm{Pb}^{2+}$ which should not exceed $10 \mathrm{ppb}$ in the drinking water. ${ }^{[3]}$ Current EPA-proven methods, such as atomic absorption spectrometry, ${ }^{[4,5]}$ inductively coupled plasma emission spectrometry, ${ }^{[6]}$ and inductively coupled plasma-mass spectrometry, ${ }^{[7]}$ have been applied for the determination of trace lead content. These conventional methods require expensive instrumentation, well-equipped lab set up and specially trained personnel, ${ }^{[8-11]}$ which may seriously limit the scope of their practical applications. Over the past two decades, considerable research efforts have been devoted to the development of fluorescence-enhancing (turn-on) or quenching (turn-off) sensory materials for detection of $\mathrm{Pb}^{2+} \cdot{ }^{[12-22]}$ Various fluorescent probes have been developed for lead ion, such as quantum dots, DNAzymes and nanoparticles, functional nucleic acids, peptides and proteins have been utilized. ${ }^{[23-27]}$ Fluorescent polymers have also been explored for lead detection. ${ }^{[28,29]}$

To detect a low level of toxic metal ions like $\mathrm{Pb}^{2+}, \mathrm{Hg}^{+}, \mathrm{Cd}^{2+}$ in water, a molecular sensor should be able to selectively bind with metal ion and highly luminescent in aqueous medium. However, in the aquatic system most of the fluorescent compounds and polymers tend to aggregate and become less or even not fluorescent, thus drastically affecting the sensing efficiency and sensitivity. On the other hand, some chromophores, e.g., tetraphenylethene (TPE), become highly fluorescent only as aggregates, due to the 
aggregation-induced emission (AIE) enhancement. ${ }^{[30]}$ Many types of AIE-active compounds have been synthesized and some are used as various chemosensors. ${ }^{[31-36]}$ For example, Tang et. al. synthesized molecular sensors containing terpyridine as a receptor and TPE as an AIE-active moiety for fluorescence turn-off detection of $\mathrm{Zn}^{2+}$ in water. ${ }^{[36 a]}$ Therefore, incorporation of TPE into a sensory polymer can impart the AIE activity to the sensory system, making it useful for highly sensitive fluorescence detection. It is also known that the aggregation of the AIE-active chromophore can be tuned by the solvent polarity and solubility. Thus, both fluorescence turn-on and turn-off sensors could be achieved by interaction with analytes in different medium. Such a dual-mode detection may offer more choices and advantages for development of practical sensing devices.

We recently synthesized a fluorescent sensor having a dicarboxylate pseudocrown (DP) as a lead ion receptor and demonstrated the fluorescence turn-on detection of $\mathrm{Pb}^{2+}$ in aqueous medium. ${ }^{[37]}$ The purpose of this work is to incorporate the DP receptor into an AIE-active TPE based polymer and explore the feasibility of dual-mode fluorescence detection of a trace amount of lead ion in aqueous medium. Our previous work revealed that binding of $\mathrm{DP}$ with $\mathrm{Pb}^{2+}$ results in a less soluble sensory compound, presumably due to a stronger ionic interaction between the $\mathrm{DP}-\mathrm{Pb}^{2+}$ complexes. Therefore, upon binding with $\mathrm{Pb}^{2+}$, the ionic interaction could alter the polymer chain folding or aggregation state of TPE segments, leading to a significant change in fluorescence intensity. The dualmode fluorescence detection could be realized as a result of formation and de-formation of the DP-TPE polymer aggregates in the presence of $\mathrm{Pb}^{2+}$, as represented conceptually in Figure 5.1. In the turn-off mode, the polymer in a more polar solvent (e.g., water) is highly emissive due to the TPE aggregation. When binding with the lead ion, the ionic 
interaction takes over the $\pi-\mathrm{CH}$ interaction between the TPE segments, causing the dissociation of the TPE aggregates and thus a decrease in fluorescence. In the turn-on mode, the polymer in a less polar solvent is non-emissive due to the chain unfolding and no TPE aggregation. After binding with lead ion, the stronger ionic interaction would cause the chain folding and TPE aggregation, which leads to an increase in fluorescence. According to the design, DP is attached to a fluorene via a double bond and the fluorene is then functionalized for further polymerization. The DP-containing fluorene monomer and the TPE monomer in different ratios are polymerized to afford a series of conjugated polymers having a different amount of the lead receptor and the AIE-active segment of TPE.
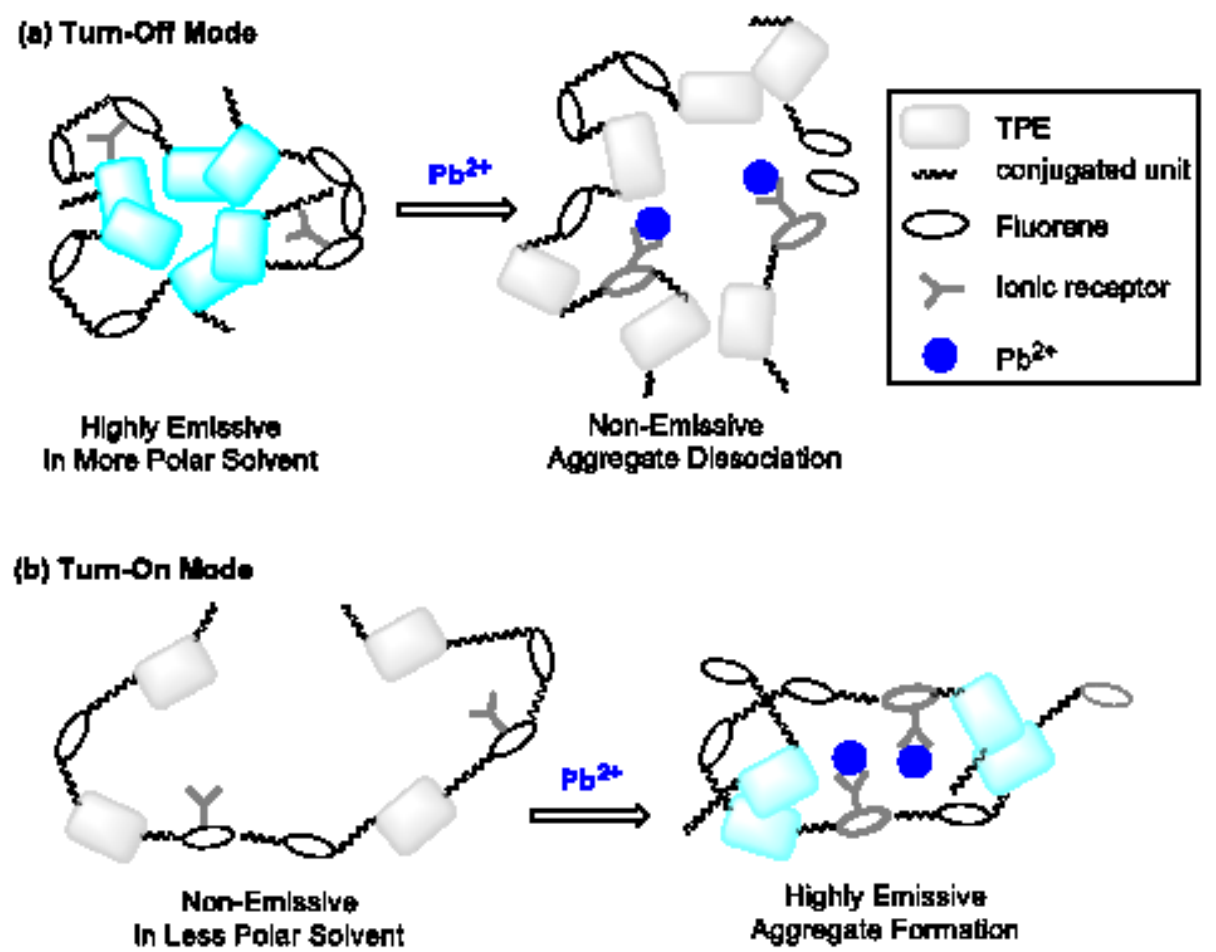

Figure 5.1 A dual-mode fluorescence detection of lead ion in aqueous solvent using AIEactive polymer. (a) Fluorescence turn-off mode and (b) fluorescence turn-on mode. 
<smiles>Brc1ccc(C(c2ccccc2)(c2ccccc2)C(c2ccccc2)(c2ccccc2)C2C=CC(Br)C2)cc1</smiles><smiles>Brc1ccc(C(=C(c2ccccc2)c2ccccc2)c2ccc(Br)cc2)cc1</smiles>

1

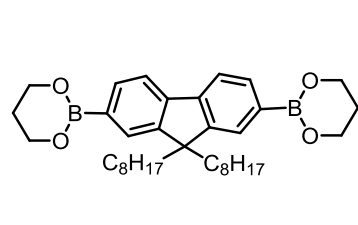

$+$
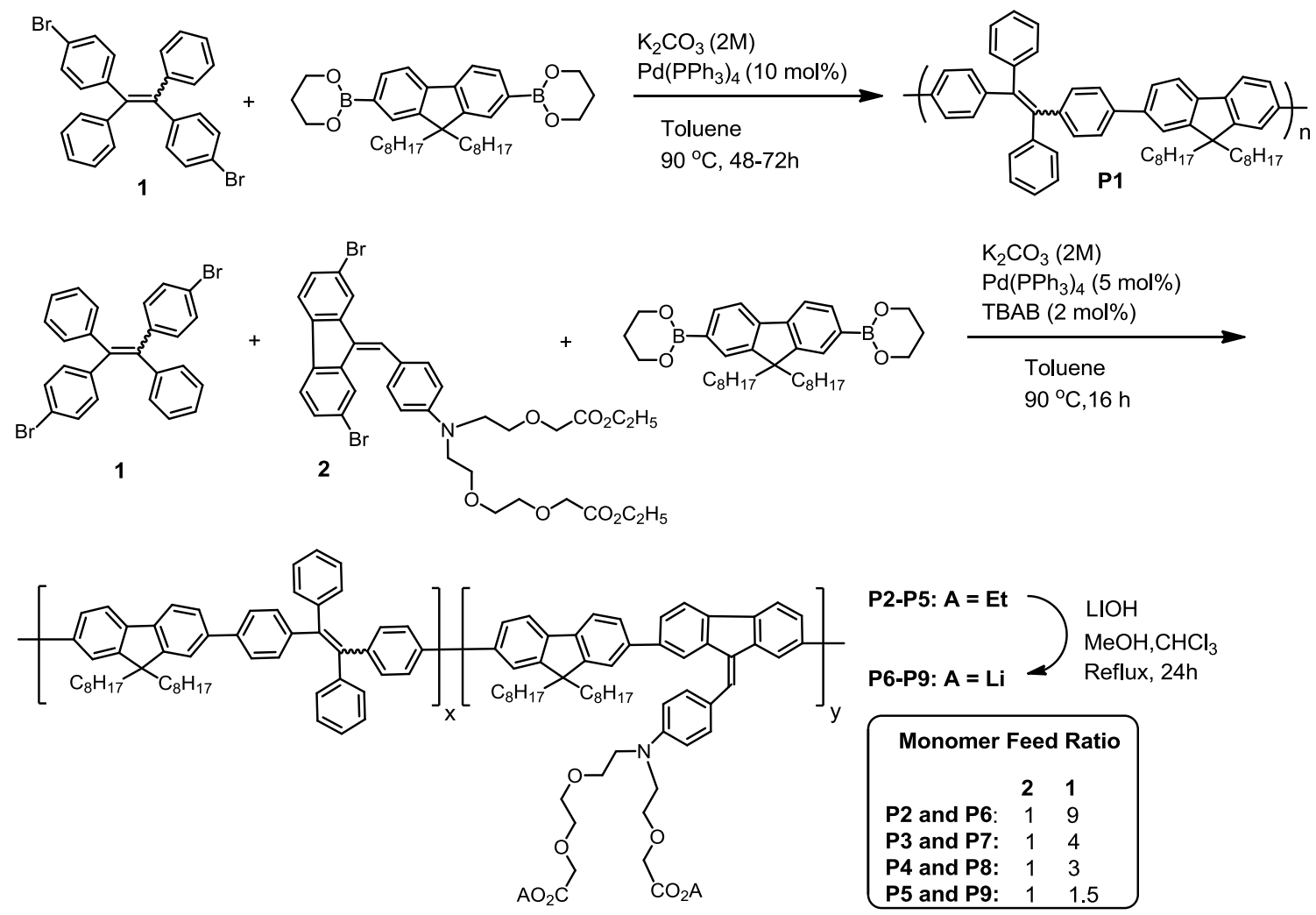

Scheme 5.1 Synthesis of polymers P1-P9.

\section{Experimental Section}

A series of DP-F-TPE polymers were synthesized according to the synthetic routes shown in Scheme 5.1. The DP and TPE content in polymers was controlled by adjusting the molar ratio between compounds $\mathbf{1}$ and $\mathbf{2}$ in polymerization. Compound $\mathbf{1}$ was synthesized in $90 \%$ yield from 4 -bromobenzophenone using $\mathrm{Zn} / \mathrm{TiCl}_{4}$ in $\mathrm{THF}^{[34,38]}$ Polymer P1 and precursor polymers P2-P5 were synthesized by the Suzuki cross coupling polymerization in toluene using $\mathrm{Pd}\left(\mathrm{PPh}_{3}\right)_{4}$ as a catalyst. ${ }^{[32,33]}$ The subsequent hydrolysis of P2-P5 using LiOH afforded P6-P9 in 90\% yield. ${ }^{[28]}$ 


\section{Results and Discussion}

All the polymers were characterized by NMR, IR and UV-vis spectroscopy. ${ }^{1} \mathrm{H}$ NMR spectra of P2-P5 show multiple peaks at 4.17 and $3.75 \mathrm{ppm}$, which are absent in the ${ }^{1} \mathrm{H}$ NMR spectrum of P1, indicating the presence of DP moiety (Appendix D, Figure S5.5). The characteristic band for the ester group in 2 (Appendix D, Figure S5.6a) and P3 (Appendix D, Figure S5.6b) are shown at $1750 \mathrm{~cm}^{-1}$, whereas the IR spectrum of polymer P7 displays a peak at $1607 \mathrm{~cm}^{-1}$ for carboxylic acid salt and no peak at $1750 \mathrm{~cm}^{-1}$ (Appendix D, Figure S6c), indicating the complete hydrolysis of ester group. The absorption spectra of P6-P9 in THF and THF/water display maximal peaks at 365-368 nm (Appendix D, Figure S5.12).

To evaluate the suitability of DP-F-TPE polymers as AIE-active fluorescence sensors for the turn-off and turn-on detection of $\mathrm{Pb}^{2+}$ in water, the emission properties of polymers and their AIE behaviors were investigated in water/THF mixture. Polymers P1 and P6-P9 are non-emissive or low emissive in THF or THF/water mixture when water content is below $30 \%$. With an increase of the water content, polymers begin to aggregate and emit the light at $500 \mathrm{~nm}$, which reaches to a maximum for P1, $\mathbf{P 6}$ and $\mathbf{P 7}$ in 90\% water in THF and for $\mathbf{P 8}$ and $\mathbf{P 9}$ in $80 \%$ water in THF (Figure 5.2 and Appendix D, Figure S5.13, S5.14). In comparison, the emission of $\mathbf{P 6}$ and $\mathbf{P 7}$ is more intense than $\mathbf{P 8}$ and $\mathbf{P 9}$ in $80-90 \%$ water in THF, due to the presence of more DP and less TPE units in the latter (Appendix D, Figure S5.13). Therefore, the solvent polarity-dependent AIE emission of DP-F-TPE polymers is offering a window of opportunity for dual-mode fluorescence detection of $\mathrm{Pb}^{2+}$. The amount of $30 \%$ water in THF represents a cut off for 
P6-P9 to be used as fluorescence turn-on detection; the amounts of $80-90 \%$ water in THF are useful for P6, P7 and P8, P9 for fluorescence turn-off detection.

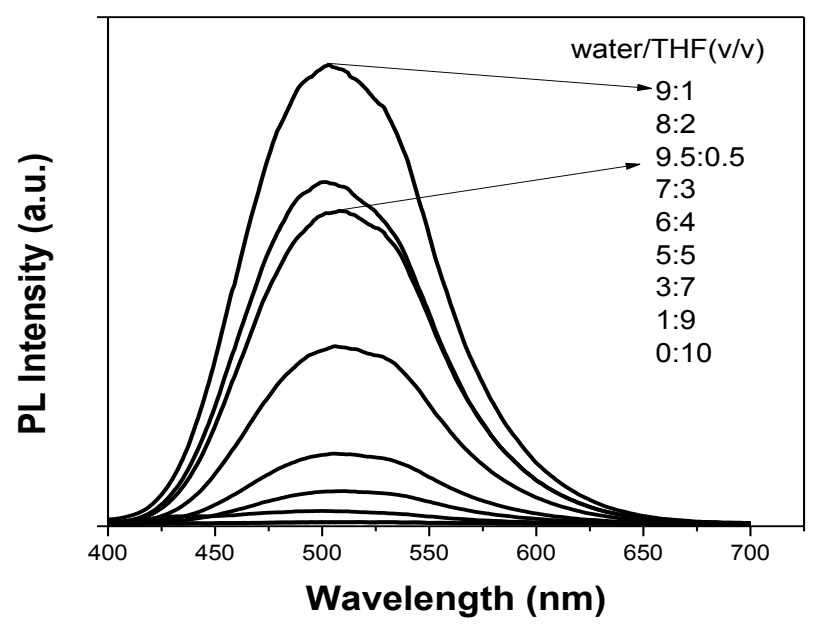

Figure 5.2 Fluorescence spectra of polymer $\mathbf{P 7}$ in water and THF.

The fluorescence turn-off detection of $\mathrm{Pb}^{2+}$ was achieved using $\mathbf{P 6}$ or $\mathbf{P 7}$ in $90 \%$ $\mathrm{H}_{2} \mathrm{O}$ in THF $\left(1.3 \times 10^{-5} \mathrm{M}\right)$. Upon the addition of $\mathrm{Pb}^{2+}$, the emission intensity of $\mathbf{P 7}$ decreased significantly and the fluorescence quenching response (FQR) reached over $90 \%$ with the $\mathrm{Pb}^{2+}$ concentration of $6 \times 10^{-7} \mathrm{M}$ (Figure 5.3a). In comparison, the FQR values of other polymers towards $\mathrm{Pb}^{2+}\left(6-8 \times 10^{-7} \mathrm{M}\right)$ are smaller. Polymer $\mathbf{P 6}$ contains $5 \%$ of DP unit displays low FQR (21\%). Polymers $\mathbf{P 8}$ and $\mathbf{P 9}$ contain a high percentage of DP receptor but relatively low AIE-active segments, giving rise to a moderate FQR (38\% and 34\%). P1 with no DP moiety has little $\mathrm{Pb}^{2+}$ quenching effect. 
To understand the observed emission turn-off process, a comparison study was further carried out using polymer P1 and a DP receptor (analogous 2).${ }^{[37]}$ Figure S16 (Appendix D) displays the emission spectrum of the mixture of polymer P1 and DP receptor in its maximum aggregation state $(90 \%$ water in THF). However, addition of $\mathrm{Pb}^{2+}$ to this mixture does not cause any change in fluorescence, indicating that the DP$\mathrm{Pb}^{2+}$ complex does not quench the AIE emission of P1. Therefore, in the above fluorescence turn-off mode where DP moiety is a part of the AIE active polymer, the DP$\mathrm{Pb}^{2+}$ complex diminishes the AIE emission. Upon addition of $\mathrm{Pb}^{2+}$ to $\mathbf{P} 7$, we believe that the formation of polar DP- $\mathrm{Pb}^{2+}$ complex within polymer could disrupt the well-packed TPE aggregation, which leads to the decrease of AIE emission (Figure 5.1). P6 with low percentage of DP is less effective than $\mathbf{P 7}$ to induce the dissociation of the TPE aggregates.
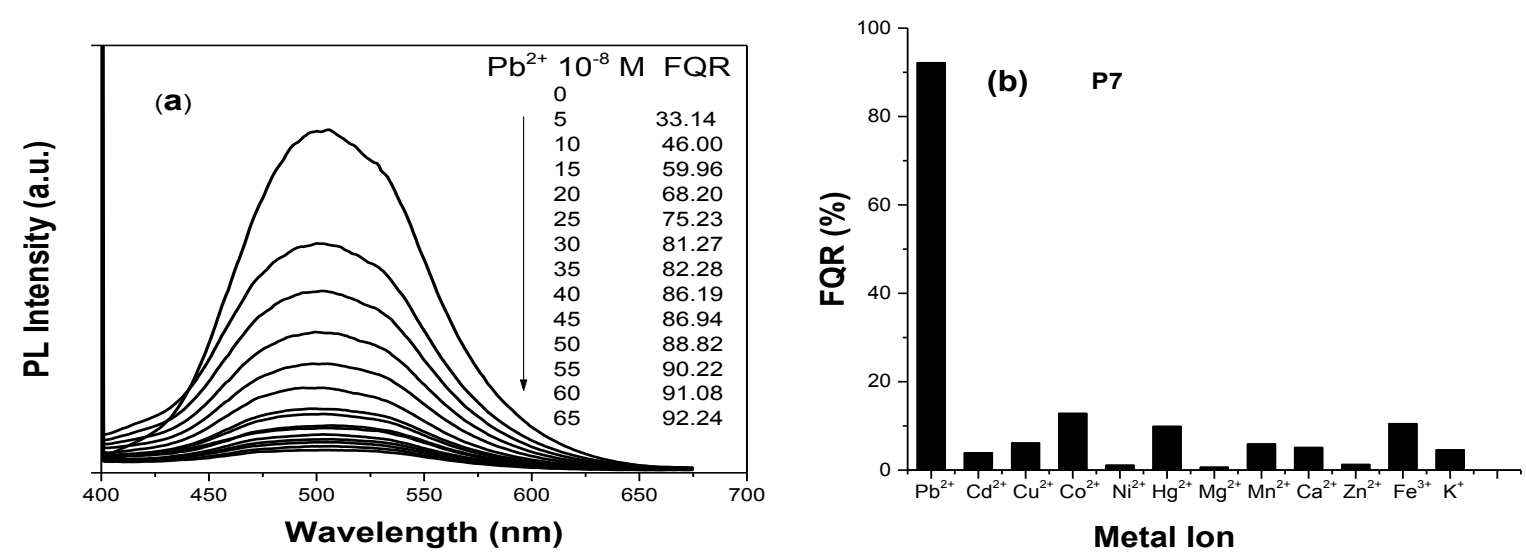

Figure 5.3 (a) Changes in fluorescence intensity (excitation at $375 \mathrm{~nm})$ of $\mathbf{P 7}\left(1.3 \times 10^{-5}\right.$ M) in $90 \%$ water/THF and (b) Relative selectivity of $\mathbf{P 7}$ towards different metal ions. 
The selectivity of polymers P7, P6 and P9 towards different alkali, alkaline earth, transition, and heavy metal ions $\left(\mathrm{K}^{+}, \mathrm{Ca}^{2+}, \mathrm{Mg}^{2+}, \mathrm{Mn}^{2+}, \mathrm{Fe}^{3+}, \mathrm{Co}^{2+}, \mathrm{Ni}^{2+}, \mathrm{Cu}^{2+}, \mathrm{Zn}^{2+}\right.$, $\left.\mathrm{Cd}^{2+}, \mathrm{Hg}^{2+}\right)$ was also investigated. Individual metal ion $\left(6-8 \times 10^{-7} \mathrm{M}\right)$ was added to the aqueous solution (90\% water in THF) of polymers P7, P6 and P9, and FQR were measured and compared against each metal ion (Figure 5.3(b), S17, Supporting Information). The results show that except for $\mathrm{Pb}^{2+}$, all the metal ions tested had no or very low quenching effect.

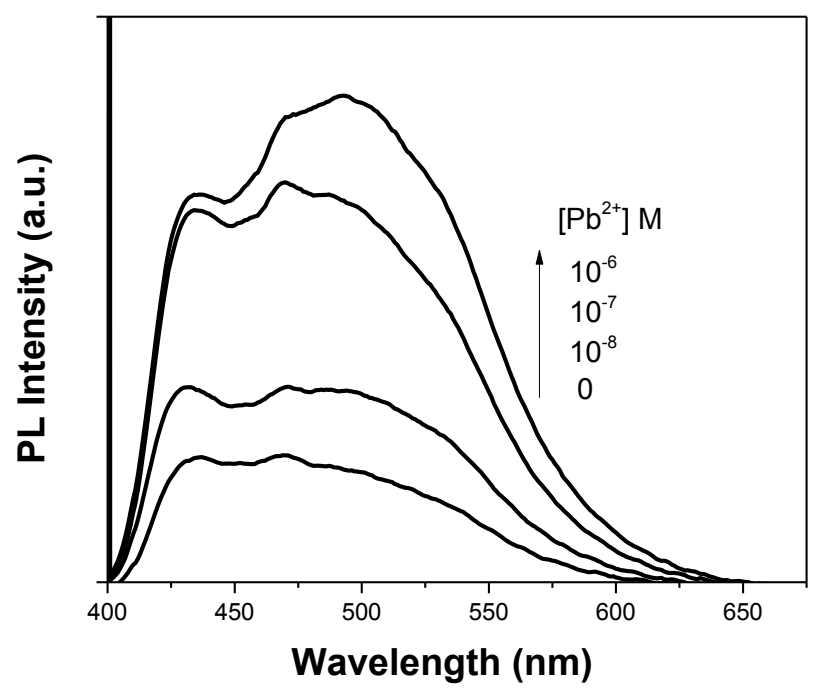

Figure 5.4 Changes of fluorescence intensity of $\mathbf{P 7}$ (excitation at $375 \mathrm{~nm}$ ) when $\mathrm{Pb}^{2+}$ in water $\left(10^{-6}, 10^{-7}, 10^{-8} \mathrm{M}\right)$ was added to P7 in THF directly (20\% water/THF mixture).

The fluorescence turn-on detection of $\mathrm{Pb}^{2+}$ was then achieved using $\mathbf{P 7}$ in a less polar medium (20\% water in THF). P7 alone emits weakly in a less polar medium but strongly in the presence of $\mathrm{Pb}^{2+}$. The fluorescence intensity increased 1.6, 3.3 and 4.2 fold when $10^{-8}, 10^{-7}$ and $10^{-6} \mathrm{M}$ of $\mathrm{Pb}^{2+}$ was added, respectively (Figure 5.4). The turn-on was noticeable even by naked eyes and solution becomes cloudy when $1 \mathrm{~mL}$ of $\mathrm{Pb}^{2+}$ in water 
$\left(10^{-6}, 10^{-7}, 10^{-8} \mathrm{M}\right)$ was added to $4 \mathrm{~mL}$ of $\mathbf{P 7}$ in THF directly (Figure S18, Supporting Information).

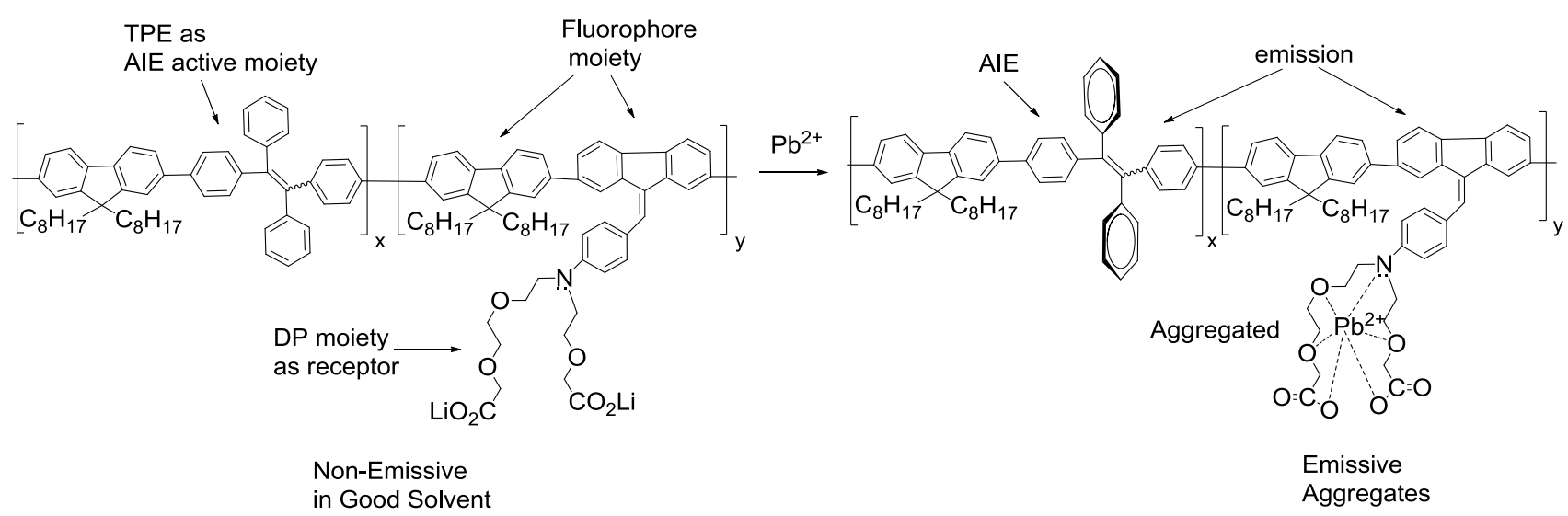

Scheme 5.2 Fluorescence turn-on mechanism of polymer in the presence of $\mathrm{Pb}^{2+}$.

In the fluorescence turn-on mode (Scheme 5.2), binding with the lead ion results in the ionic $\mathrm{DP}-\mathrm{Pb}^{2+}$ complex which is less soluble in a low polar medium, thus forcing the AIE-active segments of $\mathbf{P 7}$ to aggregate. As it could be seen visually (Appendix D, Figure S5.18), the solution of $\mathbf{P 7}$ (20\% water in THF) became turbid and emissive after mixing with $\mathrm{Pb}^{2+}$.

\section{Conclusions}

In summary, among the all the polymers tested, P7 containing 10\% DP exhibits the highest fluorescence quenching response and the best selectivity towards lead ion and can be used for both fluorescence turn-off and turn-on detection, specifically in $90 \%$ and $20 \%$ water-THF mixture, respectively. The coordination of $\mathrm{DP}$ with $\mathrm{Pb}^{2+}$ causes dissociation of the pre-packed aggregates in more polar medium and results in a significant decrease of AIE emission. In a less polar medium, the TPE units in P7 are 
mainly in a non-aggregation state and the formation of less soluble $\mathrm{DP}-\mathrm{Pb}^{2+}$ complex induces the aggregation of TPE moieties and turns on the AIE emission. This work demonstrates that the polymer structure, solvent polarity and probe-analyte complex all play jointly a critical role in the dual-mode fluorescence detection for lead ion, which provides helpful insights for future design of dual-mode detection of other analytes. 


\subsection{References}

[1] M. L. Riess, J. K. Halm, J. Gen. Intern. Med. 2007, 22, 1212.

[2] S. E. Manahan, Environmental Chemistry, Brooks/Colec, California, USA, 1984.

[3] World Health Organization, Guidelines for Drinking Water Quality, 3rd Ed., vol. 1, Geneva, 2004.

[4] A. N. Anthemidis, G. A. Zachariadis, J. A. Stratis, Talanta, 2002, 58, 831.

[5] Y. J. Cabon, Spectrochimica Acta Part B, 2002, 57, 513.

[6] M. S. Bispo, M. D. G. A. Korn, E. S. da. B. Morte, L. S. G. Teixeira, Spectrochimica Acta Part B, 2002, 57, 2175.

[7] J. Li, F. Lu, T. Umemura, k. Tsunoda, Anal. Chim. Acta, 2000, 419, 65.

[8] O. T. Butler, J. M. Cook, C. F. Harrington, S. J. Hill, J. Rieuwerts, D. L. Miles, J. Anal. At. Spectrom. 2006, 21, 217.

[9] Y. Li, C. Chen, B. Li, J. Sun, J. Wang, Y. Gao, Y. Zhao, Z. Chai, J. Anal. At. Spectrom. 2006, 21, 94.

[10] M. Leermakers, W. Baeyens, P. Quevauviller, M. Horvat, Trends Anal. Chem. $\mathbf{2 0 0 5}, 24,383$.

[11] B. Valeur, Molecular Fluorescence: Principles and Applications; Wiley-VCH Verlag GmbH, 2001.

[12] D. T. Quang, J. S. Kim, Chem. Rev. 2010, 110, 6280.

[13] H. N. Kim, W. X. Ren, J. S. Kim, J. Yoon, Chem. Soc. Rev. 2012, 41, 3210.

[14] M.-Y. Chae, J. Yoon, A. W. Czarnik, J. Mol. Recognit. 1996, 9, 297.

[15] W.-S. Xia, R. H. Schmehl, C.-J. Li, J. T. Mague, C.-P. Luo, D. M. Guldi, J. Phys. Chem. B, 2002, 106, 833. 
[16] C.-T. Chen, W.-P. Huang, J. Am. Chem. Soc. 2002, 124, 6246.

[17] T. Hayashita, D. Qing, M. Minagawa, J. C. Lee, C. H. Ku, N. Teramae, Chem. Commun. 2003, 2160.

[18] Q. He, E. W. Miller, A. P. Wong, C. J. Chang, J. Am. Chem. Soc. 2006, 128, 9316.

[19] F.-Y. Wu, S. W. Bae, J.-I. Hong, Tetrahedron Lett. 2006, 47, 8851.

[20] R. Zhou, B. Li, N. Wu, G. Gao, J. You, J. Lan, Chem. Commun. 2011, 47, 6668.

[21] J. Y. Kwon, Y. J. Jang, Y. J. Lee, K. M. Kim, M. S. Seo, W. Nam, J. Yoon, J. Am. Chem. Soc. 2005, 127, 10107.

[22] L. Marbella, B. Serli-Mitasev, P. Basu, Angew. Chem. Int. Ed. 2009, 48, 3996.

[23] a) J. Li, Y. Lu, J. Am. Chem. Soc. 2000, 122, 10466; b) C.-L. Li, K.-T. Liu, Y.-W. Lin, H.-T. Chang, Anal. Chem. 2011, 83, 225; c) T. Li, E. Wang, S. Dong, Anal. Chem. 2010, 82, 1515; d) D. Mazumdar, J. Liu, G. Lu, J. Zhou, Y. Lu, Chem. Commun. 2010, 46, 1416; e) X. Yang, J. Xu, X. Tang, H. Liu, D. Tian, Chem. Commun. 2010, 46, 3107; f) T. Li, S. Dong, E. Wang, J. Am. Chem. Soc. 2010, 132, 13156.

[24]. S. Deo, H. A. Godwin, J. Am. Chem. Soc. 2000, 122, 174.

[25] a) V. S. Shete, D. E. Benson, Biochemistry, 2009, 48, 462; b) P. Chen, B. Greenberg, S. Taghavi, C. Romano, D. van der Lelie, C. He, Angew. Chem. Int. Ed. 2005, 44, 2715.

[26] a) Q. Zheng, C. Han, H. Li, Chem. Commun. 2010, 46, 7337; b) H. Y. Lee, D. R. Bae, J. C. Park, H. Song, W. S. Han, J. H. Jung, Angew. Chem. Int. Ed. 2009, 48, 1239; c) F. Chai, C. Wang, T. Wang, L. Li, Z. Su, ACS Appl. Mater. Interfaces, 
2010, 2, 1466; d) A. Alizadeh, M. M. Khodaei, C. Karami, M. S. Workentin, M. Shamsipur, M. Sadeghi, Nanotechnology, 2010, 21, 315503.

[27] E. M. Ali, Y. Zheng, H.-H. Yu, J. Y. Ying, Anal. Chem. 2007, 79, 9452.

[28] I.-B. Kim, A. Dunkhorst, J. Gilbert, U. H. F. Bunz, Macromolecules, 2005, 38, 4560.

[29] K. M. Lee, X. Chen, W. Fang, J.-M. Kim, J. Yoon, Macromol. Rapid Commun. 2011, 32, 497.

[30] a) J. Luo, Z. Xie, J. W. Y. Lam, L. Cheng, H. Chen, C. Qiu, H. S. Kwok, X. Zhan, Y. Liu, D. Zhu, B. Z. Tang, Chem. Commun. 2001, 1740; b) B. Z. Tang, X. Zhan, G. Yu, P. P. S. Lee, Y. Liu, D. Zhu, J. Mater. Chem. 2001, 11, 2974; c) Y. Hong, J. W. J. Lam and B. Z. Tang, Chem. Soc. Rev. 2011, 40, 5361; d) Z. J. Ning, Z. Chen, Q. Zhang, Y. L. Yan, S. X. Qian, Y. Cao and H. Tian, Adv. Funct. Mater. 2007, 17, 3799; e) B. Xu, J. He, Y. Dong, F. Chen, W. Yu and W. Tian, Chem. Commun. 2011, 47, 6602.

[31] a) C. He, D. Zhu, Q. He, L. Shi, Y. Fu, D. Wen, H. Cao, J. Cheng, Chem. Commun. 2012, 48, 5739; b) Y. Suna, X. Liangb, J. Fanb, X. Yanga, J. Photochemistry and Photobiology A, 2013, 253, 81; c) X. Li, B. Xu, H. Lu, Z. Wang, J. Zhang, Y. Zhang, Y. Dong, K. Ma, S. Wen, W. Tian, Anal. Methods, 2013, 5, 438; d) M. Shellaiah, Y.-H. Wu, A. Singh, M. V. R. Raju, H.-C. Lin, J. Mater. Chem. A, 2013, 1, 1310.

[32] W. Wu, S. Ye, L. Huang, L. Xiao, Y. Fu, Q. Huang, G. Yu, Y. Liu, J. Qin, Q. Lia, Z. Li, J. Mater. Chem. 2012, 22, 6374. 
[33] R. Hu, J. L. Maldonado, M. Rodriguez, C. Deng, C. K. W. Jim, J. W. Y. Lam, M. M. F.Yuen, G. R. Ortiz, B. Z. Tang, J. Mater. Chem. 2012, 22, 232.

[34] K. R. Ghosh, S. K. Saha, J. P. Gao, Z. Y. Wang, Chem. Commun. 2014, 50, 717.

[35] Y. Liu, C. Deng, L. Tang, A. Qin, R. Hu, J. Z. Sun, B. Z. Tang, J. Am. Chem. Soc. 2011, 133, 660 .

[36] a) B. Z. Tang, Y. Hong, T. Leung, Induced Emission Luminogens for Metal Ion Detection, U.S. patent 2013/0059392 A1, March 07, 2013; b) X. Huang, X. Gu, G. Xhang, D. Xhang, Chem Commun., 2012, 48, 12195; c) J. Mei, Y. Wang, J. Tong, J. Wang, A. Qin, J. Z. Sun, B. Z. Tong, Chem. Eur. J. 2013, 19, 613.

[37] S. K. Saha, K. R. Ghosh, W, Hao, Z. Y. Wang, Y. Chiniforooshan, J. Ma, W. J. Bock, J. Mater. Chem. A, 2014, 2, 5024.

[38] T. Yamamoto, K. Takimiya, J. Am. Chem. Soc. 2007, 129, 2224. 


\section{Contribution to Knowledge}

1. Synthesized a new ladder polymer by post-polymerization annulation with an absorption edge from NIR region.

2. Studied the optical, electrochemical and solubility properties of different series of linear-ladder polymers.

3. Synthesized series of polymers by post-modification and studied their optical, electrochemical and solubility properties as well as properties in photodetector

4. Demonstrated that cyclic imide polymer does not produce ladder polymer using Lawesson's Reagent.

5. Demonstrated the dual-mode fluorescence detection of $\mathrm{Pb}^{2+}$ in water by a series of AIE active polymers 


\section{Publications}

1. Saha, S. K.; Ghosh, K. R.; Gao, J. P.; Wang, Z. Y. Highly sensitive dual-mode fluorescence detection of lead ion in water using aggregation-induced emissive polymers. Macromol. rapid commun. 2014, 35, 1592.

2. Ghosh, K. R.; Saha, S. K.; Wang, Z. Y. Ultra-sensitive detection of explosives in solution and film as well as the development of thicker film effectiveness by tetraphenylethene moiety in AIE active fluorescent conjugated polymer. Polym. Chem. 2014, 5, 5638 .

3. Saha, S. K.; Ghosh, K. R.; Hao, W.; Gao, J. P.; Wang, Z. Y.; Ma, J.; Chiniforooshan, Y.; Bock, W. J. Highly sensitive and selective fluorescence turn-on detection of lead Ion in water using fluorene-based compound and polymer. J. Mater. Chem A. 2014, 2, 5024. 4. Ghosh, K. R.; Saha, S. K.; Gao, J. P.; Wang, Z. Y Wang Direct detection of ultralow trace amounts of isocyanates in air using a fluorescent conjugated polymer. Chem. Commun. 2014, 50, 176.

\section{Seminar and Conference Presentations}

1. Saha, S. K.; Ghosh, K. R.; Mia, S.; Wang, Z. Y. Design, Synthesis and Characterization of Low Band Gap Polymers.. Oral presentation, $98^{\text {th }}$ Canadian Chemistry Conference and Exhibition, Ottawa University, Ottawa, ON, June 2015.

2. Saha, S. K.; Ghosh, K. R.; Gao, J. P.; Wang, Z. Y. Amplified Fluorescence Detection of Isocyanates, Nitro-Explosives and Toxic Metals Using Aggregation-Induced Emissive 
Polymers. Oral presentation, Ottawa-Carleton Chemistry Institute Conference day, Carleton University, Ottawa, ON, May 2014.

3. Ghosh, K. R.; Saha, S. K.; Gao, J. P.; Wang, Z. Y. Ultra Low Detection of Isocyanates and Nitro-Explosives Using Aggregation-Induced Emission Active Fluorescent Conjugated Polymer. Poster presentation, Ottawa-Carleton Chemistry Institute Conference day, Carleton University, Ottawa, ON, May 2014. 


\section{Appendix A: Spectra of synthesized compounds and polymers in chapter 2}

sk-21-13
1D proton

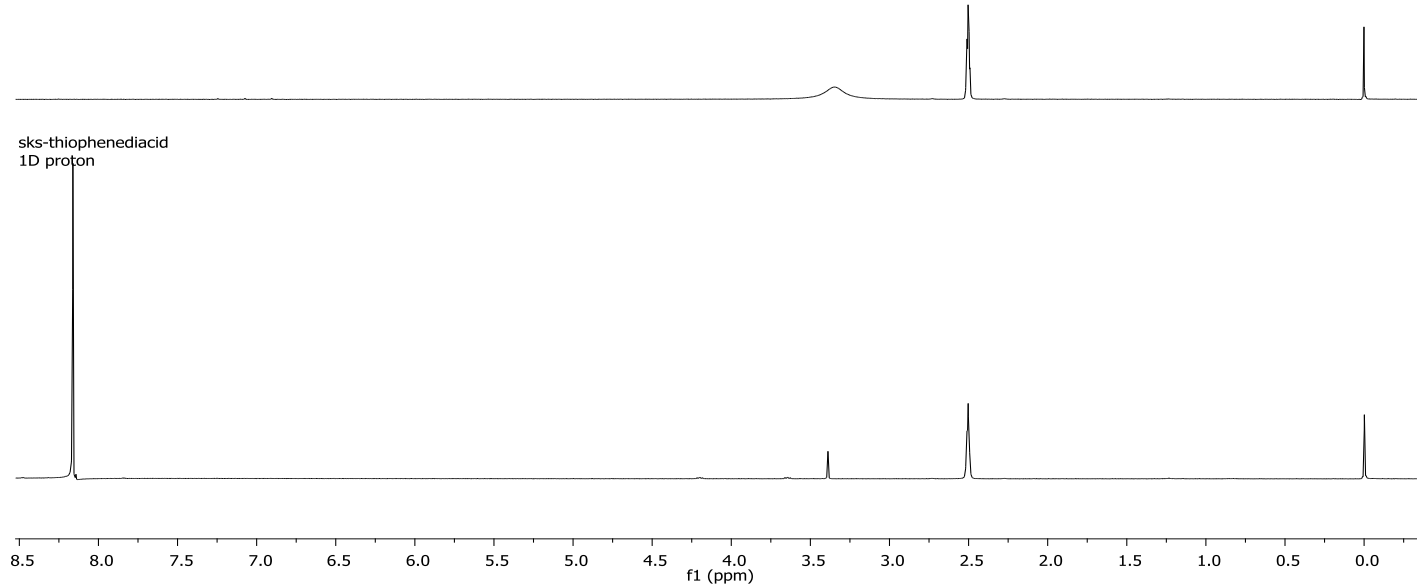

Figure S2.1 ${ }^{1} \mathrm{H}$ NMR spectrum (300 MHz, DMSO-D $)$ of compound thiophene-3,4dicarboxalic acid and 2,5-dibromothiophene-3,4-dicarboxalic acid (1).

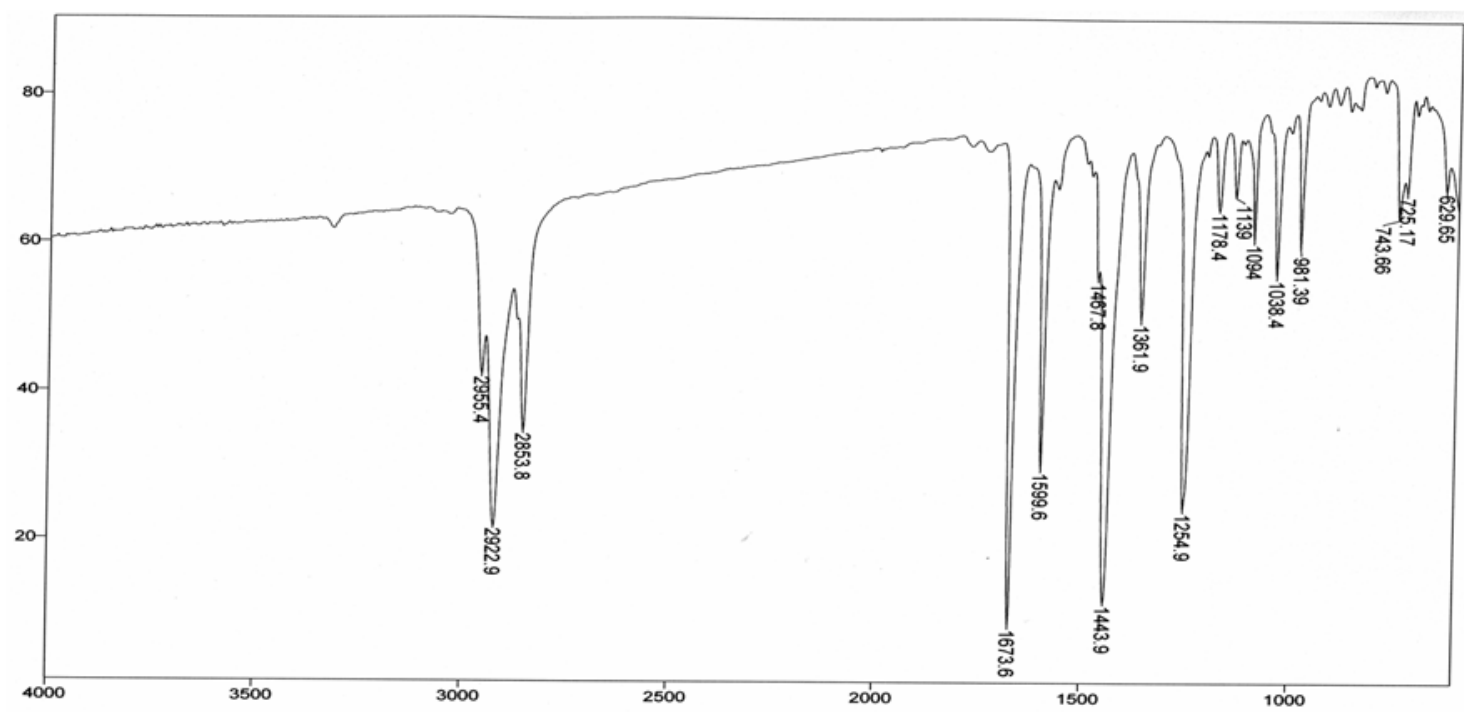

Figure S2.2 IR spectrum of monomer $\mathbf{M 1}$ on $\mathrm{NaCl}$ plate. 

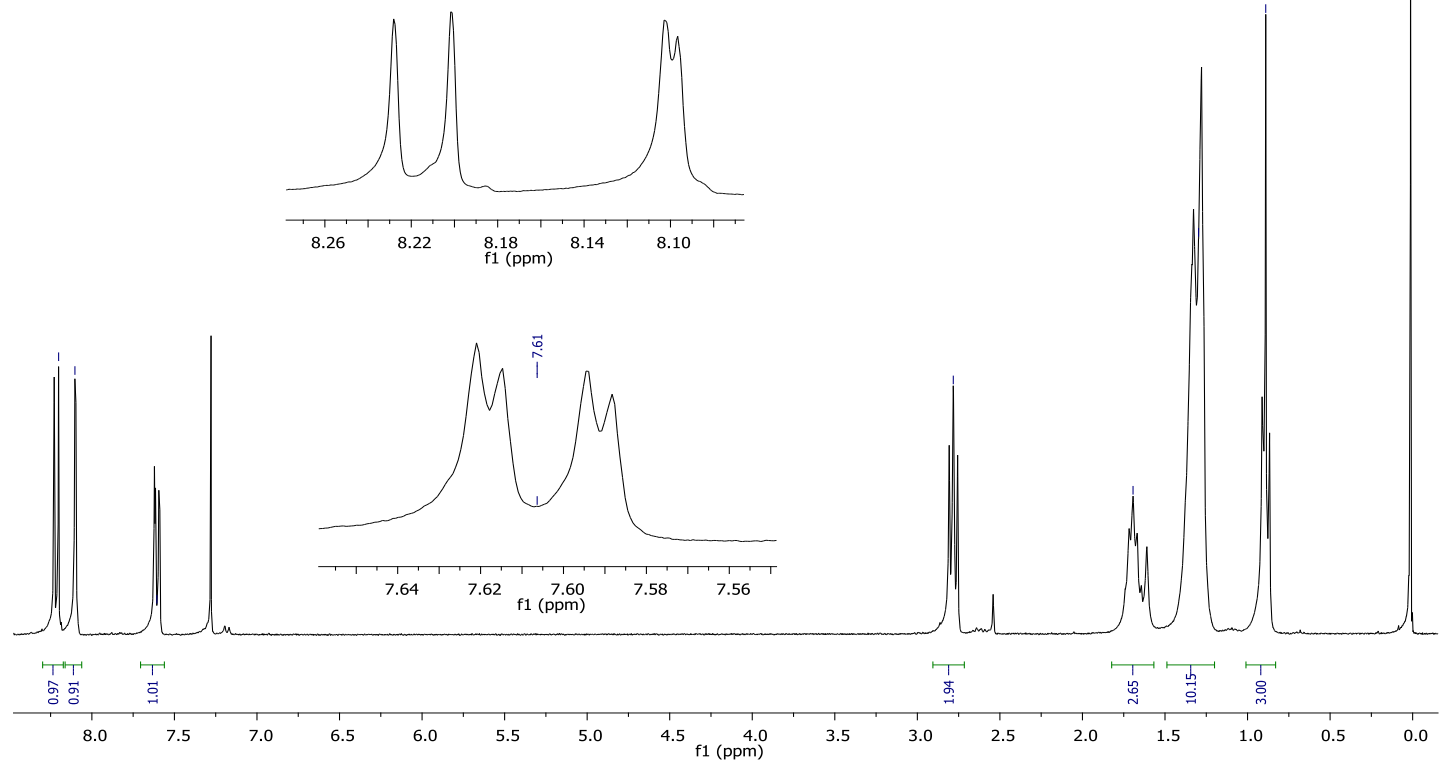

Figure S2.3 ${ }^{1} \mathrm{H}$ NMR spectrum $\left(300 \mathrm{MHz}, \mathrm{CDCl}_{3}\right.$ ) of compound M1.
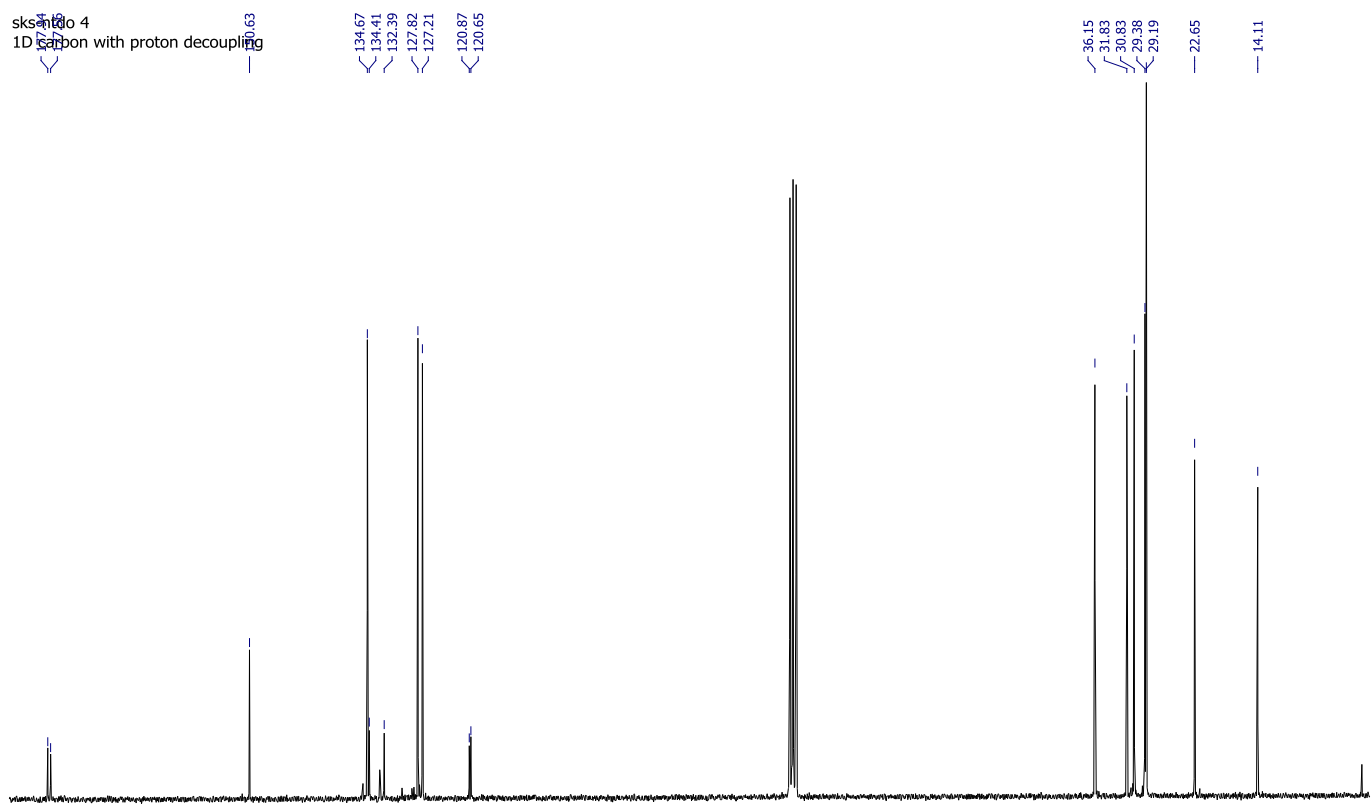

$180 \quad 170$

Figure S2.4. ${ }^{13} \mathrm{C}$ NMR spectrum $\left(75 \mathrm{MHz}, \mathrm{CDCl}_{3}\right.$ ) of compound M1. 
$2 \mathrm{C} 8814$ Scan $10 \mathrm{RT}=1: 55 \quad 100 \%=13161 \mathrm{mv} 31-\mathrm{Jan}-2014 \quad 10: 25$ HRP + EI 3
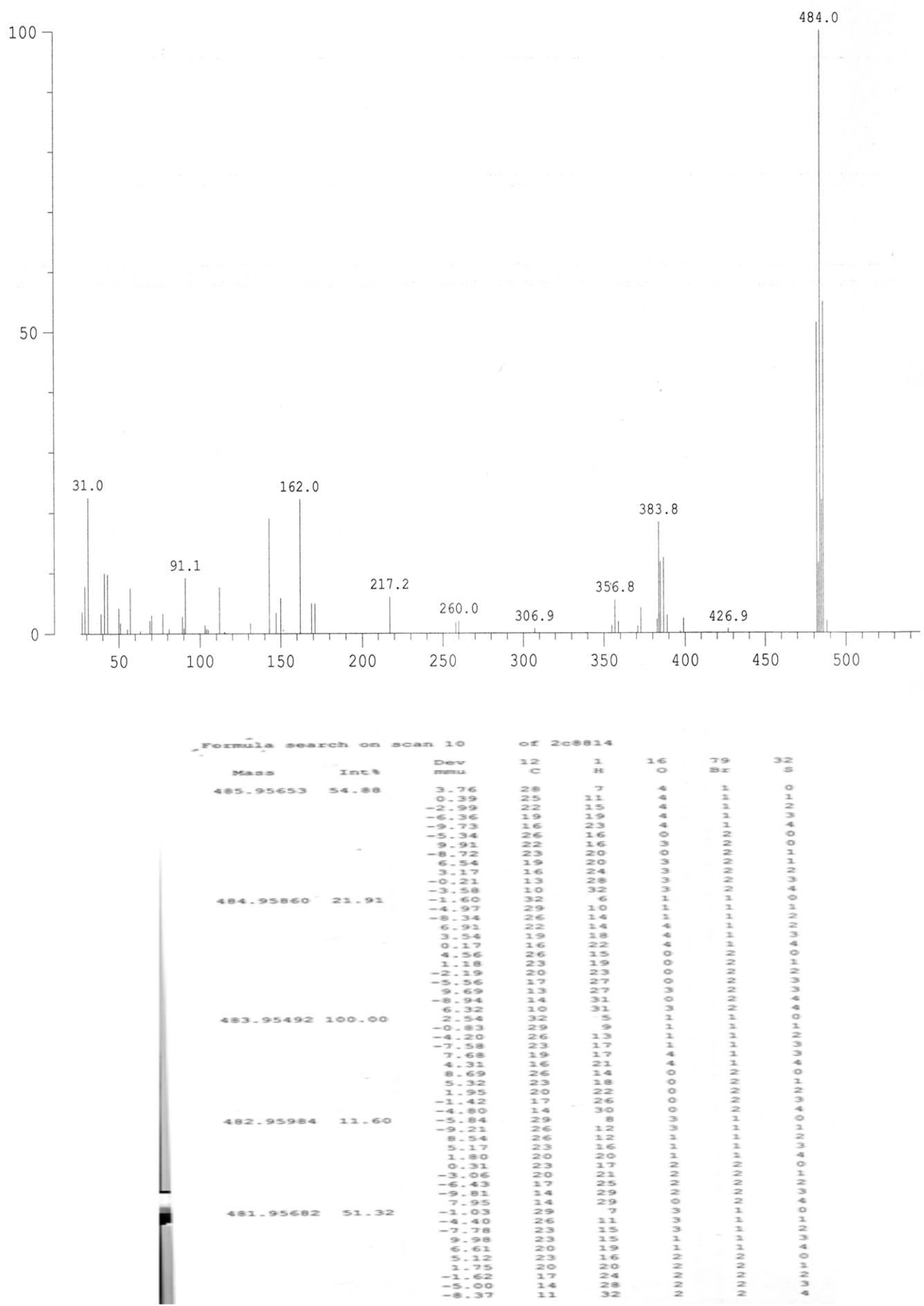

Figure S2.5 Mass spectrum of monomer M1. 


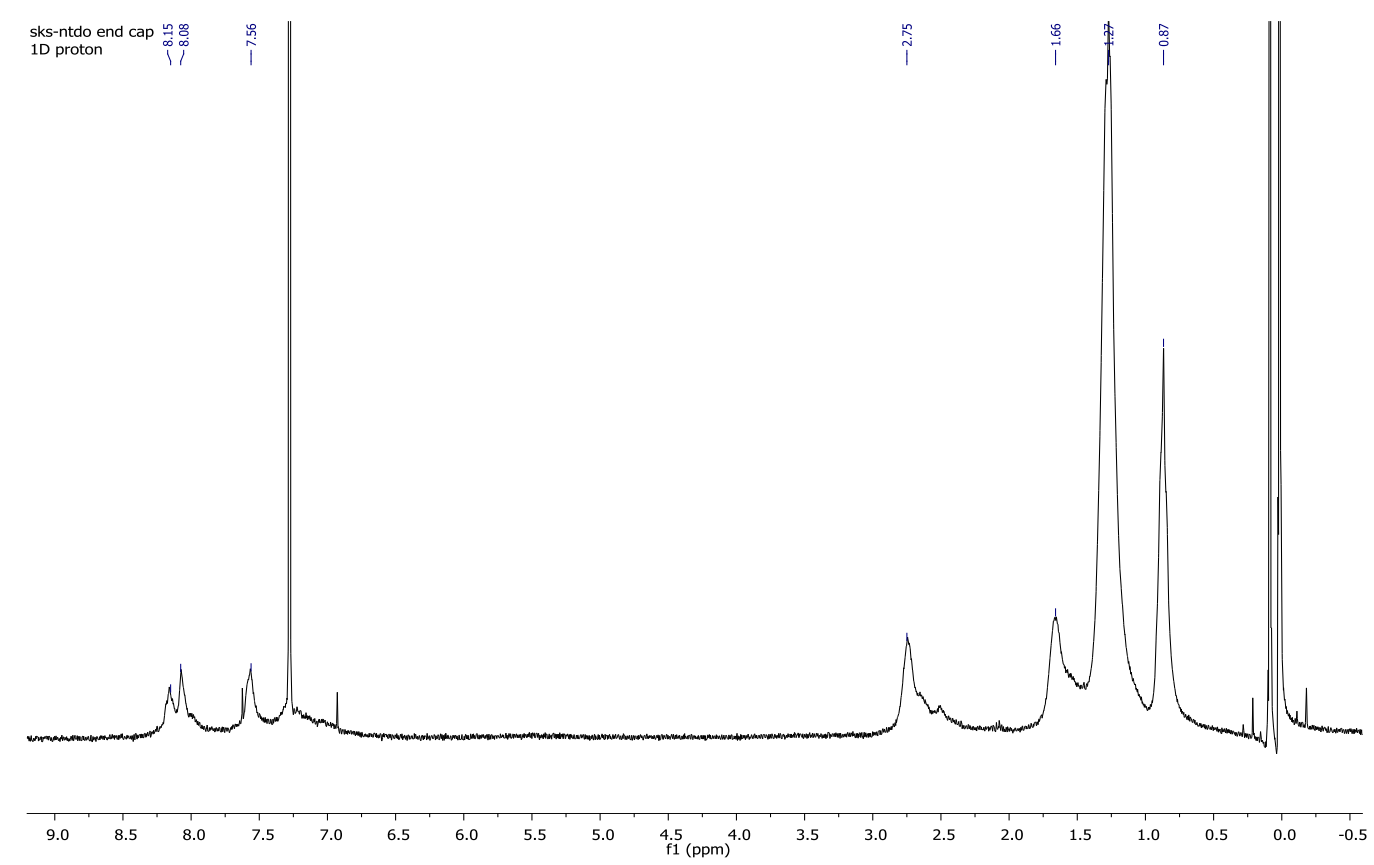

Figure S2. $6{ }^{1} \mathrm{H}$ NMR spectrum $\left(300 \mathrm{MHz}, \mathrm{CDCl}_{3}\right)$ of polymer P1.

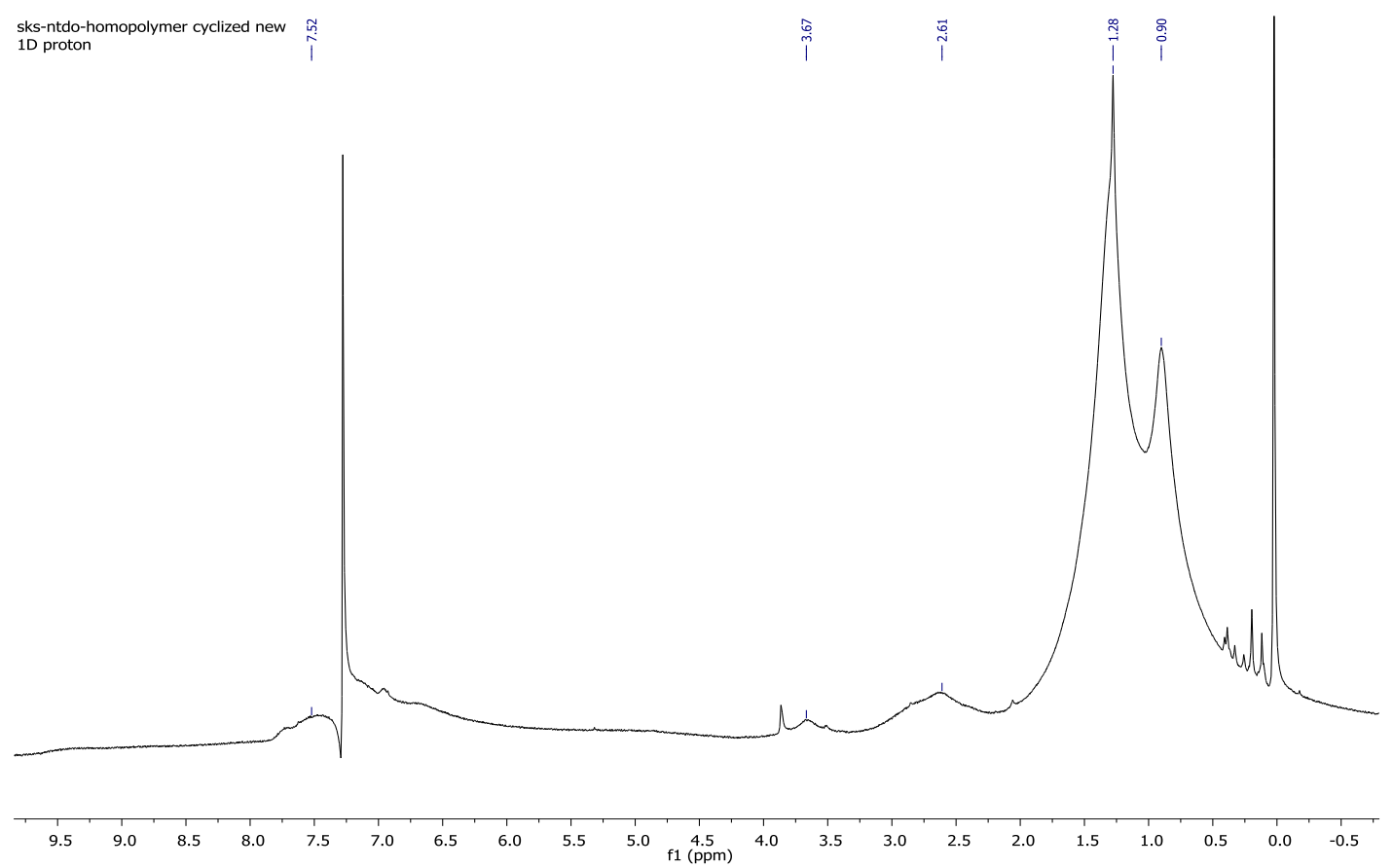

Figure S2. $7{ }^{1} \mathrm{H}$ NMR spectrum $\left(300 \mathrm{MHz}, \mathrm{CDCl}_{3}\right.$ ) of polymer P2. 


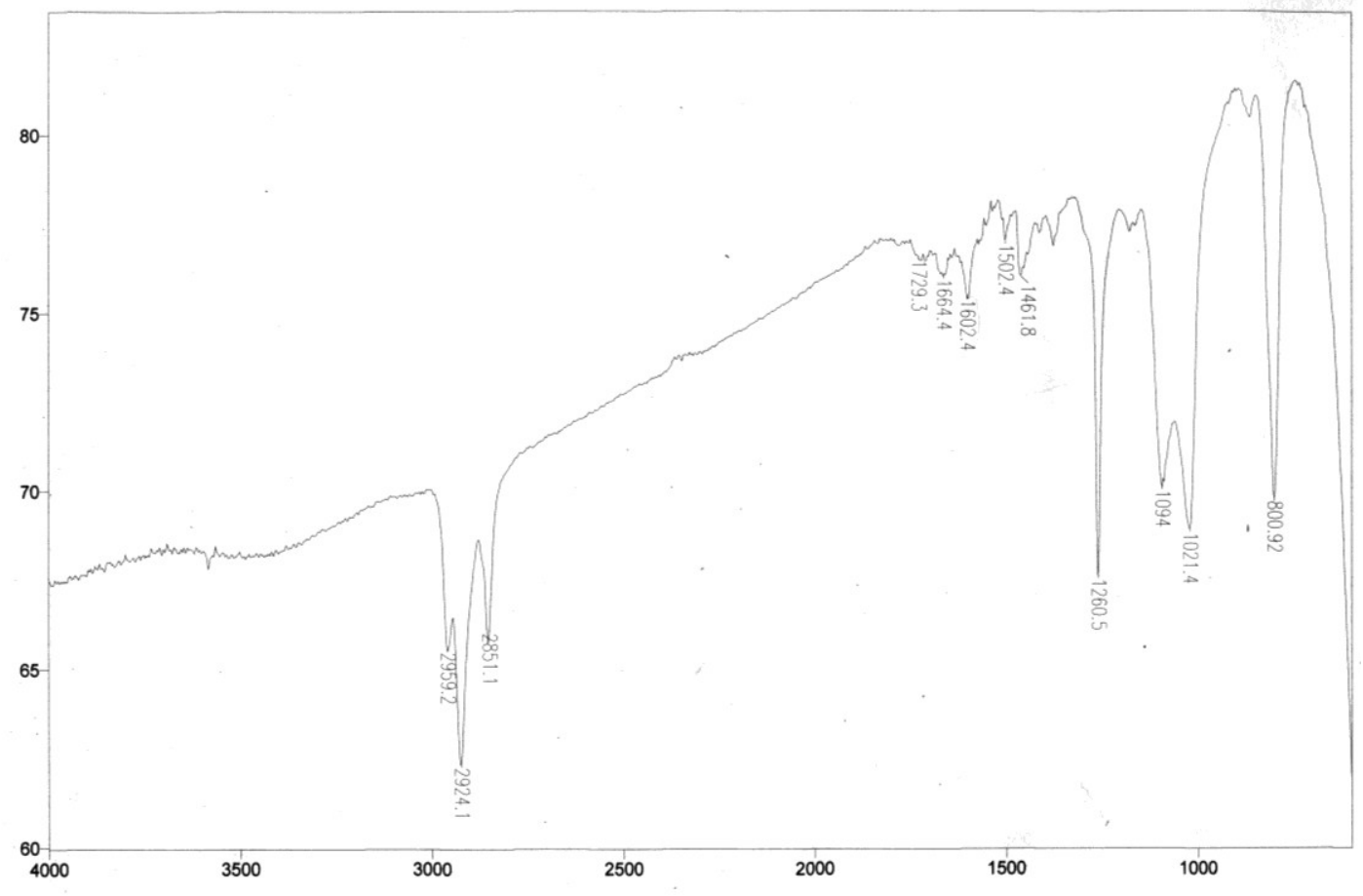

Figure S2.8 IR of desulfurization reaction of polymer $\mathbf{P 2}$ on $\mathrm{NaCl}$ plate.

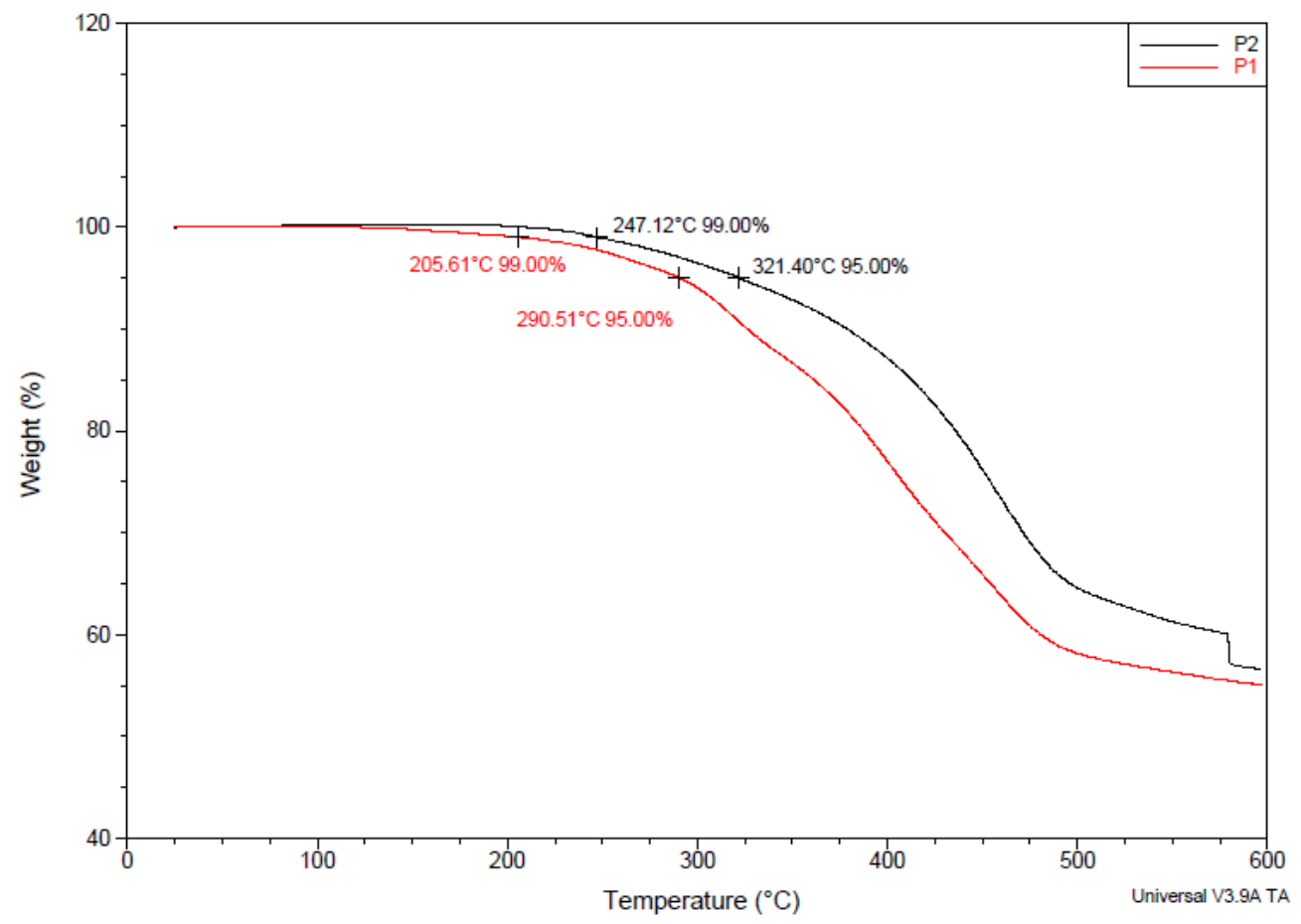

Figure S2.9 TGA plots of polymers $\mathbf{P 1}$ and $\mathbf{P 2}$ with a heating rate of $10{ }^{\circ} \mathrm{C} / \mathrm{min}$ under nitrogen atmosphere. 


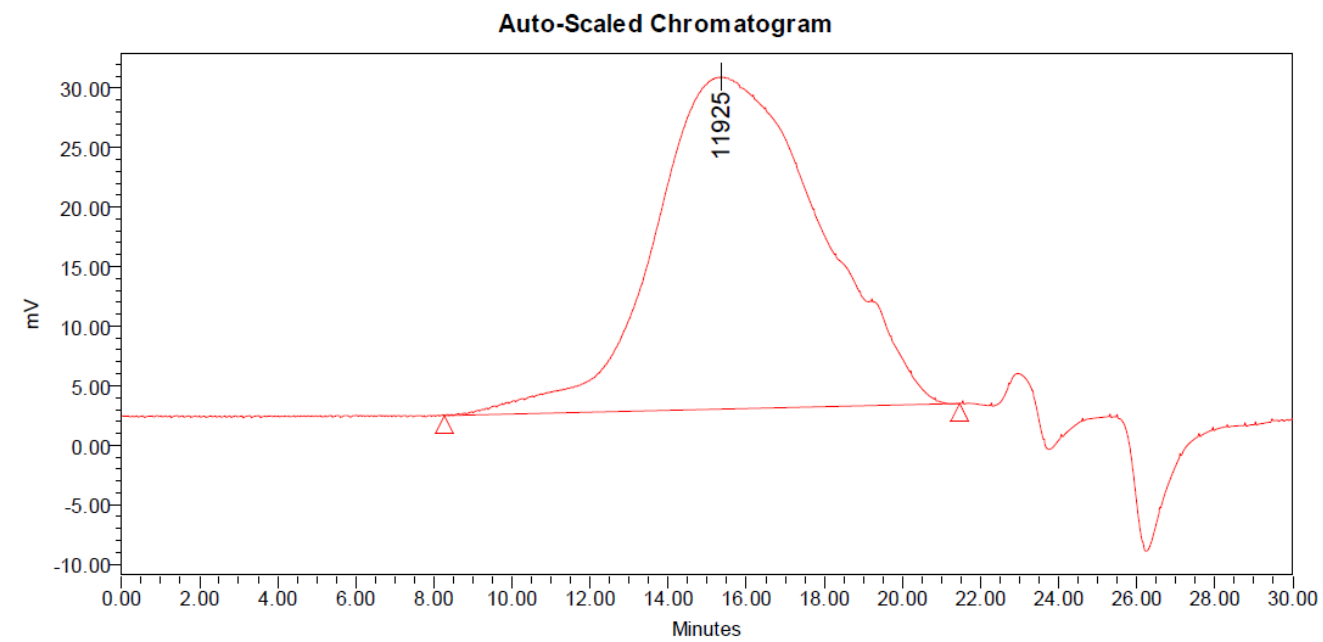

GPC Results

\begin{tabular}{|c|c|c|c|c|c|c|c|c|c|c|}
\hline & Dist Name & $\mathrm{Mn}$ & $\mathrm{Mw}$ & $\mathrm{MP}$ & $\mathrm{Mz}$ & $\mathrm{Mz}+1$ & $\mathrm{Mv}$ & Poly dispersity & MW Marker 1 & MW Marker 2 \\
\hline 1 & & 6216 & 21229 & 11925 & 127151 & 336667 & & 3.415047 & & \\
\hline
\end{tabular}

Figure S2.10 GPC result for polymer P1
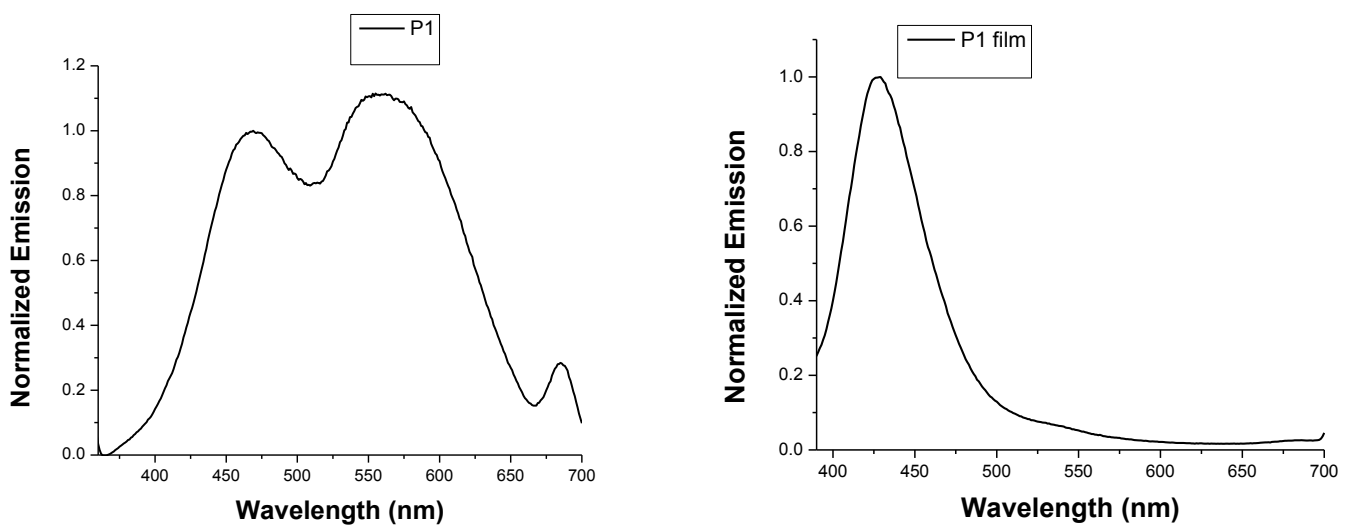

Figure S2.11 Normalized emission of polymer $\mathbf{P 1}$ solution in $\mathrm{CHCl}_{3}$ and film on glass substrate. 


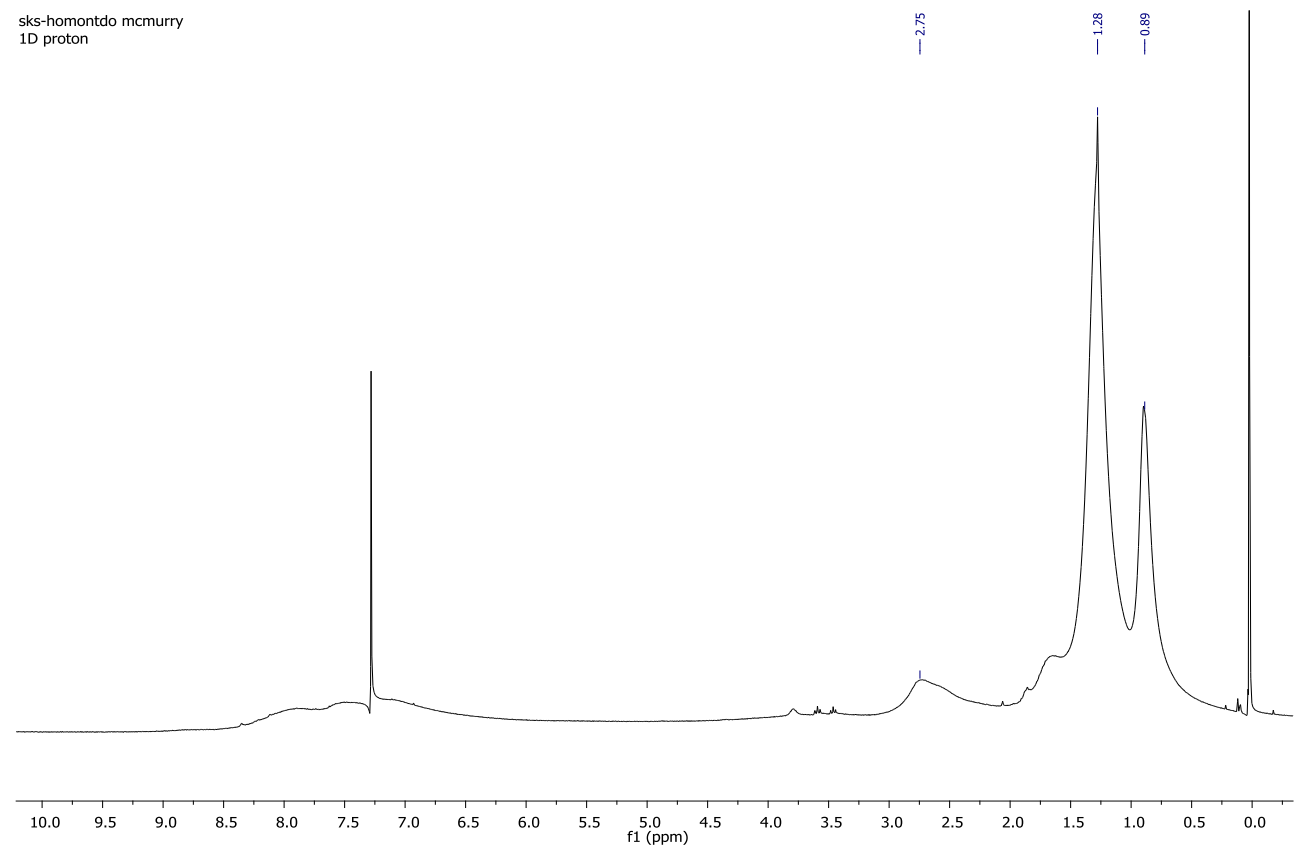

Figure S2.12 ${ }^{1} \mathrm{H}$ NMR spectrum $\left(300 \mathrm{MHz}, \mathrm{CDCl}_{3}\right.$ ) of polymer $\mathbf{P 2}$ by McMurry.

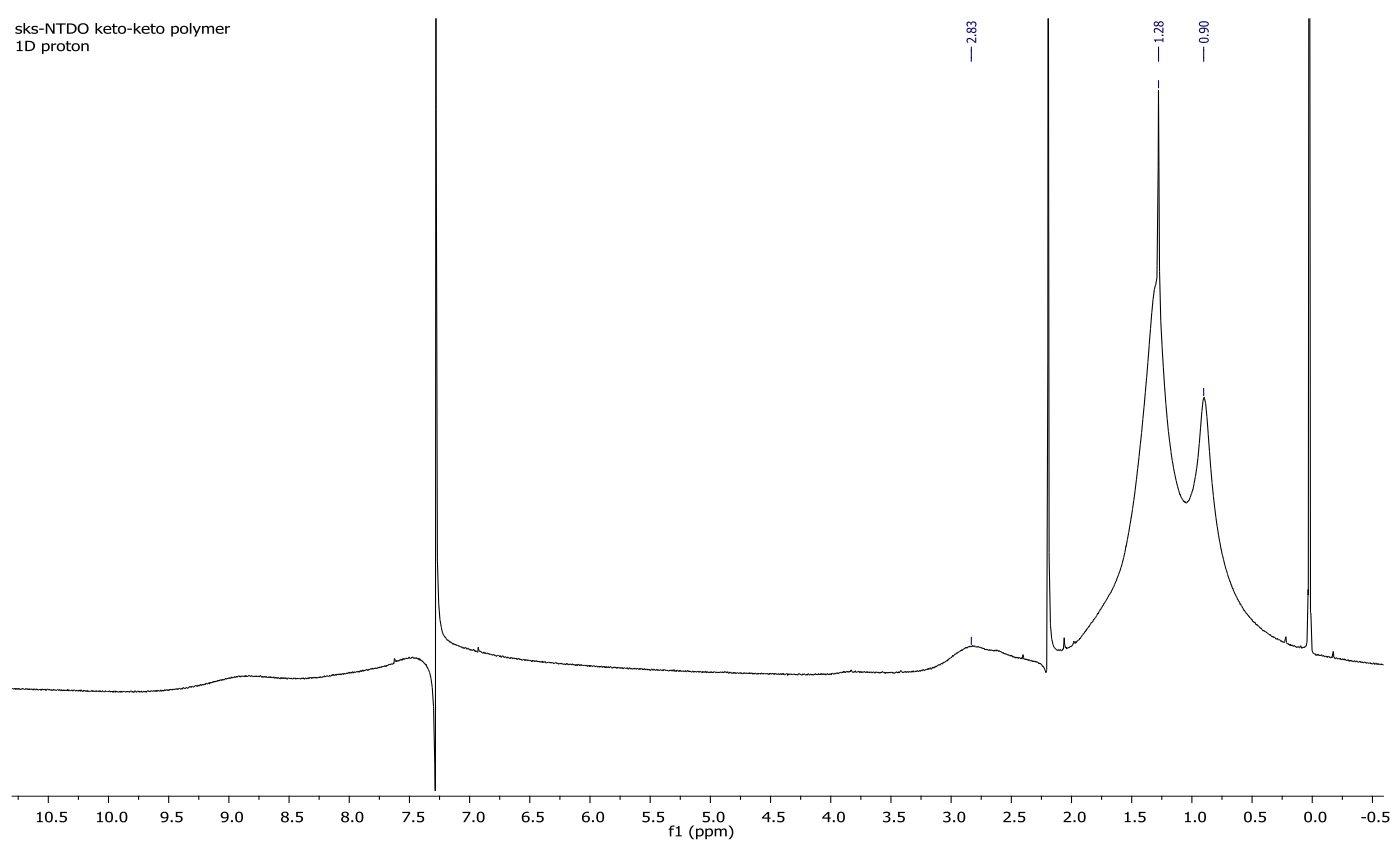

Figure S2.13 ${ }^{1} \mathrm{H}$ NMR spectrum $\left(300 \mathrm{MHz}, \mathrm{CDCl}_{3}\right)$ of polymer P3. 


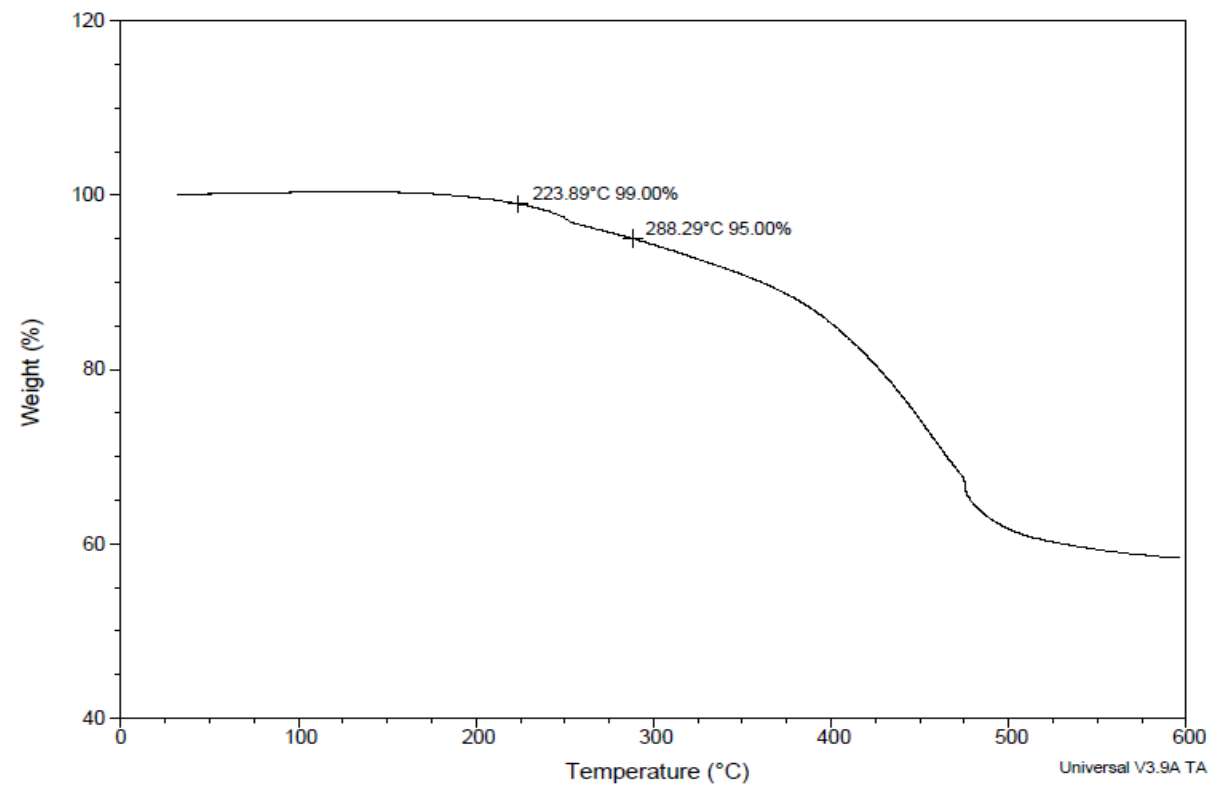

Figure S2.14 TGA plots of polymer $\mathbf{P 3}$ with a heating rate of $10{ }^{\circ} \mathrm{C} / \mathrm{min}$ under nitrogen atmosphere.

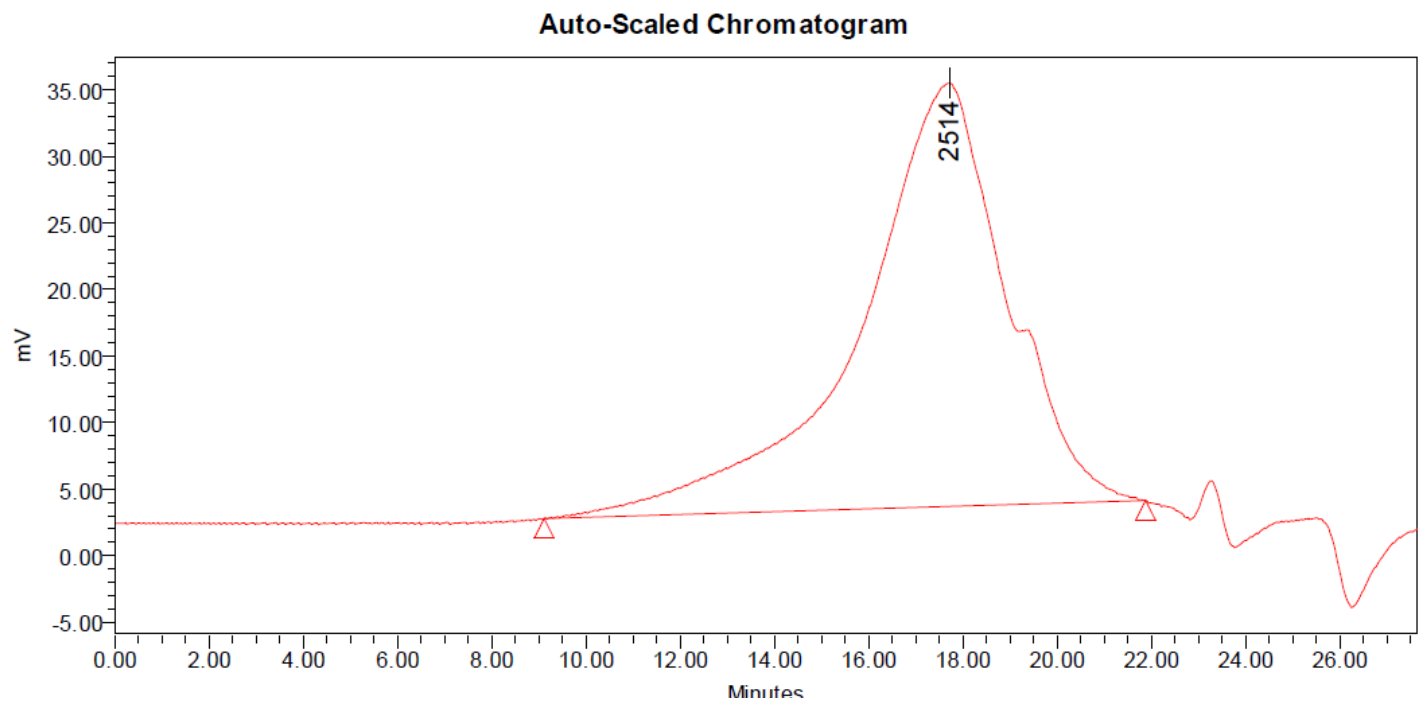

GPC Results

\begin{tabular}{|c|c|c|c|c|c|c|c|r|r|l|}
\hline & Dist Name & Mn & Mw & MP & Mz & Mz+1 & Mv & Poly dispersity & MW Marker 1 & MW Marker 2 \\
\hline 1 & & 3609 & 14254 & 2514 & 137236 & 329765 & & 3.949299 & & \\
\hline
\end{tabular}

Figure S2.15: GPC result for polymer P3. 


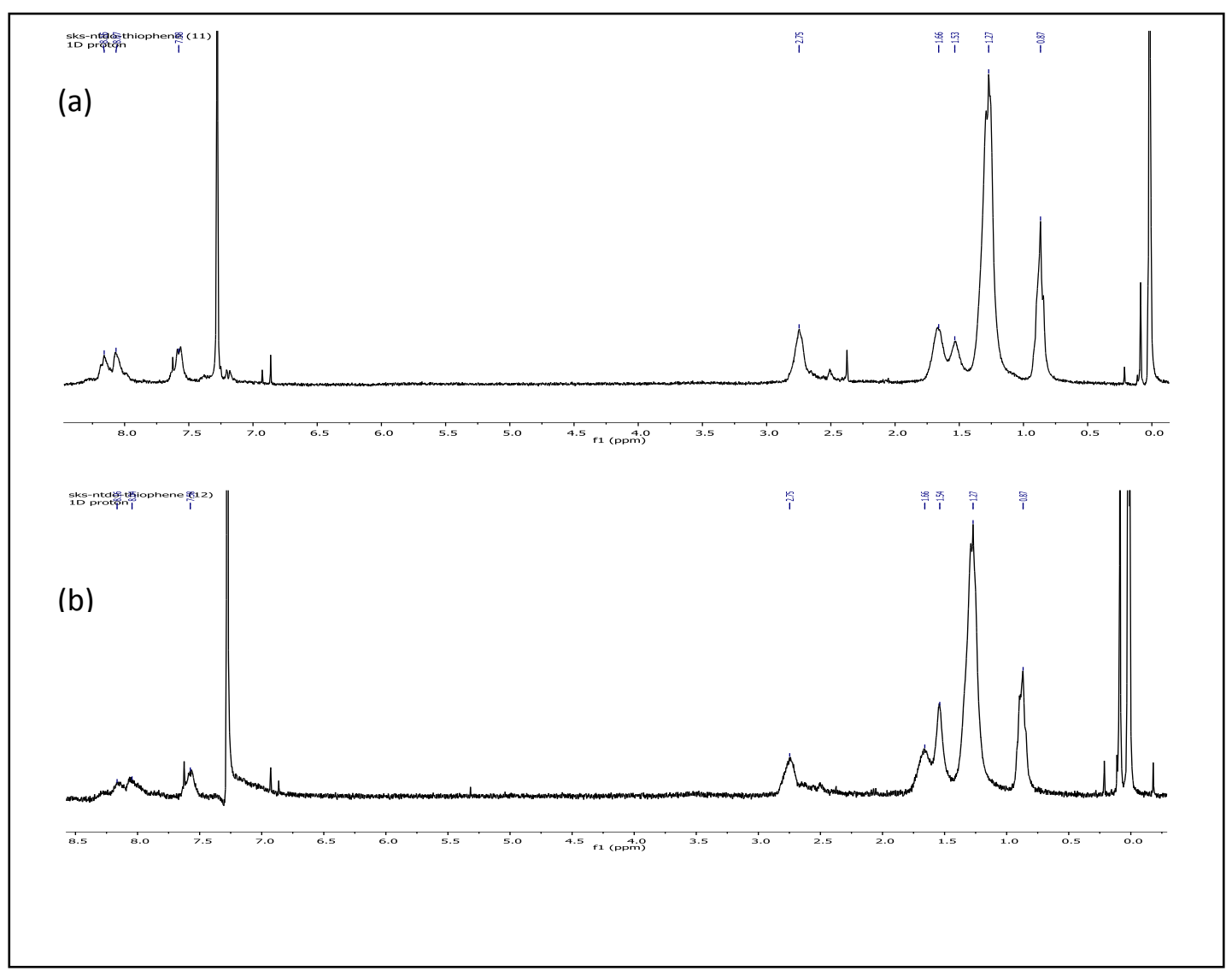

Figure S2.16 ${ }^{1} \mathrm{H}$ NMR for Polymer (a) P4, and (b) P5 (300 MHz, CDCl 3 ).

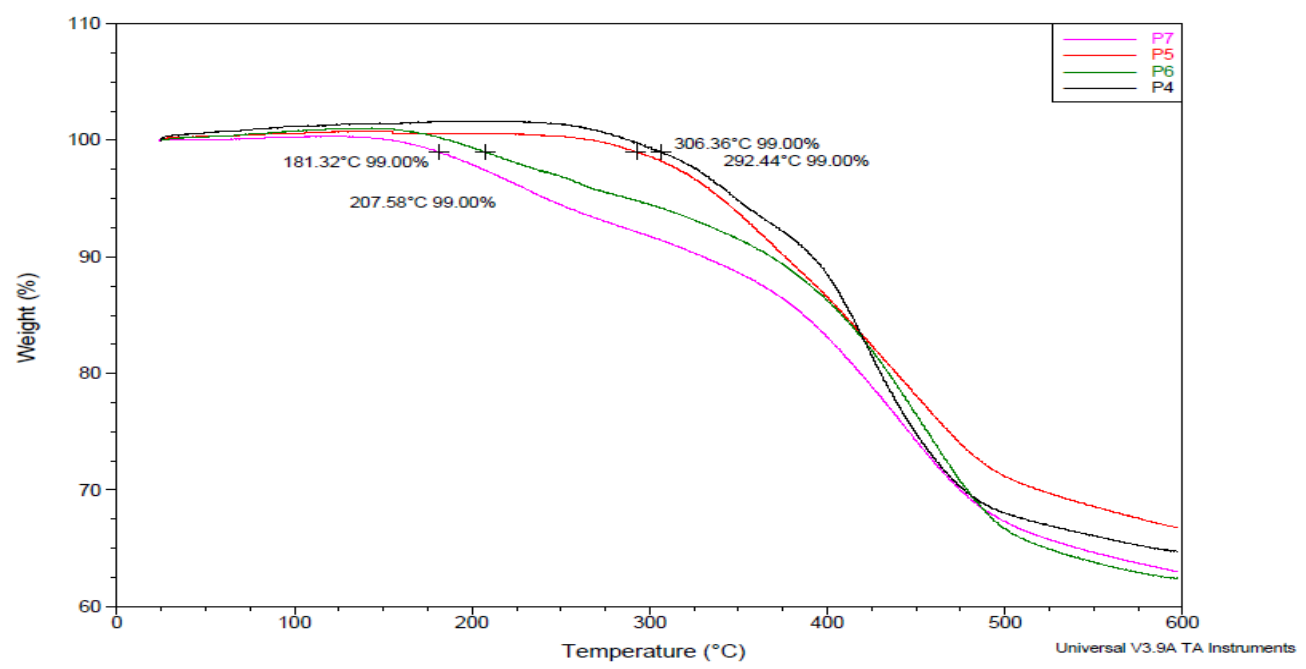

Figure S2.17 TGA plots of the polymers with a heating rate of $10^{\circ} \mathrm{C} / \mathrm{min}$ under an inert atmosphere. 


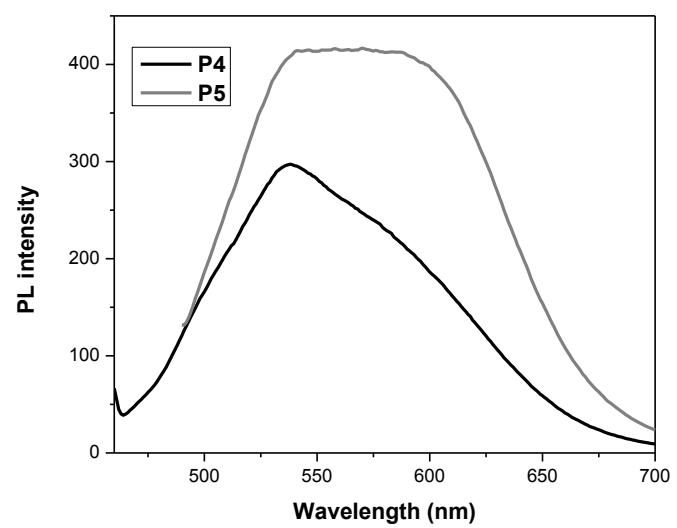

Figure S2.18 Emission of polymer P4 and P5 solution in TCB.

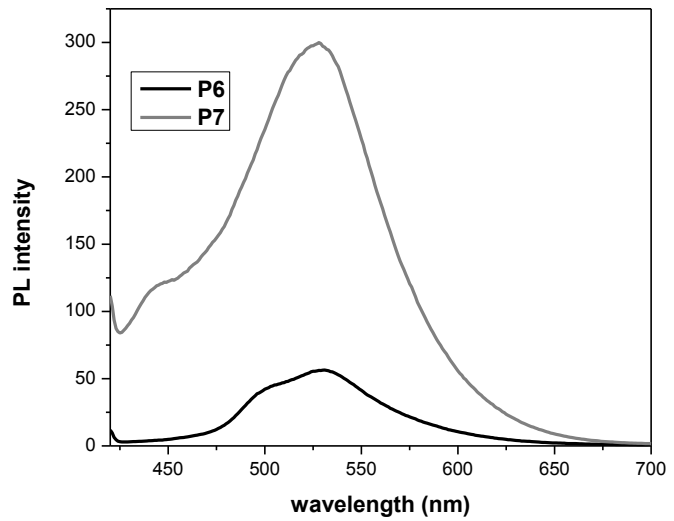

Figure S2.19 Emission of polymers P6 and P7 solution in TCB and TCE, respectively. 


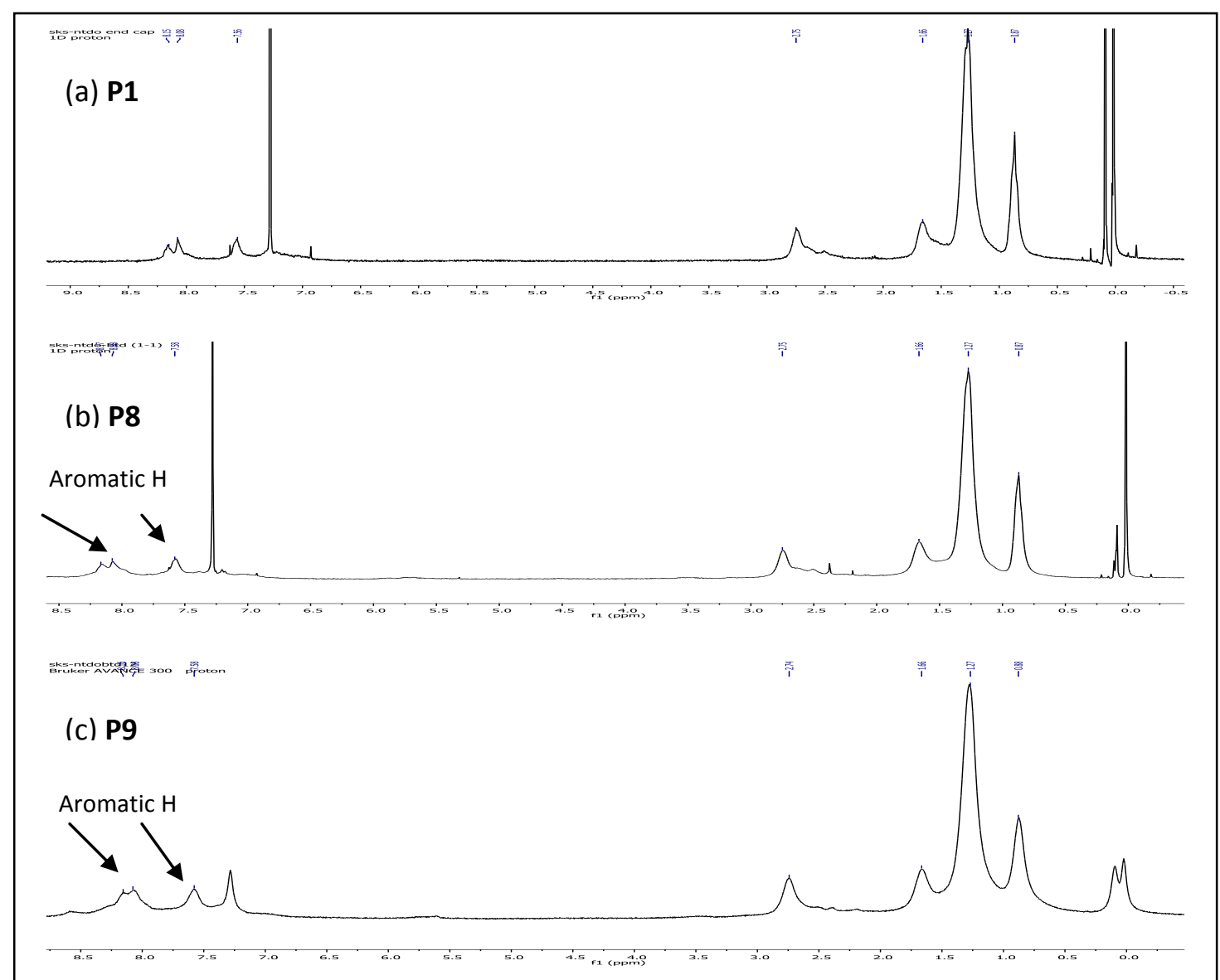

Figure S2.20 ${ }^{1} \mathrm{H}$ NMR for polymers (a) P1, (b) $\mathbf{P 8}$ and (c) P9 (300 MHz, CDCl 3 ). 


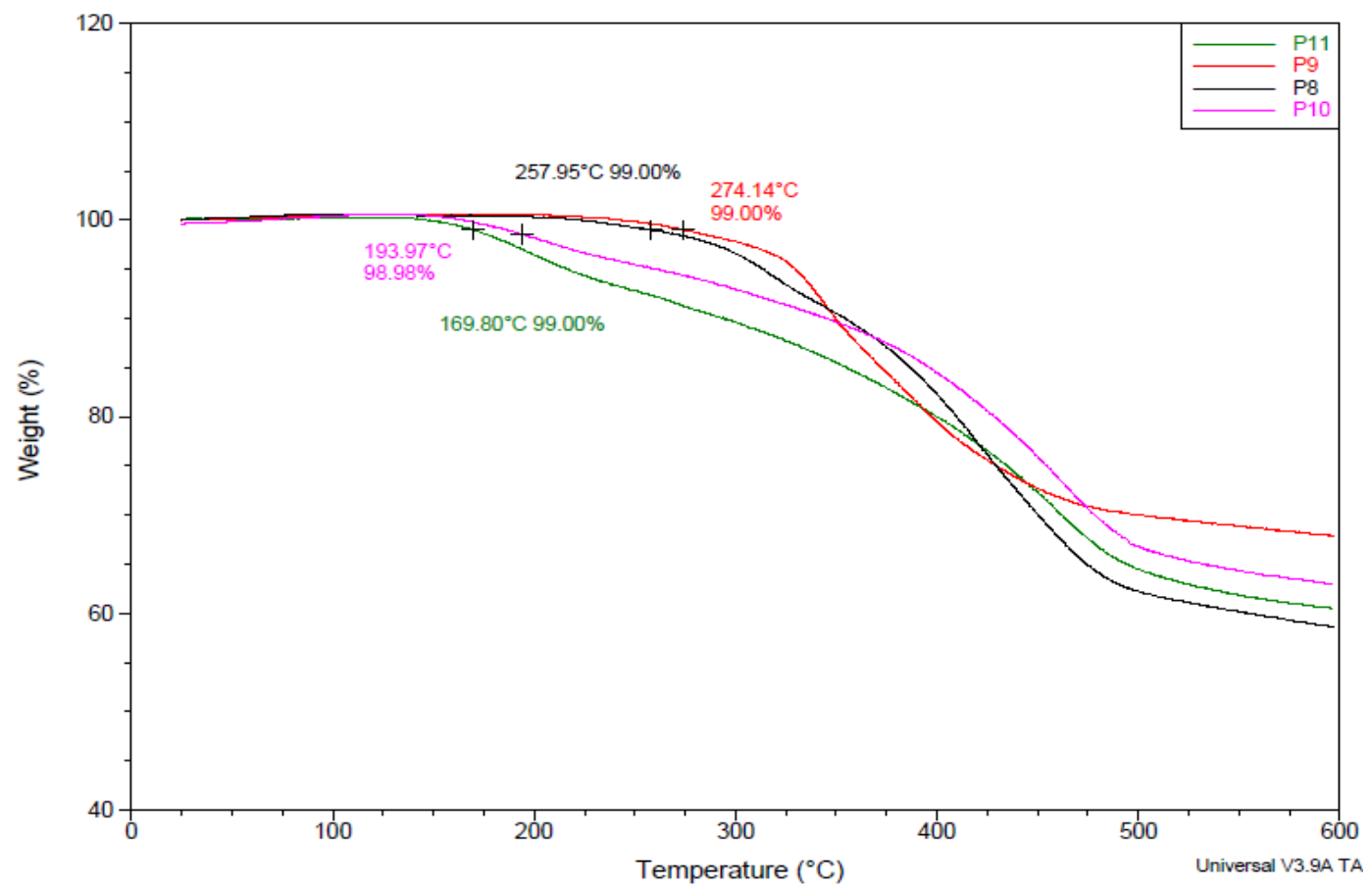

Figure S2.21 TGA plots of the polymers with a heating rate of $10^{\circ} \mathrm{C} / \mathrm{min}$ under an inert atmosphere.

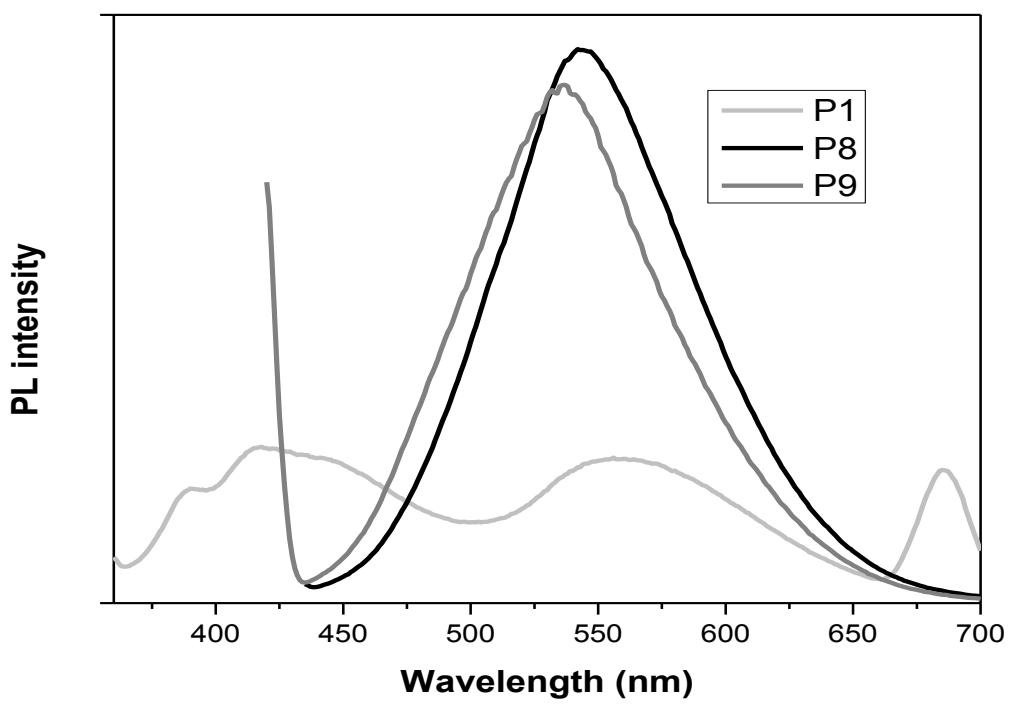

Figure S2.22 Emission of polymers $\mathbf{P 1}, \mathbf{P 8}$ and $\mathbf{P 9}$ solution in $\mathrm{CHCl}_{3}$. 


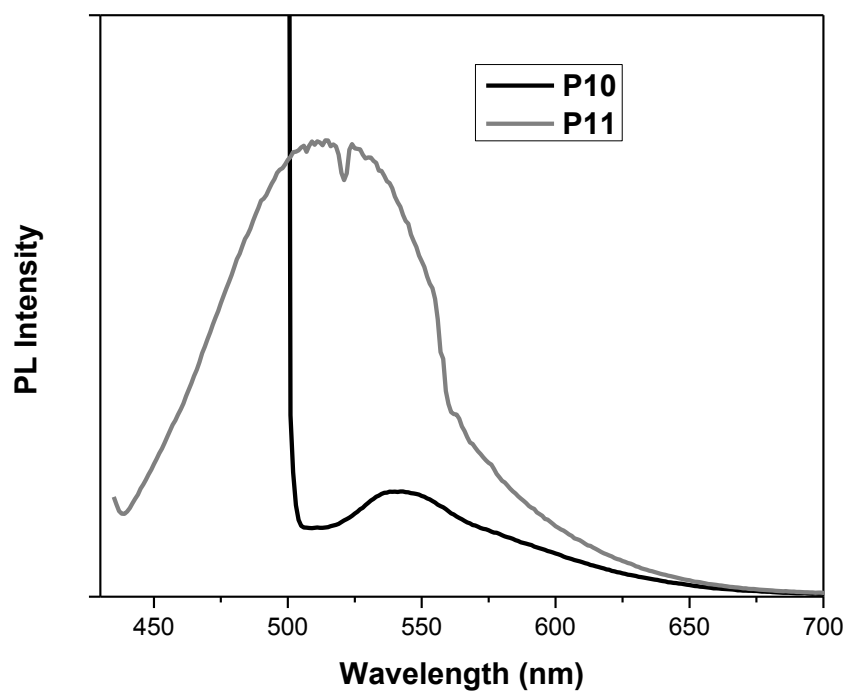

Figure S2.23 Emission of polymers P10 and P11 solution in CB and NMP, respectively.

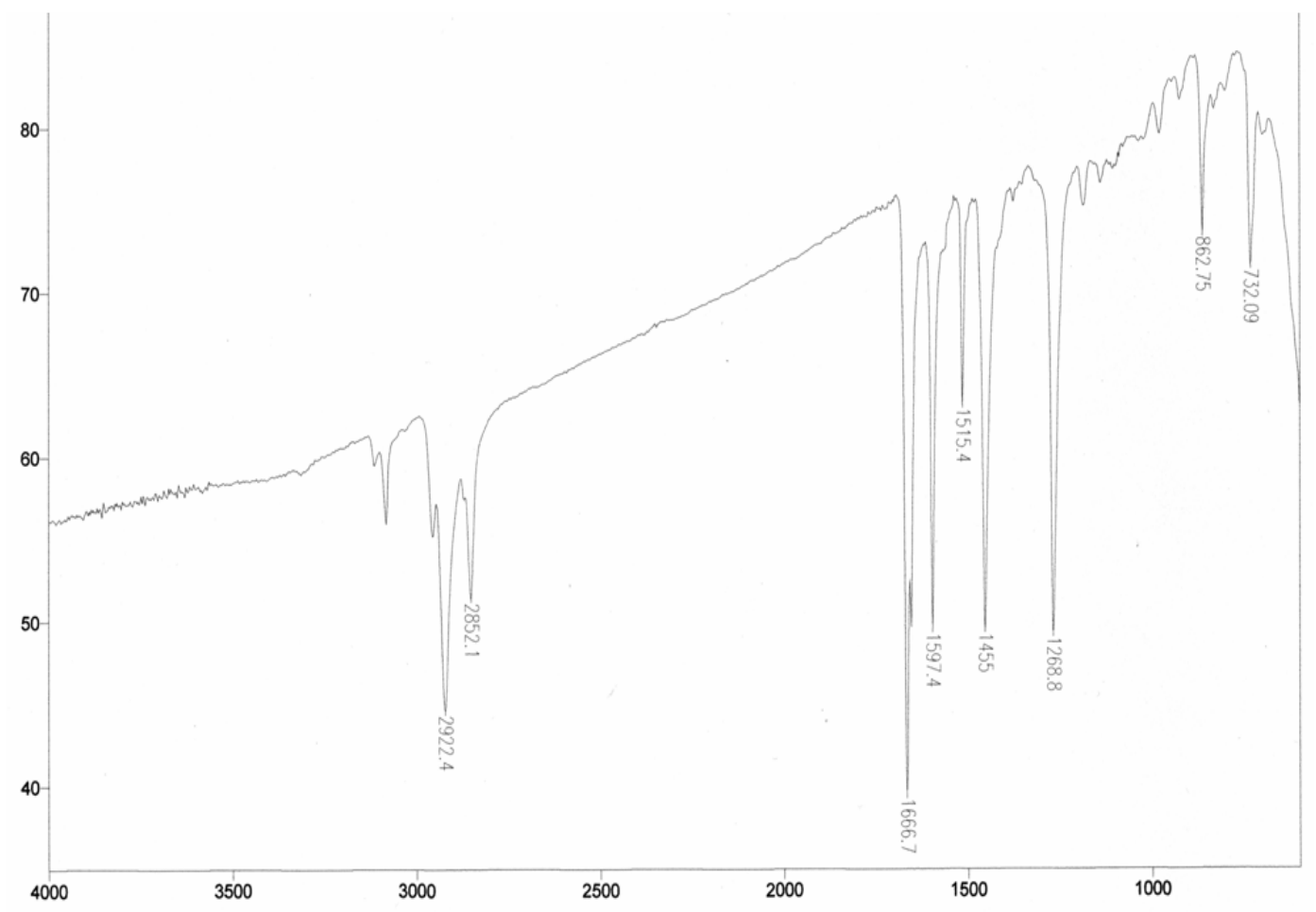

Figure S2.24 IR spectrum of monomer $\mathbf{M} 2$ on $\mathrm{NaCl}$ plate. 


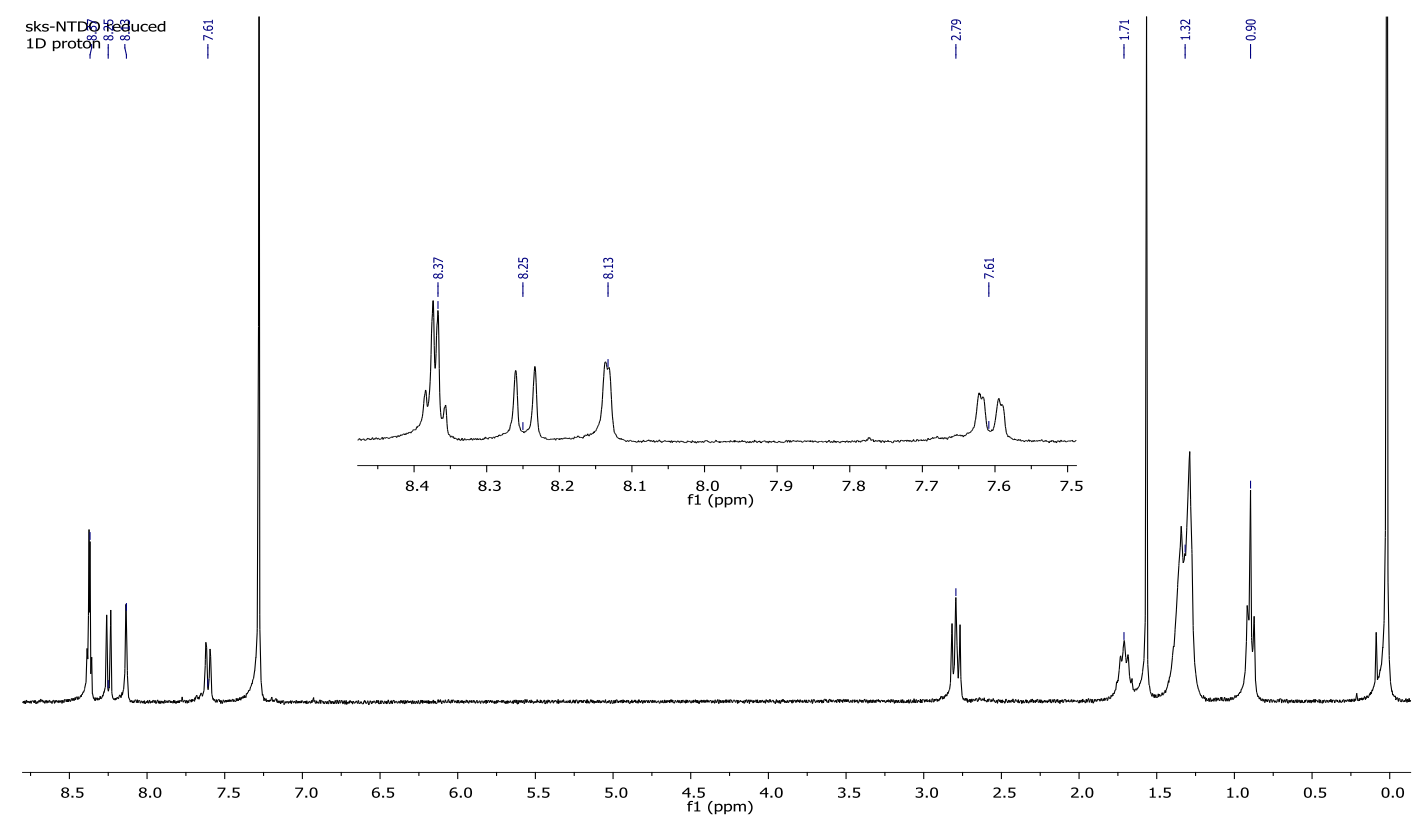

Figure S2.25. ${ }^{1} \mathrm{H}$ NMR spectrum $\left(300 \mathrm{MHz}, \mathrm{CDCl}_{3}\right.$ ) of compound $\mathbf{M 2}$.
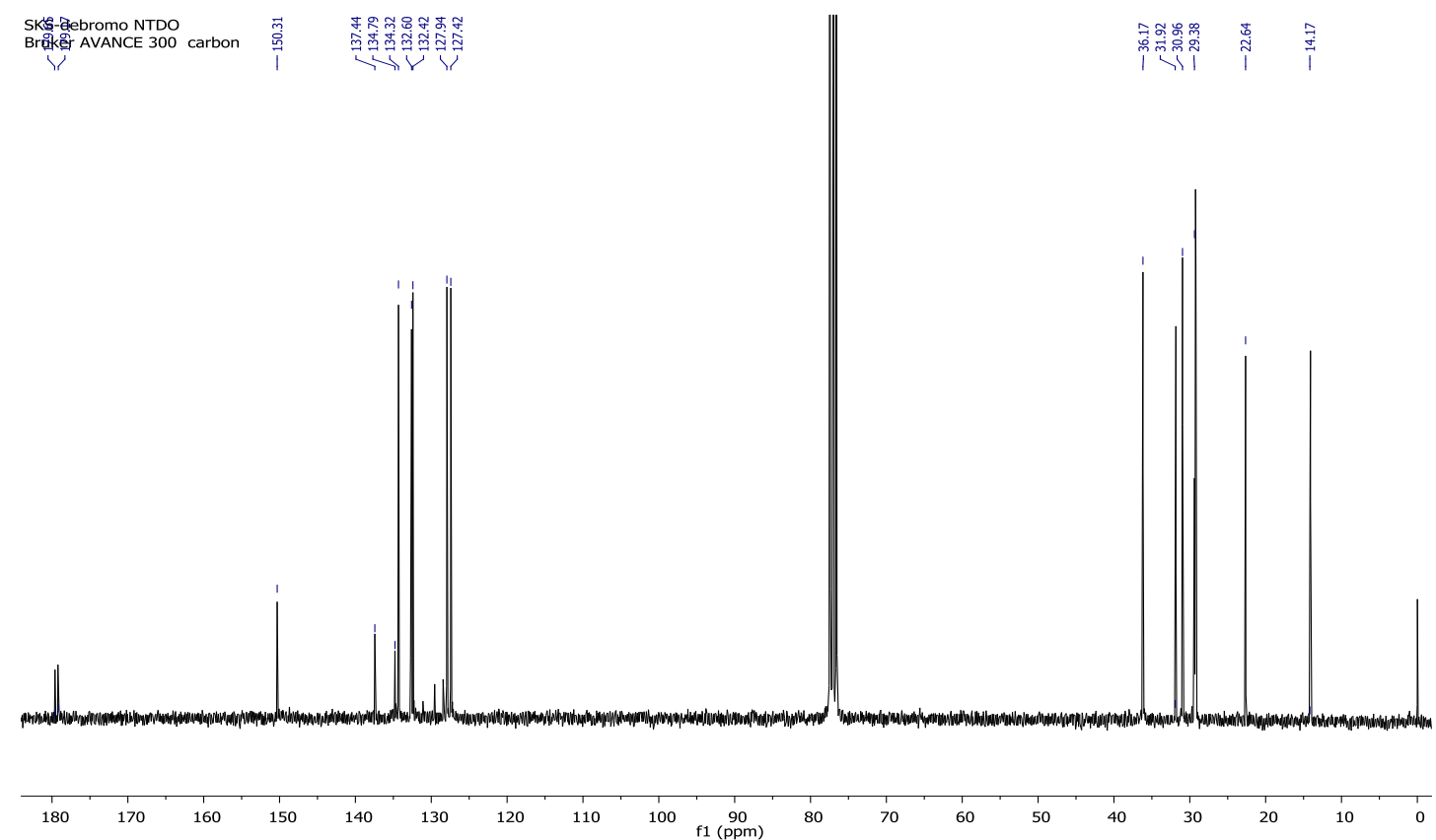

Figure S2.26. ${ }^{13} \mathrm{C}$ NMR spectrum $\left(75 \mathrm{MHz}, \mathrm{CDCl}_{3}\right)$ of compound $\mathbf{M 2}$. 


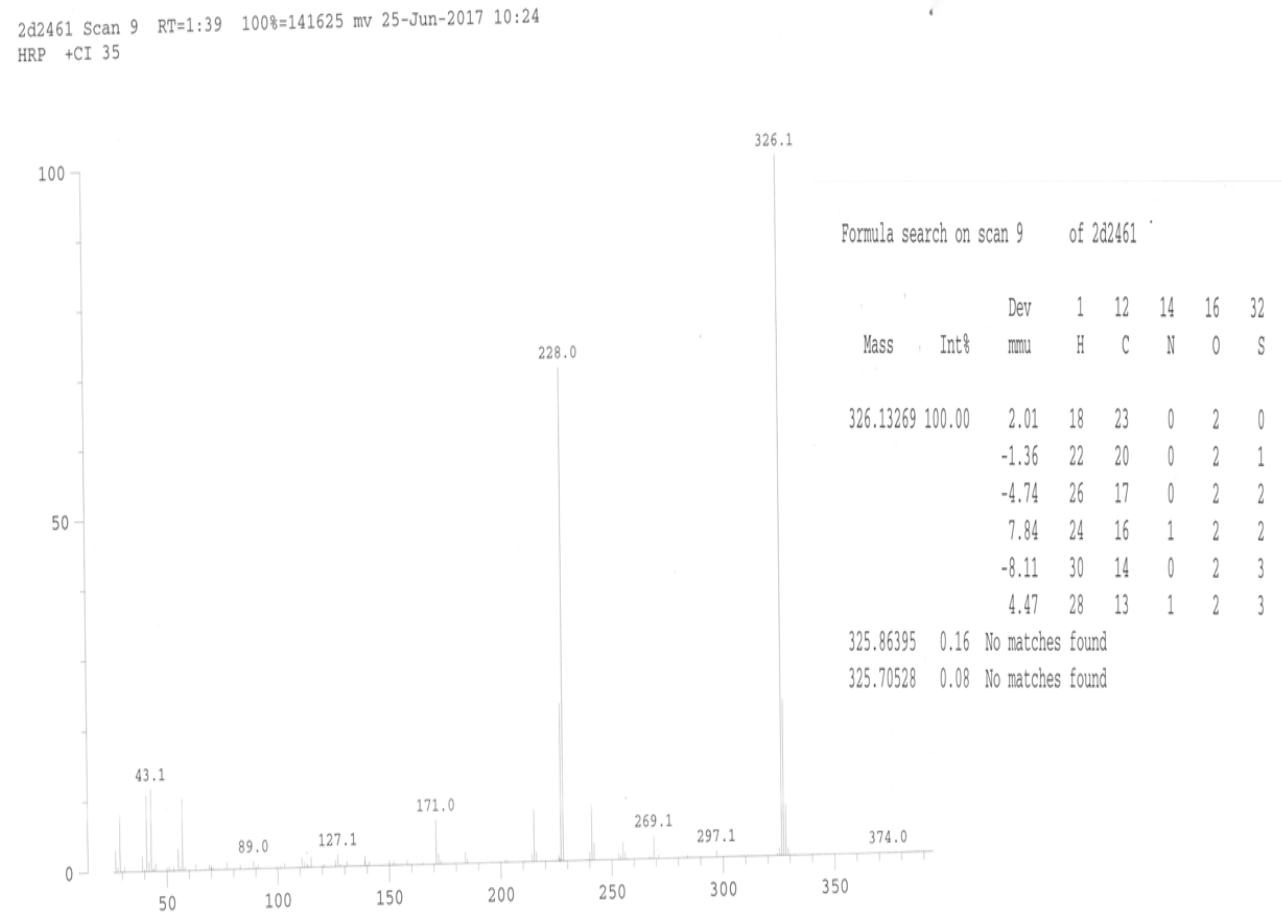

Figure S2.27 Mass spectrum of monomer M2.

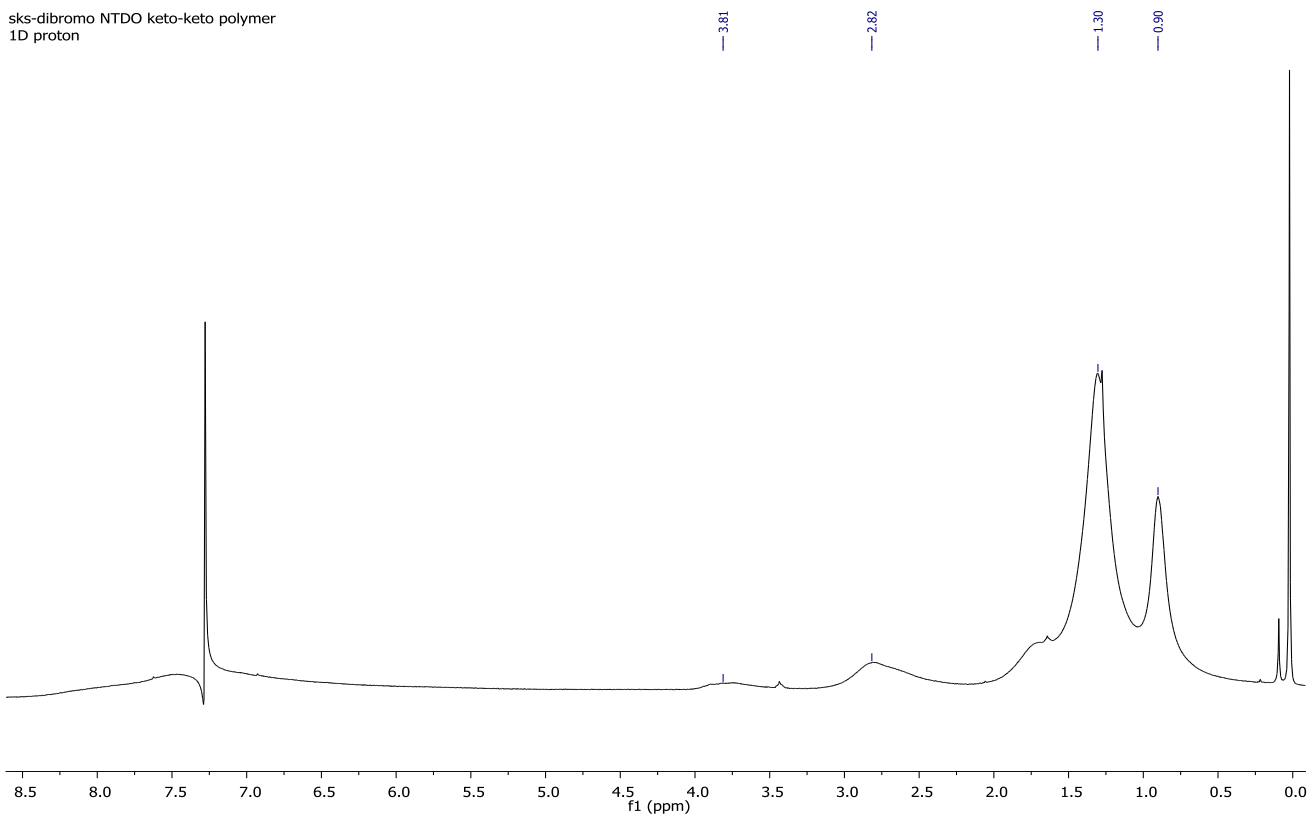

Figure S28 ${ }^{1} \mathrm{H}$ NMR spectrum $\left(300 \mathrm{MHz}, \mathrm{CDCl}_{3}\right)$ of polymer P12. 


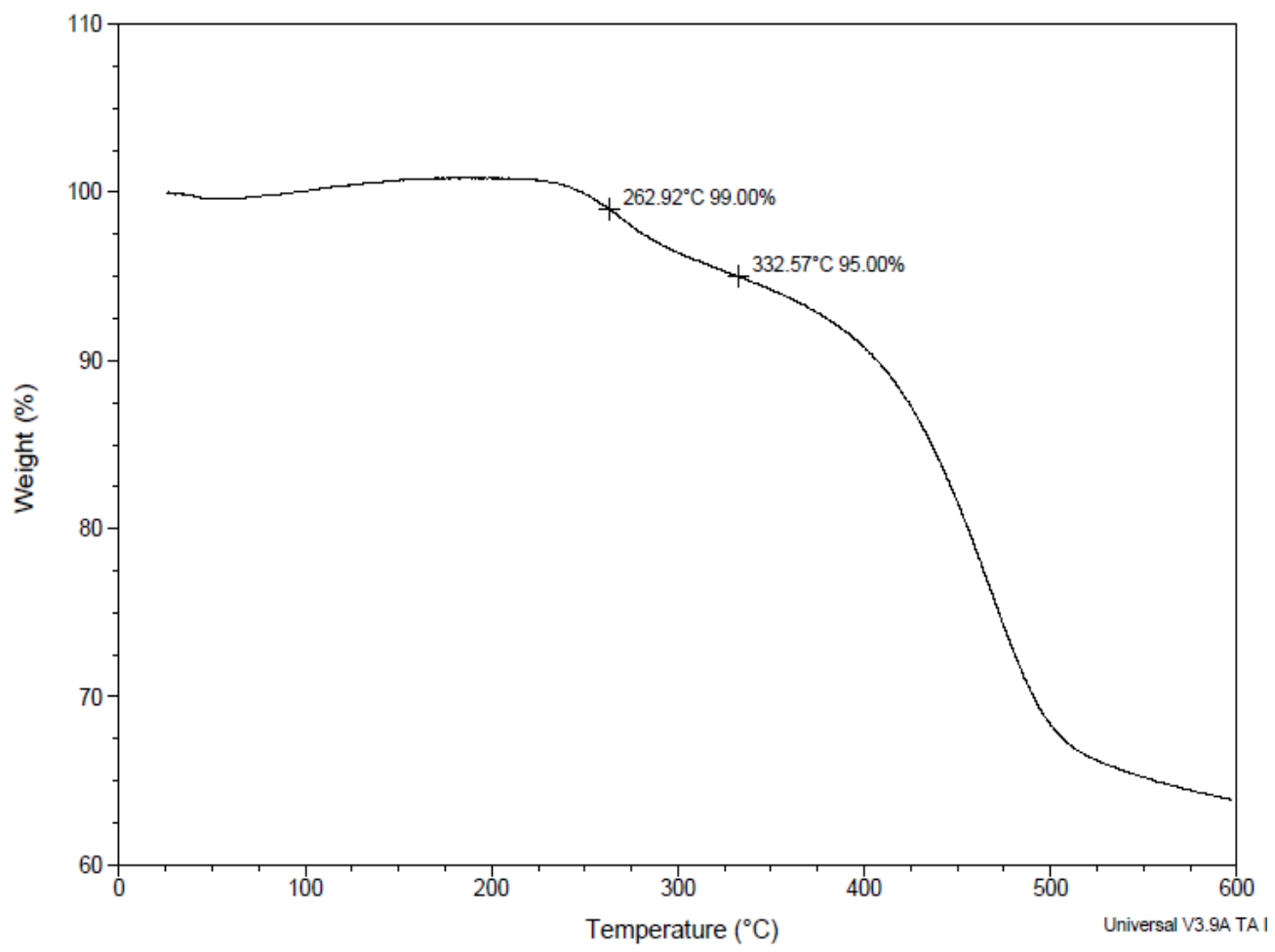

Figure S2.29 TGA plot of polymer $\mathbf{P 1 2}$ with a heating rate of $10{ }^{\circ} \mathrm{C} / \mathrm{min}$ under an inert atmosphere.

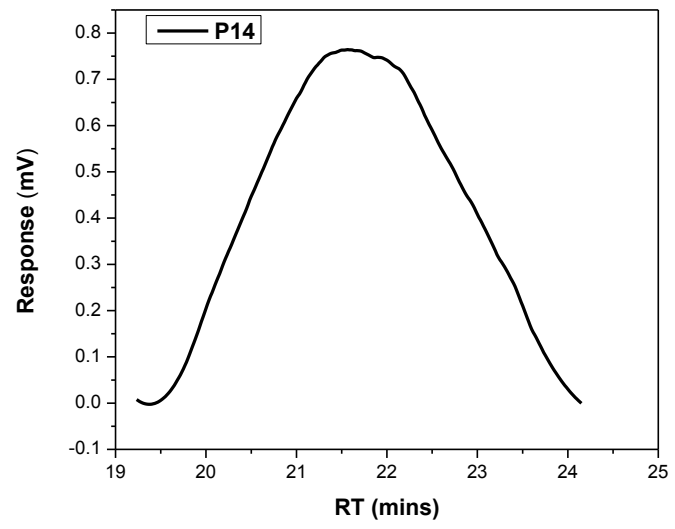




\section{GPC result}

$\begin{array}{llllllll}\text { Peak No } & \text { Mp } & \text { Mn } & \text { Mw } & \text { Mz } & \text { Mz+1 } & \text { Mv } & \text { PD } \\ 1 & 13795 & 6762 & 15565 & 29177 & 42050 & 13725 & 2.30183\end{array}$

Figure S2.30: GPC result for polymer P12.

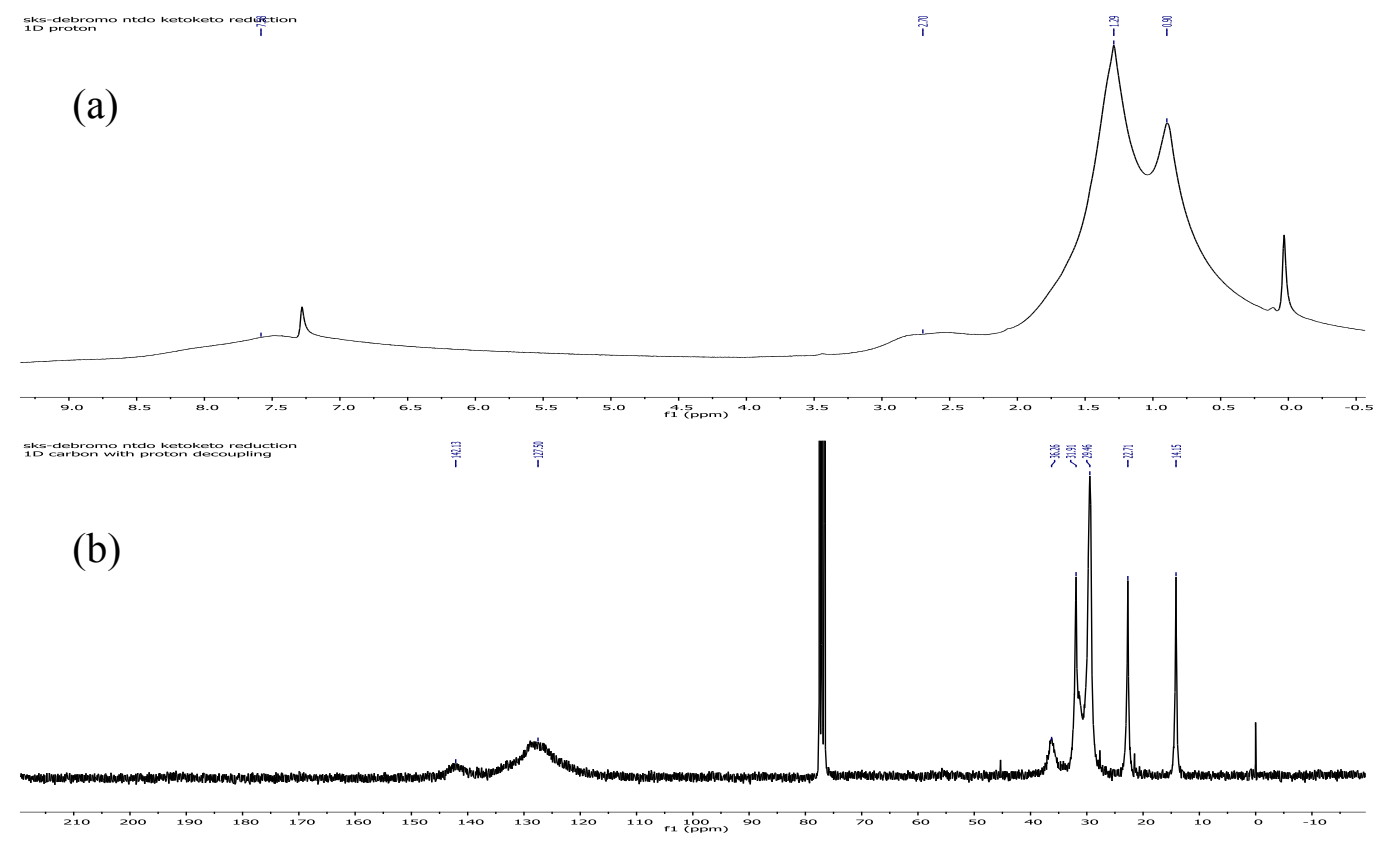

Figure S2.31 ${ }^{1} \mathrm{H}$ NMR and ${ }^{13} \mathrm{C}$ NMR spectra $\left(300 \mathrm{MHz}, \mathrm{CDCl}_{3}\right)$ of oligomer 1. 
Appendix B: Spectra of synthesized compounds, polymers and device properties in chapter 3

sks-babingew
10 profor
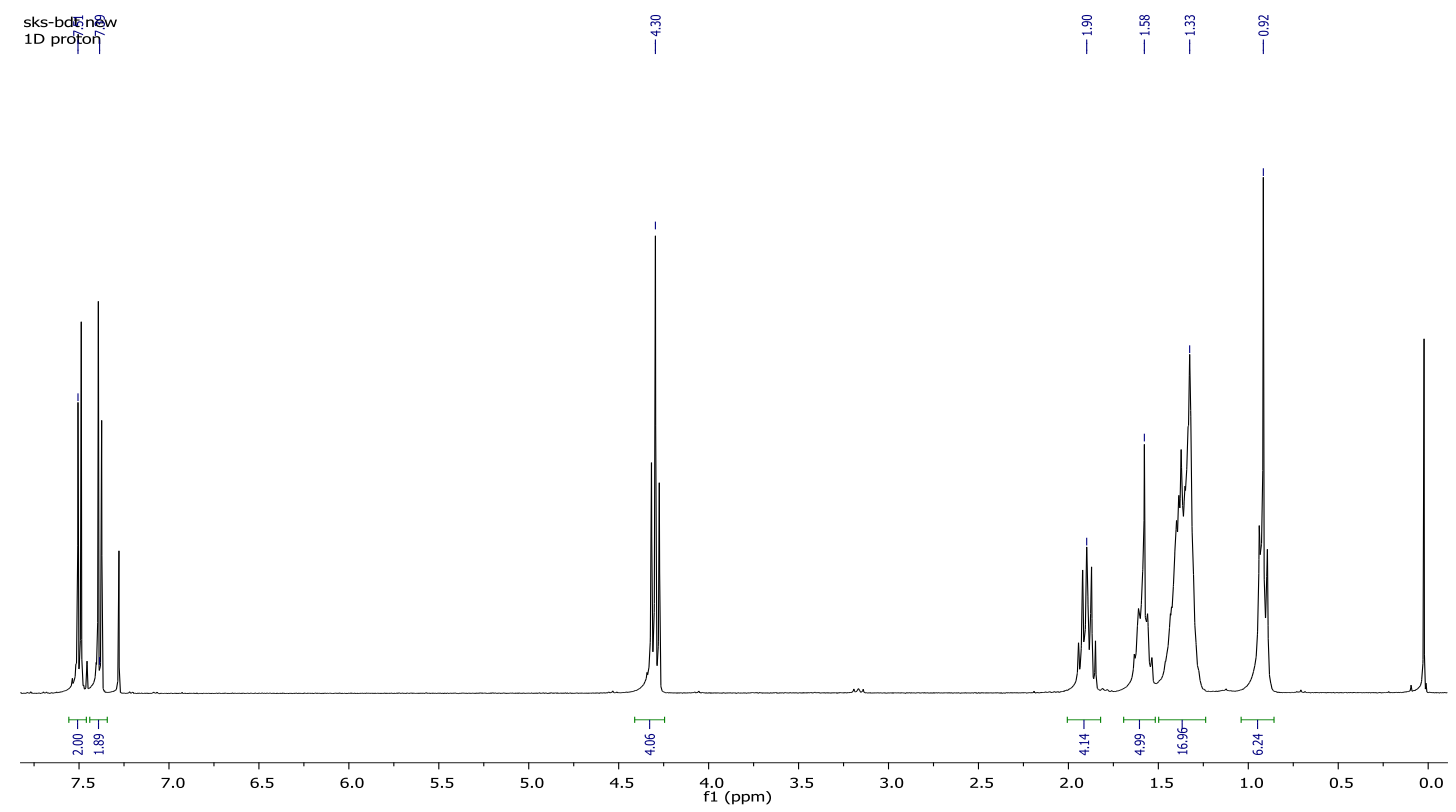

Figure $\mathrm{S} 3.1{ }^{1} \mathrm{H}$ NMR spectrum $\left(300 \mathrm{MHz}, \mathrm{CDCl}_{3}\right)$ of compound $\mathbf{5}$.

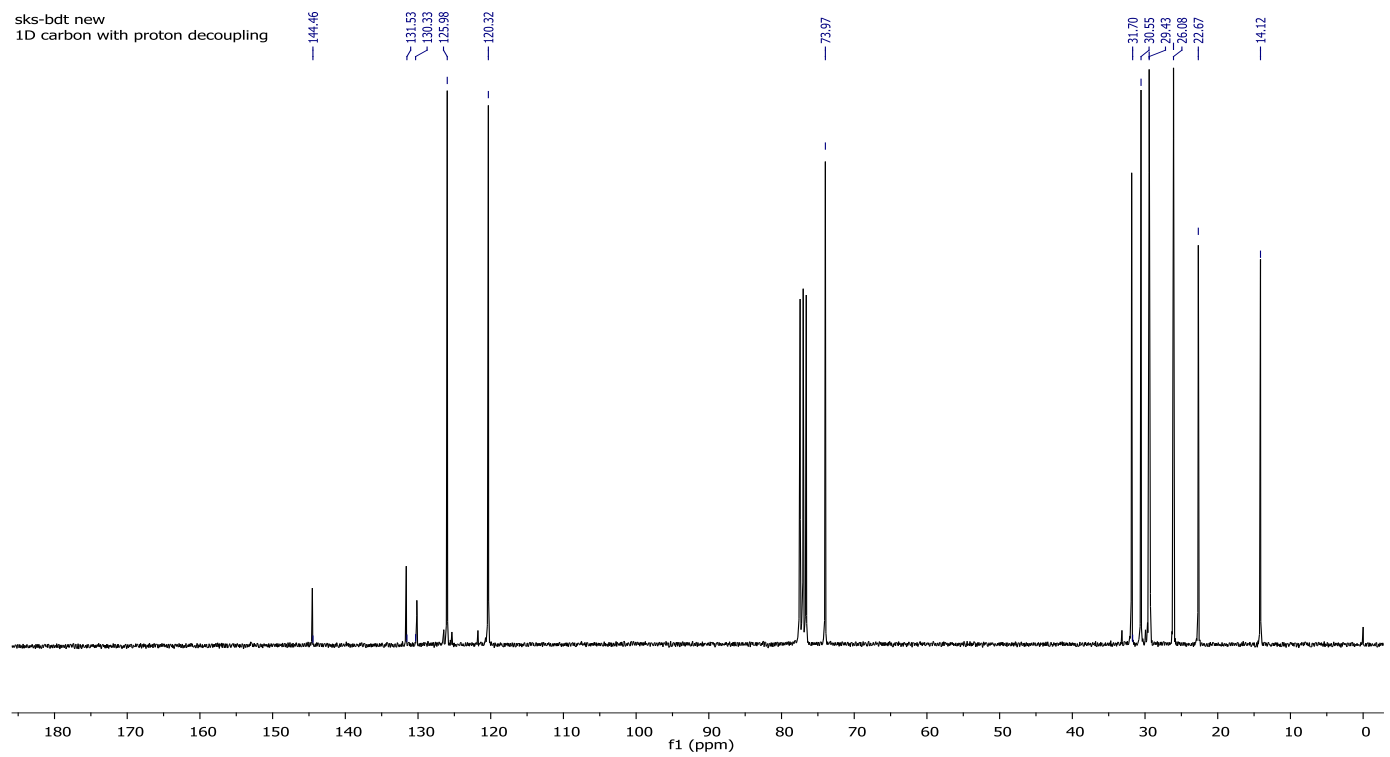

Figure S3.2. ${ }^{13} \mathrm{C}$ NMR spectrum $\left(75 \mathrm{MHz}, \mathrm{CDCl}_{3}\right)$ of compound $\mathbf{5}$. 


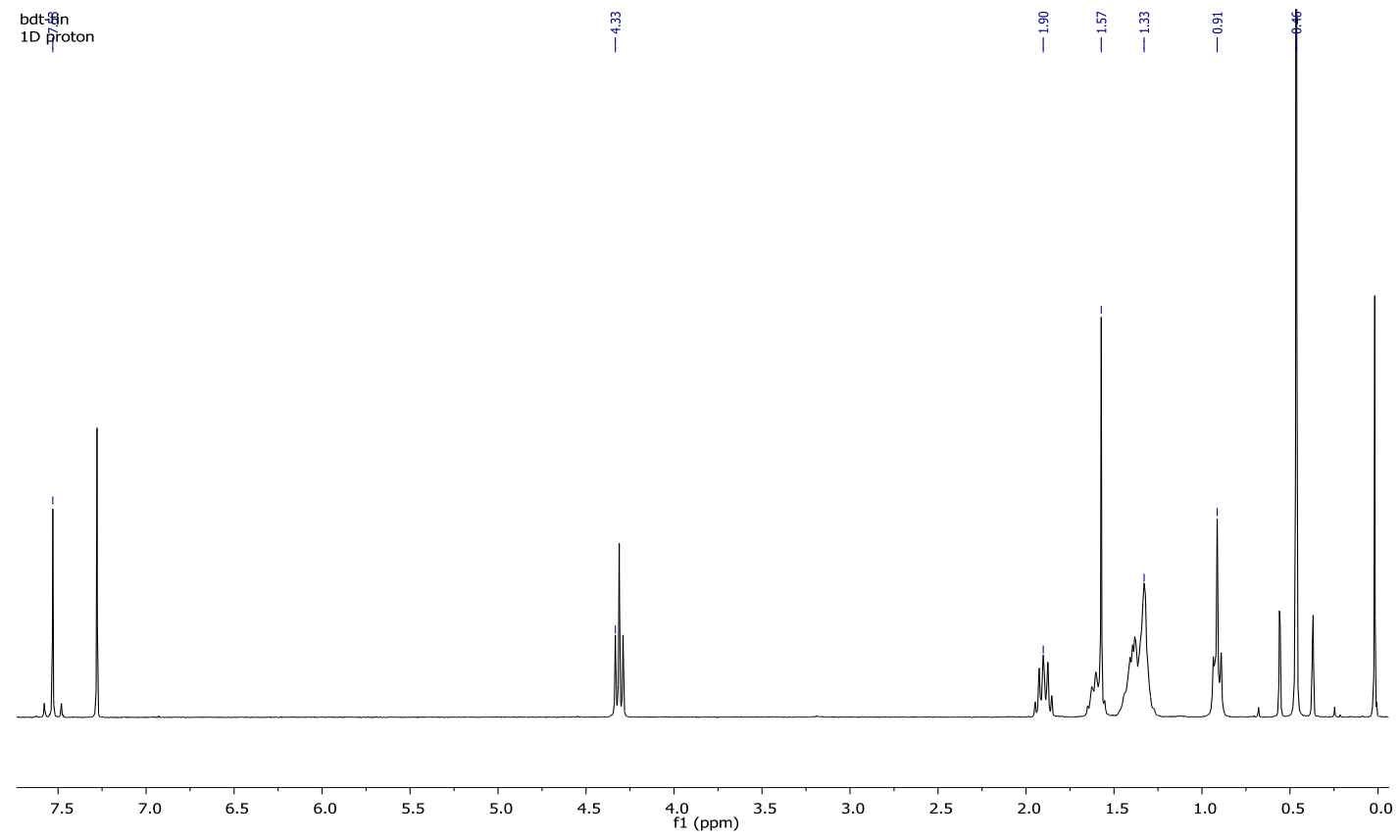

Figure $\mathrm{S} 3.3{ }^{1} \mathrm{H}$ NMR spectrum $\left(300 \mathrm{MHz}, \mathrm{CDCl}_{3}\right)$ of monomer M4.

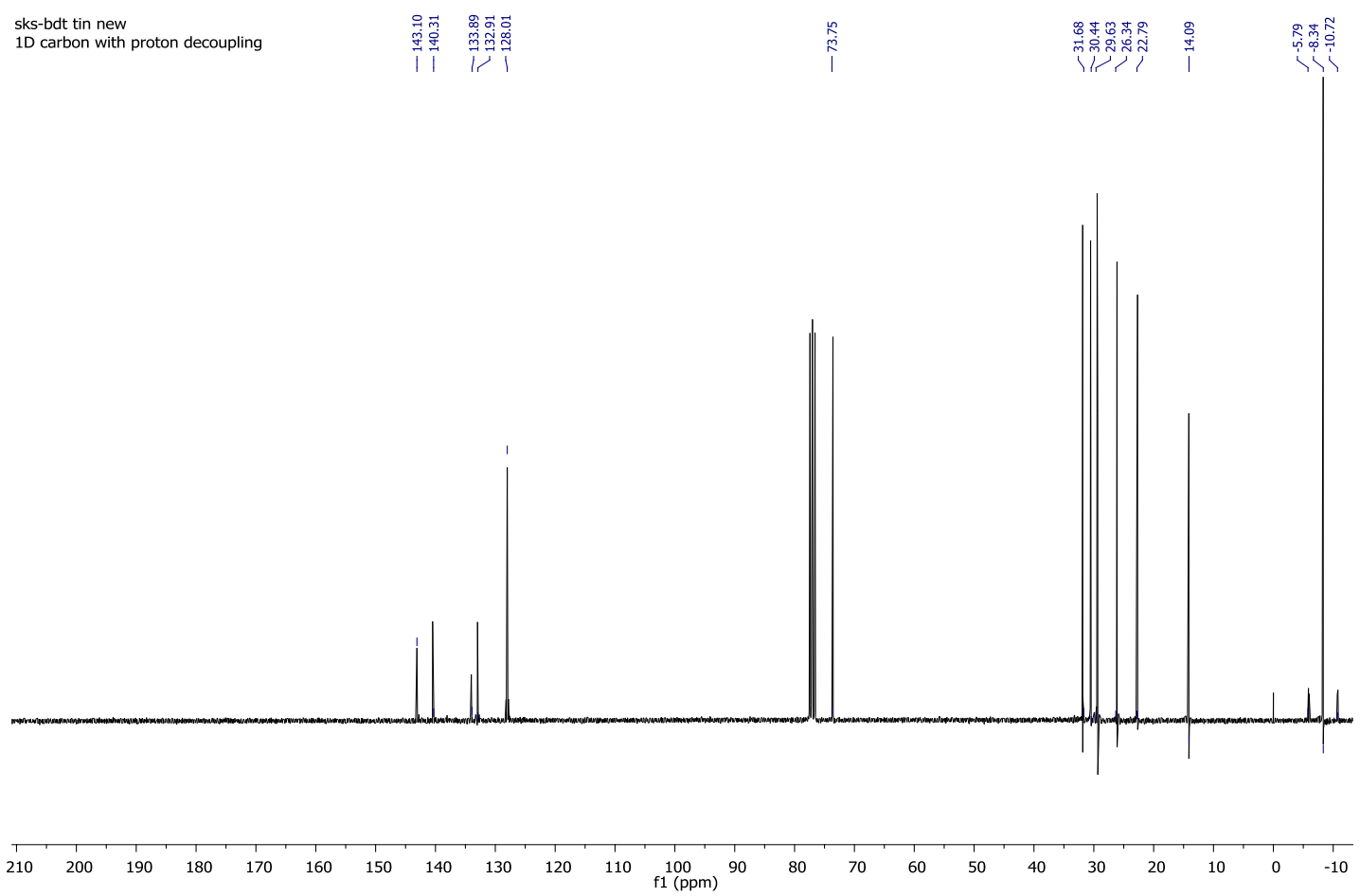

Figure S3.4. ${ }^{13} \mathrm{C}$ NMR spectrum $\left(75 \mathrm{MHz}, \mathrm{CDCl}_{3}\right)$ monomer M4. 


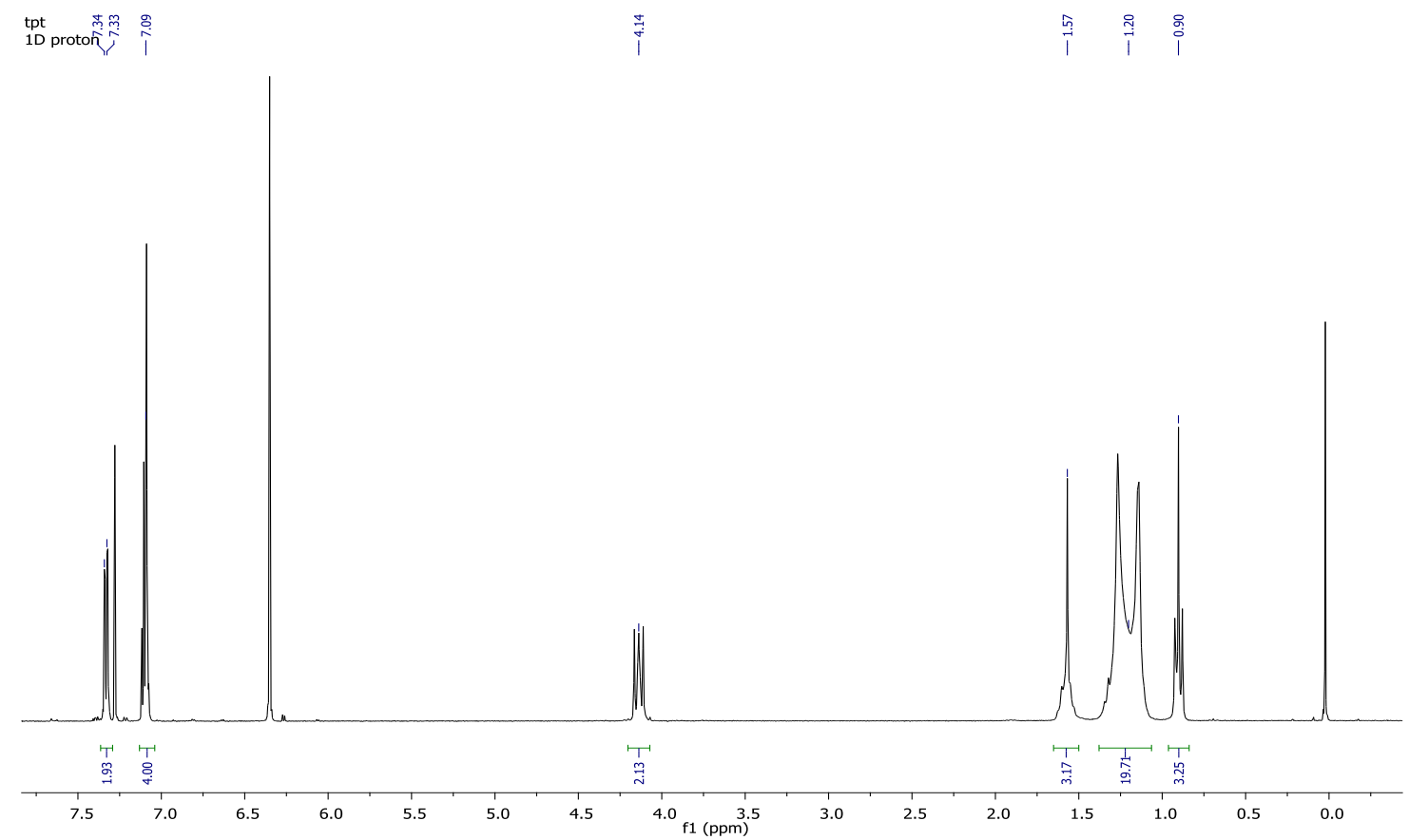

Figure $\mathrm{S} 3.5{ }^{1} \mathrm{H}$ NMR spectrum $\left(300 \mathrm{MHz}, \mathrm{CDCl}_{3}\right)$ of compound 7 .

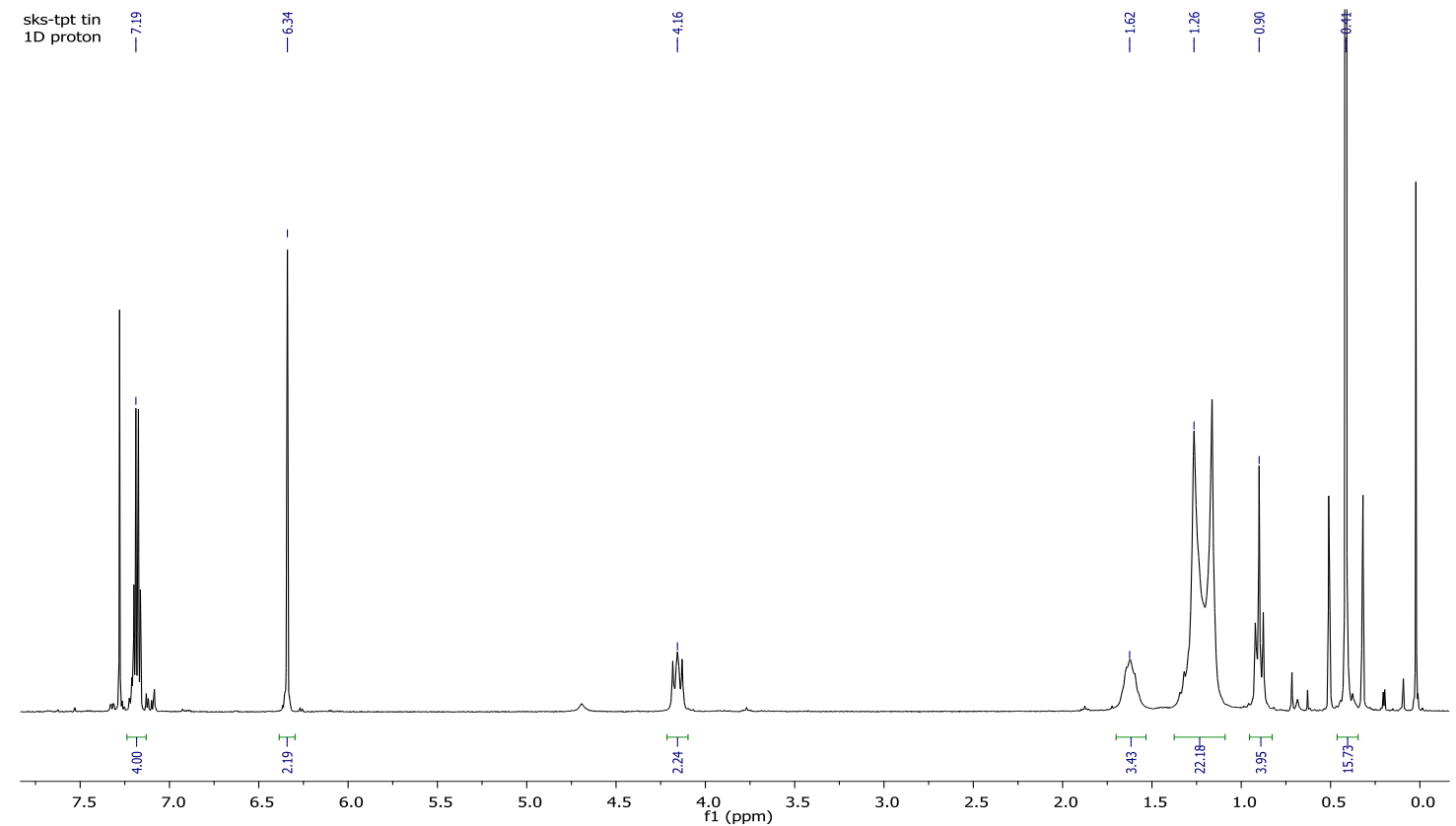

Figure S3. $6{ }^{1} \mathrm{H}$ NMR spectrum $\left(300 \mathrm{MHz}, \mathrm{CDCl}_{3}\right)$ of monomer $\mathbf{M 5}$. 


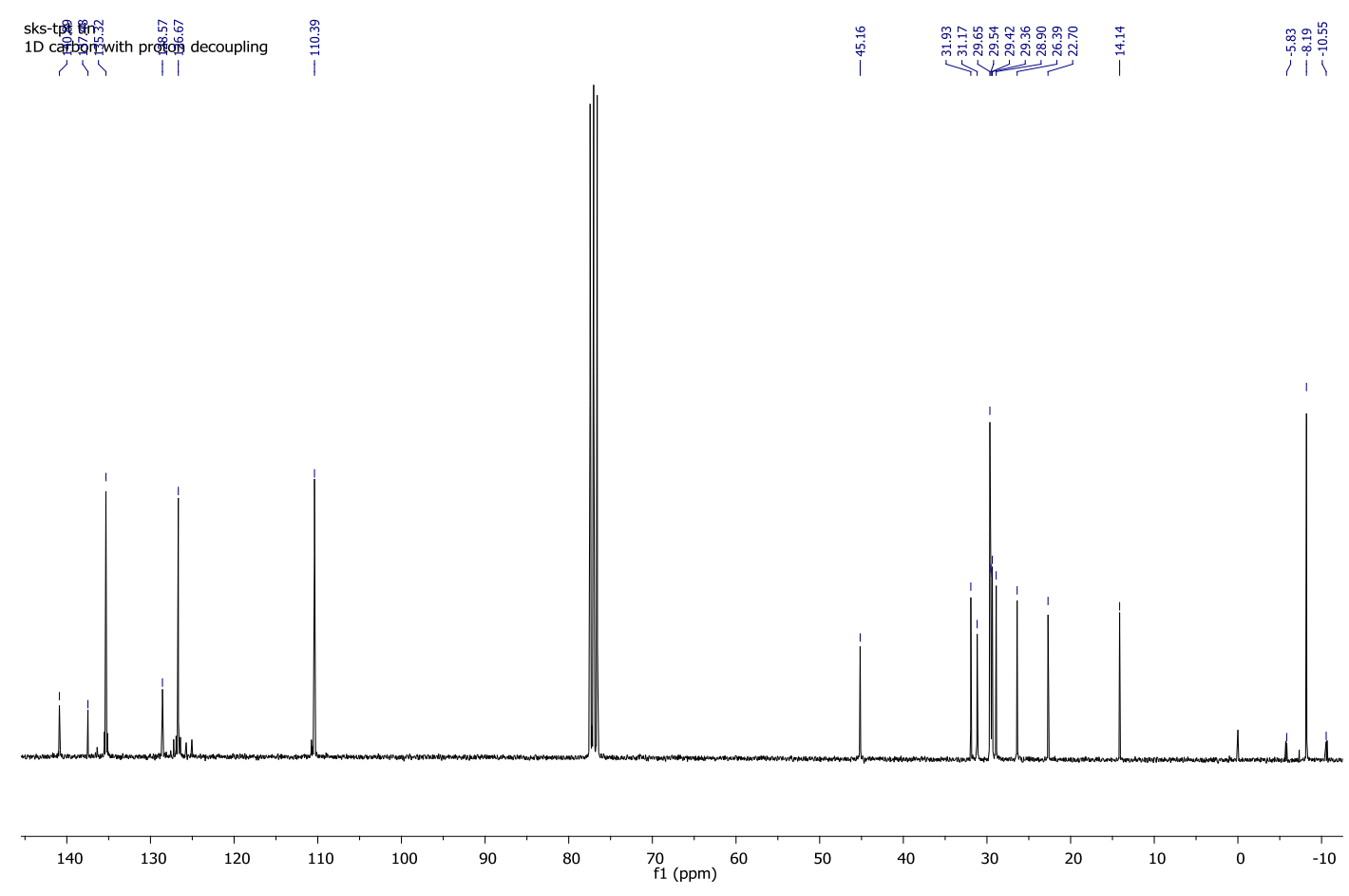

Figure S3.7 ${ }^{13} \mathrm{C}$ NMR spectrum $\left(75 \mathrm{MHz}, \mathrm{CDCl}_{3}\right)$ of monomer $\mathbf{M 5}$.

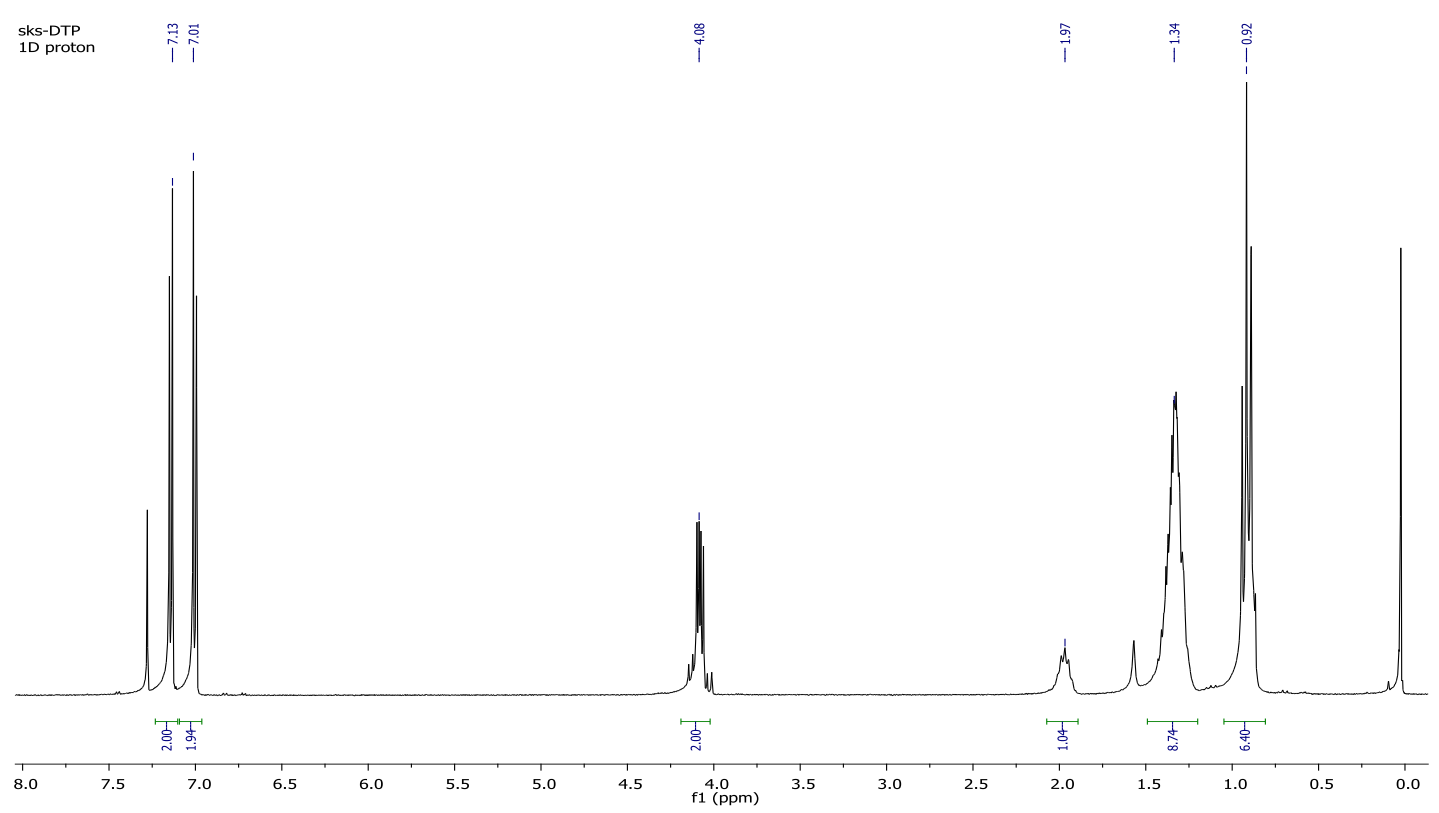

Figure $\mathrm{S} 3.8{ }^{1} \mathrm{H}$ NMR spectrum $\left(300 \mathrm{MHz}, \mathrm{CDCl}_{3}\right)$ of compound 9. 


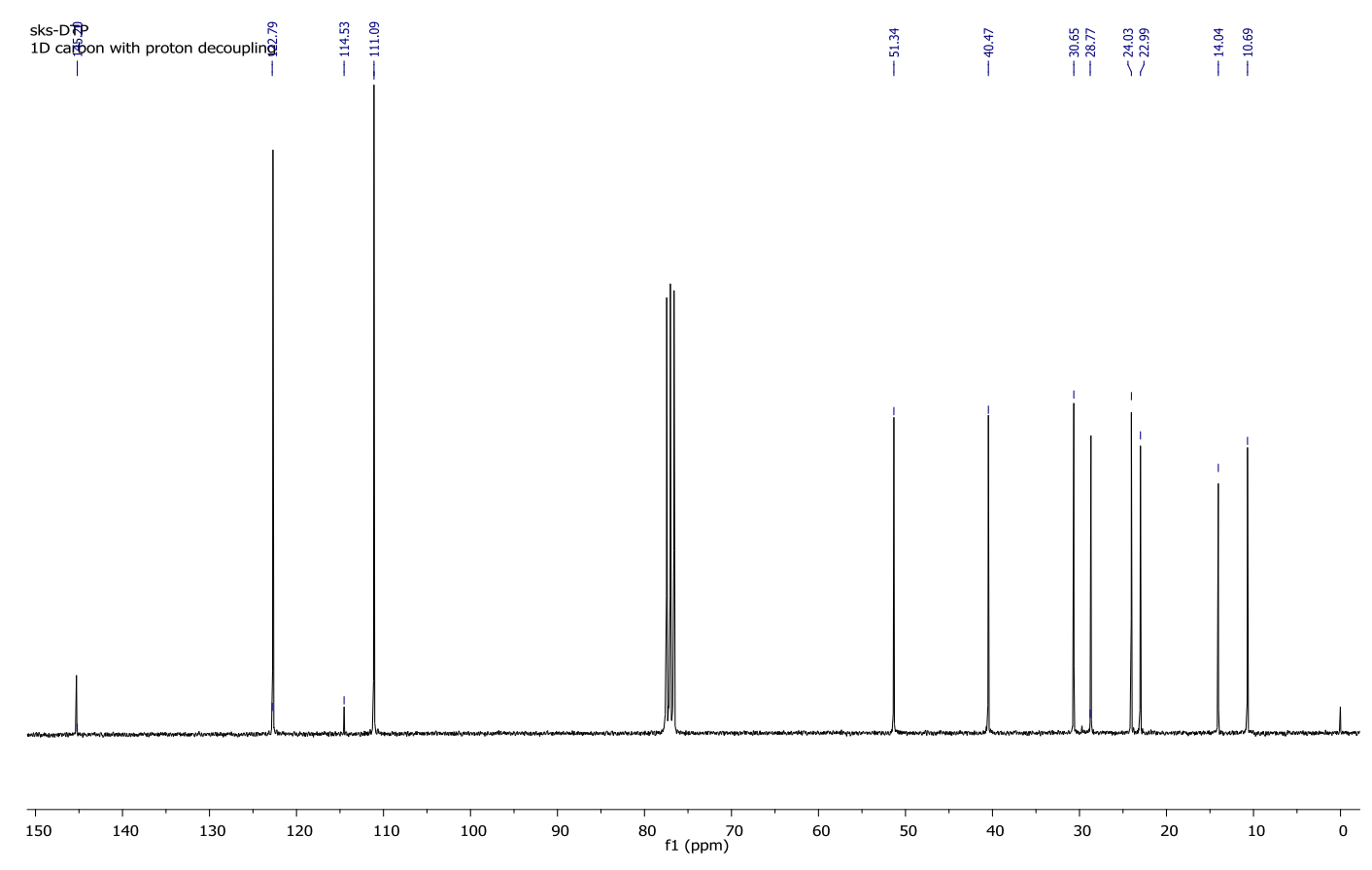

Figure S3.9 ${ }^{13} \mathrm{C}$ NMR spectrum $\left(75 \mathrm{MHz}, \mathrm{CDCl}_{3}\right)$ of monomer compound 9.

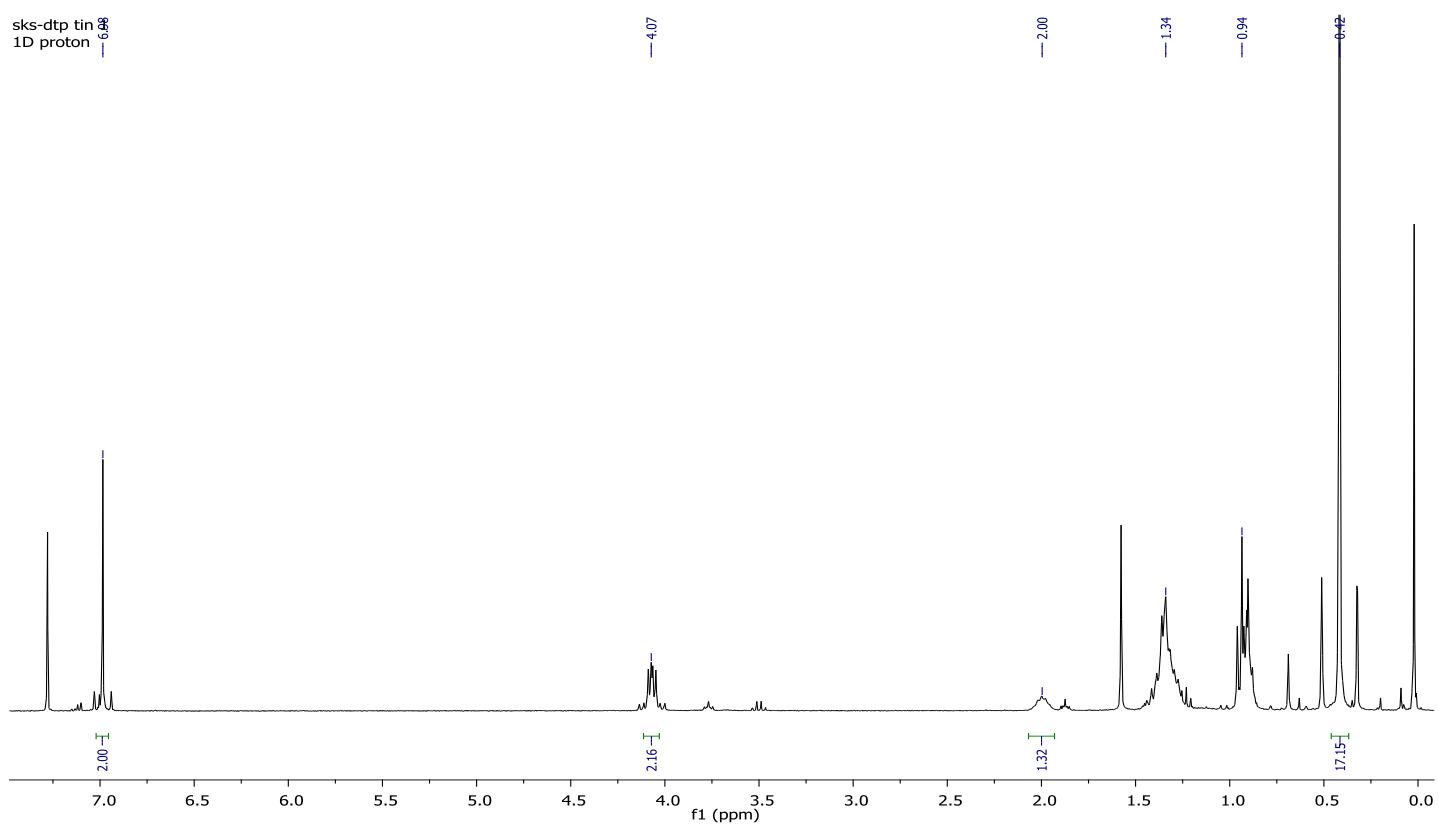

Figure $\mathrm{S} 3.10{ }^{1} \mathrm{H}$ NMR spectrum $\left(300 \mathrm{MHz}, \mathrm{CDCl}_{3}\right)$ of monomer M6. 


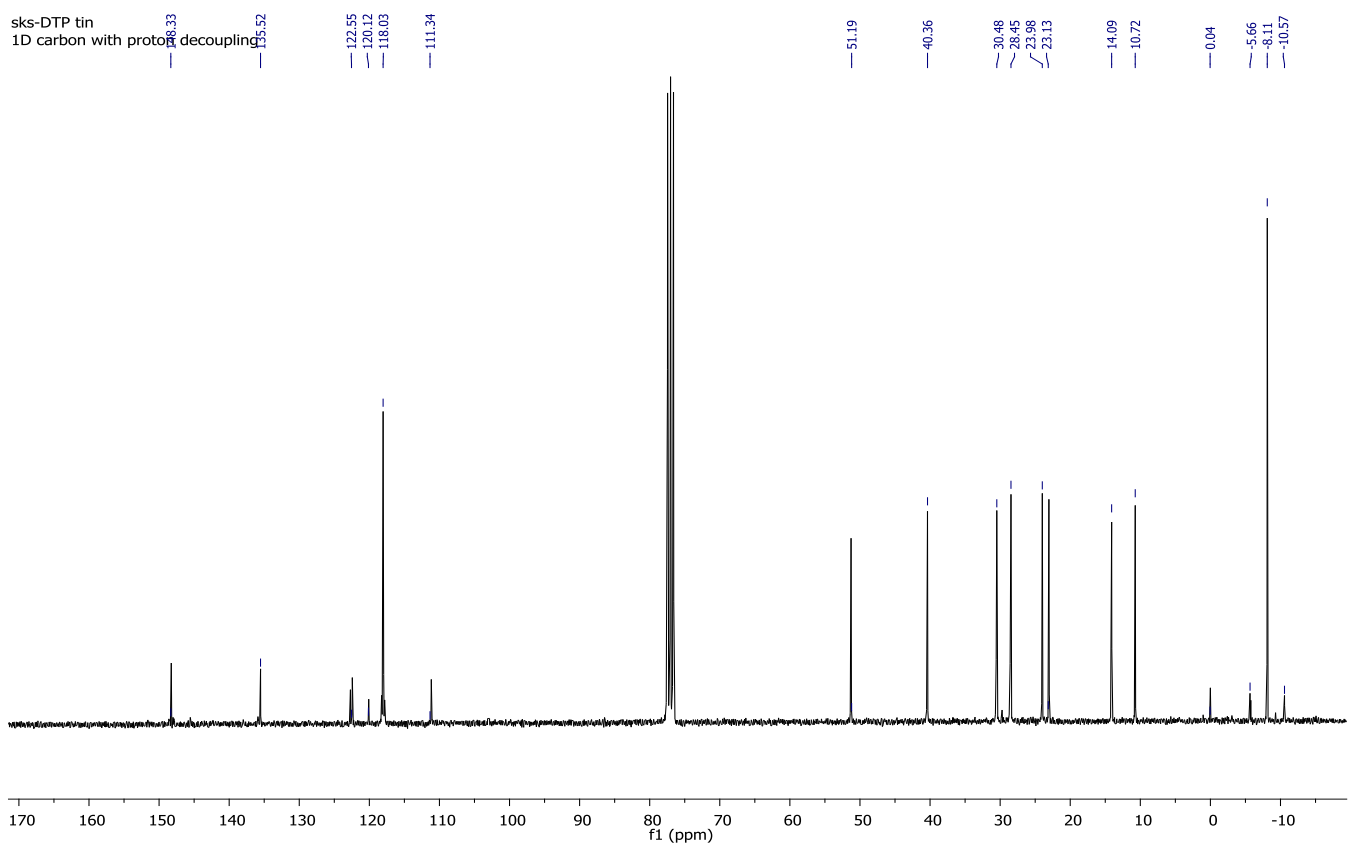

Figure S3.11 ${ }^{13} \mathrm{C}$ NMR spectrum $\left(75 \mathrm{MHz}, \mathrm{CDCl}_{3}\right)$ of monomer M6.
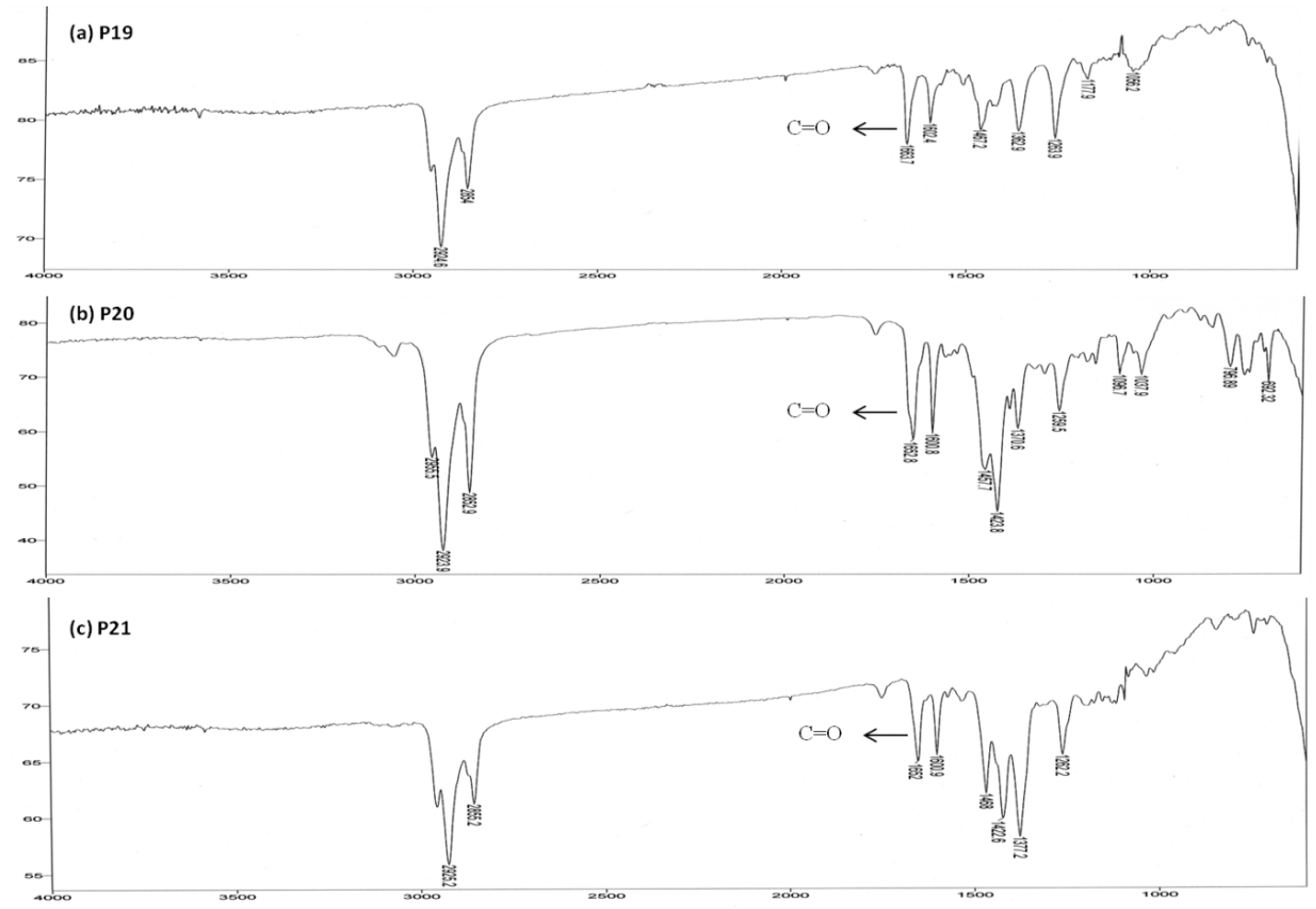

Figure S3.12 IR spectra polymers P19 to P21 on $\mathrm{NaCl}$ plate. 


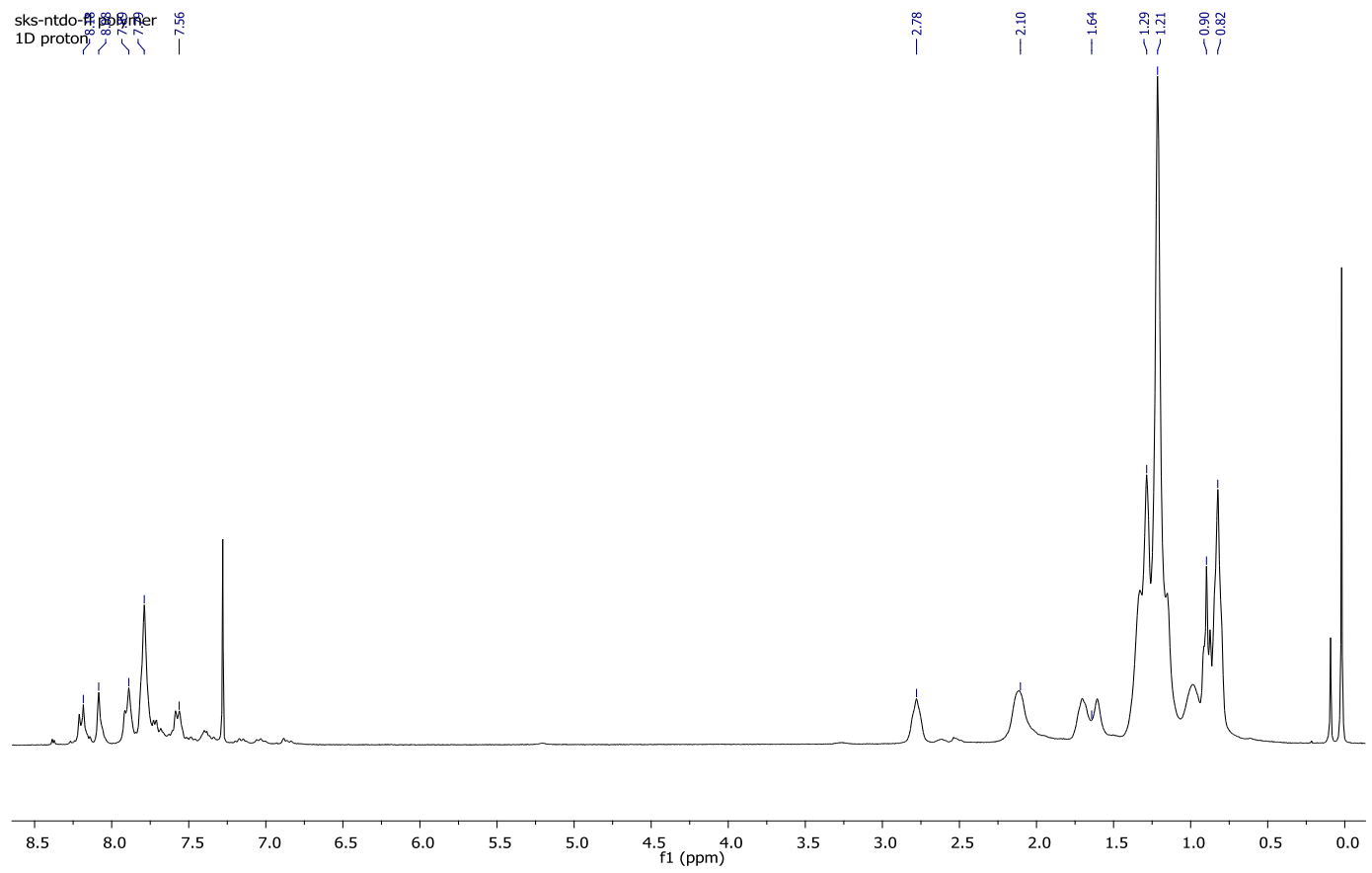

Figure S3.13. ${ }^{1} \mathrm{H}$ NMR spectrum (300 MHz, $\left.\mathrm{CDCl}_{3}\right)$ of polymer $\mathbf{P 1 8}$.

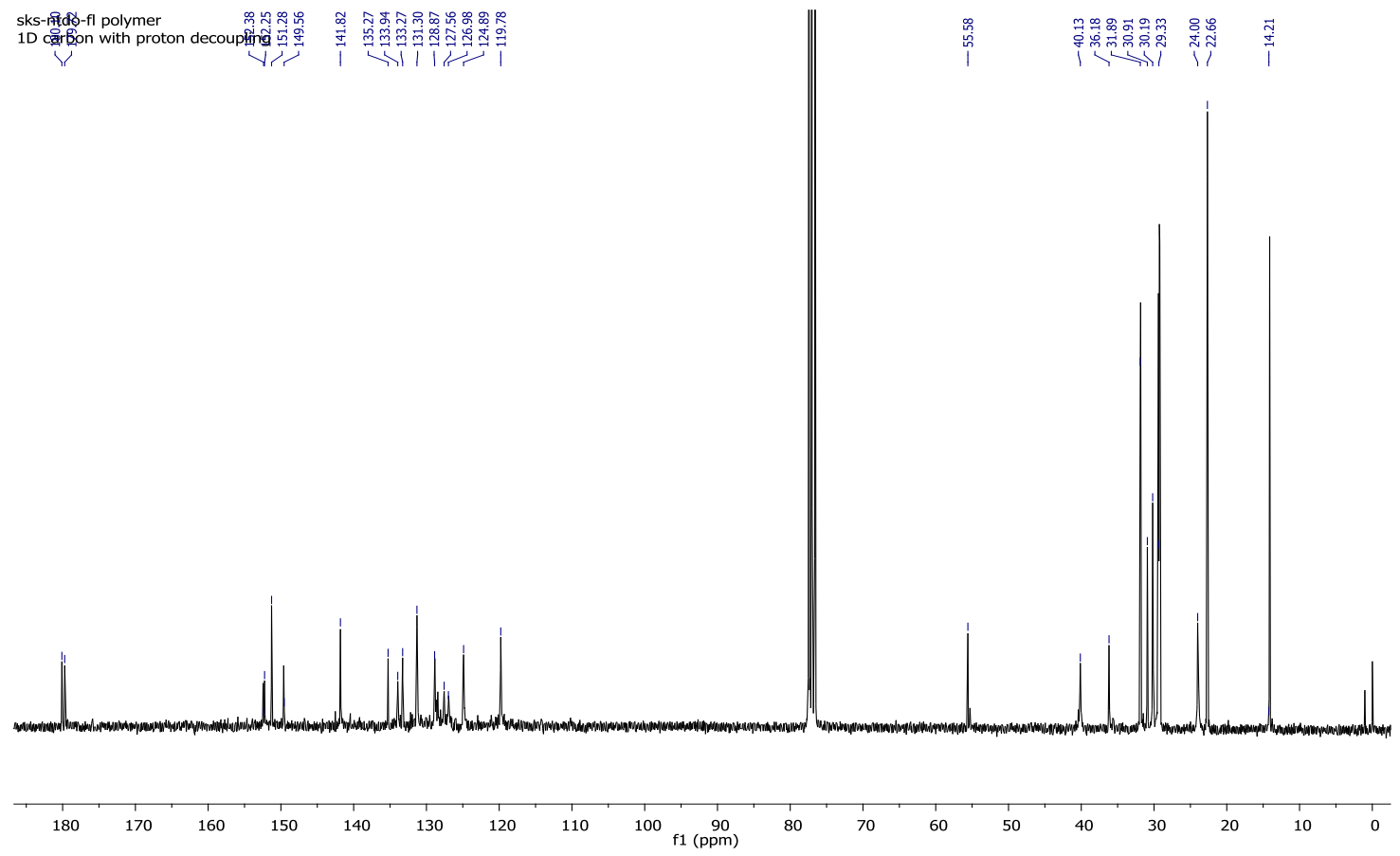

Figure S3.14. ${ }^{13} \mathrm{C}$ NMR spectrum $\left(75 \mathrm{MHz}, \mathrm{CDCl}_{3}\right.$ ) of polymer $\mathbf{P 1 8}$. 


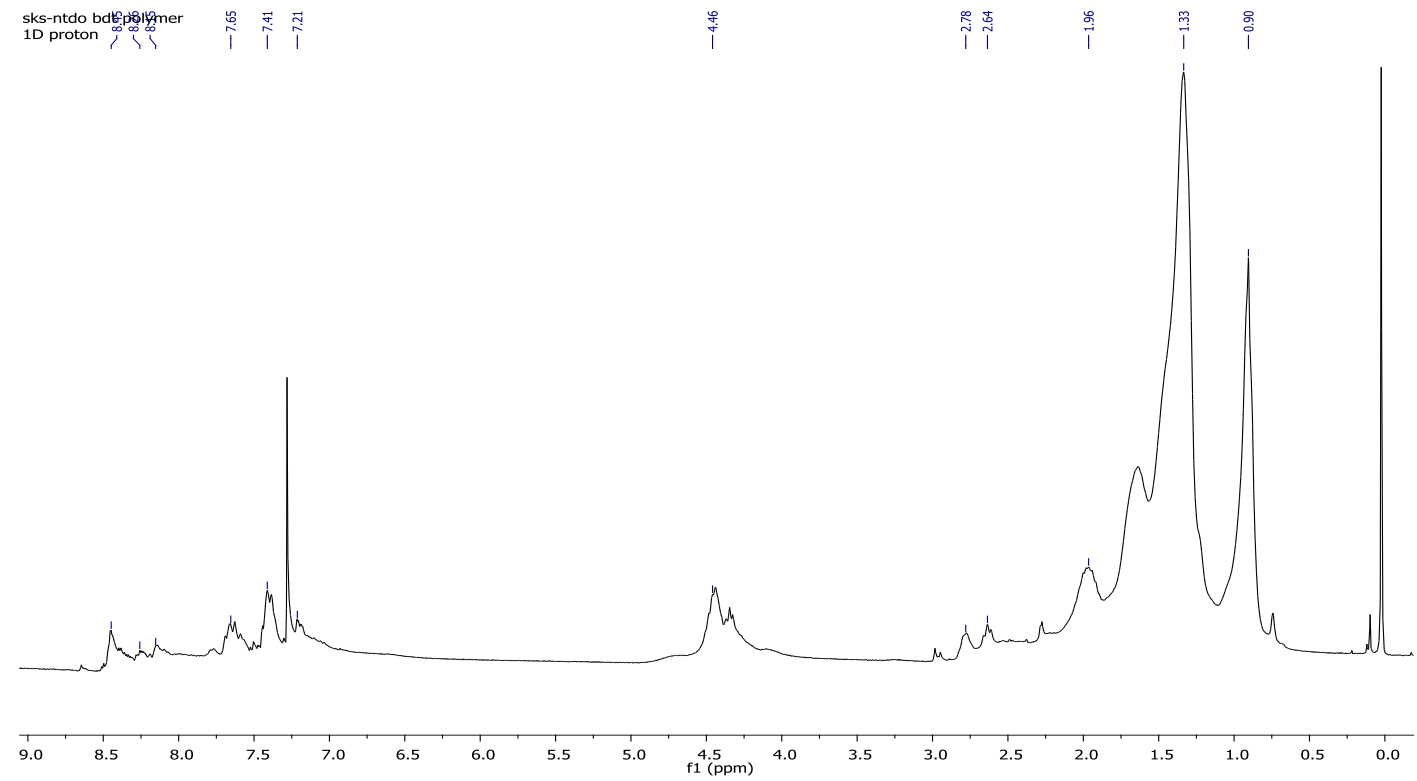

Figure S3.15. ${ }^{1} \mathrm{H}$ NMR spectrum $\left(300 \mathrm{MHz}, \mathrm{CDCl}_{3}\right)$ of polymer P19.

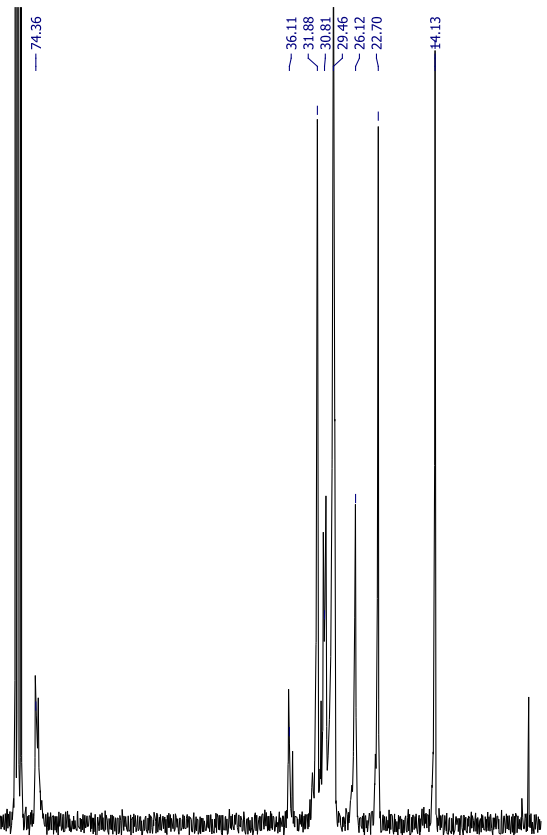

$210 \quad 200$
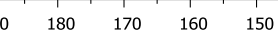

$140 \quad 130 \quad 120$

${ }_{\mathrm{f} 1(\mathrm{ppm})}^{100}$

Figure S3.16. ${ }^{13} \mathrm{C}$ NMR spectrum $\left(75 \mathrm{MHz}, \mathrm{CDCl}_{3}\right)$ of polymer $\mathbf{P 1 9}$. 


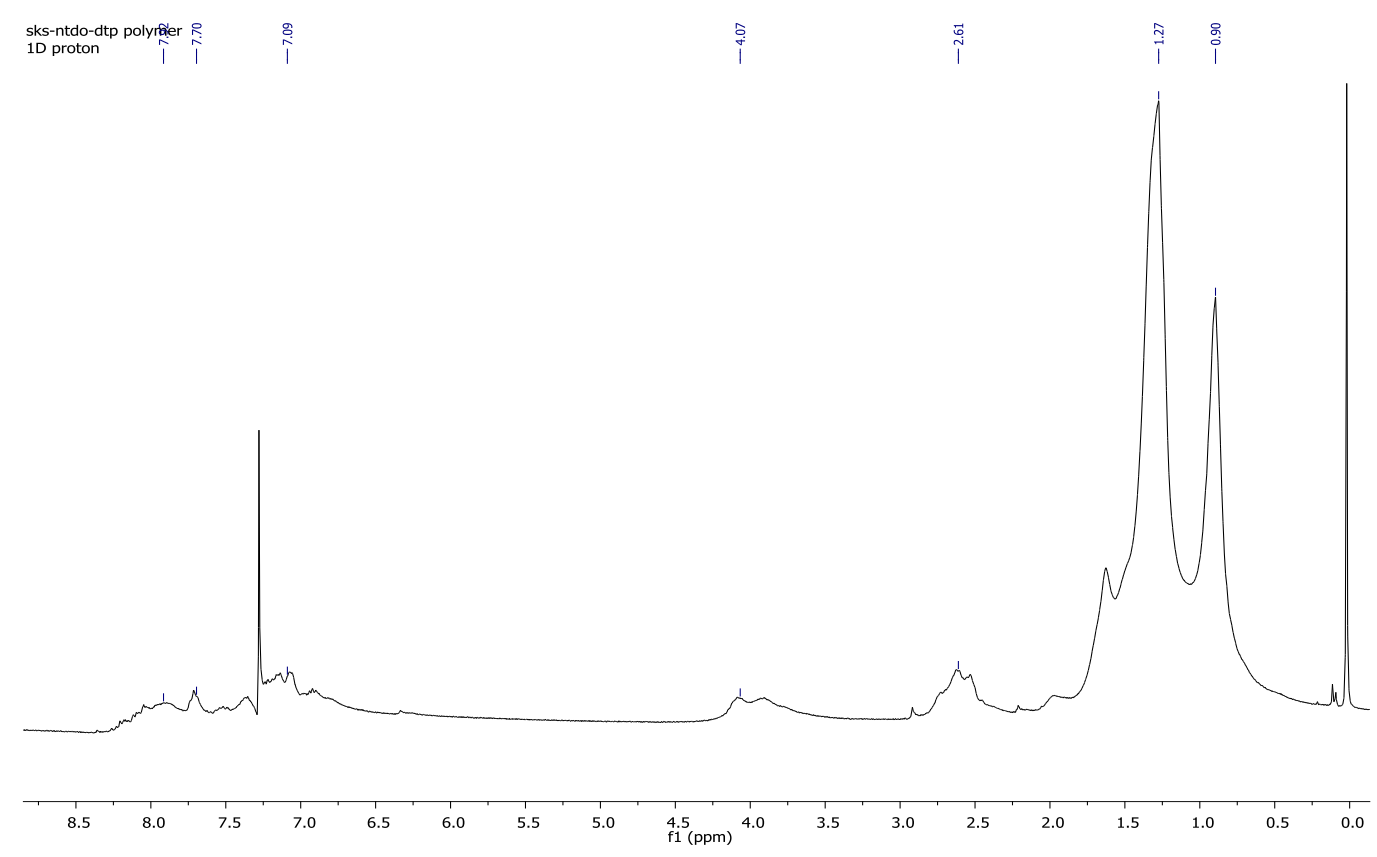

Figure S3.17. ${ }^{1} \mathrm{H}$ NMR spectrum $\left(300 \mathrm{MHz}, \mathrm{CDCl}_{3}\right)$ of polymer $\mathbf{P 2 1}$.

sks-ntdo-dtp polymer
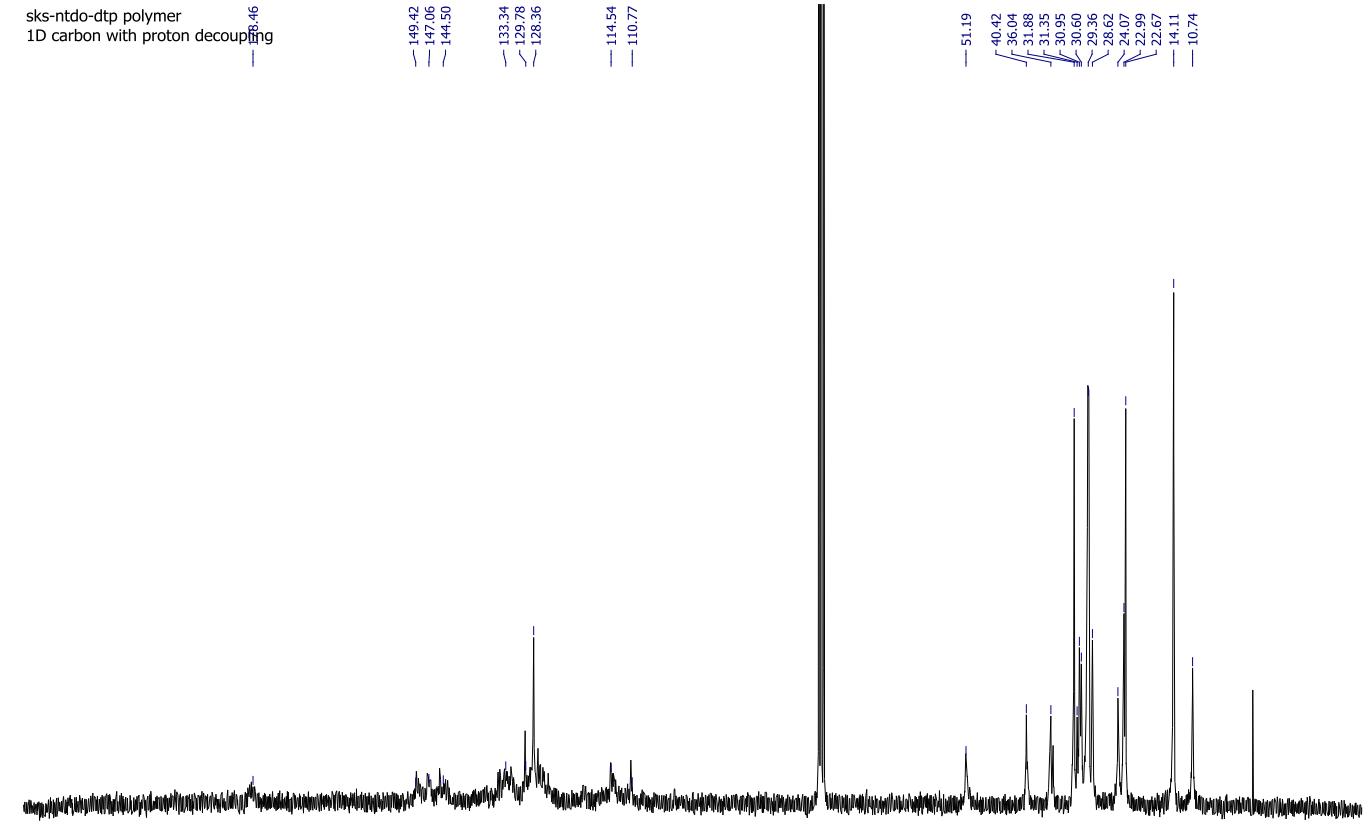

$\begin{array}{llllllllllllllllllllllllllllllll}210 & 200 & 190 & 180 & 170 & 160 & 150 & 140 & 130 & 120 & 110 & 100 & 90 & 80 & 70 & 60 & 50 & 40 & 30 & 20 & 10 & 0 & -10\end{array}$

Figure S3.18. ${ }^{13} \mathrm{C}$ NMR spectrum $\left(75 \mathrm{MHz}, \mathrm{CDCl}_{3}\right)$ of polymer $\mathbf{P 2 1}$. 


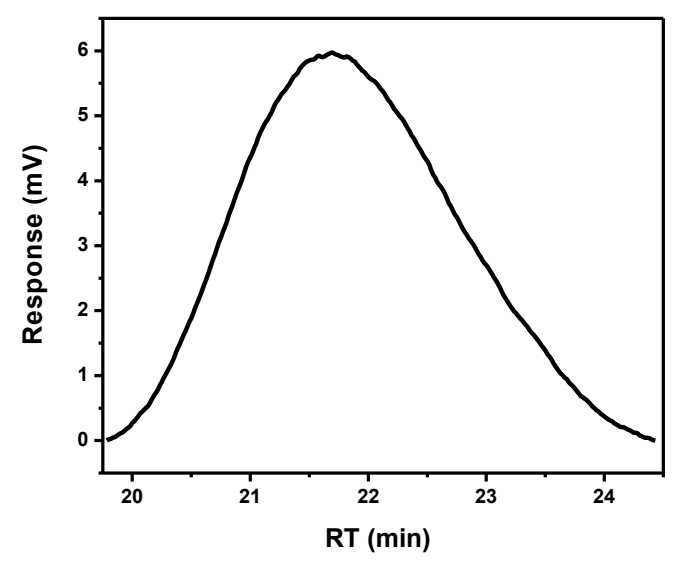

\begin{tabular}{|l|l|l|l|l|l|l|l|}
\hline Polymer & Mp & Mn & Mw & Mz & Mz+1 & Mv & PD \\
\hline P18 & 12241 & 7109 & 13963 & 22979 & 31812 & 12671 & 1.96413 \\
\hline
\end{tabular}

Figure S3.19 GPC result for polymer P18.

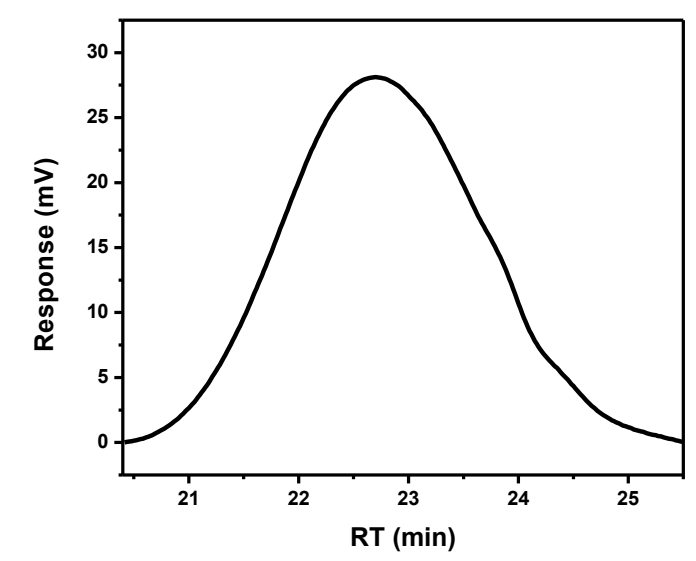

\begin{tabular}{|l|l|l|l|l|l|l|l|}
\hline Polymer & $\mathrm{Mp}$ & $\mathrm{Mn}$ & $\mathrm{Mw}$ & $\mathrm{Mz}$ & $\mathrm{Mz}+1$ & $\mathrm{Mv}$ & $\mathrm{PD}$ \\
\hline P19 & 4578 & 2936 & 5700 & 9821 & 14571 & 5154 & 1.94142 \\
& & & & & & & \\
\hline
\end{tabular}

Figure S3.20 GPC result for polymer P19. 


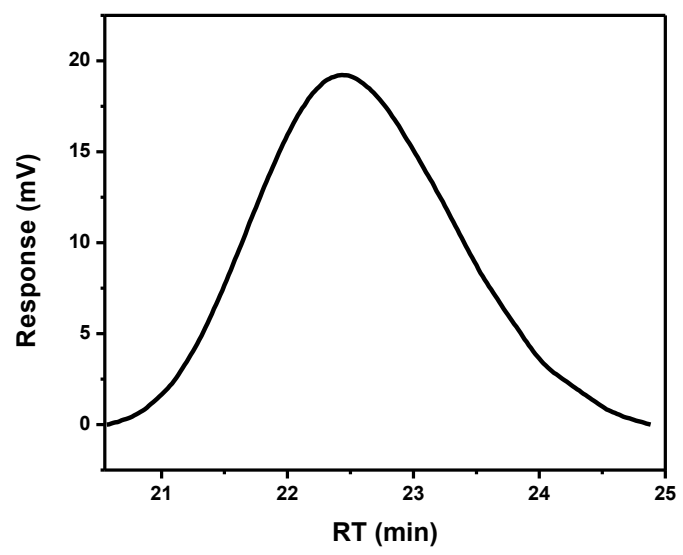

\begin{tabular}{|l|l|l|l|l|l|l|l|}
\hline Polymer & Mp & Mn & Mw & Mz & Mz+1 & Mv & PD \\
\hline P20 & 5951 & 3920 & 6595 & 10031 & 13686 & 6103 & 1.6824 \\
\end{tabular}

Figure S3.21 GPC result for polymer P20.

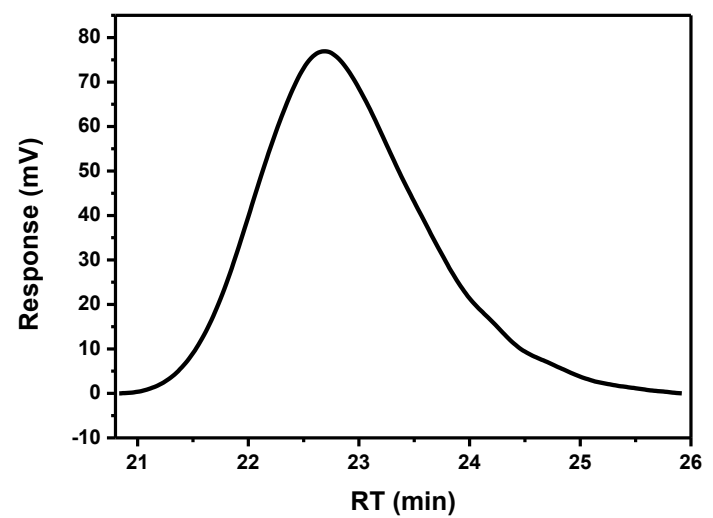

\begin{tabular}{|l|l|l|l|l|l|l|l|}
\hline Polymer & $\mathrm{Mp}$ & $\mathrm{Mn}$ & $\mathrm{Mw}$ & $\mathrm{Mz}$ & $\mathrm{Mz}+1$ & $\mathrm{Mv}$ & $\mathrm{PD}$ \\
\hline P21 & 4653 & 2695 & 4714 & 6927 & 9203 & 4380 & 1.74917 \\
& & & & & & & \\
\hline
\end{tabular}

Figure S3.22 GPC result for polymer P21. 


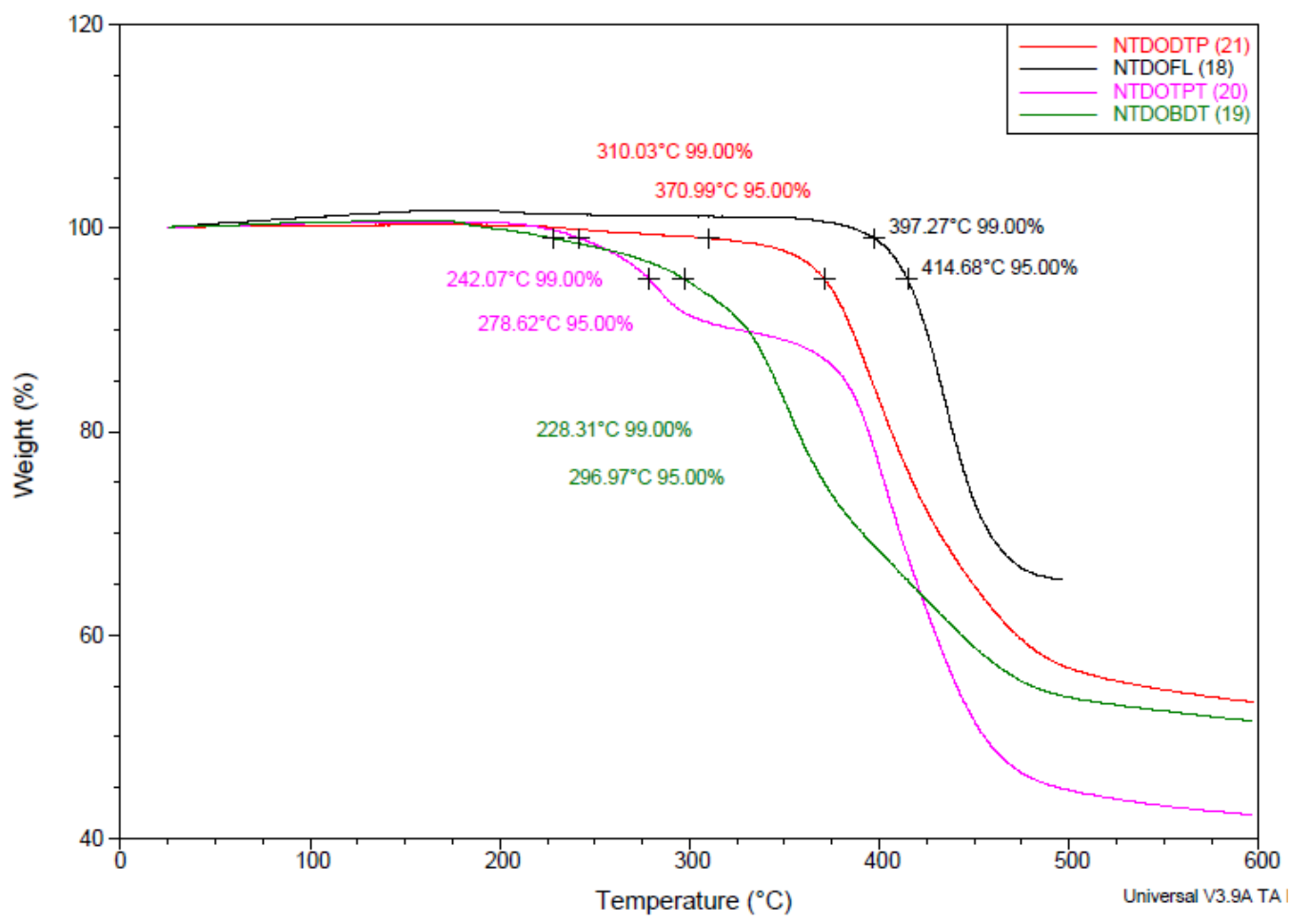

Figure S3.23 TGA plots of polymers $\mathbf{P 1 8}$ to $\mathbf{P 2 1}$ with a heating rate of $10{ }^{\circ} \mathrm{C} / \mathrm{min}$ under nitrogen atmosphere.
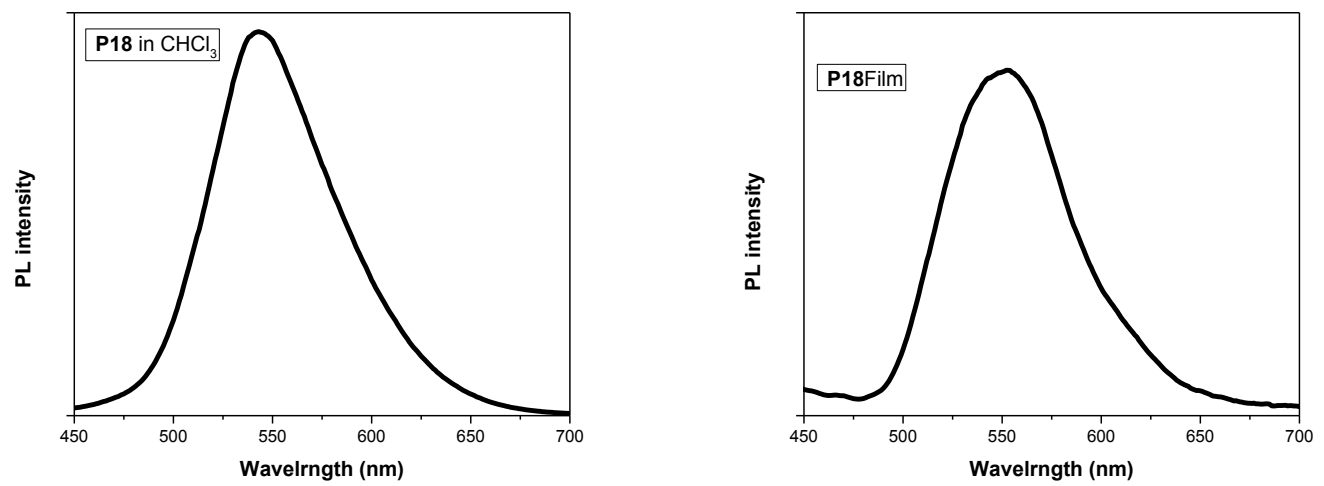

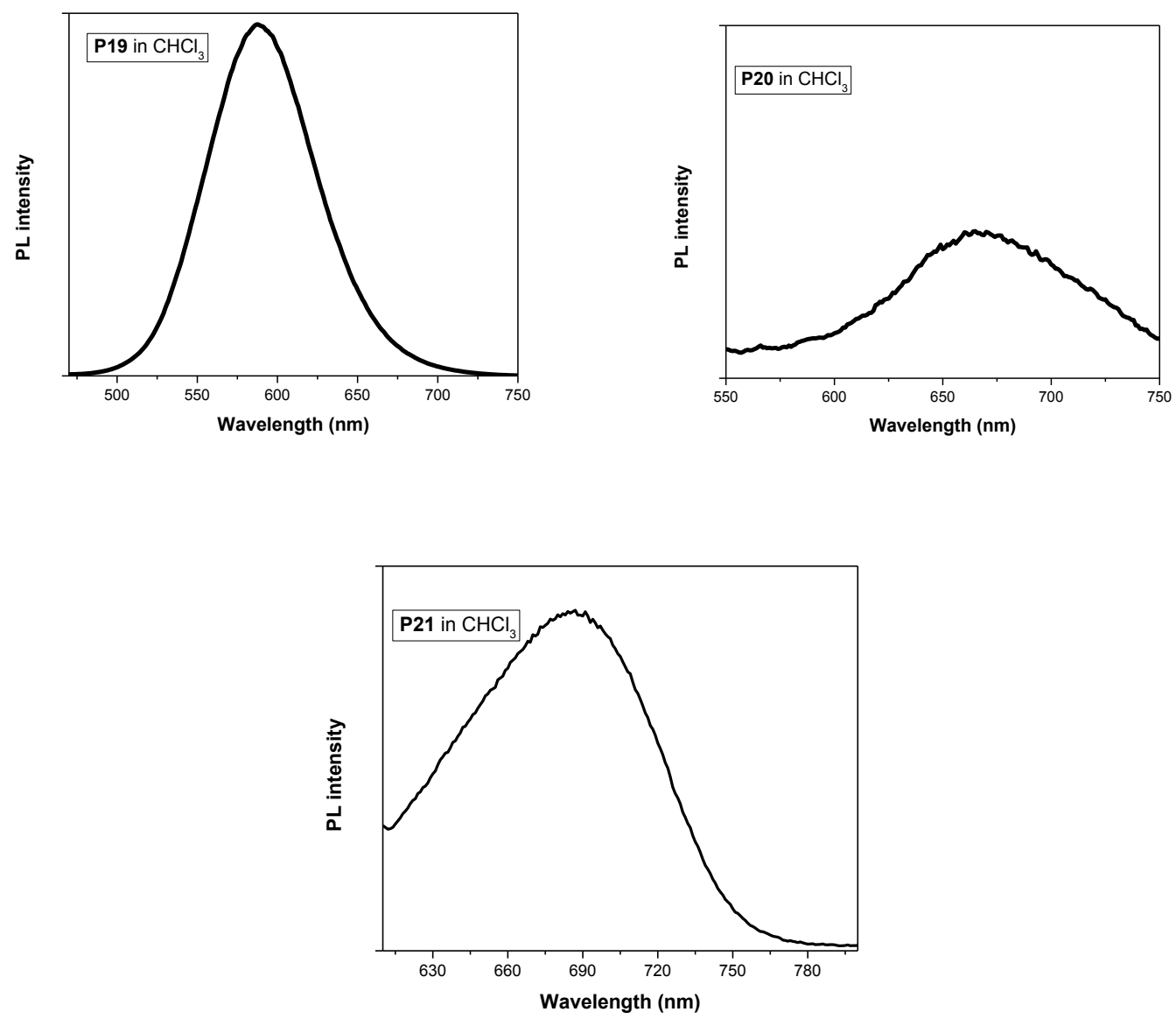

Figure S3.24 Emission spectra for polymers P18-P21. 


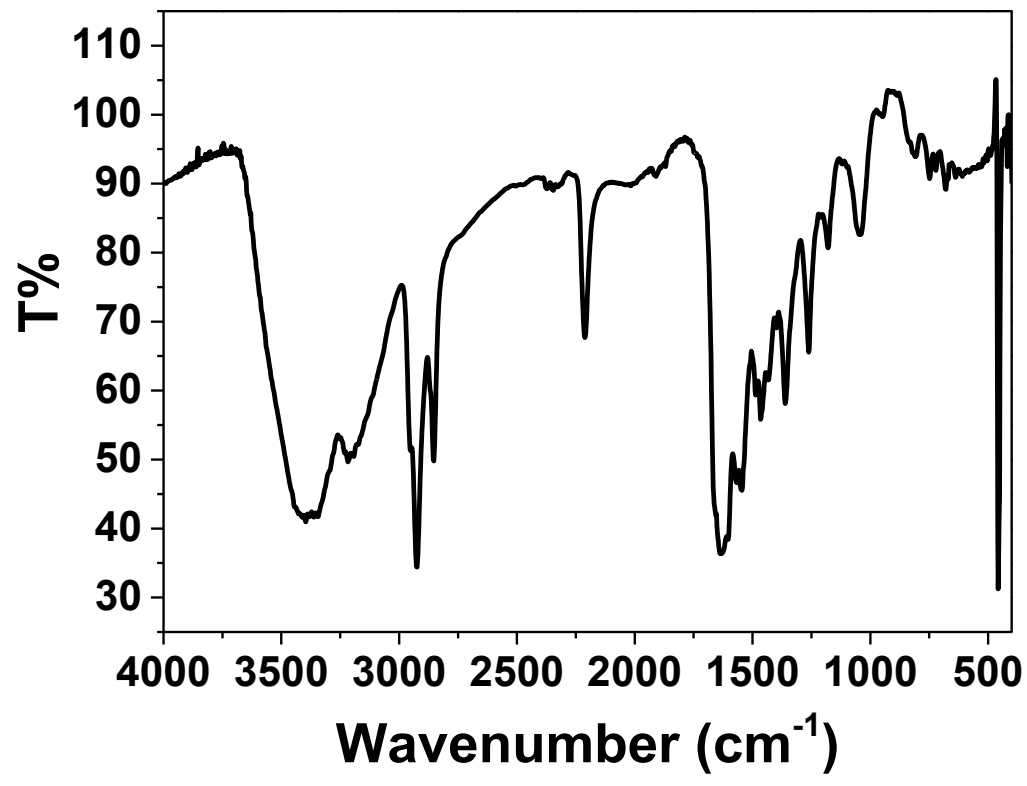

Figure S3.25 IR spectrum of polymer $\mathbf{P 2 3}$ in KBr pellet.

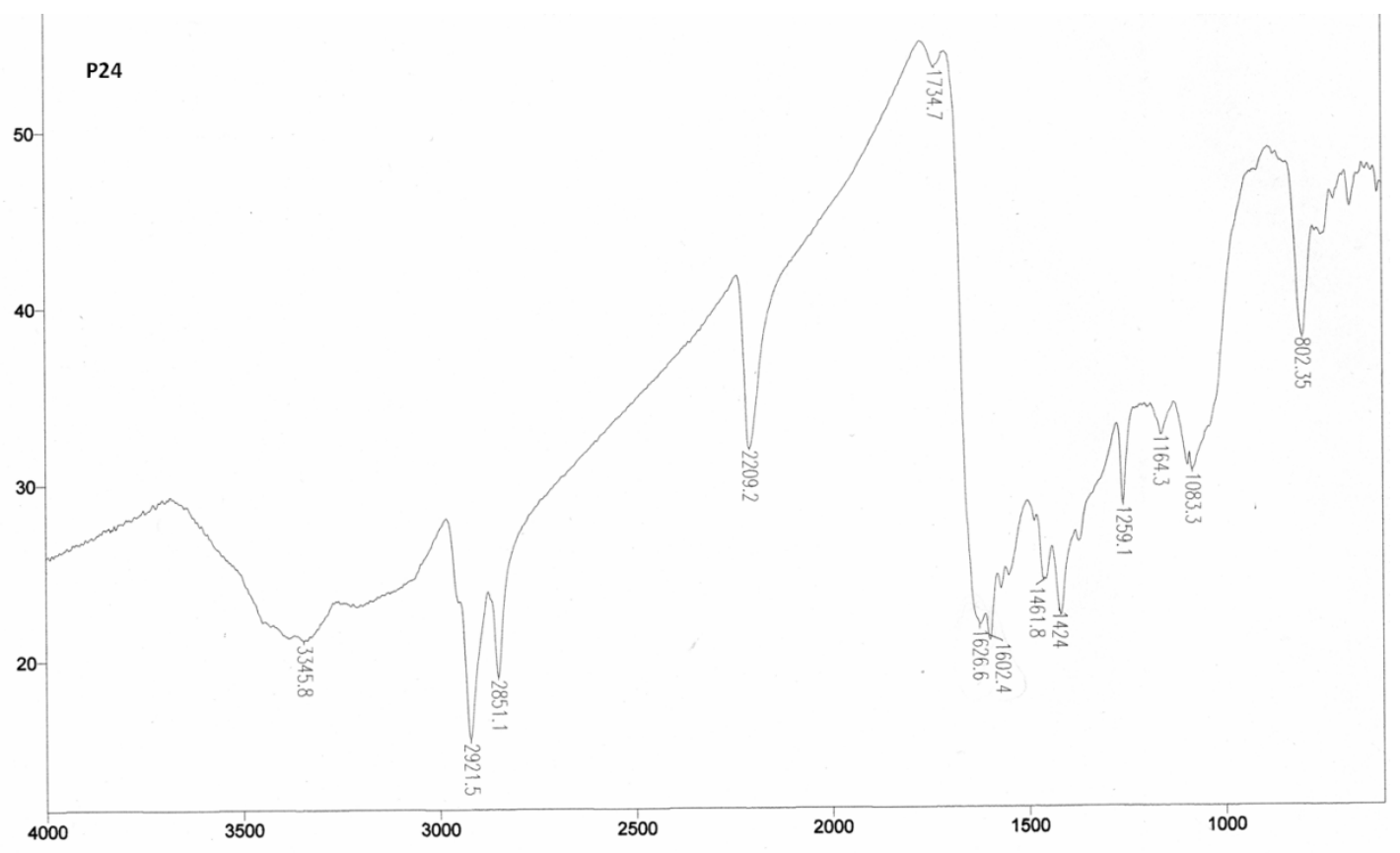

Figure S3.26. IR spectrum of polymer P24 in KBr pellet. 


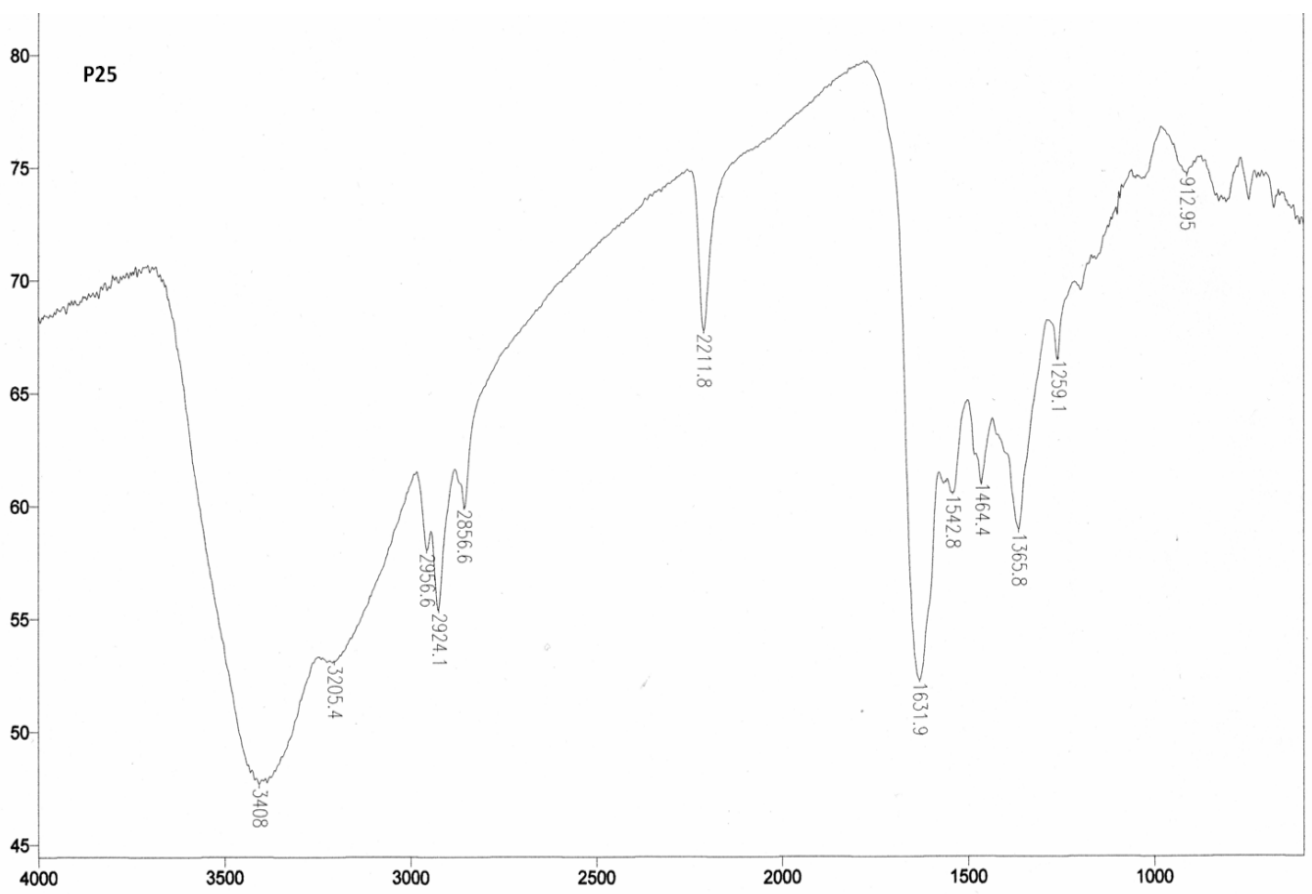

Figure S3.27 IR spectrum of polymer P25 in KBr pellet.

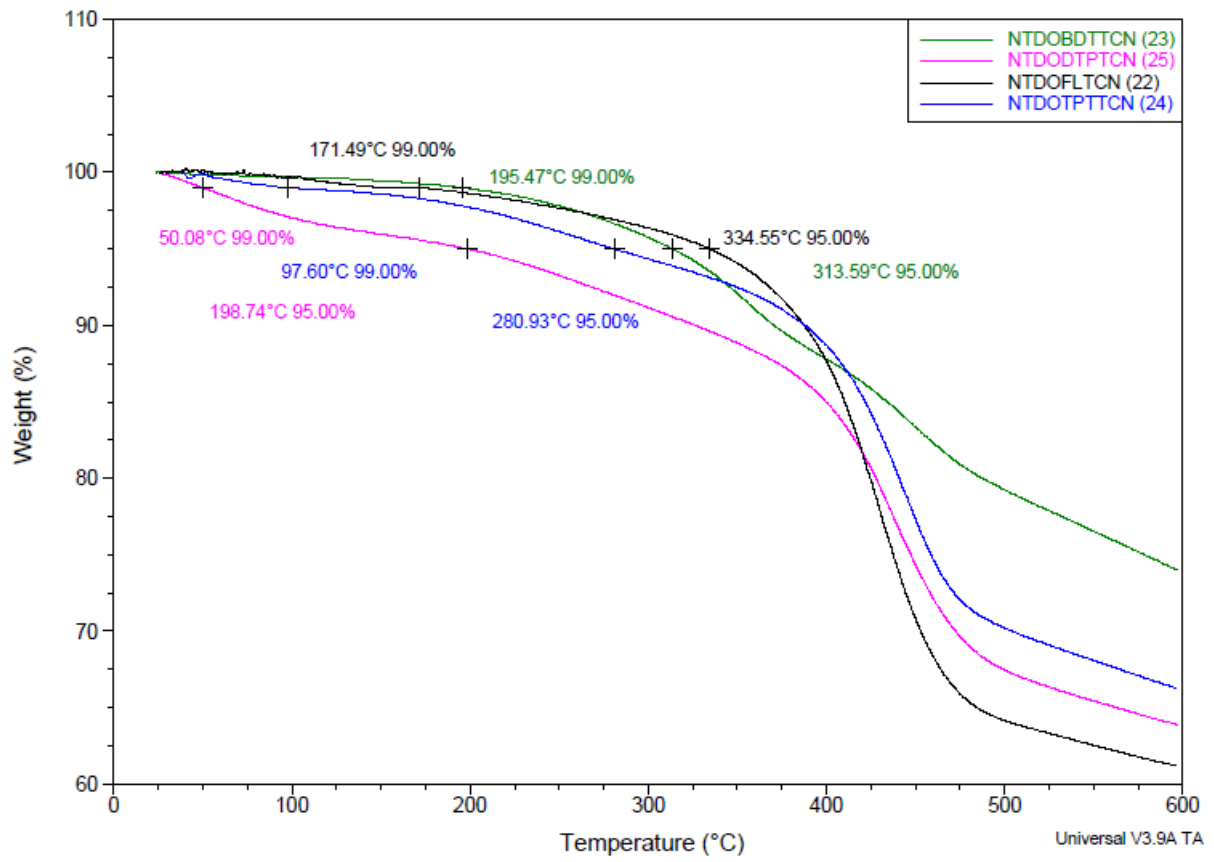

Figure S3.28 TGA plots of the polymers $\mathbf{P 2 2}$ to $\mathbf{P 2 5}$ with a heating rate of $10^{\circ} \mathrm{C} / \mathrm{min}$ under nitrogen atmosphere. 

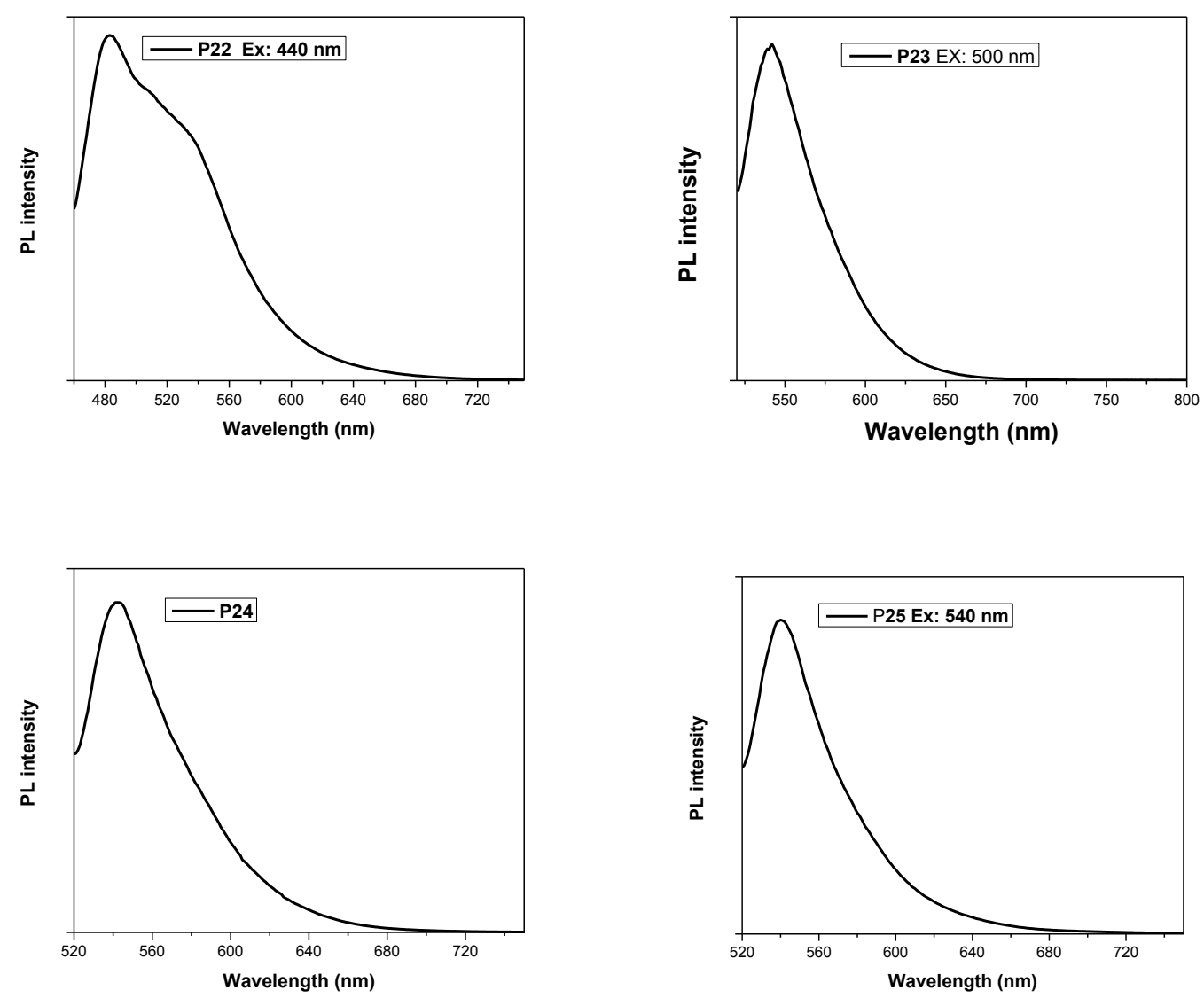

Figure S2.29 Emission of polymers P25 and P28 solution in TCB.
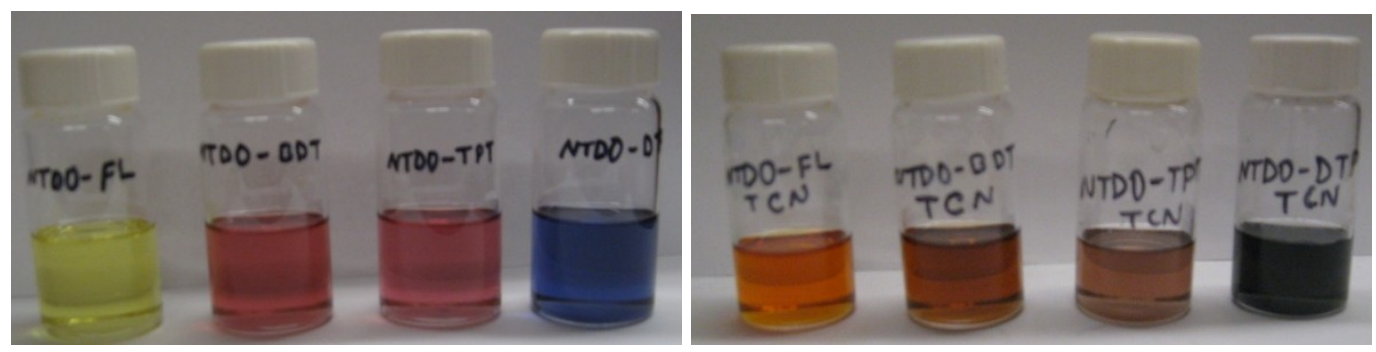

Figure S3.30 Photograph of polymers P18 to P25 solution.

\section{Device preparation}

The mixture of polymer $(10 \mathrm{mg})$ and $\mathrm{PC}_{61} \mathrm{BM}$ with a weight ratio of 1:2 was dissolved in NMP in a concentration of $30 \mathrm{mg} / \mathrm{mL}$ and stirred at $80{ }^{\circ} \mathrm{C}$ for $4 \mathrm{~h}$ under air. The ITOcoated glass was cleaned and treated with plasma of oxygen. PEDOT:PSS was spin- 
coated onto the ITO surface at $3000 \mathrm{rpm}$ for $60 \mathrm{~s}$ to obtain a thin film of $30 \mathrm{~nm}$ and dried at $110{ }^{\circ} \mathrm{C}$ for $30 \mathrm{~min}$. The active layer was spin coated onto the PEDOT:PSS film at 600 rpm for $120 \mathrm{~s}$ under air. Finally, thin layers of BCP $(3 \mathrm{~nm})$ and $\mathrm{Al}(120 \mathrm{~nm})$ were deposited by thermal evaporation in vacuum.
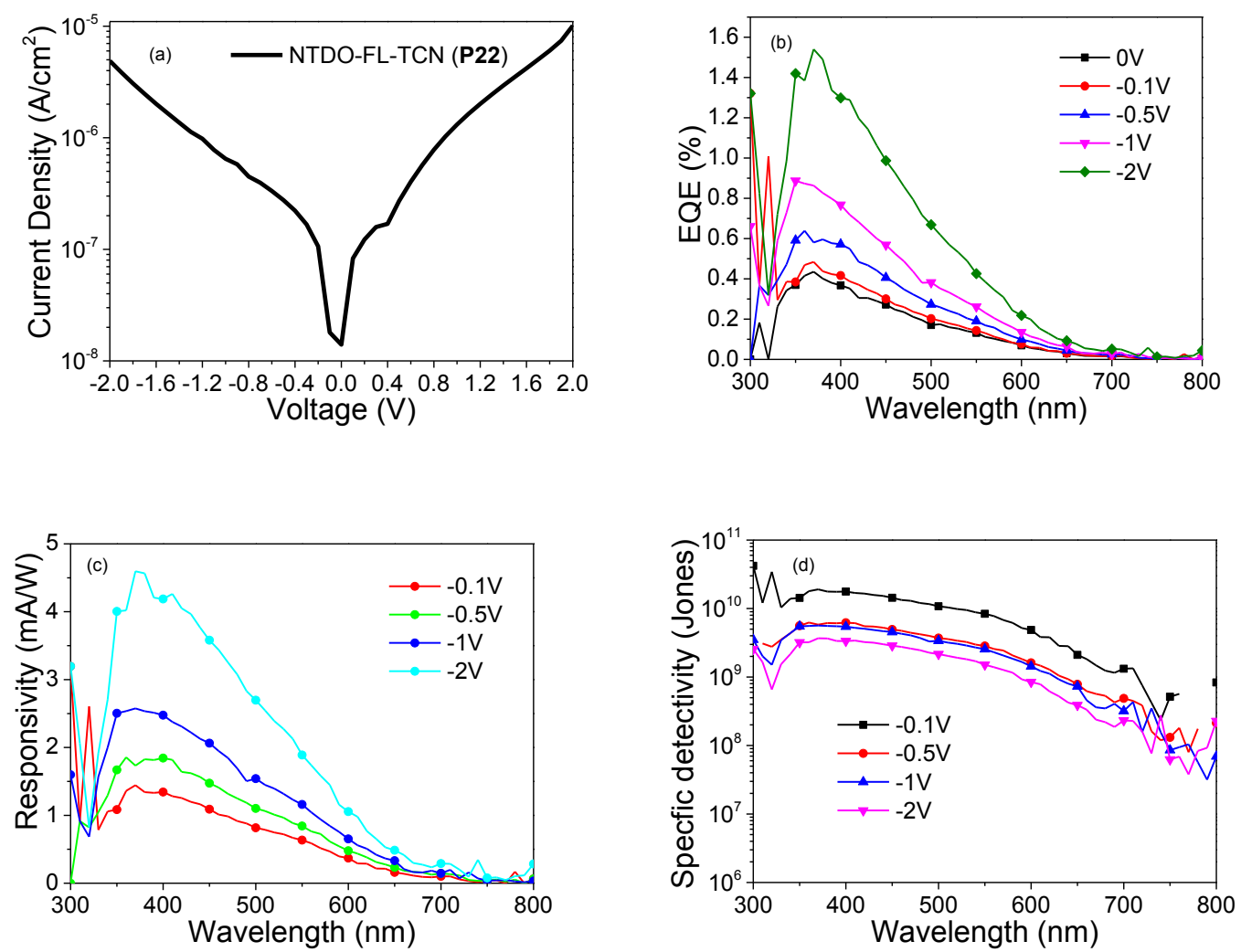

Figure S3.31 Photodetector properties of polymer P22 (a) dark current (b) EQE (c) responsivity $(\mathrm{d})$ detectivity.
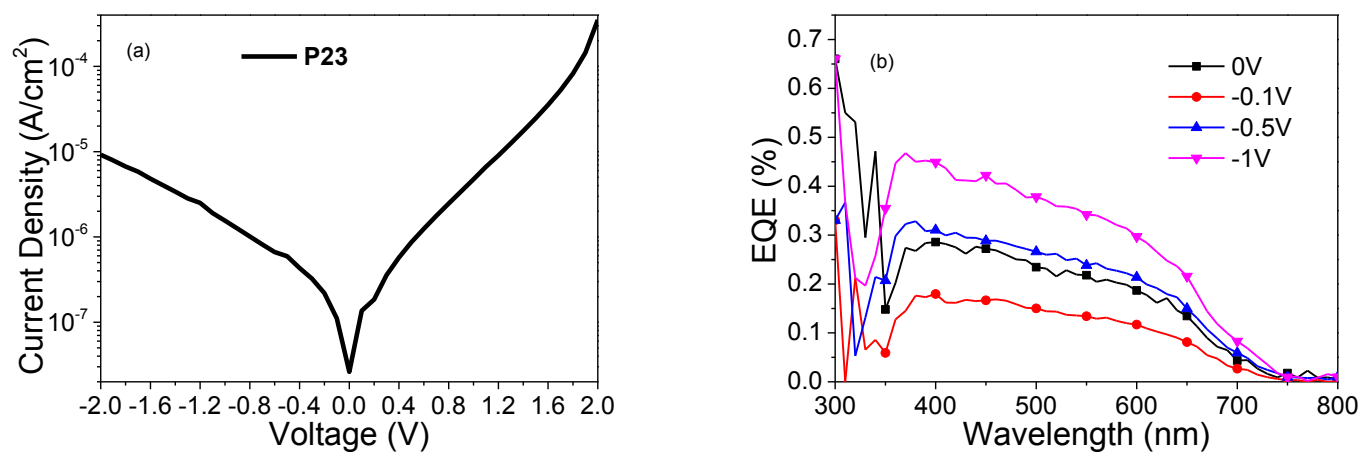

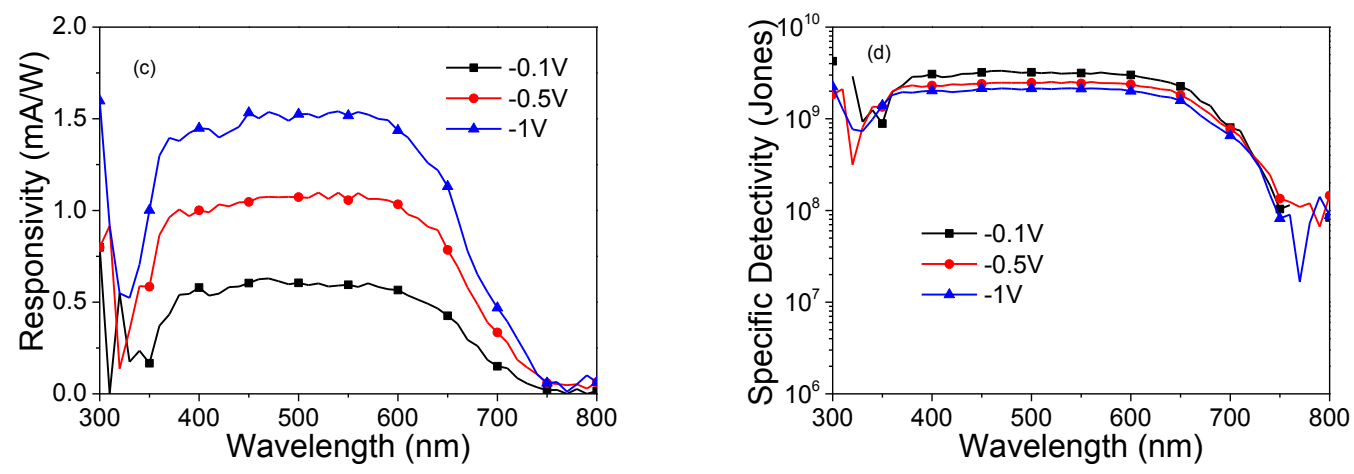

Figure S3.32 Photodetector properties of polymer P23 (a) dark current (b) EQE (c) responsivity (d) detectivity.
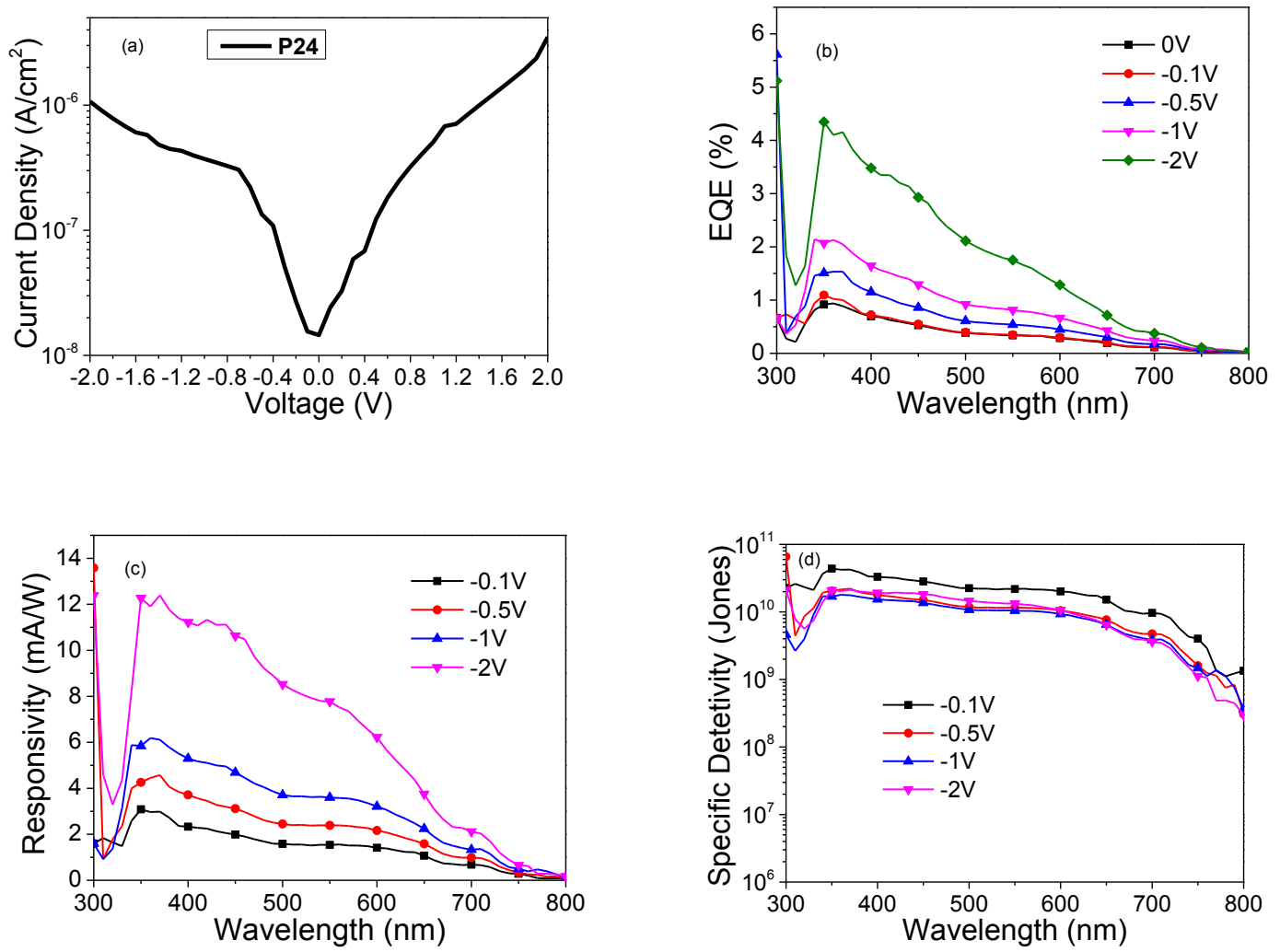

Figure S3.33 Photodetector properties of polymer P24 (a) dark current (b) EQE (c) responsivity (d) detectivity. 

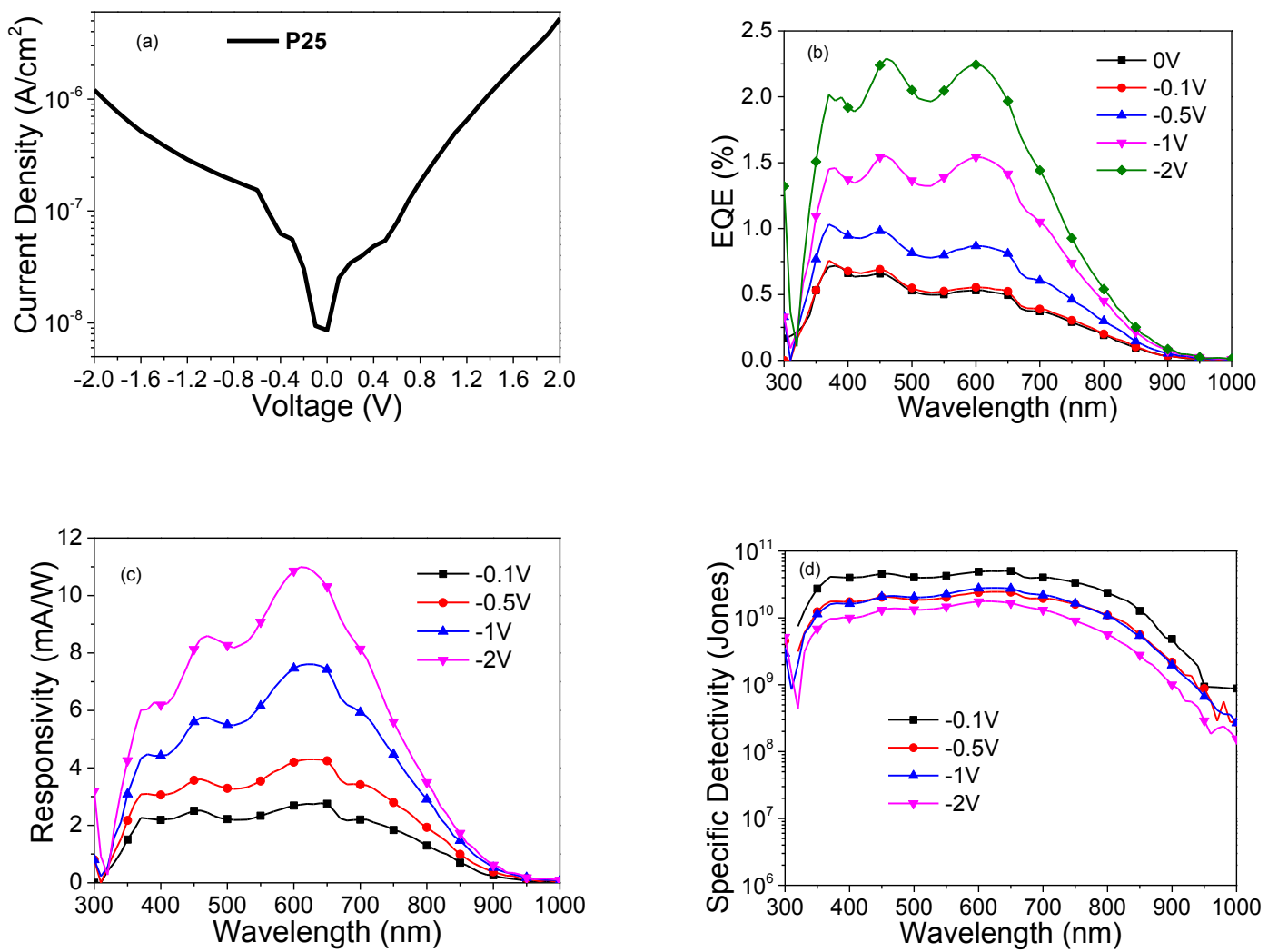

Figure S3.34 Photodetector properties of polymer P25 (a) dark current (b) EQE (c) responsivity (d) detectivity. 
Appendix C: Spectra of synthesized compounds and polymers in chapter 4

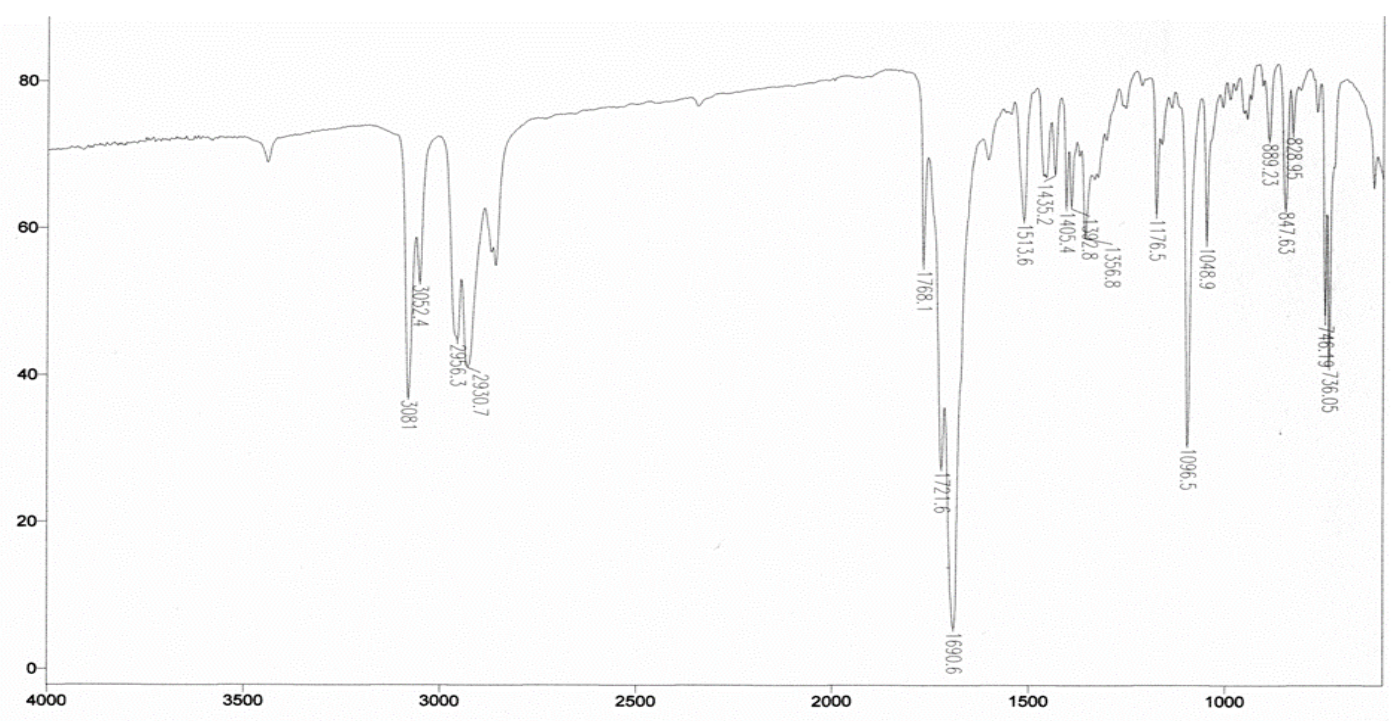

Figure S4.1 IR spectrum of Compound $\mathbf{1 0}$ (on $\mathrm{NaCl}$ plate).

sks-thiopheneimices

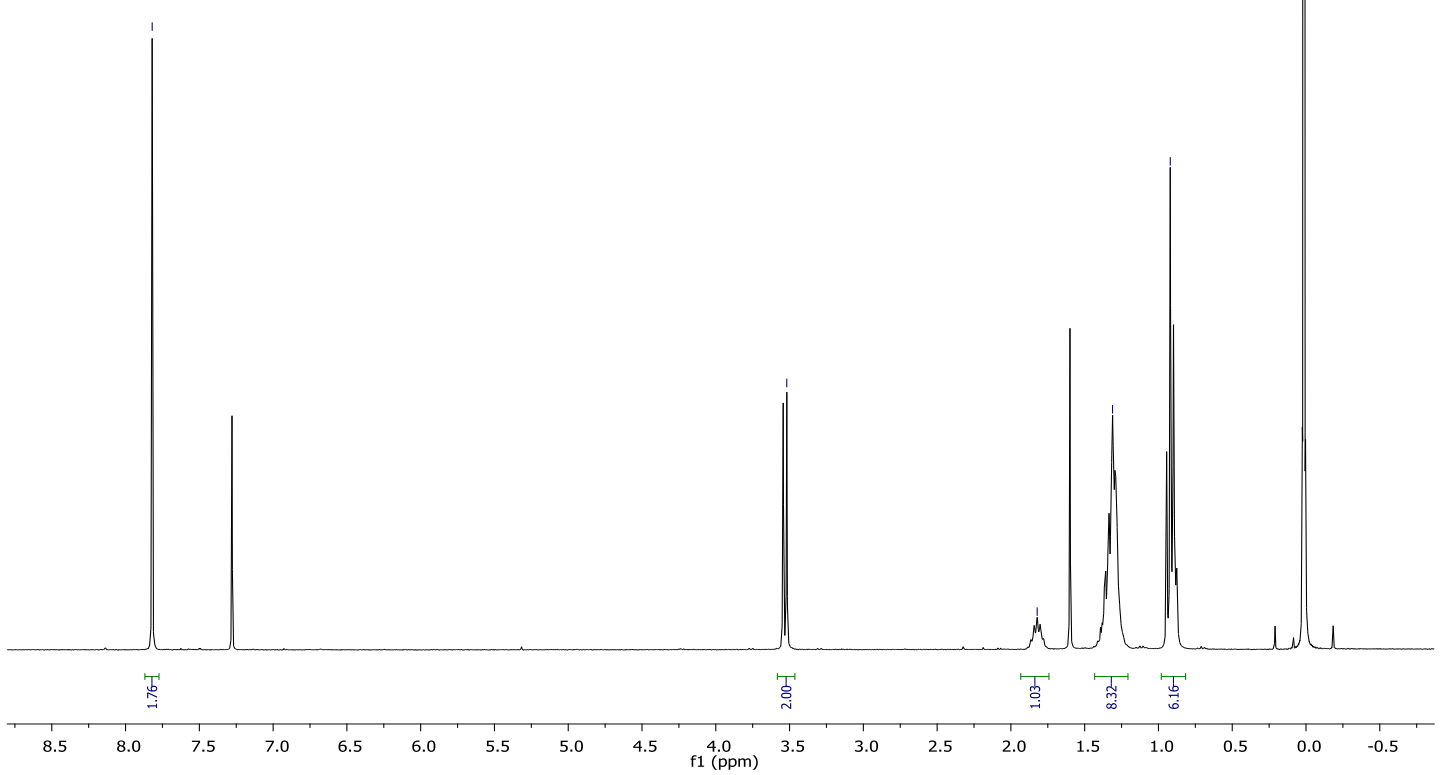

Figure $\mathrm{S} 4.2{ }^{1} \mathrm{H}$ NMR spectrum $\left(300 \mathrm{MHz}, \mathrm{CDCl}_{3}\right)$ of compound $\mathbf{1 0 .}$ 

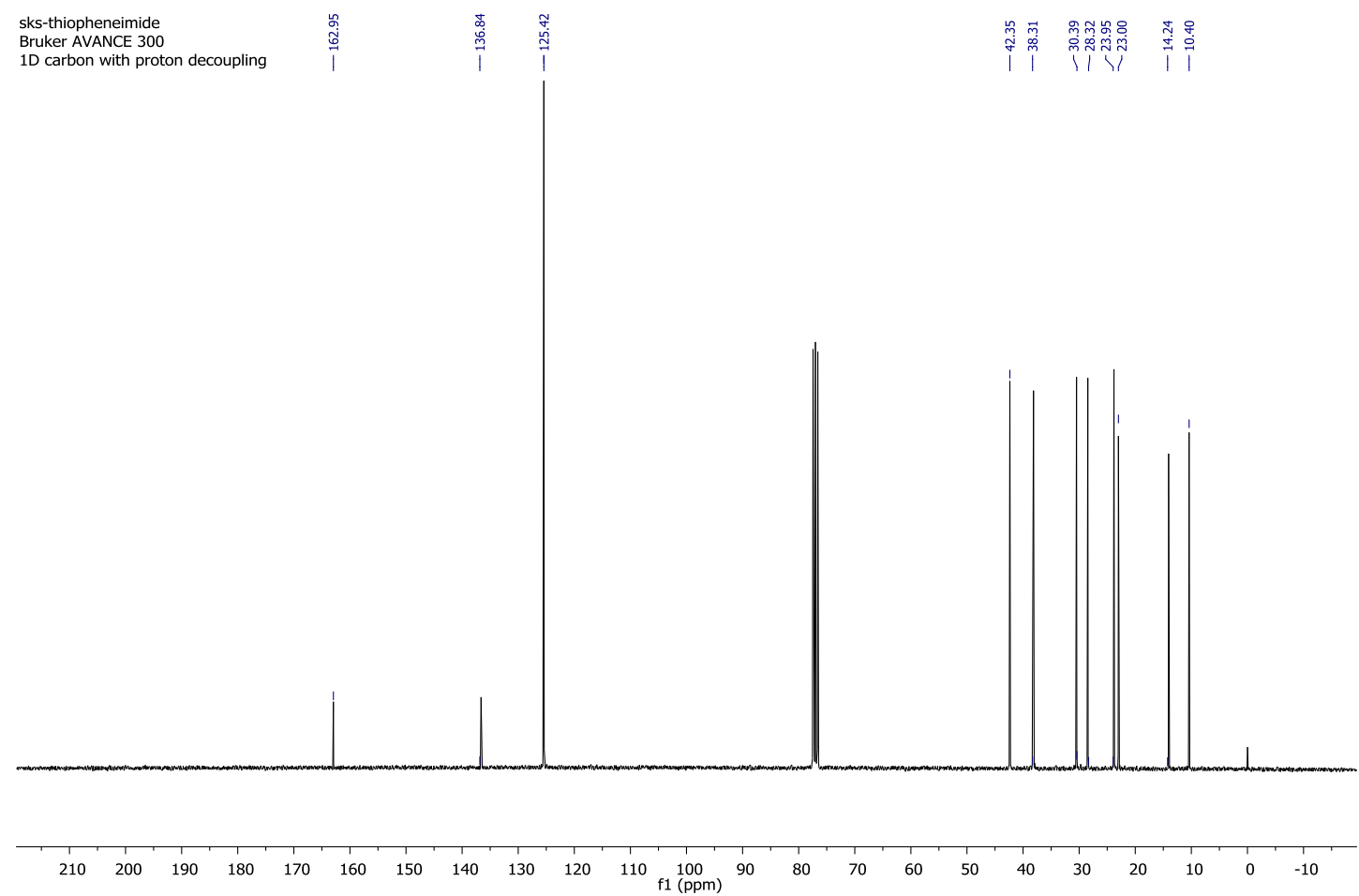

Figure $\mathrm{S} 4.3{ }^{13} \mathrm{C}$ NMR spectrum $\left(75 \mathrm{MHz}, \mathrm{CDCl}_{3}\right)$ of compound $\mathbf{1 0}$.

2 d0037 Scan $8 \quad$ RT $=1: 28 \quad 100 \%=30170$ mV $14-A p r-2015 \quad 09: 34$ HRP +EI ac3-14

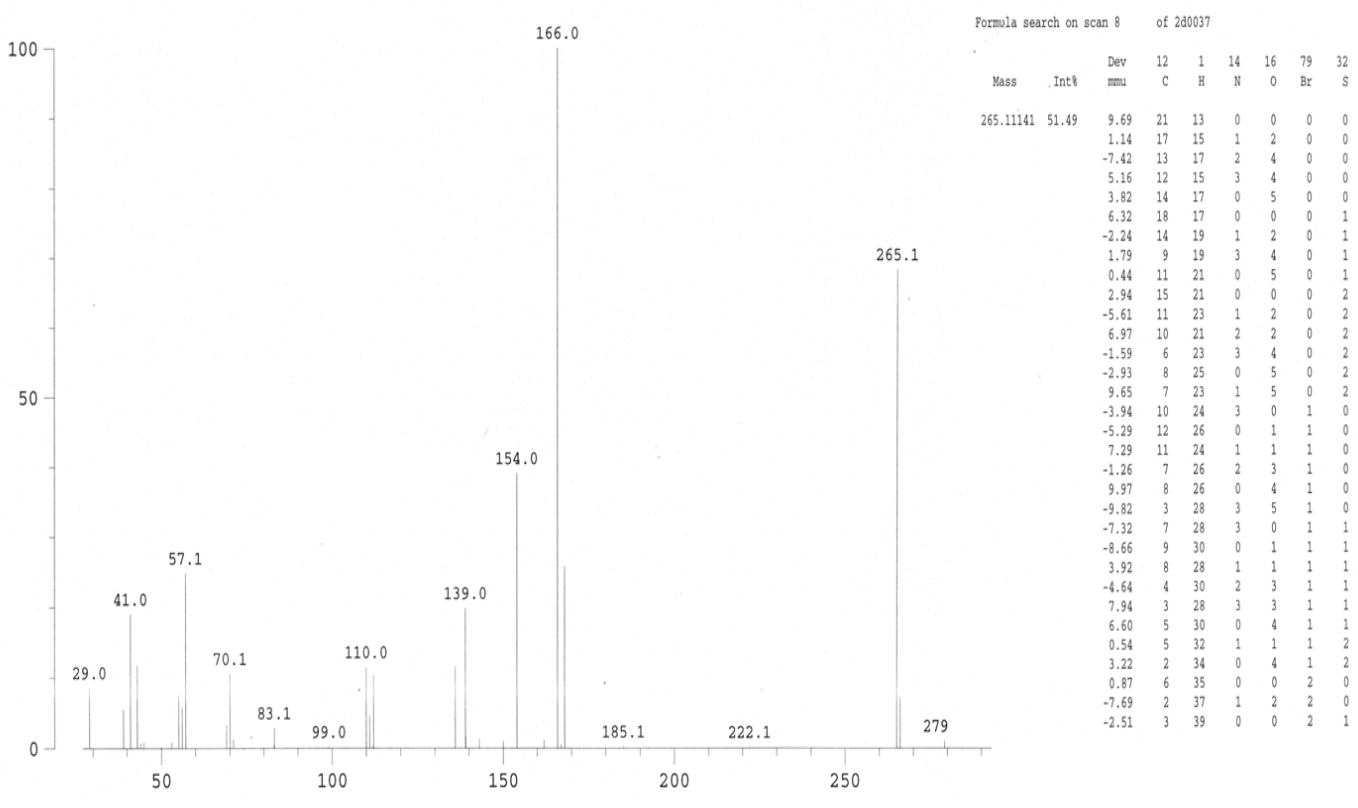

Figure S4.4 Mass spectrum of compound 10. 


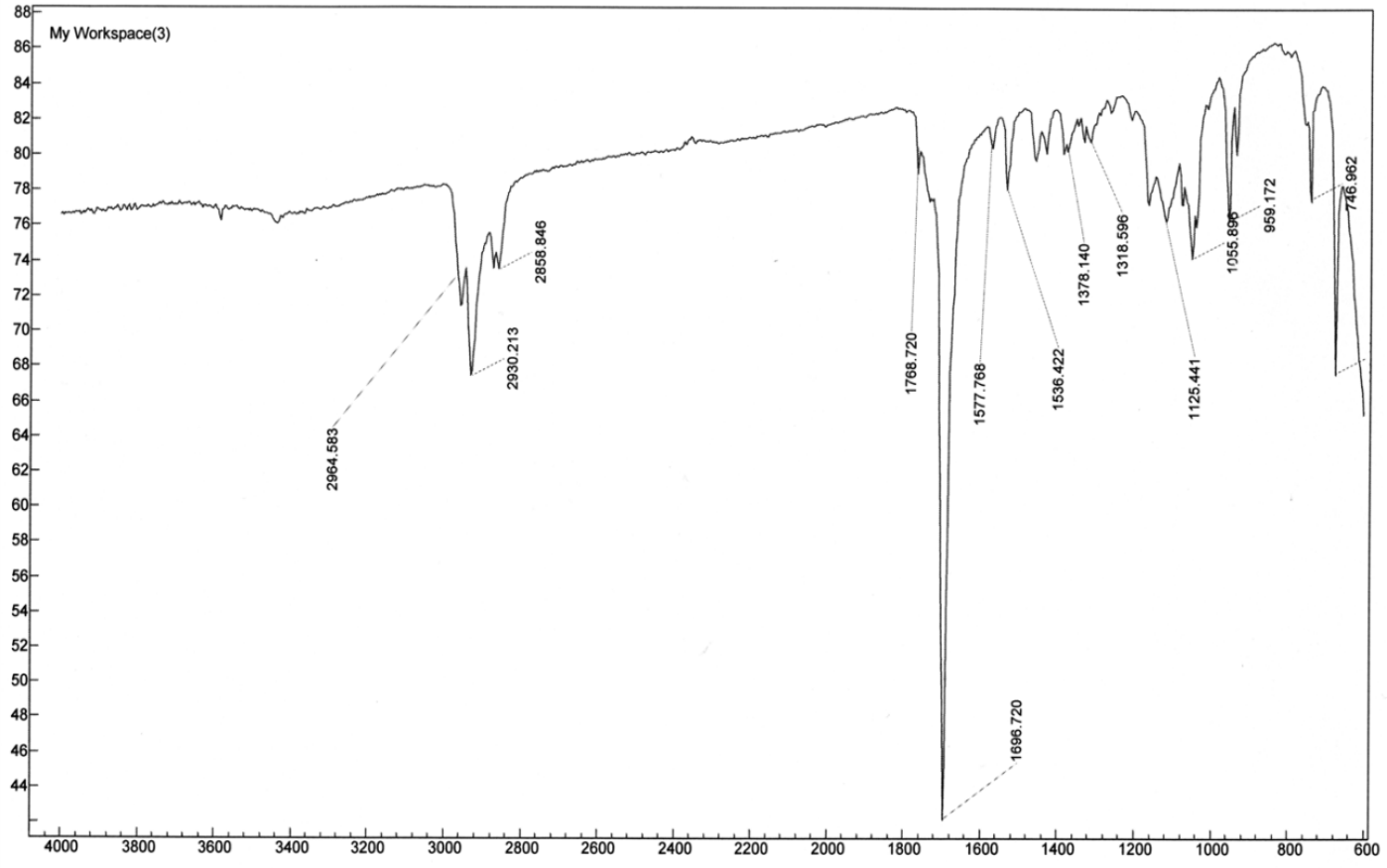

Figure S4.5 IR spectrum of compound $\mathbf{M 7}$ on $\mathrm{NaCl}$ plate.

sks-2,5 dibromothiophen\&imide
Bruker AVANCE 300 proton
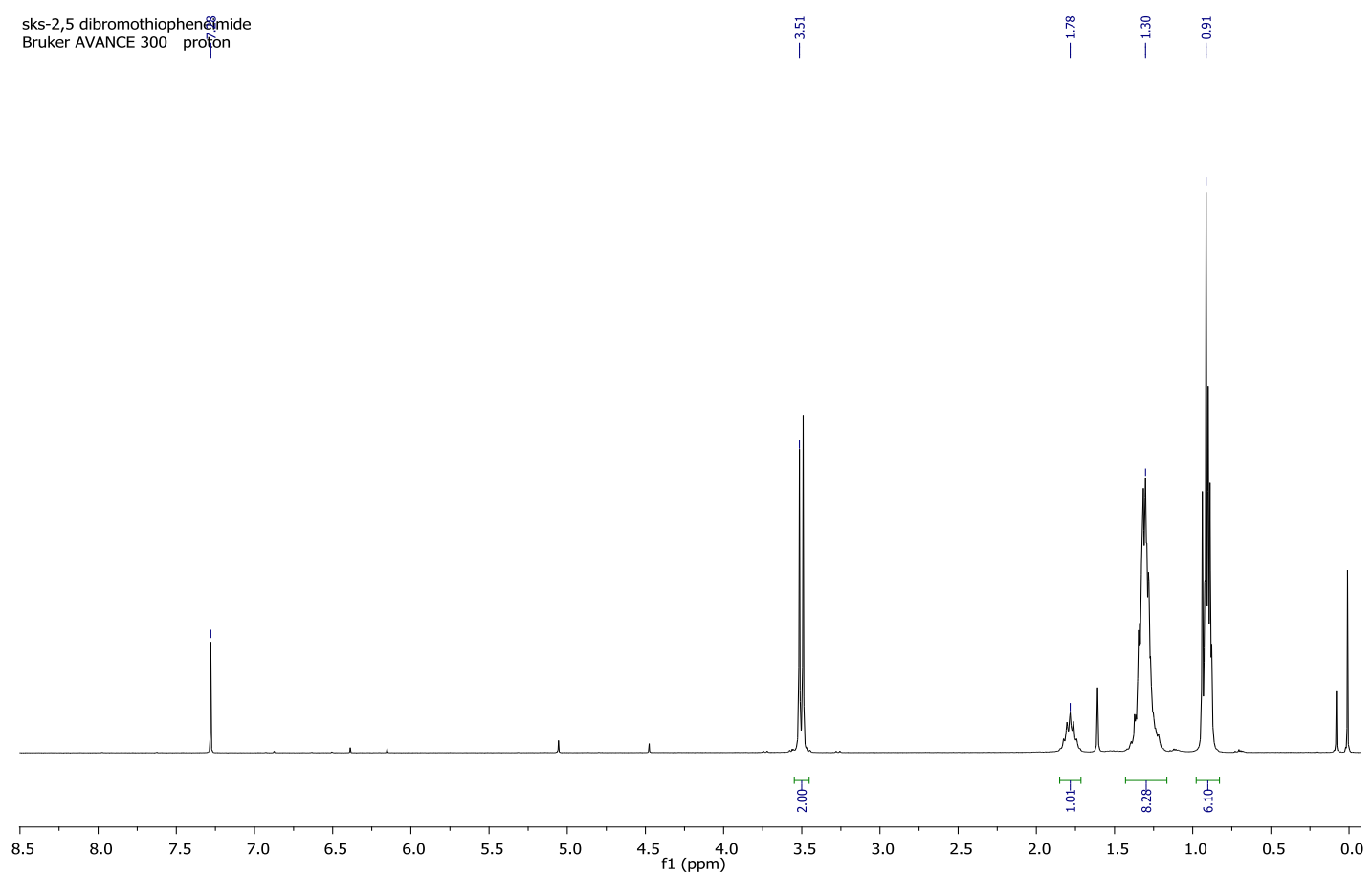

Figure S4. $6{ }^{1} \mathrm{H}$ NMR spectrum $\left(300 \mathrm{MHz}, \mathrm{CDCl}_{3}\right)$ of compound $\mathbf{M} 7$. 


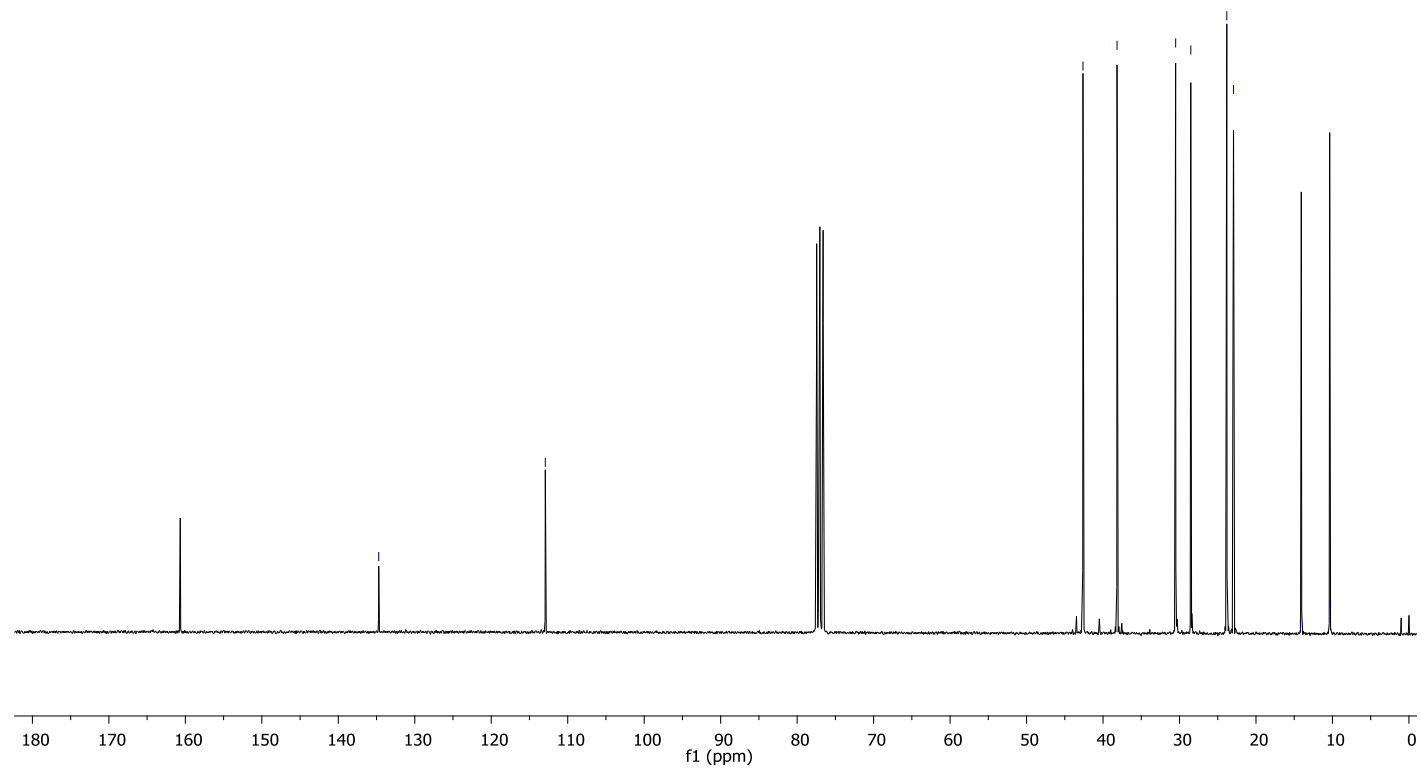

Figure $\mathrm{S} 4.7{ }^{13} \mathrm{C}$ NMR spectrum $\left(75 \mathrm{MHz}, \mathrm{CDCl}_{3}\right)$ of compound $\mathbf{M} 7$.

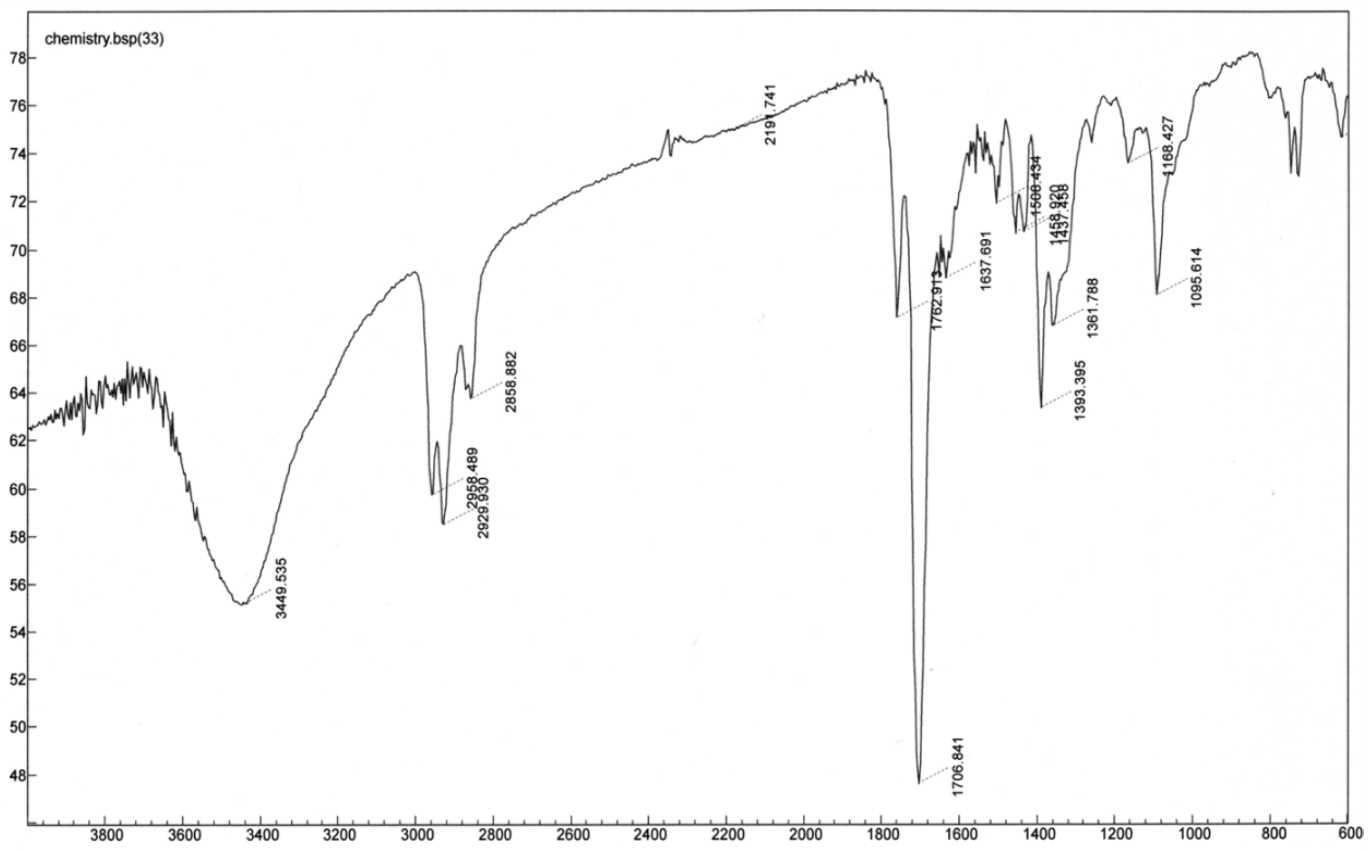

Figure S4.8 IR spectrum of polymer P26 (in KBr Pellet). 


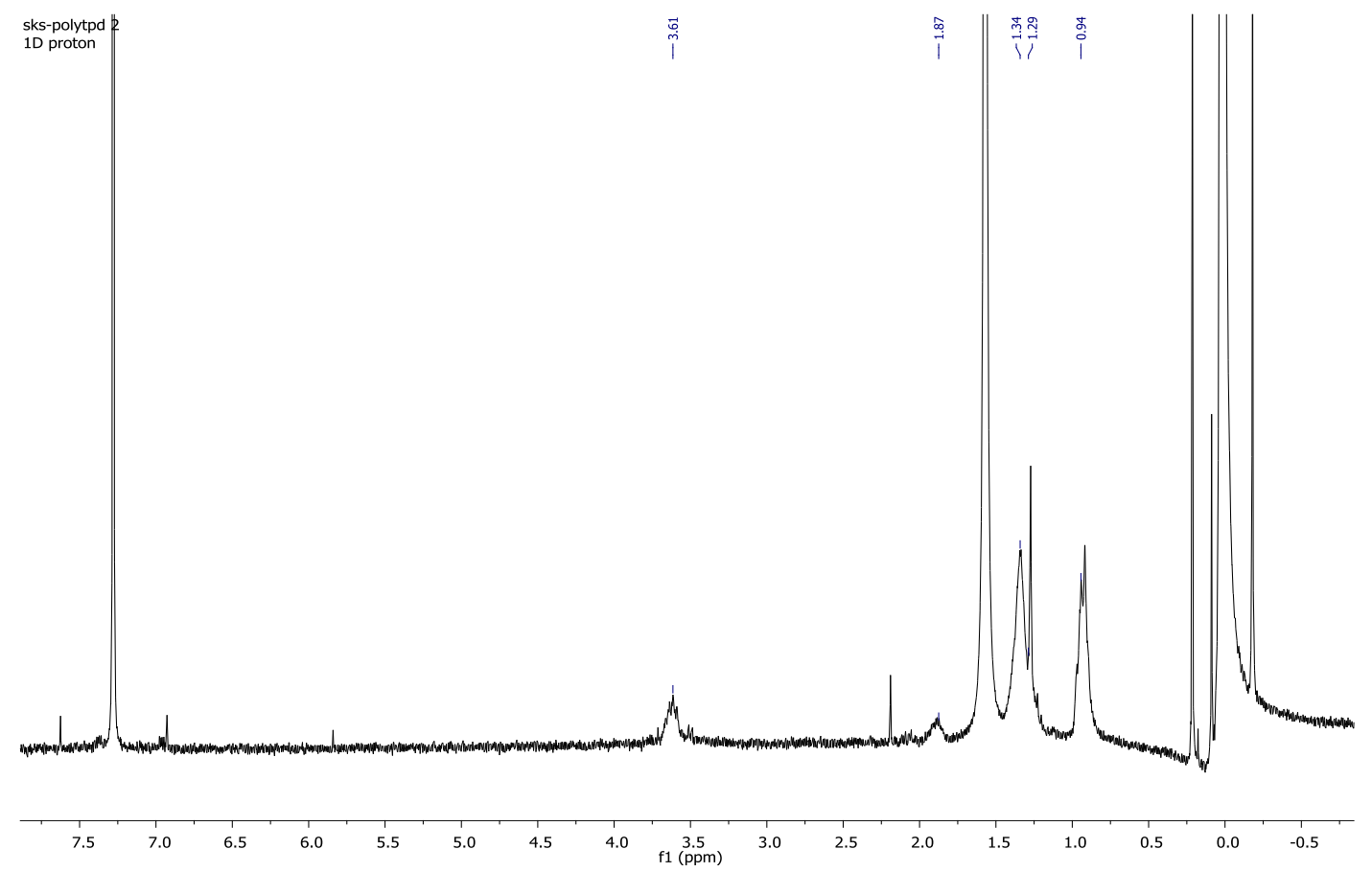

Figure S4. $9{ }^{1} \mathrm{H}$ NMR spectrum $\left(300 \mathrm{MHz}, \mathrm{CDCl}_{3}\right.$ ) of polymer $\mathbf{P 2 6}$.

TPD-homopolymer-2
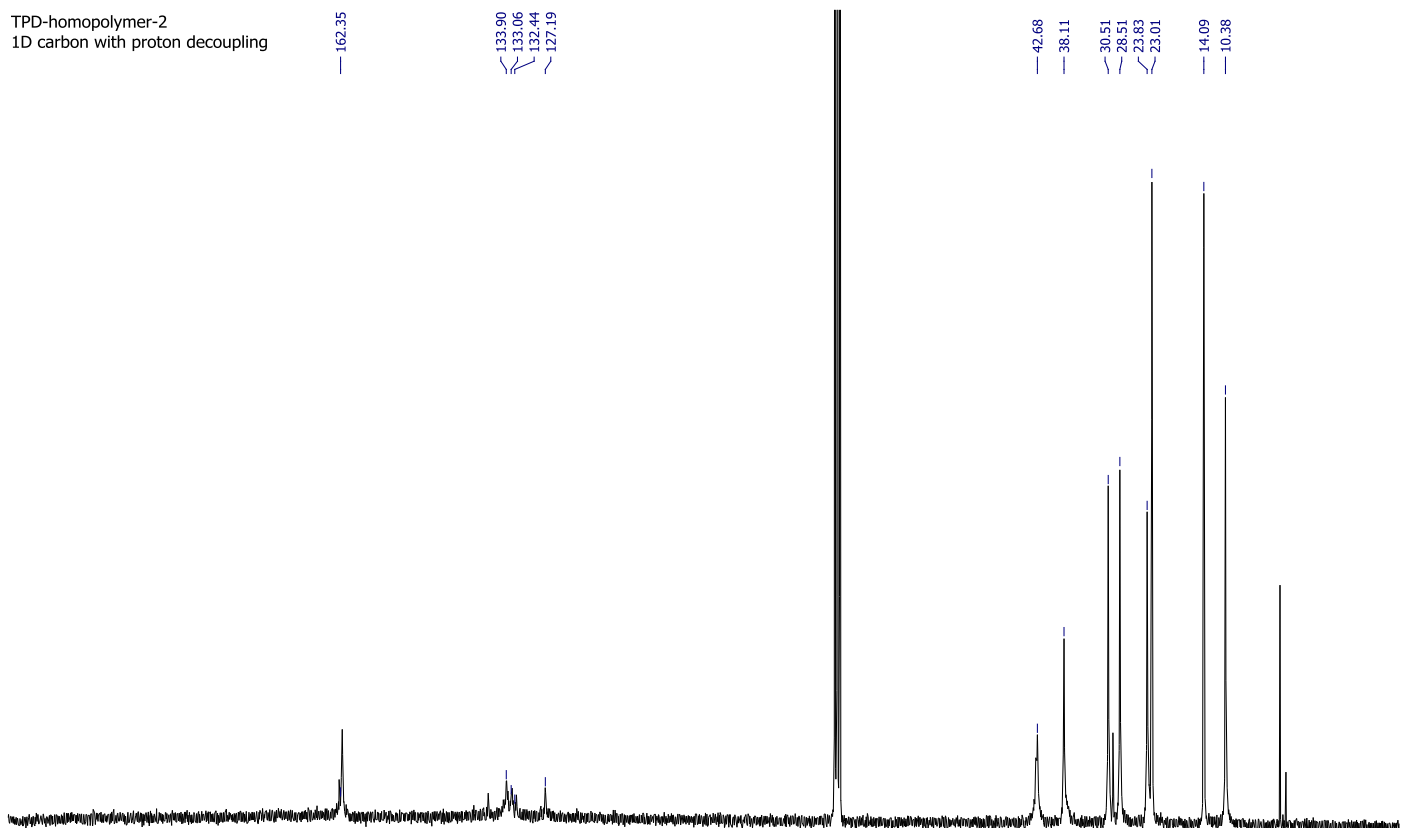

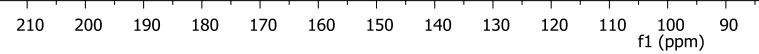

Figure $\mathrm{S} 4.10{ }^{13} \mathrm{C}$ NMR spectrum $\left(75 \mathrm{MHz}, \mathrm{CDCl}_{3}\right.$ ) of polymer $\mathbf{P 2 6}$. 


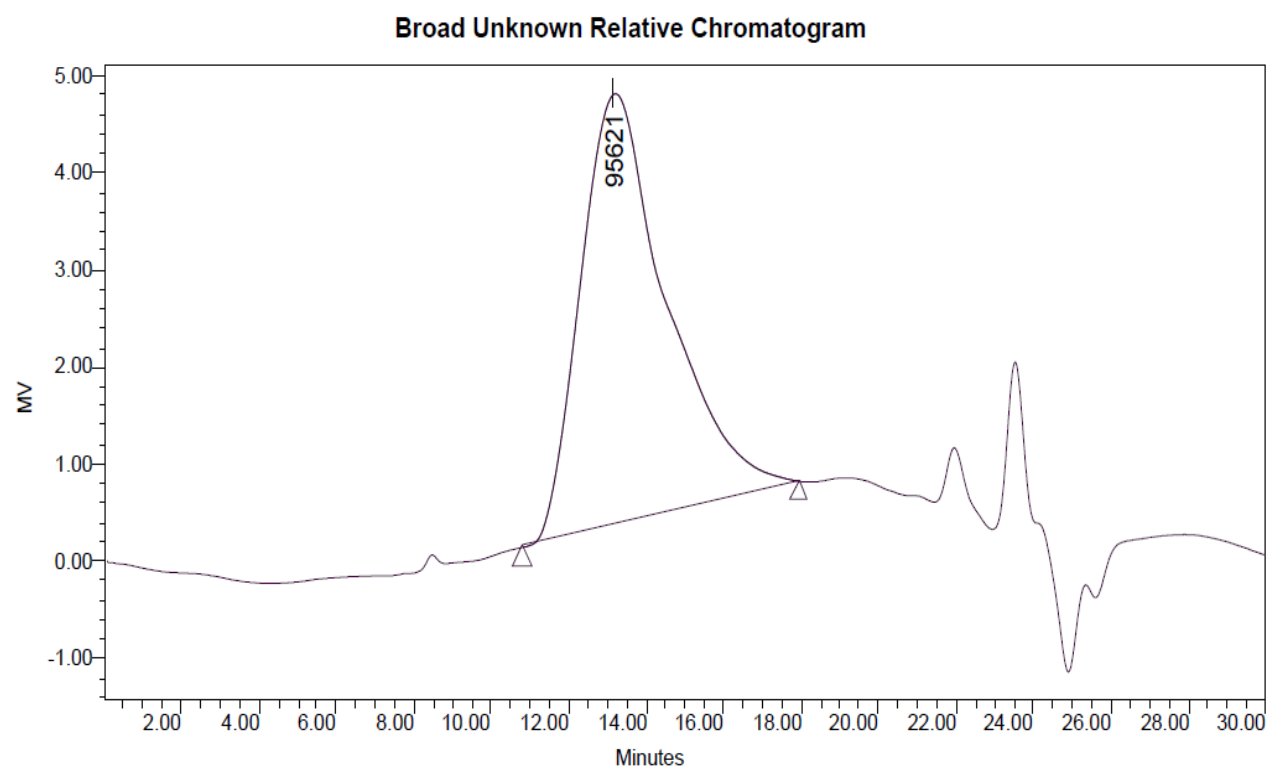

Broad Unknown Relative Peak Table

\begin{tabular}{|c|c|r|r|r|r|r|r|r|r|}
\hline & $\begin{array}{c}\text { Distribution } \\
\text { Name }\end{array}$ & $\begin{array}{c}\mathrm{Mn} \\
\text { (Daltons) }\end{array}$ & $\begin{array}{c}\mathrm{Mw} \\
\text { (Daltons) }\end{array}$ & $\begin{array}{c}\mathrm{MP} \\
\text { (Daltons ) }\end{array}$ & $\begin{array}{c}\mathrm{Mz} \\
\text { (Daltons) }\end{array}$ & $\begin{array}{c}\mathrm{Mz}+1 \\
\text { (Daltons) }\end{array}$ & Polydispersity & $\mathrm{Mz} / \mathrm{Mw}$ & $\mathrm{Mz}+1 / \mathrm{Mw}$ \\
\hline 1 & & 49869 & 103617 & 95621 & 186278 & 286555 & 2.077763 & 1.797755 & 2.765531 \\
\hline
\end{tabular}

Figure S4.11 GPC result for polymer P26.

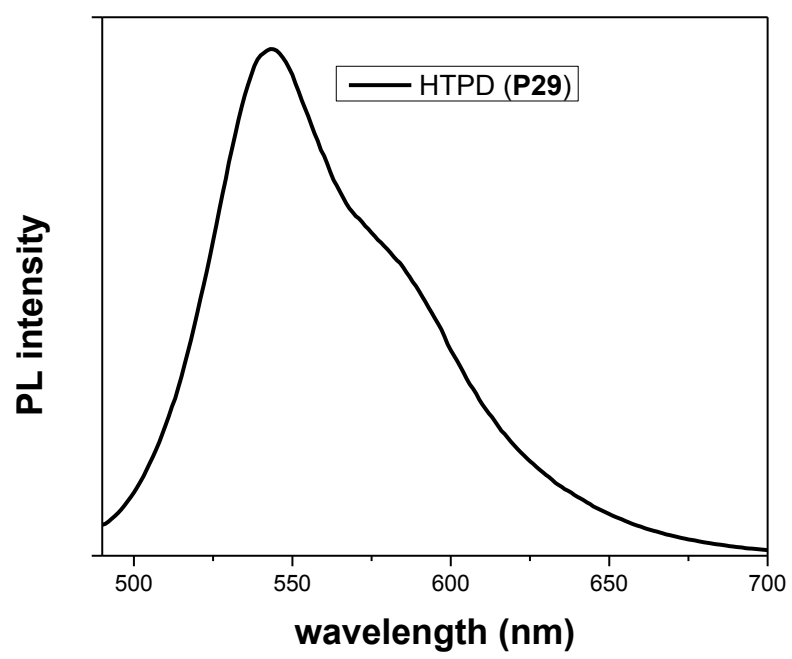

Figure S4.12 Emission spectrum of polymer HTPD (P26), excitation at $470 \mathrm{~nm}$. 


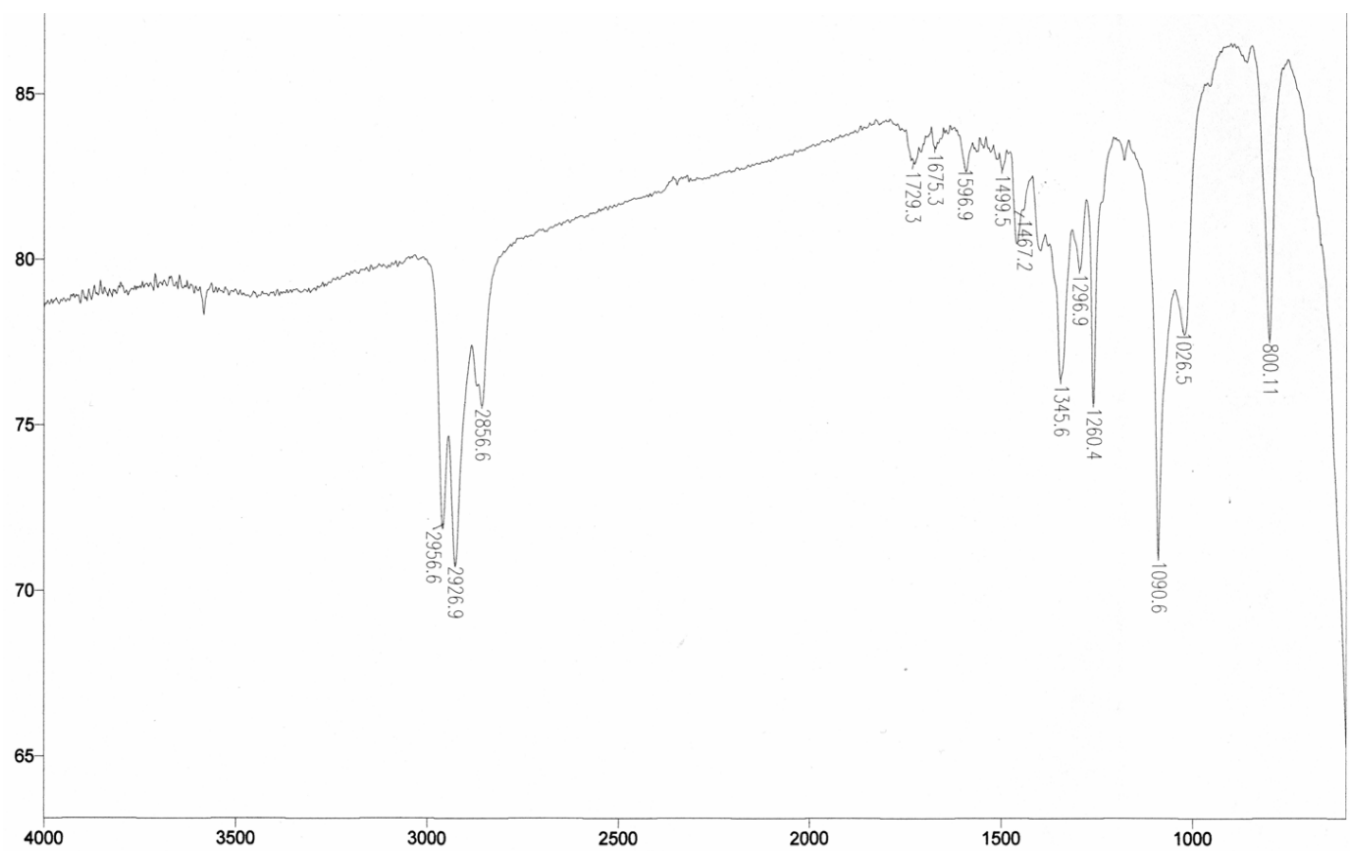

Figure S4.13 IR spectrum of polymer $\mathbf{P 2 7}$ (on $\mathrm{NaCl}$ plate).
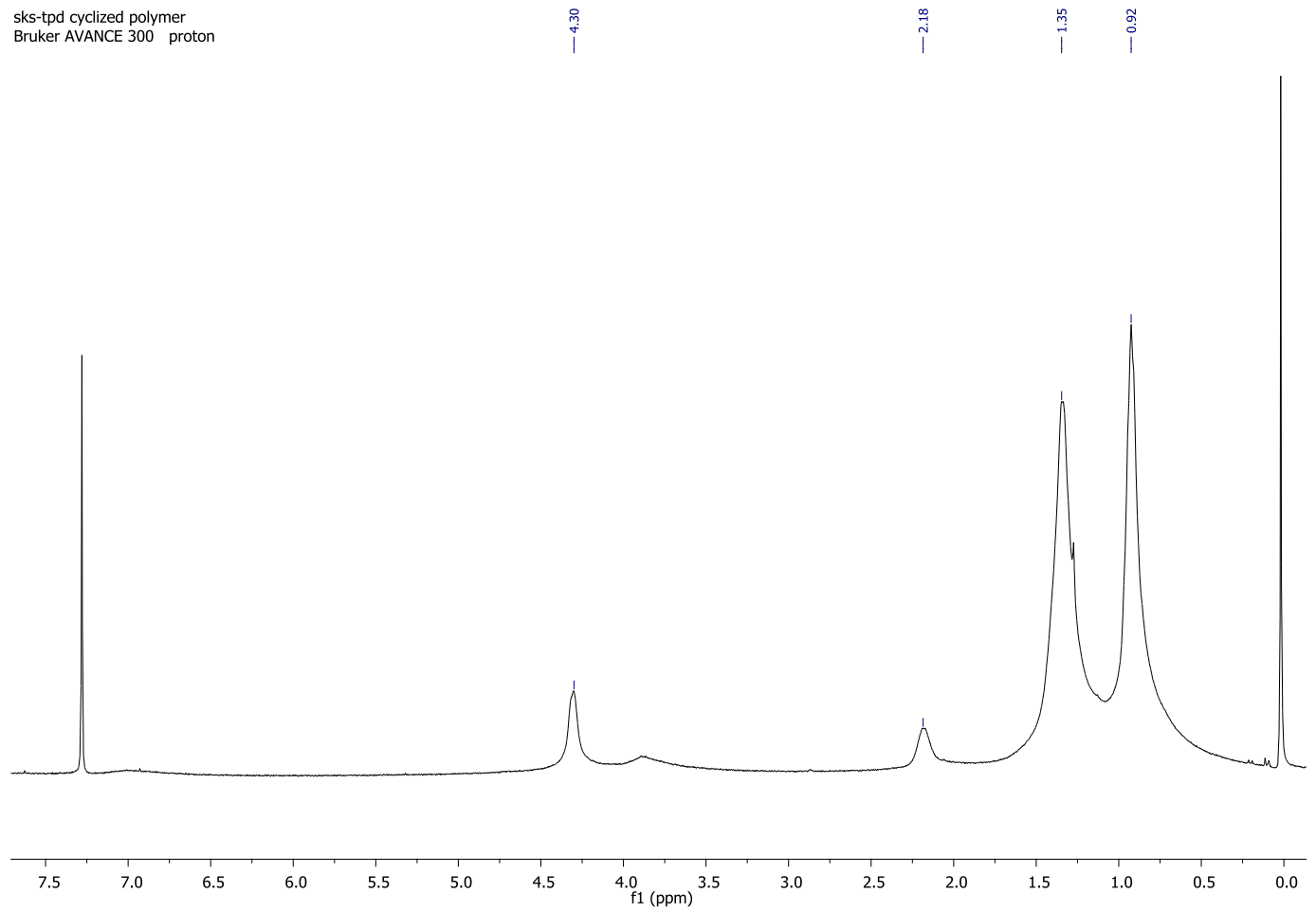

Figure S4.14 ${ }^{1} \mathrm{H}$ NMR spectrum $\left(300 \mathrm{MHz}, \mathrm{CDCl}_{3}\right)$ of $\mathbf{P 2 7}$. 


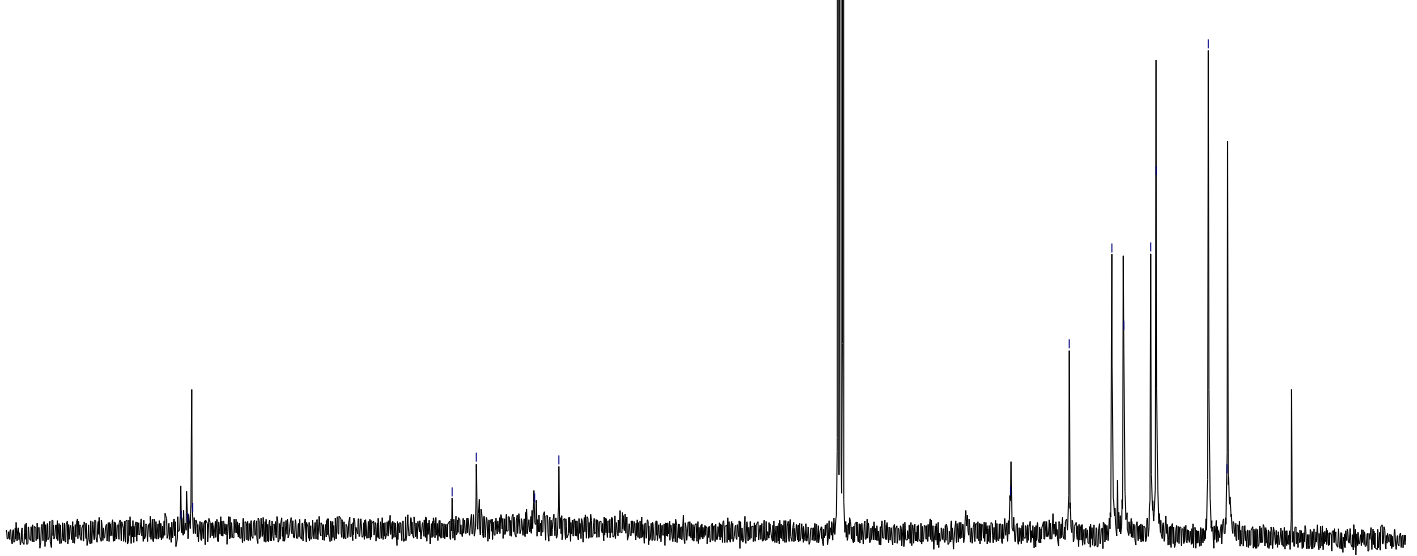

$\begin{array}{lllllllllll}210 & 200 & 190 & 180 & 170 & 160 & 150 & 140 & 130 & 120 & 110 \underset{\mathrm{f} 1(\mathrm{ppm})}{100} 90\end{array}$

Figure $\mathrm{S} 4.15{ }^{13} \mathrm{C}$ NMR spectrum $\left(75 \mathrm{MHz}, \mathrm{CDCl}_{3}\right.$ ) of $\mathbf{P 2 7}$.

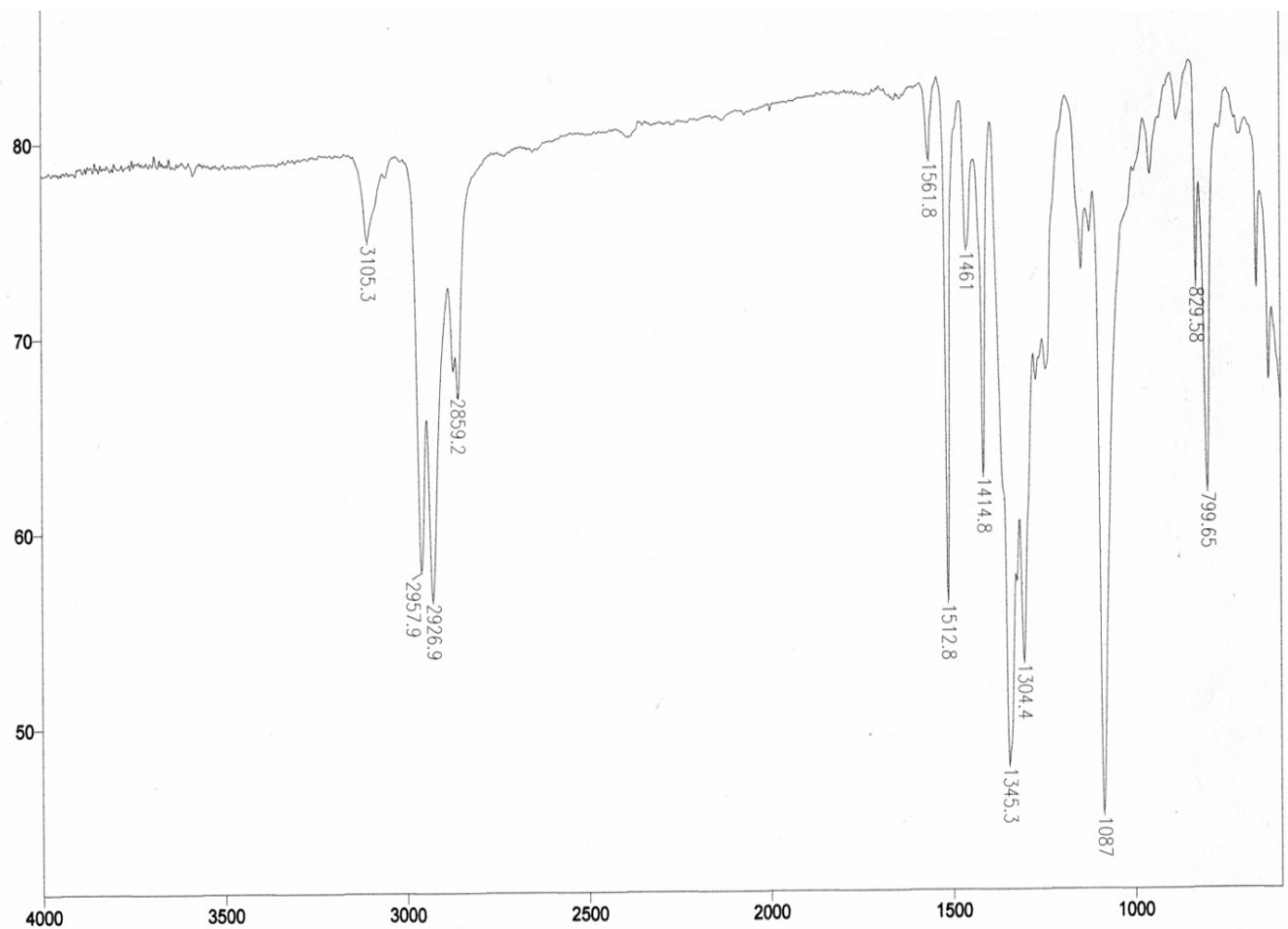

Figure S4.16 IR spectrum of compound $\mathbf{1 1}$ (on $\mathrm{NaCl}$ plate). 


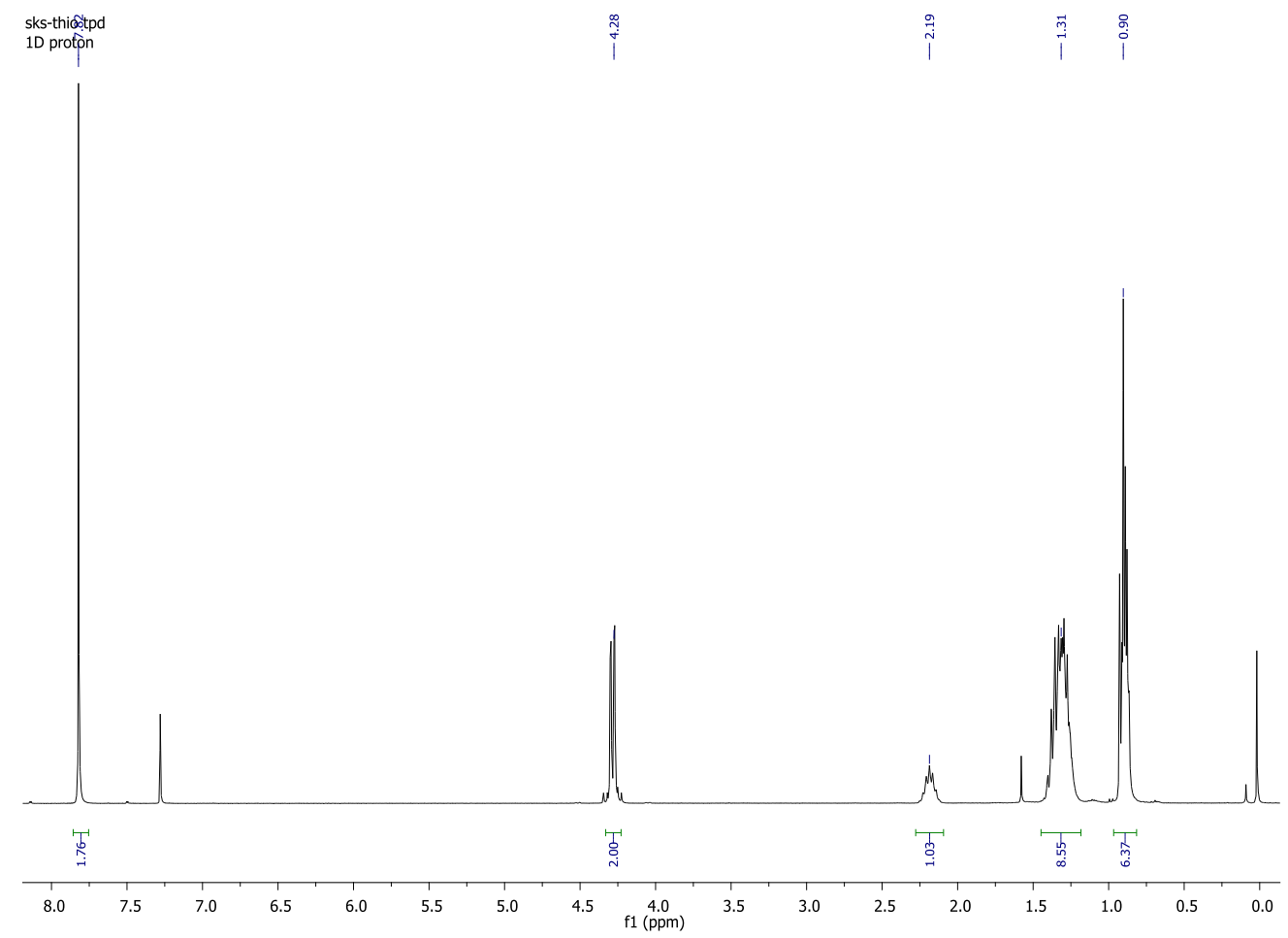

Figure $\mathrm{S} 4.17{ }^{1} \mathrm{H}$ NMR spectrum $\left(300 \mathrm{MHz}, \mathrm{CDCl}_{3}\right.$ ) of compound 11.

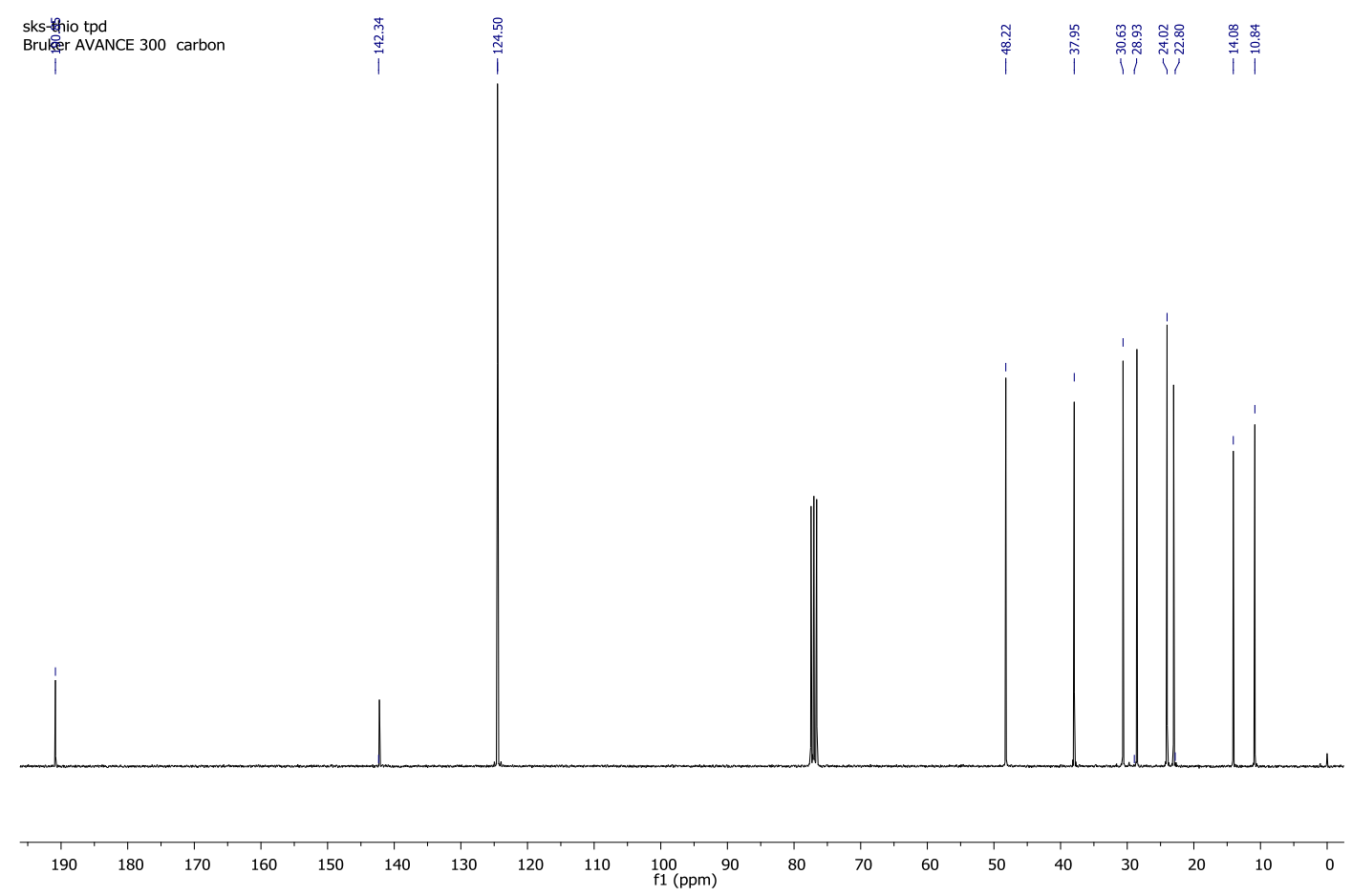

Figure $\mathrm{S} 4.18{ }^{13} \mathrm{C}$ NMR spectrum $\left(300 \mathrm{MHz}, \mathrm{CDCl}_{3}\right)$ of compound 11 . 


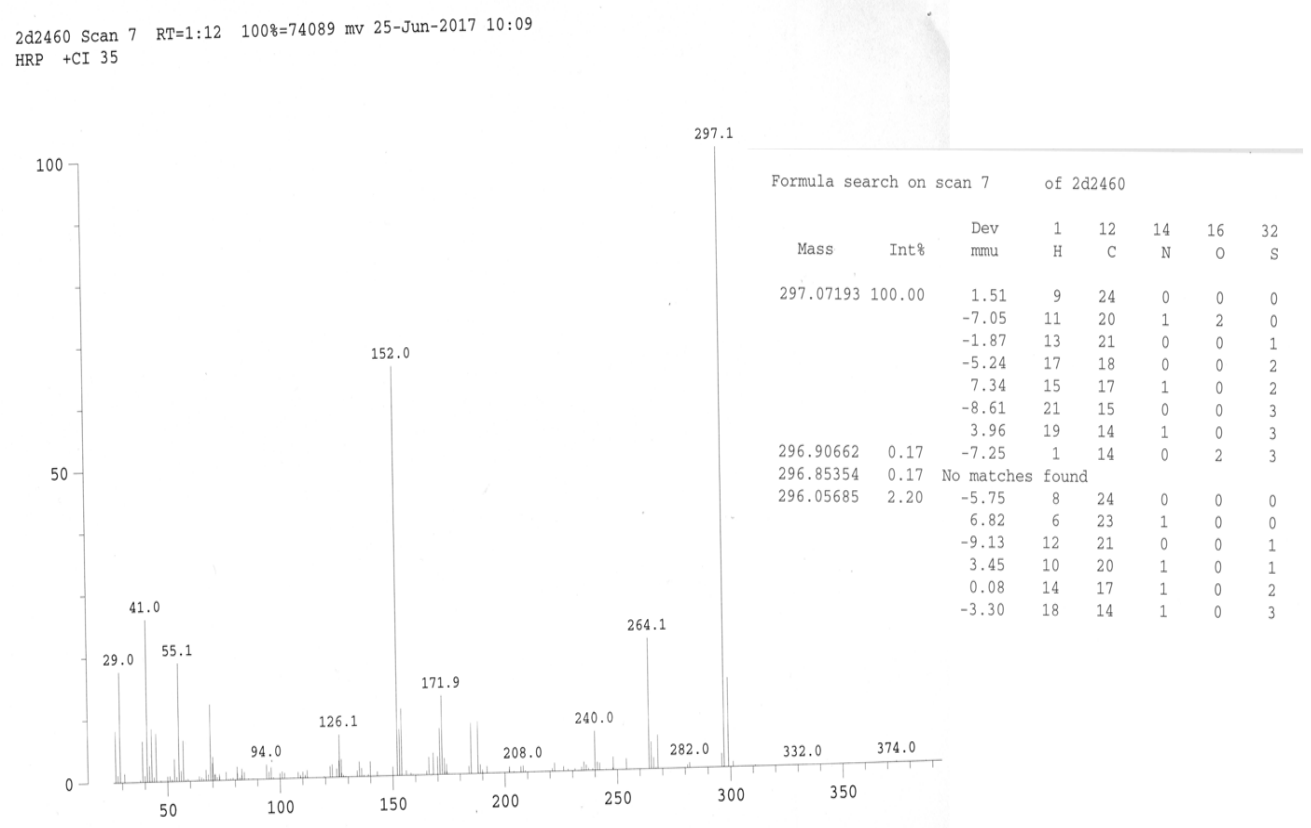

Figure S4.19 Mass spectrum of compound 11.

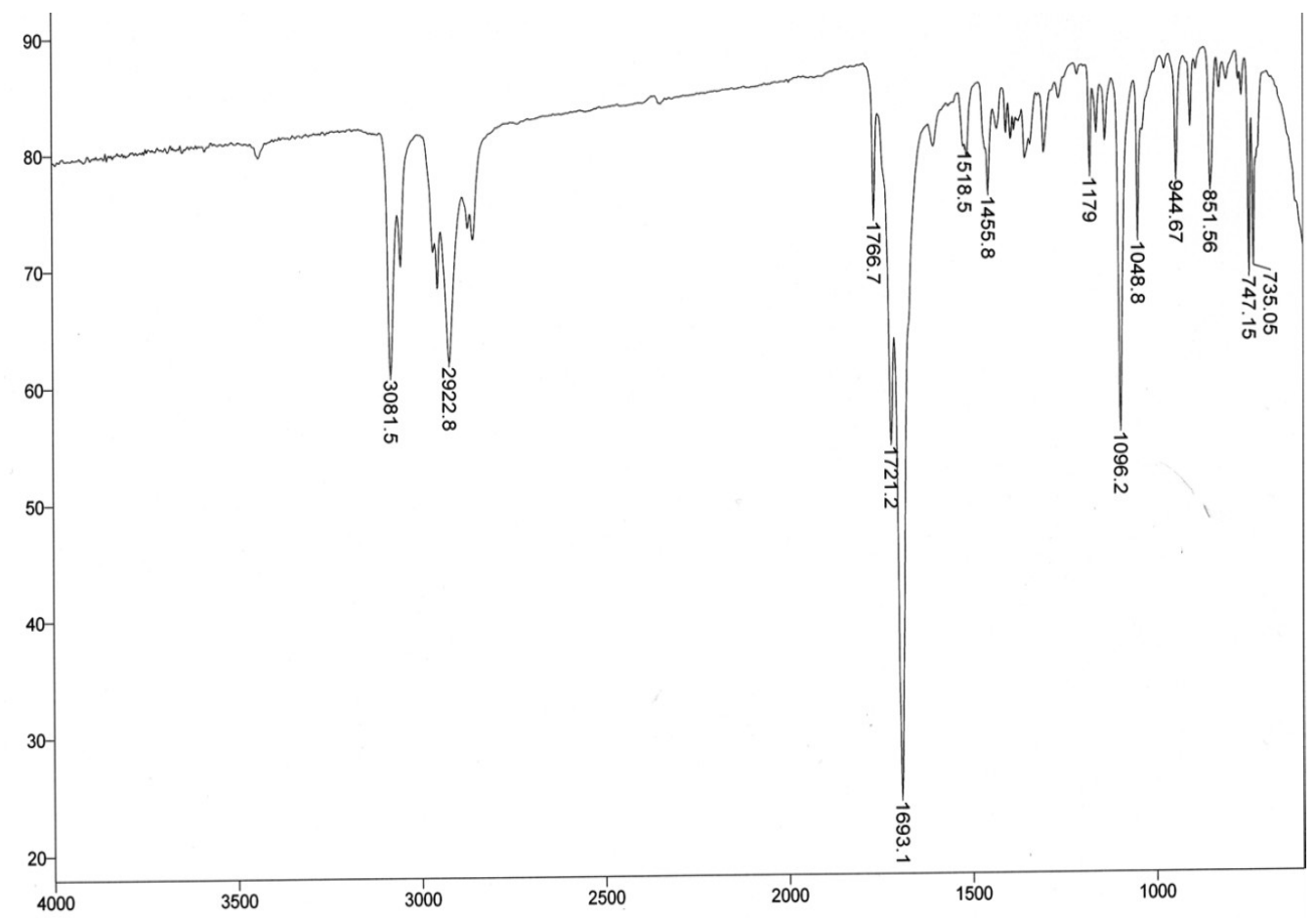

Figure S4.20 IR spectrum of compound $\mathbf{1 2}$ on $\mathrm{NaCl}$ plate. 


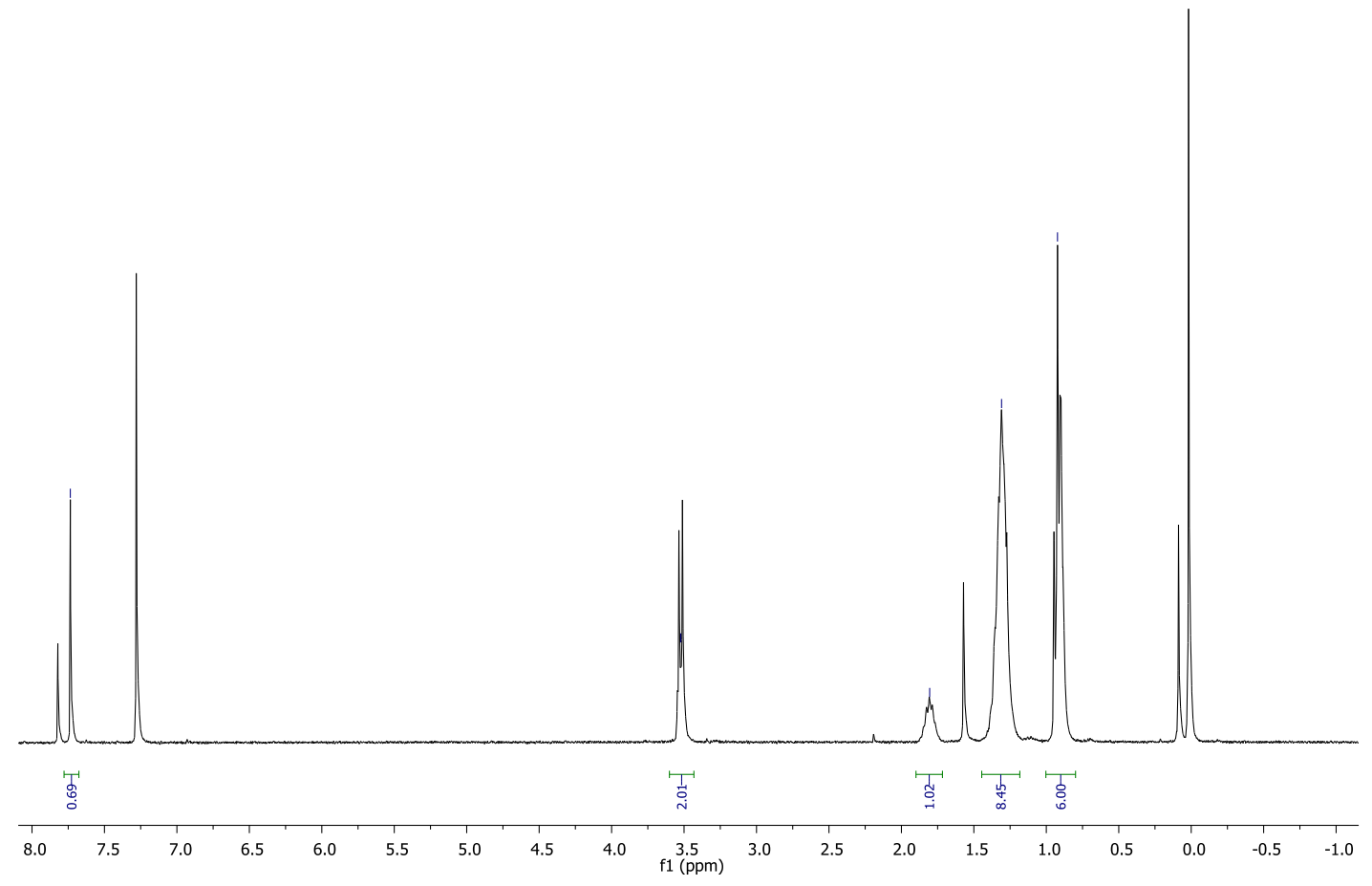

Figure S21 ${ }^{1} \mathrm{H}$ NMR spectrum $\left(300 \mathrm{MHz}, \mathrm{CDCl}_{3}\right)$ of compound 12 .

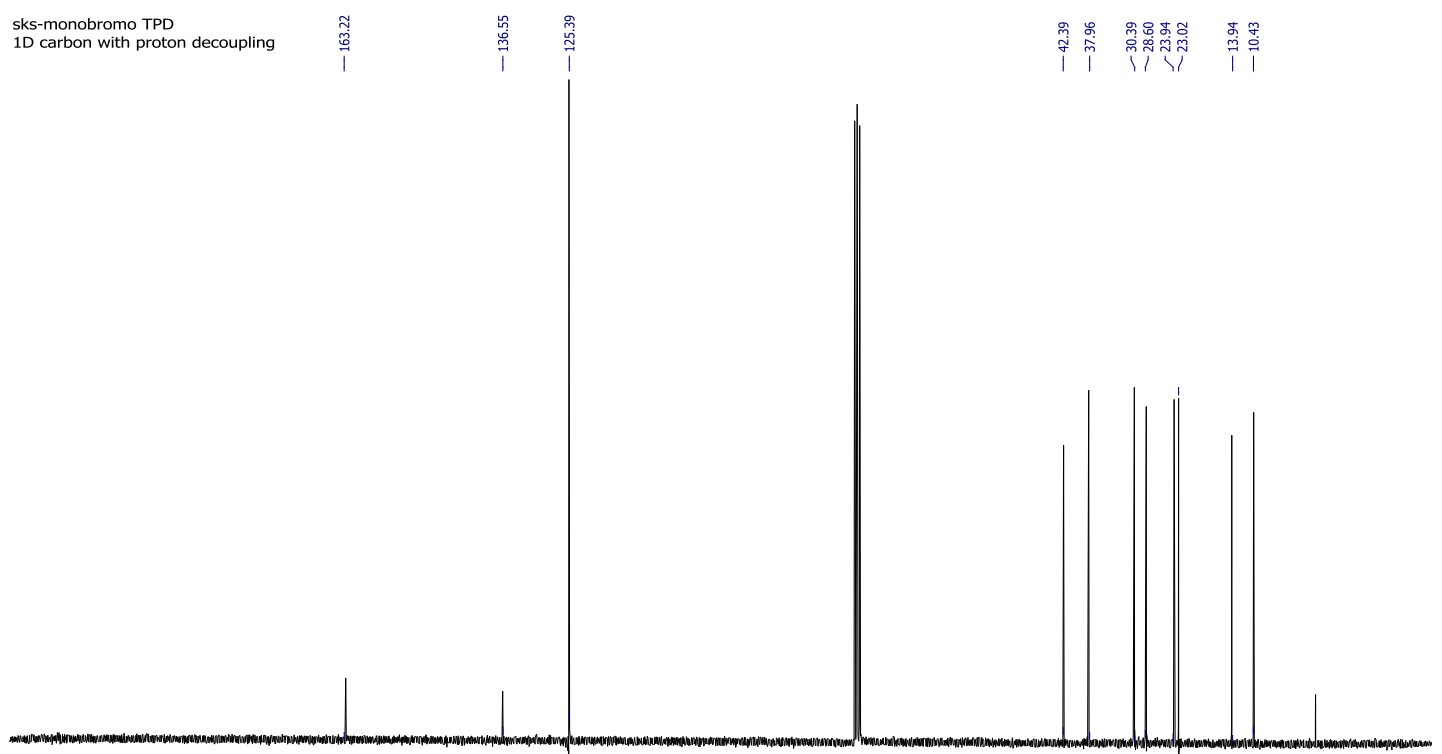

$\begin{array}{llllllllllllllllllllllll}1 & 210 & 200 & 190 & 180 & 170 & 160 & 150 & 140 & 130 & 120 & 110 & 100 & 100 & 80 & 70 & 60 & 50 & 40 & 30 & 20 & 10 & 0 & -10\end{array}$

Figure $\mathrm{S} 22{ }^{13} \mathrm{C}$ NMR spectrum $\left(75 \mathrm{MHz}, \mathrm{CDCl}_{3}\right)$ of compound 12. 


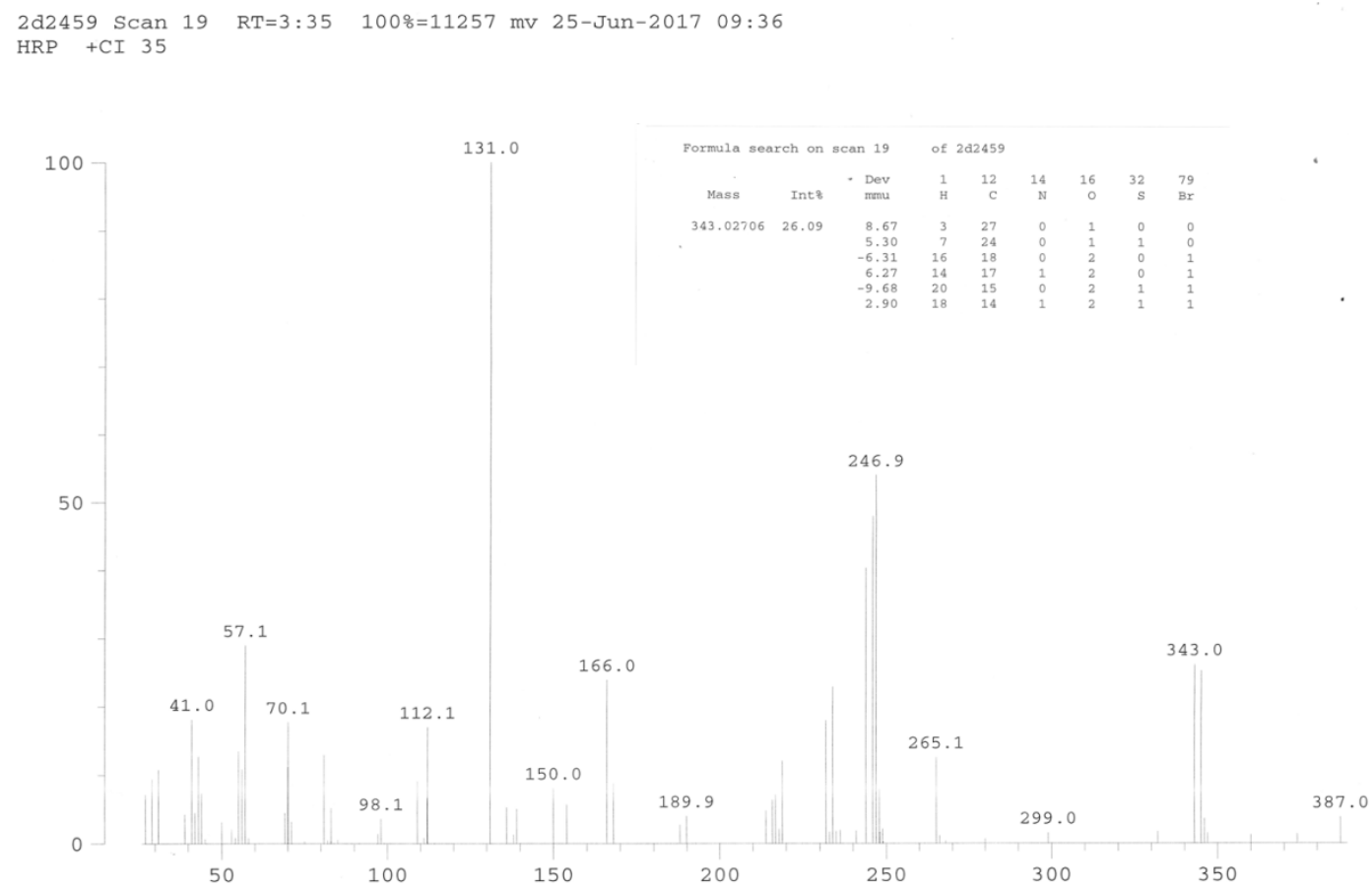

Figure S23 Mass spectrum of compound 12.

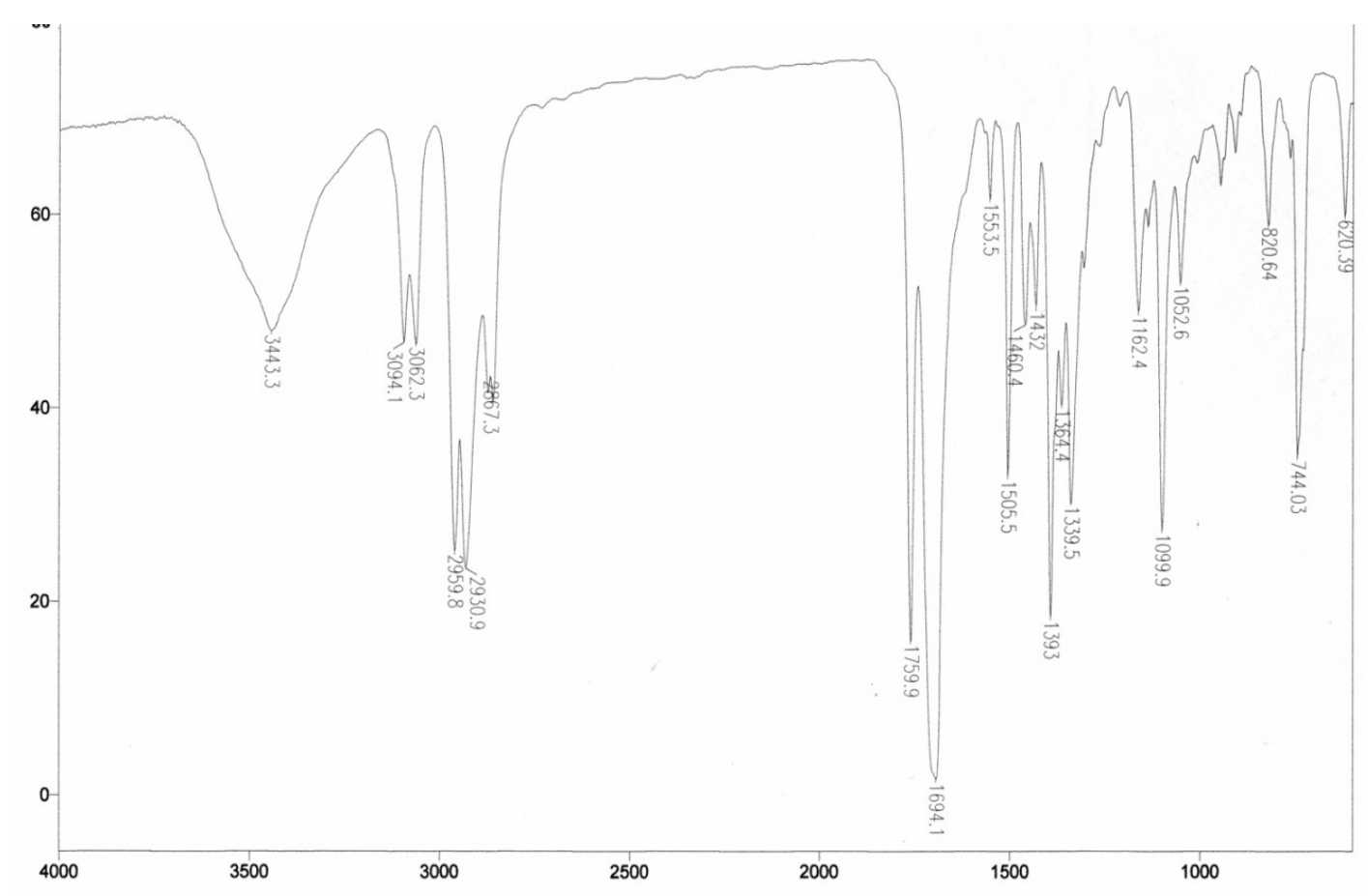

Figure S4.24 IR spectrum of compound $\mathbf{1 3}$ (in $\mathrm{KBr}$ pellet) 


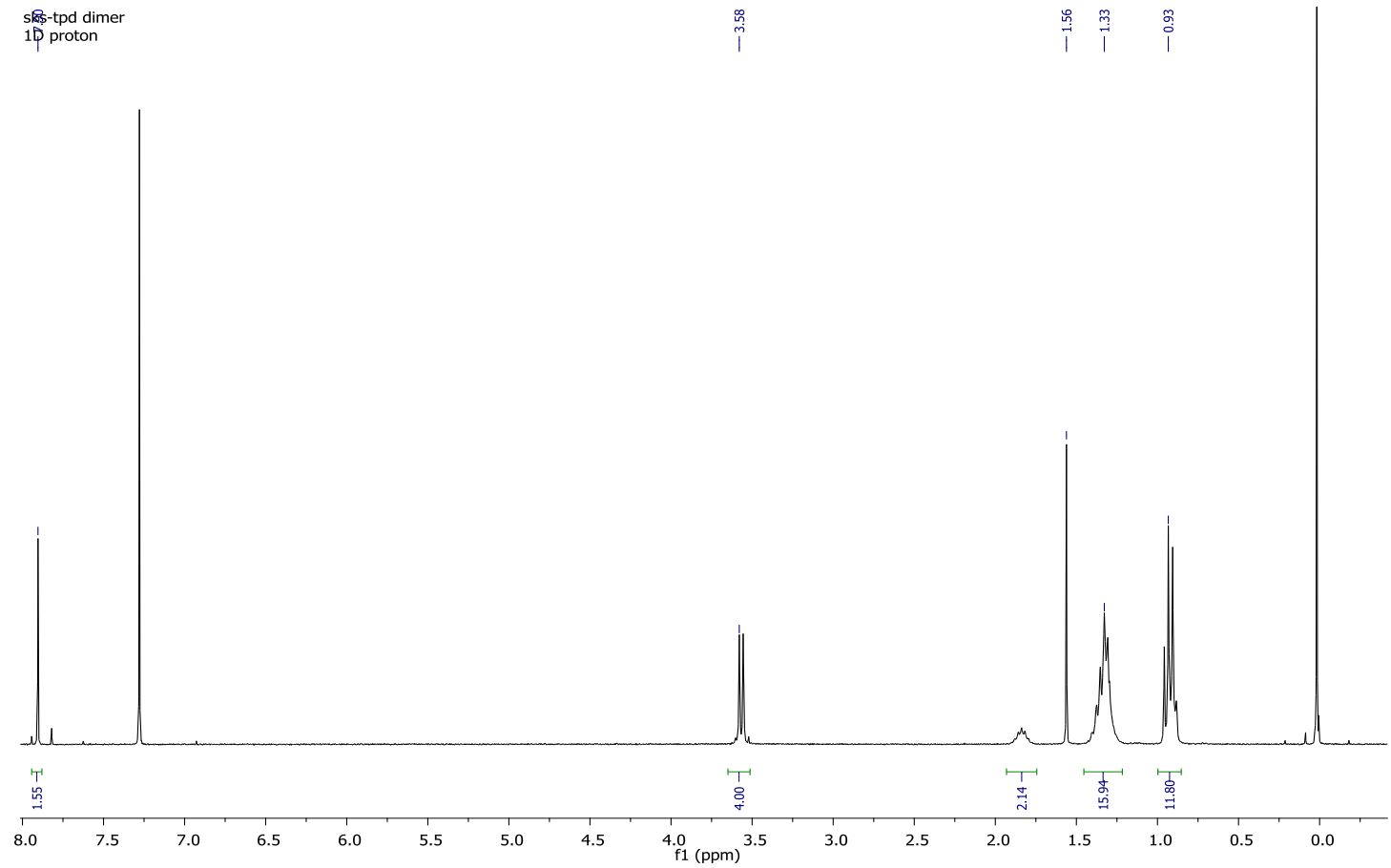

Figure $\mathrm{S} 4.25{ }^{1} \mathrm{H}$ NMR spectrum $\left(300 \mathrm{MHz}, \mathrm{CDCl}_{3}\right)$ of compound 13.
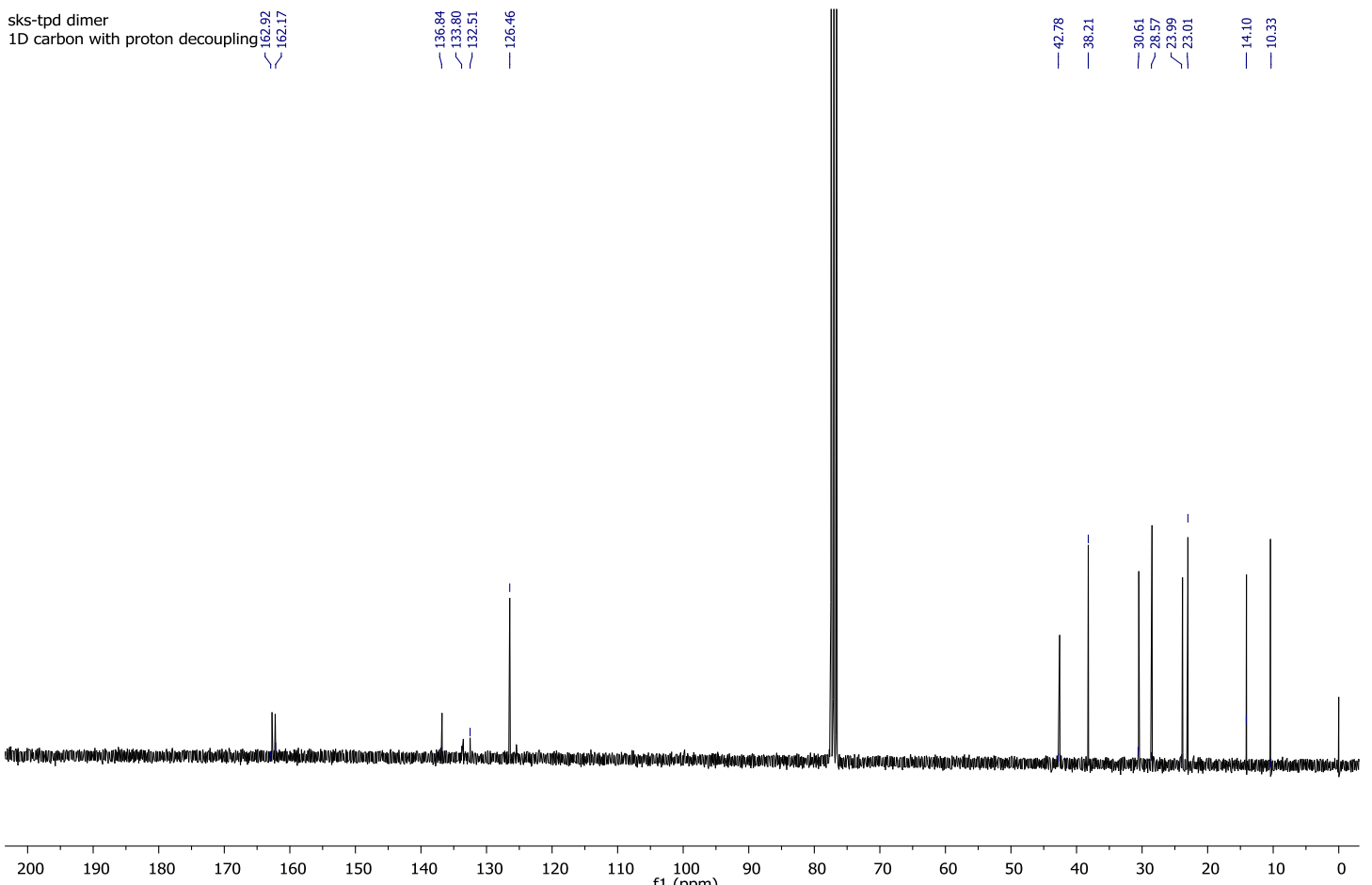

Figure S $4.26{ }^{13} \mathrm{C}$ NMR spectrum $\left(75 \mathrm{MHz}, \mathrm{CDCl}_{3}\right.$ ) of compound 13. 
2d0068 Scan $8 \quad$ RT $=1: 27 \quad 100 \%=83887 \mathrm{mv} 23-$ Apr $-2015 \quad 10: 52$

HRP +EI ac3-14

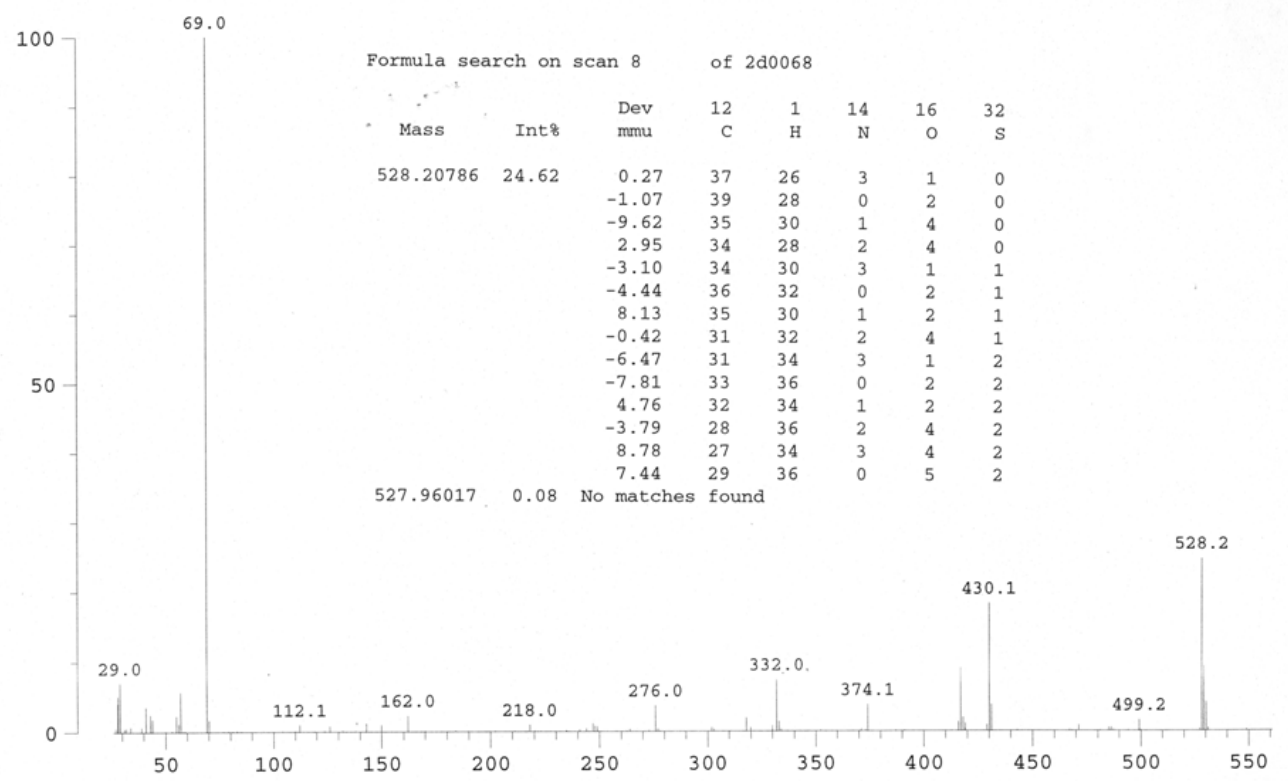

Figure S4.27 Mass spectrum of compound 13.

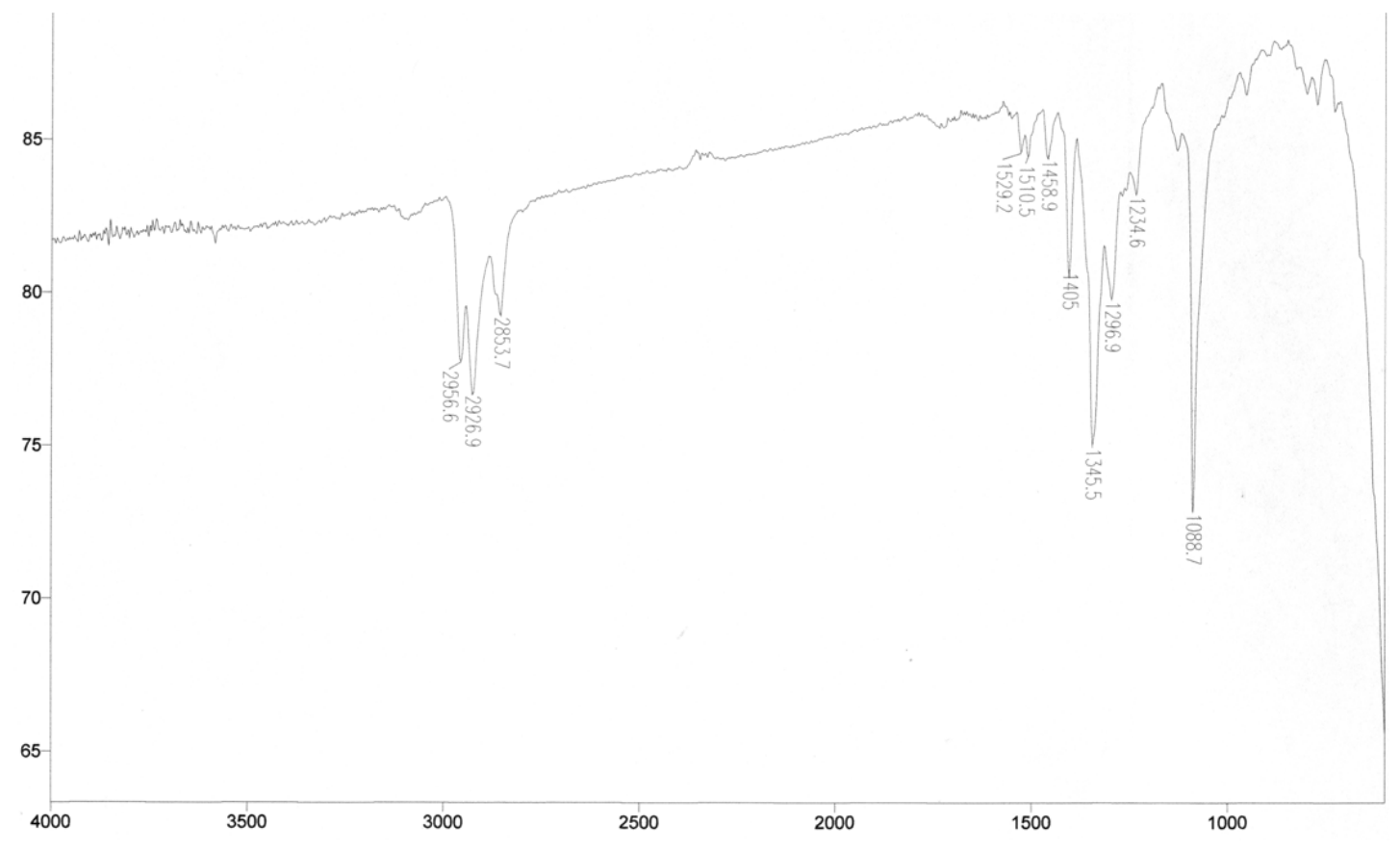

Figure S4.28 IR spectrum of compound $\mathbf{1 4}$ (on $\mathrm{NaCl}$ plate). 


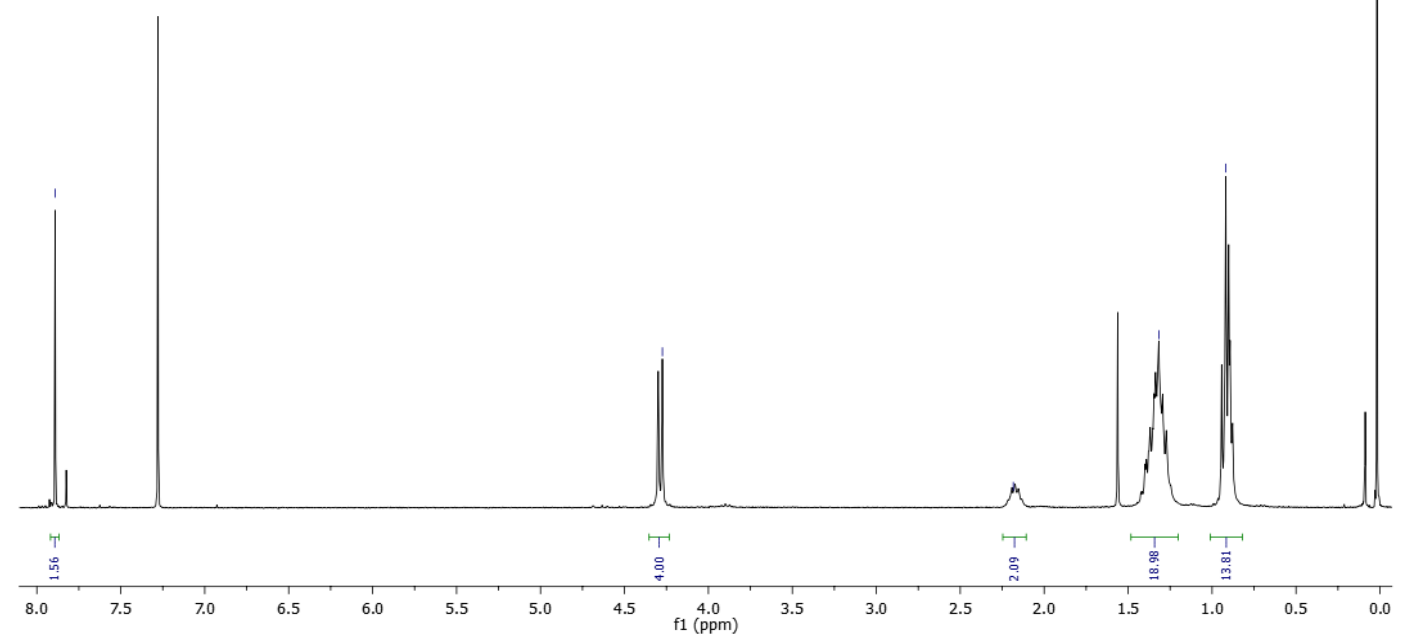

Figure S4.29 ${ }^{1} \mathrm{H}$ NMR spectrum $\left(300 \mathrm{MHz}, \mathrm{CDCl}_{3}\right)$ of compound 14.

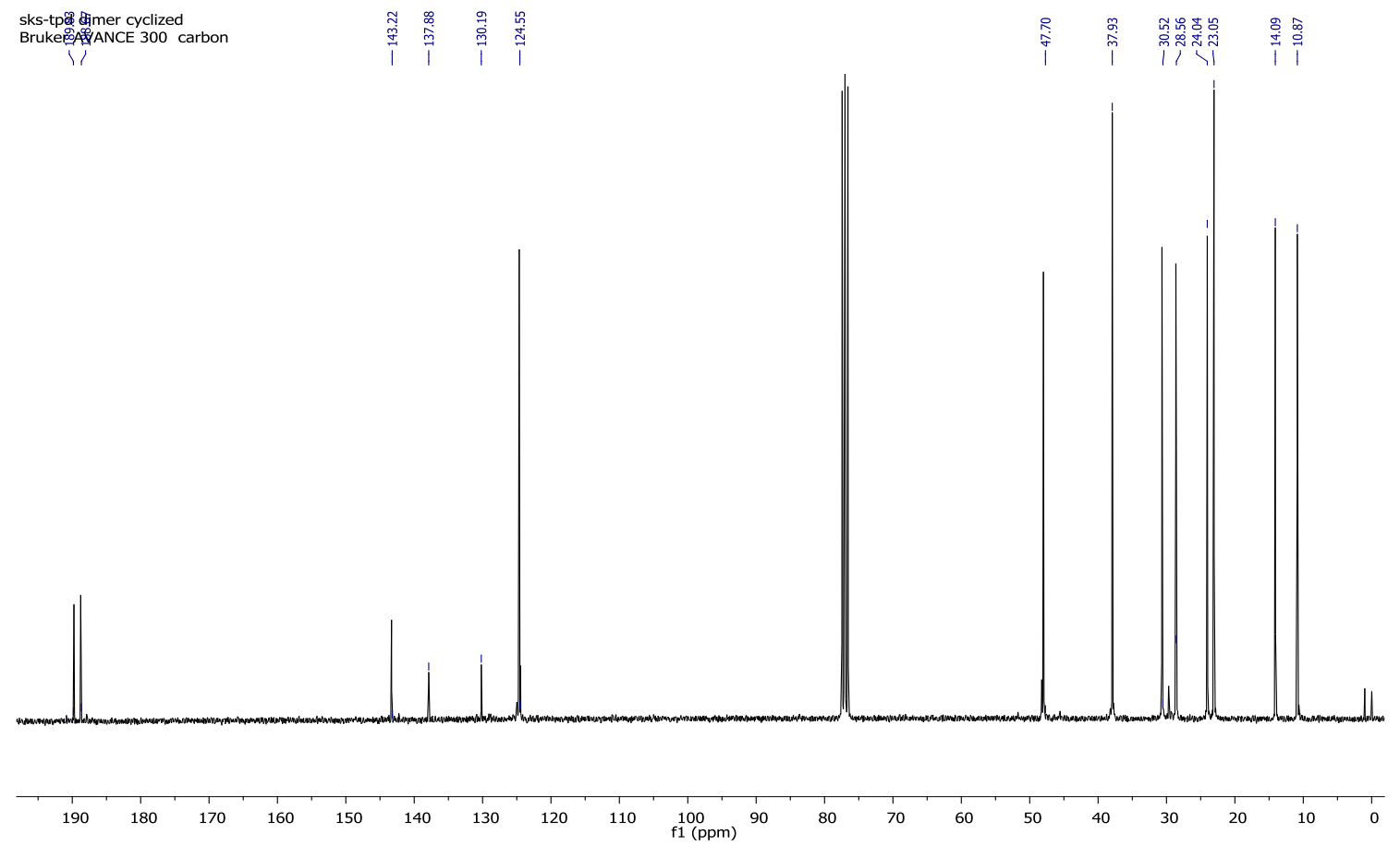

Figure $\mathrm{S} 4.30{ }^{13} \mathrm{C}$ NMR spectrum $\left(75 \mathrm{MHz}, \mathrm{CDCl}_{3}\right)$ of compound 14. 


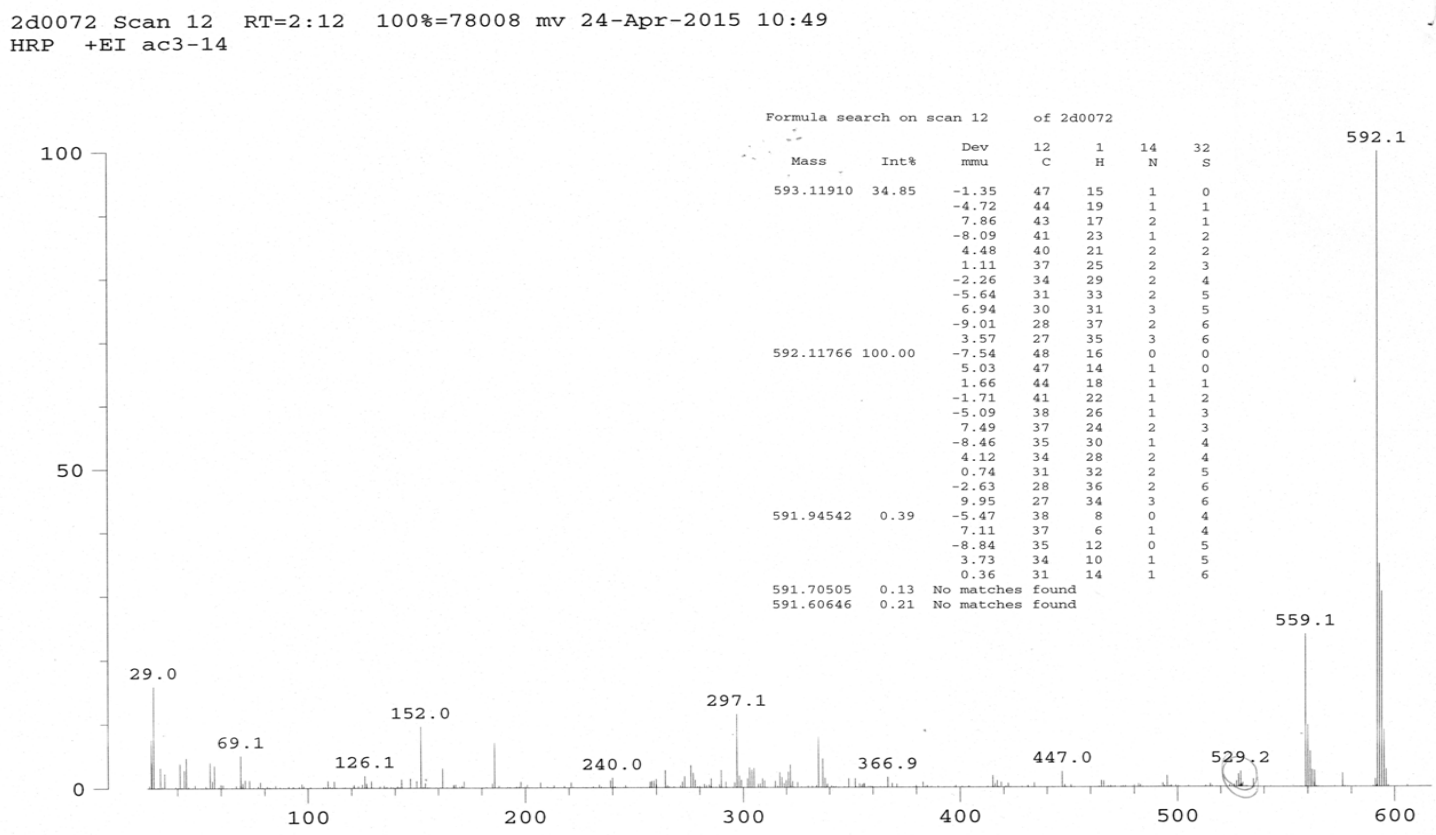

Figure S4.31 Mass spectrum of compound 14. 


\section{Appendix D: Supporting information for chapter 5}

\section{Highly Sensitive Dual-Mode Fluorescence Detection of Lead Ion in Water Using}

\section{Aggregation-Induced Emissive Polymers}

Dear Sukanta Kumar Saha,

We hereby grant permission for the requested use expected that due credit is given to the original source.

If material appears within our work with credit to another source, authorisation from that source must be obtained.

Credit must include the following components:

Journals: Author(s) Name(s): Title of the Article. Name of the Journal. Publication year. Volume. Page(s). Copyright Wiley-VCH Verlag GmbH \& Co. KGaA. Reproduced with permission.

If you also wish to publish your thesis in electronic format, you may use the article according to the Copyright transfer agreement:

\section{Final Published Version.}

Wiley-VCH hereby licenses back to the Contributor the following rights with respect to the final published version of the Contribution:

a. $[\ldots]$

b. Re-use in other publications. The right to re-use the final Contribution or parts thereof for any publication authored or edited by the Contributor (excluding journal articles) where such re-used material constitutes less than half of the total material in such publication. In such case, any modifications should be accurately noted.

Kind regards

Bettina Loycke

Senior Rights Manager

Rights \& Licenses

Wiley-VCH Verlag GmbH \& Co. KGaA

Boschstraße 12

69469 Weinheim,Germany

www.wiley-vch.de

$\mathrm{T}+(49) 6201606-280$

$\mathrm{F}+(49) 6201$ 606-332

rightsDE@wiley.com 


\section{Experimental Section}

Materials. Fluorene, 4-bromobenzophenone, 9,9-dioctylfluorene-2,7-diboronic acid bis(1,3-propanediol) ester, titanium(IV) chloride, zinc, tetrakis(triphenylphosphine)palladium(0), tetrabutylammonium bromide (TBAB), lead acetate trihydrate, copper(II) chloride, nickel bromide, cobalt chloride hexahydrate, calcium chloride, cadmium chloride, potassium bromide, ferric chloride, zinc sulfate, manganese chloride, magnesium chloride, lithium chloride, mercury chloride and all other reagents were purchased from Aldrich Chemicals Co. 9,9-Dioctylfluorene-2,7-diboronic acid bis(1,3propanediol) ester was recrystallized in hexane before using. Toluene was dried and distilled over $\mathrm{CaH}_{2}$ under an atmosphere of dry argon. Tetrahydofuran (THF) was distilled over sodium under an atmosphere of dry argon. The water used in this work was purified using Millipore ${ }^{\mathrm{TM}}$ Milli- $\mathrm{Q}^{\mathrm{TM}}$ Advantage A10 water purification system from Fisher Scientific. Column chromatography was done using silica gel (Silicycle Chemical Division, 70-230 mesh) as the stationary phase.

Measurements. ${ }^{1} \mathrm{H}$ and ${ }^{13} \mathrm{C}$ NMR spectra were recorded on a Bruker Avance Digital 300 and $400 \mathrm{MHz}$. Resonances were quoted on the $\delta$ scale relative to tetramethylsilane (TMS, $\delta=0$ ) as an internal standard. Infrared measurements were performed on a Varian 1000 FT-IR Scirinitar series spectrophotometer. Mass spectra were measured with a Micromass Quattro LC ESI (EI). The UV-Vis spectra were recorded on a Perkin-Elmer Lambda 900 UV-Vis-NIR spectrometer at room temperature. The fluorescence spectra of all samples were measured in a quartz cuvette with a path length $1 \mathrm{~cm}$ in Shimadzu RF- 
1501 spectroflurometer. The fluorescence spectra were recorded at room temperature at the excitation wavelengths of $375 \mathrm{~nm}$ for polymers P6-P9.

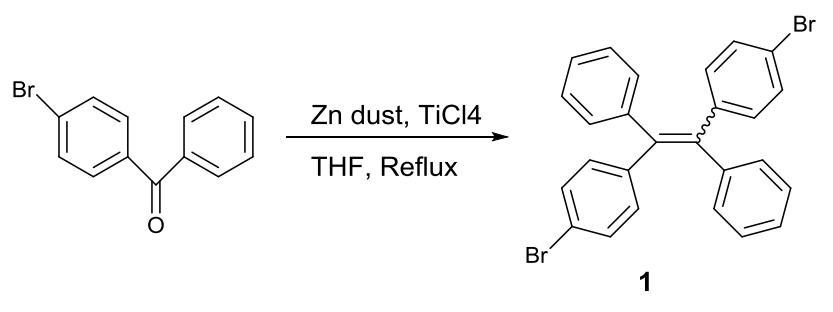

Scheme S5.1 Synthesis of compound 1.

\section{Synthesis}

1,2-Bis(4-bromophenyl)-1,2-diphenylethene (1). In a flame-dried three-neck flask under argon atmosphere, an ice-cooled $\left(-5^{\circ} \mathrm{C}\right)$ suspension of zinc powder $(12.50 \mathrm{~g}$, $191.19 \mathrm{mmol})$ in THF $(240 \mathrm{~mL})$ was prepared and titanium tetrachloride $(20.00 \mathrm{~mL}$, $181.81 \mathrm{mmol}$ ) was added to the suspension. The resulting mixture was refluxed for $4 \mathrm{~h}$. After cooling to the room temperature, a solution of 4-bromobenzophenone (10.00 g, $38.30 \mathrm{mmol})$ in THF $(50 \mathrm{~mL})$ was s added and the mixture was then refluxed overnight. After cooling to room temperature, the mixture was diluted with saturated aqueous sodium hydrogen carbonate solution and dichloromethane and the solution was stirred for $5 \mathrm{~h}$. The mixture was filtered through a Celite pad, and the filtrate was separated into organic and aqueous layers. The aqueous layer was extracted twice with dichloromethane and the combined organic layer was dried over MgSO4. The solvent was removed under the reduced pressure. The resulting white crude product was purified by silica gel column chromatography using hexane as eluting solvent. Compound $\mathbf{1}$ was obtained as white powder ( $8.50 \mathrm{~g}, 90.6 \%$ yield) after removing the solvent. Compound 1 was further purified by multiple recrystalization in ehanol:toluene (3:2) and hexane:ethyl acetate 
(8:2) and obtained as white crystal. ${ }^{1} \mathrm{H}$ NMR $\left(300 \mathrm{MHz}, \mathrm{CDCl}_{3}, \mathrm{ppm}\right): \delta 7.26(4 \mathrm{H}, \mathrm{m})$, $7.15(6 \mathrm{H}, \mathrm{m}), 7.01(4 \mathrm{H}, \mathrm{m}), 6.90(4 \mathrm{H}, \mathrm{m}) ;{ }^{13} \mathrm{C} \mathrm{NMR}\left(100 \mathrm{MHz}, \mathrm{CDCl}_{3}\right): 142.93,142.83$, $142.39,142.29,140.31,132.87,131.21,131.11,130.91,128.02,127.82,126.95,126.83$, 120.80, 120.66; EI-MS calculated for $\left[\mathrm{M}^{+}\right]$490.23, found 490.0. IR (KBr Pellet, $\mathrm{cm}^{-1}$ ): $1640\left(v_{\mathrm{C}=\mathrm{C}}\right)$.

Polymer P1. To a mixture of compound 1 (0.300 g, $0.612 \mathrm{mmol})$ and 9,9dioctylfluorene-2,7-diboronic acid bis(1,3-propanediol) ester $(0.342 \mathrm{~g}, 0.612 \mathrm{mmol})$ in toluene $(4.0 \mathrm{~mL})$ was added $2.5 \mathrm{~mL}$ of $2 \mathrm{M}$ aqueous solution of $\mathrm{K}_{2} \mathrm{CO}_{3}$. After degassed and purged with argon for $30 \mathrm{~min}, \mathrm{Pd}\left(\mathrm{PPh}_{3}\right)_{4}(0.035 \mathrm{~g}, 0.035 \mathrm{mmol})$ was added. The mixture was heated at $90{ }^{\circ} \mathrm{C}$ for $72 \mathrm{~h}$ under argon. The solution was then poured into methanol with vigorous stirring. After stirring $2 \mathrm{~h}$ a greenish precipitated as polymer P1 was collected by filtration. The polymer was purified by being dissolved in $\mathrm{CHCl}_{3}$ and reprecipitated into methanol and then was further purified by a Soxhlet extraction in acetone for $24 \mathrm{~h}$. The resultant polymer was dissolved in $\mathrm{CHCl}_{3}$ and precipitated with methanol. After drying under vacuum at $60{ }^{\circ} \mathrm{C}$ overnight, the polymers were obtained $\left(0.37 \mathrm{~g}, 84.1 \%\right.$ yield) as greenish powder. ${ }^{1} \mathrm{H} \mathrm{NMR}\left(300 \mathrm{MHz}, \mathrm{CDCl}_{3}, \mathrm{ppm}\right): \delta 7.71-7.16$ (aromatic $\mathrm{CH}$ ), 2.0 ( $\alpha-\mathrm{CH}_{2}$ of octyl group at 9-position of dioctylfluorene unit), 1.05 $\left(\mathrm{CH}_{2}\right), 0.74\left(\mathrm{CH}_{3}\right) . \mathrm{IR}\left(\mathrm{NaCl}\right.$ plate, $\left.\mathrm{cm}^{-1}\right): 3026,2926,2854,1598,1500$

Polymer P2. To a mixture of compound 1 (0.208 g, $0.425 \mathrm{mmol})$, compound 2 (0.017 g, $0.022 \mathrm{mmol})$ and 9,9-dioctylfluorene-2,7-diboronic acid bis(1,3-propanediol) ester (0.250 $\mathrm{g}, 0.448 \mathrm{mmol})$ in toluene $(5.0 \mathrm{~mL})$ was added $2.5 \mathrm{~mL}$ of $2 \mathrm{M}$ aqueous solution of 
$\mathrm{K}_{2} \mathrm{CO}_{3}$. After degassed and purged with argon for $30 \mathrm{~min}, \mathrm{Pd}\left(\mathrm{PPh}_{3}\right)_{4}(0.026 \mathrm{~g}, 0.022$ $\mathrm{mmol})$ and TBAB $(0.003 \mathrm{~g}, 0.009 \mathrm{mmol})$ were added. The mixture was heated at $90{ }^{\circ} \mathrm{C}$ for $48 \mathrm{~h}$ under argon. The solution was then poured into methanol with vigorous stirring. After stirring $2 \mathrm{~h}$ a yellow precipitated as polymer $\mathbf{P} 2$ was collected by filtration. The polymer was purified by being dissolved in $\mathrm{CHCl}_{3}$ and re-precipitated into methanol and then was further purified by a Soxhlet extraction in acetone for $24 \mathrm{~h}$. The resultant polymer was dissolved in $\mathrm{CHCl}_{3}$ and precipitated with methanol. After drying under vacuum at $60{ }^{\circ} \mathrm{C}$ for $5 \mathrm{~h}$, the polymers were obtained $(0.300 \mathrm{~g}, 91.0 \%$ yield $)$ as greenishyellow powder. ${ }^{1} \mathrm{H}$ NMR (300 MHz, $\left.\mathrm{CDCl}_{3}, \mathrm{ppm}\right)$ : $\delta$ 7.70-7.16 (aromatic $\left.\mathrm{CH}\right) 4.18\left(\mathrm{NH}_{2}\right.$ and $\mathrm{O}-\mathrm{CH}_{2}-\mathrm{C}=\mathrm{O}$ moiety from compound 2), $3.74\left(\mathrm{O}-\mathrm{CH}_{2}\right.$ moiety from compound 2), $2.02\left(\alpha-\mathrm{CH}_{2}\right.$ of octyl group at 9-position of dioctylfluorene unit), $1.08\left(\mathrm{CH}_{2}\right.$ from dioctylfluorene and $\mathrm{CH}_{3}$ from compound 2), $0.75\left(\mathrm{CH}_{3}\right)$. IR ( $\mathrm{NaCl}$ plate, $\left.\mathrm{cm}^{-1}\right)$ : 3026, 2926, 2853, 1750 (ester $\mathrm{C}=\mathrm{O}$ from compound 2), 1597, 1513

Polymer P3. To a mixture of compound $1(0.270 \mathrm{~g}, 0.551 \mathrm{mmol})$, compound 2 ( $0.045 \mathrm{~g}$, $0.0612 \mathrm{mmol}$ ) and 9,9-dioctylfluorene-2,7-diboronic acid bis(1,3-propanediol) ester $(0.342 \mathrm{~g}, 0.612 \mathrm{mmol})$ in toluene $(5.0 \mathrm{~mL})$ was added $2.5 \mathrm{~mL}$ of $2 \mathrm{M}$ aqueous solution of $\mathrm{K}_{2} \mathrm{CO}_{3}$. After degassed and purged with argon for $30 \mathrm{~min}, \mathrm{Pd}\left(\mathrm{PPh}_{3}\right)_{4}(0.035 \mathrm{~g}, 0.035$ mmol $)$ and TBAB $(0.004 \mathrm{~g}, 0.011 \mathrm{mmol})$ were added. The mixture was heated at $90{ }^{\circ} \mathrm{C}$ for $72 \mathrm{~h}$ under argon. The polymers were obtained $(0.220 \mathrm{~g}, 48.2 \%$ yield $)$ as yellow powder. ${ }^{1} \mathrm{H}$ NMR (300 MHz, $\left.\mathrm{CDCl}_{3}, \mathrm{ppm}\right): \delta$ 7.70-7.16 (aromatic $\left.\mathrm{CH}\right) 4.17\left(\mathrm{NH}_{2}\right.$ and $\mathrm{O}-$ $\mathrm{CH}_{2}-\mathrm{C}=\mathrm{O}$ moiety from compound 2$), 3.76\left(\mathrm{O}-\mathrm{CH}_{2}\right.$ moiety from compound 2$), 2.0(\alpha-$ $\mathrm{CH}_{2}$ of octyl group at 9-position of dioctylfluorene unit), $1.05\left(\mathrm{CH}_{2}\right.$ from dioctylfluorene 
and $\mathrm{CH}_{3}$ from compound 2), $0.74\left(\mathrm{CH}_{3}\right)$. IR $\left(\mathrm{NaCl}\right.$ plate, $\left.\mathrm{cm}^{-1}\right)$ : 3021, 2922, 2850, 1750 (ester $\mathrm{C}=\mathrm{O}$ from compound 2), 1597, 1513.

Polymer P4. To a mixture of compound 1 ( $0.229 \mathrm{~g}, 0.468 \mathrm{mmol})$, compound 2 ( $0.060 \mathrm{~g}$, $0.0826 \mathrm{mmol}$ ) and 9,9-dioctylfluorene-2,7-diboronic acid bis(1,3-propanediol) ester $(0.307 \mathrm{~g}, 0.551 \mathrm{mmol})$ in toluene $(5.0 \mathrm{~mL})$ was added $2.8 \mathrm{~mL}$ of $2 \mathrm{M}$ aqueous solution of $\mathrm{K}_{2} \mathrm{CO}_{3}$. After degassed and purged with argon for $30 \mathrm{~min}, \mathrm{Pd}\left(\mathrm{PPh}_{3}\right)_{4}(0.032 \mathrm{~g}, 0.028$ mmol) and TBAB $(0.004 \mathrm{~g}, 0.011 \mathrm{mmol})$ were added. The mixture was heated at $90{ }^{\circ} \mathrm{C}$ for $24 \mathrm{~h}$ under argon. The polymers were obtained $(0.380 \mathrm{~g}, 93.9 \%$ yield $)$ as yellowishgreen powder. ${ }^{1} \mathrm{H}$ NMR (300 MHz, $\mathrm{CDCl}_{3}, \mathrm{ppm}$ ): $\delta$ 7.70-7.16 (aromatic $\left.\mathrm{CH}\right) 4.16\left(\mathrm{NH}_{2}\right.$ and $\mathrm{O}-\mathrm{CH}_{2}-\mathrm{C}=\mathrm{O}$ moiety from compound 2$), 3.75\left(\mathrm{O}-\mathrm{CH}_{2}\right.$ moiety from compound 2$), 2.0$ $\left(\alpha-\mathrm{CH}_{2}\right.$ of octyl group at 9-position of dioctylfluorene unit), $1.08\left(\mathrm{CH}_{2}\right.$ from dioctylfluorene and $\mathrm{CH}_{3}$ from compound 2), $0.74\left(\mathrm{CH}_{3}\right)$. IR ( $\mathrm{NaCl}$ plate, $\left.\mathrm{cm}^{-1}\right)$ : 3025, 2926, 2853, 1756 (ester C=O from compound 2), 1598, 1516.

Polymer P5. To a mixture of compound 1 (0.176 g, $0.358 \mathrm{mmol})$, compound 2 ( $0.066 \mathrm{~g}$, $0.090 \mathrm{mmol})$ and 9,9-dioctylfluorene-2,7-diboronic acid bis(1,3-propanediol) ester (0.250 g, $0.448 \mathrm{mmol})$ in toluene $(5.0 \mathrm{~mL})$ was added $2.8 \mathrm{~mL}$ of $2 \mathrm{M}$ aqueous solution of $\mathrm{K}_{2} \mathrm{CO}_{3}$. After degassed and purged with argon for $30 \mathrm{~min}, \mathrm{Pd}\left(\mathrm{PPh}_{3}\right)_{4}(0.035 \mathrm{~g}, 0.035$ mmol $)$ and TBAB $(0.003 \mathrm{~g}, 0.009 \mathrm{mmol})$ were added. The mixture was heated at $90{ }^{\circ} \mathrm{C}$ for $24 \mathrm{~h}$ under argon. The polymers were obtained $(0.320 \mathrm{~g}, 92.2 \%$ yield) as yellowishgreen powder. ${ }^{1} \mathrm{H}$ NMR (300 MHz, $\mathrm{CDCl}_{3}, \mathrm{ppm}$ ): $\delta$ 7.70-7.16 (aromatic $\left.\mathrm{CH}\right) 4.16\left(\mathrm{NH}_{2}\right.$ and $\mathrm{O}-\mathrm{CH}_{2}-\mathrm{C}=\mathrm{O}$ moiety from compound 2$), 3.75\left(\mathrm{O}-\mathrm{CH}_{2}\right.$ from compound 2$), 2.0\left(\alpha-\mathrm{CH}_{2}\right.$ 
of octyl group at 9-position of dioctylfluorene unit), $1.08\left(\mathrm{CH}_{2}\right.$ from dioctylfluorene and $\mathrm{CH}_{3}$ from compound 2), $0.74\left(\mathrm{CH}_{3}\right)$. IR ( $\mathrm{NaCl}$ plate, $\left.\mathrm{cm}^{-1}\right): 3025,2926,2853,1755$ (ester $\mathrm{C}=\mathrm{O}$ from compound 2), 1601, 1516.

Polymer P6. A solution of $\mathrm{LiOH}(0.020 \mathrm{~g}, 0.835 \mathrm{mmol})$ in methanol $(6 \mathrm{~mL})$ was added to a solution of polymer $\mathbf{P 2}(0.20 \mathrm{~g}, 0.273 \mathrm{mmol})$ in $\mathrm{CHCl}_{3}(20 \mathrm{~mL})$ and the mixture was refluxed overnight. Then the solvent was evaporated by reduced pressure. A concentrated $\mathrm{CHCl}_{3}$ solution was prepared and added dropwise into methanol. A greenish-yellow precipitate was collected by filtration $(0.190 \mathrm{~g}, 95.3 \%$ yield $)$ and dried in vacuum oven for $12 \mathrm{~h}$ at $60^{\circ} \mathrm{C}$; IR $\left(\mathrm{KBr}, \mathrm{cm}^{-1}\right): 3026,2926,2853,1597,1500,1465$

Polymer P7. A solution of $\mathrm{LiOH}(0.040 \mathrm{~g}, 1.67 \mathrm{mmol})$ in methanol $(6 \mathrm{~mL})$ was added to a solution of polymer $\mathbf{P 3}(0.150 \mathrm{~g}, 0.202 \mathrm{mmol})$ in $\mathrm{CHCl}_{3}(20 \mathrm{~mL})$ and the mixture was refluxed overnight. Polymer P7 was obtained (0.138 g, 92.6\% yield) as yellow powder. IR (KBr, cm $\left.{ }^{-1}\right): 3024,2926,2853,1597,1515,1465$.

Polymer P8. A solution of $\mathrm{LiOH}(0.060 \mathrm{~g}, 2.51 \mathrm{mmol})$ in methanol $(7 \mathrm{~mL})$ was added to a solution of polymer $\mathbf{P 4}(0.250 \mathrm{~g}, 0.331 \mathrm{mmol})$ in $\mathrm{CHCl}_{3}(20 \mathrm{~mL})$ and the mixture was refluxed overnight. Polymer P8 was obtained (0.235 g, 94.8\% yield) as yellowish-green powder. IR $\left(\mathrm{KBr}, \mathrm{cm}^{-1}\right):$ 3056, 3025, 2926, 2853, 1608, 1518, 1465.

Polymer P9. LiOH $(0.100 \mathrm{~g}, 2.51 \mathrm{mmol})$ in methanol $(7 \mathrm{~mL})$ was added to a solution of polymer P5 $(0.250 \mathrm{~g}, 0.325 \mathrm{mmol})$ in $\mathrm{CHCl}_{3}(20 \mathrm{~mL})$ and the mixture was refluxed 
overnight. The polymers were obtained $(0.230 \mathrm{~g}, 93.1 \%$ yield $)$ as yellowish-green powder. IR ( $\left.\mathrm{KBr}, \mathrm{cm}^{-1}\right):$ 3056, 3025, 2926, 2854, 1613, 1513, 1465.

\section{Preparation of Analyte Solution}

Stock aqueous solutions of $\mathrm{Pb}^{2+}, \mathrm{Cd}^{2+}, \mathrm{Co}^{2+}, \mathrm{Ni}^{2+}, \mathrm{Ca}^{2+}, \mathrm{Mg}^{2+}, \mathrm{Hg}^{2+}, \mathrm{Mn}^{2+}, \mathrm{Zn}^{2+}$, $\mathrm{Fe}^{3+}, \mathrm{Cu}^{2+}$ and $\mathrm{K}^{+}$were prepared using purified water. Stock solutions of the polymers were prepared using distilled THF. Polymer THF solutions were mixed with purified water under vigorous stirring to give water/THF mixture of different fraction $\left(f_{w}=0-95\right)$. Fluorescence turn-off detection of aqueous $\mathrm{Pb}^{2+}$ was carried out in $80-90 \%$ water mixture $\left(\mathrm{H}_{2} \mathrm{O} / \mathrm{THF}\right)$. For the fluorescence turn-on measurement, $1 \mathrm{~mL}$ of $\mathrm{Pb}^{2+}$ in water $\left(10^{-6}, 10^{-7}\right.$, $\left.10^{-8} \mathrm{M}\right)$ was added to $4 \mathrm{~mL}$ of $\mathbf{P 7}$ in THF directly with vigorous stirring to give $20 \%$ water mixture. 


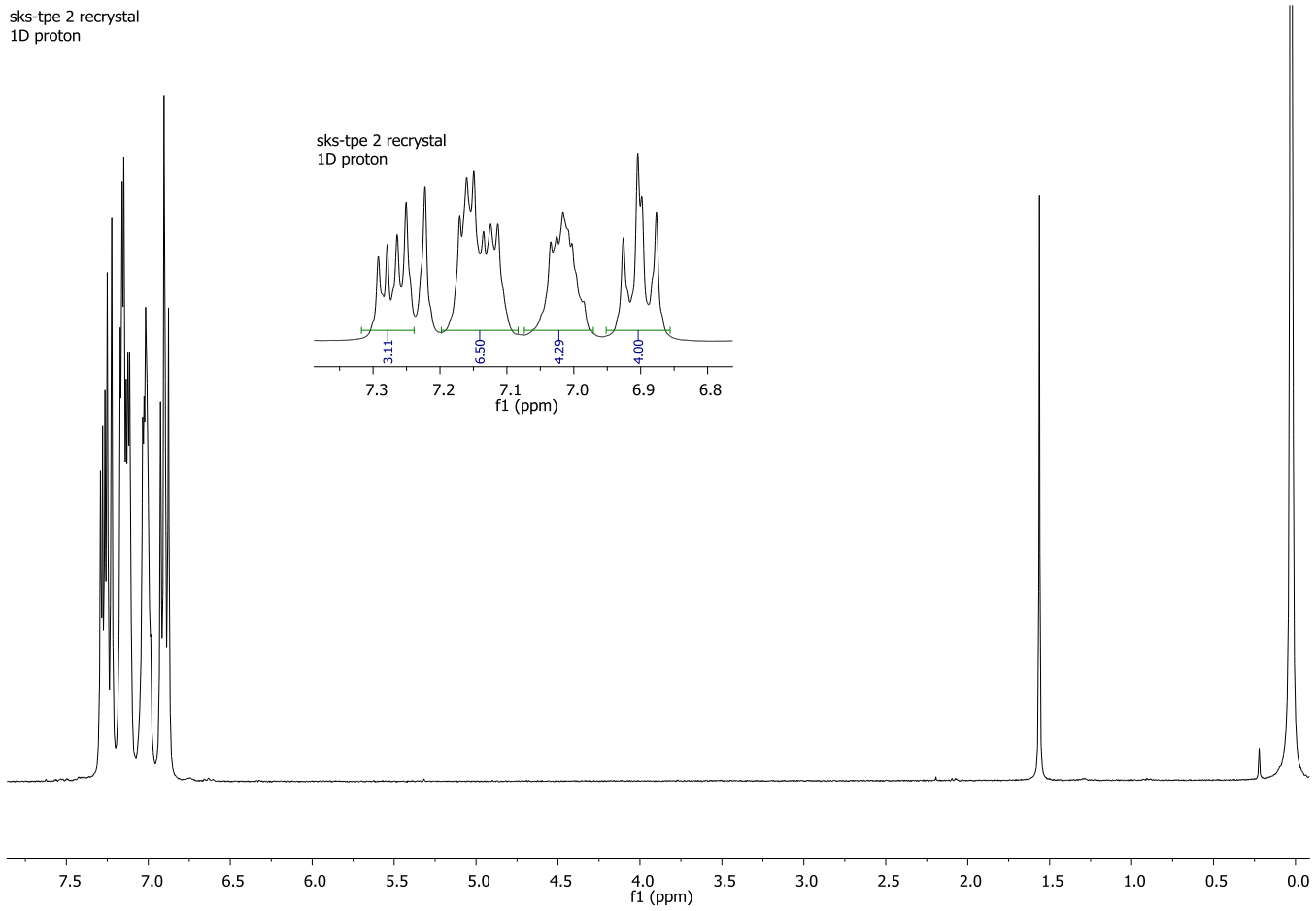

Figure S5.1 ${ }^{1} \mathrm{H}-\mathrm{NMR}$ spectrum $\left(300 \mathrm{MHz}, \mathrm{CDCl}_{3}\right)$ of compound $\mathbf{1}$.

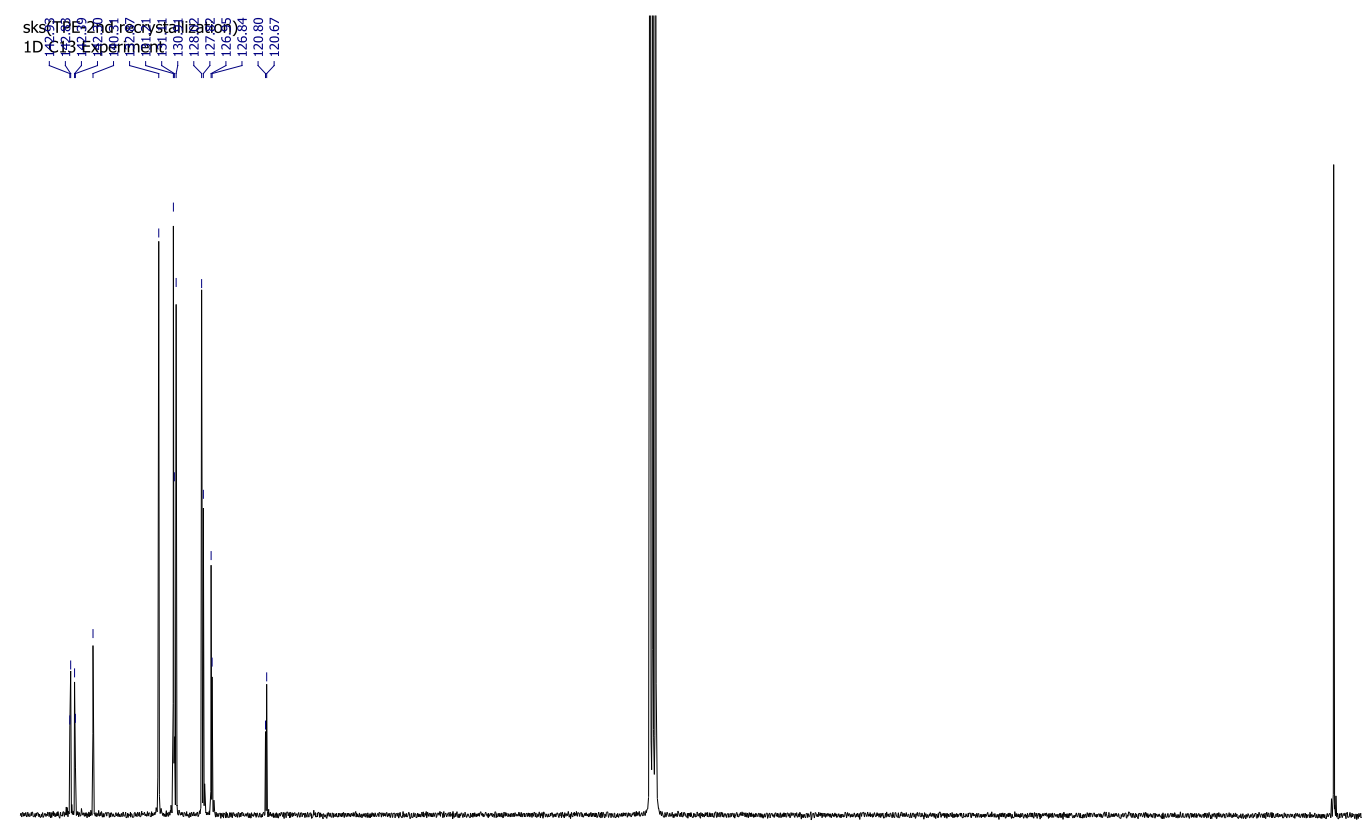

$\begin{array}{llllllllllllllllllllllllllllll}145 & 140 & 135 & 130 & 125 & 120 & 115 & 110 & 105 & 100 & 95 & 90 & 85 & 80 & 75 & 70 & 65 & 60 & 55 & 50 & 45 & 40 & 35 & 30 & 25 & 20 & 15 & 10 & 5 & 0\end{array}$

Figure S5.2 ${ }^{13} \mathrm{C}-\mathrm{NMR}$ spectrum $\left(100 \mathrm{MHz}, \mathrm{CDCl}_{3}\right)$ of compound $\mathbf{1}$ 


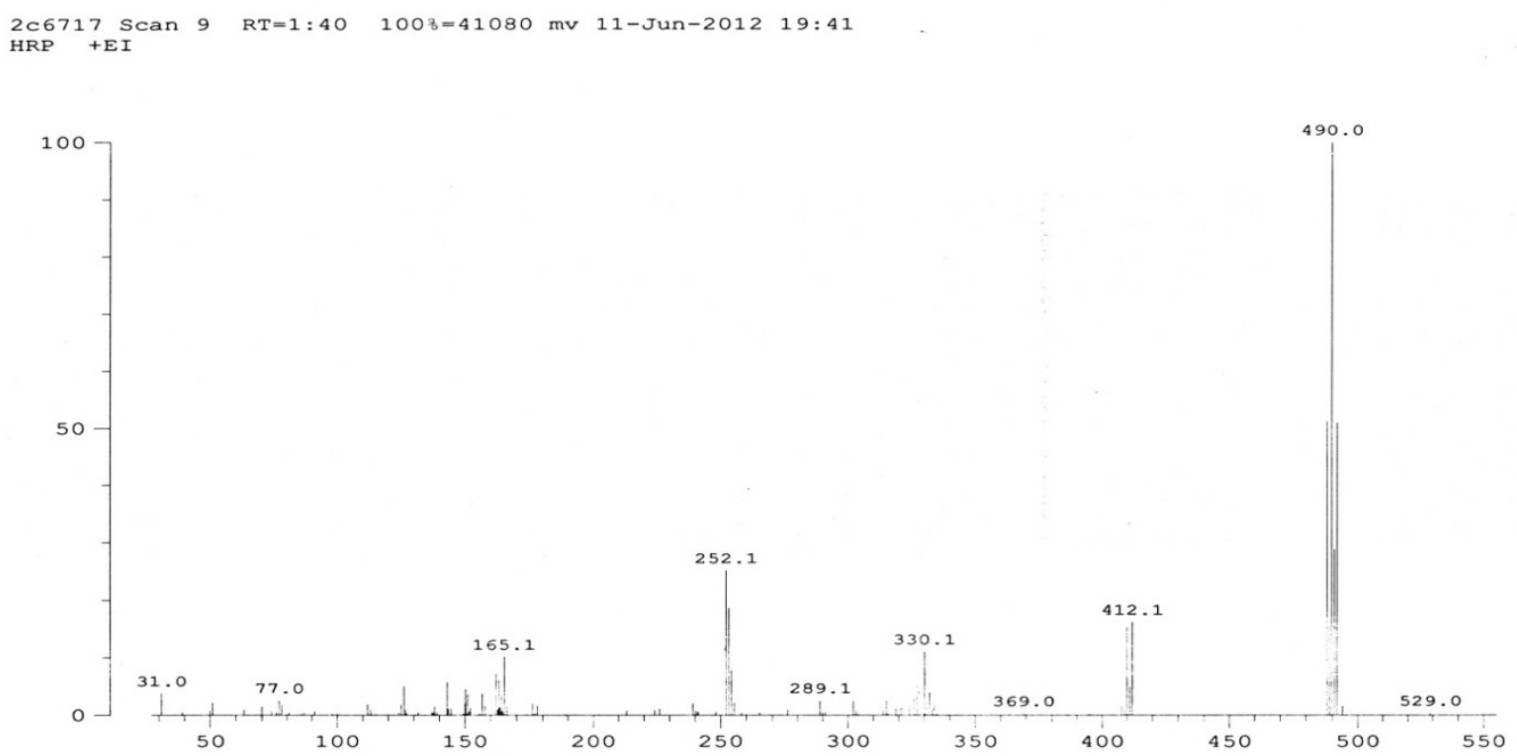

Figure S5.3 Mass spectrum of compound 1.

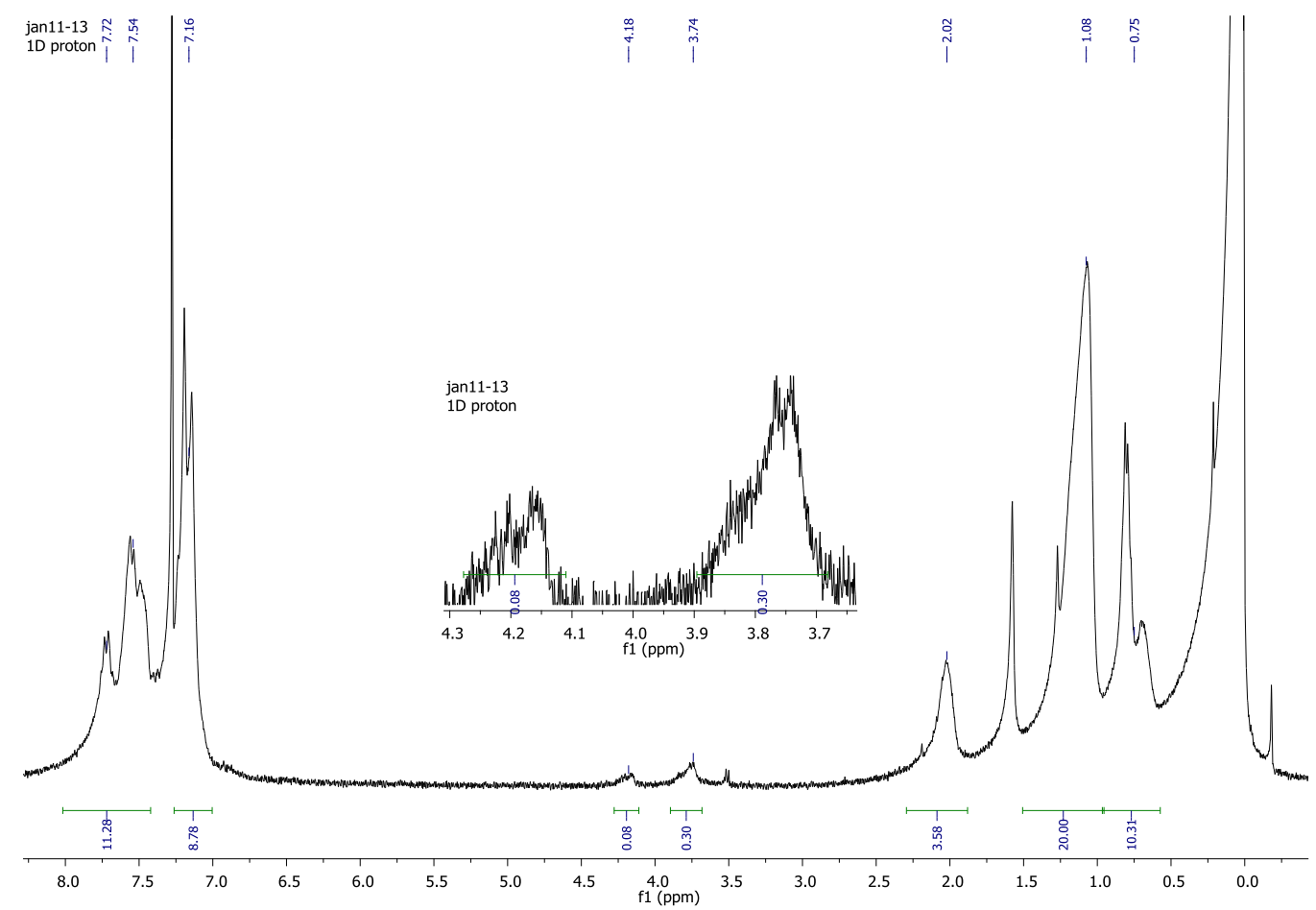

Figure S5.4 ${ }^{1} \mathrm{H}-\mathrm{NMR}$ spectrum $\left(300 \mathrm{MHz}, \mathrm{CDCl}_{3}\right)$ of polymer P2. 

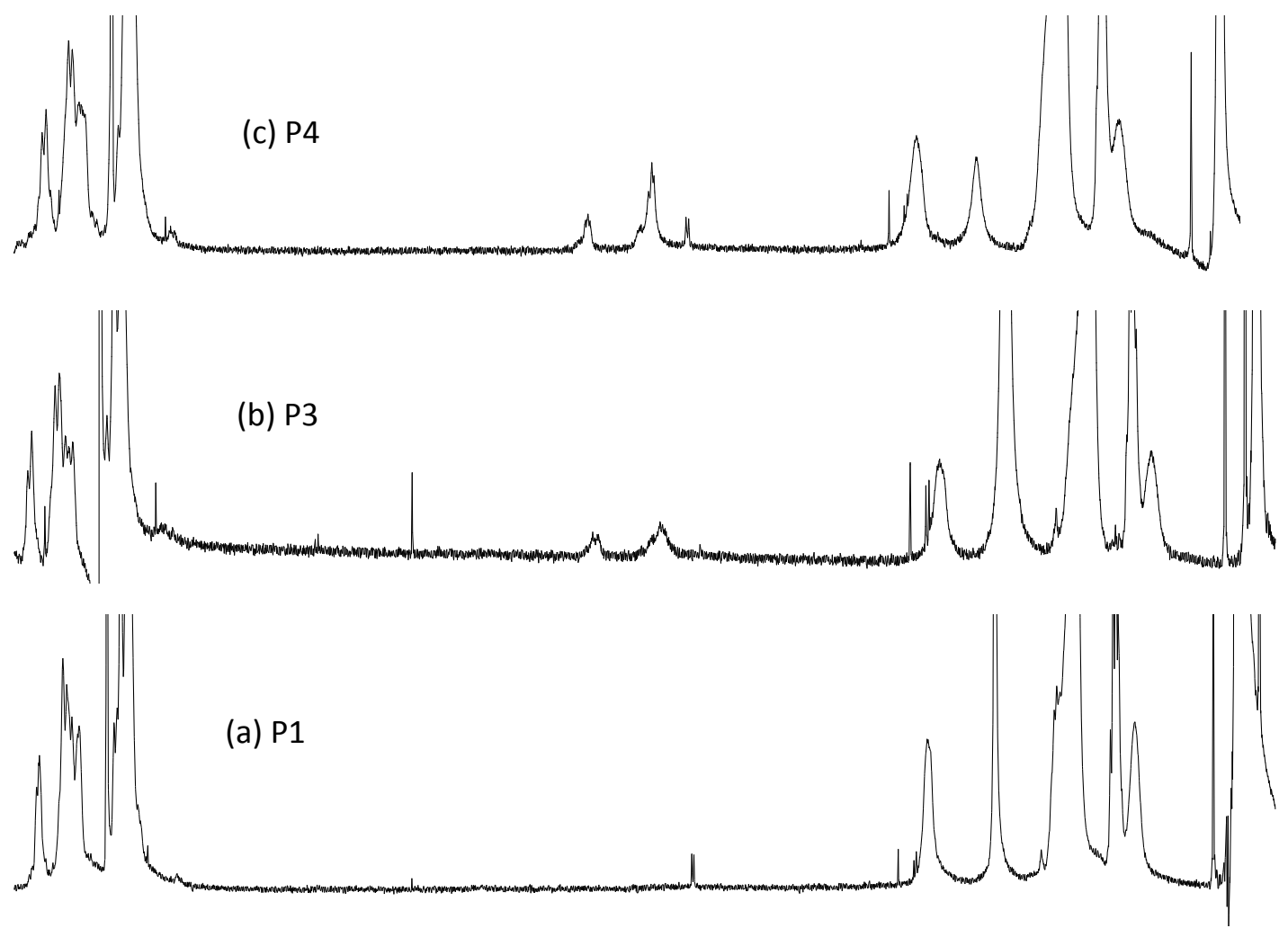

Figure S5.5 ${ }^{1} \mathrm{H}-\mathrm{NMR}\left(300 \mathrm{MHz}, \mathrm{CDCl}_{3}\right)$ spectra of polymers P1, P3, P4.

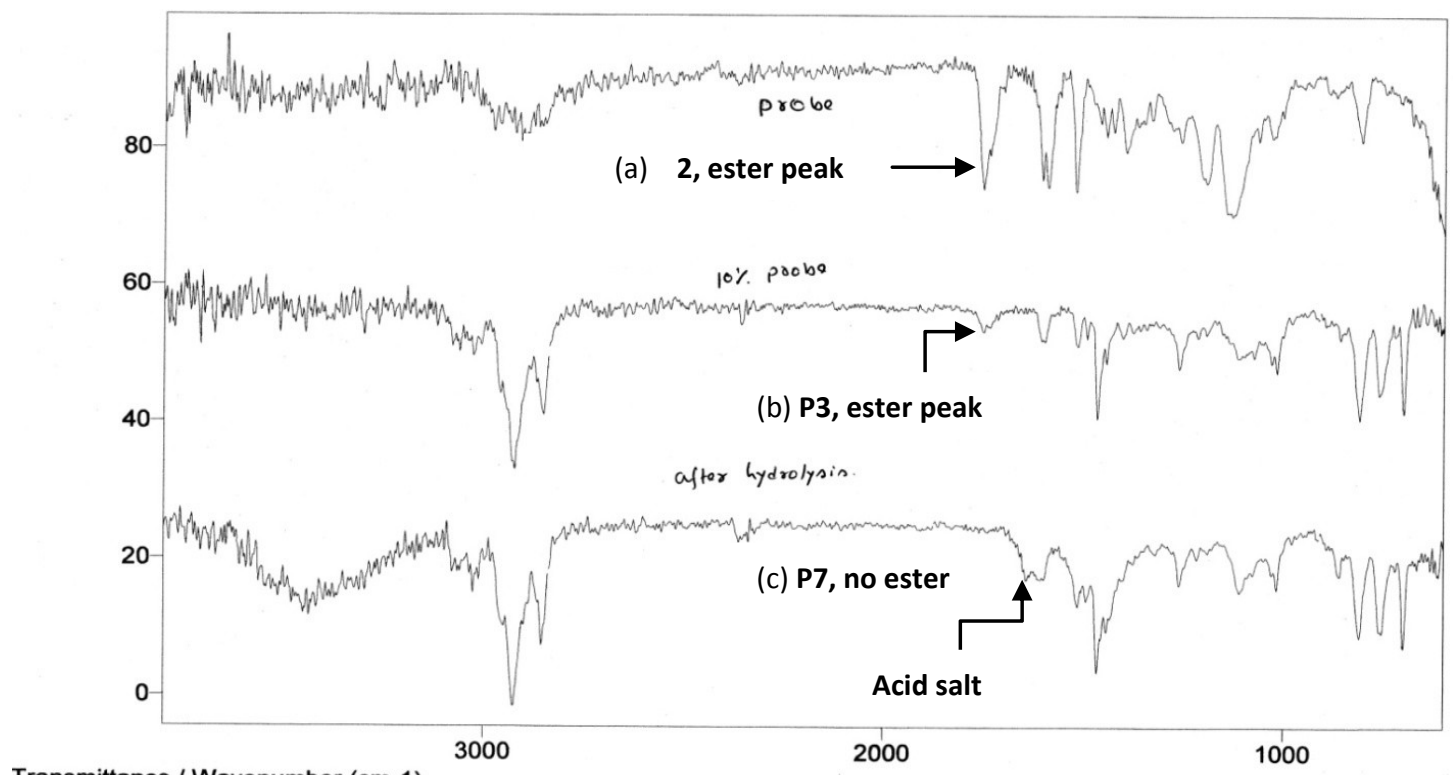

Figure S5.6 IR spectra of compound $\mathbf{2}$ and polymer $\mathbf{P 3}$ and $\mathbf{P} 7$ (on $\mathrm{NaCl}$ plate). 
Varian Resolutions

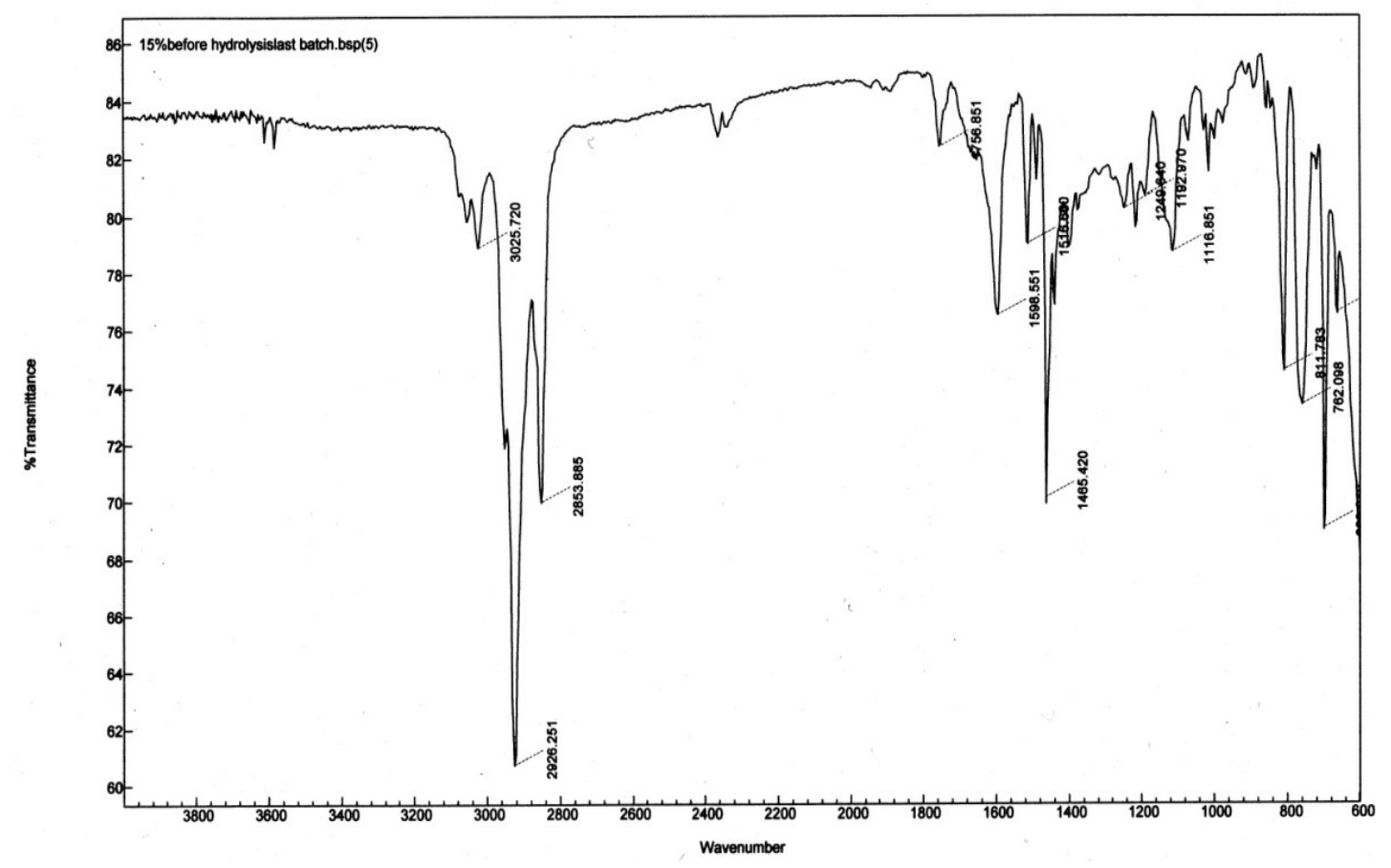

Figure S5.7 IR spectrum of polymer $\mathbf{P 4}$ (on $\mathrm{NaCl}$ plate).

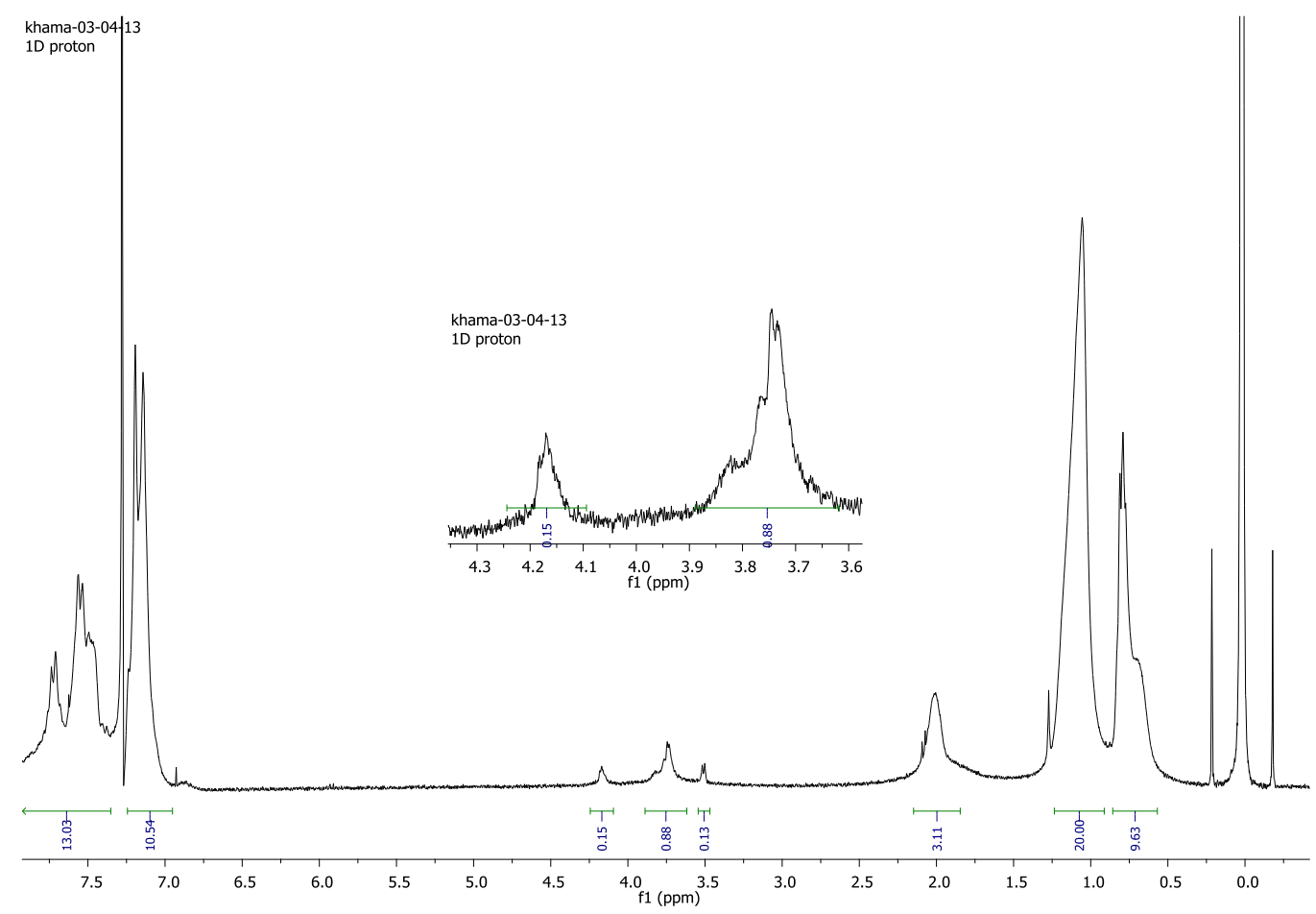

Figure S5.8 ${ }^{1} \mathrm{H}-\mathrm{NMR}$ spectrum $\left(300 \mathrm{MHz}, \mathrm{CDCl}_{3}\right)$ of polymer P5. 


\section{Varian Resolutions}

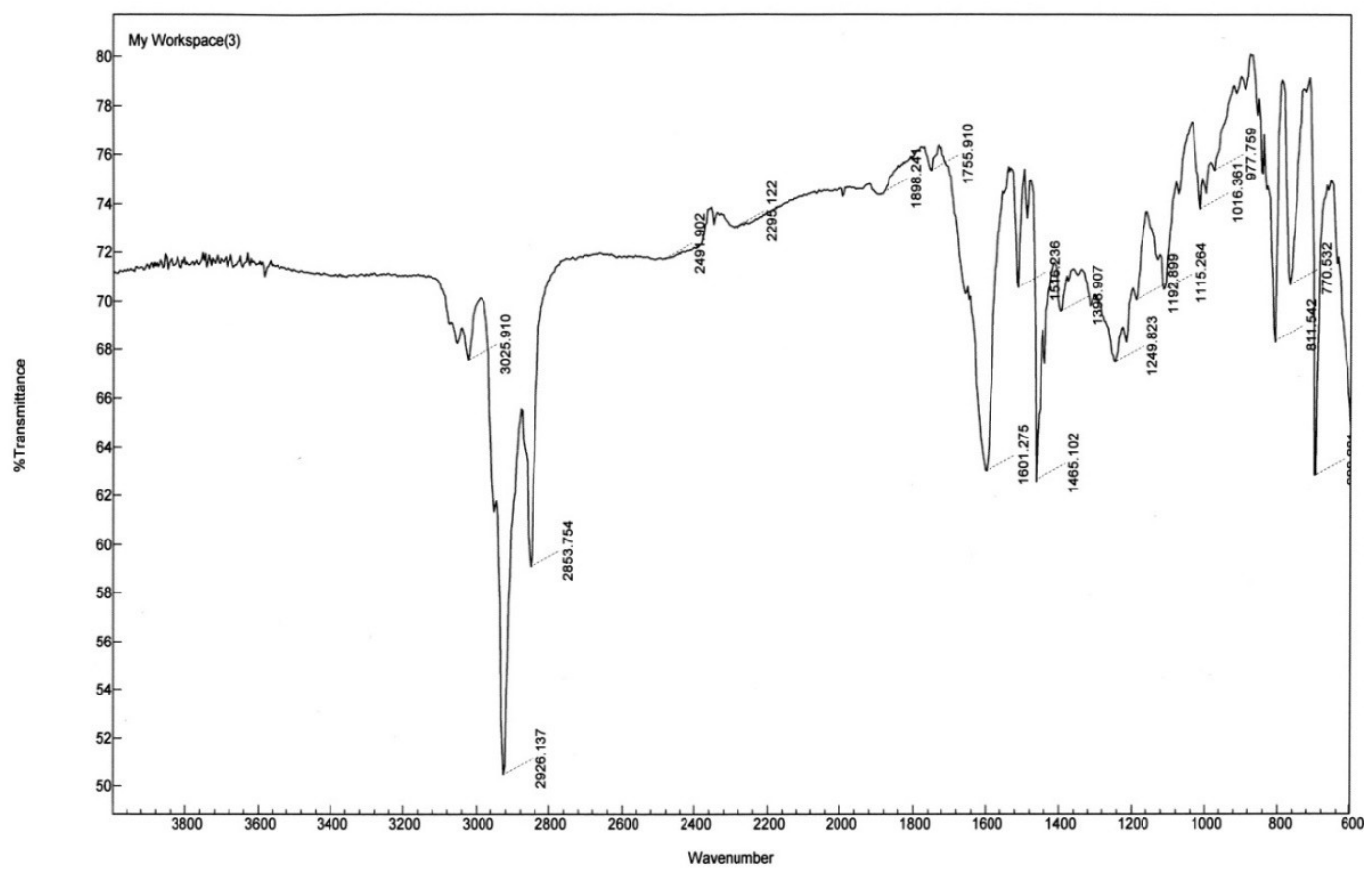

Figure S5.9 IR spectrum of polymer P5 (on NaCl plate).

Table S5.1 Molecular weight (g/mol) of polymers P2-P5.

\begin{tabular}{cccc}
\hline Polymer & Mw & Mn & PDI \\
\hline P2 & 9184 & 5643 & 1.6273 \\
P3 & 17394 & 9784 & 1.7777 \\
P4 & 11411 & 6030 & 1.8925 \\
P5 & 12471 & 6131 & 2.0340 \\
\hline
\end{tabular}




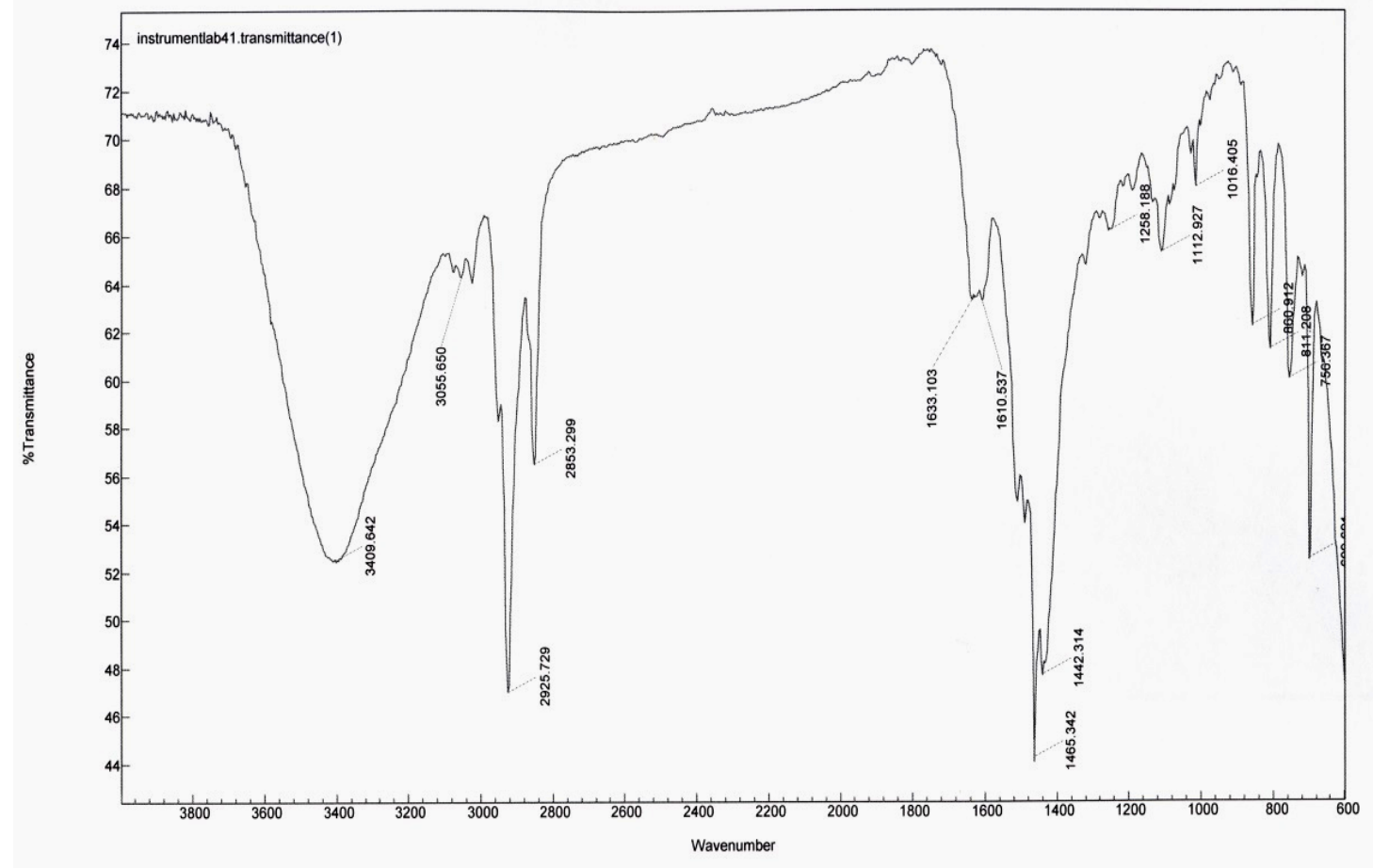

Figure S5.10 IR spectrum of polymer $\mathbf{P 8}$ (on $\mathrm{NaCl}$ plate).

\section{Varian Resolutions}

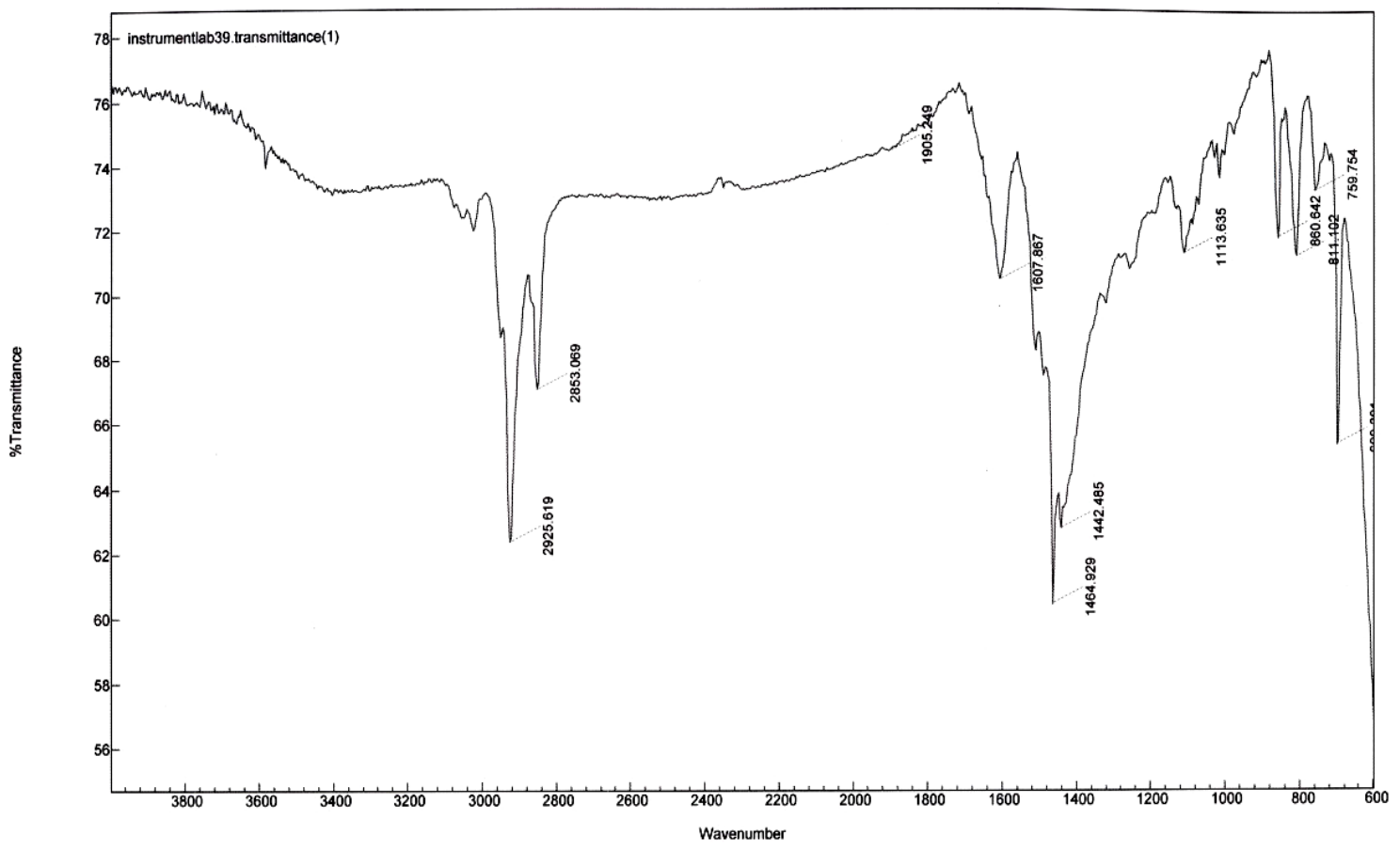

Figure. S5.11 IR spectrum of polymer $\mathbf{P 9}$ (on $\mathrm{NaCl}$ plate). 

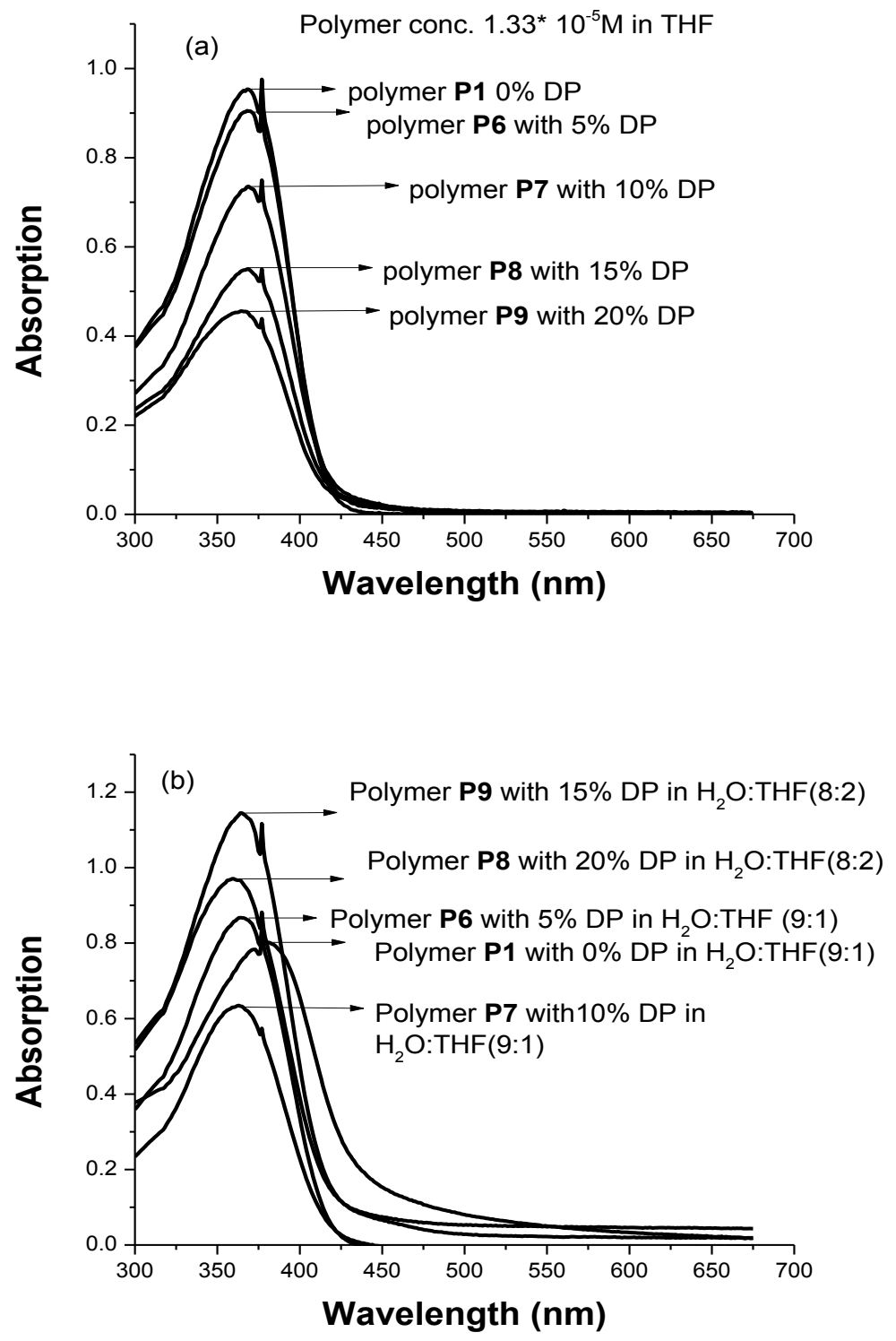


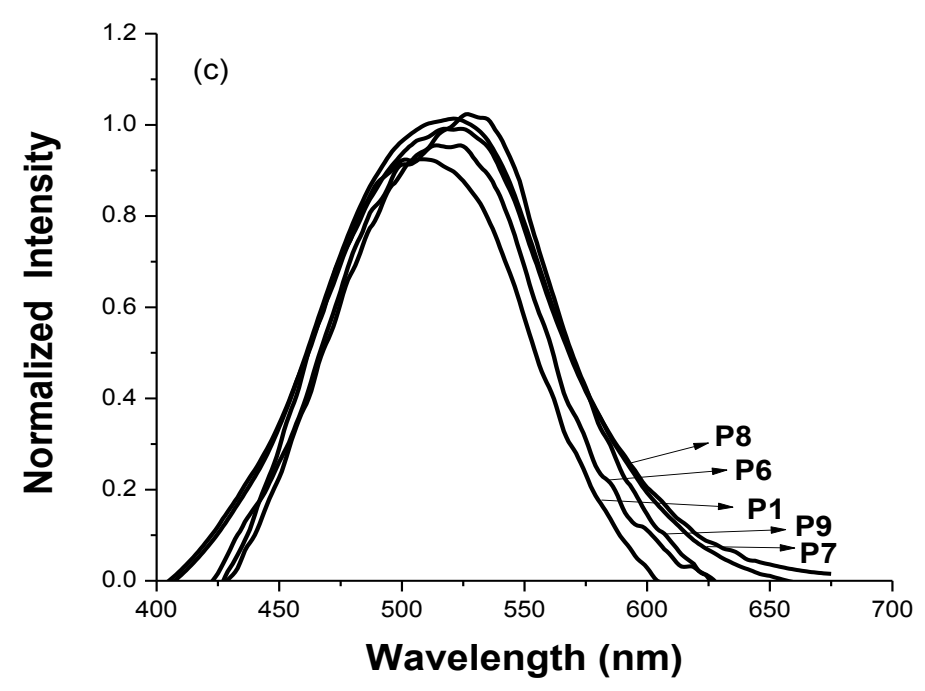

Figure S5.12 Absorption spectra of polymers (a) in THF and (b) in water/THF. Emission spectra of polymers (c) in THF.
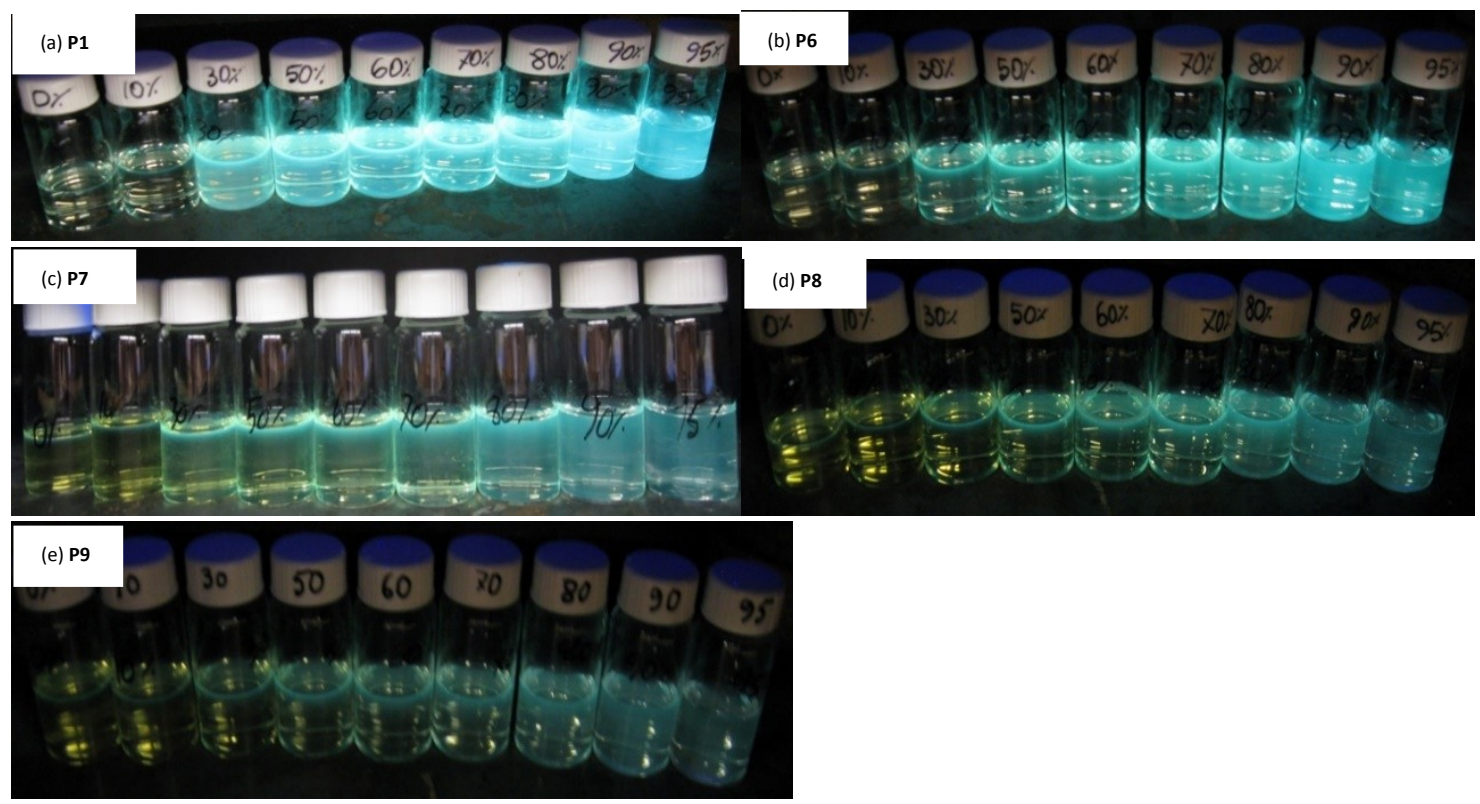

Figure S5.13 Photographs of polymers P1 and P6-P9 in water/THF (v/v) mixtures (from left to right $0,10,20,30,50,60,70,80,90,95 \%$ of water) taken under $365 \mathrm{~nm}$ illumination. 

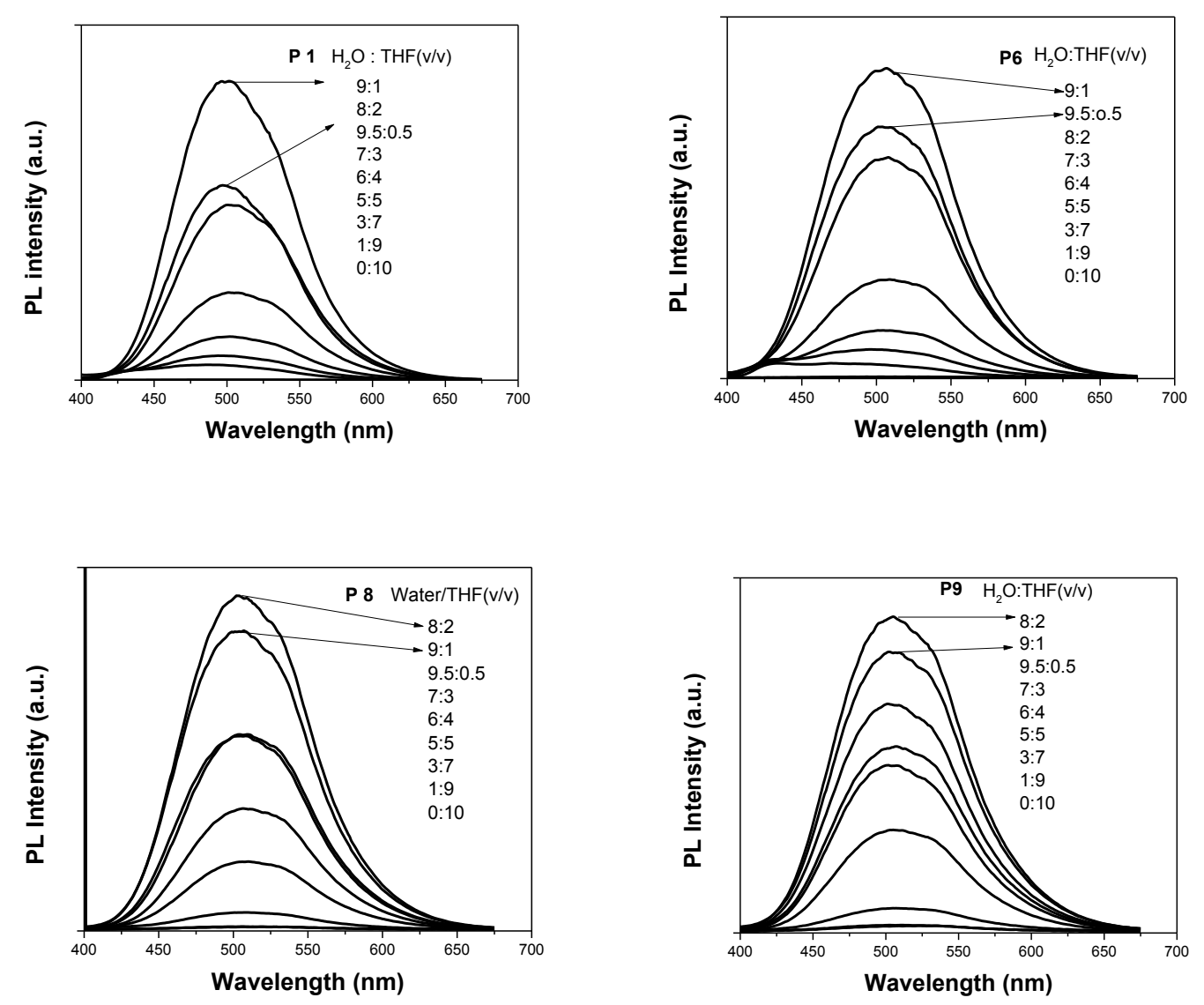

Figure S5.14 Emission spectra of polymers P1, P6 P8 and P9 in water/THF.
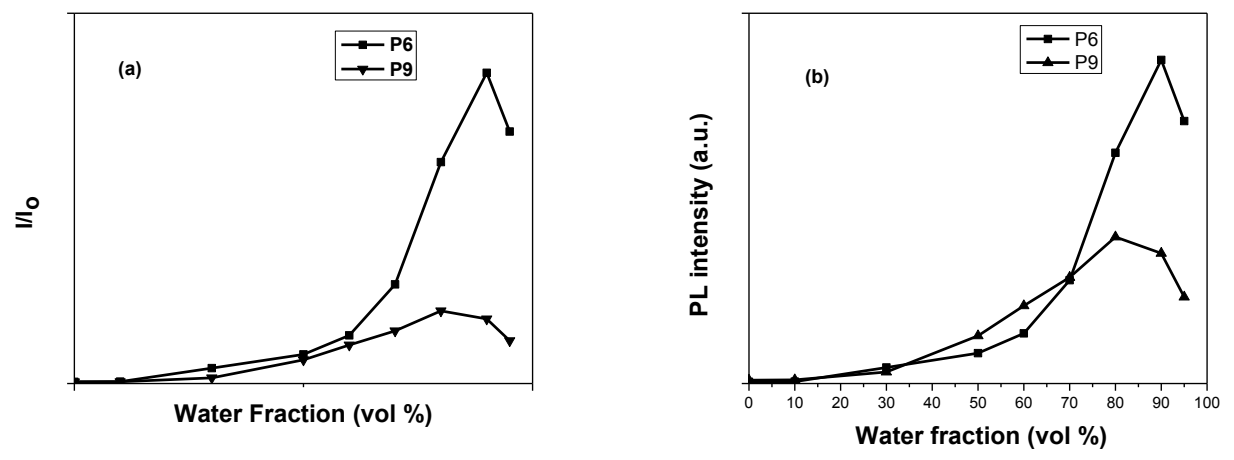

Figure S5.15 (a) Plot of (I/Io) values versus the compositions of the aqueous mixtures. $\mathrm{I}_{\mathrm{o}}$ is the emission intensity in pure THF solution. Excitation wavelength at $375 \mathrm{~nm}$. (b) PL intensity of polymers P6-P9 versus different water fraction. 


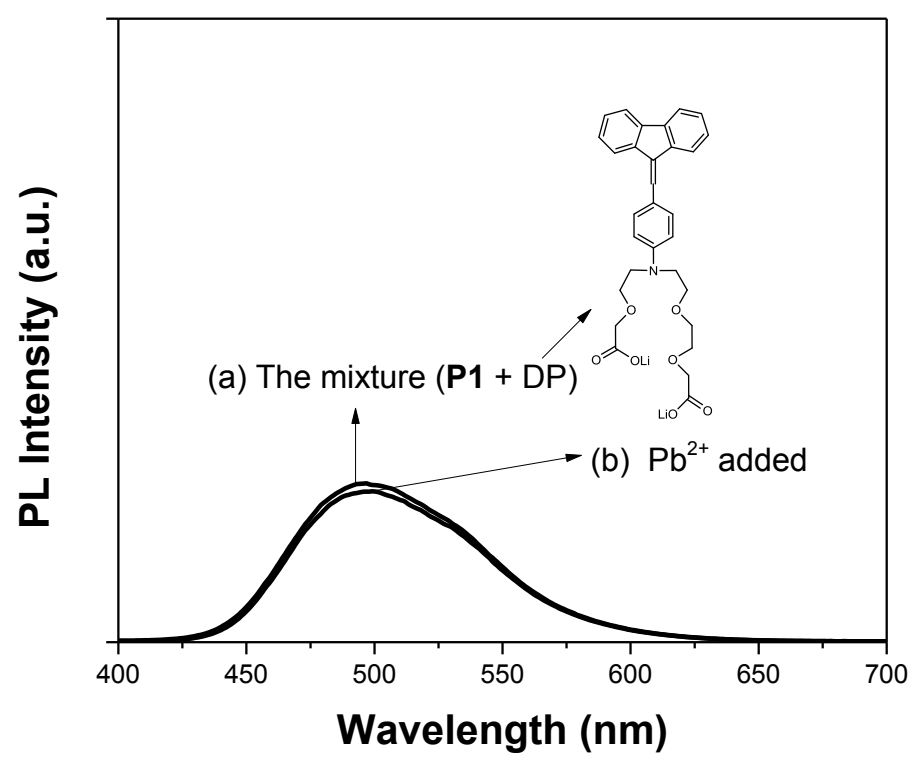

Figure S5.16 Emission spectra for a mixture of $\mathbf{P} \mathbf{1}$ and $\mathrm{DP}$ receptor upon addition of $\mathrm{Pb}^{2+}$ $\left(33 \times 10^{-6} \mathrm{M}\right)$

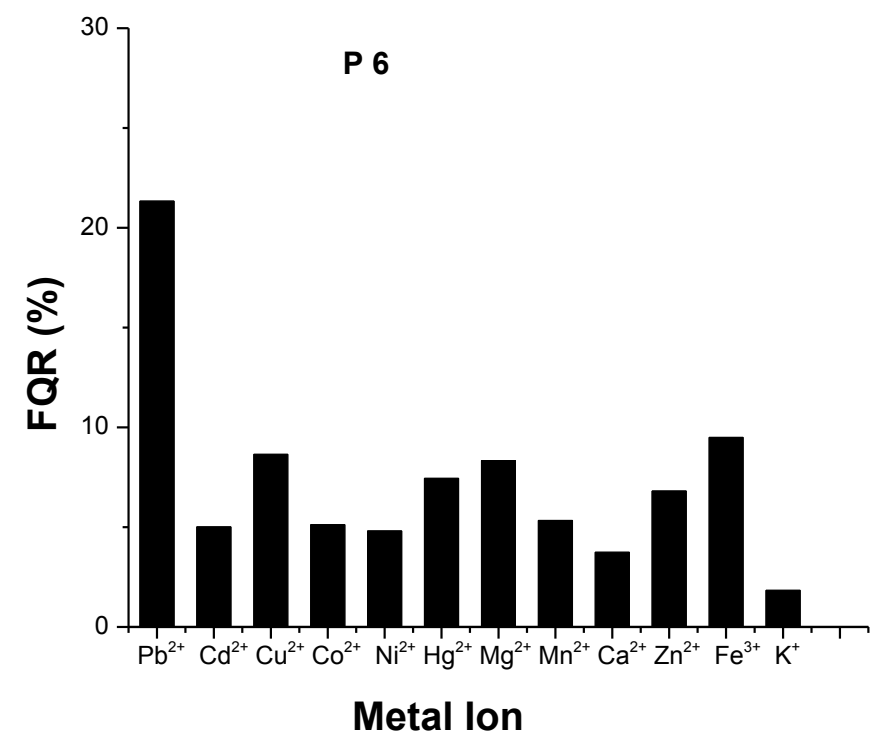



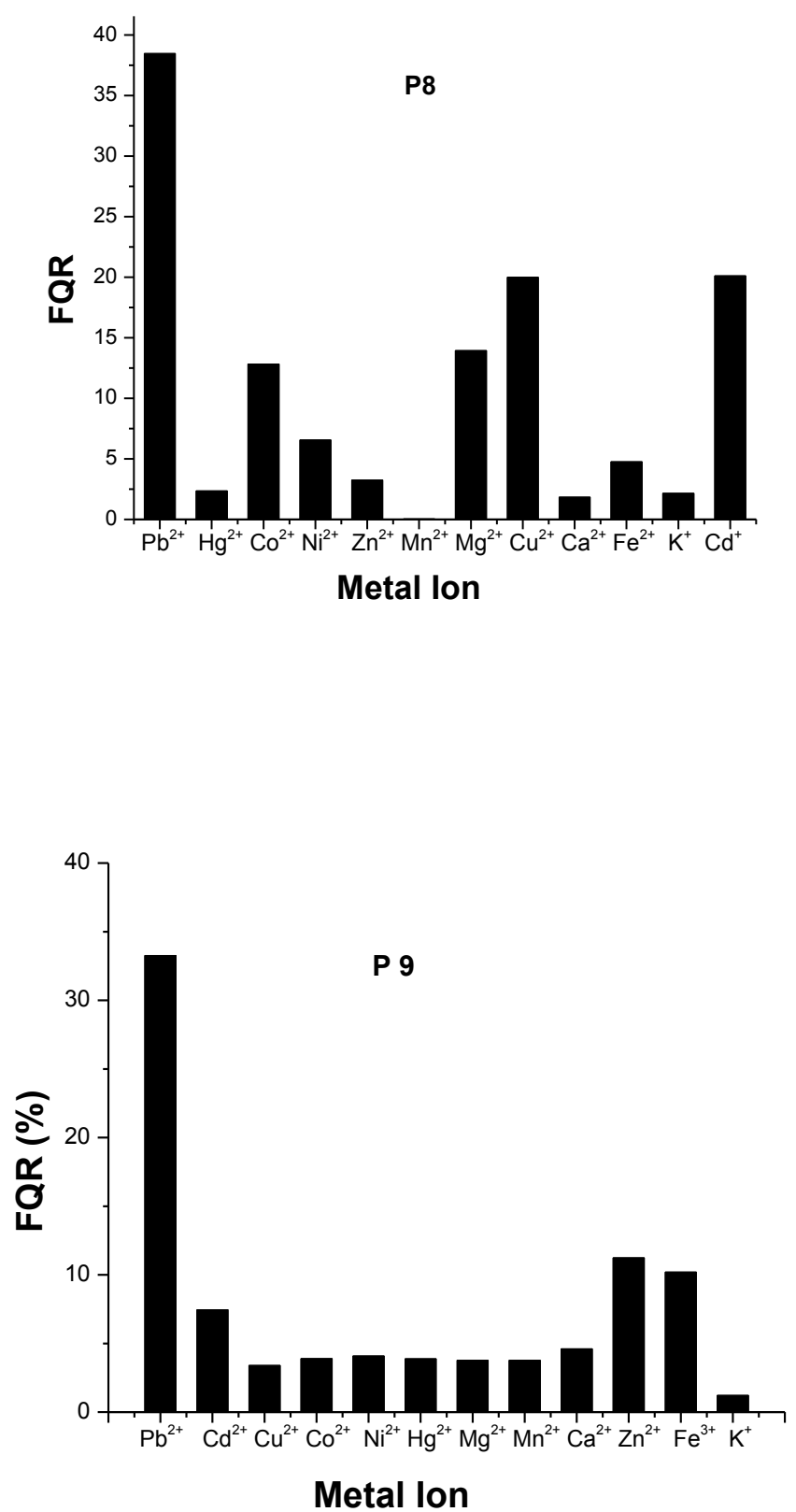

Figure S5.17 Relative selectivity of $\mathbf{P 6}$ and $\mathbf{P 9}$ towards different metal ions. 


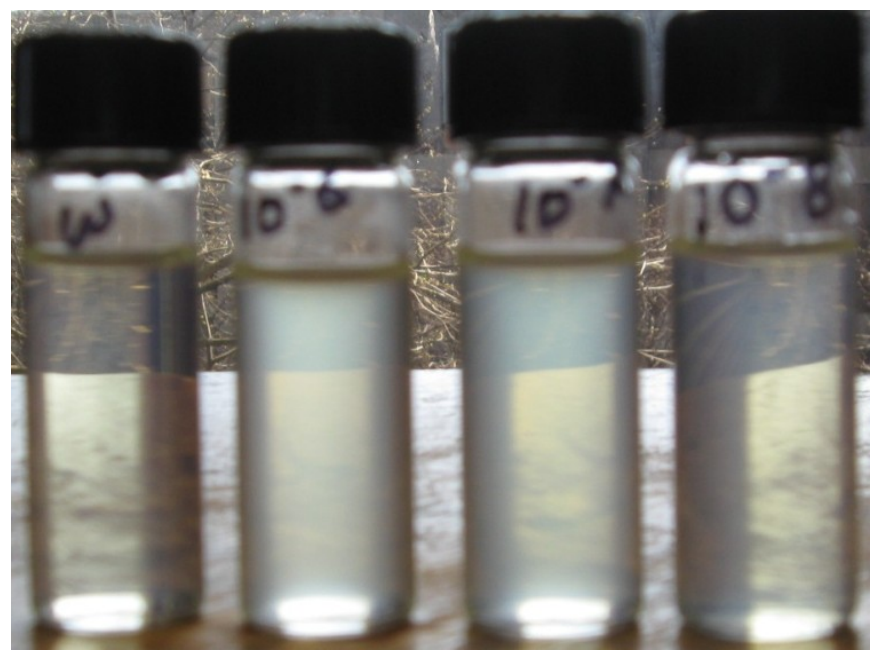

Figure S5.18 Photographs of polymer $\mathbf{P 7}$ in $20 \%$ water $/ \mathrm{THF}$ and with different $\mathrm{Pb}^{2+}$ concentrations $\left(10^{-6}, 10^{-7}, 10^{-8}\right)$ under sunlight. 